\title{
Assessment of the LV-S2 \& LV-S3 Stack Sampling Probe Locations for Compliance with ANSI/HPS N13.1-1999
}

\author{
JA Glissmeyer \\ EJ Antonio \\ JE Flaherty \\ BG Amidan
}

September 2014 


\title{
DISCLAIMER
}

This report was prepared as an account of work sponsored by an agency of the United States Government. Neither the United States Government nor any agency thereof, nor Battelle Memorial Institute, nor any of their employees, makes any warranty, express or implied, or assumes any legal liability or responsibility for the accuracy, completeness, or usefulness of any information, apparatus, product, or process disclosed, or represents that its use would not infringe privately owned rights. Reference herein to any specific commercial product, process, or service by trade name, trademark, manufacturer, or otherwise does not necessarily constitute or imply its endorsement, recommendation, or favoring by the United States Government or any agency thereof, or Battelle Memorial Institute. The views and opinions of authors expressed herein do not necessarily state or reflect those of the United States Government or any agency thereof.

\author{
PACIFIC NORTHWEST NATIONAL LABORATORY \\ operated by \\ BATTELLE \\ for the \\ UNITED STATES DEPARTMENT OF ENERGY \\ under Contract DE-AC05-76RL01830
}

Printed in the United States of America

Available to DOE and DOE contractors from the

Office of Scientific and Technical Information,

P.O. Box 62, Oak Ridge, TN 37831-0062;

ph: (865) 576-8401

fax: $(865)$ 576-5728

email: reports@adonis.osti.gov

Available to the public from the National Technical Information Service

5301 Shawnee Rd., Alexandria, VA 22312

ph: (800) 553-NTIS (6847)

email: orders/antis.gov <http://www.ntis.gov/about/form.aspx>

Online ordering: http://www.ntis.gov 
PNNL-23386

WTP-RPT-231, Rev. 0

\section{Assessment of the LV-S2 \& LV-S3 Stack Sampling Probe Locations for Compliance with ANSI/HPS N13.1-1999}

JA Glissmeyer

EJ Antonio

JE Flaherty

BG Amidan

September 2014

Test Specification:

N/A

Statement of Work

24590-QL-SRA-W000-00101

Work Authorization:

WA \#009

Test Plan:

TP-WTPSP-104, Rev 0.0

Test Exceptions:

N/A

Test Scoping Statement(s):

NA

QA Technology Level:

Developmental Work

Prepared for

the U.S. Department of Energy

under Contract DE-AC05-76RL01830 and

Subcontract Number 24590-QL-HC9-WA49-00001

Pacific Northwest National Laboratory

Richland, Washington 99352 

WTP-RPT-231

\section{Completeness of Testing}

This report describes the results of work and testing specified by test plan TP-WTPSP-104. The work and any associated testing followed the quality assurance requirements outlined in the test plan. The descriptions provided in this test report are an accurate account of both the conduct of the work and the data collected. Test plan results are reported. Also reported are any unusual or anomalous occurrences that are different from expected results. The test results and this report have been reviewed and verified.

\section{Approved:}

Reid A. Peterson, Manager

Date

WTP Support Project 



\section{Summary}

This document reports on a series of tests conducted to assess the proposed air sampling locations for the Hanford Tank Waste Treatment and Immobilization Plant (WTP) Group 1-2A exhaust stacks with respect to the applicable criteria regarding the placement of an air sampling probe. The LV-C2, LV-S2, and LV-S3 exhaust stacks were tested together as a group (Test Group 1-2A). This report only covers the results of LV-S2 and LV-S3; LV-C2 will be reported on separately. Federal regulations ${ }^{1}$ require that a sampling probe be located in the exhaust stack according to the criteria established by the American National Standards Institute/Health Physics Society (ANSI/HPS) N13.1-1999, Sampling and Monitoring Releases of Airborne Radioactive Substances from the Stack and Ducts of Nuclear Facilities. ${ }^{2}$ These criteria address the capability of the sampling probe to extract a sample that represents the effluent stream.

The testing on scale models of the stacks conducted for this project was part of the River Protection Project-Waste Treatment Plant Support Program under Contract No. DE-AC05-76RL01830 according to the statement of work issued by Bechtel National, Inc. (BNI, 24590-QL-SRA-W000-00101, N13.1-1999 Stack Monitor Scale Model Testing and Qualification, Revision 1, 9/12/2007) and Work Authorization 09 of Memorandum of Agreement 24590-QL-HC9-WA49-00001. The internal Pacific Northwest National Laboratory (PNNL) project for this task is 53024, Work for Hanford Contractors Stack Monitoring. The testing described in this document was further guided by the Test Plan Air Sampling Probe Location Tests for Waste Treatment Plant LAW LV-C2, LV-S2 and LV-S3 (Group 1-2A) Air Exhaust Systems (TP-WTPSP-104).

The tests conducted by PNNL during 2013 on the Group 1-2A scale model systems are described in this report. The series of tests consists of various measurements taken over a grid of points in the duct cross section at the designed sampling probe locations. The ANSI/HPS N13.1-1999 qualification criteria concern the following properties of the air flowing through the ducts where the air sampling probes are to be located:

1. Uniform Air Velocity-The gas momentum across the stack cross section where the sample is extracted should be well-mixed or uniform. The uniformity is expressed as the variability of the measurements about the mean, expressed as the percent coefficient of variance (\%COV). It is calculated as the standard deviation divided by the mean and expressed as a percentage - the lower the \%COV value, the more uniform the velocity.

2. Angular Flow - The purpose of this test is to determine whether the air velocity vector is aligned with the sampling nozzle.

3. Uniform Concentration of Tracer Gases-A uniform contaminant concentration in the sampling plane enables the extraction of samples that represent the true concentration.

\footnotetext{
${ }^{1}$ Title 40 of the Code of Federal Regulations (CFR), Part 61, National Emissions Standards for Hazardous Air Pollutants (NESHAP), Subpart H, National Emission Standard for Emissions of Radionuclides Other Than Radon From Department of Energy Facilities.

${ }^{2}$ Health Physics Society, McLean, VA 22101. The standard has been reaffirmed in 2011 and is identical to the 1999 version. The regulations have not been updated yet, so the 1999 version is still referenced.
} 
4. Uniform Concentration of Tracer Particles-Uniformity in contaminant concentration at the sampling probe was further demonstrated using tracer particles large enough to exhibit inertial effects.

Particles of $10-\mu \mathrm{m}$ aerodynamic diameter were used.

The LV-S2 stack will have one sampling location, while the LV-S3 stack will have two sampling locations. The test results for the successfully-tested configurations are summarized in Table S.1. The details of the successful configurations are given in the report.

Table S.1. Summary of Sampling Probe Location Results for the LV-S2 and LV-S3 Scale Model Stacks

\begin{tabular}{|c|c|c|c|c|c|}
\hline & \multirow{2}{*}{$\begin{array}{l}\text { Acceptance } \\
\text { Criteria }\end{array}$} & \multirow{2}{*}{ Units } & \multirow{2}{*}{$\frac{\text { LV-S2 }}{\text { Test Port } 1}$} & \multicolumn{2}{|c|}{ LV-S3 } \\
\hline & & & & Test Port 1 & Test Port 2 \\
\hline $\begin{array}{l}\text { Velocity } \\
\text { Uniformity }\end{array}$ & $\leq 20$ & $\% \mathrm{COV}$ & $4.0-5.5$ & $5.1-8.6$ & $5.8-9.3$ \\
\hline Flow Angle & $\leq 20$ & Degrees & $3.1-5.3$ & $2.2-10.7$ & $2.1-10.3$ \\
\hline Gas Tracer & $\leq 20$ & $\% \mathrm{COV}$ & $0.2-8.3$ & $0.2-1.8$ & $0.2-1.3$ \\
\hline Uniformity & $\leq 30$ & $\begin{array}{l}\text { Maximum \% } \\
\text { Deviation from Mean }\end{array}$ & $0.6-19.7$ & $0.4-3.9$ & $0.4-1.7$ \\
\hline $\begin{array}{l}\text { Particle Tracer } \\
\text { Uniformity }\end{array}$ & $\leq 20$ & Normalized \%COV & $5.2-19.2$ & $3.8-11.0$ & $4.0-11.0$ \\
\hline
\end{tabular}

Based on these scale model tests, the locations proposed for the air sampling probes in each of the scale model stacks meet the requirements of the ANSI/HPS N13.1-1999 standard for velocity uniformity, flow angle, gas tracer and particle tracer uniformity. Additional velocity uniformity and flow angle tests on the actual stacks will be necessary during cold startup to confirm the validity of the scale model results in representing the actual stacks. In particular, the velocity uniformity test results for the actual stacks must be within $5 \% \mathrm{COV}$ of the range of results listed for the scale model so that scale model results can be said to be representative of the stack. If the velocity uniformity results on the actual stack fall within these bounds, and flow angle test results fall within qualification criteria (mean angle $\leq 20^{\circ}$ ) the scale model results may be used as a substitute for results from the actual stack. 


\section{Quality Assurance}

The PNNL quality assurance (QA) program is based on the requirements defined in the U.S. Department of Energy Order 414.1D, Quality Assurance, and 10 CFR 830, Energy/Nuclear Safety Management, and Subpart A-Quality Assurance Requirements (a.k.a. the Quality Rule). PNNL has chosen to implement the following consensus standards in a graded approach:

- ASME NQA-1-2000, Quality Assurance Requirements for Nuclear Facility Applications, Part I, "Requirements for Quality Assurance Programs for Nuclear Facilities."

- ASME NQA-1-2000, Part II, Subpart 2.7, Quality Assurance Requirements for Computer Software for Nuclear Facility Applications.

- ASME NQA-1-2000, Part IV, Subpart 4.2, Guidance on Graded Application of Quality Assurance (QA) Requirements for Nuclear-Related Research and Development.

The procedures necessary to implement the requirements are documented through PNNL's "How Do I...?” (HDI), which is a system for managing the delivery of laboratory-level policies, requirements, and procedures.

The Waste Treatment Plant Support Program (WTPSP) implements an NQA-1-2000 QA program, using a graded approach as presented in NQA-1-2000, Part IV, Subpart 4.2. The WTPSP Quality Assurance manual (QA-WTPSP-0002) describes the technology life cycle stages under the WTPSP QA plan (QA-WTPSP-0001). The technology life cycle includes the progression of technology development, commercialization, and retirement in process phases of basic and applied research and development (R\&D), engineering and production, and operation until process completion. The life cycle is characterized by flexible and informal QA activities in basic research, which becomes more structured and formalized through the applied R\&D stages. The work described in this report has been completed under the QA Technology level of Developmental Work as the data will be used for applying for air discharge permits.

- DEVELOPMENTAL WORK-Developmental work consists of research tasks moving toward technology commercialization. These tasks still require a degree of flexibility, and there is still a degree of uncertainty that exists in many cases. The role of quality on developmental work is to make sure that adequate controls exist to support movement into commercialization.

WTPSP addresses internal verification and validation activities by conducting an Independent Technical Review of the final data report in accordance with WTPSP's procedure QA-WTPSP-0601, Document Preparation and Change. This review verifies that the reported results are traceable, that inferences and conclusions are soundly based, and the reported work satisfies the test plan objectives. Appendix A lists the reviewed test plan, test instructions, and calculation packages used for the tests documented in this report. 



\section{Acronyms}

\begin{tabular}{|c|c|}
\hline acfm & actual cubic feet per minute \\
\hline $\mathrm{AD}$ & aerodynamic diameter \\
\hline afpm & actual feet per minute \\
\hline ANOVA & Analysis of Variance \\
\hline ANSI & American National Standards Institute \\
\hline Atm & Atmosphere \\
\hline $\mathrm{AOV}$ & Analysis of Variance \\
\hline ASME & American Society of Mechanical Engineers \\
\hline BNI & Bechtel National, Inc. \\
\hline $\mathrm{C} 2 \mathrm{~V}$ & $\mathrm{C} 2 \mathrm{~V}$ ventilation system \\
\hline C5V & C5V ventilation system \\
\hline CAM & continuous air monitor \\
\hline СCP & computer-assisted calculation package \\
\hline CFR & Code of Federal Regulations \\
\hline $\mathrm{CO}_{2}$ & carbon dioxide \\
\hline $\mathrm{COV}$ & Coefficient of variance \\
\hline DOE & U.S. Department of Energy \\
\hline DV & hydraulic diameter $\times$ mean velocity \\
\hline EPA & U.S. Environmental Protection Agency \\
\hline FA & flow angle test \\
\hline FC & flow control test \\
\hline $\mathrm{ft}$ & feet \\
\hline GE & Gas equivalency \\
\hline GT & gaseous tracer test \\
\hline $\mathrm{H}_{2} \mathrm{O}$ & water \\
\hline HDI & “How Do I...?” \\
\hline HEPA & high-efficiency particulate air (filter) \\
\hline HPS & Health Physics Society \\
\hline $\mathrm{Hz}$ & Hertz \\
\hline LAW & low-activity waste \\
\hline LVP & LAW secondary offgas / vessel vent process system \\
\hline LV-C2 & low-activity waste C2V ventilation system \\
\hline LV-S2 & low-activity waste C5V ventilation system \\
\hline LV-S3 & low-activity waste melter offgas emission unit \\
\hline MaxDev & Maximum Deviation \\
\hline $\min$ & minute \\
\hline
\end{tabular}


MS

$\mathrm{N}_{2} \mathrm{O}$

NESHAP

OPC

$\% \mathrm{COV}$

PNNL

PT

PVC

QA

$\mathrm{R} \& \mathrm{D}$

RSD

scfm

$\mathrm{SF}_{6}$

sfpm

TI

VT

WTP

WTPSP
Mean Square

nitrous oxide

National Emissions Standards for Hazardous Air Pollutants

optical particle counter

percent coefficient of variation

Pacific Northwest National Laboratory

particulate tracer test

polyvinyl chloride

quality assurance

research and development

relative standard deviation

standard cubic feet per minute

sulfur hexafluoride

standard feet per minute

test instruction

velocity uniformity test

Hanford Tank Waste Treatment and Immobilization Plant

Waste Treatment Plant Support Program 


\section{Acknowledgments}

Preparing, executing, and post-processing these scale model measurements involved a number of Pacific Northwest National Laboratory staff. We would like to particularly acknowledge the support of our quality engineer, Kirsten Meier, and the administrative support from Chrissy Charron and Mona Champion. We would also like to express our appreciation to scientific staff members Carmen Arimescu, Carolyn Burns, Tristan Hay, Susan Sande, Sandra Snyder, and Yin-Fong Su, who conducted measurements under a variety of weather conditions. Additionally, Matthew Barnett, Carmen Arimescu, and Elizabeth Golovich provided technical reviews. Megan Peters provided editorial support for this report.

Funding for this effort was provided by the Department of Energy's Waste Treatment and Immobilization Plant Project through a subcontract with Bechtel National, Inc. Pacific Northwest National Laboratory is operated for the U.S. Department of Energy by Battelle under Contract DE-AC05-76RL01830. 



\section{Contents}

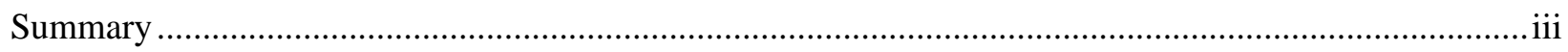

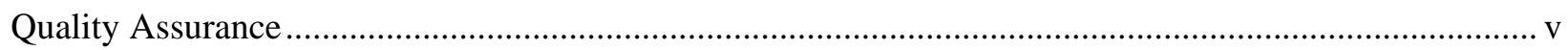

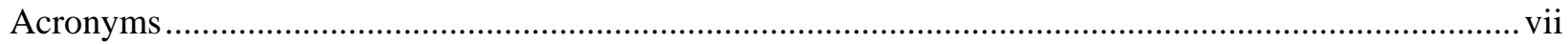

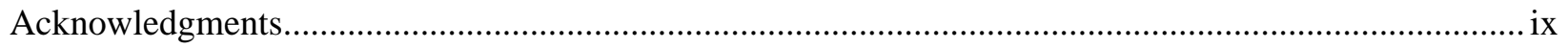

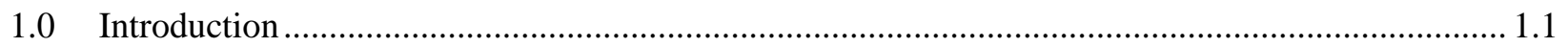

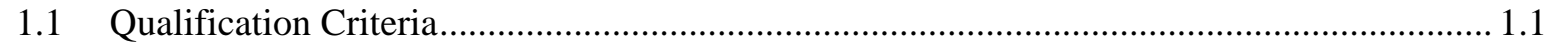

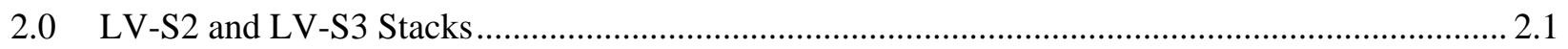

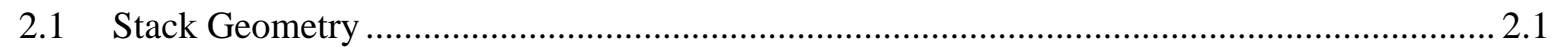

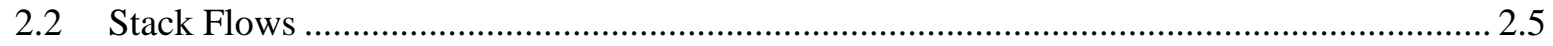

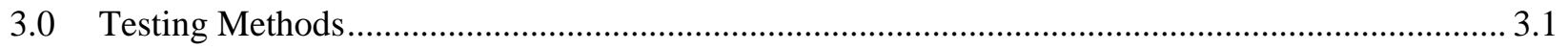

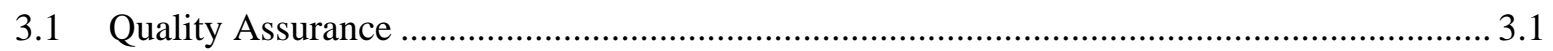

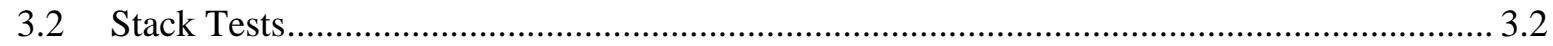

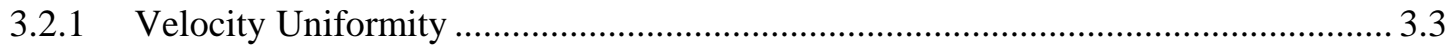

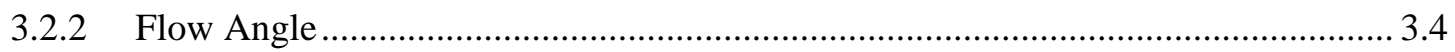

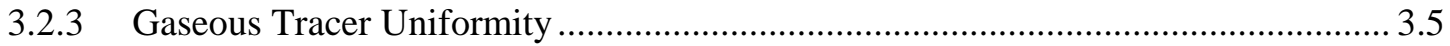

3.2.4 Particle Tracer Uniformity .................................................................................. 3.9

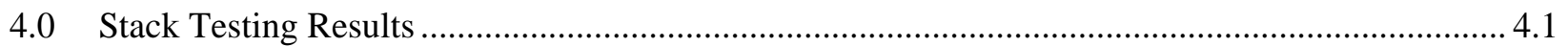

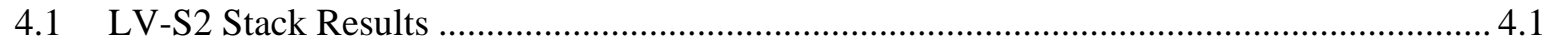

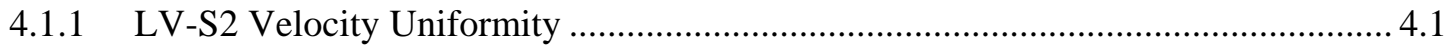

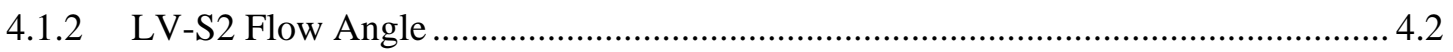

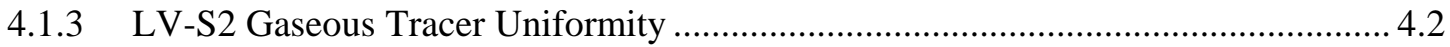

4.1.4 LV-S2 Particle Tracer Uniformity …….............................................................. 4.5

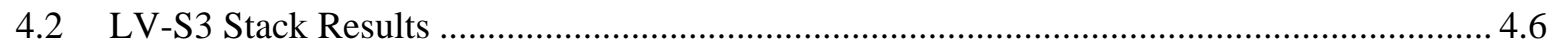

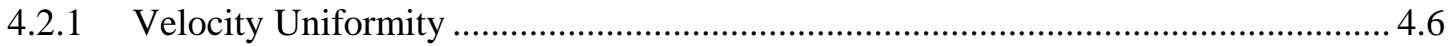

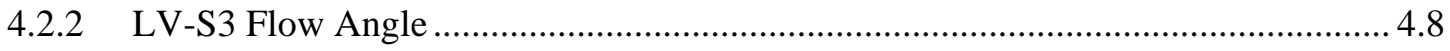

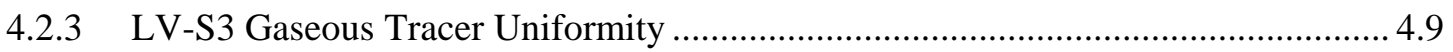

4.2.4 LV-S3 Particle Tracer Uniformity ……............................................................... 4.11

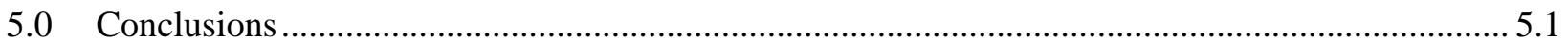

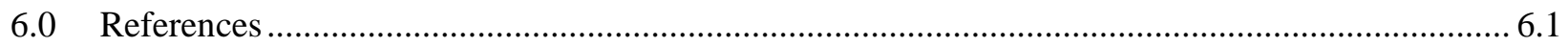

Appendix A: LV-S2 and LV-S3 Scale Model Testing Supporting Documents List ............................. A.1

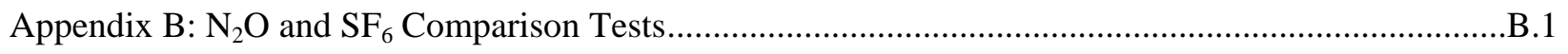

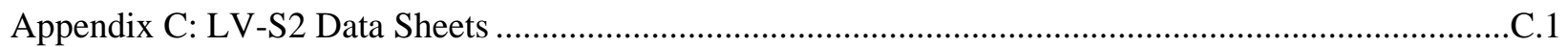

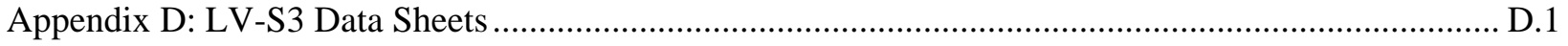




\section{Figures}

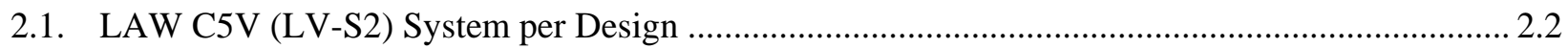

2.2. LAW LVP (LV-S3) System per Design .................................................................................... 2.3

2.3. Layout of the LAW C5V (LV-S2) Scale Model Test System ...................................................... 2.4

2.4. Layout of LAW LVP (LV-S3) Scale Model Test System .......................................................... 2.4

3.1. Cross Section of the Duct at the Testing Ports with Measurement Points ...................................... 3.3

3.2. Equipment Used for the Velocity Uniformity Test ......................................................................... 3.4

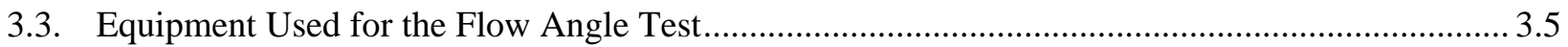

3.4. Equipment Used for the Gaseous Tracer Injection, Injection Probe Installed in the LV-S2 Scale Model, and Cylinders of Pure $\mathrm{N}_{2} \mathrm{O}$ with Heater and Regulator............................................ 3.6

3.5. Illustration of Five Injection Points in a Circular Duct............................................................... 3.7

3.6. Illustration of Five Injection Points in a Rectangular Duct......................................................... 3.7

3.7. Equipment Used for the Gaseous Tracer Sampling ................................................................ 3.8

3.8. Equipment Used for Particle Injection (LV-S3). ..................................................................... 3.9

3.9. Particle Counters Used for the Particle Sampling ......................................................................... 3.10

4.1. Measurement and Reference Particle Test Data from PT-32 on the LV-S3 Scale Model Stack.

4.2. Linear Regression Results of Average Velocity Versus \%COV for Test Port 1 and 2 Data Presented in Table 4.9

\section{Tables}

2.1. Scaling Factor for 12-in.-Diameter Scale Model Stack ................................................................ 2.5

2.2. Summary of Flow Parameters for Scale Model Stacks ................................................................ 2.6

2.3. Summary of DV Values for Scale Model Stacks ........................................................................ 2.6

4.1. Summary of LV-S2 Velocity Uniformity Tests ........................................................................ 4.2

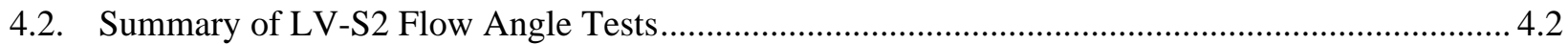

4.3. Summary of LV-S2 Gas Tracer Uniformity Tests .................................................................... 4.4

4.4. Summary of LV-S2 Particle Tracer Uniformity Tests ............................................................... 4.6

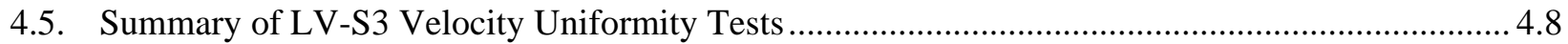

4.6. Summary of LV-S3 Flow Angle Tests....................................................................................... 4.9

4.7. Summary of LV-S3 Gas Tracer Uniformity at Test Ports 1 and 2 ............................................ 4.10

4.8. Summary of LV-S3 Particle Tracer Uniformity Tests with Particle Injection at I5 .................... 4.13

4.9. Summary of Velocity Variation Tests at Test Ports 1 and 2 ....................................................14

4.10. Summary of Tests Moving Injection Port Upstream................................................................... 4.15

4.11. Summary of Additional Testing to Verify B\&C Max and Norm Test Results ............................ 4.15 
4.12. Summary of Tests with Dual Fan Combinations with Particle Injection at I7 ............................. 4.16

5.1. Summary of Results for the LV-S2 and LV-S3 Scale Model Stacks............................................ 5.1 



\subsection{Introduction}

This series of scale model tests was performed to document whether the current designs for the air monitoring locations at three of the air exhaust stacks in the Hanford Tank Waste Treatment and Immobilization Plant (WTP) meet the applicable regulatory criteria governing effluent monitoring systems. This group (Test Group 1-2A) originally consisted of 3 Low-Activity Waste (LAW) facility stacks: LV-C2, LV-S2, and LV-S3 (i.e., the emission units for the LAW C2V, LAW C5V, and LAW LVP ventilation systems, respectively). These three stacks are located at the LAW vitrification building. The LV-C2 stack, however, requires further testing and will be reported on separately.

The emissions from these low-activity waste facility air exhaust stacks may exceed the 0.1-millirem per year threshold limit given in Title 40 of the Code of Federal Regulations (CFR), Part 61, National Emissions Standards for Hazardous Air Pollutants (NESHAP), Subpart H, National Emission Standard for Emissions of Radionuclides Other Than Radon From Department of Energy Facilities. The NESHAP rule requires that a sampling probe be located in the exhaust stack according to criteria established by the American National Standards Institute/Health Physics Society (ANSI/HPS) N13.1-1999, Sampling and Monitoring Releases of Airborne Radioactive Substances from the Stack and Ducts of Nuclear Facilities. ${ }^{1}$ The capability of the sampling probe locations to meet this standard has been demonstrated with a series of tests on scale models. These data will be used by BNI as input to the air discharge permitting process.

This work is performed as part of the River Protection Project-Waste Treatment Plant Support Program under Contract No. DE-AC05-76RL01830 according to the statement of work issued by BNI, 24590-QL-SRA-W000-00101, N13.1-1999 Stack Monitor Scale Model Testing and Qualification, Revision 1, 09/12/2007 and Work Authorization 09 of Memorandum of Agreement 24590-QL-HC9-WA49-00001. The internal Pacific Northwest National Laboratory (PNNL) project for this task is 53024, Work for Hanford Contractors Stack Monitoring.

PNNL personnel conducted these scale model tests during 2013. No BNI personnel were directly involved in the tests. The BNI WTP point of contact and facility engineers provided the most current engineering input to support PNNL's tests. BNI retains responsibility for the technical design of the stack discharge and air monitoring systems.

\subsection{Qualification Criteria}

The qualification criteria for the location of a stack air monitoring probe are taken from ANSI/HPS N13.1-1999, Section 5.2.2 and are paraphrased as follows:

1. Uniform Air Velocity - It is important that the gas velocity be fairly uniform across the stack cross section where the sample is extracted. Consequently, the velocity is measured at several discrete points in the duct cross section at the proposed location of the sampling nozzle. The uniformity is expressed as the variability of the measurements about the mean. This is expressed using the percent coefficient of variation (\%COV), ${ }^{2}$ which is the standard deviation divided by the mean and expressed

\footnotetext{
${ }^{1}$ Health Physics Society, McLean, VA 22101. The standard has been reaffirmed in 2011 and is identical to the 1999 version. The regulations have not been updated yet, so the 1999 version is still referenced.

${ }^{2}$ Coefficient of variation is considered "dated" terminology. The modern terminology is percent relative standard deviation. However, because the standard uses the older terminology, it will likewise be used here.
} 
as a percentage - the lower the \%COV value, the more uniform the velocity. The qualification criterion is that the \%COV of the air velocity must be $\leq 20 \%$ in the center two-thirds of the duct cross section where the sampling probe is to be located.

2. Angular Flow - Sampling nozzles are typically aligned with the axis of the stack. If the air travels through the stack in cyclonic fashion, the air velocity vector approaching a sampling nozzle could be sufficiently misaligned with the nozzle to impair extraction of particles. Consequently, the flow angle is measured at the proposed location of the sampling probe. The average of the flow angle measurements (made at the same grid of points as the velocity measurements) should not exceed $20^{\circ}$ relative to the sampling nozzle axis.

3. Uniform Concentration of Tracer Gases-A uniform contaminant concentration in the sampling plane enables the extraction of samples that represent the true concentration within the duct. The uniformity of the concentration is first tested with a tracer gas to represent gaseous effluents. The fan is a good mixer, so injecting the tracer downstream of the fan provides worst-case results. The qualification criteria are that 1 ) the \%COV of the measured tracer gas concentration is $\leq 20 \%$ across the center two-thirds of the duct cross section at the sampling location, and that 2) the concentrations at all the measurement points cannot deviate from the mean by $>30 \%$.

4. Uniform Concentration of Tracer Particles - The second set of tests addressing contaminant concentration uniformity at the sampling position uses tracer particles large enough to exhibit inertial effects. Tracer particles of $10-\mu \mathrm{m}$ aerodynamic diameter (AD) are used by default unless it is known that larger contaminant particles will be present in the airstream. The qualification criterion is that the $\% \mathrm{COV}$ of particle concentration is $\leq 20 \%$ across the center two-thirds of the duct at the sampling location.

Tests to determine if criteria 1 through 4 were met were conducted on two scale models of the LV-S2 and LV-S3 at the proposed sampling locations along the exhaust ducts. By conducting tests on scale models of the exhaust systems, the designed air sampling locations can be qualified before cold commissioning, and compensatory measures could be made in the design if testing results were not satisfactory. All of the tracer concentration, velocity, and flow angle measurements were made using the same grid of points in a given cross section of the duct. The ANSI/HPS N13.1-1999 standard sets additional qualification criteria for the use of a scale model as a substitute for the actual stack:

- The scale model and its sampling location must be geometrically similar to the actual stack.

- The product of the hydraulic diameter and the mean velocity (DV) for the scale model must be within a factor of six of the DV for the actual stack.

- The Reynolds number for the actual and model stacks must be $>10,000$.

- The scale model results are considered valid if it is further shown that:

- $\quad$ The velocity profile in the actual stack meets the uniformity criterion (\%COV $\leq 20 \%)$.

- $\quad$ The velocity uniformity COV values for the actual and model stacks agree within $5 \% \mathrm{COV}$.

- $\quad$ The flow angle criterion (with a mean value less than or equal to $20^{\circ}$ ) is met.

The tests to determine the validity of the scale model testing will be performed during cold startup testing on the actual WTP stacks under separate test plans. The scale model testing conducted, as well as the results of these tests, are described in subsequent sections of this report. 


\subsection{LV-S2 and LV-S3 Stacks}

\subsection{Stack Geometry}

In the LV-S2 and LV-S3 stacks, the designed sampling probes will be located in horizontal sections of duct. Figure 2.1 and Figure 2.2 show the layout for each of the two stack designs, from the fan outlet to the outlet of the vertical duct. Figure 2.3 and Figure 2.4 show the scale model layout for each stack design. The simplified models are based on assumptions about the necessary simulation detail. These assumptions are listed below:

- Geometric simulation of the components upstream of the backdraft damper was ignored. Backdraft damper blades do not usually open fully. The partially open blades direct the air velocity vector toward one side of the duct, resulting in considerable disruption to the air flow. Consequently, it was assumed that the air velocity and tracer uniformity downstream of the dampers would not be greatly influenced by equipment upstream of the dampers. This assumption has not been tested; however, this assumption had the benefit of reducing the cost of the models by using a single fan/filter/heater arrangement and the elimination of the control damper.

- Components several duct diameters downstream of the sampling point are not modeled. It was assumed that the only effect of any components downstream of the sampling probe location would be to slightly change the pressure at the sampling port. While this assumption was not tested, stack components generally do not significantly influence flow patterns upstream.

The same fans were used for each of the two scale models. The fans were connected to a flexible duct that was connected to the backdraft damper. The backdraft damper was connected to each of the scale models for testing.

For both of the scale model stacks, Test Port 1 (Figure 2.3 and Figure 2.4) represents the planned location for the record sampling systems (Figure 2.1 and Figure 2.2) according to the current WTP BNI designs. $^{1,2}$ The LV-S3 system has a second test port, located approximately seven duct diameters downstream of Test Port 1 , which is the planned location for the continuous air monitor system. Both scale model systems have an additional Particle Reference Port located five duct diameters downstream of the last Test Port. This was used for a fixed-position OPC used to monitor the output of the aerosol generator during the particle tracer uniformity testing.

The ratio of the prototype dimensions to the scale model dimensions varies with each system. Each scale model was constructed with a primary duct diameter of 12 in. for convenience and to maintain the ability to re-use the duct sections for subsequent stack designs. Table 2.1 lists the diameter of the actual stack with the scaling factor for the 12-in. scale model diameter. The calculations of the key scale model dimensions were performed in spreadsheets and then verified and validated in accordance with appropriate quality assurance (QA) procedures. ANSI/HPS N13.1-1999 requires that the models be geometrically similar to the actual stacks. Acceptable deviations in key dimensions of the scale model arising from scaling and fabrication errors are within about $\pm 5 \%$ for cross-sectional dimensions and about

\footnotetext{
${ }^{1}$ Haukur Hazen (BNI) to Dean Kurath (PNNL), CCN 252037 "Transmittal of CCN 251800 Documentation Supporting ANSI/HPS N13.1-1999 LAW C5V (LV-S2) Scale Model Testing,” dated October 2, 2012.

${ }^{2}$ Haukur Hazen (BNI) to Dean Kurath (PNNL), CCN 251525 "Transmittal of CCN 249983 Documentation Supporting ANSIIHPS N13.1-1999 LAW LVP (LV-S3) Scalie Model Testing,” dated September 7, 2012.
} 
$25 \%$ of a duct diameter in overall length between the sampling point and the flow disturbances. These deviations would have less impact on the test results than the normal standard deviation of repeat tests. The key scale model dimensions for the as-built scale models were measured and recorded by testing staff.

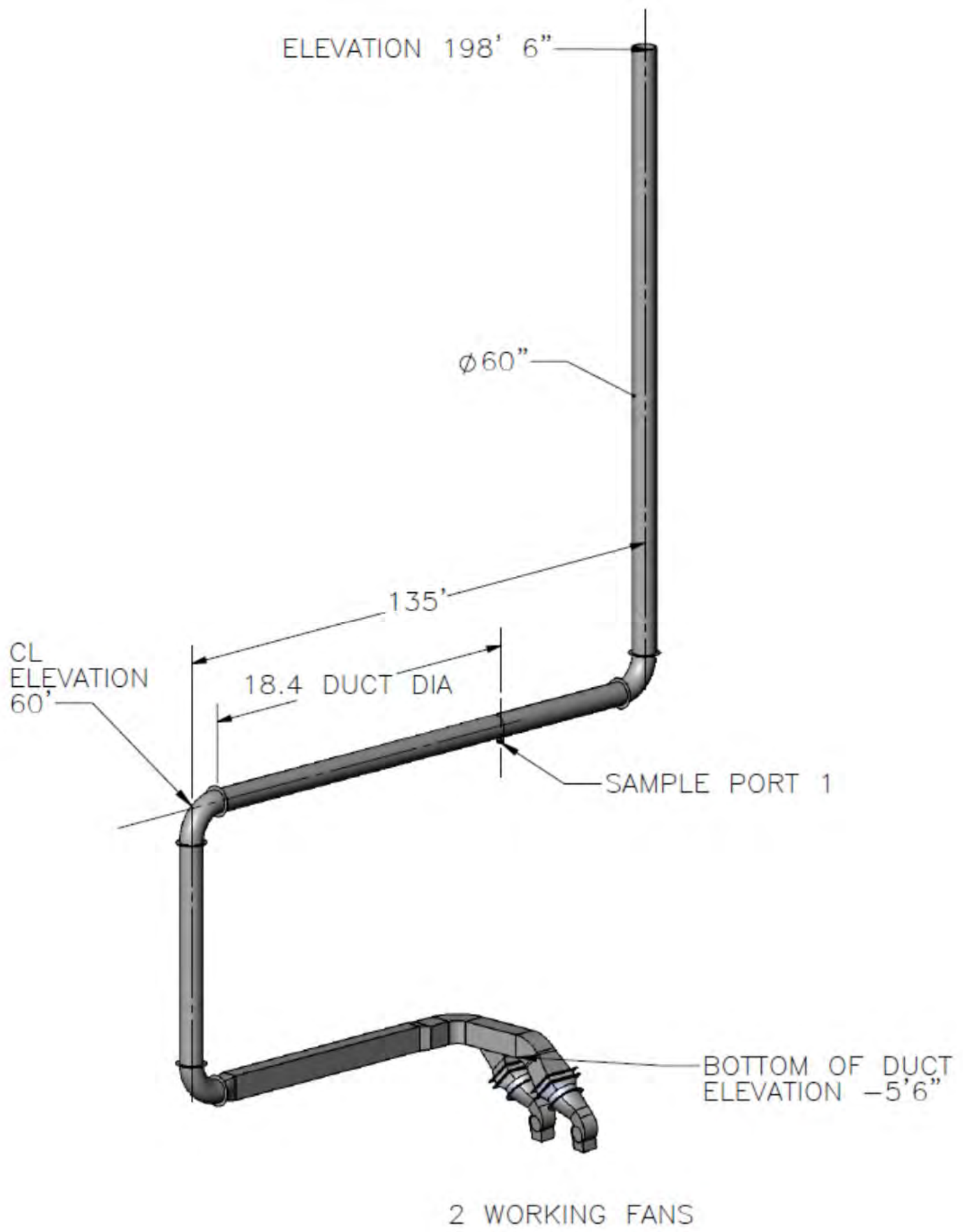

Figure 2.1. LAW C5V (LV-S2) System per Design 


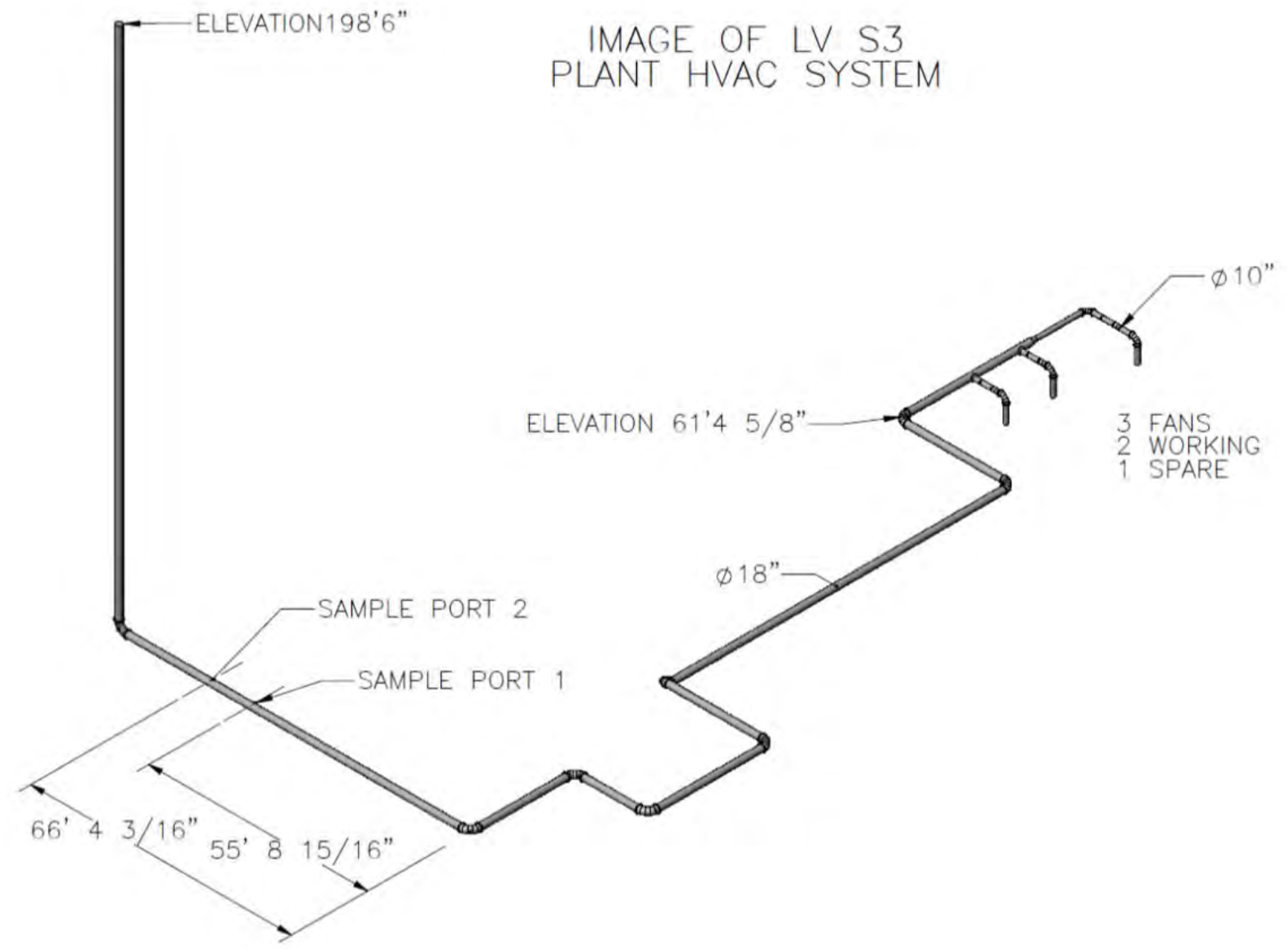

Figure 2.2. LAW LVP (LV-S3) System per Design 


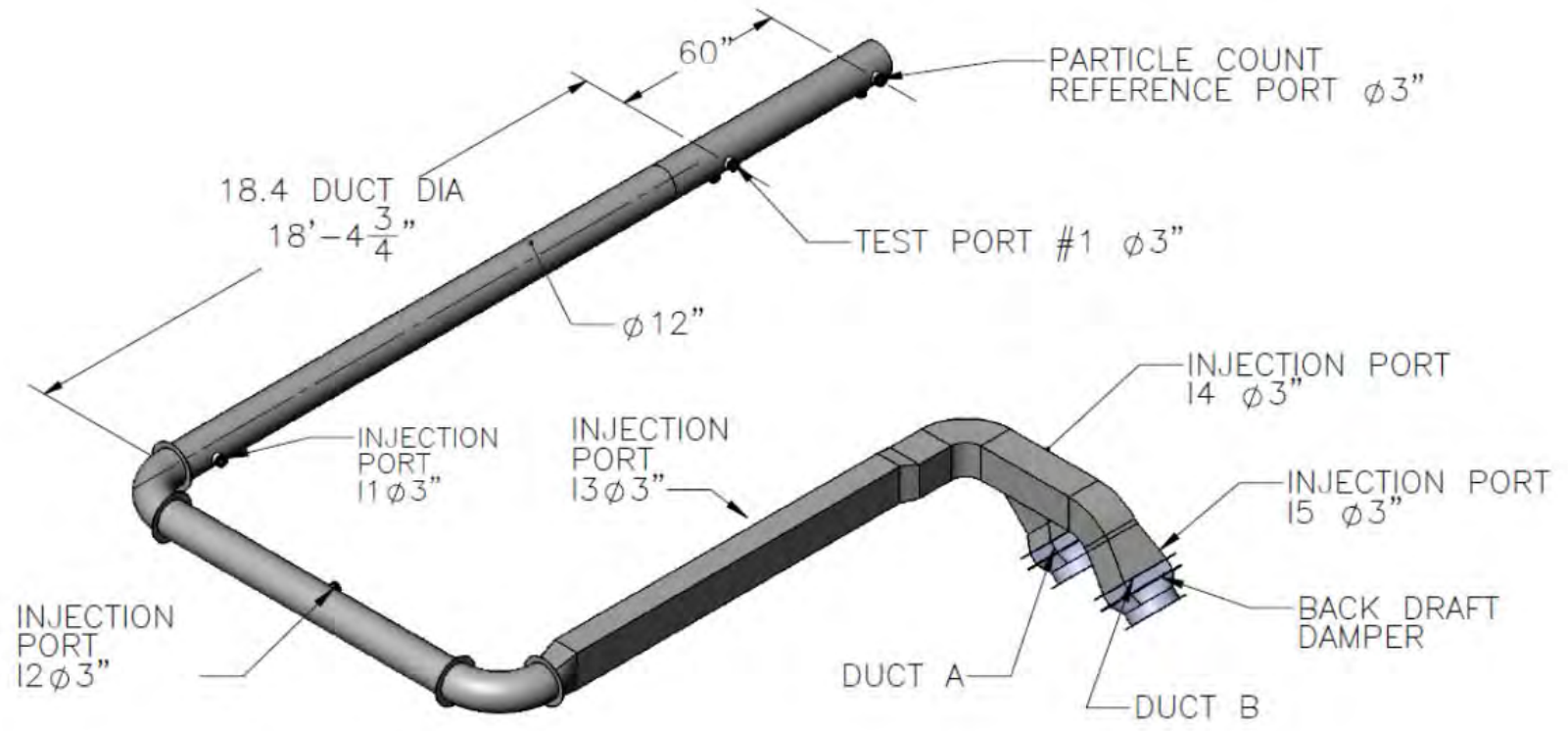

Figure 2.3. Layout of the LAW C5V (LV-S2) Scale Model Test System

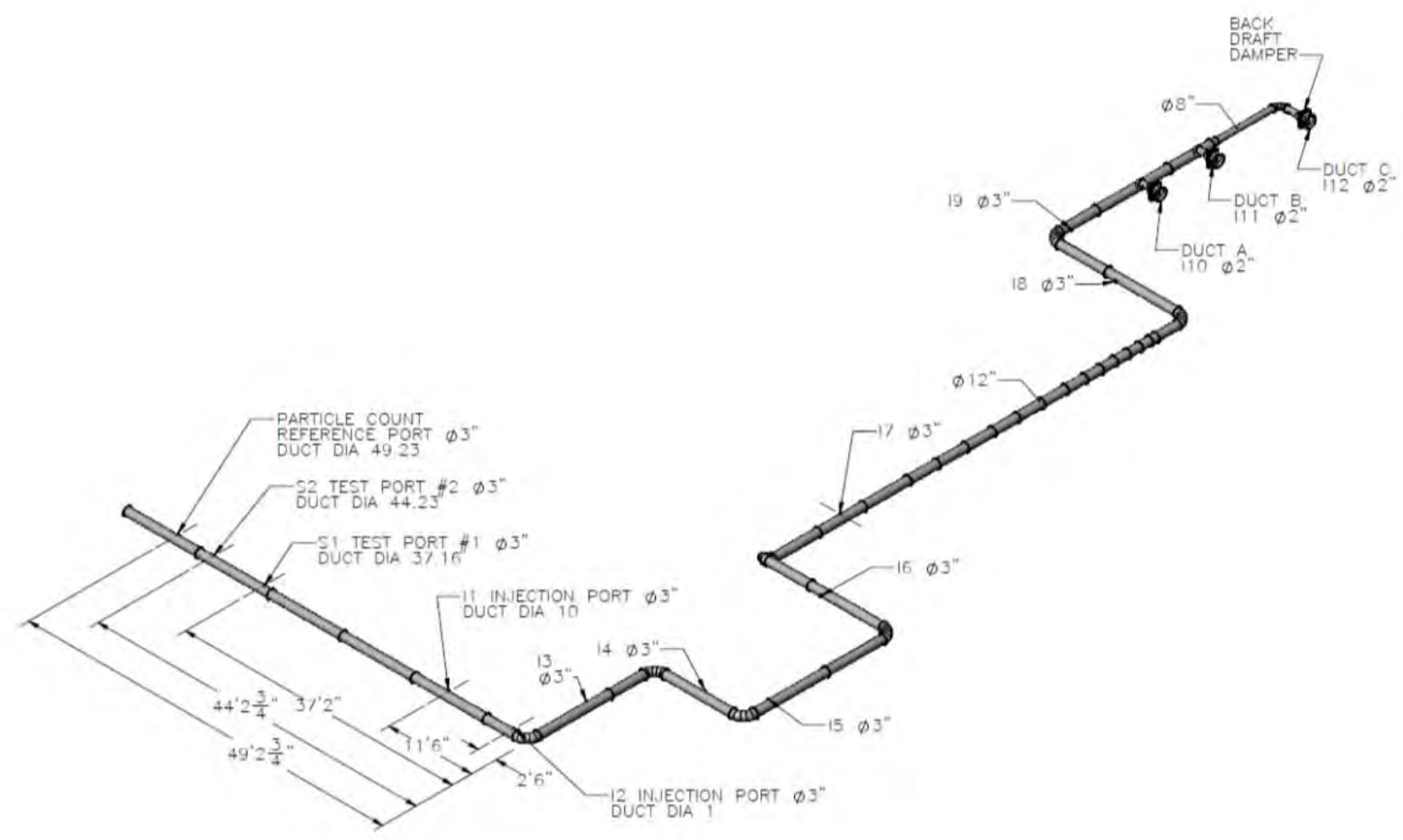

Figure 2.4. Layout of LAW LVP (LV-S3) Scale Model Test System 
Table 2.1. Scaling Factor for 12-in.-Diameter Scale Model Stack

\begin{tabular}{ccc}
\hline & Actual Diameter & Scaling Factor \\
\hline LV-S2 & 60 in. & 5.00 \\
LV-S3 & 18 in. & 1.50 \\
\hline
\end{tabular}

\subsection{Stack Flows}

Tests of scale model stacks were conducted at flow rates that bracket the range of expected normal and accident flow rates and operating configurations. Various combinations of flow rates and operating fans were tested. BNI provided normal, minimum, and maximum flow rates.

Additional considerations come from the ANSI/HPS N13.1-1999 standard. The standard requires that the DV of the scale model be within a factor of six of the actual stack. For stacks with a circular cross section, this is equivalent to requiring that the ratio of flow rate to stack diameter be within a factor of six of the actual stack. The standard also requires that the Reynolds number for the prototype and model stacks must both exceed 10,000.

There are two fans available to power the LAW C5V (LV-S2) exhaust system, which exhausts the air from the C5 ventilation system of the low-activity waste facility. One fan will be operated at a time, and one will be on standby. Each fan is capable of providing the design maximum flow rate, and is equipped with an adjustable-speed drive to compensate for filter loading and pressure variations.

Three fans are available to power the LAW LVP (LV-S3) exhaust system (Figure 2.2). This system discharges air from the melter and associated process vessels. Normally, two fans will be operated at a time, and one will be on standby. However, under off- normal conditions, a single fan could be in operation. The likelihood of all three fans operating is very remote. Two fans are required to provide the maximum design flowrate and the system is equipped with adjustable-speed drives.

Table 2.2 lists the flow conditions for the actual stack as well as the scale model stack. The minimum air flow (in actual cubic feet per minute [acfm]) and air velocity (in actual feet per minute [afpm]) to achieve the assumed minimum and maximum actual stack flow are listed. The tabulated values of flow and velocity in the "Scale Model Minimum" columns are the minimum scaled values that will meet the criterion listed in Section 1.1 that the DV product be within a factor of six of the prototype. The scale model Reynolds numbers are calculated for those minima. One of the qualification criteria listed in Section 1.1 is that the Reynolds number for both the actual and scale model stack must be greater than 10,000. Therefore, the Reynolds numbers for the actual and scale model stacks at the minimum and maximum flow rates are included in Table 2.2. The conditions prescribed for these scale model tests fulfill the criterion of a Reynolds number greater than 10,000.

As listed in Section 1.1, the qualification criteria include a constraint on the DV value of the scale model relative to the actual stack. For comparison between the tests on the scale models and the acceptable DV range, DV values are tabulated in Table 2.3. They are calculated for each of the three flow conditions for each stack. The acceptable DV range (1/6 to $6 \mathrm{X}$ that of the actual stack) is quite large, and the upper end of the range is impractical with real-world blowers. Usually, during testing, an upper velocity approximately equal to that of the actual stack is selected for use. With the use of variable frequency drives to control the blowers, the velocity corresponding to the lower end of the DV range is practical, and this velocity (or a slightly higher velocity) is typically used. 
Table 2.2. Summary of Flow Parameters for Scale Model Stacks

\begin{tabular}{llccccc}
\hline & \multicolumn{2}{c}{ Air Flow (acfm) } & \multicolumn{2}{c}{ Air Velocity (afpm) } & \multicolumn{2}{c}{ Reynolds Number } \\
\cline { 2 - 7 } Flow & $\begin{array}{c}\text { Actual } \\
\text { Stack }\end{array}$ & $\begin{array}{c}\text { Model } \\
\text { Minimum }\end{array}$ & $\begin{array}{c}\text { Actual } \\
\text { Stack }\end{array}$ & $\begin{array}{c}\text { Scale Model } \\
\text { Minimum }\end{array}$ & $\begin{array}{c}\text { Actual Stack } \\
\text { Scale Model } \\
\text { Minimum }\end{array}$ \\
\hline LV-S2 & & & & & \\
\hline Max flow & 91,019 & 3,034 & 4,636 & 3,863 & $1.8 \mathrm{E}+06$ & $3.7 \mathrm{E}+05$ \\
Normal flow & 60,117 & 2,004 & 3,062 & 2,551 & $1.2 \mathrm{E}+06$ & $2.0 \mathrm{E}+05$ \\
Min flow & 35,294 & 1,176 & 1,798 & 1,498 & $7.2 \mathrm{E}+05$ & $1.2 \mathrm{E}+05$ \\
\hline LV-S3 & 6,258 & 695 & 3,541 & 885 & $3.3 \mathrm{E}+05$ & $2.2 \mathrm{E}+05$ \\
\hline Max flow & 5,631 & 626 & 3,181 & 797 & $3.0 \mathrm{E}+05$ & $5.0 \mathrm{E}+04$ \\
Normal flow & 2,632 & 292 & 1,489 & 372 & $1.4 \mathrm{E}+05$ & $2.3 \mathrm{E}+04$ \\
Min flow & &
\end{tabular}

Both scale model stack diameters are 1 foot, so the DV values in Table 2.3 are equal to the velocity values for the scale model. For example, the LV-S2 minimum DV, which is $1498 \mathrm{ft}^{2} / \mathrm{min}$, corresponds to a velocity of $1498 \mathrm{ft} / \mathrm{min}$ for the 1 -ft-diameter scale model. The practical ranges of test velocities are also included in Table 2.3. As described above, the lower end of this range is defined by the minimum DV, and the upper end is practically implemented as the actual stack velocity for the given operating condition. Referring to Table 2.2, for example, the minimum LV-S2 stack velocity is $1798 \mathrm{ft} / \mathrm{min}$, so this value is listed for the Test Velocity Range in Table 2.3. Again, the velocity value is identical to the DV value for 1-ft-diameter stacks, so the upper velocity value is the DV value. Note that, for the LV-S3 model, where the model diameter and the stack diameter are nearly the same, the ranges of test velocities overlap between the flow conditions. This allows some latitude in the operating conditions for the tests, and the full-scale stack conditions that they represent.

Table 2.3. Summary of DV Values for Scale Model Stacks

\begin{tabular}{llccc} 
Stack & DV, $\mathrm{ft}^{2} / \mathrm{min}$ & Min & Norm & Max \\
\hline \multirow{4}{*}{ LV-S2 } & Actual Stack & 8990 & 15,310 & 23,180 \\
& Min. for Model & 1498 & 2552 & 3863 \\
& Max. for Model & 53,940 & 91,860 & 139,080 \\
\cline { 2 - 5 } & Test Velocity Range, & & & \\
& $\mathrm{ft} / \mathrm{min}$ & $1498-1798$ & $2552-3062$ & $3863-4636$ \\
\hline \multirow{5}{*}{ LV-S3 } & Actual Stack & 2234 & 4772 & 5312 \\
& Min. for Model & 372 & 795 & 885 \\
& Max. for Model & 13,401 & 28,629 & 31,869 \\
\cline { 2 - 5 } & Test Velocity Range, & & & \\
& $\mathrm{ft} /$ min & $372-1489$ & $795-3181$ & $885-3541$ \\
\hline
\end{tabular}




\subsection{Testing Methods}

The testing methods were based on the requirements of ANSI/HPS N13.1-1999. A test plan, TP-WTPSP-104, Air Sampling Probe Location Tests for Waste Treatment Plant LAW LV-C2, LV-S2 and LV-S3 (Group 1-2A) Air Exhaust Systems, was prepared by PNNL and approved by BNI. This plan referenced the use of PNNL procedures, which define how the test should be conducted in general. A test instruction (TI) was prepared for each test type and for each scale model stack. These TIs contain specific instructions pertaining to the tests that are not addressed in the general procedures. Such information includes the following:

- Layout of measurement points

- Locations of tracer injection points

- List of equipment and instrumentation

- Safety requirements

- List of minimum test runs

- Test description and measurement data sheets with hand entries

- Table of preliminary results.

Because the final data sheets and a description of the test methods are included in this report, the TIs are not included here. The QA program that is implemented for this project is described in Section 3.1 and a summary of the stack testing methods used for each of the four test types is presented in Section 3.2.

\subsection{Quality Assurance}

The PNNL QA program is based on the requirements defined in the U.S. Department of Energy Order 414.1D, Quality Assurance, and 10 CFR 830, Energy/Nuclear Safety Management, and Subpart A-Quality Assurance Requirements (a.k.a. the Quality Rule). PNNL has chosen to implement the following consensus standards in a graded approach:

- ASME NQA-1-2000, Quality Assurance Requirements for Nuclear Facility Applications, Part I, "Requirements for Quality Assurance Programs for Nuclear Facilities."

- ASME NQA-1-2000, Part II, Subpart 2.7, Quality Assurance Requirements for Computer Software for Nuclear Facility Applications.

- ASME NQA-1-2000, Part IV, Subpart 4.2, Guidance on Graded Approach Application of Quality Assurance (QA) Requirements for Nuclear-Related Research and Development.

The procedures necessary to implement the requirements are documented through PNNL's "How Do I...?” (HDI), which is a system for managing the delivery of laboratory-level policies, requirements, and procedures.

The Waste Treatment Plant Support Program (WTPSP) implements an NQA-1-2000 QA program, using a graded approach as presented in NQA-1-2000, Part IV, Subpart 4.2. The WTPSP Quality Assurance manual (QA-WTPSP-0002) describes the technology life cycle stages under the WTPSP QA 
plan (QA-WTPSP-0001). The technology life cycle includes the progression of technology development, commercialization, and retirement in process phases of basic and applied research and development (R\&D), engineering and production, and operation until process completion. The life cycle is characterized by flexible and informal QA activities in basic research, which becomes more structured and formalized through the applied R\&D stages. The work described in this report has been completed under the QA Technology level of Developmental Work as the data will be used for applying for air discharge permits.

- DEVELOPMENTAL WORK-Developmental work consists of research tasks moving toward technology commercialization. These tasks still require a degree of flexibility, and there is still a degree of uncertainty that exists in many cases. The role of quality on developmental work is to make sure that adequate controls exist to support movement into commercialization.

WTPSP addresses internal verification and validation activities by conducting an Independent Technical Review of the final data report in accordance with WTPSP's procedure QA-WTPSP-0601, Document Preparation and Change. This review verifies that the reported results are traceable, that inferences and conclusions are soundly based, and the reported work satisfies the test plan objectives. Appendix A lists the reviewed test plan, test instructions, and calculation packages used for the tests documented in this report.

\subsection{Stack Tests}

The tests described in the following subsections were conducted under scale flow conditions designed for each stack, which were listed in Table 2.2. The test matrix included with the test plan described the minimum number of tests that were planned for each stack. The actual number of tests typically exceeded those planned because tests were added to confirm results that had to be repeated.

Before conducting the tests to determine whether the four qualification criteria described in Section 1.1 were met for each stack, two other measurement sets were made. First, the major features of the stack were measured. The longitudinal distances from the fans to the bends, duct reducers, and ports were determined in addition to the duct diameter at each measurement port. The second set of measurements determined the fan frequency settings needed to achieve the desired flow rates. For these measurements, the location within the duct cross section that had velocity measurements closest to the mean velocity was determined for the test port. Then, velocity measurements were made at this single measurement point at 5- $\mathrm{Hz}$ increments in the fan frequency setting. By developing a

frequency-vs.-velocity relationship for the scale model stack, the frequency setting needed to achieve the flow conditions could be pre-determined. The data sheets from these velocity calibration tests are included in Appendices B.1 and C.1.

A common grid of measurement points in the duct cross section was used for each of the qualification criteria tests described in the following subsections. The number and distance between measurement points were based on the U.S. Environmental Protection Agency (EPA) procedure 40 CFR 60, Appendix A, Method 1, for circular stacks. For a 12-in. duct diameter, eight traverse points are required at the relative positions shown in Figure 3.1. Measurements also were made at the centerpoint. In lieu of making the two measurement points nearest to the walls at $3.2 \%$ of the duct diameter from the duct walls, the minimum distance from the wall was set to 0.5 in., as prescribed by EPA Method 1. The measurement point closest to the port was Point 1, while the point farthest from the port was Point 8. 


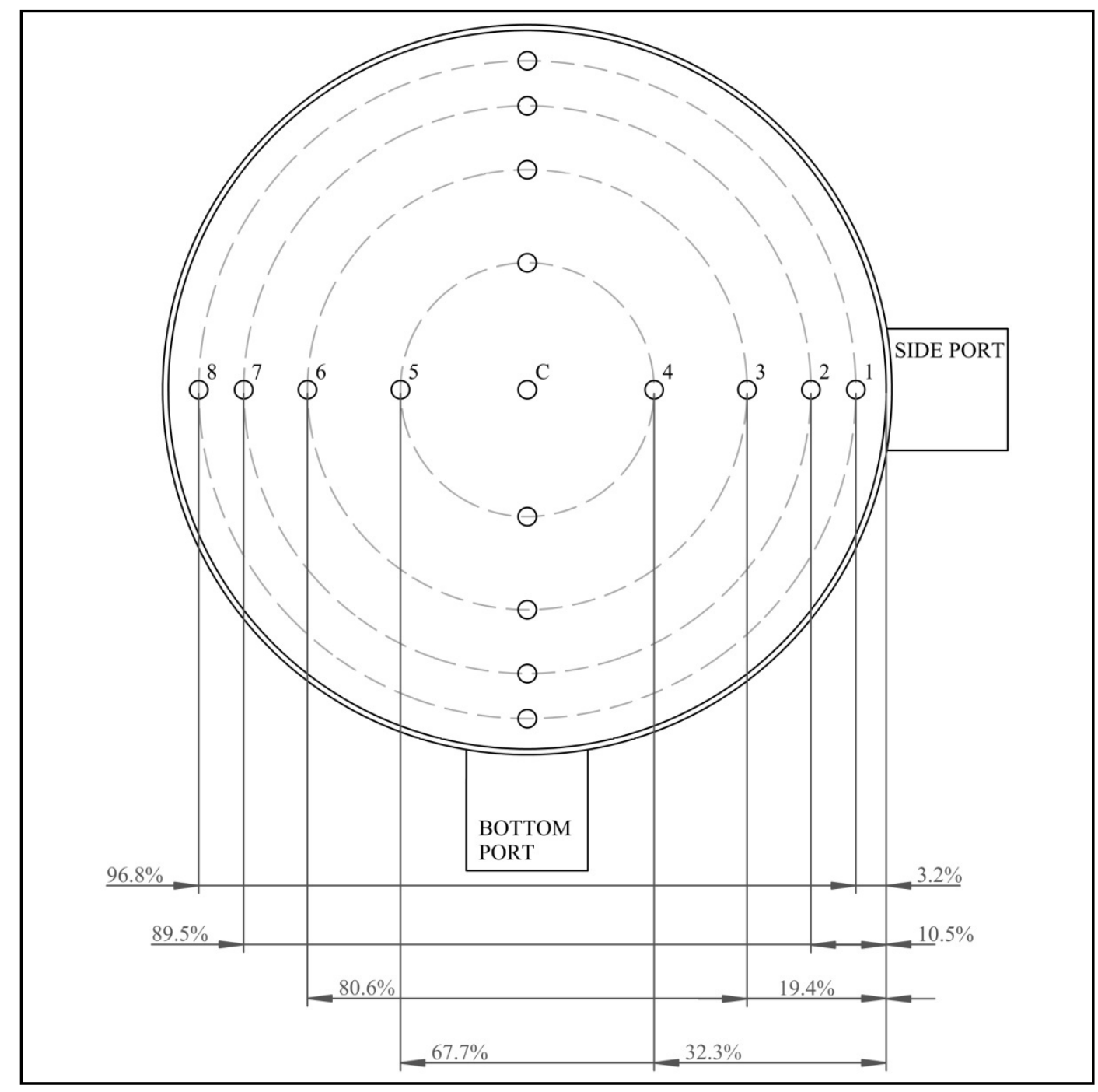

Figure 3.1. Cross Section of the Duct at the Testing Ports with Measurement Points

\subsubsection{Velocity Uniformity}

The uniformity of air velocity at the stack monitoring location indicates whether the momentum in the stack is well-mixed. The method used to conduct the velocity uniformity tests was based on 40 CFR 60, Appendix A, Method 1. The velocity uniformity criterion is that the $\%$ COV should be less than $20 \%$ in the center two-thirds of the duct (measurement points 2-7).

For each test run, three air velocity readings were obtained at each measurement point in the cross section of the duct. The measured velocity was the average of the three readings. The measured velocity for each point was used to determine the mean and standard deviation of the velocity across the cross-sectional plane. The \%COV (a.k.a., the percent relative standard deviation) was calculated as 100 times the standard deviation divided by the mean.

Air velocity measurements were made using a handheld thermal anemometer (TSI, Inc. Model 9545, Shoreview, MN). Duct air temperature measurements also were made with the same handheld thermal 
anemometer. The thermal anemometer is capable of reporting velocity in standard feet per minute (sfpm), with standard conditions defined as $1 \mathrm{~atm}$ and $70^{\circ} \mathrm{F}$, or in actual feet per minute (afpm) using the actual air temperature measured by the thermal anemometer and the actual barometric pressure measured with another instrument and input to the anemometer. Figure 3.2 shows the thermal anemometer used for this test. The procedure EMS-JAG-04 and test instructions TI-WTPSP-114 and TI-WTPSP-120 were followed to conduct this test for the scale models.
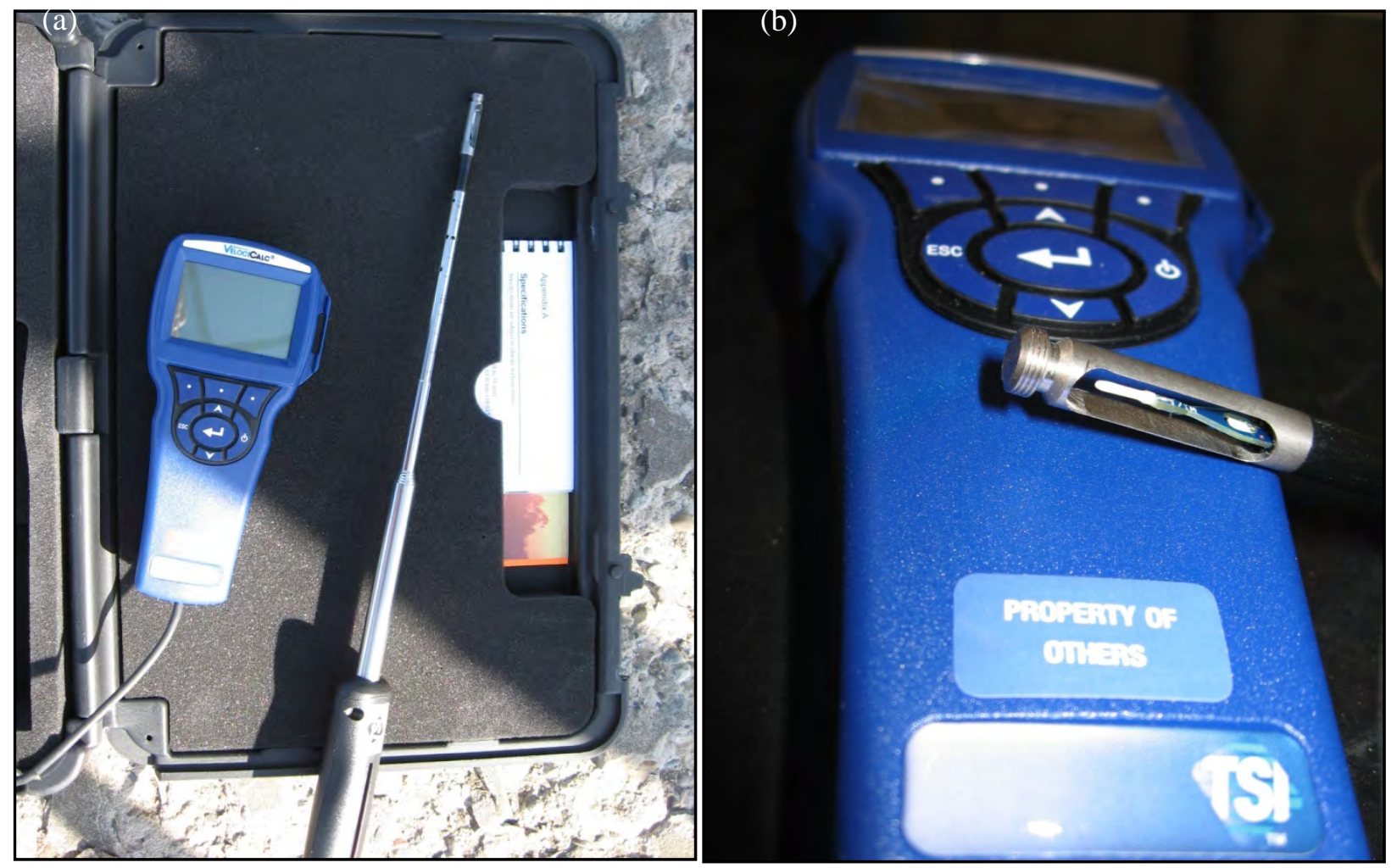

Figure 3.2. Equipment Used for the Velocity Uniformity Test (a) Thermal Anemometer, and (b) Close-Up View of Thermal Anemometer Probe Tip

\subsubsection{Flow Angle}

The air velocity vector approaching the sample nozzle should be aligned with the axis of the nozzle within an acceptable range so that the sample extraction performance is not degraded. The test method is based on 40 CFR 60, Appendix A, Method 1, Section 11.4, "Verification of the Absence of Cyclonic Flow." The term "flow angle” refers to the angle between the velocity vector of the flow in the duct and the axis of the sampling nozzle. For the stack testing activities, the flow angle was measured at a grid of nine points across two axes in a cross section of the duct (see Figure 3.1). The qualification criterion for the flow angle test is that the average angle should not exceed $20^{\circ}$.

The flow angle measurements were made using an S-type Pitot tube (Dwyer Instruments, 160S-36, Michigan City, IN) attached by flexible tubing to a slant-tube manometer (Dwyer Instruments, 400-5, Michigan City, IN) and an angle-indicating device attached to the sampling port as shown in Figure 3.3. For this test, the S-type Pitot tube is rotated so that the planes of the two open ends of the two tubes are parallel to the long axis of the duct. The pitot tube is then rotated about its long axis until the differential 
pressure across the open ends of the tubes reads zero on the manometer. The rotation angle is read from the angle-indicating device. The measured flow angle for each point is the average of three readings. These measured values are used to calculate the mean absolute value of the flow angle across the duct. The procedure EMS-JAG-05 and test instructions TI-WTPSP-115 and TI-WTPSP-121 were used to conduct this test for each of the scale models.
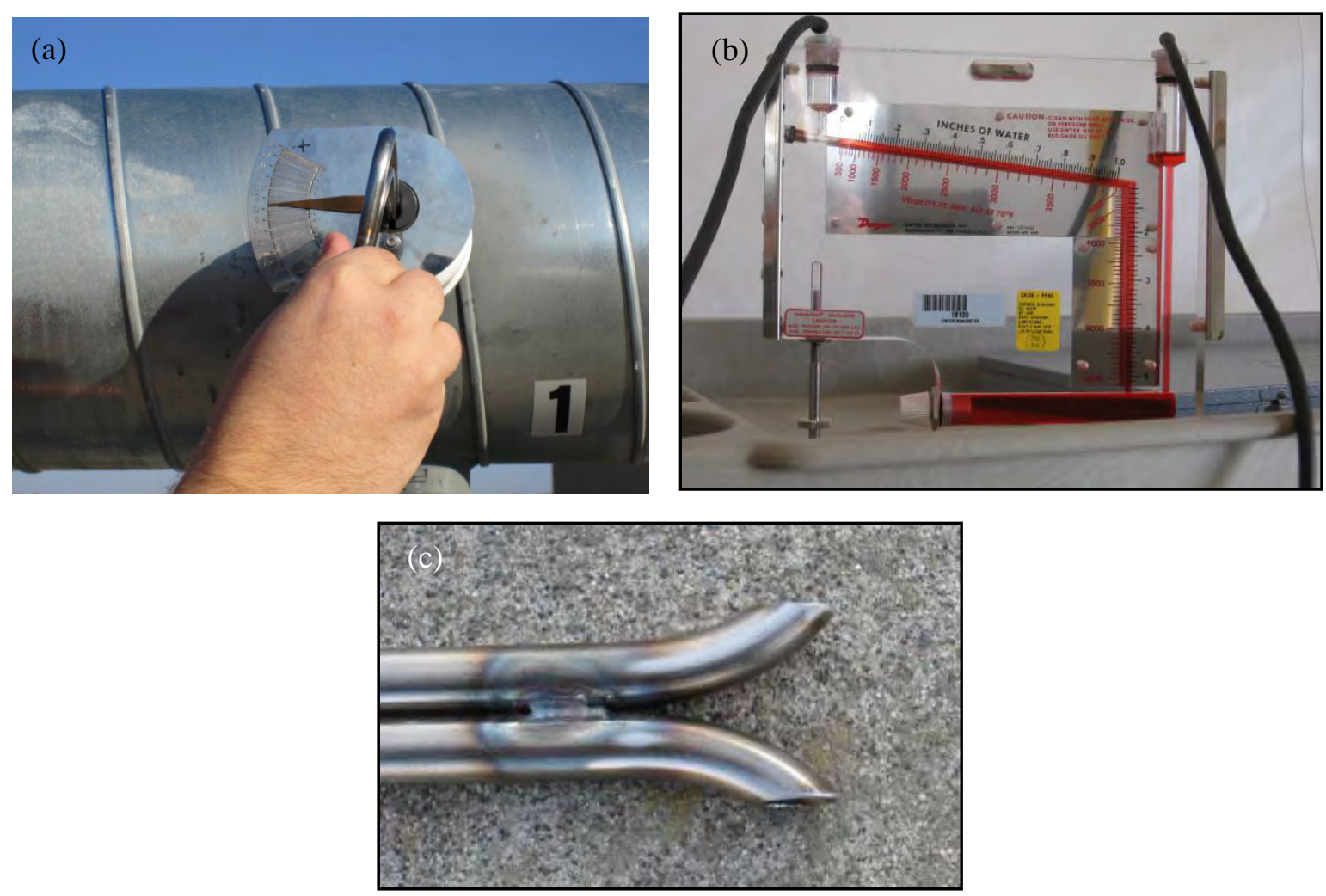

Figure 3.3. Equipment Used for the Flow Angle Test: (a) S-type Pitot Tube Inserted in a Test Port with the Angle-Indicating Device, (b) Slant-Tube Manometer, and (c) Openings at Tip of S-Type Pitot Tube

\subsubsection{Gaseous Tracer Uniformity}

The gaseous contaminant concentration uniformity was demonstrated using the tracer gas nitrous oxide $\left(\mathrm{N}_{2} \mathrm{O}\right)$. In the past, PNNL has used sulfur hexafluoride $\left(\mathrm{SF}_{6}\right)$ gas for the gaseous tracer uniformity tests. $\mathrm{SF}_{6}$ is a well-established gas tracer; however, it is also a potent greenhouse gas with a global warming potential 22,800 times that of $\mathrm{CO}_{2}$. For each scale model test, approximately 1-5 pounds of $\mathrm{SF}_{6}$ is used. Nitrous oxide is also a greenhouse gas; however its global warming potential is only 298 times that of $\mathrm{CO}_{2}$. Although the global warming potential of $\mathrm{N}_{2} \mathrm{O}$ is nearly two orders of magnitude lower than that of $\mathrm{SF}_{6}$, the background concentration of $\mathrm{N}_{2} \mathrm{O}$ is an order of magnitude greater than the detection limit of $\mathrm{SF}_{6}$ using the available instrumentation, which increases the emission rate of $\mathrm{N}_{2} \mathrm{O}$ relative to $\mathrm{SF}_{6}$. Additionally, the measurement of $\mathrm{N}_{2} \mathrm{O}$, using the available instrumentation requires the measurement of both $\mathrm{H}_{2} \mathrm{O}$ and $\mathrm{CO}_{2}$, which results in an incremental increase in the measurement time and, consequently, the gas emission time. Despite these tradeoffs, an evaluation of alternative technologies and tracer gases 
was performed, and substituting $\mathrm{SF}_{6}$ with $\mathrm{N}_{2} \mathrm{O}$ and using existing measurement equipment was found to be the most cost-effective method of reducing the global warming impact of the gaseous tracer uniformity tests. An evaluation of gaseous tracer uniformity test results using the two gases was performed under both well-mixed and poorly-mixed conditions to ensure that the results of the uniformity qualification are not impacted by the use of a different gas. An Analysis of Variance (ANOVA) showed that there were no statistically significant differences between the use of $\mathrm{N}_{2} \mathrm{O}$ and $\mathrm{SF}_{6}$ (see Appendix B).

A compressed gas cylinder and a flow controller were used to deliver a constant stream of $\mathrm{N}_{2} \mathrm{O}$ into the duct. The gaseous tracer was typically injected into the duct at a point downstream of the fans. Figure 3.4 shows the injection locations with an injection probe positioned in the port. For separate test runs, the injection probe is positioned at one of five different locations in the duct cross section as illustrated in Figure 3.5 for circular ducts and in Figure 3.6 for rectangular ducts. The LV-S2 tests used both circular and rectangular duct injection locations. The LV-S3 scale model had only a circular duct injection location. For some tests, just the centerline position is used. The remaining four injection locations are within a specified distance of the duct wall. For a nominally 12-in.-diameter duct, the four "wall” injection locations were located within 2.4 in. of the wall. For a nominally 12.75 in. by 9.25 in. rectangular duct, the four "wall” injection locations are located with 2.7 in. of the corners.

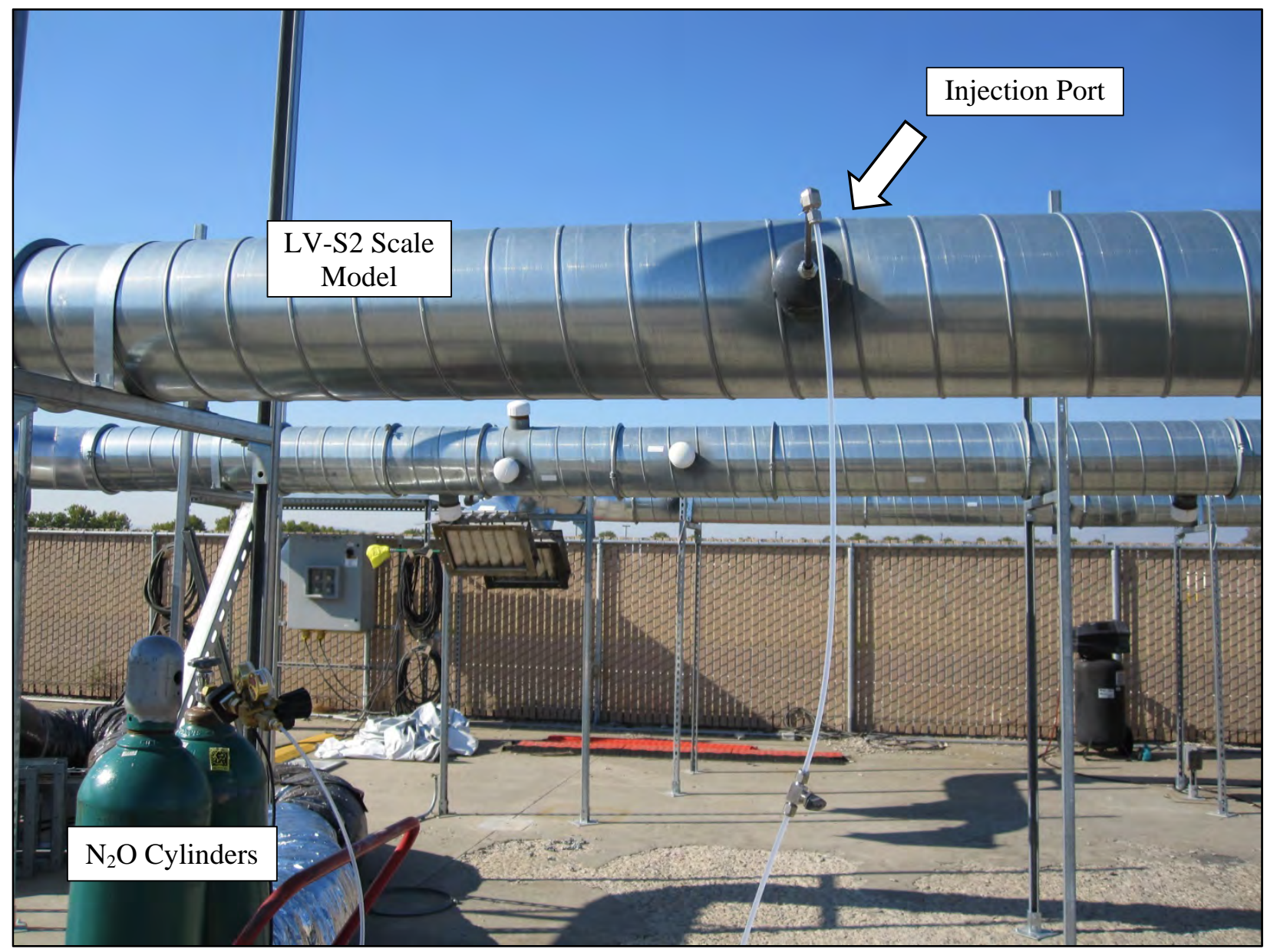

Figure 3.4. Equipment Used for the Gaseous Tracer Injection, Injection Probe Installed in the LV-S2 Scale Model, and Cylinders of Pure $\mathrm{N}_{2} \mathrm{O}$ with Heater and Regulator 


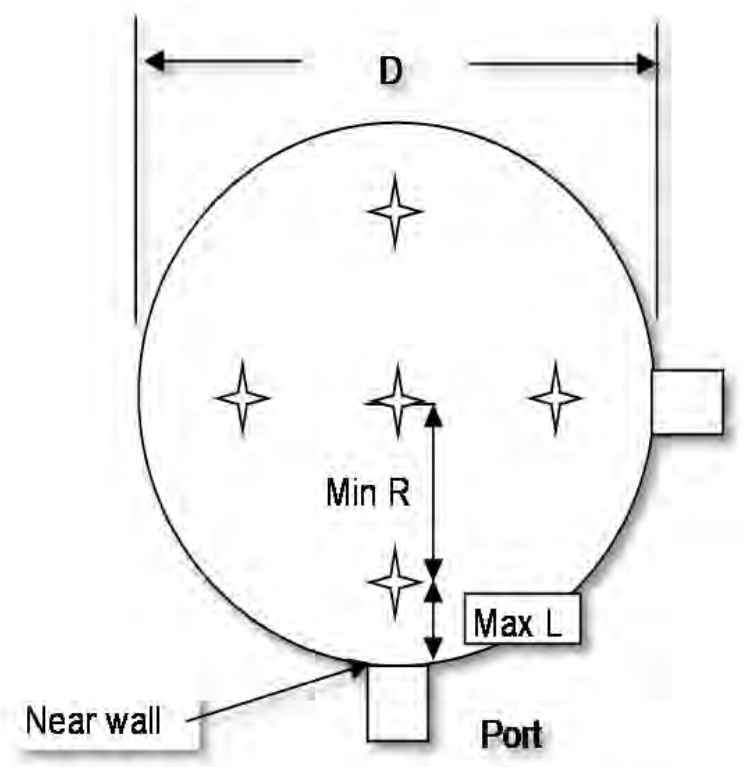

Figure 3.5. Illustration of Five Injection Points in a Circular Duct. Note: Max L is the maximum distance from the wall, which is $20 \%$ of the hydraulic diameter. Therefore, Min R, the minimum radius from the duct center, is $80 \%$ of the hydraulic diameter. In the case of a circular duct, the hydraulic diameter is equal to the physical diameter (D).

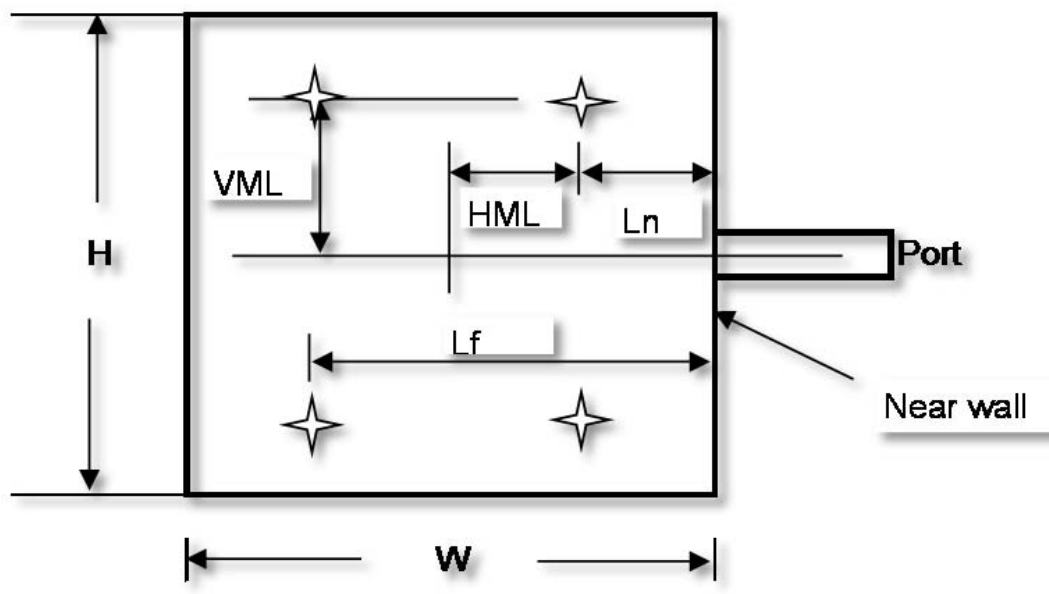

Figure 3.6. Illustration of Five Injection Points in a Rectangular Duct. Note: VML is the vertical minimum length off-center, HML is the horizontal minimum length off-center; $\mathrm{Ln}$ is the minimum length from near wall; and Lf is the minimum length from far wall. In the case of a rectangular duct, the hydraulic diameter is equal to four times the cross-sectional area divided by the rectangle's perimeter.

For each test run, the tracer concentration was read three times at each of the measurement points across the duct. The measured concentration for each point is the average of the three readings. These measured concentrations are used to calculate the overall mean, standard deviation, and \%COV. These calculations also are performed just for the measurement points in the center two-thirds of the duct. The qualification criteria for the gaseous tracer test are that 1 ) the $\%$ COV should be $\leq 20 \%$ within the center 
two-thirds of the duct, and 2) the concentration at any measurement point should not deviate from the overall mean by more than $30 \%$.

A photoacoustic gas analyzer (Brüel \& Kjær, Model 1302, Ballerup, Denmark) was used to measure tracer gas concentrations. The concentration variation is the important result for this test, so calibration bias is not important in the test results. However, the analyzer response was checked with calibration standards before and after conducting the test series (as well as weekly during the test series) to verify an adequate instrument response. The response was considered acceptable if the concentration from the instrument was within $10 \%$ of the calibration standard.

A simple probe was used to extract the sample and deliver it to the gas analyzer. A small pump drew air from within the stack through the probe. The gas analyzers then sampled the air from the sample line for analysis (Figure 3.7). The procedure EMS-JAG-01, Rev 4 and test instructions TI-WTPSP-117 and TI-WTPSP-122 were used to conduct this test for each scale model.

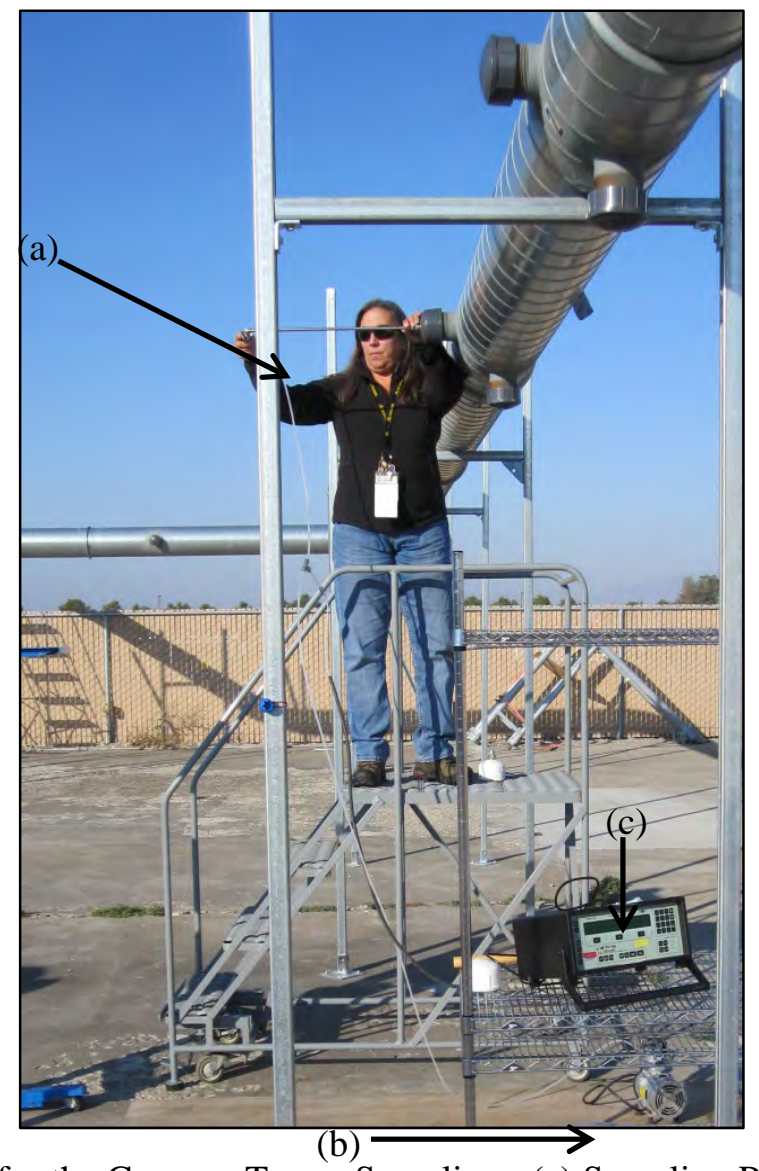

Figure 3.7. Equipment Used for the Gaseous Tracer Sampling: (a) Sampling Probe Installed in a Port, (b) Sampling Pump, and (c) Gas Analyzer 


\subsubsection{Particle Tracer Uniformity}

The uniformity of the particulate contaminant concentration was demonstrated using polydisperse pump oil particles as a particle tracer. Vacuum pump oil was drawn into a spray nozzle (driven by compressed air) housed in a stainless steel chamber. These aerosol particles were injected into the duct air at an injection point downstream of the fans as shown in Figure 3.8. Figure 3.8 shows the equipment setup for an aerosol injection in the LV-S3 scale model stack. The stainless steel chamber and spray nozzle assembly is also referred to as the aerosol generator. In previous tests, an aerosol generator of similar design, except constructed out of PVC, was used. These are the first scale model tests to utilize the new all-metal aerosol generator design. The aerosol was injected at the centerline of the duct, and this test was repeated to gain some sense of the variability of the results.
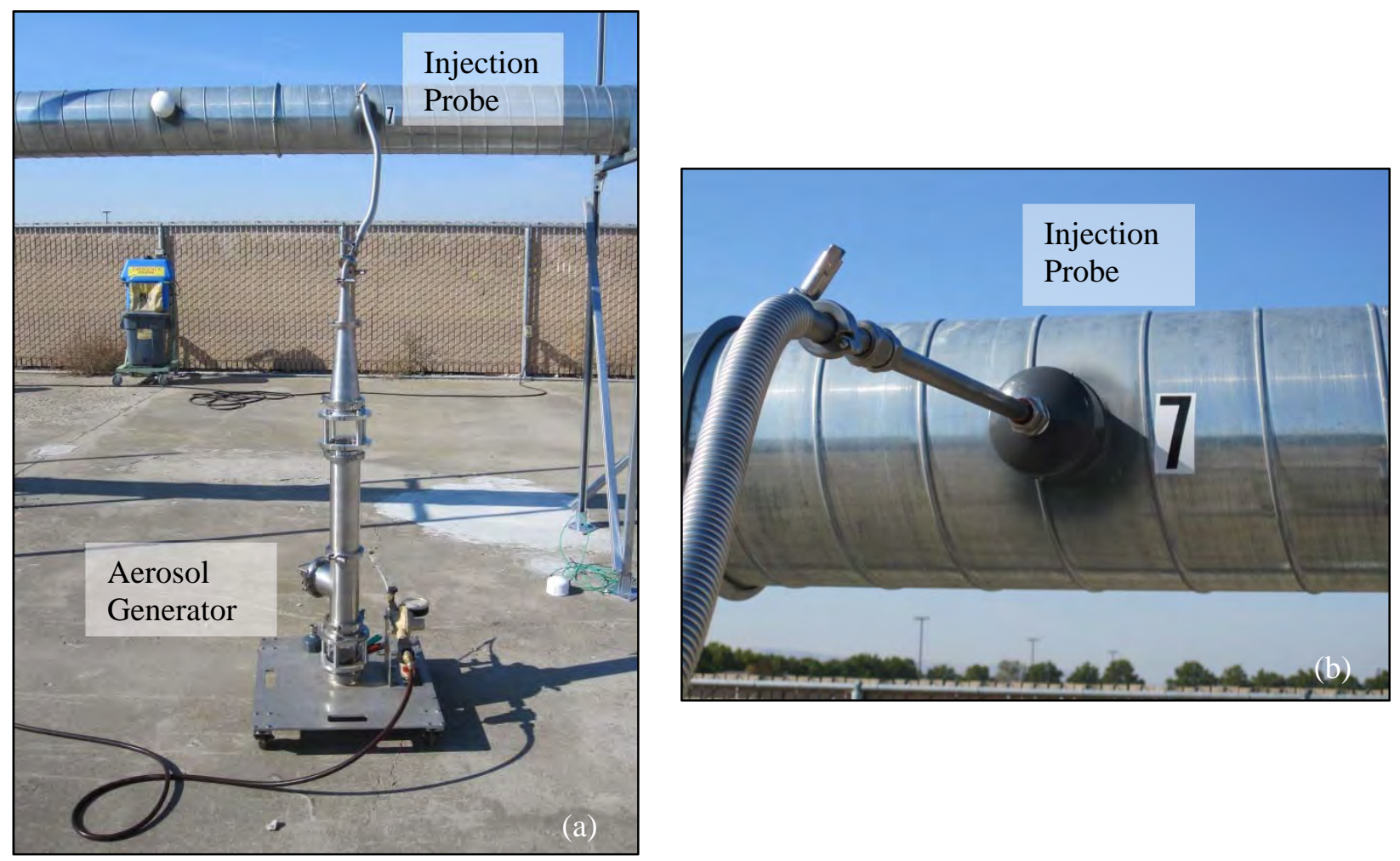

Figure 3.8. Equipment Used for Particle Injection (LV-S3). a) shows Aerosol Generator with Injection Probe placed in Injection Port 7; b) Close up of Injection Probe and Injection Port 7.

The concentration of the particles is measured at the sampling grid points with a calibrated optical particle counter (OPC) (Hach, Met-One Model 3415, Loveland, CO). A simple probe was used to extract the sample and deliver it to the OPC. Figure 3.9 shows the sampling setup with the simple probe connected to the OPC. To identify potential inconsistencies in the aerosol output, tests were conducted with a reference instrument measuring the particle concentration at a location downstream of the test port. The OPC sorts the particles into six size channels. As mentioned in Section 1.1, the particles of interest have an $\mathrm{AD}$ of $10 \mu \mathrm{m}$. Therefore, only data in the 9- to $11-\mu \mathrm{m}$ channel of the OPC were used. 
The particle concentration was read three times at each of the measurement points across the cross section of the duct. The measured concentration for each point is the average of the three readings. From these measurements, the overall mean, standard deviation, and \%COV were calculated for all of the points and also just for those within the center two-thirds of the duct. The qualification criterion for the particle tracer test is that the \%COV should be less than or equal to $20 \%$ within the center two-thirds of the duct. The procedure EMS-JAG-02 and test instructions TI-WTPSP-116 and TI-WTPSP-123 were used to conduct this test for the scale models.
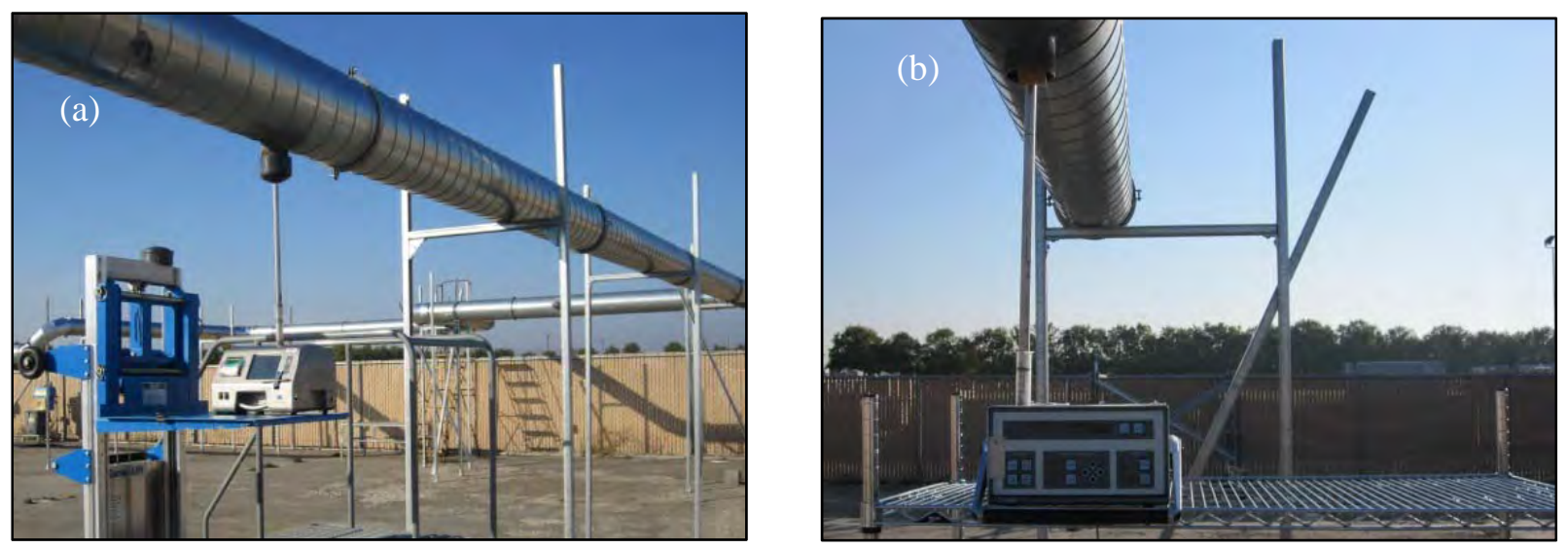

Figure 3.9. Particle Counters Used for the Particle Sampling. (a) Optical Particle Counter for Measurement Data in Bottom Port of LV-S3 Scale Model System; (b) Reference Optical Particle Counter in Bottom of LV-S3 Reference Port. 


\subsection{Stack Testing Results}

This section summarizes the results of the stack testing activities for two scale model stacks in Group 1-2A (LV-S2 and LV-S3). The primary, reportable results are the data and data calculations to confirm that the requirements of the ANSI/HPS N13.1-1999 standard have been met. Independent reviews were performed to verify the data transcription and calculations. These calculations were performed using Excel $(2007,2010)$ and documented in computer-assisted calculation packages (CCPs) in accordance with WTPSP procedures. The final data sheets for the LV-S2 and LV-S3 tests are included in Appendices $\mathrm{C}$ and $\mathrm{D}$, respectively. Appendix A contains a list of supporting documentation (such as the test plan and test instructions) used with this scale model test group. Appendix B contains documentation on gas tracer comparison and justification for switching to $\mathrm{N}_{2} \mathrm{O}$.

Each scale model stack underwent a series of velocity uniformity tests (designated VT), flow angle tests (designated FA), gas tracer tests (designated GT) and particle tracer tests (designated PT). Tables summarizing the results of tests for each scale model are presented in subsections of this chapter. During some tests the scale model velocity values were higher than the actual stack flow for the conditions the test was meant to represent. This is acceptable because the DV value was still within a factor of six of the stack design values, and the facility flow conditions are estimates and may vary significantly from the design conditions at times for a variety of reasons.

\subsection{LV-S2 Stack Results}

Summary tables of the data for Test Port 1 for LV-S2 flow angle, velocity, gas tracer, and particle tracer test results are presented in the following subsections. Some test combinations were repeated (i.e., performed more than once at different times) to quantify the testing and response measurement uncertainty.

\subsubsection{LV-S2 Velocity Uniformity}

Table 4.1 lists the results for the velocity uniformity tests performed on the scale model LV-S2 stack. In all cases, the results were well within the criterion of \%COV values $\leq 20 \%$. Velocity uniformity results were typically around 5\%. The velocity in the stack ranged from 1523 to 4645 afpm (1190 to $3630 \mathrm{acfm})$. Table 2.2 lists the desired range of minimum scale model flow rates as 1498 to $3863 \mathrm{afpm}$ (1176 to $3034 \mathrm{acfm}$ ). The desired testing conditions were between the minimum scale model flow rate and the actual stack velocity. With these flow conditions, the scale model meets both the Reynolds number and DV criteria required to represent the actual stack. The completed data sheets from these tests are available in Appendix C, Subsection C.2. 
Table 4.1. Summary of LV-S2 Velocity Uniformity Tests

\begin{tabular}{cccccc}
\hline \multirow{2}{*}{ Operating Fan } & $\begin{array}{c}\text { Flow } \\
\text { Condition }\end{array}$ & Run No. & $\begin{array}{c}\text { Flow } \\
(\mathrm{acfm})\end{array}$ & $\begin{array}{c}\text { Approx. Air } \\
\text { Velocity } \\
(\mathrm{afpm})\end{array}$ & \%COV \\
\hline \multirow{3}{*}{ A } & \multirow{3}{*}{ Max } & VT-7 & 3630 & 4645 & 4.1 \\
& & VT-8 & 3395 & 4345 & 4.6 \\
& & VT-9 & 3417 & 4373 & 5.5 \\
\cline { 3 - 6 } & \multirow{3}{*}{ Min } & VT-4 & 1294 & 1656 & 4.6 \\
& & VT-5 & 1294 & 1656 & 4.7 \\
& Max & VT-6 & 1306 & 1671 & 4.5 \\
\hline \multirow{3}{*}{ B } & \multirow{3}{*}{ Min } & VT-10 & 3541 & 4532 & 4.0 \\
& & VT-1 & 1195 & 1530 & 5.1 \\
& & VT-2 & 1193 & 1526 & 5.2 \\
& & VT-3 & 1190 & 1523 & 5.0 \\
\hline
\end{tabular}

Note: Individual and replicate sets of tests are alternately shaded and unshaded.

\subsubsection{LV-S2 Flow Angle}

Table 4.2 lists the results for the flow angle tests performed on the scale model LV-S2 stack. The results for all tests were well within the criterion of flow angle values $\leq 20^{\circ}$. Fan A flow angles ranged from $3.1^{\circ}$ to $5.3^{\circ}$, while Fan $B$ flow angles range from $3.6^{\circ}$ to $4.9^{\circ}$. The completed data sheets from these tests are available in Appendix C, Subsection C.3.

Table 4.2. Summary of LV-S2 Flow Angle Tests

\begin{tabular}{ccccc}
\hline \multirow{2}{*}{ Operating Fan } & $\begin{array}{c}\text { Flow } \\
\text { Condition }\end{array}$ & $\begin{array}{c}\text { Approx. Air No. } \\
\text { Relocity (afpm) }\end{array}$ & $\begin{array}{c}\text { Flow Angle } \\
\text { (Degrees) }\end{array}$ \\
\hline \multirow{3}{*}{ A } & \multirow{3}{*}{ Max } & FA-5 & 4196 & 3.1 \\
& & FA-6 & 4512 & 3.3 \\
& Min & FA-7 & 4504 & 4.3 \\
\cline { 2 - 5 } & \multirow{3}{*}{ Max } & FA-1 & 1680 & 5.3 \\
\hline \multirow{3}{*}{ B } & FA-9 & 4510 & 4.9 \\
& & FA-10 & 4520 & 4.5 \\
& \multirow{3}{*}{ Min } & FA-2 & 1718 & 3.6 \\
\hline & & FA-3 & 1630 & 4.6 \\
& & FA-4 & 1594 & 4.1 \\
\hline
\end{tabular}

Note: Individual and replicate sets of tests are alternately shaded and unshaded.

\subsubsection{LV-S2 Gaseous Tracer Uniformity}

During the gas tracer testing, the responses of the gas analyzers were checked against calibration standards of appropriate concentrations, and the results met the requirements of the procedure. The data sheets from these calibration checks can be found in the first portion of Appendices B.3 and C.4. 
The LV-S2 scale model had five injection ports, however, only two were employed during testing. They are injection port 2 (I2) and injection port 3 (I3) shown in Figure 2.3. Injection port 2 is in a circular section of duct and the five injection points within the circular duct are shown in Figure 3.5. Injection port 3 is located in a rectangular portion of the duct and the five injection points within this section of duct are illustrated in Figure 3.6.

Table 4.3 lists the results for all of the gaseous tracer uniformity tests performed on the scale model LV-S2 stack. No contingency sampling port was tested for this scale model; all tests were performed at the proposed test port location.

Gaseous uniformity tests using Fan A ranged from 1.3 to $6.9 \% \mathrm{COV}$ for gas injection at I2, and 0.2 to $2.9 \% \mathrm{COV}$ with gas injection at I3. Greater mixing, and lower \%COV values are expected at I3 because it is both upstream of and separated by a bend from I2, which allows more mixing to occur. Gas uniformity tests using Fan B ranged from 1.9 to 8.3 \%COV with gas injection occurring at I2 and 0.6 to $1.5 \% \mathrm{COV}$ with gas injection at I3. This greater difference in \%COV may be due, in part, to the fact that the Fan B runs with gas injection at I3 occurred during minimum velocity fan conditions.

In all cases, the gas tracer was well-mixed, with results well within the qualification criteria of $\% \mathrm{COV}$ values less than or equal to $20 \%$ and absolute value of maximum deviation less than or equal to $30 \%$. COV values were typically less than $9 \%$, with maximum deviation values typically less than $20 \%$. The least amount of mixing was observed for the Fan B maximum flow conditions. For this condition, the COV ranged from $1.9 \%$ to $8.3 \%$, while the deviation from the mean ranged from $4.4 \%$ to $19.7 \%$. The completed data sheets are available in Appendix C, Subsection C.4. 
Table 4.3. Summary of LV-S2 Gas Tracer Uniformity Tests

\begin{tabular}{|c|c|c|c|c|c|c|}
\hline $\begin{array}{c}\text { Operating } \\
\text { Fan }\end{array}$ & $\begin{array}{c}\text { Flow } \\
\text { Condition } \\
\end{array}$ & $\begin{array}{c}\text { Injection Port \& } \\
\text { Location }\end{array}$ & Run No. & $\begin{array}{c}\text { Avg } \\
\text { Velocity } \\
\text { (afpm) }\end{array}$ & $\% \mathrm{COV}$ & $\begin{array}{l}\text { Absolute \% } \\
\text { Max. Dev. } \\
\text { from Mean }\end{array}$ \\
\hline \multirow{11}{*}{ Fan A } & \multirow{11}{*}{ Max } & I2, Center & GT-16 & 4553 & 6.9 & 14.6 \\
\hline & & I2, Near & GT-17 & 4517 & 1.6 & 4.1 \\
\hline & & I2, Far & GT-18 & 4517 & 3.1 & 8.3 \\
\hline & & I2, Bottom & GT-19 & 4530 & 3.4 & 8.4 \\
\hline & & I2, Top & GT-20 & 4457 & 4.5 & 6.9 \\
\hline & & I3, Center & GT-26 & 4635 & 2.2 & 7.6 \\
\hline & & I3, Near Top & GT-27 & 4549 & 0.3 & 0.8 \\
\hline & & I3, Far Top & GT-30 & 4749 & 1.0 & 1.4 \\
\hline & & I3, Near Bottom & GT-28 & 4456 & 0.2 & 0.6 \\
\hline & & I3, Far Bottom & GT-29 & 4421 & 0.6 & 0.8 \\
\hline & & I3 Center & GT-32 & 4462 & 0.3 & 06 \\
\hline \multirow{8}{*}{ Fan B } & \multirow{8}{*}{ Max } & I2, Center & GT-7 & 4708 & 1.9 & 4.4 \\
\hline & & I2, Top & GT-9 & 4772 & 6.6 & 15.2 \\
\hline & & I2 Far & GT-10 & 4812 & 6.9 & 15.6 \\
\hline & & I2, Near & GT-11 & 4688 & 4.8 & 11.5 \\
\hline & & I2, Bottom & GT-8 & 4684 & 4.8 & 9.2 \\
\hline & & I2, Far & GT-14 & 4578 & 6.7 & 15.7 \\
\hline & & I2, Far & GT-15 & 4094 & 8.3 & 19.7 \\
\hline & & I3, Center & GT-1 & 4671 & 0.7 & 1.2 \\
\hline \multirow{6}{*}{ Fan A } & \multirow{6}{*}{ Min } & I2, Center & GT-21 & 1758 & 2.6 & 7.2 \\
\hline & & I2, Top & GT-23 & 1779 & 2.7 & 5.6 \\
\hline & & I2, Far & GT-22 & 1785 & 3.9 & 10.1 \\
\hline & & I2, Near & GT-24 & 1760 & 3.5 & 6.4 \\
\hline & & I2, Bottom & GT-25 & 1809 & 1.3 & 4.4 \\
\hline & & I2, Far & GT-31 & 1759 & 5.4 & 12.0 \\
\hline \multirow{7}{*}{ Fan B } & \multirow{7}{*}{ Min } & I3, Center & GT-2 & 1760 & 0.8 & 1.3 \\
\hline & & I3, Near Top & GT-3 & 1784 & 0.6 & 1.5 \\
\hline & & I3, Far Top & GT-4 & 1775 & 1.5 & 2.3 \\
\hline & & I3, Near Bottom & GT-5 & 1731 & 1.4 & 2.8 \\
\hline & & I3, Far Bottom & GT-6 & 1759 & 1.0 & 2.5 \\
\hline & & I3, Near Bottom & GT-12 & 1690 & 0.8 & 1.4 \\
\hline & & I3, Near Bottom & GT-13 & 1732 & 1.4 & 2.1 \\
\hline
\end{tabular}

Note: Individual and replicate sets of tests are alternately shaded and unshaded. 


\subsubsection{LV-S2 Particle Tracer Uniformity}

Table 4.4 lists the results of the LV-S2 particle tracer uniformity tests, which include non-normalized $\% \mathrm{COV}$, normalized \%COV, and absolute value of the maximum deviation from the mean for each run. The column of maximum deviation from the mean is included for information only, and is not a criterion for qualifying the sampling location. The non-normalized \%COV utilizes the particulate concentration data directly, while the normalized \%COV results from a data adjustment that has been performed for this and previous tests based on previous test experience. Typically, a concentration bias is encountered between the two traverse directions because the probe orientation is vertical through one port and horizontal through the side port. The bias is removed by adjusting the data from the traverse with the lower concentration upward by a factor to match the concentrations at the center of the duct (the common point between the two traverses). These results were then termed "normalized." In interpreting the table of particle tracer uniformity results, the normalized data are considered the pertinent result. The non-normalized data are used in comparison to the normalized result as an indication of the concentration bias.

In all but one case (PT-2), the uniformity criterion was met. Based on the normalized \%COV, the result for PT-2 was, at $20.5 \% \mathrm{COV}$, just slightly higher than the $20 \%$ criterion. However, with several repeats of that test condition, it was determined that, on average, the test condition with Fan A at maximum flow and particle injection at I3 meets the tracer uniformity criterion. The four repeat tests were all less than $20 \% \mathrm{COV}$, and the average of the five tests for this configuration was $18.0 \% \mathrm{COV}$. The poorest mixing overall was observed under Fan A Max (I3 injection) conditions. The particulate tracer uniformity for this condition ranged from 15.1 to $20.5 \%$ COV. Fan B maximum conditions, on the other hand, ranged from 14.4 to $17.3 \% \mathrm{COV}$. Results at the minimum flow conditions were generally less than $10 \% \mathrm{COV}$. The completed data sheets from these tests are available in Appendix C, Subsection C.5. 
Table 4.4. Summary of LV-S2 Particle Tracer Uniformity Tests

\begin{tabular}{|c|c|c|c|c|c|c|c|}
\hline $\begin{array}{c}\text { Operating } \\
\text { Fan }\end{array}$ & $\begin{array}{c}\text { Injection } \\
\text { Port }\end{array}$ & $\begin{array}{c}\text { Flow } \\
\text { Condition }\end{array}$ & Run No. & $\begin{array}{c}\text { Avg } \\
\text { Velocity } \\
\text { (afpm) }\end{array}$ & $\begin{array}{c}\text { Non- } \\
\text { Normalized } \\
\% \mathrm{COV}\end{array}$ & $\begin{array}{c}\text { Normalized } \\
\% \mathrm{COV}\end{array}$ & $\begin{array}{l}\text { Absolute \% } \\
\text { Max. Dev. } \\
\text { from Mean }\end{array}$ \\
\hline \multirow{11}{*}{ A } & \multirow{4}{*}{$\mathrm{I} 2$} & \multirow{4}{*}{ Max } & PT-16 & 3884 & 24.0 & 18.5 & 73.3 \\
\hline & & & PT-17 & 3852 & 20.2 & 15.3 & 65.1 \\
\hline & & & PT-18 & 2966 & 13.1 & 8.9 & 34.2 \\
\hline & & & PT-19 & 4608 & 19.2 & 19.2 & 60.7 \\
\hline & \multirow{6}{*}{ I3 } & \multirow{5}{*}{ Max } & PT-1 & 4543 & 32.7 & 17.9 & 71.4 \\
\hline & & & PT-2 & 4286 & 23.5 & 20.5 & 62.4 \\
\hline & & & PT-3 & 4254 & 21.2 & 19.9 & 71.6 \\
\hline & & & PT-20 & 3983 & 18.4 & 16.3 & 56.0 \\
\hline & & & PT-21 & 3747 & 15.1 & 15.1 & 54.2 \\
\hline & & Min & PT-4 & 1547 & 21.8 & 5.8 & 30.5 \\
\hline & I2 & Min & PT-15 & 1593 & 6.9 & 6.4 & 18.3 \\
\hline \multirow{10}{*}{ B } & $\mathrm{I} 2$ & Max & PT-11 & 3954 & 14.1 & 14.4 & 50.4 \\
\hline & \multirow{3}{*}{ I3 } & \multirow{3}{*}{ Max } & PT-8 & 4059 & 30.3 & 17.3 & 71.8 \\
\hline & & & PT-9 & 3873 & 23.9 & 16.8 & 68.5 \\
\hline & & & PT-10 & 3814 & 15.9 & 16.5 & 60.3 \\
\hline & \multirow{3}{*}{ I2 } & \multirow{3}{*}{ Min } & PT-12 & 1586 & 15.5 & 13.1 & 34.3 \\
\hline & & & PT-13 & 1599 & 8.5 & 8.8 & 15.3 \\
\hline & & & PT-14 & 1567 & 17.7 & 7.8 & 29.5 \\
\hline & \multirow{3}{*}{ I3 } & \multirow{3}{*}{ Min } & PT-5 & 1593 & 16.8 & 7.0 & 37.4 \\
\hline & & & PT-6 & 1717 & 30.0 & 7.7 & 51.5 \\
\hline & & & PT-7 & 1744 & 27.6 & 5.2 & 38.6 \\
\hline
\end{tabular}

Note: Individual and replicate sets of tests are alternately shaded and unshaded.

\subsection{LV-S3 Stack Results}

Summary tables for LV-S3 velocity, flow angle, gas tracer, and particle tracer test results are presented in the following subsections. Some test combinations were repeated (performed more than once at different times) to quantify the testing and response measurement uncertainty. Tests on the LV-S3 scale model stack include two test ports for the two different sampling systems; Test Port 1 is approximately seven duct diameters upstream of Test Port 2.

\subsubsection{Velocity Uniformity}

The initial test to determine the fan frequency setting for the LV-S3 model to achieve the desired flow conditions is included in Appendix D, Subsection D.1. Table 4.5 lists the results of the 25 velocity uniformity runs performed with the LV-S3 scale model. Table 2.2 lists the range of minimum scale model flow rates as 372 to $885 \mathrm{afpm}$ (292 to $695 \mathrm{acfm}$ ). The desired testing conditions were between the minimum scale model flow rate and the actual stack velocity. With these flow conditions, the scale 
model meets both the Reynolds number and DV criteria required to represent the actual stack with one operating fan.

All results were within the qualification criterion of \%COV values $\leq 20 \%$. COV values were typically less than $8 \%$, with values ranging from 5.1 to $9.3 \% \mathrm{COV}$. The largest COV value occurred for the $\mathrm{AB}$ fan combination at Port 2 under a minimum flow condition. The completed data sheets from these tests are available in Appendix D, Subsection D.2. 
Table 4.5. Summary of LV-S3 Velocity Uniformity Tests

\begin{tabular}{|c|c|c|c|c|c|c|}
\hline $\begin{array}{c}\text { Operating } \\
\text { Fan(s) }\end{array}$ & $\begin{array}{c}\text { Flow } \\
\text { Condition } \\
\end{array}$ & $\begin{array}{l}\text { Test } \\
\text { Port } \\
\end{array}$ & Run No. & $\begin{array}{l}\begin{array}{l}\text { Flow } \\
(\mathrm{acfm})\end{array} \\
\end{array}$ & $\begin{array}{c}\begin{array}{l}\text { Velocity } \\
\text { (afpm) }\end{array} \\
\end{array}$ & $\% \mathrm{COV}$ \\
\hline \multirow{8}{*}{$\mathrm{AB}$} & Max & 1 & VT-1 & 2308 & 2977 & 5.9 \\
\hline & Norm & 1 & VT-26 & 2143 & 2764 & 6.5 \\
\hline & \multirow{6}{*}{ Min } & \multirow{3}{*}{1} & VT-20 & 1129 & 1456 & 7.5 \\
\hline & & & VT-21 & 1127 & 1454 & 7.2 \\
\hline & & & VT-22 & 1120 & 1445 & 6.9 \\
\hline & & \multirow{3}{*}{2} & VT-23 & 1136 & 1465 & 8.4 \\
\hline & & & VT-24 & 1150 & 1484 & 9.3 \\
\hline & & & VT-25 & 1124 & 1451 & 8.0 \\
\hline \multirow{8}{*}{ BC } & \multirow{6}{*}{ Max } & \multirow{3}{*}{2} & VT-2 & 2577 & 3324 & 6.1 \\
\hline & & & VT-3 & 2555 & 3296 & 5.8 \\
\hline & & & VT-4 & 2559 & 3301 & 6.0 \\
\hline & & \multirow{3}{*}{1} & VT-5 & 2606 & 3361 & 5.6 \\
\hline & & & VT-6 & 2598 & 3351 & 5.7 \\
\hline & & & VT-7 & 2589 & 3340 & 5.3 \\
\hline & Norm & 1 & VT-8 & 2398 & 3093 & 6.0 \\
\hline & Min & 1 & VT-9 & 1238 & 1598 & 7.0 \\
\hline \multirow{2}{*}{$\mathrm{AC}$} & Max & 1 & VT-18 & 2574 & 3321 & 5.1 \\
\hline & Min & 1 & VT-19 & 1238 & 1597 & 7.1 \\
\hline \multirow{4}{*}{ A } & \multirow{4}{*}{ Norm } & \multirow{3}{*}{1} & VT-14 & 1306 & 1683 & 8.3 \\
\hline & & & VT-15 & 1300 & 1677 & 8.6 \\
\hline & & & VT-16 & 1281 & 1653 & 6.9 \\
\hline & & 2 & VT-17 & 1288 & 1662 & 7.3 \\
\hline \multirow{3}{*}{ B } & \multirow{3}{*}{ Min } & \multirow{3}{*}{1} & VT-10 & 665 & 858 & 7.3 \\
\hline & & & VT-11 & 674 & 869 & 7.3 \\
\hline & & & VT-12 & 747 & 964 & 7.9 \\
\hline C & Min & 1 & VT-13 & 941 & 1214 & 7.6 \\
\hline
\end{tabular}

\subsubsection{LV-S3 Flow Angle}

Table 4.6 lists the results of the flow angle tests for the LV-S3 scale model. The qualification criterion of $\leq 20^{\circ}$ was met in all cases. The largest results of $10.7^{\circ}$ and $10.3^{\circ}$ occurred during tests of minimum flow conditions for Fan B and Fan C, respectively. Flow angle results were typically less than $7^{\circ}$. The completed data sheets from these tests are available in Appendix D, Subsection D.3. 
Table 4.6. Summary of LV-S3 Flow Angle Tests

\begin{tabular}{|c|c|c|c|c|c|}
\hline $\begin{array}{c}\text { Operating } \\
\text { Fan }\end{array}$ & $\begin{array}{c}\text { Flow } \\
\text { Condition }\end{array}$ & $\begin{array}{l}\text { Test } \\
\text { Port }\end{array}$ & Run No. & $\begin{array}{c}\text { Approx. Air } \\
\text { Velocity (afpm) }\end{array}$ & $\begin{array}{l}\text { Mean Absolute } \\
\text { Flow Angle }\left({ }^{\circ}\right)\end{array}$ \\
\hline \multirow{8}{*}{$\mathrm{B} \& \mathrm{C}$} & \multirow{6}{*}{ Max } & \multirow{3}{*}{1} & FA-24 & 3334 & 2.8 \\
\hline & & & FA-25 & 3296 & 2.8 \\
\hline & & & FA-26 & 3577 & 3.3 \\
\hline & & \multirow{3}{*}{2} & FA-27 & 3668 & 3.2 \\
\hline & & & FA-28 & 3668 & 3.9 \\
\hline & & & FA-29 & 3569 & 2.9 \\
\hline & Normal & 1 & FA-23 & 3311 & 3.0 \\
\hline & Min & 1 & FA-22 & 1587 & 2.2 \\
\hline \multirow{8}{*}{$A \& B$} & Max & 1 & FA-1 & 3196 & 7.4 \\
\hline & Normal & 1 & FA-2 & 3104 & 6.8 \\
\hline & \multirow{6}{*}{ Min } & \multirow{3}{*}{1} & FA-3 & 1517 & 6.3 \\
\hline & & & FA-4 & 1533 & 9.8 \\
\hline & & & FA-5 & 1533 & 5.0 \\
\hline & & \multirow{3}{*}{2} & FA-11 & 1516 & 2.1 \\
\hline & & & FA-12 & N.A. & 2.6 \\
\hline & & & FA-13 & 1544 & 2.3 \\
\hline \multirow{3}{*}{$\mathrm{A} \& \mathrm{C}$} & Max & 1 & FA-21 & 3549 & 4.0 \\
\hline & \multirow{2}{*}{ Min } & 1 & FA-20 & 1636 & 5.8 \\
\hline & & 2 & FA-14 & 1744 & 3.2 \\
\hline \multirow{4}{*}{ A } & \multirow{4}{*}{ Normal } & 1 & FA-7 & 1800 & 7.8 \\
\hline & & \multirow{3}{*}{2} & FA-8 & 1710 & 6.1 \\
\hline & & & FA-9 & 1699 & 4.9 \\
\hline & & & FA-10 & 1577 & 3.1 \\
\hline $\mathrm{B}$ & Min & 1 & FA-6 & 797 & 10.7 \\
\hline \multirow{5}{*}{$\mathrm{C}$} & \multirow{5}{*}{ Min } & \multirow{3}{*}{1} & FA-17 & 1032 & 5.4 \\
\hline & & & FA-18 & 1032 & 6.3 \\
\hline & & & FA-19 & 1060 & 8.8 \\
\hline & & \multirow[b]{2}{*}{2} & FA-15 & 1091 & 8.6 \\
\hline & & & FA-16 & 1184 & 10.3 \\
\hline
\end{tabular}

Note: Individual and replicate sets of tests are alternately shaded and unshaded.

\subsubsection{LV-S3 Gaseous Tracer Uniformity}

Fifty-one gaseous tracer tests were conducted with the LV-S3 scale model. Table 4.7 lists the test results for the tests that are considered to have valid results. Gas tracer tests $27 \& 28$ are not included in Table 4.7 due to potential errors in the injection probe position. Test results were typically less than $1 \% \mathrm{COV}$, with similarly small \%maximum deviation values, which indicates a high level of tracer mixing at both Test Ports. This result was expected, given the number of bends and duct runs between the injection and sampling points. The completed data sheets from the LV-S3 gas tracer tests are available in Appendix D, Subsection D.4. 
Table 4.7. Summary of LV-S3 Gas Tracer Uniformity at Test Ports 1 and 2

\begin{tabular}{|c|c|c|c|c|c|c|c|}
\hline $\begin{array}{l}\text { Operating } \\
\text { Fan(s) }\end{array}$ & $\begin{array}{l}\text { Test } \\
\text { Port } \\
\end{array}$ & $\begin{array}{c}\text { Flow } \\
\text { Condition } \\
\end{array}$ & Injection Point & Run No. & $\begin{array}{c}\text { Avg } \\
\text { Velocity } \\
\text { (afpm) }\end{array}$ & $\% \mathrm{COV}$ & $\begin{array}{l}\text { Abs. \% Max. Dev. } \\
\text { from Mean }\end{array}$ \\
\hline \multirow{19}{*}{$\mathrm{B} \& \mathrm{C}$} & \multirow{7}{*}{2} & \multirow{7}{*}{ Max } & Center & GT-1 & 3590 & 0.9 & 1.1 \\
\hline & & & Center & GT-22 & 3298 & 0.7 & 0.8 \\
\hline & & & \multirow{2}{*}{$\begin{array}{c}\text { Center } \\
\text { Top }\end{array}$} & GT-23 & 3307 & 0.8 & 1.1 \\
\hline & & & & GT-2 & 3463 & 0.3 & 0.7 \\
\hline & & & \multirow{2}{*}{$\begin{array}{l}\text { Bottom } \\
\text { Far }\end{array}$} & GT-3 & 3323 & 0.2 & 0.5 \\
\hline & & & & GT-4 & 3323 & 0.4 & 0.7 \\
\hline & & & Near & GT-5 & 3441 & 0.2 & 0.4 \\
\hline & \multirow{7}{*}{1} & \multirow{7}{*}{ Max } & Near & GT-6 & 3464 & 0.7 & 1.1 \\
\hline & & & Near & GT-20 & 3332 & 0.4 & 0.8 \\
\hline & & & Near & GT-21 & 3351 & 1.4 & 1.7 \\
\hline & & & Center & GT-7 & 3410 & 0.2 & 0.7 \\
\hline & & & Far & GT-8 & 3404 & 0.3 & 0.6 \\
\hline & & & Top & GT-9 & 3655 & 0.2 & 0.6 \\
\hline & & & Bottom & GT-10 & 3839 & 0.2 & 0.4 \\
\hline & \multirow{2}{*}{1} & \multirow{2}{*}{ Normal } & Center & GT-18 & 3267 & 0.6 & 0.9 \\
\hline & & & Center & GT-19 & 3171 & 0.3 & 0.7 \\
\hline & \multirow{3}{*}{1} & \multirow{3}{*}{ Min } & Center & GT-15 & 1564 & 0.3 & 0.7 \\
\hline & & & Center & GT-16 & 1555 & 0.4 & 0.7 \\
\hline & & & Center & GT-17 & 1646 & 0.3 & 0.8 \\
\hline \multirow{15}{*}{$A \& B$} & \multirow{4}{*}{1} & \multirow{2}{*}{ Max } & Top & GT-49 & 3046 & 1.2 & 1.7 \\
\hline & & & Top & GT-50 & 3022 & 0.4 & 1.0 \\
\hline & & \multirow{2}{*}{ Normal } & Top & GT-46 & 2872 & 1.0 & 1.3 \\
\hline & & & Top & GT-47 & 2922 & 0.3 & 0.7 \\
\hline & \multirow{9}{*}{2} & \multirow{9}{*}{ Min } & Center & GT-37 & 1532 & 0.6 & 1.1 \\
\hline & & & Near Wall & GT-38 & 1541 & 0.6 & 0.9 \\
\hline & & & Far Wall & GT-39 & 1513 & 0.3 & 0.6 \\
\hline & & & Top & GT-40 & 1513 & 0.7 & 1.0 \\
\hline & & & Top & GT-48 & 1532 & 0.4 & 0.8 \\
\hline & & & Bottom & GT-41 & 1519 & 0.3 & 0.9 \\
\hline & & & Bottom & GT-51 & 1509 & 1.0 & 1.3 \\
\hline & & & Top & GT-42 & 1507 & 0.4 & 0.8 \\
\hline & & & Top & GT-43 & 1495 & 0.3 & 1.0 \\
\hline & \multirow{2}{*}{1} & \multirow{2}{*}{ Min } & Top & GT-44 & 1374 & 1.6 & 2.0 \\
\hline & & & Top & GT-45 & 1372 & 0.3 & 0.6 \\
\hline \multirow{7}{*}{$A \& C$} & & & Center & GT-35 & 3483 & 1.1 & 1.4 \\
\hline & 1 & Max & Center & GT-36 & 3485 & 0.3 & 0.7 \\
\hline & & & Center & GT-26 & 1614 & 0.3 & 0.7 \\
\hline & & & Far & GT-29 & 1657 & 0.3 & 0.5 \\
\hline & 1 & Min & Bottom & GT-30 & 1627 & 0.3 & 0.6 \\
\hline & & & Тор & GT-31 & 1665 & 0.3 & 0.7 \\
\hline & & & Near & GT-32 & 1593 & 0.5 & 0.9 \\
\hline & 1 & Normal & Center & GT-33 & 1616 & 0.3 & 0.7 \\
\hline$A$ & 1 & Normal & Center & GT-34 & 1610 & 0.3 & 0.7 \\
\hline A & 2 & & Center & GT-24 & 1579 & 0.8 & 1.2 \\
\hline & 2 & Normal & Center & GT-25 & 1541 & 1.3 & 1.7 \\
\hline$R$ & & & Center & GT-11 & 956 & 0.5 & 1.3 \\
\hline B & 1 & Min & Center & GT-12 & 946 & 0.4 & 0.9 \\
\hline & & & Center & GT-13 & 1086 & 0.4 & 0.7 \\
\hline C & 1 & Min & Center & GT-14 & 1041 & 1.8 & 3.9 \\
\hline
\end{tabular}




\subsubsection{LV-S3 Particle Tracer Uniformity}

Particle tracer uniformity tests were performed with the LV-S3 scale model stack at two sampling ports using several injection ports. Table 4.8 shows particle tracer uniformity test results with and without normalization applied for the LV-S3 scale model tests using injection port I5. Normalization refers to the removal of the concentration bias observed between the measurements from the bottom and side ports at a test location, as described in Section 4.1.4. As an example of the effect of normalization, measured particle concentration data from PT-32 is presented in Figure 4.1. The column of maximum deviation from the mean is included in these summary tables for information only, and is not a criterion for qualifying the sampling location. The completed data sheets from all tests are available in Appendix D, Subsection D.5.

As shown in Table 4.8, the first few particle uniformity tests failed to meet the criterion of $\leq 20 \%$ COV. PT-1 through PT-4 had normalized COV values of about $22 \%$. Particulate uniformity results greater than $20 \% \mathrm{COV}$ were not expected on this rather long stack, particularly since the gaseous tracer uniformity results had such low values. As described previously, for any particular stack flow condition, there is a range of acceptable scale model velocity conditions that can be used to represent that condition. As a result, test run PT-6, which had a lower velocity (than PT-1 through PT-3) and which still represents a maximum stack condition, resulted in a significantly lower (and within qualification criterion) uniformity result of $12.3 \% \mathrm{COV}$. Consequently, additional testing with the BC fan combination was performed to measure the particulate tracer uniformity as a function of stack velocity. Particulate mixing had a high positive correlation to the average velocity in the stack. Table 4.9 presents particle tracer uniformity data collected versus variable air velocity, with data collected at both Port 1 and Port 2 . Figure 4.2 graphically presents the data from Table 4.9 , and the linear fit for the data from each port. Although the linear correlation is an unexpected result, and the high level of correlation was unanticipated.

Since some unforeseen results were encountered within the initial and velocity varying tests, additional tests (PT-36 through PT-40) were performed to examine effects of moving the injection port further upstream from I5. Injection port numbers with higher numerical values are further upstream (closer to the fans). Results from these tests are presented in Table 4.10. As expected, these data show that, as the injection port is moved further upstream from the test port, mixing is more uniform. There is a rather significant increase in tracer uniformity (or reduction in \%COV) between I7 and I8. While there is a bend between I5 and I6, and again between I6 and I7, there is a significant length of stack duct as well as a bend between I7 and I8, which appears to increase tracer mixing significantly.

The first test among the injection location tests was PT-40, a repeat of the "failed" tests PT- 1 through PT-3. However, PT-40 had a normalized \%COV of $16.0 \%$, which is both below the $20 \%$ criterion and lower than the average value of $21.8 \% \mathrm{COV}$ from PT-1 through PT-3. Furthermore, a separate test, PT-41 was a repeat of the "failed" test run PT-4. PT-41 had a moderately low result of $13.5 \% \mathrm{COV}$, compared with the $21.1 \% \mathrm{COV}$ result from PT-4. Additional tests were conducted to verify these results. These were the first scale model tests to use an aerosol generator with an all-metal design. To explore the possibility that this new aerosol generator design was contributing to large variability in the tracer uniformity test results, two tests were performed using the previous PVC aerosol generator design. PT-42 and PT-43 used the PVC aerosol generator; normalized COV values were acceptable and comparable to the PT-40 result. However, the PT-42 and PT-43 test results had unusually high normalization factors (1.53 and 3.54, respectively), so subsequent testing with the PVC aerosol generators was discontinued. 
Instead, the all-metal aerosol generator was used for the remaining tests, and PT-44 and PT-46, which were performed under the same stack conditions as PT-42 and PT-43, provided reasonably comparable results without the high normalization factor. Results of all the I5 repeat tests (PT-41 though PT-46) are summarized in Table 4.11 .

Although these later tests (in Table 4.11) passed the tracer uniformity criterion, there was no simple way to reconcile the difference between the original (PT-1 through PT-4) test results. Consequently, additional tests at an alternate injection location were performed to provide independent verification of the stack qualification metric for these two sampling port locations. Although all injection locations installed on the scale model stack are acceptable for evaluating the stack, locations that are accessible at the facility itself are preferred in case stack testing on the actual stack is later required. As a result of a site visit, injection port I7 was selected as an alternative injection location for scale model testing. Table 4.12 presents the results of tests performed with the I7 injection port under dual fan combination conditions, at two different fan settings. All tests resulted in uniformity values that were well within the $20 \%$ COV uniformity criterion, with most values less than $10 \%$ COV. As a result, this stack and test condition combination is overall qualified under the particle tracer uniformity requirement. 
Table 4.8. Summary of LV-S3 Particle Tracer Uniformity Tests with Particle Injection at I5

\begin{tabular}{|c|c|c|c|c|c|c|c|}
\hline $\begin{array}{c}\text { Operating } \\
\text { Fan(s) }\end{array}$ & $\begin{array}{l}\text { Test } \\
\text { Port }\end{array}$ & $\begin{array}{c}\text { Flow } \\
\text { Condition }\end{array}$ & Run No. & $\begin{array}{c}\text { Avg } \\
\text { Velocity } \\
\text { (afpm) }\end{array}$ & $\begin{array}{c}\text { Non- } \\
\text { normalized } \\
\% \text { COV }\end{array}$ & $\begin{array}{c}\text { Normalized } \\
\% \text { COV }\end{array}$ & $\begin{array}{l}\text { Absolute \% } \\
\text { Max. Dev. } \\
\text { from Mean }\end{array}$ \\
\hline \multirow{9}{*}{$\mathrm{B} \& \mathrm{C}$} & \multirow{4}{*}{1} & \multirow{4}{*}{ Max } & $P T-1$ & 3467 & 22.3 & 22.2 & 56.8 \\
\hline & & & $P T-2$ & 3475 & 22.6 & 21.8 & 52.5 \\
\hline & & & $P T-3$ & 3643 & 23.8 & 21.4 & 53.0 \\
\hline & & & PT-6 & 2289 & 12.7 & 12.3 & 27.7 \\
\hline & \multirow{3}{*}{2} & \multirow{3}{*}{ Max } & PT-7 & 2131 & 12.5 & 12.0 & 28.3 \\
\hline & & & PT-8 & 2230 & 12.7 & 12.0 & 29.8 \\
\hline & & & PT-9 & 2235 & 12.6 & 12.6 & 25.8 \\
\hline & 1 & Normal & $P T-4$ & 3133 & 21.1 & 21.1 & 48.9 \\
\hline & 1 & Min & PT-5 & 1620 & 9.1 & 7.9 & 20.4 \\
\hline \multirow{12}{*}{$A \& B$} & 1 & Max & PT-24 & 2515 & 15.3 & 12.9 & 36.9 \\
\hline & \multirow{2}{*}{2} & \multirow{2}{*}{ Max } & PT-28 & 3121 & 17.9 & 17.9 & 36.2 \\
\hline & & & PT-35 & 3079 & 12.5 & 12.7 & 28.7 \\
\hline & \multirow{3}{*}{1} & \multirow{3}{*}{ Normal } & PT-25 & 2103 & 10.7 & 10.2 & 23.9 \\
\hline & & & PT-26 & 1953 & 10.3 & 10.0 & 21.5 \\
\hline & & & PT-27 & 1944 & 9.7 & 9.5 & 23.1 \\
\hline & \multirow{3}{*}{1} & \multirow{3}{*}{ Min } & PT-21 & 1278 & 4.2 & 4.2 & 9.9 \\
\hline & & & PT-22 & 1254 & 5.7 & 5.4 & 12.7 \\
\hline & & & PT-23 & 1290 & 5.0 & 4.8 & 14.2 \\
\hline & \multirow{3}{*}{2} & \multirow{3}{*}{ Min } & PT-18 & 1277 & 6.6 & 4.1 & 15.7 \\
\hline & & & PT-19 & 1247 & 8.4 & 5.8 & 17.2 \\
\hline & & & PT-20 & 1278 & 6.8 & 4.7 & 14.7 \\
\hline \multirow{2}{*}{$A \& C$} & \multirow{2}{*}{1} & Max & PT-12 & 2126 & 13.4 & 11.8 & 35.3 \\
\hline & & Min & PT-14 & 1313 & 6.8 & 6.8 & 16.4 \\
\hline \multirow{4}{*}{ A } & 1 & Normal & PT-13 & 1873 & 8.8 & 9.0 & 19.8 \\
\hline & \multirow{3}{*}{2} & \multirow{3}{*}{ Normal } & PT-15 & 1836 & 11.5 & 9.1 & 26.3 \\
\hline & & & PT-16 & 1884 & 9.6 & 6.7 & 22.0 \\
\hline & & & PT-17 & 1910 & 10.2 & 8.6 & 24.1 \\
\hline B & 1 & Min & PT-10 & 921 & 9.6 & 4.2 & 15.1 \\
\hline $\mathrm{C}$ & 1 & Min & PT-11 & 1202 & 7.1 & 5.6 & 16.1 \\
\hline
\end{tabular}

Note: Individual or replicate sets of tests are alternately shaded and unshaded. Italicized results indicate tests that exceed the qualification criterion. 


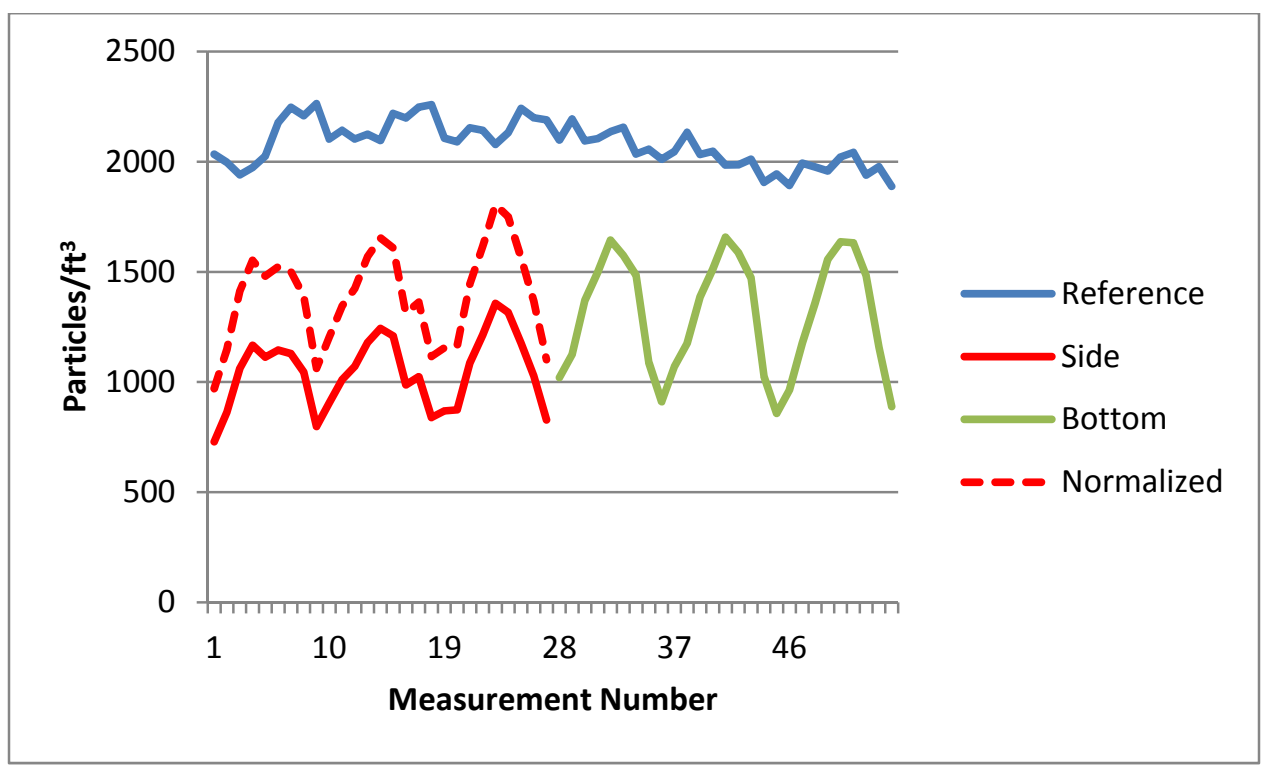

Figure 4.1. Measurement and Reference Particle Test Data from PT-32 on the LV-S3 Scale Model Stack. The side and bottom lines represent measurement traverses from the side and bottom of Test Port 1, while the reference line represents the concentration at a fixed point of the Reference Port. Normalized data are show with dotted line. The data collected from the side port have been adjusted up (normalized) by a factor of 1.33 .

Table 4.9. Summary of Velocity Variation Tests at Test Ports 1 and 2. Particle injection occurred at Injection Port 5.

\begin{tabular}{|c|c|c|c|c|c|c|}
\hline Test Port & $\begin{array}{c}\text { Fan Setting } \\
(\mathrm{Hz})\end{array}$ & Run No. & $\begin{array}{c}\text { Avg } \\
\text { Velocity } \\
\text { (afpm) }\end{array}$ & $\begin{array}{c}\text { Non- } \\
\text { normalized } \\
\% \mathrm{COV}\end{array}$ & $\begin{array}{c}\text { Normalized } \\
\% \mathrm{COV}\end{array}$ & $\begin{array}{l}\text { Absolute \% } \\
\text { Max. Dev. } \\
\text { from Mean }\end{array}$ \\
\hline \multirow{9}{*}{1} & \multirow{3}{*}{60} & PT-1 & 3467 & 22.3 & 22.2 & 56.8 \\
\hline & & PT-2 & 3475 & 22.6 & 21.8 & 52.5 \\
\hline & & PT-3 & 3643 & 23.8 & 21.4 & 53.0 \\
\hline & 55 & PT-4 & 3133 & 21.1 & 21.1 & 48.9 \\
\hline & 50 & PT-33 & 2858 & 17.1 & 16.3 & 43.9 \\
\hline & 45 & PT-34 & 2530 & 13.9 & 13.7 & 37.5 \\
\hline & 41 & PT-32 & 2107 & 18.0 & 12.6 & 39.8 \\
\hline & 40 & PT-6 & 2289 & 12.7 & 12.3 & 27.7 \\
\hline & 30 & PT-5 & 1636 & 9.1 & 7.9 & 18.5 \\
\hline \multirow{6}{*}{2} & 60 & PT-29 & 3581 & 20.2 & 19.1 & 51.9 \\
\hline & 55 & PT-31 & 2958 & 19.5 & 18.0 & 48.7 \\
\hline & 41 & PT-30 & 2263 & 11.5 & 11.5 & 29.3 \\
\hline & \multirow{3}{*}{40} & PT-7 & 2131 & 12.5 & 12.0 & 28.3 \\
\hline & & PT-8 & 2230 & 12.7 & 12.0 & 29.8 \\
\hline & & PT-9 & 2235 & 12.6 & 12.6 & 25.8 \\
\hline
\end{tabular}

Note: Individual or replicate sets of tests are alternately shaded and unshaded 


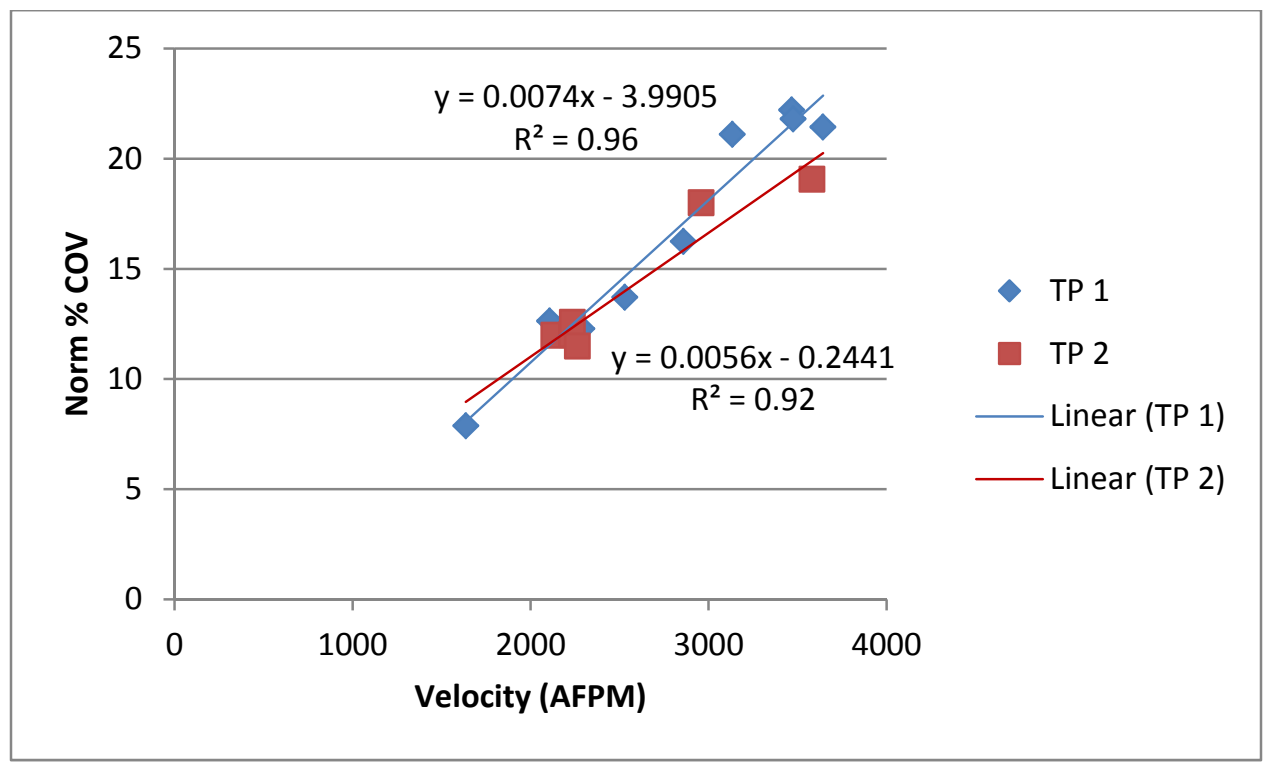

Figure 4.2. Linear Regression Results of Average Velocity Versus \%COV for Test Port 1 and 2 Data Presented in Table 4.9

Table 4.10. Summary of Tests Moving Injection Port Upstream

\begin{tabular}{|c|c|c|c|c|c|c|c|}
\hline Test Port & $\begin{array}{c}\text { Fan Setting } \\
(\mathrm{Hz})\end{array}$ & $\begin{array}{l}\text { Injection } \\
\text { Port }\end{array}$ & Run No. & $\begin{array}{l}\text { Avg Velocity } \\
\text { (afpm) }\end{array}$ & $\begin{array}{c}\text { Non- } \\
\text { normalized } \\
\% \text { COV }\end{array}$ & $\begin{array}{l}\text { Normalized } \\
\% \mathrm{COV}\end{array}$ & $\begin{array}{l}\text { Absolute \% } \\
\text { Max. Dev. } \\
\text { from Mean }\end{array}$ \\
\hline \multirow{6}{*}{1} & \multirow{6}{*}{60} & I5 & PT-40 & 3351 & 17.0 & 15.9 & 42.7 \\
\hline & & I6 & PT-36 & 3505 & 13.6 & 13.7 & 30.9 \\
\hline & & I7 & PT-37 & 3733 & 12.2 & 11.6 & 25.2 \\
\hline & & I8 & PT-38 & 3391 & 7.6 & 6.7 & 16.5 \\
\hline & & \multirow{2}{*}{ I9 } & PT-39 & 3523 & 5.8 & 5.8 & 15.7 \\
\hline & & & PT-47 & 3310 & 5.7 & 5.3 & 12.4 \\
\hline
\end{tabular}

Note: Individual or replicate sets of tests are alternately shaded and unshaded.

Table 4.11. Summary of Additional Testing to Verify B\&C Max and Norm Test Results

\begin{tabular}{|c|c|c|c|c|c|c|c|}
\hline Test Port & $\begin{array}{l}\text { Injection } \\
\text { Port }\end{array}$ & $\begin{array}{c}\text { Fan } \\
\text { Setting } \\
(\mathrm{Hz})\end{array}$ & Run No. & $\begin{array}{l}\text { Avg Velocity } \\
\text { (afpm) }\end{array}$ & $\begin{array}{c}\text { Non- } \\
\text { normalized } \\
\% \mathrm{COV}\end{array}$ & $\begin{array}{l}\text { Normalized } \\
\% \text { COV }\end{array}$ & $\begin{array}{l}\text { Absolute \% } \\
\text { Max. Dev. } \\
\text { from Mean }\end{array}$ \\
\hline \multirow{6}{*}{1} & \multirow{6}{*}{ I5 } & \multirow{4}{*}{60} & $P T-42$ & 3423 & 25.9 & 18.5 & 56.8 \\
\hline & & & $P T-43$ & 3505 & 55.5 & 16.7 & 97.6 \\
\hline & & & PT-44 & 3406 & 18.7 & 17.1 & 47.4 \\
\hline & & & PT-46 & 3789 & 17.6 & 17.4 & 43.6 \\
\hline & & \multirow{2}{*}{55} & PT-41 & 2969 & 13.4 & 13.5 & 31.5 \\
\hline & & & PT-45 & 3146 & 14.1 & 14.4 & 28.8 \\
\hline
\end{tabular}

Note: Individual or replicate sets of tests are alternately shaded and unshaded.

Italicized results indicate tests with unusually high normalization factors. 
Table 4.12. Summary of Tests with Dual Fan Combinations with Particle Injection at I7

\begin{tabular}{|c|c|c|c|c|c|c|c|}
\hline $\begin{array}{c}\text { Fan } \\
\text { Combination }\end{array}$ & $\begin{array}{c}\text { Fan Setting } \\
(\mathrm{Hz})\end{array}$ & Test Port & Run No. & $\begin{array}{c}\text { Avg } \\
\text { Velocity } \\
\text { (afpm) }\end{array}$ & $\begin{array}{c}\text { Non- } \\
\text { normalized } \\
\% \mathrm{COV}\end{array}$ & $\begin{array}{c}\text { Normalized } \\
\% \mathrm{COV}\end{array}$ & $\begin{array}{l}\text { Absolute \% } \\
\text { Max. Dev. } \\
\text { from Mean }\end{array}$ \\
\hline \multirow{4}{*}{$\mathrm{BC}$} & \multirow{2}{*}{60} & 1 & PT-48 & 3685 & 11.6 & 10.6 & 31.1 \\
\hline & & 2 & PT-50 & 3282 & 9.2 & 9.1 & 21.4 \\
\hline & \multirow{2}{*}{25} & 1 & PT-49 & 1259 & 5.9 & 4.5 & 13.2 \\
\hline & & 2 & PT-51 & 1235 & 9.0 & 4.2 & 15.3 \\
\hline \multirow{4}{*}{ AC } & \multirow{2}{*}{60} & 1 & PT-55 & 3519 & 11.0 & 11.0 & 27.2 \\
\hline & & 2 & PT-52 & 3375 & 11.4 & 11.0 & 24.8 \\
\hline & \multirow{2}{*}{25} & 1 & PT-54 & 1295 & 4.1 & 3.9 & 8.9 \\
\hline & & 2 & PT-53 & 1251 & 14.5 & 5.3 & 22.6 \\
\hline \multirow{4}{*}{$\mathrm{AB}$} & \multirow{2}{*}{60} & 1 & PT-56 & 2999 & 9.8 & 9.7 & 23.1 \\
\hline & & 2 & PT-59 & 3019 & 14.5 & 9.9 & 31.6 \\
\hline & \multirow{2}{*}{25} & 1 & PT-57 & 1166 & 4.9 & 3.8 & 11.6 \\
\hline & & 2 & PT-58 & 1194 & 6.7 & 4.0 & 12.5 \\
\hline
\end{tabular}

Note: Individual or replicate sets of tests are alternately shaded and unshaded. 


\subsection{Conclusions}

The results of the stack qualification tests performed with the LV-S2 and LV-S3 scale model stack are summarized in Table 5.1. The criteria for sampling probe locations given in ANSI/HPS N13.1-1999, Sampling and Monitoring Releases of Airborne Radioactive Substances from the Stack and Ducts of Nuclear Facilities, were met in all cases. These criteria address the capability of the sampling probe to extract a sample that represents the effluent stream. The range of results presented in Table 5.1 for the LV-S2 stack covers the designed location for the air sampling probe, Test Port 1. For the LV-S3 stack, tests were conducted at two locations; Test Port 1 represents the Record Sample location and seven duct diameters downstream is the continuous air monitor or CAM sampling location, Test Port 2. The results for both Test Ports are presented in Table 5.1.

Table 5.1. Summary of Results for the LV-S2 and LV-S3 Scale Model Stacks

\begin{tabular}{|c|c|c|c|c|c|}
\hline & \multirow{2}{*}{$\begin{array}{c}\text { Acceptance } \\
\text { Criteria }\end{array}$} & \multirow{2}{*}{ Units } & \multirow{2}{*}{$\begin{array}{c}\text { LV-S2 } \\
\text { Test Port } 1\end{array}$} & \multicolumn{2}{|c|}{ LV-S3 } \\
\hline & & & & Test Port 1 & Test Port 2 \\
\hline $\begin{array}{l}\text { Velocity } \\
\text { Uniformity }\end{array}$ & $\leq 20$ & $\% \mathrm{COV}$ & $4.0-5.5$ & $5.1-8.6$ & $5.8-9.3$ \\
\hline Flow Angle & $\leq 20$ & Degrees & $3.1-5.3$ & $2.2-10.7$ & $2.1-10.3$ \\
\hline \multirow{2}{*}{$\begin{array}{l}\text { Gas Tracer } \\
\text { Uniformity }\end{array}$} & $\leq 20$ & $\% \mathrm{COV}$ & $0.2-8.3$ & $0.2-1.8$ & $0.2-1.3$ \\
\hline & $\leq 30$ & $\begin{array}{l}\text { Maximum \% } \\
\text { Deviation from Mean }\end{array}$ & $0.6-19.7$ & $0.4-3.9$ & $0.4-1.7$ \\
\hline $\begin{array}{l}\text { Particle Tracer } \\
\text { Uniformity }\end{array}$ & $\leq 20$ & Normalized \%COV & $5.2-19.2$ & $3.8-11.0$ & $4.0-11.0$ \\
\hline
\end{tabular}

Based on these scale model tests, the locations proposed for the air sampling probes in each of the LV-S2 and LV-S3 stacks meet the requirements of the ANSI/HPS N13.1-1999 standard. Additional velocity uniformity and flow angle tests on the actual stacks will be necessary during cold startup to confirm the validity of the scale model results in representing the actual stacks. In particular, the velocity uniformity test results for the actual stacks must be within $5 \% \mathrm{COV}$ of the range of results listed above for the scale model so that scale model results can be said to be representative of the stack. For example, if the actual LV-S2 stack sampling probe is located in a position corresponding to Test Port 1, the measured velocity uniformity \%COV should be between 0.0 and $10.5 \% \mathrm{COV}$ (non-negative value for $4.0-5=0.0$, and $5.5+5=10.5$ ). The velocity uniformity test results summarized in Table 5.1 cover a range of flow conditions that are expected to bracket the conditions of the actual stack. For cold startup tests, the DV value and Reynolds number should meet the criteria listed in Section 1.1 (i.e., DV within a factor of six and Reynolds number $>10,000$ ). The velocity uniformity acceptance range would be constructed using the scale model results that correspond to the probe location and fan operating conditions present during the test on the actual stack.

If in-plant qualification testing becomes necessary, results comparable to this report are more likely when the tracer injection locations are comparable to those used for these tests. For the LV-S2 system either injection port (I2 or I3) would be acceptable; however, for the LV-S3 system, I-7 is preferred. 



\subsection{References}

10 CFR 830, Subpart A. 2008. “Quality Assurance Requirements.” Code of Federal Regulations, U.S. Department of Energy.

40 CFR 60, Appendix A, Method 1. "Method 1-Sample and Velocity Traverses for Stationary

Sources.” Code of Federal Regulations, U.S. Environmental Protection Agency.

40 CFR 61, Subpart H. "National Emission Standard for Emissions of Radionuclides Other Than Radon From Department of Energy Facilities.” Code of Federal Regulations, U.S. Environmental Protection Agency.

ANSI/HPS N13.1-1999. Sampling and Monitoring Releases of Airborne Radioactive Substances from the Stacks and ducts of Nuclear Facilities. American National Standards Institute and the Health Physics Society, McLean, VA (reaffirmed in 2011 as ANSI/HPS N13.1-2011).

American Society of Mechanical Engineers (ASME). 2001. Quality Assurance Requirements for Nuclear Facility Applications. NQA-1-2000, New York, NY.

DOE Order 414.1D. “Quality Assurance.” U.S. Department of Energy, Washington, D.C. 



\section{Appendix A}

LV-S2 and LV-S3 Scale Model Testing Supporting Documents List 



\section{Appendix A: Document List}

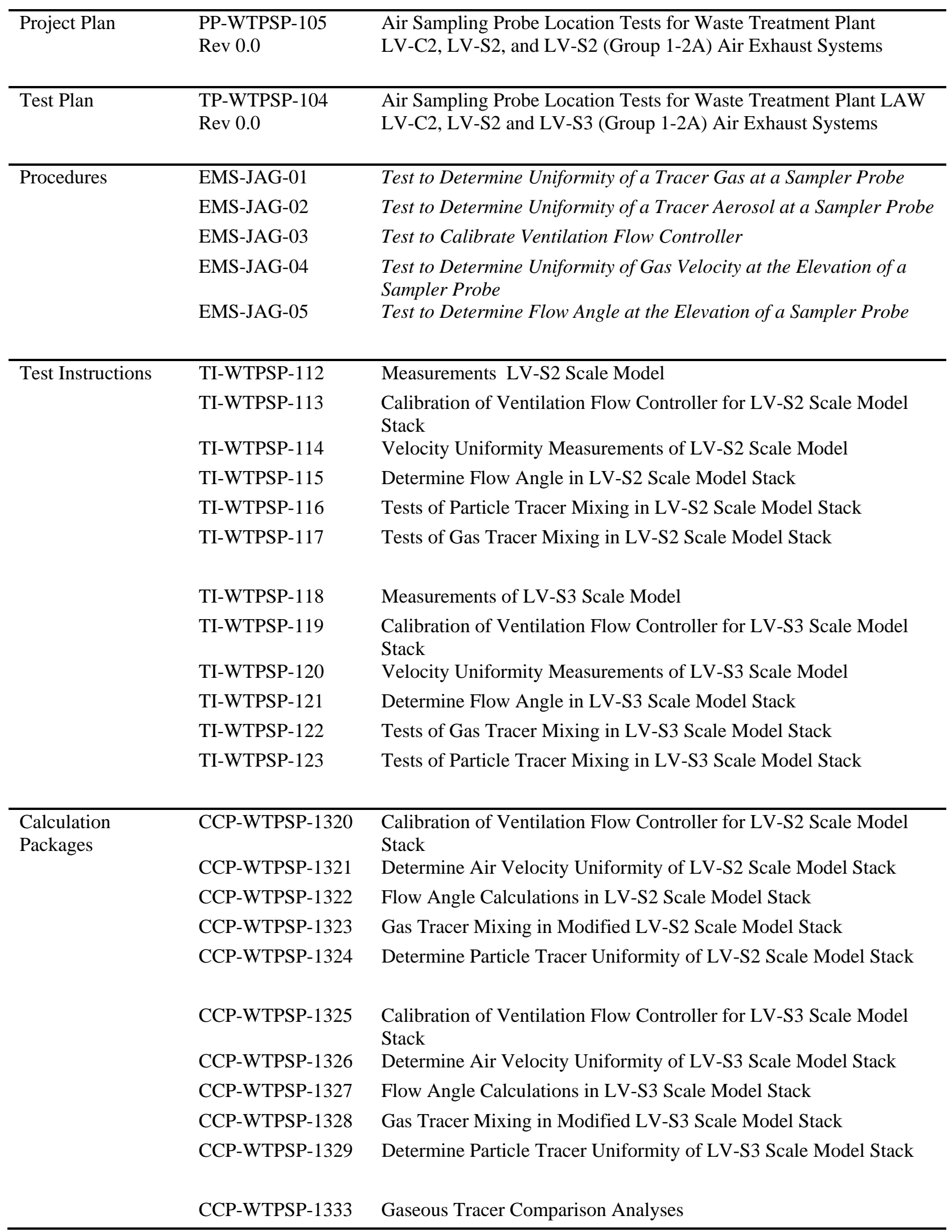



Appendix B

\section{$\mathrm{N}_{2} \mathrm{O}$ and $\mathrm{SF}_{6}$ Comparison Tests}





\section{Appendix $\mathrm{B}: \mathrm{N}_{2} \mathrm{O}$ and $\mathrm{SF}_{6}$ Comparison Tests}

The objective of replacing $\mathrm{SF}_{6}$ with $\mathrm{N}_{2} \mathrm{O}$ for the tracer gas in the gaseous tracer uniformity testing was primarily to reduce the global warming effects of these tests. An added benefit to the use of $\mathrm{N}_{2} \mathrm{O}$ is that, after initial investments in equipment upgrades, the cost of the gas itself is lower than $\mathrm{SF}_{6}$, so modest cost savings are realized. Although, in principle, all tracer gases should behave in an identical manner for measuring mixing within a stack, a series of tests with a basis in statistics was performed to demonstrate the equivalence of $\mathrm{N}_{2} \mathrm{O}$ testing to $\mathrm{SF}_{6}$ testing in the context of these qualification tests. This Appendix contains three sets of information, which describe the evaluation performed.

Subsection B.1 contains the sampling plan that was drafted to develop a set of tests that would determine whether $\mathrm{N}_{2} \mathrm{O}$ and $\mathrm{SF}_{6}$ are equivalent gases in the context of these stack qualification tests. Several sets of paired tests, where the $\mathrm{N}_{2} \mathrm{O}$ and $\mathrm{SF}_{6}$ were used in back-to-back tests, were outlined to determine, with statistical significance, whether the outcome of the stack qualification test would be unchanged by the use of this alternative gas. Subsection B.2 contains a summary and analysis of the tests that were performed. This demonstrates that the $\mathrm{N}_{2} \mathrm{O}$ gas tests were equivalent to the $\mathrm{SF}_{6}$ gas tests, and was the basis for proceeding with the exclusive use of $\mathrm{N}_{2} \mathrm{O}$ for the tests described in the body of this report. Subsection B.3 contains a summary of the conclusions that were made in the N2O and SF6 comparison. Finally, Subsection B.4 contains the data sheets from the tests that are summarized in Subsection B.2. Statistical and experimental terms that are used in the following sections have been defined in Table B.1. 
Table B.1. Statistical and Experimental Terms Used in these Appendix Sections

\begin{tabular}{|c|c|}
\hline $\begin{array}{l}\text { Analysis of Variance } \\
(\text { AOV) }\end{array}$ & A statistical test determining whether the means of groups are equal. \\
\hline$\% C O V$ & $\begin{array}{l}\text { The standard deviation divided by the mean for a given set of sample data. Also known as } \\
\text { the relative standard deviation (RSD). }\end{array}$ \\
\hline Max \% Deviation & The largest absolute deviation of a single data point from the mean. \\
\hline Response Variable & $\begin{array}{l}\text { The data value or values that are measured that help test the hypotheses. In this case } \\
\% \mathrm{COV} \text { and Max \% Deviation are being measured for each gas, } \mathrm{N}_{2} \mathrm{O} \text { and } \mathrm{SF}_{6} \text {. Because } \\
\text { the hypothesis looks at the difference between the gases, the response variable is actually } \\
\text { the difference between the gases with respect to \%COV and also Max \% Deviation. }\end{array}$ \\
\hline Statistical Power & $\begin{array}{l}\text { The probability that the test will conclude there is a significant difference between the } \\
\text { gases, given that there truly is a difference between the gases. Increasing the sample size } \\
\text { will increase the power. Ideally, enough samples should be taken to have at least } 80 \% \\
\text { statistical power. }\end{array}$ \\
\hline Null Hypothesis & $\begin{array}{l}\text { What the experimenter is trying to disprove. In this case, the null hypothesis is that there } \\
\text { is no difference between the gases. }\end{array}$ \\
\hline Delta & $\begin{array}{l}\text { The minimum amount of differences between the gas measurements that would indicate } \\
\text { that the gases are in fact significantly different. For example, how much bigger or smaller } \\
\text { should the \%COV or Max \% Deviation values of } \mathrm{N}_{2} \mathrm{O} \text { be from } \mathrm{SF}_{6} \text { to be convinced that } \\
\mathrm{N}_{2} \mathrm{O} \text { and } \mathrm{SF}_{6} \text { are in fact different? }\end{array}$ \\
\hline$d f$ & Degrees of freedom. \\
\hline Standard Deviation & The average distance each data value is from the mean. \\
\hline Significance Level & $\begin{array}{l}\text { Otherwise known as } 1 \text { - confidence level. This is the probability of a type I error, which } \\
\text { is concluding to reject the null hypothesis when the null was actually true. The } \\
\text { significance level was set to } 0.05 \text {, meaning that the confidence level was } 0.95 \text { ( } 95 \% \\
\text { confidence). }\end{array}$ \\
\hline Sum of Squares (SS) & $\begin{array}{l}\text { An intermediate step needed in the AOV to help calculate the test statistic. It is an } \\
\text { unscaled measure of variability. }\end{array}$ \\
\hline Mean Square (MS) & $\begin{array}{l}\text { An intermediate step needed in the AOV to help calculate the test statistic equal to the } \\
\text { SS/df. It is measure of the estimated variance. }\end{array}$ \\
\hline F & Test statistic based on an F distribution and is a ratio of mean square (MS) values. \\
\hline p-value & $\begin{array}{l}\text { The probability of obtaining a test statistic at least as extreme as the one that was actually } \\
\text { observed from the sample data, assuming that the null hypothesis is true. }\end{array}$ \\
\hline
\end{tabular}

\section{B.1 $\mathrm{N}_{2} \mathrm{O}$ and $\mathrm{SF}_{6}$ Comparison Sampling Plan}

The goal of the sampling plan was to determine if $\mathrm{N}_{2} \mathrm{O}$ and $\mathrm{SF}_{6}$ perform similarly in the role of being a tracer during mixing tests. To achieve this goal, the test conditions needed to be determined, as well as the number of samples. 
Preliminary data were collected to help determine the proper test conditions and the necessary number of samples. These data were acquired from the LB-S1 tests performed using $\mathrm{SF}_{6}$, as well as two additional $\mathrm{N}_{2} \mathrm{O}$ tests. Two different responses were recorded during each mixing test: 1) \%COV (also referred to as \%RSD), and 2) \%Max Deviation (\%MaxDev) (see definitions above). Preliminary data were collected by varying the levels of three different variables: 1) fan configuration, 2) injection position, and 3) fan control frequency (airflow control). Two of three fans (labeled A, B, and C) were used during each test, so the fan configuration levels were $\mathrm{AB}, \mathrm{AC}$, and $\mathrm{BC}$. Five injection positions were tested: bottom, center, far wall, near wall, and top. Three airflow levels (fan control frequencies) were tested: max, normal, and min. The scale model used for these tests is described in report WTP-RPT-227, Assessment of Waste Treatment Plant Lab C3V (LB-S1) Stack Sampling Probe Location for Compliance with ANSI/HPS N13.1-1999.

Preliminary data were analyzed using analysis of variance (AOV) and the following AOV model:

$$
Y=\mu+f_{i}+p_{j}+c_{k}
$$

where $Y$ is the response variable (\%COV or \%MaxDev), $\mu$ is the overall mean, $f_{i}$ is the fan configuration, $p_{j}$ is the injection position, and $c_{k}$ is the fan control frequency. Significant differences were found between the fan configurations and between the injection positions. There were no significant differences in \%COV and \%MaxDev as a function of airflow (flow control). The boxplots below (Figure B.1) show which levels resulted in higher or lower \%COV and \%MaxDev values.

These analyses are useful in determining which test conditions should be run, so that the sampling plan will provide varying airflow conditions to best compare $\mathrm{N}_{2} \mathrm{O}$ to $\mathrm{SF}_{6}$. Because there were no significant differences between the airflow conditions (fan control settings), this variable does not need to be varied during the tests. All tests should then be performed at one flowrate level (e.g., "normal”). The fan configuration analysis shows that using the A-C fan combination is quite different from the A-B and B-C combinations. Injection position analyses showed that the bottom injection is generally higher with a little larger variability, and the center is generally smaller, with the other three levels very similar. To represent the widest range in flow conditions, the best levels to select for injection position would be bottom and center.

Statistical power (see definition in previous section) analyses were performed to help determine how many samples are needed. The necessary inputs to calculate statistical power are: 1) number of samples, 2) delta, 3) standard deviation, and 4) significance level (definitions for each input are found in previous section above). Significance level was set to 5\%, meaning that the confidence level was set to 95\% (very common level). The preliminary data of the differences between the gases showed that a conservative estimate for the standard deviation is 0.7 (when using \%COV or \%MaxDev). This means that on average the difference between $\mathrm{N}_{2} \mathrm{O}$ and $\mathrm{SF}_{6}$ values (under common testing conditions) varies from the mean by 0.7 (using either \%COV or \%MaxDev). The number of samples was varied between 2 and 10 . Each sample was actually a pair of samples (one for each gas). The delta was also varied from 1 to 2.5 by 0.25 intervals. 
Fan Configuration

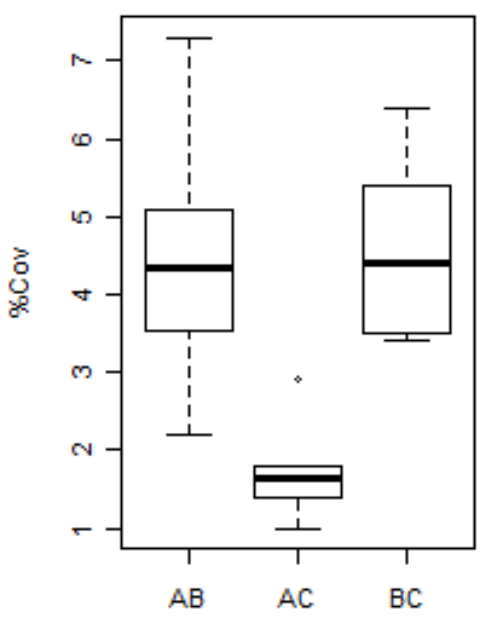

Fan Configuration

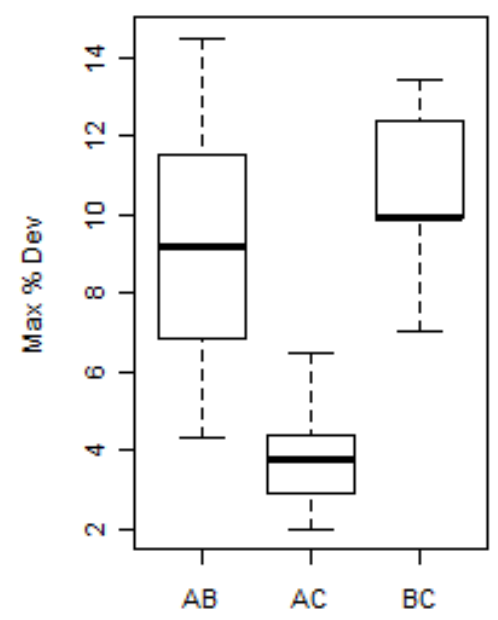

Injection Position

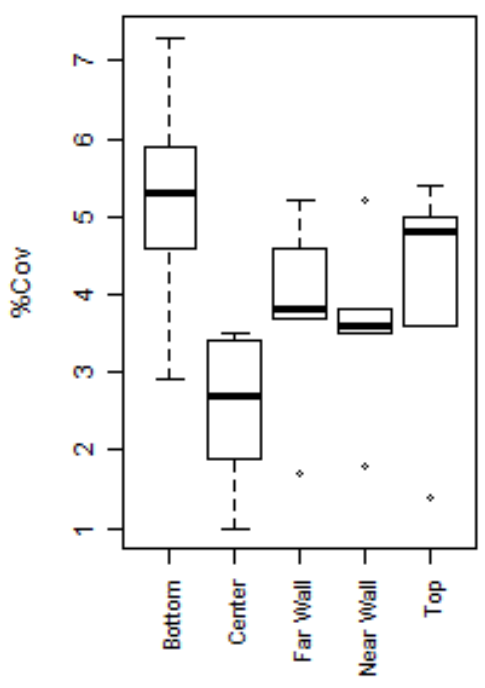

Injection Position

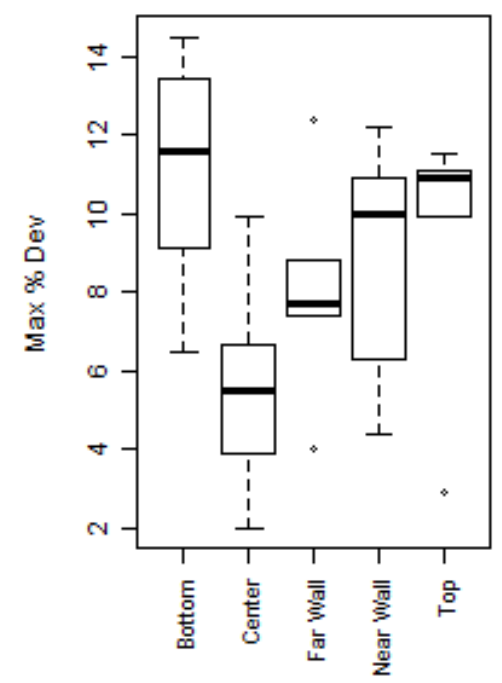

Fan Control

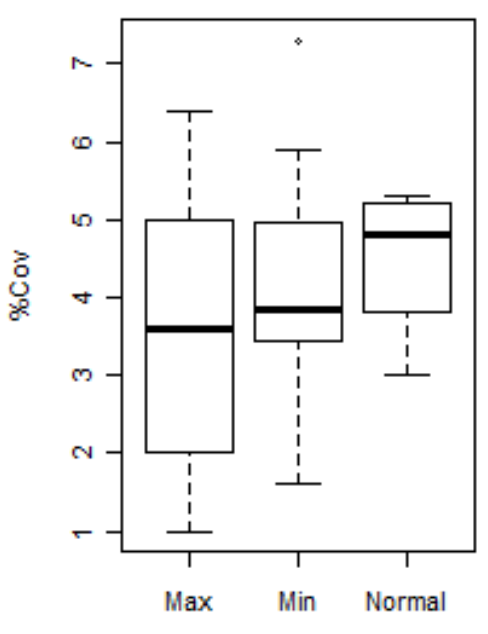

Fan Control

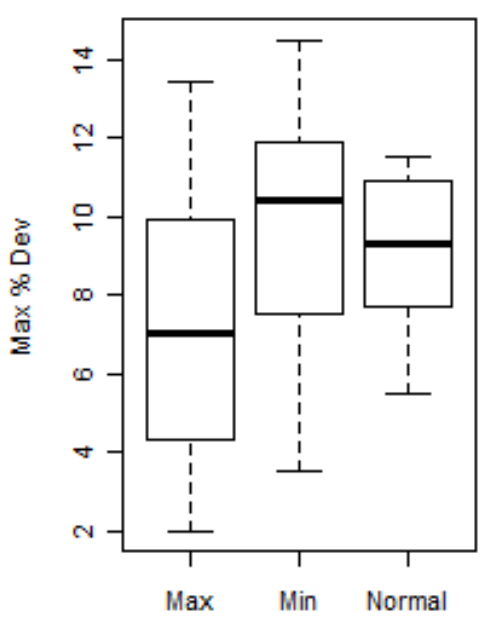

Figure B.1. Box Plots of the Preliminary Data from the LB-S1 Gaseous Tracer Tests

Figure B.2 shows how the statistical power increases as the sample size increases for each of the measures of delta assuming a standard deviation of the difference values to be 0.7 . For example, if the user wants to conclude that any difference larger than $1 \% \mathrm{COV}$ as statistically different (this is what delta measures) and do this with an $80 \%$ statistical power probability, then the user would need to take six samples per pair. (Note: because sample numbers cannot be partial, number of samples should always be rounded up.)

With $99 \%$ power a difference of two would be detectable with six sampling pairs. A delta of two is a reasonable assumption; based on previous experience, if the sample data from the two gases differed by less than $2 \% \mathrm{COV}$, the gases would not be considered to be significantly different. Six sampling pairs 
would amount to three days of testing, where one test pair is run in the morning and another test pair is run in the afternoon. Six pairs also fit well with the possible test conditions. There are two different fan configurations that should be included and two injection positions plus an additional injection position not run during the preliminary tests (called "Other"). This additional position of "Other" is one that would mix poorly and give a better opportunity to see differences between the gases (if differences exist) in the extreme case. A complete experiment would then consist of two fan configurations and three injection points, which would amount to six total test pairs.

These power calculations were made assuming a standard deviation of 0.7 , which was based on a small number of paired differences between the gases. An analysis of previous data calculated the expected random standard deviation which occurs when using one gas. This standard deviation was 1.3. This value could be considered a worst-case value for the standard deviation of the difference between the gases, because when taking the standard deviation of differences between two groups, the uncertainty (standard deviation) decreases. Using a standard deviation of 1.3, a worst-case scenario was calculated and the results are shown in Figure B.3. From this it would be concluded that with six sample pairs and a delta of $2 \% \mathrm{COV}$, the test would be expected to have at least $85 \%$ statistical power. This is a further indication that six sample pairs should be sufficient.

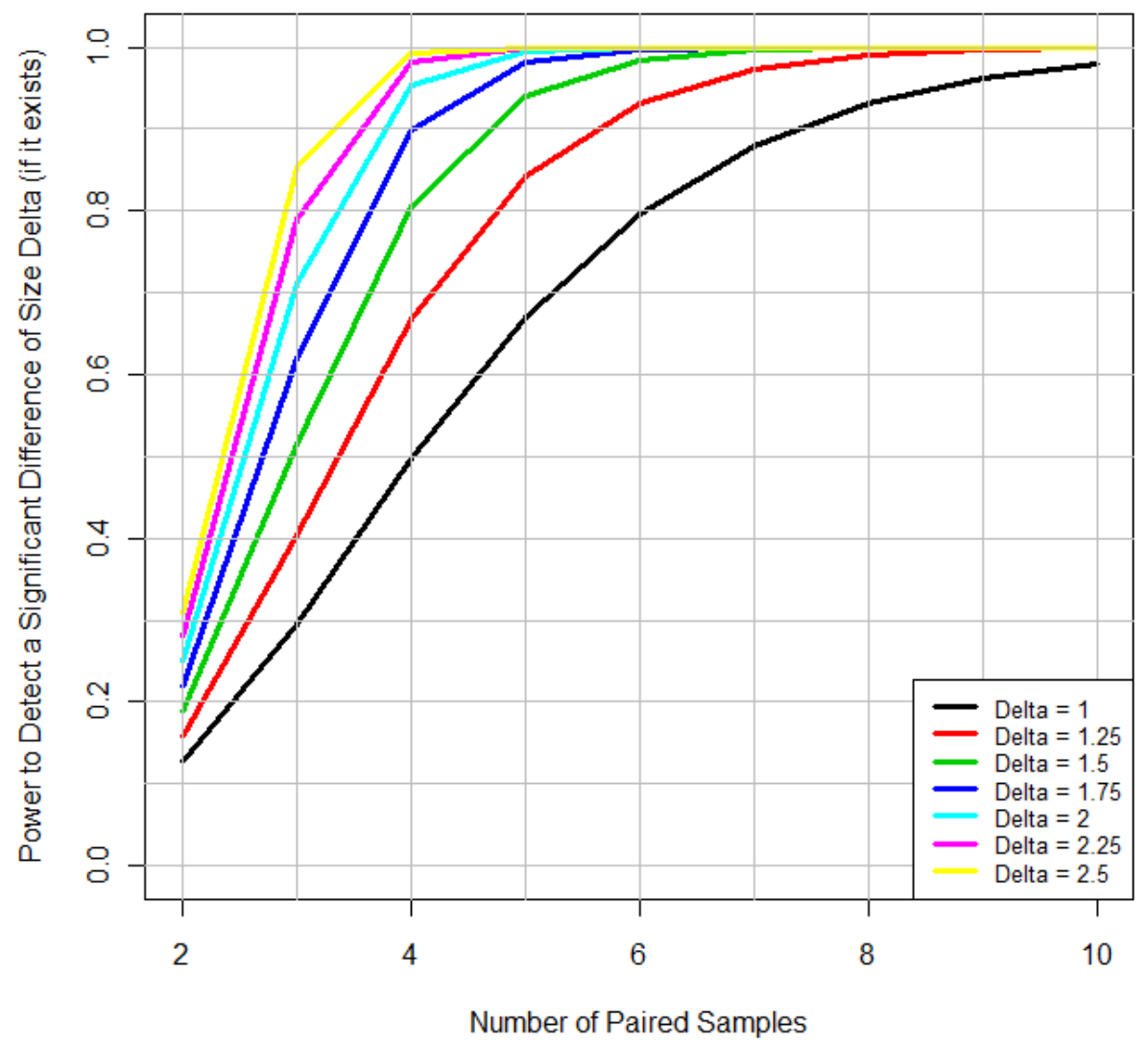

Figure B.2. Power and Sample Size Curves for Each Delta Assuming St. Dev. $=0.7$ 


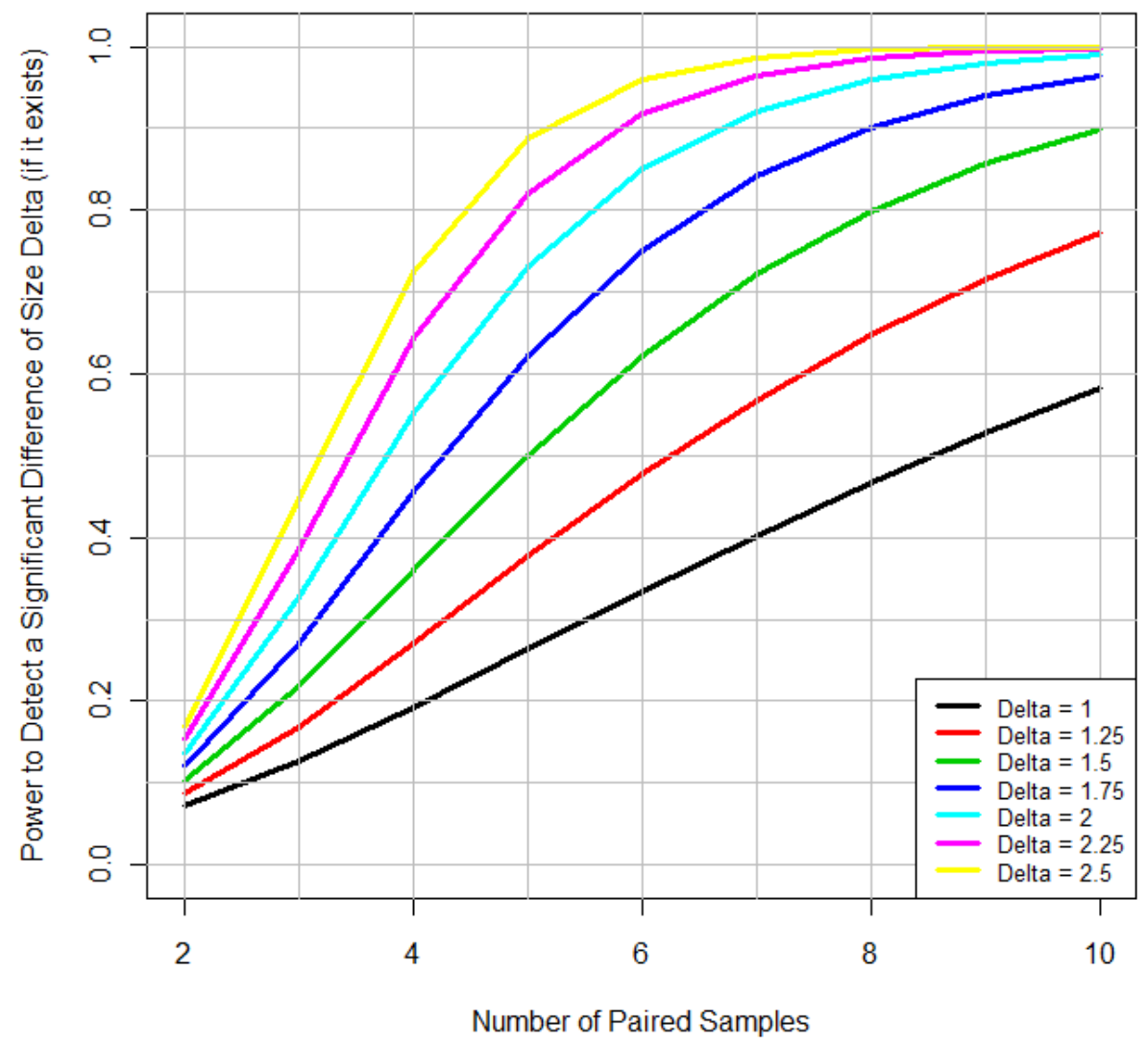

Figure B.3. Power and Sample Size Curves for Each Delta Assuming St. Dev. $=1.3$

It is important to remember that the purpose of this test was to determine if $\mathrm{N}_{2} \mathrm{O}$ is similar to $\mathrm{SF}_{6}$. Running tests at varying flow conditions should be done to help the researcher measure how consistent that comparison is during varying flow conditions. However, the purpose of the varying flow conditions is not to determine if these conditions vary the response (\%COV and \%MaxDev). Varying flow conditions do vary the response. The varying conditions instead act as a blocking or pairing variable commonly used in randomized block AOV or paired t-test analyses. The variable that best varied the flow conditions was injection position. Because of this, the varying flow conditions will be created by changing the injection position, while holding the fan configuration and fan control setting constant.

Another factor that may cause differences in the responses between the two gases is the concentration. Usually, $\mathrm{N}_{2} \mathrm{O}$ tests are performed at a higher concentration than $\mathrm{SF}_{6}$ because the background is much higher. It is common that higher means, resulting from higher concentrations, will result in lower \%COV values because the mean is part of the denominator of the \%COV calculation. For this reason, just running them at their usual, or optimal, concentrations may result in a bias in the difference of their \%COV values. To better quantify this, it was decided to also include tests in which both gases are run at the same concentrations. Table B.2 lists the rules to be followed in determining the concentration levels for each gas during testing, where $b_{s}$ is the instrument-measured background level for $\mathrm{SF}_{6}$ and $b_{n}$ is the background for $\mathrm{N}_{2} \mathrm{O}$. (The $\mathrm{N}_{2} \mathrm{O}$ background is significantly larger than that of $\mathrm{SF}_{6}$.) Example calculations of the concentration levels are shown in the last two columns, assuming nominal background values of $b_{s}=0.02$ and $b_{n}=0.5$. 
Table B.2. Rules to Determine Gas Concentration Levels for Testing

\begin{tabular}{|c|c|c|c|c|}
\hline \multirow{2}{*}{$\begin{array}{c}\text { Concentration } \\
\text { Variable Levels }\end{array}$} & & & \multicolumn{2}{|c|}{ If $b_{s}=0.02 \& b_{n}=0.5$} \\
\cline { 4 - 5 } & $\mathrm{SF}_{6}$ & $\mathrm{~N}_{2} \mathrm{O}$ & $\mathrm{SF}_{6}$ & $\mathrm{~N}_{2} \mathrm{O}$ \\
\hline Same & $b_{n} * 100$ & $b_{n} * 100$ & 50 & 50 \\
\hline Optimal & $b_{s} * 50$ & $b_{n} * 50$ & 1 & 25 \\
\hline
\end{tabular}

Table B.3 below shows the sampling plan. Fan configuration and flowrate will remain constant throughout the tests (control variables). The two levels of concentration and three injection positions will be varied such that all six combinations are run. Each gas was run at each of the six combinations, so that a difference in the gas \%COV and \%MaxDev values may be analyzed.

Table B.3. Proposed Sampling Plan for Gas Comparison

\begin{tabular}{|c|c|c|c|c|c|c|}
\hline $\begin{array}{c}\text { Test } \\
\text { Number }\end{array}$ & $\begin{array}{c}|c| \\
\text { Control Variables } \\
\text { Configuration }\end{array}$ & Airflow & Concentration & $\begin{array}{c}\text { Injection } \\
\text { Position }\end{array}$ & First Gas & $\begin{array}{c}\text { Second } \\
\text { Gas }\end{array}$ \\
\hline $1-2$ & AB & Normal & Same & Center & $\mathrm{N}_{2} \mathrm{O}$ & $\mathrm{SF}_{6}$ \\
\hline $3-4$ & $\mathrm{AB}$ & Normal & Optimal & Other & $\mathrm{SF}_{6}$ & $\mathrm{~N}_{2} \mathrm{O}$ \\
\hline $5-6$ & $\mathrm{AB}$ & Normal & Optimal & Center & $\mathrm{SF}_{6}$ & $\mathrm{~N}_{2} \mathrm{O}$ \\
\hline $7-8$ & $\mathrm{AB}$ & Normal & Same & Bottom & $\mathrm{N}_{2} \mathrm{O}$ & $\mathrm{SF}_{6}$ \\
\hline $9-10$ & $\mathrm{AB}$ & Normal & Optimal & Bottom & $\mathrm{N}_{2} \mathrm{O}$ & $\mathrm{SF}_{6}$ \\
\hline $11-12$ & $\mathrm{AB}$ & Normal & Same & Other & $\mathrm{SF}_{6}$ & $\mathrm{~N}_{2} \mathrm{O}$ \\
\hline
\end{tabular}

After the 12 tests (six pairs) were run, an initial analysis was performed to determine which tests would benefit from being repeated. Two to four of the test conditions were to provide replication.

Only one randomization constraint was added to the sampling plan. This was that the order of which gas was tested first for the first test of each day was randomized, but the second test of that day was to start with the same gas that was last used in the previous paired test. For example, test \# 2 used $\mathrm{SF}_{6}$, so therefore the second set of tests, test \# 3, would also start with $\mathrm{SF}_{6}$. This was done to minimize the number of times the gas was changed, because changing gases requires additional time and effort.

\section{B.2 $\mathrm{N}_{2} \mathrm{O}$ and $\mathrm{SF}_{6}$ Comparison Results and Discussion}

The testing strategy described in Subsection B.1 was performed using a modified version of the LB-S1 scale model stack, shown in Figure B.4. This modification included replacing one duct section with two sections that included two ports. This substitution was performed to utilize one of the ports for an injection location that was expected to result in poor mixing at the sampling location. This port is represented as the "Other" injection point in Table B.4 below. The modification to the LB-S1 stack resulted in less than a 1-inch reduction in the distance to the measurement port. The two sections that replaced the single section were nearly the same length. 

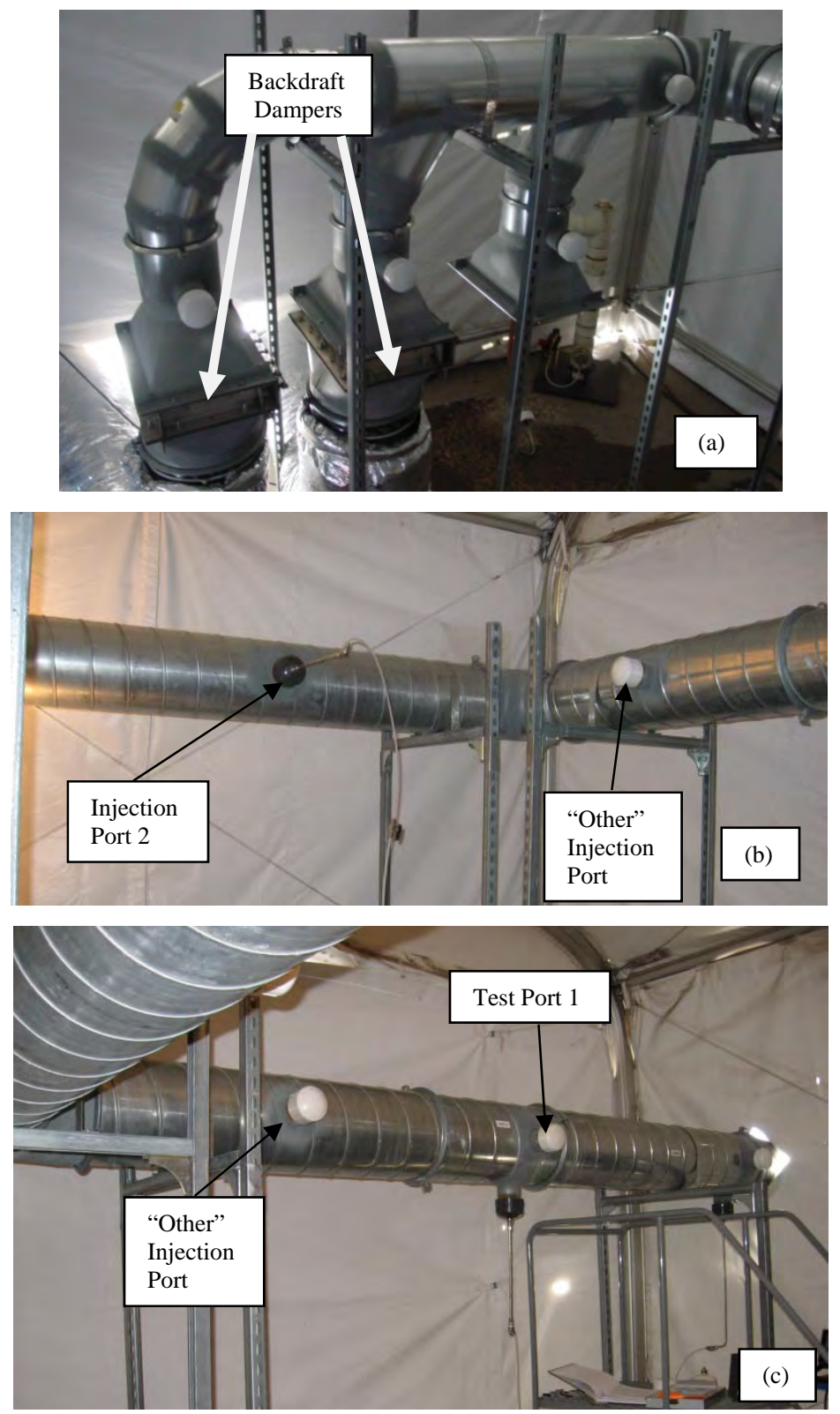

Figure B.4. Pictures of Modified LB-S1 Scale Model: a) Upstream section with backdraft dampers; b) Center section with Injection Port 2; c) Downstream section with “Other” Injection Port and Sampling Port 2.

Table B.4 summarizes the results of the tests performed (see Figure 3.1 for sampling points). All tests were performed with fans A \& B, and at a velocity that was nominally the expected normal stack velocity, and measurements were made at Test Port 1 . The variables that were adjusted in these tests were the injection location, the gas concentration, and whether $\mathrm{N}_{2} \mathrm{O}$ or $\mathrm{SF}_{6}$ was used first for each pair of tests. Gas equivalency (GE) tests 1 through 12 were the six pairs of base tests, and three of these tests were repeated. The shading in Table B.4 highlights the pairs of tests that were repeated. Note that the \%COV values for any given pair of tests are comparable, and there is no systematic bias in the $\mathrm{N}_{2} \mathrm{O}$ results compared with the $\mathrm{SF}_{6}$ results. A statistical evaluation of these results is presented below. 
Table B.4. Summary of Gas Equivalency Test Results. Each test was performed as part of a pair of tests, one with $\mathrm{SF}_{6}$ and one with $\mathrm{N}_{2} \mathrm{O}$. The shaded rows highlight tests that were repeated.

\begin{tabular}{|c|c|c|c|c|c|c|c|c|c|}
\hline $\begin{array}{l}\text { Run } \\
\text { No. }\end{array}$ & Fans & Injection & $\begin{array}{l}\text { Test } \\
\text { Port }\end{array}$ & $\begin{array}{c}\text { Velocity } \\
\text { (sfpm) }\end{array}$ & Gas & $\% \mathrm{COV}$ & \%MaxDev & $\begin{array}{l}\text { Mean } \\
\text { Conc } \\
(\mathrm{ppm})\end{array}$ & $\begin{array}{l}\text { Conc } \\
\text { Level }\end{array}$ \\
\hline GE-1 & A \& B & 2 - Center & 1 & 3331 & $\mathrm{~N}_{2} \mathrm{O}$ & 4.5 & -8.8 & 41 & Same \\
\hline GE-2 & A \& B & 2 - Center & 1 & 3317 & $\mathrm{SF}_{6}$ & 4.3 & -7.5 & 41 & Same \\
\hline GE-3 & A \& B & Other & 1 & 3336 & $\mathrm{SF}_{6}$ & 119.8 & 258.8 & 0.776 & Optimal \\
\hline GE-4 & A \& B & Other & 1 & 3353 & $\mathrm{~N}_{2} \mathrm{O}$ & 110.0 & 274.1 & 51 & Optimal \\
\hline GE-5 & A \& B & 2 - Center & 1 & 3308 & $\mathrm{SF}_{6}$ & 2.4 & 5.0 & 0.776 & Optimal \\
\hline GE-6 & A \& B & 2 - Center & 1 & 3330 & $\mathrm{~N}_{2} \mathrm{O}$ & 1.7 & 4.8 & 53 & Optimal \\
\hline GE-7 & $A \& B$ & 2-Bottom & 1 & 3437 & $\mathrm{SF}_{6}$ & 5.3 & -16.2 & 42 & Same \\
\hline GE-8 & A \& B & 2-Bottom & 1 & 3299 & $\mathrm{~N}_{2} \mathrm{O}$ & 4.8 & -12.9 & 43 & Same \\
\hline GE-9 & A \& B & 2-Bottom & 1 & 3322 & $\mathrm{SF}_{6}$ & 5.0 & -9.6 & 0.793 & Optimal \\
\hline GE-10 & A \& B & 2-Bottom & 1 & 3395 & $\mathrm{~N}_{2} \mathrm{O}$ & 4.8 & -11.4 & 41 & Optimal \\
\hline GE-11 & A \& B & Other & 1 & 3331 & $\mathrm{~N}_{2} \mathrm{O}$ & 69.0 & 159.7 & 38.63 & Same \\
\hline GE-12 & $A \& B$ & Other & 1 & 3291 & $\mathrm{SF}_{6}$ & 69.5 & 157 & 33.7 & Same \\
\hline GE-13 & $A \& B$ & Other & 1 & 3380 & $\mathrm{SF}_{6}$ & 71.4 & 181.8 & 0.747 & Optimal \\
\hline GE-14 & A \& B & Other & 1 & 3350 & $\mathrm{~N}_{2} \mathrm{O}$ & 67.5 & 149.2 & 11.7 & Optimal \\
\hline GE-15 & A \& B & 2-Bottom & 1 & 3408 & $\mathrm{SF}_{6}$ & 4.5 & -8.3 & 43.5 & Same \\
\hline GE-16 & $A \& B$ & 2-Bottom & 1 & 3288 & $\mathrm{~N}_{2} \mathrm{O}$ & 4.3 & -9.1 & 43.6 & Same \\
\hline GE-17 & A \& B & 2-Center & 1 & 3318 & $\mathrm{~N}_{2} \mathrm{O}$ & 1.3 & 4.0 & 43.4 & Same \\
\hline GE-18 & $A \& B$ & 2-Center & 1 & 3351 & $\mathrm{SF}_{6}$ & 1.1 & 4.5 & 45.1 & Same \\
\hline
\end{tabular}

AOV was run to determine if there were differences between the two gases in \%COV and maximum deviation (\%MaxDev). The AOV also looked for differences in the three injection points and the two different levels of concentration (optimal and same), as well as any interactions between the three factors. This resulted in the following AOV model being used:

$$
Y=\mu+p_{i}+g_{k}+p g_{i k}+l_{j}+p l_{i j}+\lg _{j k}
$$

where $Y$ is the response variable (\%COV or \%MaxDev), $\mu$ is the overall mean, $p_{i}$ is the injection point, $l_{j}$ is the level of concentration, $g_{k}$ is the gas, and $p l_{i j}, p g_{i k}$, and $\lg _{j k}$ are the two-way interactions.

Table B.5 shows the AOV results when analyzing \%COV, while Table B.6 shows the AOV results when analyzing \%MaxDev. Factors with p-values less than 0.05 indicate significant differences between the different levels with 95\% confidence. As expected, with both \%COV and \%MaxDev, there were significant differences between the three injection points (\%COV p-value $=0.0010$, \%MaxDev p-value $=0.0005$ ). This was expected because the "Other" injection point was specifically selected because it was quite different from the other two injection points. 
The AOV results show that there were no significant differences between the gases of $\mathrm{N}_{2} \mathrm{O}$ and $\mathrm{SF}_{6}$ $(\% \mathrm{COV}$ p-value $=0.5522, \% \mathrm{MaxDev} \mathrm{p}$-value$=0.3439)$. There were also no significant interactions between the gases and injection point or concentration. This means that the two gases acted similarly across all of the tested injection points and the two concentration levels. The lack of interaction between gas and injection point can be seen in
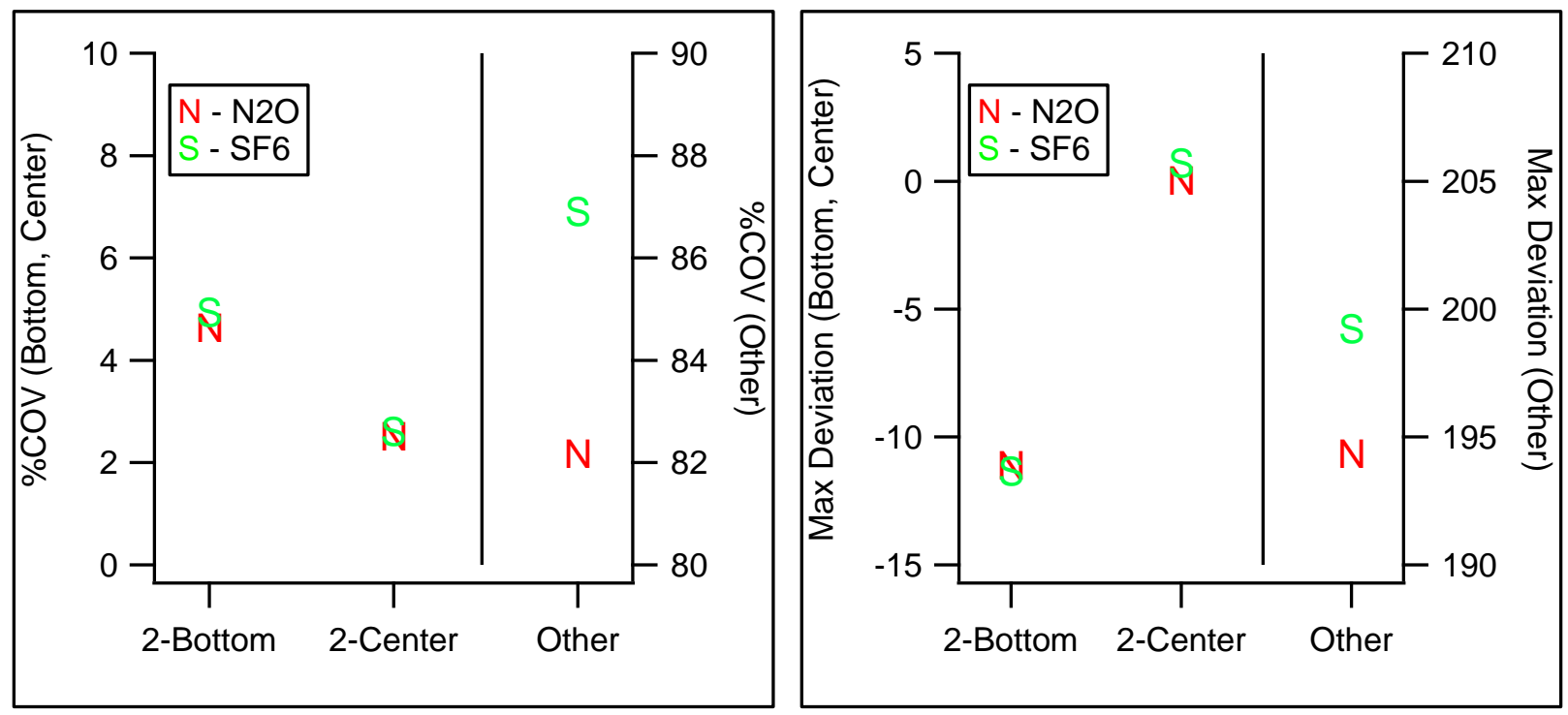

Figure B.5.

\section{B.3 $\mathrm{N}_{2} \mathrm{O}$ and $\mathrm{SF}_{6}$ Comparison Conclusions}

Preliminary data were used to determine what factors influence \%COV and \%MaxDev, as well as to help determine the number of runs that would be needed in an experiment comparing multiple gases. It was found that injection point and fan configuration influenced \%COV and \%MaxDev, but fan control frequency (airflow control) had no effect. Using statistical power calculations it was determined that running six samples per pair (paired because two gases were going to be tests) would supply 99\% statistical power of finding a significant difference between the gases if the gases were in fact different by more than $2 \%$ COV and 2 \%MaxDev.

An experimental was designed with six paired samples to study the differences in the gases. It was decided to look at three different injection points and to look at two concentration levels, because the gases ran at such significantly different concentration levels. After the six paired samples were performed, three repeat pairs were performed for those runs that showed the greatest variation. Analysis of Variance (AOV) was performed on the experimental data and significant differences were found between the three injection points for both \%COV and \%MaxDev (p-values $<0.01$ ). There were no significant differences found between the two gases for either \%COV (p-value $=0.5522$ ) or \%MaxDev $(\mathrm{p}$-value $=0.3439)$. With no significant differences found between the two gases when performing an experiment specifically designed with high statistical power to find differences if differences truly existed, it can be stated with high confidence that the gases perform similarly in these test conditions. 
Table B.5. Analysis of \%COV on Full Data

\begin{tabular}{|c|c|c|c|c|c|}
\hline Source & df & SS & MS & F & p-value \\
\hline Injection & 2 & 9698.75 & 4849.38 & 18.534 & $0.0010^{*}$ \\
\hline Gas & 1 & 3.173 & 3.173 & 0.502 & 0.5522 \\
\hline Injection x Gas & 2 & 12.649 & 6.324 & 0.024 & 0.9762 \\
\hline Conc & 1 & 1.017 & 1.017 & 0.004 & 0.9540 \\
\hline Injection x Conc & 2 & 479.522 & 239.761 & 0.916 & 0.4382 \\
\hline Gas x Conc & 1 & 5.601 & 5.601 & 0.021 & 0.8873 \\
\hline Error & 8 & 2093.21 & 261.651 & & \\
\hline
\end{tabular}

* Indicates the factor has significant differences between the levels (p-value $<0.05$ )

Table B.6. Analysis of \%MaxDev on Full Data

\begin{tabular}{|c|c|c|c|c|c|}
\hline Source & df & SS & MS & F & p-value \\
\hline Injection & 2 & 60828.48 & 60828.48 & 22.155 & $0.0005^{*}$ \\
\hline Gas & 1 & 7.112 & 7.112 & 1.511 & 0.3439 \\
\hline Injection x Gas & 2 & 9.412 & 4.706 & 0.003 & 0.9966 \\
\hline Conc & 1 & 1.342 & 1.342 & 0.001 & 0.9772 \\
\hline Injection x Conc & 2 & 2579.167 & 1289.584 & 0.939 & 0.4301 \\
\hline Gas x Conc & 1 & 20.854 & 20.854 & 0.015 & 0.9049 \\
\hline Error & 8 & 10982.201 & 1372.775 & & \\
\hline
\end{tabular}

* Indicates the factor has significant differences between the levels (p-value $<0.05$ )
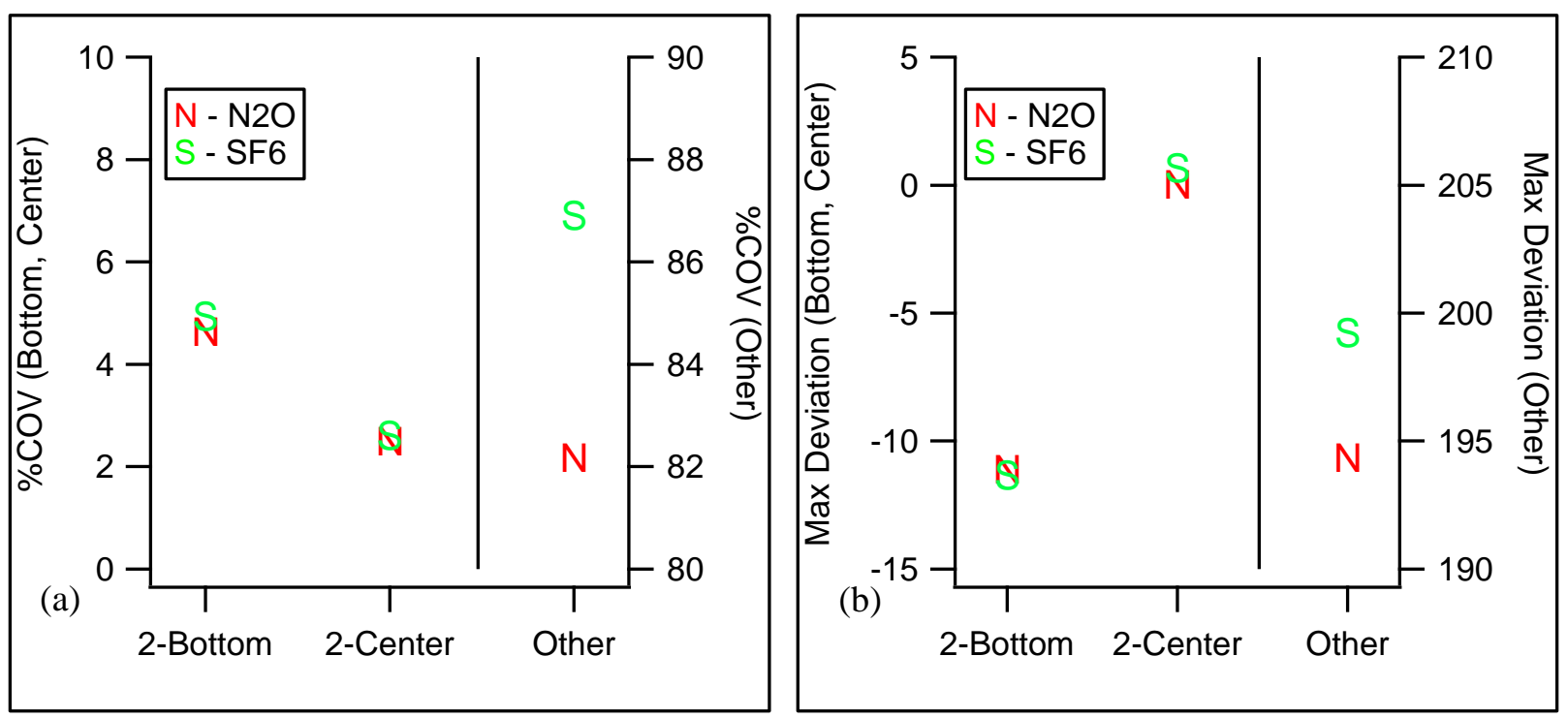

Figure B.5. The Mean \%COV (a) and Maximum Deviation (b) for Each Gas at Each Injection Point 


\section{B.4 $\mathrm{N}_{2} \mathrm{O}$ and $\mathrm{SF}_{6}$ Comparison Datasheets}

SULFUR HEXAFLUORIDE AND NITROUS OXIDE GAS INSTRUMENT CALIBRATION

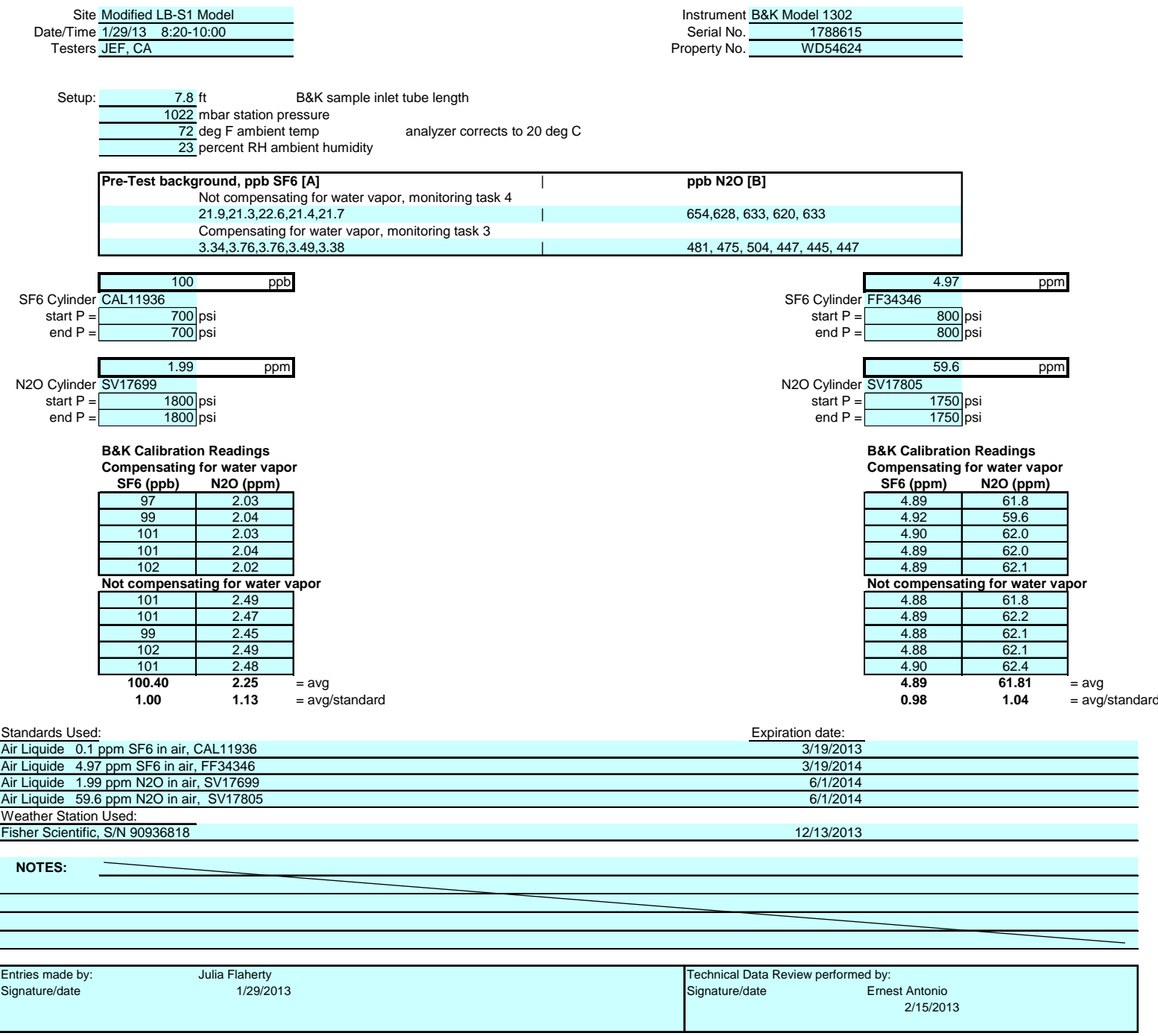




\section{SULFUR HEXAFLUORIDE AND NITROUS OXIDE GAS INSTRUMENT CALIBRATION}

Site Modified LB-S1 Model

Date/Time 2/2/2013 15:10 - 16:20

Testers JEF
Instrument B\&K Model 1302

Serial No. 1788615

Property No. $\quad$ WD54624

Setup:

$7.8 \mathrm{ft}$

B\&K sample inlet tube length

1030 mbar station pressure

71 deg F ambient temp analyzer corrects to $20 \operatorname{deg} \mathrm{C}$

24 percent $\mathrm{RH}$ ambient humidity

\begin{tabular}{|c|c|}
\hline Pre-Test background, pp & ppb N2O [B] \\
\hline Not comp & vapor, monitoring task 4 \\
\hline $44,47,51,46,47$ & I $1004,978,1000,984,906$ \\
\hline Compens & or, monitoring task 3 \\
\hline $0.29,0.90,0.87,-1.0,-1.3$ & $430,442,429,433,435$ \\
\hline
\end{tabular}

\begin{tabular}{|c|c|c|}
\hline \multirow{4}{*}{$\begin{array}{r}\text { SF6 Cylinder } \\
\text { start } \mathrm{P}= \\
\text { end } \mathrm{P}=\end{array}$} & 100 & $\mathrm{ppb}$ \\
\hline & CAL11936 & \\
\hline & 700 psi & \\
\hline & 700 psi & \\
\hline \multirow{4}{*}{$\begin{array}{r}\mathrm{N} 2 \mathrm{O} \text { Cylinder } \\
\text { start } \mathrm{P}= \\
\text { end } \mathrm{P}=\end{array}$} & 1.99 & $\mathrm{ppm}$ \\
\hline & SV17699 & \\
\hline & 1800 psi & \\
\hline & 1800 psi & \\
\hline
\end{tabular}

B\&K Calibration Readings Compensating for water vapor

\begin{tabular}{|c|c|}
\multicolumn{1}{c|}{ SF6 (ppb) } & N2O (ppm) \\
\hline 99 & 2.00 \\
\hline 102 & 2.05 \\
\hline 101 & 2.04 \\
\hline 98 & 2.04 \\
\hline 104 & 2.01 \\
\hline
\end{tabular}

Not compensating for water vapor

\begin{tabular}{|c|c|}
\hline 111 & $\mathrm{~N} / \mathrm{A}$ \\
\hline 114 & $\mathrm{~N} / \mathrm{A}$ \\
\hline 110 & $\mathrm{~N} / \mathrm{A}$ \\
\hline 108 & $\mathrm{~N} / \mathrm{A}$ \\
\hline 102 & $\mathrm{~N} / \mathrm{A}$ \\
\hline 104.94 & $\mathbf{2 . 0 3}=$ avg \\
1.05 & $1.02 \quad=$ avg/standard
\end{tabular}

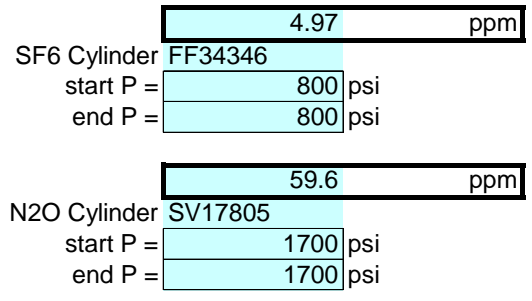

B\&K Calibration Readings Compensating for water vapor

\begin{tabular}{|c|c|}
\multicolumn{1}{|c|}{ SF6 (ppm) } & N2O (ppm) \\
\hline 4.86 & 60.7 \\
\hline 4.88 & 60.9 \\
\hline 4.90 & 60.7 \\
\hline 4.91 & 60.9 \\
\hline 4.92 & 61.0 \\
\hline
\end{tabular}

Not compensating for water vapor

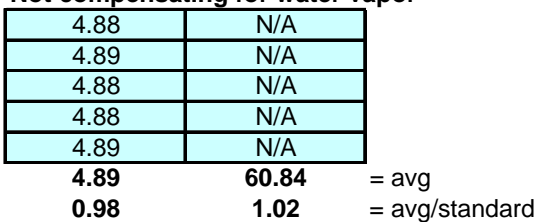

Standards Used:

\begin{tabular}{lll}
\hline Air Liquide & 0.1 ppm SF6 in air, CAL11936 & Expiration date: \\
\hline Air Liquide & 4.97 ppm SF6 in air, FF34346 & $3 / 19 / 2013$ \\
\hline Air Liquide & 1.99 ppm N2O in air, SV17699 & $3 / 19 / 2014$ \\
\hline Air Liquide & 59.6 ppm N2O in air, SV17805 & $6 / 1 / 2014$ \\
\hline Weather Station Used: & $6 / 1 / 2014$ \\
\hline Fisher Scientific, S/N 90936818 & $12 / 13 / 2013$ \\
\hline
\end{tabular}

NOTES: Don't calibrate / check N2O without $\mathrm{H} 2 \mathrm{O}$ compensation - values don't make sense and aren't used.

\begin{tabular}{|c|c|c|c|}
\hline Entries made by: & \multicolumn{2}{|c|}{ Julia Flaherty } & Technical Data Review performed by: \\
\hline Signature/date & On File w/ Original & $2 / 2 / 2013$ & $\begin{array}{l}\text { Ernest Antonio } \\
2 / 15 / 2013\end{array}$ \\
\hline
\end{tabular}


SULFUR HEXAFLUORIDE AND NITROUS OXIDE GAS INSTRUMENT CALIBRATION

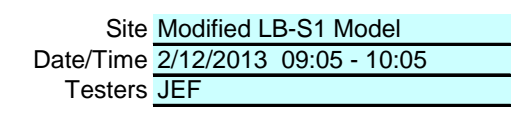

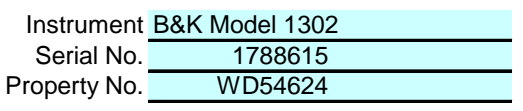

WD54624

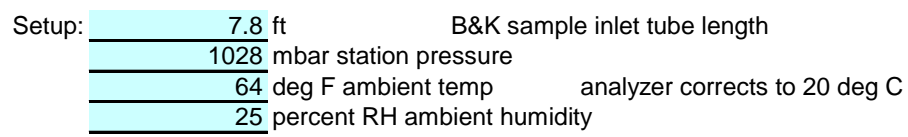

1028 mbar station pressure

64 deg F ambient temp analyzer corrects to 20 deg $\mathrm{C}$

25 percent $\mathrm{RH}$ ambient humidity

\begin{tabular}{|c|c|}
\hline \begin{tabular}{c|c} 
Pre-Test background, ppb SF6 [A] & ppb N2O [B] \\
Not compensating for water vapor, monitoring task 4
\end{tabular} \\
\begin{tabular}{l|c}
$20,22,18,20,19$ & $750,798,713,694,715$ \\
& Compensating for water vapor, monitoring task 3
\end{tabular} \\
$9,7,12,4,1$ & $599,626,562,637,564$ \\
\hline
\end{tabular}

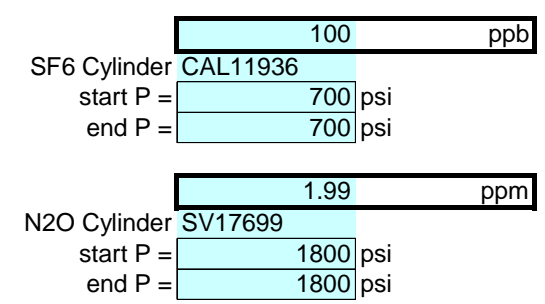

B\&K Calibration Readings

Compensating for water vapor

\begin{tabular}{|c|c|}
\hline SF6 (ppb) & N2O (ppm) \\
\hline 99.1 & 2.03 \\
\hline 100 & 2.05 \\
\hline 98.6 & 2.04 \\
\hline 101 & 2.05 \\
\hline 97.7 & 2.06 \\
\hline
\end{tabular}

\begin{tabular}{|c|c|}
\hline Not compensating for water vapor \\
\hline 101 & N/A \\
\hline 101 & N/A \\
\hline 99.9 & N/A \\
\hline 101 & N/A \\
\hline 98.5 & N/A \\
\hline 99.78 & $2.05 \quad=a v g$ \\
1.00 & $1.03 \quad=a v g /$ standard
\end{tabular}

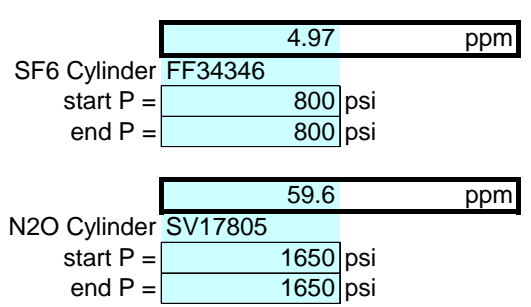

B\&K Calibration Readings Compensating for water vapor

\begin{tabular}{|c|c|}
\hline SF6 (ppm) & N2O (ppm) \\
\hline 4.83 & 61.8 \\
\hline 4.91 & 62.2 \\
\hline 4.91 & 62.2 \\
\hline 4.88 & 62.1 \\
\hline 4.88 & 62.1 \\
\hline
\end{tabular}

Not compensating for water vapor

\begin{tabular}{|c|c|}
\hline 4.87 & N/A \\
\hline 4.86 & N/A \\
\hline 4.89 & N/A \\
\hline 4.89 & N/A \\
\hline 4.90 & N/A \\
\hline 4.88 & $\mathbf{6 2 . 0 8} \quad=$ avg \\
$\mathbf{0 . 9 8}$ & $1.04 \quad=a v g /$ standard
\end{tabular}

Standards Used: Air Liquide 0.1 ppm SF6 in air, CAL11936

Air Liquide 4.97 ppm SF6 in air, FF34346

Air Liquide 1.99 ppm N2O in air, SV17699

Air Liquide 59.6 ppm N2O in air, SV17805

Weather Station Used:

Fisher Scientific, S/N 90936818

Expiration date:

$3 / 19 / 2013$

$3 / 19 / 2014$

$6 / 1 / 2014$

$6 / 1 / 2014$

$12 / 13 / 2013$

NOTES: $\quad$ Modify Task $3 \& 4$ on B\&K to measure SF6 for background check. Do not run $\mathrm{N} 2 \mathrm{O}$ cal gas w/o water compensation. Numbers don't make sense.

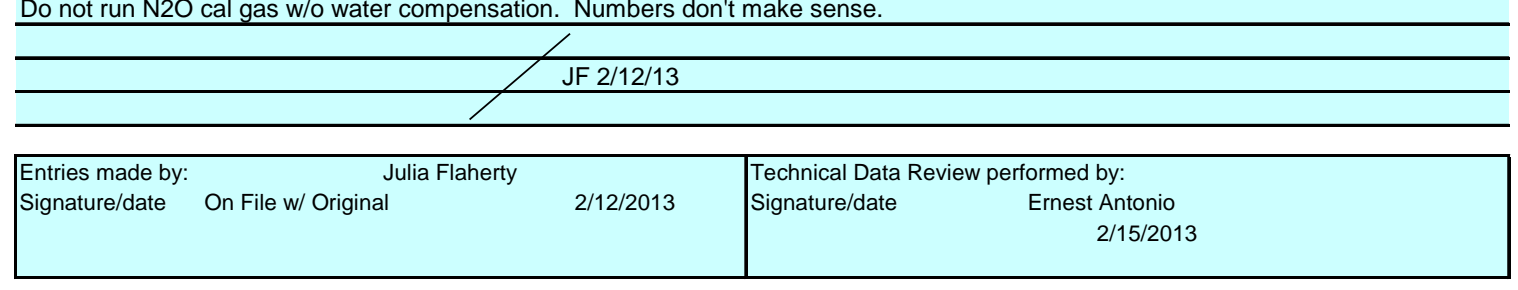


SULFUR HEXAFLUORIDE AND NITROUS OXIDE GAS INSTRUMENT CALIBRATION

$\begin{aligned} \text { Site } & \text { Modified LB-S1 Model } \\ \text { Date/Time } & \frac{2 / 18 / 2013 \quad 09: 00-10: 40}{X Y Y} \\ \text { Testers } & \text { XY }\end{aligned}$

Setup:

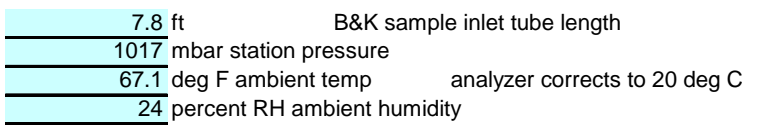

\begin{tabular}{|l|l}
\hline Pre-Test background, ppb SF6 [A] & ppb N2O [B]
\end{tabular}

$2,2,2,2,-0.6$

Not compensating for water vapor, monitoring task 4

\begin{tabular}{l}
$4,4,2,2,4 \quad$ Compensating for water vapor, monitoring task 3 \\
$\qquad \quad 511,475,468,454,465$ \\
\hline
\end{tabular}
Instrument B\&K Model 1302

Serial No. $\frac{1788615}{12054624}$

Property No. WD54624

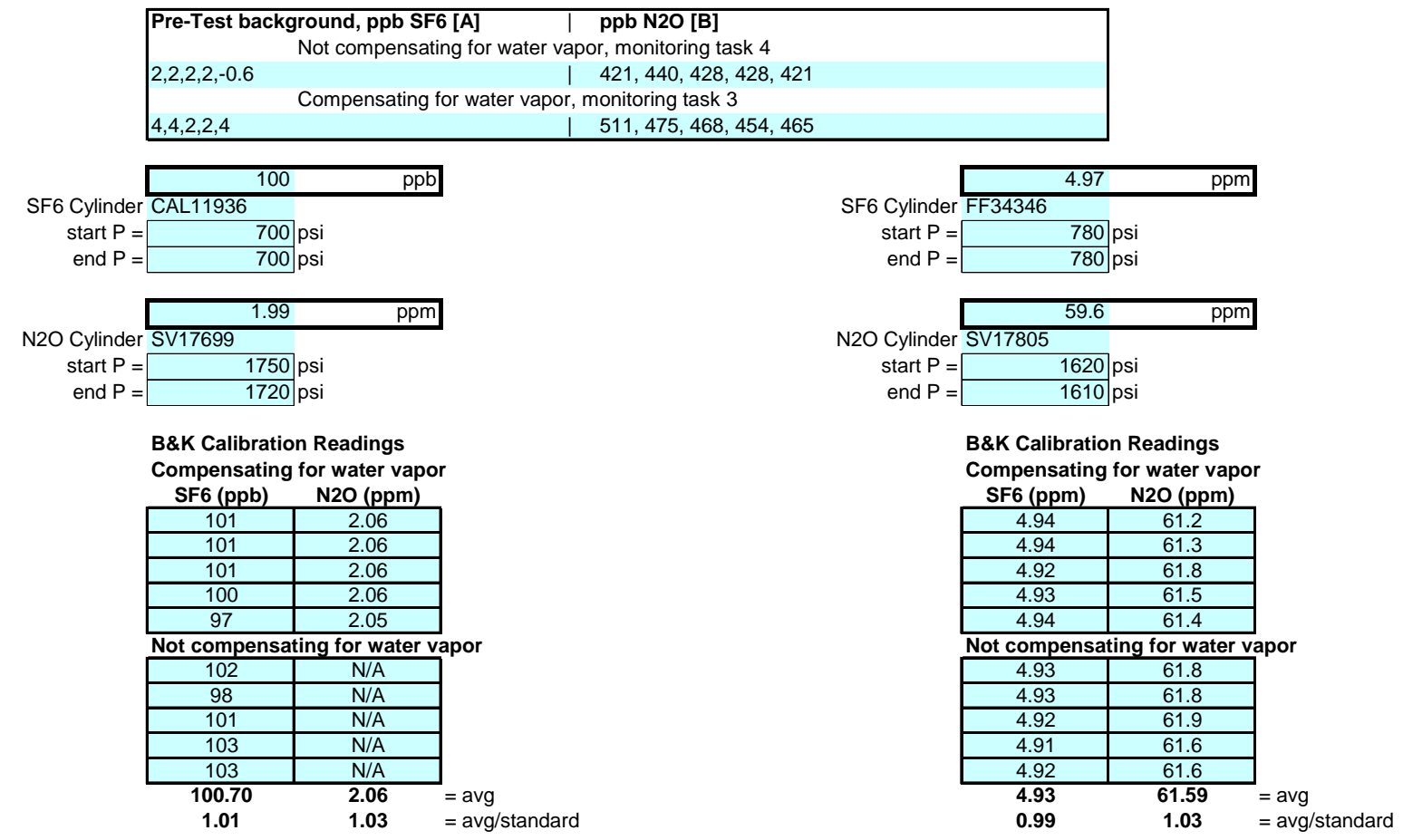

Standards Used:

Air Liquide $0.1 \mathrm{ppm}$ SF6 in air CAL11936

Expiration date:

Air Liquide 4.97 ppm SF6 in air, FF34346

Air Liquide 1.99 ppm N2O in air, SV17699

Air Liquide 59.6 ppm N2O in air, SV17805

Weather Station Used:

Fisher Scientific, S/N 90936818

= avg/standard

$3 / 19 / 2013$

$3 / 19 / 2014$

$6 / 1 / 2014$

$12 / 13 / 2013$

NOTES: $\quad$ Results of $2 \mathrm{ppm}$ without water compensation are much higher than $2 \mathrm{ppm}$, i.e., $\sim 2.5 \mathrm{ppm}$. Not sure why. XYY 2/18/13. Checkwed with Julia, she has seen similar results, so we will not use these.

\begin{tabular}{|c|c|c|c|}
\hline & & \multicolumn{2}{|c|}{1} \\
\hline & & \multicolumn{2}{|c|}{$X Y Y 2 / 18 / 13$} \\
\hline Entries made by: & \multicolumn{2}{|c|}{ Xiao-Ying Yu } & Technical Data Review performed by: \\
\hline Signature/date & On File w/ Original & $2 / 18 / 2013$ & $\begin{array}{rr}\text { Signature/date } & \text { Ernest Antonio } \\
2 / 15 / 2013\end{array}$ \\
\hline
\end{tabular}




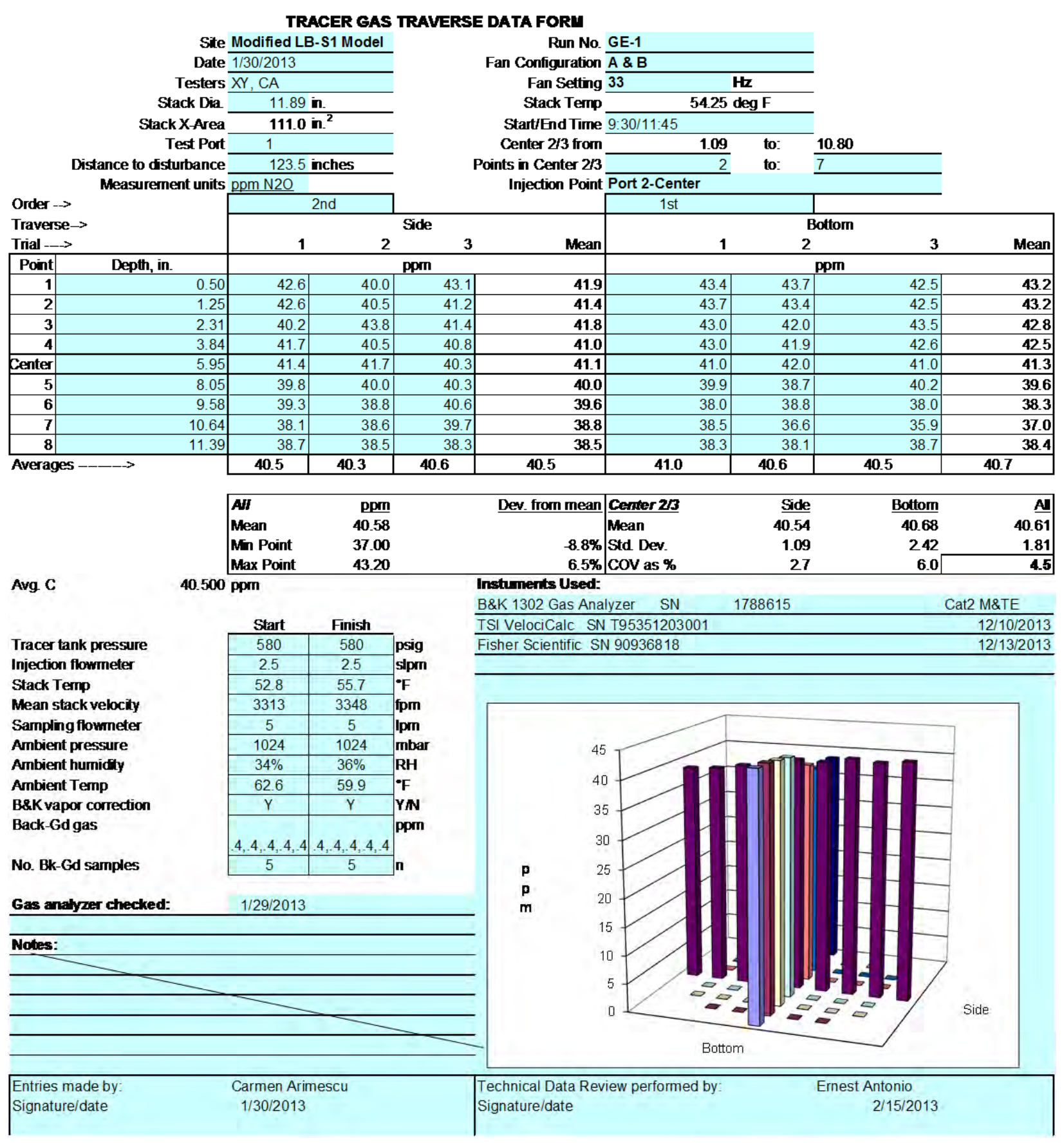




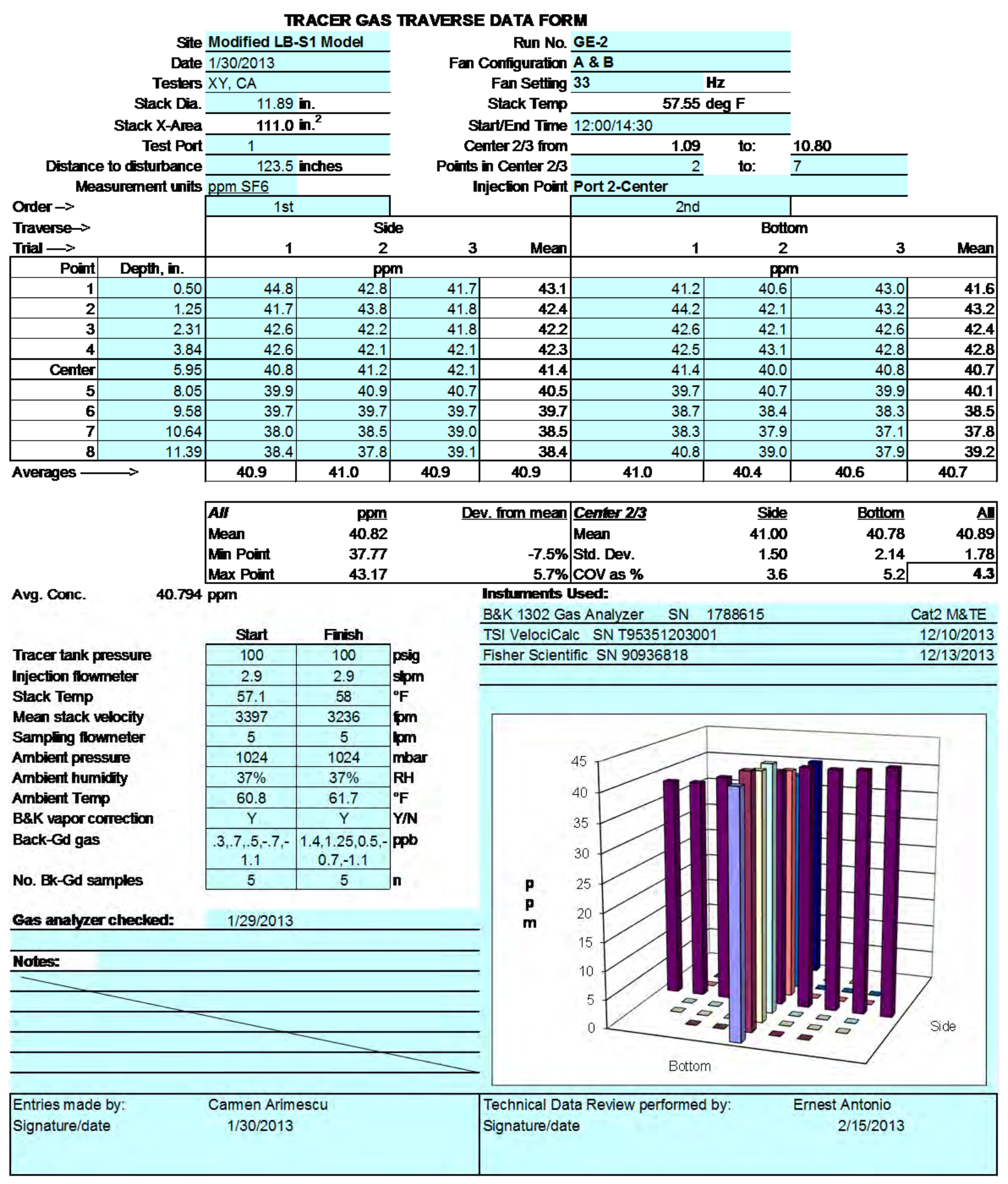




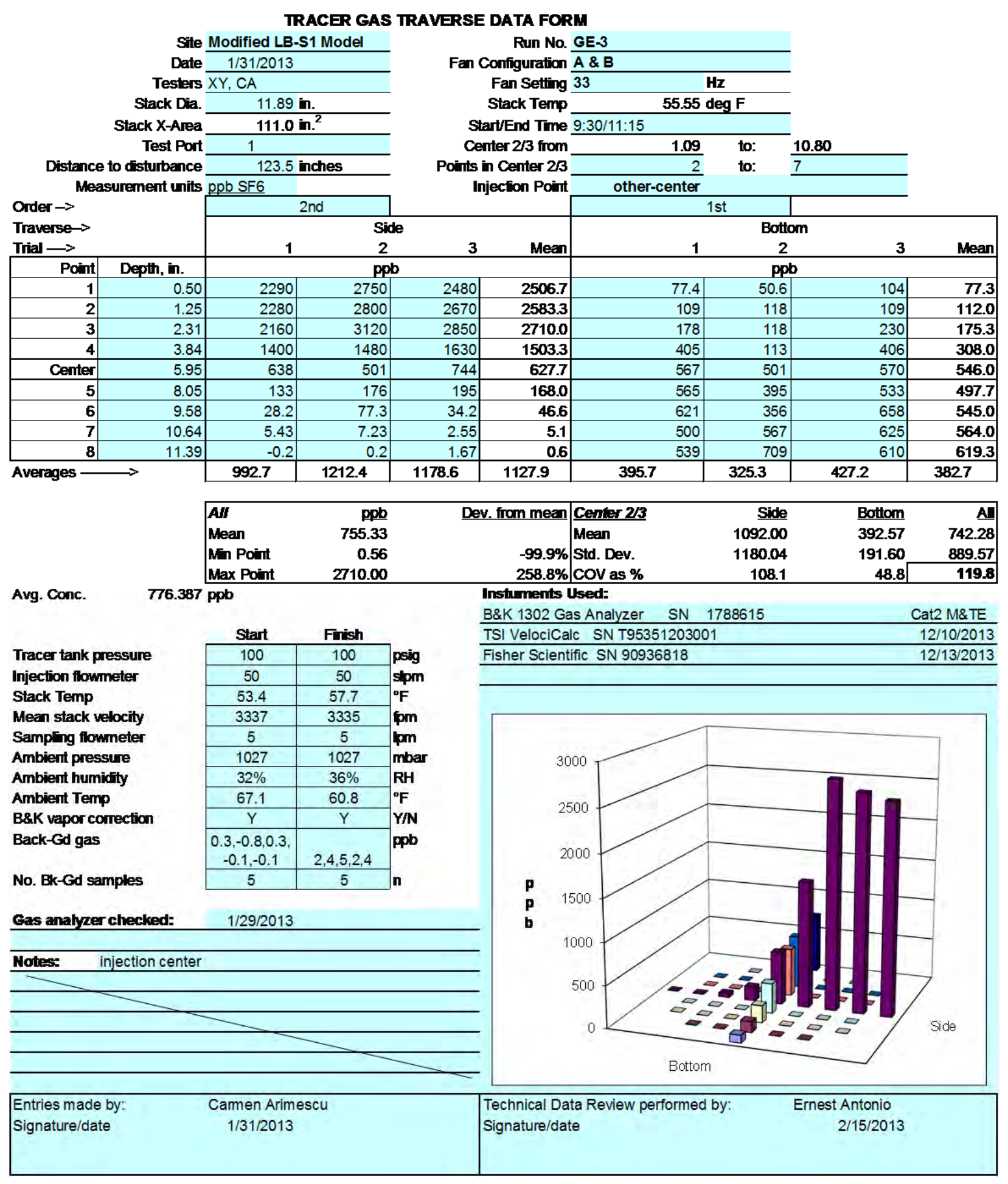




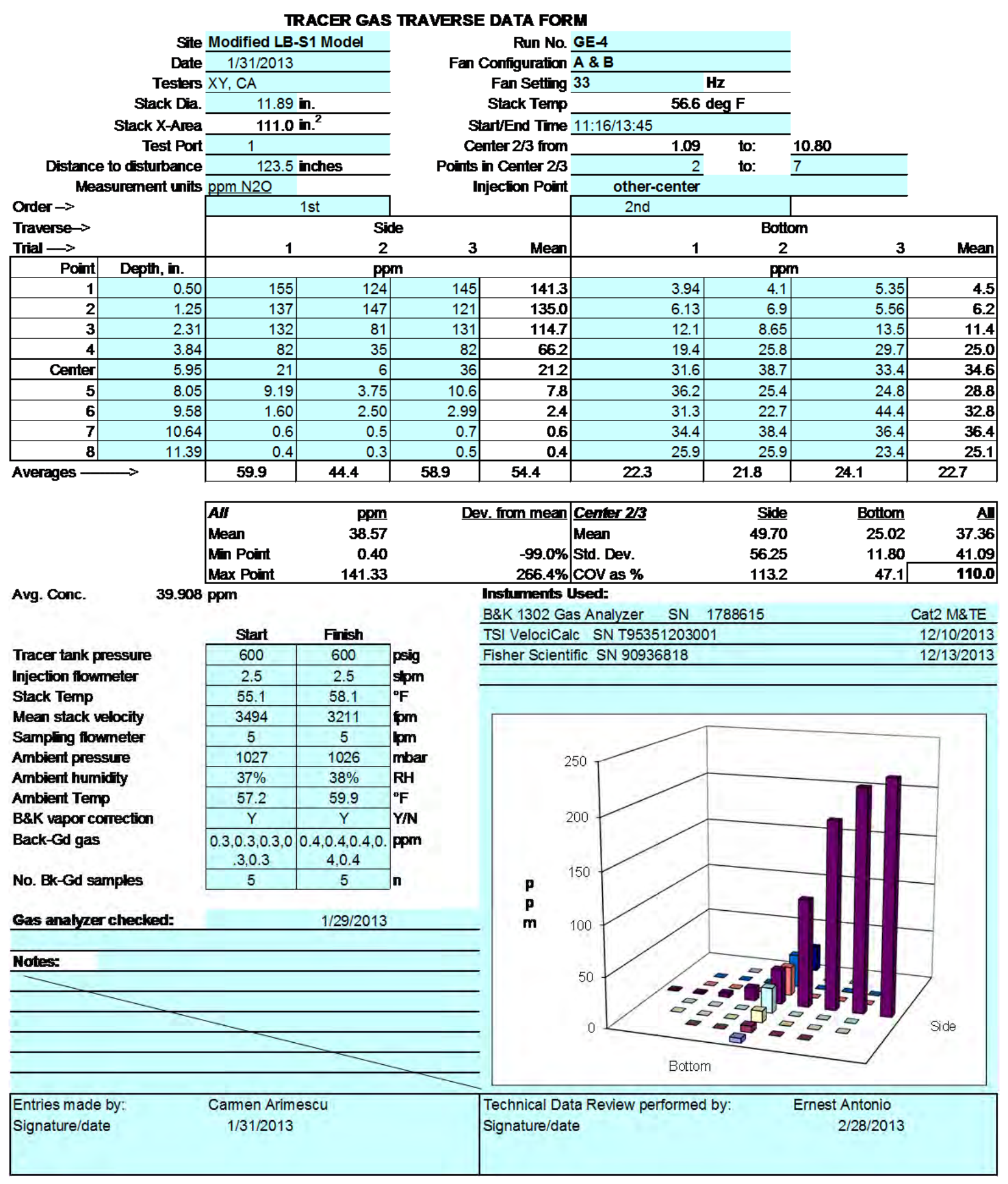




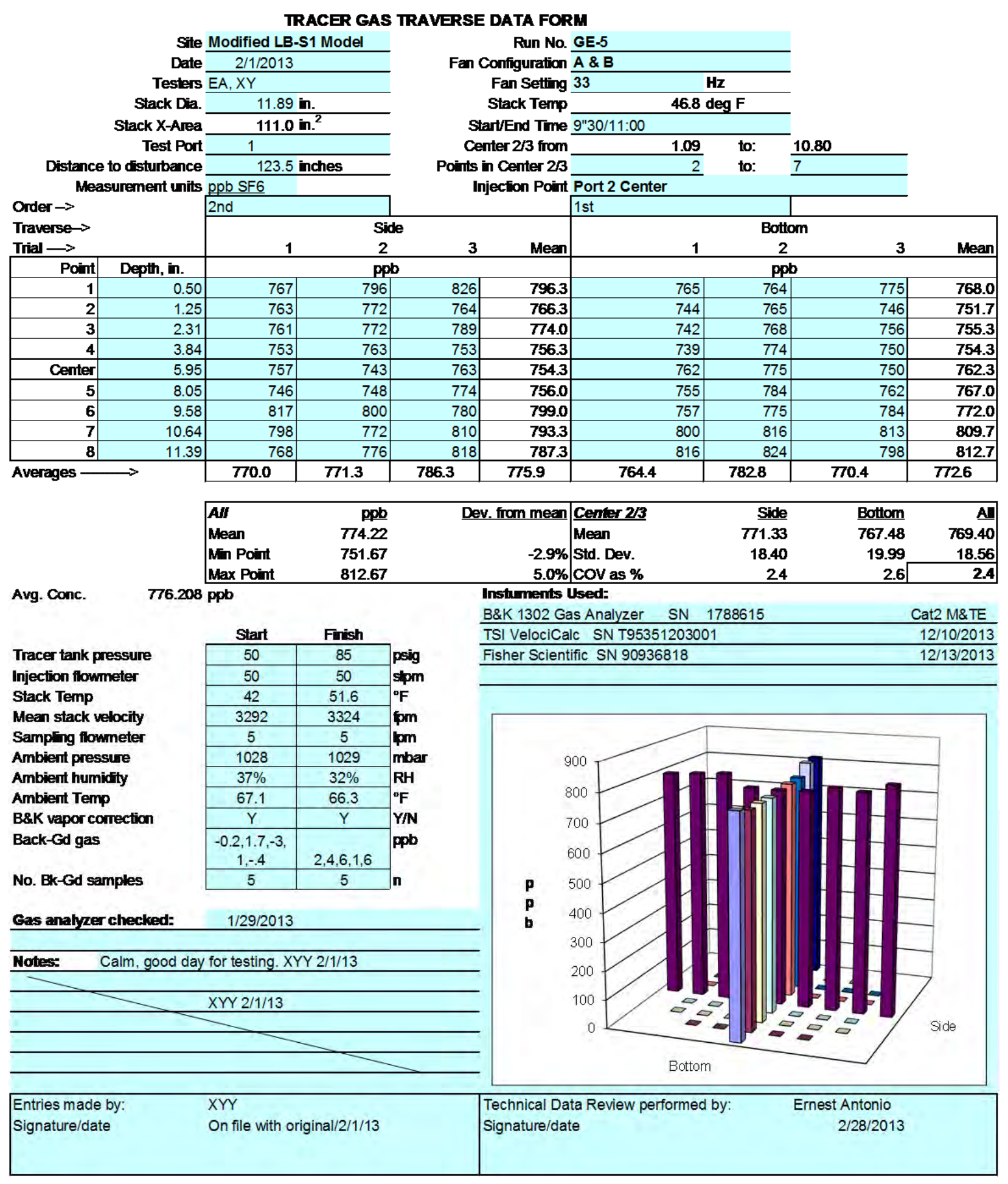


TRACER GAS TRAVERSE DATA FORM

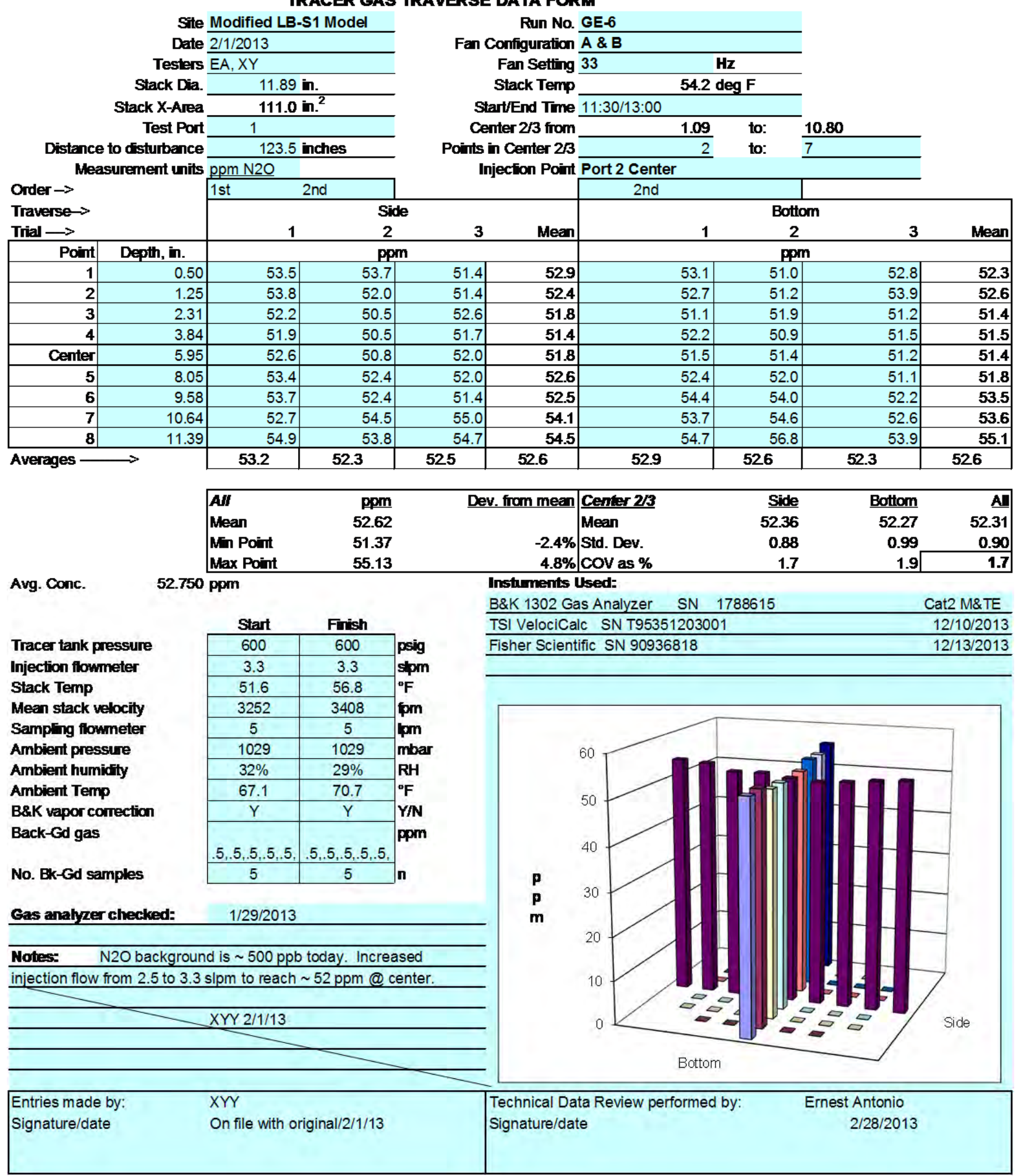




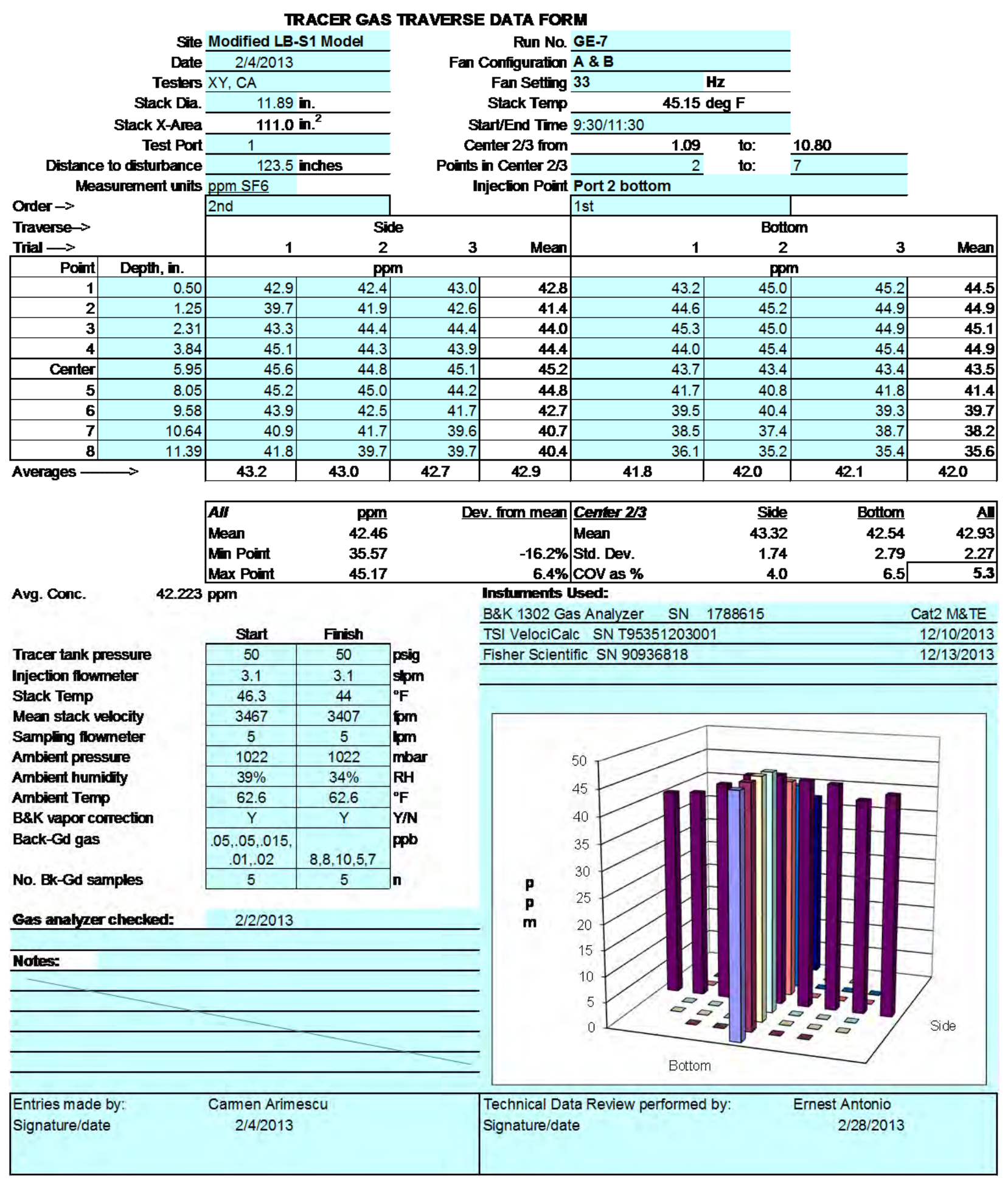




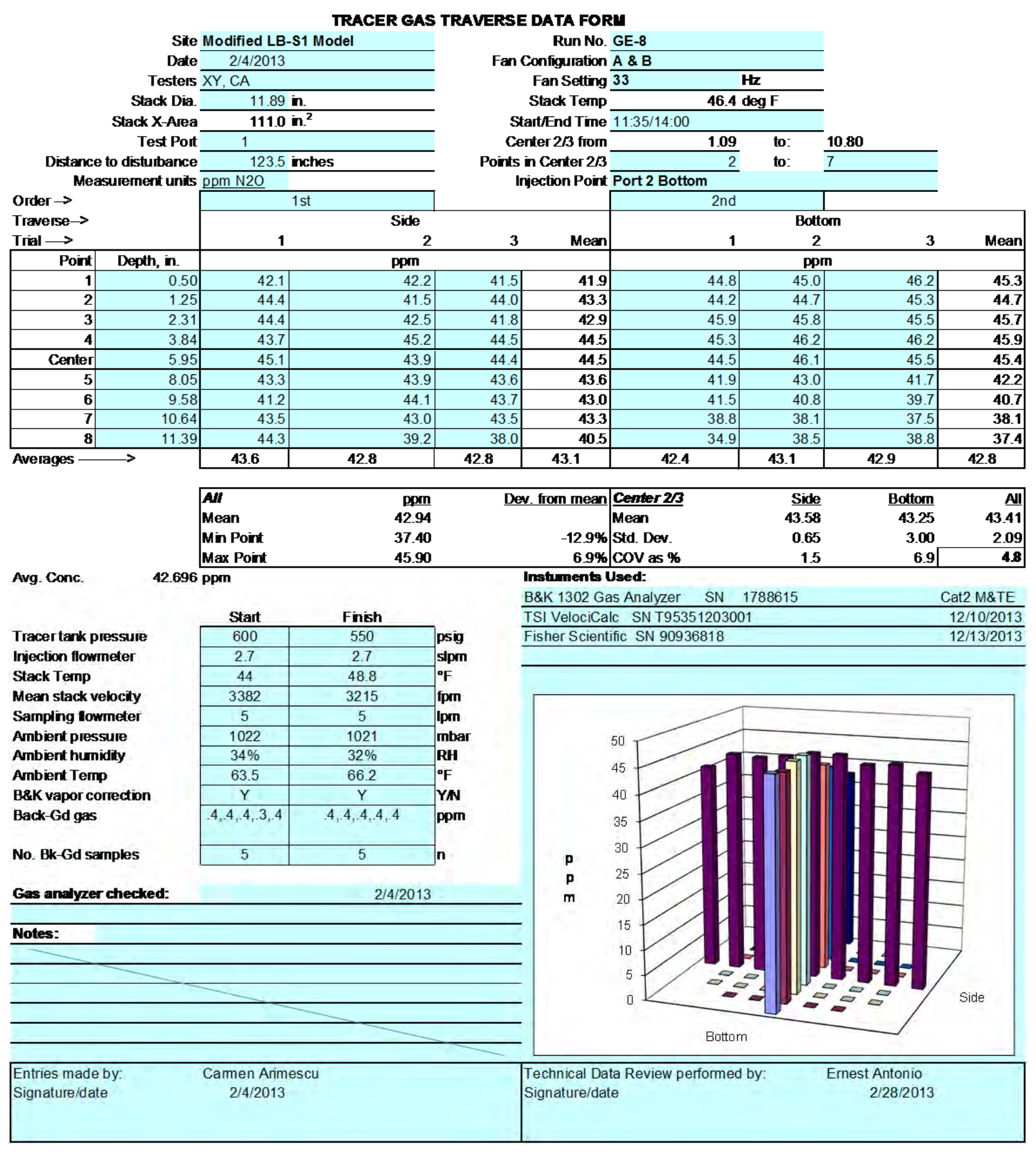




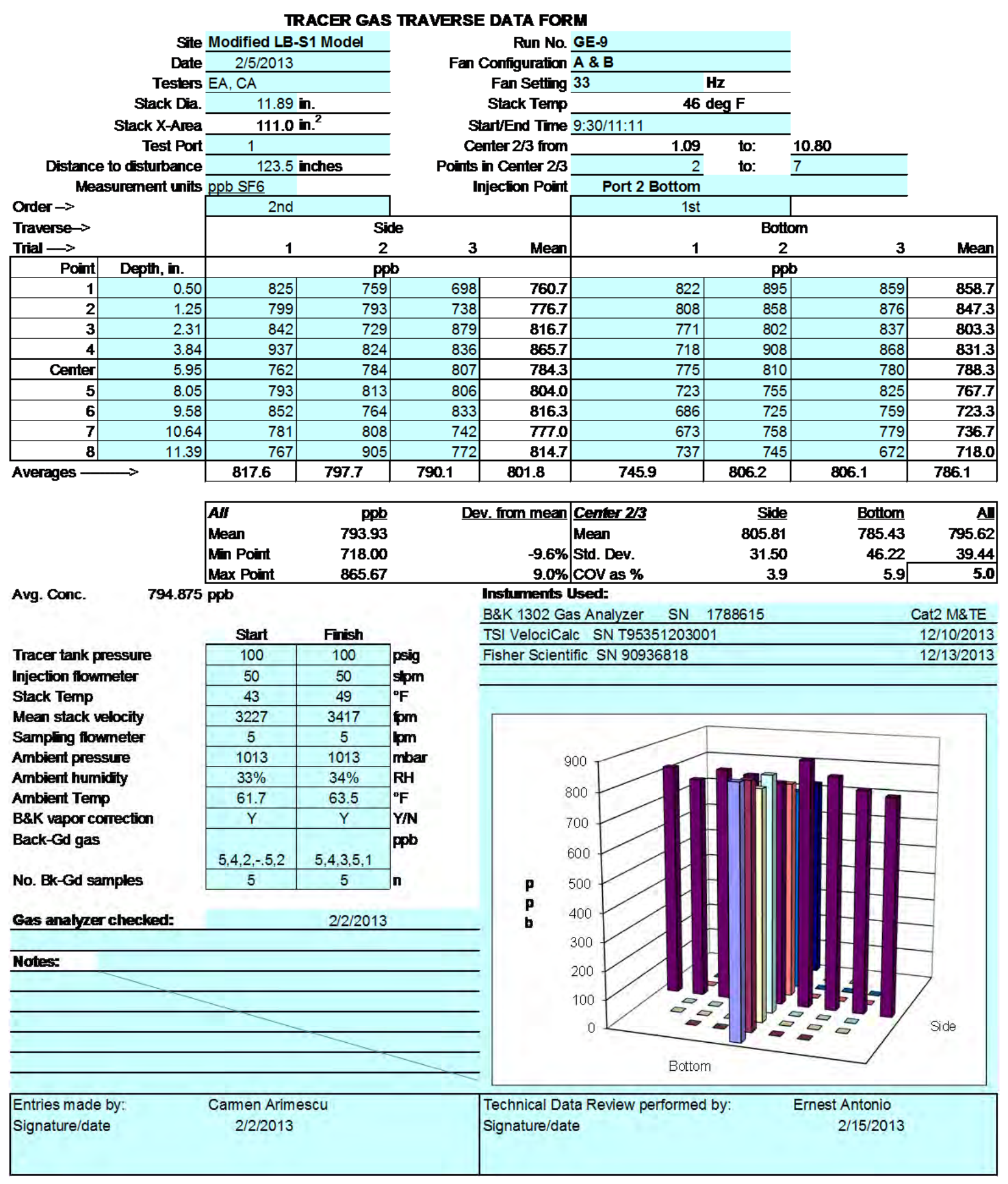




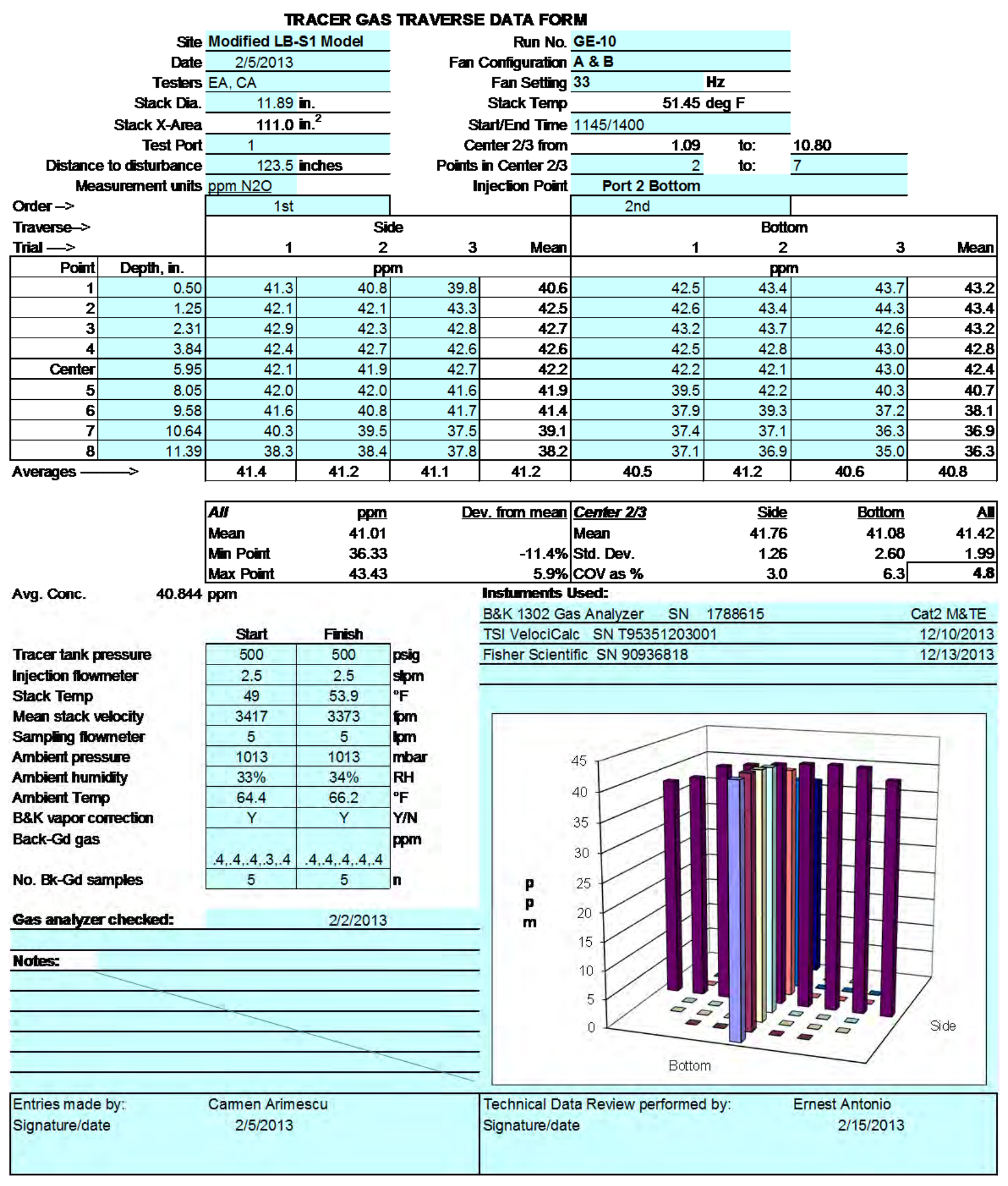




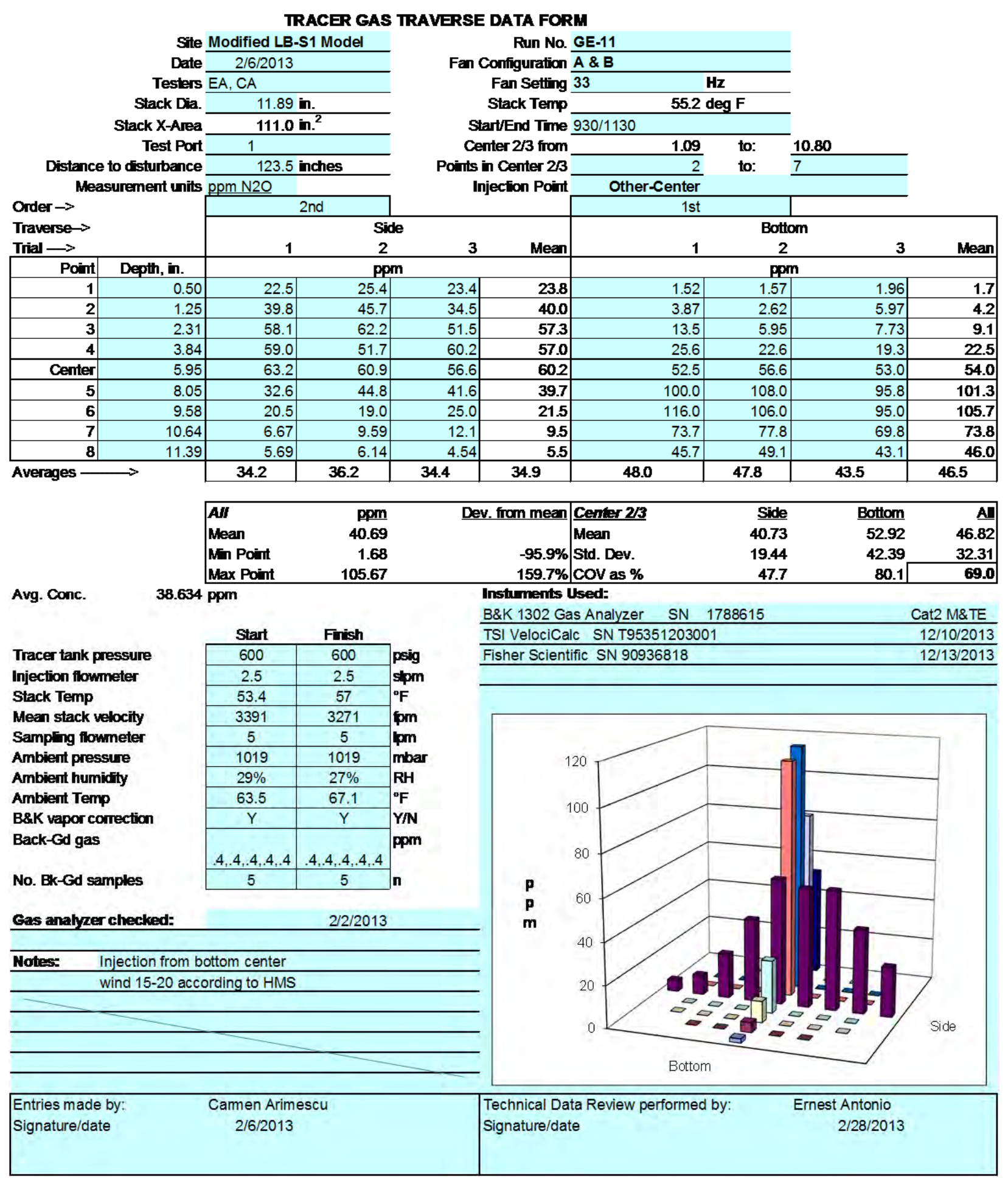




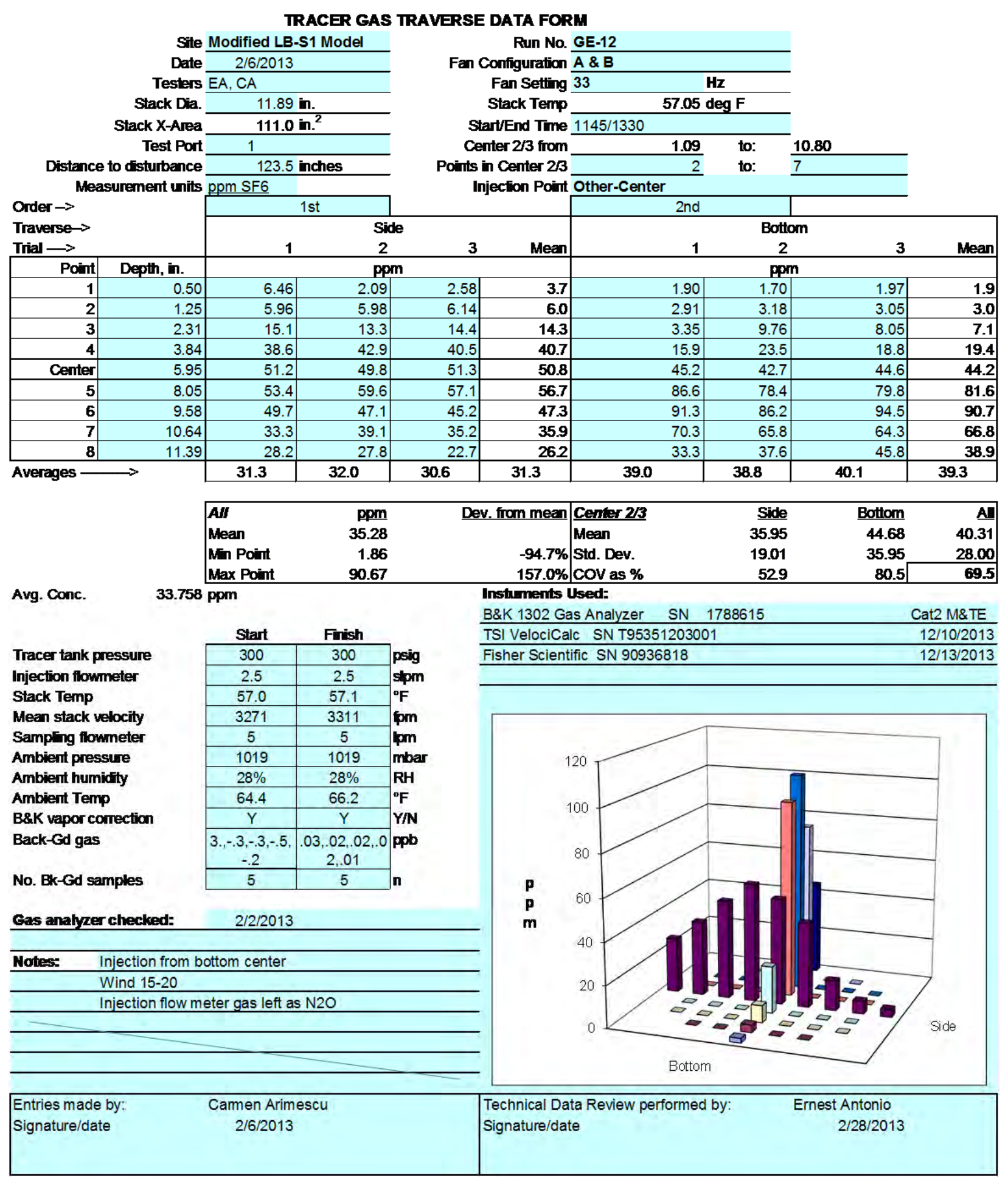




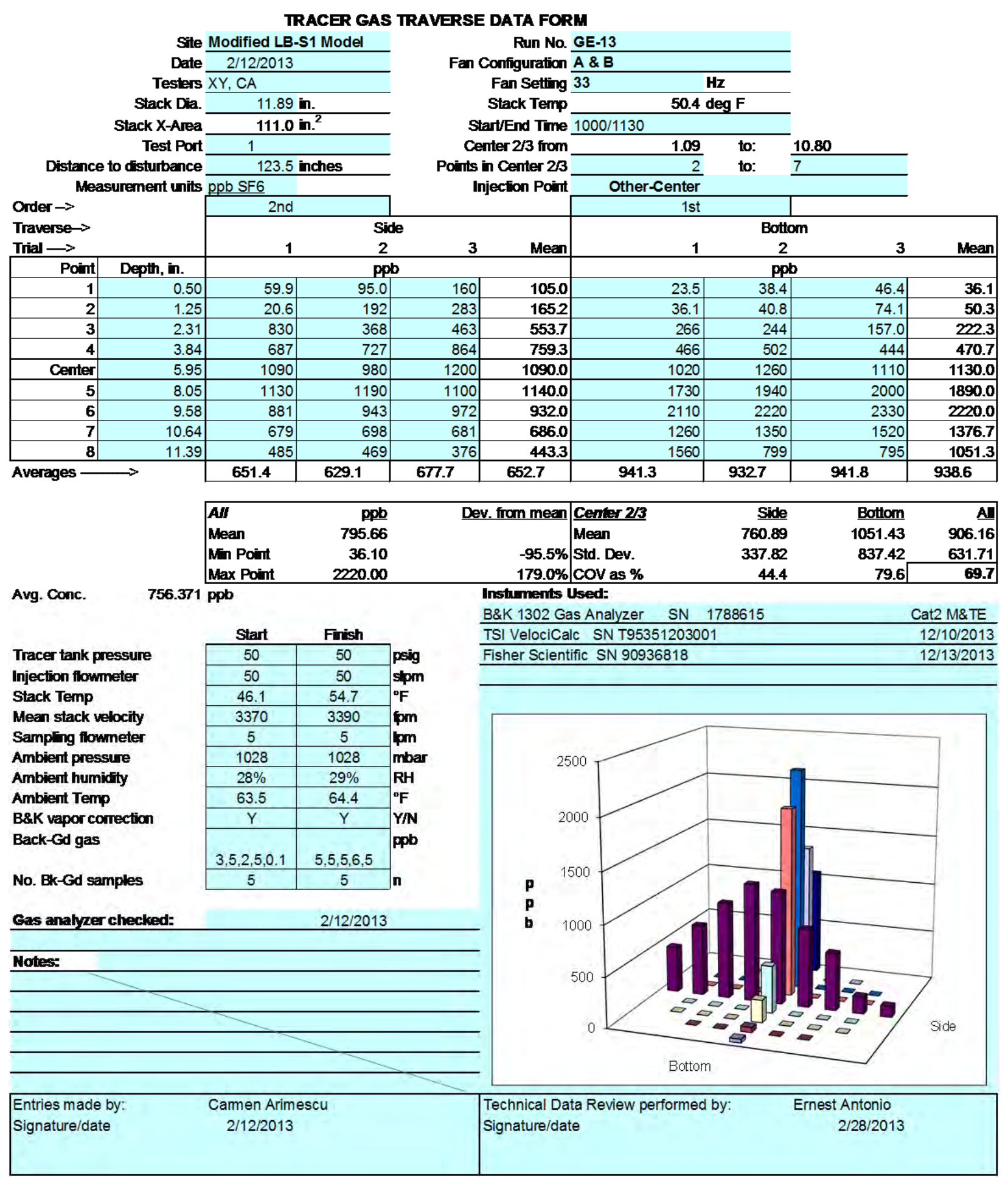




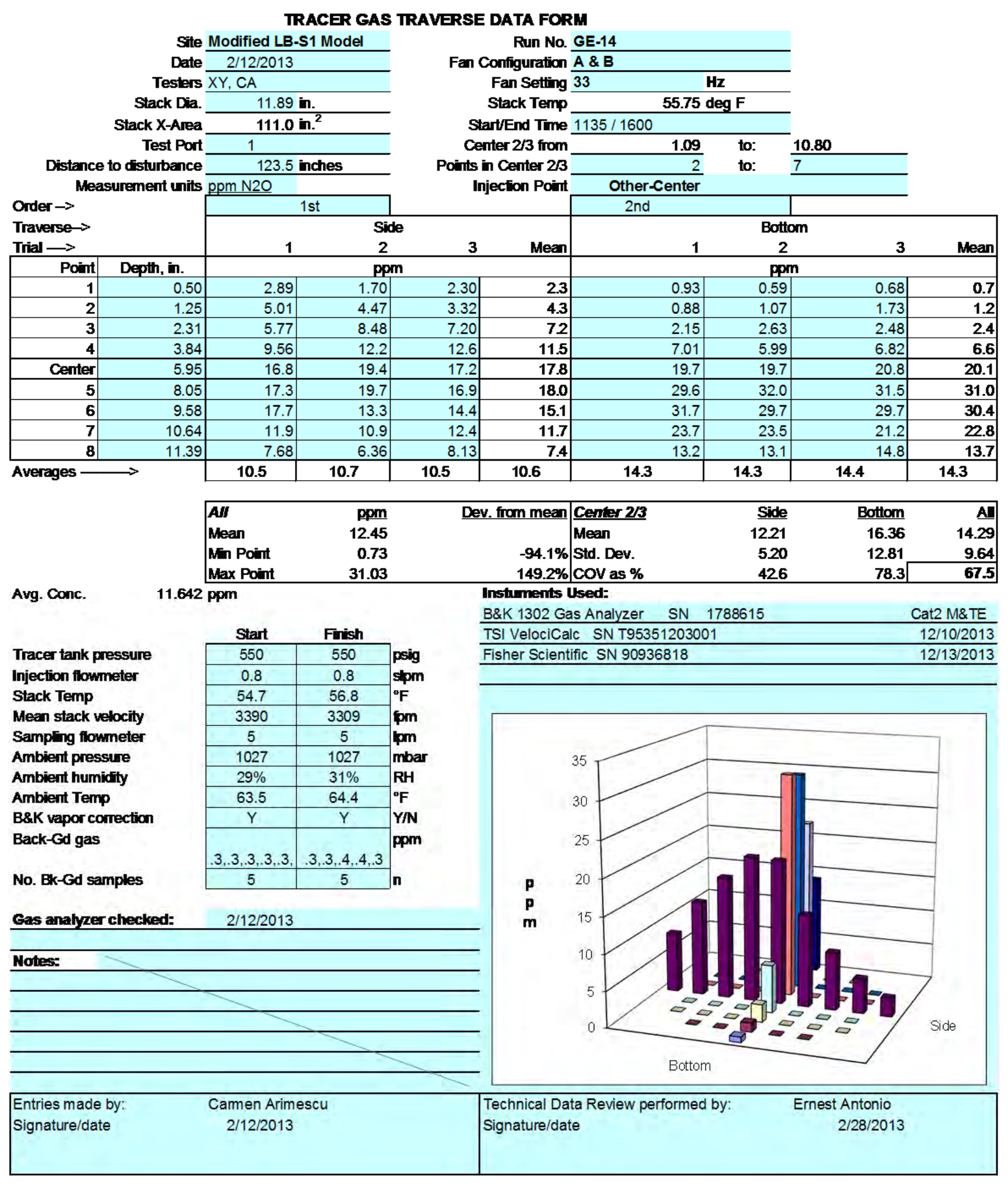




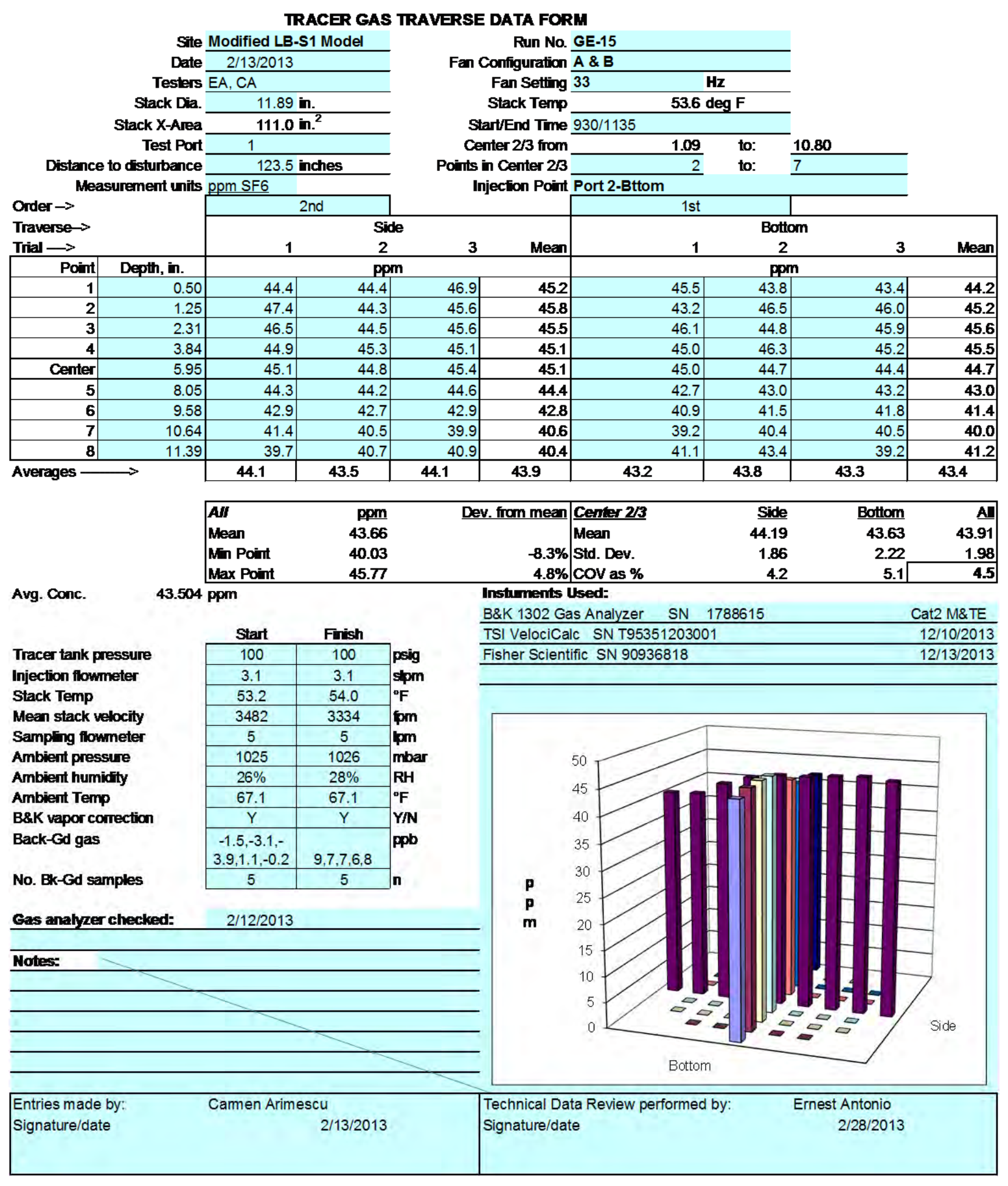




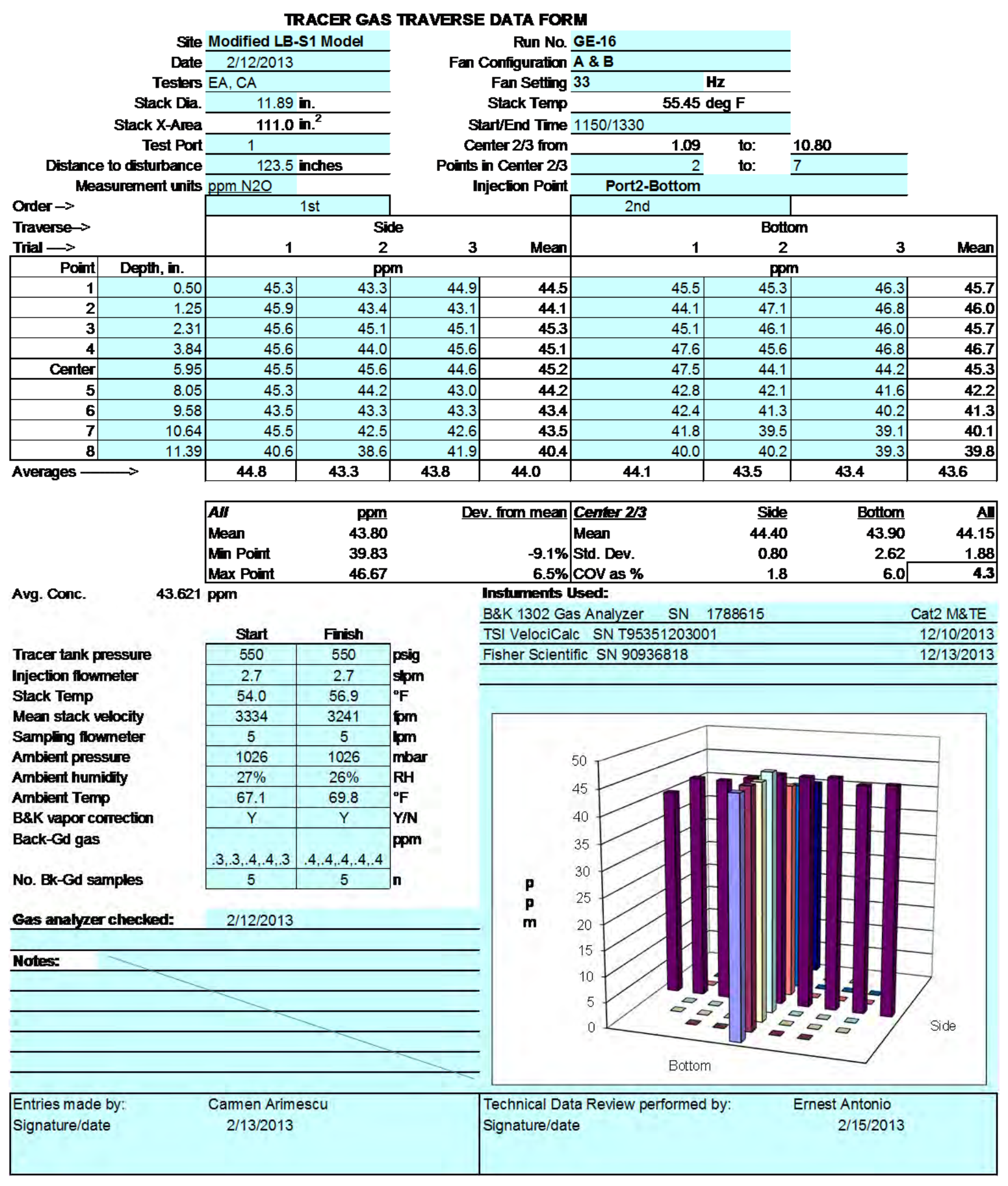




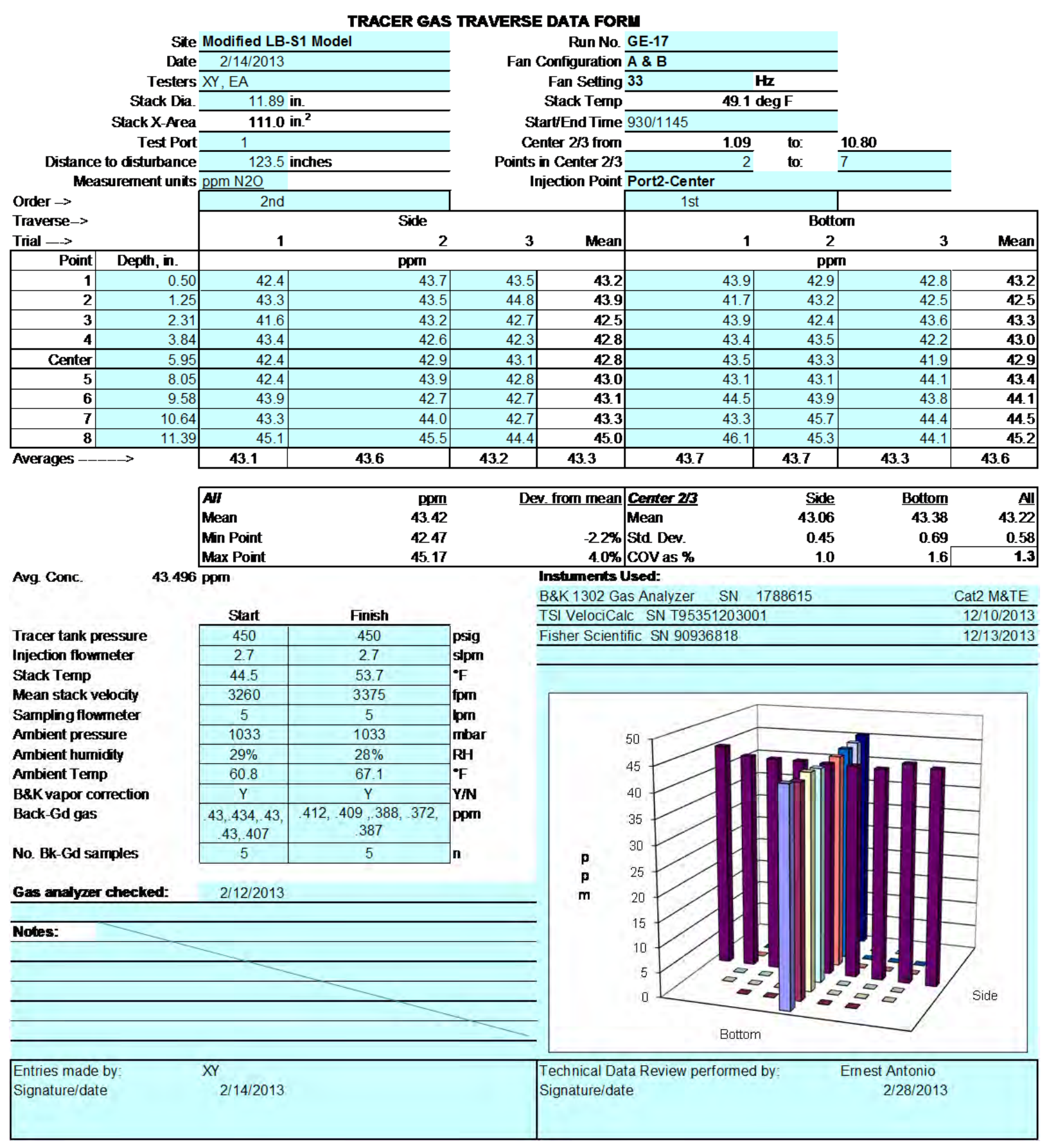




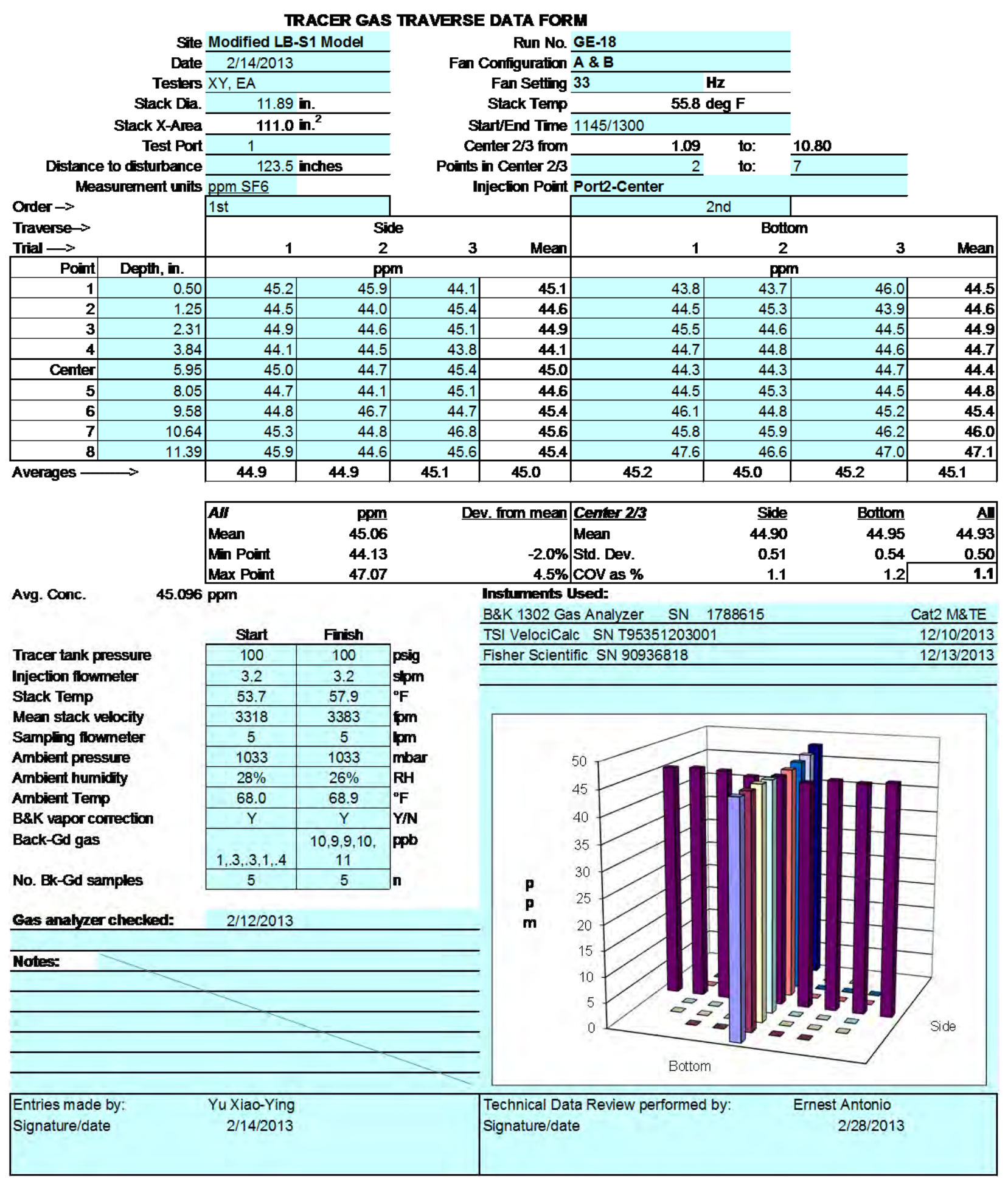



Appendix C

LV-S2 Data Sheets 



\section{C.1 LV-S2 Calibration of Ventilation Flow Controller}

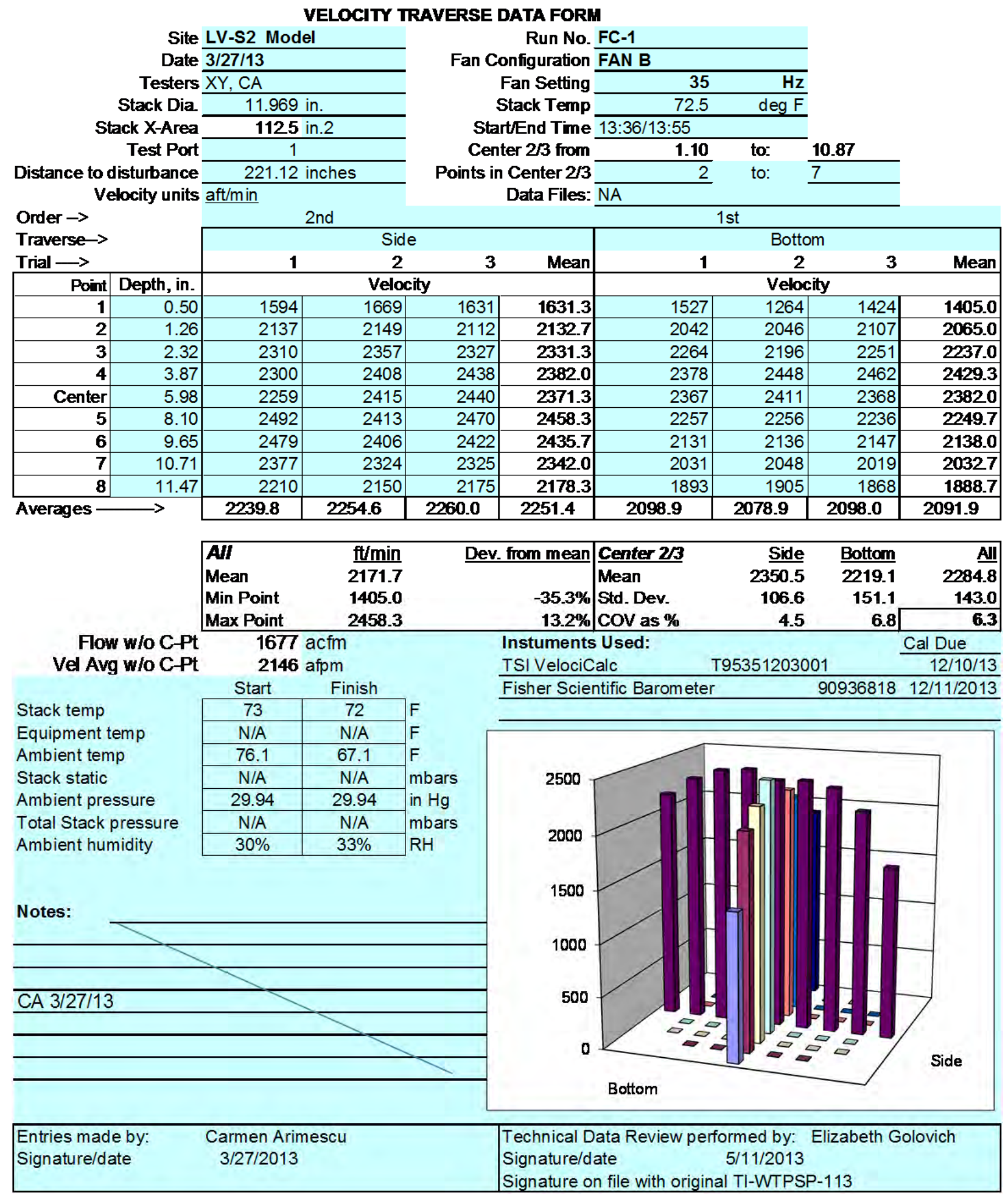


VELOCITY TRAVERSE DATA FORM

Site LV-S2 Model

Date 3/27/13

Testers $\mathrm{XY}, \mathrm{CA}$

Stack Dia.

Stack X-Area

Test Port

\begin{tabular}{|c|}
\hline 11.969 in. \\
\hline 1125 in. 2 \\
\hline 1 \\
\hline
\end{tabular}

Distance to disturbance

Velocity units aft/min

Run No. FC-2

Fan Configuration FAN A

Fan Setting

Stack Temp

Start/End Time

Center 2/3 from

Points in Center 2/3

Data Files: $\frac{\mathrm{NA}}{2 \mathrm{nd}}$

\begin{tabular}{|c|c|}
\hline $\begin{array}{l}\text { Order } \rightarrow \\
\text { Traverse-> } \\
\text { Trial } \longrightarrow\end{array}$ & \\
\hline Point & Depth, in \\
\hline 1 & 0.5 \\
\hline 2 & 1.2 \\
\hline 3 & 2.3 \\
\hline 4 & 3.8 \\
\hline Center & 5.9 \\
\hline 5 & 8.1 \\
\hline 6 & 9.6 \\
\hline 7 & 10.7 \\
\hline 8 & 11. \\
\hline
\end{tabular}

$1 \mathrm{st}$

\begin{tabular}{|rr}
\multicolumn{2}{c}{1 st } \\
& Sid \\
$\mathbf{1}$ & $\mathbf{2}$
\end{tabular}

$\begin{array}{cc}\text { Side } & \\ 2 & 3\end{array}$

3 Mean

2nd

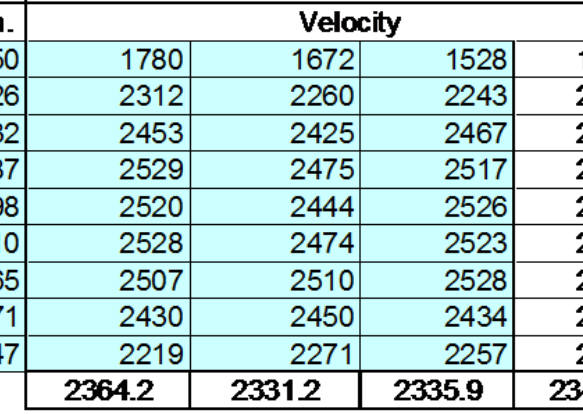

\begin{tabular}{|l|}
\hline 1660.0 \\
\hline 2271.7 \\
\hline 2448.3 \\
\hline 2507.0 \\
\hline 2496.7 \\
\hline 2508.3 \\
\hline 2515.0 \\
\hline 2438.0 \\
\hline 2249.0 \\
\hline 343.8 \\
\hline
\end{tabular}

\begin{tabular}{|c|c|c|c|}
\hline 1 & 2 & 3 & Mean \\
\hline \multicolumn{4}{|c|}{ Velocity } \\
\hline 1631 & 1256 & 1242 & 1376.3 \\
\hline 2157 & 2177 & 2162 & 2165.3 \\
\hline 2356 & 2380 & 2378 & 2371.3 \\
\hline 2489 & 2486 & 2484 & 2486.3 \\
\hline 2456 & 2414 & 2424 & 2431.3 \\
\hline 2421 & 2339 & 2370 & 2376.7 \\
\hline 2290 & 2368 & 2290 & 2316.0 \\
\hline 2177 & 2227 & 2177 & 2193.7 \\
\hline 2024 & 2004 & 2072 & 2033.3 \\
\hline 2222.3 & 2183.4 & 2177.7 & 2194.5 \\
\hline
\end{tabular}

\begin{tabular}{|lr}
\hline $\boldsymbol{A} I$ & $\underline{\mathrm{ft} / \mathrm{min}}$ \\
Mean & $\mathbf{2 2 6 9 . 1}$ \\
Min Point & $\mathbf{1 3 7 6 . 3}$ \\
Max Point & $\mathbf{2 5 1 5 . 0}$ \\
\hline
\end{tabular}

\begin{tabular}{|c|c|c|c|c|}
\hline Dev. from mean & Center 2/3 & Side & Bottom & All \\
\hline & Mean & 2455.0 & 2334.4 & 2394.7 \\
\hline$-39.3 \%$ & Std. Dev. & 86.4 & 118.6 & 117.7 \\
\hline $10.8 \%$ & cov as $\%$ & 3.5 & 5.1 & 4.9 \\
\hline
\end{tabular}

Flow w/o C-Pt

$1754 \mathrm{acfm}$ 2245 afpm

Vel Avg w/o C-Pt Start Finish

Stack temp

Equipment temp Ambient temp

Stack static

Ambient pressure

Total Stack pressure

Ambient humidity

\begin{tabular}{|c|c|l|}
\hline 72 & 72 & F \\
\cline { 1 - 2 } N/A & N/A & F \\
\hline 66.2 & 67.1 & F \\
\cline { 1 - 2 } N/A & N/A & mbars \\
\cline { 1 - 2 } 29.94 & 29.94 & in Hg \\
\cline { 1 - 2 } N/A & N/A & mbars \\
\cline { 1 - 2 } $33 \%$ & $34 \%$ & RH \\
\hline
\end{tabular}

Notes:

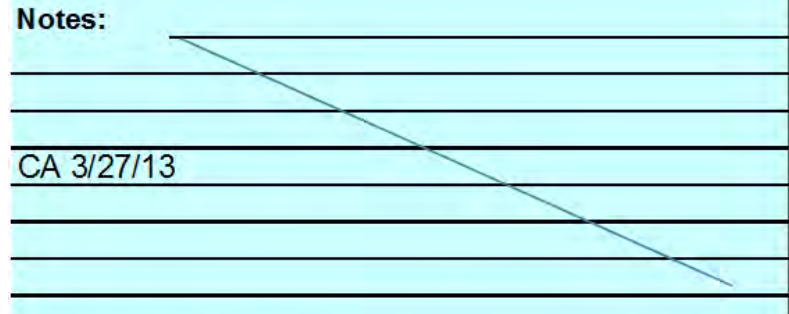
Instuments Used:

TSI VelociCalc $\quad$ T95351203001

Cal Due

Fisher Scientific Barometer

$90936818 \quad 12 / 11 / 2013$

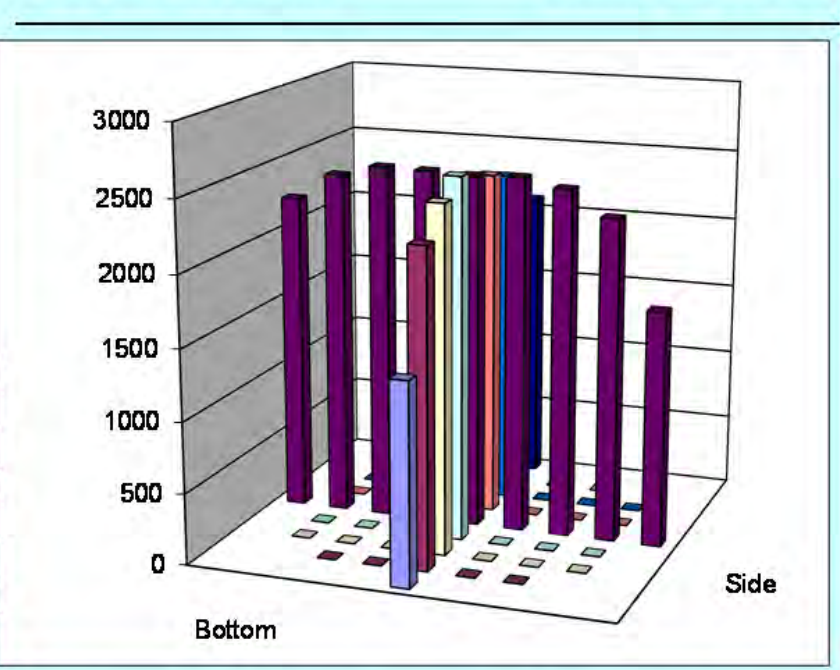

Technical Data Review performed by: Elizabeth Golovich Signature/date

Signature on file with original TI-WTPSP-113 


\section{VELOCITY TRAVERSE DATA FORM}

Site LV-S2 Model

Date 4/9/13

Testers EA

Stack Dia. Stack X-Area

Test Port

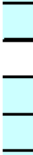

11.969 in 1125 in. 2

Distance to disturbance Velocity units aft/min
Run No. FC-3

Fan Configuration Fan A

Fan Setting 25

Stack Temp

Start/End Time 1100/1135

Center 233 from

Points in Center 2/3

Data Files: NA

$\begin{aligned} & \text { Order } \rightarrow \\
& \text { Traverse } \rightarrow\end{aligned}$
Trial $\longrightarrow$.
\begin{tabular}{|r|r|}
\hline Point & Depth, in. \\
\hline $\mathbf{1}$ & 0.50 \\
\hline $\mathbf{2}$ & 1.26 \\
\hline $\mathbf{3}$ & 2.32 \\
\hline $\mathbf{4}$ & 3.87 \\
\hline Center & 5.98 \\
\hline $\mathbf{5}$ & 8.10 \\
\hline $\mathbf{6}$ & 9.65 \\
\hline $\mathbf{7}$ & 10.71 \\
\hline $\mathbf{8}$ & 11.47 \\
\hline
\end{tabular}

\begin{tabular}{|r|r|r|r|}
\hline \multicolumn{4}{|c|}{$\begin{array}{c}\text { Side } \\
\mathbf{2}\end{array}$} \\
\hline \multicolumn{3}{|c|}{ Velocity } \\
\hline 1019 & 1357 & 1286 & $\mathbf{3}$ \\
\hline 1979 & 1904 & 1937 & \\
\hline 2069 & 2076 & 2109 & \\
\hline 2095 & 2117 & 2116 & \\
\hline 2118 & 2095 & 2067 & \\
\hline 2109 & 2135 & 2102 & \\
\hline 2108 & 2113 & 2113 & \\
\hline 2077 & 2079 & 2038 & \\
\hline 1915 & 1869 & 1912 & $\mathbf{1 9}$ \\
\hline $\mathbf{1 9 4 3 . 2}$ & $\mathbf{1 9 7 1 . 7}$ & $\mathbf{1 9 6 4 . 4}$ & $\mathbf{1 9}$ \\
\hline
\end{tabular}

\begin{tabular}{r|r|r|r|r|}
\cline { 2 - 5 } Mean & \multicolumn{5}{|c|}{ 1st } \\
& $\mathbf{4}$ & \multicolumn{3}{c|}{$\mathbf{2}$} \\
$\mathbf{1 2 2 0 . 7}$ & 1204 & 1620 & 1612 & $\mathbf{1 4 7 8 . 7}$ \\
\hline $\mathbf{1 9 4 0 . 0}$ & 1909 & 1978 & 1972 & $\mathbf{1 9 5 3 . 0}$ \\
\hline $\mathbf{2 0 8 4 . 7}$ & 2144 & 2165 & 2045 & $\mathbf{2 1 1 8 . 0}$ \\
\hline $\mathbf{2 1 0 9 . 3}$ & 2264 & 2220 & 2174 & $\mathbf{2 2 1 9 . 3}$ \\
\hline $\mathbf{2 0 9 3 . 3}$ & 2198 & 2183 & 2098 & $\mathbf{2 1 5 9 . 7}$ \\
\hline $\mathbf{2 1 1 5 . 3}$ & 2215 & 2111 & 2040 & $\mathbf{2 1 2 2 0}$ \\
\hline $\mathbf{2 1 1 1 . 3}$ & 2123 & 2038 & 2001 & $\mathbf{2 0 5 4 . 0}$ \\
\hline $\mathbf{2 0 6 4 . 7}$ & 1964 & 1968 & 1910 & $\mathbf{1 9 4 7 . 3}$ \\
\hline $\mathbf{1 8 9 8 . 7}$ & 1855 & 1843 & 1785 & $\mathbf{1 8 2 7 . 7}$ \\
\hline $\mathbf{1 9 5 9 . 8}$ & $\mathbf{1 9 8 6 . 2}$ & $\mathbf{2 0 1 4 . 0}$ & $\mathbf{1 9 5 9 . 7}$ & $\mathbf{1 9 8 6 . 6}$ \\
\hline
\end{tabular}

\begin{tabular}{|c|c|c|c|c|c|c|}
\hline $\boldsymbol{A} \boldsymbol{I}$ & ft/min & Dev. from mean & Center 2/3 & Side & Bottom & All \\
\hline Mean & 1973.2 & & Mean & 2074 & 2081.9 & 2078.0 \\
\hline Min Point & 1220.7 & $-38.1 \%$ & Std. Dev. & 61.8 & 1028 & 81.5 \\
\hline Max Point & 2219.3 & $125 \%$ & Cov as \% & 3.0 & 4.9 & 3.9 \\
\hline
\end{tabular}

Flow w/o C-Pt Vel Avg w/o C-Pt

1527 acfm
1954 afpm

$125 \%$ COV as $\%$

1.10

$\mathrm{Hz}$
$67.0 \quad \operatorname{deg} \mathrm{F}$

10 to: 10.87

Instuments Used: Cal Due

TSI VelociCalc $\quad$ T95351203001 $\quad 12 / 10 / 13$

Fisher Scientific Barometer $90936818 \quad 12 / 11 / 2013$

Stack temp

Equipment temp Ambient temp

Stack static

Ambient pressure

Total Stack pressure

Ambient humidity

\begin{tabular}{|c|c|l|}
\hline \multicolumn{1}{c|}{ Start } & Finish & \\
\hline 68 & 66 & F \\
\hline NA & NA & F \\
\hline 55 & 60 & F \\
\hline NA & NA & mbars \\
\hline 30.15 & 30.18 & in Hg \\
\hline NA & NA & mbars \\
\hline $44 \%$ & 38.00 & RH \\
\hline
\end{tabular}

Notes: The Wye intersection was installed this morning combining both fans into one output. The output was Connected to Fan A back draft damper.
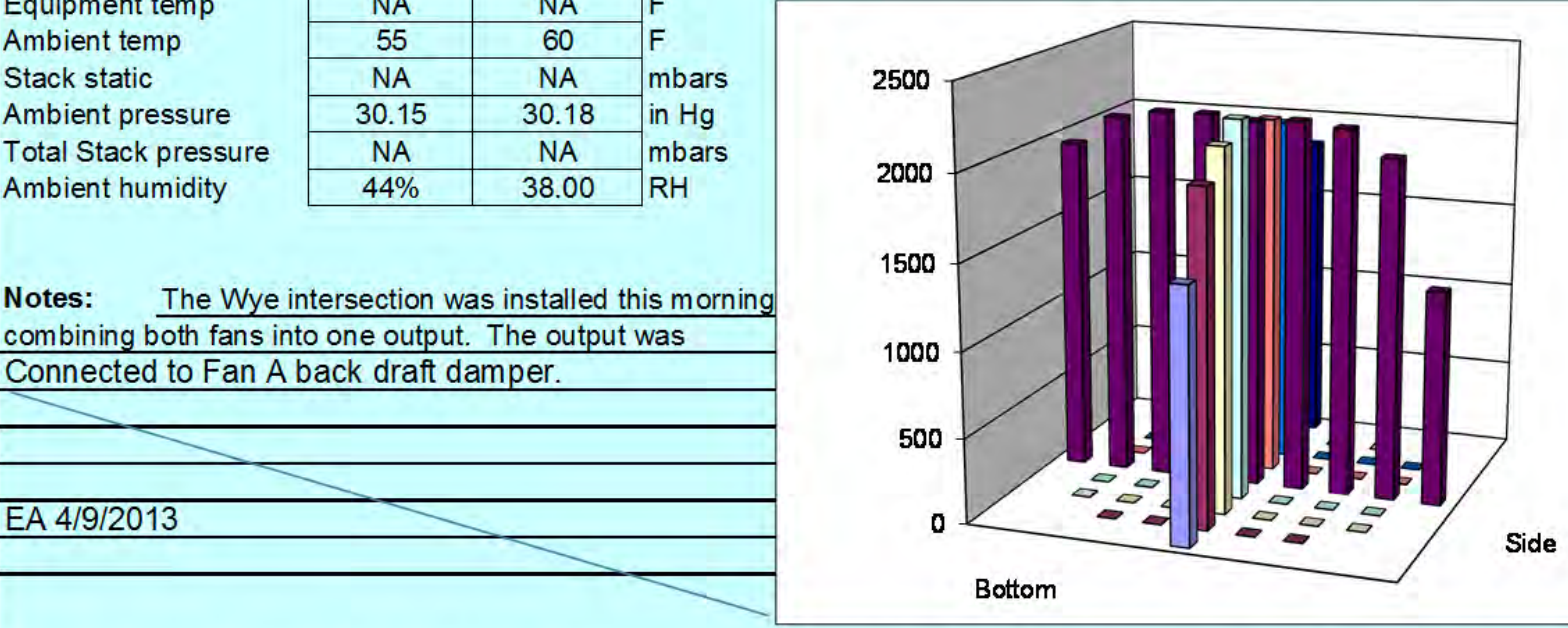

Technical Data Review performed by: Elizabeth Golovich Signature/date

Signature on file with original TI-WTPSP-113 
VELOCITY TRAVERSE DATA FORM

Site LV-S2 Model

Date 4/17/13

Testers EA, CA

Stack Dia

Stack X-Area

Test Port

\begin{tabular}{c}
\hline 11.969 in. \\
\hline $\mathbf{1 1 2 5}$ in. 2 \\
\hline 1 \\
\hline 221.12 inches
\end{tabular}

Distance to disturbance

Velocity units aft/min
Run No. FC-4

Fan Configuration Fan B

Fan Setting 35

Stack Temp

Start/End Time $1108 / 1130$

Center 2/3 from

Points in Center 2/3

Data Files: NA

\begin{tabular}{|c|c|}
\hline $\begin{array}{l}\text { Order } \rightarrow \\
\text { Traverse-> } \\
\text { Trial } \longrightarrow\end{array}$ & \\
\hline Point & Depth, in. \\
\hline 1 & 0.50 \\
\hline 2 & 1.2 \\
\hline 3 & 2.3 \\
\hline 4 & 3.8 \\
\hline Center & 5.9 \\
\hline 5 & 8.10 \\
\hline 6 & 9.6 \\
\hline 7 & 10.71 \\
\hline 8 & 11.4 \\
\hline
\end{tabular}

\begin{tabular}{|c|c|}
\hline \multicolumn{2}{|l|}{ 2nd } \\
\hline \multicolumn{2}{|c|}{1} \\
\hline & \\
\hline 1931 & $\leq$ \\
\hline 2761 & 2 \\
\hline 2890 & 2 \\
\hline 2953 & 2 \\
\hline 2953 & 2 \\
\hline 2969 & 2 \\
\hline 2982 & 2 \\
\hline 2923 & 2 \\
\hline 2762 & 2 \\
\hline 91.6 & 20.3 \\
\hline
\end{tabular}

\begin{tabular}{|c|c|c|}
\hline \multicolumn{3}{|c|}{ Side } \\
\hline 2 & 3 & \\
\hline \multicolumn{3}{|c|}{ Velocity } \\
\hline 2298 & 2187 & \\
\hline 2744 & 2711 & \\
\hline 2922 & 2890 & \\
\hline 2985 & 2978 & \\
\hline 2967 & 2955 & \\
\hline 2970 & 2975 & \\
\hline 2967 & 2969 & \\
\hline 2920 & 2928 & \\
\hline 2748 & 2756 & \\
\hline 5.7 & 2816.6 & 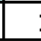 \\
\hline
\end{tabular}

\begin{tabular}{r|r|r|r|r|}
\cline { 2 - 5 } Mean & \multicolumn{5}{|c|}{$\begin{array}{c}\text { Bst } \\
\mathbf{2}\end{array}$} \\
\hline $\mathbf{2 1 3 8 . 7}$ & 1653 & 2290 & 2101 & $\mathbf{2 0 1 4 . 7}$ \\
\hline $\mathbf{2 7 3 8 . 7}$ & 2690 & 2672 & 2656 & $\mathbf{2 6 7 2 7}$ \\
\hline $\mathbf{2 9 0 0 . 7}$ & 2905 & 2890 & 2870 & $\mathbf{2 8 8 8 . 3}$ \\
\hline $\mathbf{2 9 7 2 . 0}$ & 3144 & 3199 & 3005 & $\mathbf{3 1 1 6 . 0}$ \\
\hline $\mathbf{2 9 5 8 . 3}$ & 3113 & 3196 & 2939 & $\mathbf{3 0 8 2 7}$ \\
\hline $\mathbf{2 9 7 1 . 3}$ & 2975 & 3166 & 2930 & $\mathbf{3 0 2 3 . 7}$ \\
\hline $\mathbf{2 9 7 2 . 7}$ & 2952 & 3089 & 2837 & $\mathbf{2 9 5 9 . 3}$ \\
\hline $\mathbf{2 9 2 3 . 7}$ & 2758 & 2798 & 2717 & $\mathbf{2 7 5 7 . 7}$ \\
\hline $\mathbf{2 7 5 5 . 3}$ & 2524 & 2623 & 2555 & $\mathbf{2 5 6 7 . 3}$ \\
\hline $\mathbf{2 8 1 4 . 6}$ & $\mathbf{2 7 4 6 . 0}$ & $\mathbf{2 8 8 0 . 3}$ & $\mathbf{2 7 3 4 . 4}$ & $\mathbf{2 7 8 6 . 9}$ \\
\hline
\end{tabular}

\begin{tabular}{|lrr|lrrr|}
\hline$A I I$ & $\underline{\text { tt/min }}$ & Dev. from mean & Center 2/3 & Side & Bottom & All \\
\cline { 3 - 3 } Mean & 2800.8 & & Mean & 2919.6 & 2928.6 & 2924.1 \\
Min Point & 2014.7 & $-28.1 \%$ & Std. Dev. & 84.5 & 165.9 & 126.6 \\
Max Point & 3116.0 & $11.3 \%$ & COV as \% & 2.9 & 5.7 & 4.3 \\
\hline
\end{tabular}

Flow w/o C-Pt Vel Avg w/o C-Pt

Stack temp Equipment temp Ambient temp Stack static Ambient pressure Total Stack pressure Ambient humidity

\begin{tabular}{|c|c|c|}
\hline & & \\
\hline Start & Finish & \multirow[b]{2}{*}{$\mathrm{F}$} \\
\hline 61 & 62 & \\
\hline NA & NA & $F$ \\
\hline 52.7 & 54.1 & $\mathrm{~F}$ \\
\hline NA & NA & mbars \\
\hline 30.30 & 30.30 & in $\mathrm{Hg}$ \\
\hline NA & NA & mbars \\
\hline $29 \%$ & 29.00 & $\mathrm{RH}$ \\
\hline
\end{tabular}

Notes: The Fans output were routed to the Wye Junctic and the output of the Wye Juction was connected to Fan B back draft damper.

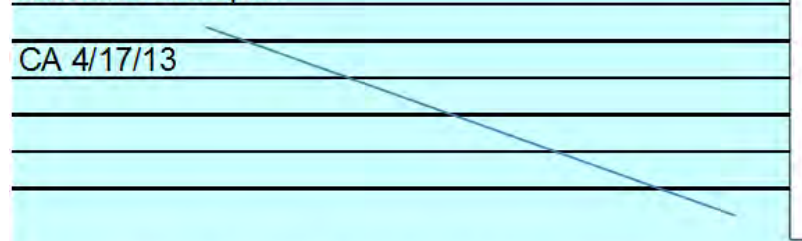

$\mathrm{Hz}$
$61.5 \quad \operatorname{deg} \mathrm{F}$

1.10 to: 10.87

1.10 to: $\quad 10.87$

2 to: $\frac{10}{7}$

TSI VelociCalc $\quad$ T95351203001 $\quad 12 / 10 / 13$

Fisher Scientific Barometer $90936818 \quad 12 / 11 / 2013$

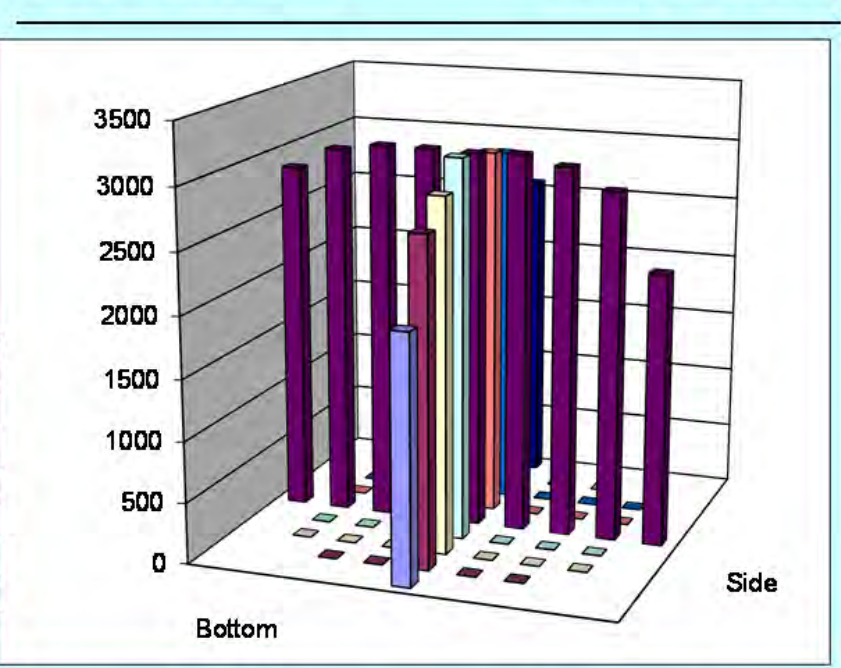

Technical Data Review performed by: Elizabeth Golovich Signature/date $\quad 5 / 27 / 2013$

Signature on file with original TI-WTPSP-113 
VELOCITY vS. FREQUENCY DATA FORM

\begin{tabular}{rlr} 
Site & LV-S2 Model \\
\cline { 2 - 3 } Date & \multicolumn{2}{c}{$3 / 27 / 2013$} \\
\cline { 2 - 2 } Tester & XY, CA \\
\cline { 2 - 3 } Stack Dia. & 11.969 & in. \\
\cline { 2 - 3 } Stack X-Area & 112.5 & in2 \\
Test Port & 1 &
\end{tabular}

\begin{aligned} Run No. VF-1 \\ Stack Temp \\ Stack RH\% \\ \cline { 2 - 2 } Baro Press \\ \cline { 2 - 2 } an Configuration \\ Start/End Time \end{aligned}

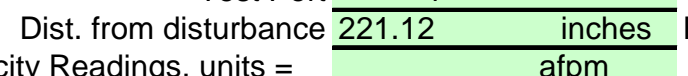

Velocity Readings, units =

Reference point from velocity test VC : Side 8

\begin{tabular}{|c|c|c|c|c|c|c|c|c|}
\hline & & & & & \multirow{6}{*}{$\begin{array}{l}\text { max } \\
\text { normal } \\
\min \end{array}$} \\
\hline & & & & & Target & Target & Estmtd & \\
\hline & & & & & acfm & afpm & $\mathrm{Hz}$ & \\
\hline & & & & & 3034 & 4636 & 73.1 & \\
\hline & & & & & 2004 & 3062 & 48.8 & \\
\hline & \multicolumn{4}{|c|}{ afpm } & 1176 & 1798 & 29.3 & \\
\hline $\mathrm{Hz}$ & 1 & 2 & 3 & Mean & StDev & 2 StDev & cfm & \\
\hline 5 & 82 & 218 & 210 & 170.00 & 76.32 & 152.63 & 132.83 & \\
\hline 10 & 554 & 520 & 569 & 547.67 & 25.11 & 50.21 & 427.92 & \\
\hline 15 & 865 & 838 & 835 & 846.00 & 16.52 & 33.05 & 661.02 & \\
\hline 20 & 1216 & 1173 & 1152 & 1180.33 & 32.62 & 65.25 & 922.25 & \\
\hline 25 & 1562 & 1496 & 1466 & 1508.00 & 49.11 & 98.22 & 1178.27 & \\
\hline 30 & 1908 & 1850 & 1835 & 1864.33 & 38.55 & 77.11 & 1456.69 & \\
\hline 35 & 2240 & 2195 & 2179 & 2204.67 & 31.63 & 63.26 & 1722.61 & \\
\hline 40 & 2606 & 2518 & 2513 & 2545.67 & 52.31 & 104.62 & 1989.05 & \\
\hline 45 & 2915 & 2860 & 2848 & 2874.33 & 35.73 & 71.45 & 2245.85 & \\
\hline 50 & 3306 & 3142 & 3152 & 3200.00 & 91.93 & 183.87 & 2500.31 & \\
\hline 55 & 3386 & 3429 & 3434 & 3416.33 & 26.39 & 52.78 & 2669.34 & \\
\hline 60 & 3522 & 3758 & 3706 & 3662.00 & 124.00 & 248.00 & 2861.29 & \\
\hline \multicolumn{7}{|c|}{ Instuments Used: } & \multicolumn{2}{|c|}{ Cal Exp. Date: } \\
\hline \multicolumn{2}{|c|}{ TSI VelociCalc } & \multicolumn{3}{|c|}{ T95351203001 } & & & \multicolumn{2}{|c|}{$12 / 10 / 2013$} \\
\hline \multicolumn{2}{|c|}{ Fisher Scientific Barometer } & 90936 & & & & & & $12 / 11 / 2013$ \\
\hline
\end{tabular}

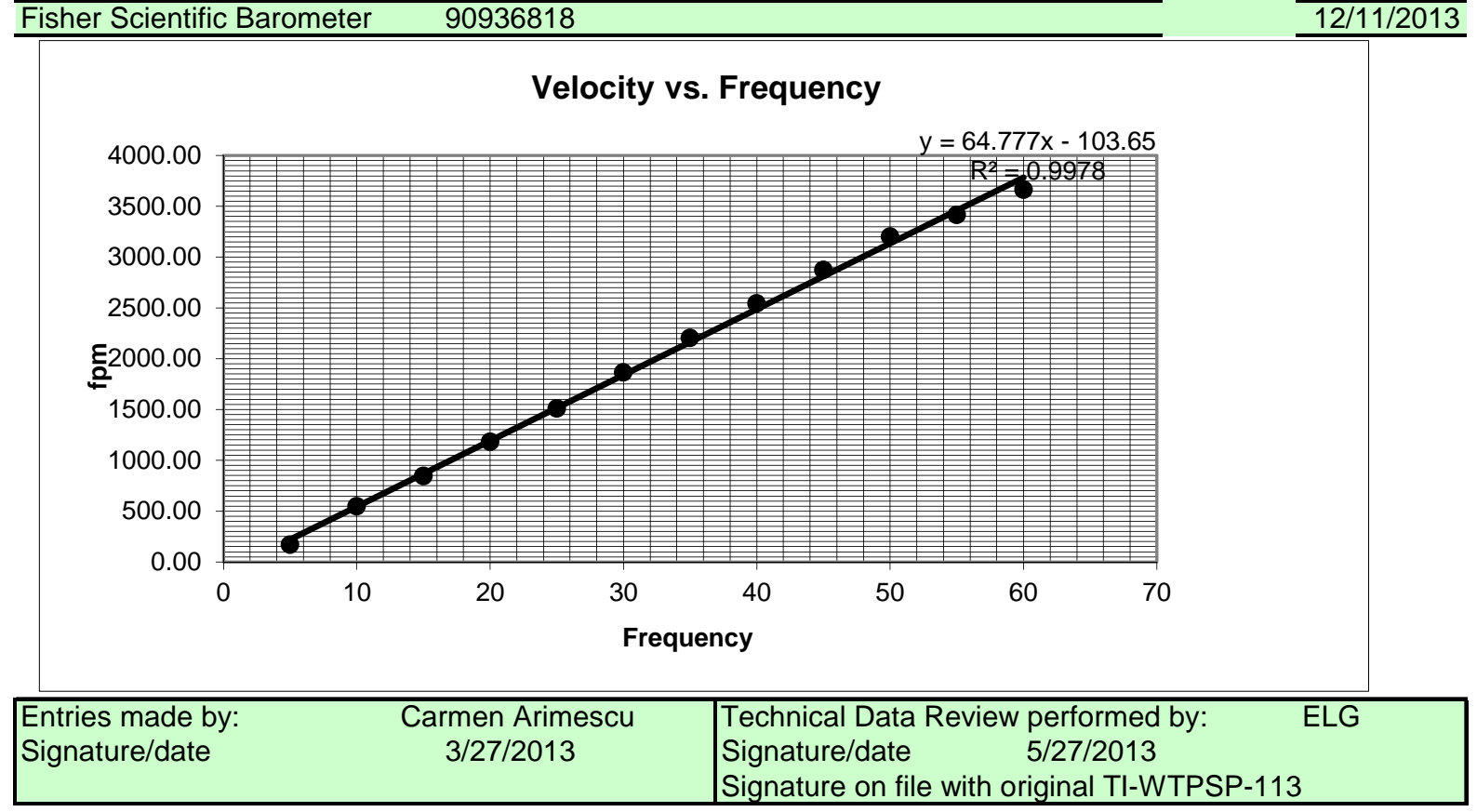

C. 5 


\section{VELOCITY vS. FREQUENCY DATA FORM}

\begin{tabular}{|c|c|c|}
\hline Site & \multirow{2}{*}{\multicolumn{2}{|c|}{$\frac{\text { LV-S2 model }}{3 / 27 / 2013}$}} \\
\hline Date & & \\
\hline Tester & \multicolumn{2}{|c|}{$\frac{3 / 27 / 2013}{E A, C A}$} \\
\hline Stack Dia. & 11.969 & in. \\
\hline Stack X-Area & 112.5 & in2 \\
\hline Test Port & 1 & \\
\hline isturbance & 221.12 & inches \\
\hline units - & & afpm \\
\hline
\end{tabular}

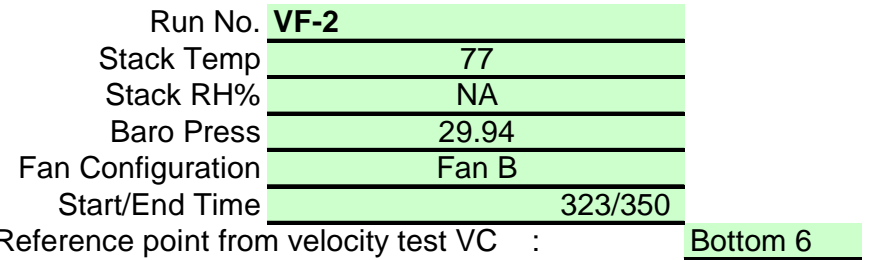

Velocity Readings, units =

\begin{tabular}{|c|c|c|c|c|c|c|}
\hline & & & & & \multirow{5}{*}{\multicolumn{2}{|c|}{ 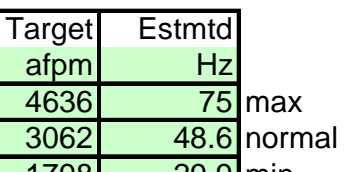 }} \\
\hline & & & Target & Target & Estmtd & \\
\hline & & & acfm & afpm & $\mathrm{Hz}$ & \\
\hline & & & 3034 & 4636 & 75 & \\
\hline & & & 2004 & 3062 & & \\
\hline \multicolumn{3}{|c|}{ afpm } & 1176 & 1798 & 29.9 & $\min$ \\
\hline 2 & 3 & Mean & StDev & 2 StDev & cfm & \\
\hline 274 & 192 & 228.67 & 41.68 & 83.36 & 178.67 & \\
\hline 520 & 505 & 511.00 & 7.94 & 15.87 & 399.27 & \\
\hline 872 & 827 & 838.33 & 29.67 & 59.34 & 655.03 & \\
\hline 1201 & 1160 & 1168.67 & 28.99 & 57.98 & 913.13 & \\
\hline 1500 & 1469 & 1488.67 & 17.10 & 34.20 & 1163.16 & \\
\hline 1794 & 1798 & 1802.67 & 11.72 & 23.44 & 1408.51 & \\
\hline 2103 & 2121 & 2121.33 & 18.50 & 37.00 & 1657.49 & \\
\hline 2409 & 2481 & 2487.00 & 81.17 & 162.33 & 1943.21 & \\
\hline 2771 & 2819 & 2786.00 & 28.62 & 57.24 & 2176.83 & \\
\hline 3043 & 3070 & 3057.33 & 13.58 & 27.15 & 2388.83 & \\
\hline 3368 & 3361 & 3362.33 & 5.13 & 10.26 & 2627.14 & \\
\hline 3674 & 3574 & 3626.67 & 50.21 & 100.43 & 2833.68 & \\
\hline
\end{tabular}

\section{Instuments Used:}

\section{TSI VelociCalc $\quad$ T95351203001}

Fisher Scientific Barometer 90936818

Exp. Date:

$12 / 10 / 2013$

$12 / 11 / 2013$

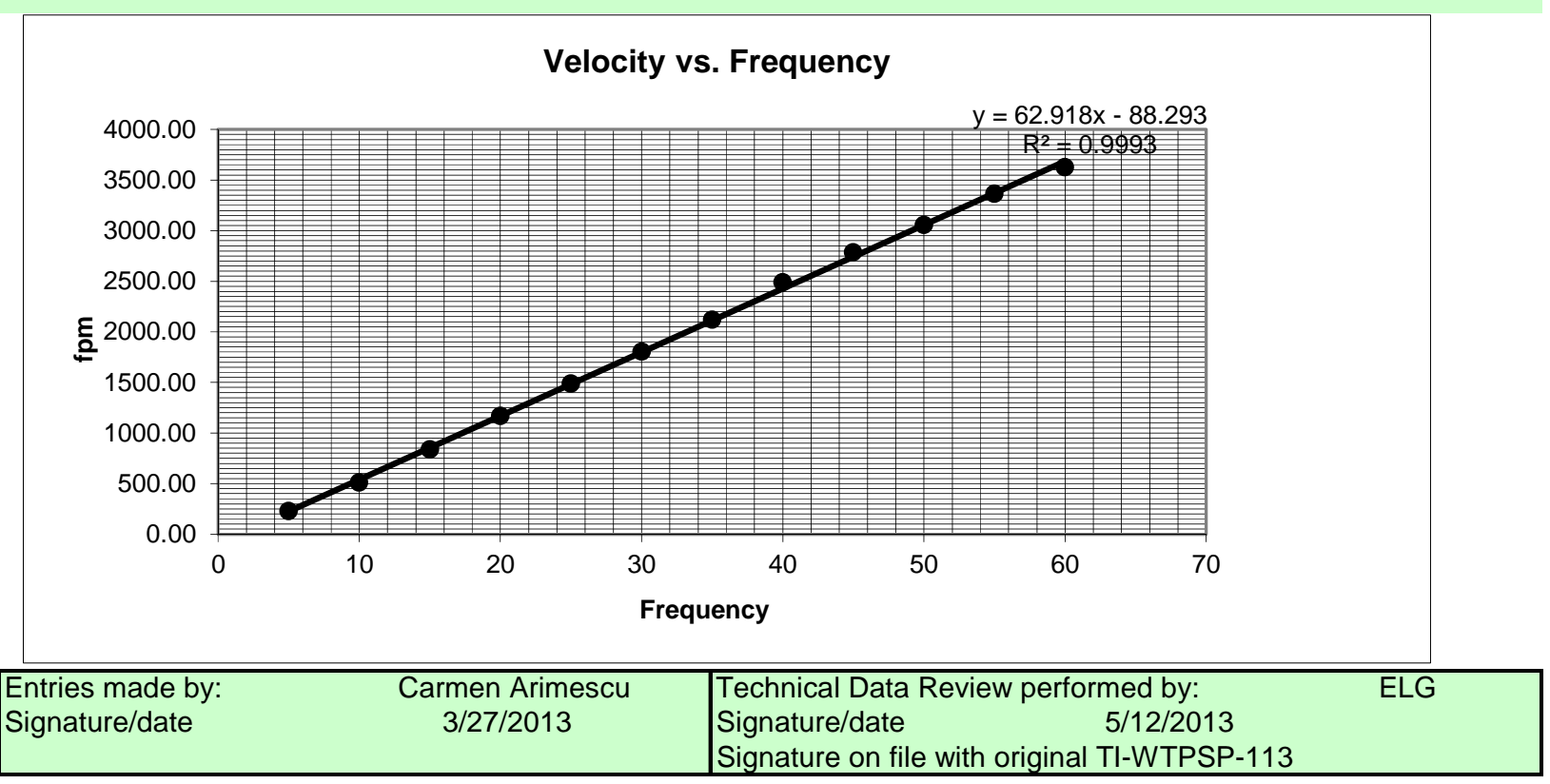




\section{VELOCITY vS. FREQUENCY DATA FORM}

\begin{tabular}{|c|c|c|}
\hline Site & \multicolumn{2}{|c|}{ LV-S2 model } \\
\hline Date & \multicolumn{2}{|c|}{$4 / 9 / 2013$} \\
\hline Tester & \multicolumn{2}{|c|}{ EA, CA } \\
\hline Stack Dia. & 11.969 & in. \\
\hline Stack X-Area & 112.5 & in2 \\
\hline Test Port & 1 & \\
\hline sturbance & 221.12 & inches \\
\hline
\end{tabular}

Run No. VF-3

Stack Temp Stack RH\% Baro Press

Fan Configuration Start/End Time

\begin{tabular}{c}
\hline 67 \\
\hline NA \\
\hline 30.21 \\
\hline Fan A \\
\hline $131 / 220$ \\
\hline
\end{tabular}
$\begin{array}{cc}\text { Dist. from disturbance } & \frac{221.12}{\text { inches }} \\ \text { Velocity Readings, units }= & \text { afpm }\end{array}$ Reference point from velocity test VC

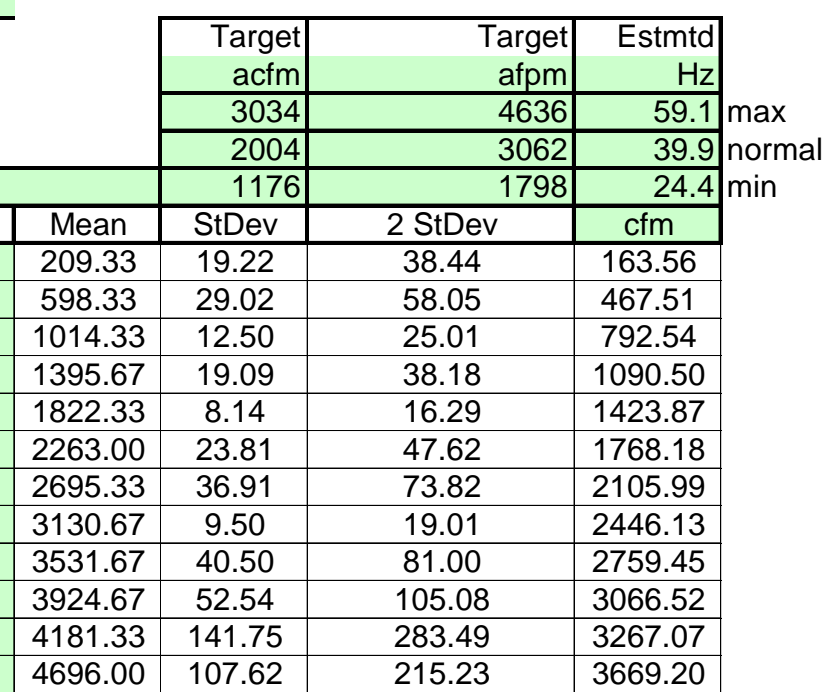

\section{Instuments Used:}

\section{TSI VelociCalc $\quad$ T95351203001}

Cal Exp. Date:

Fisher Scientific Barometer 90936818

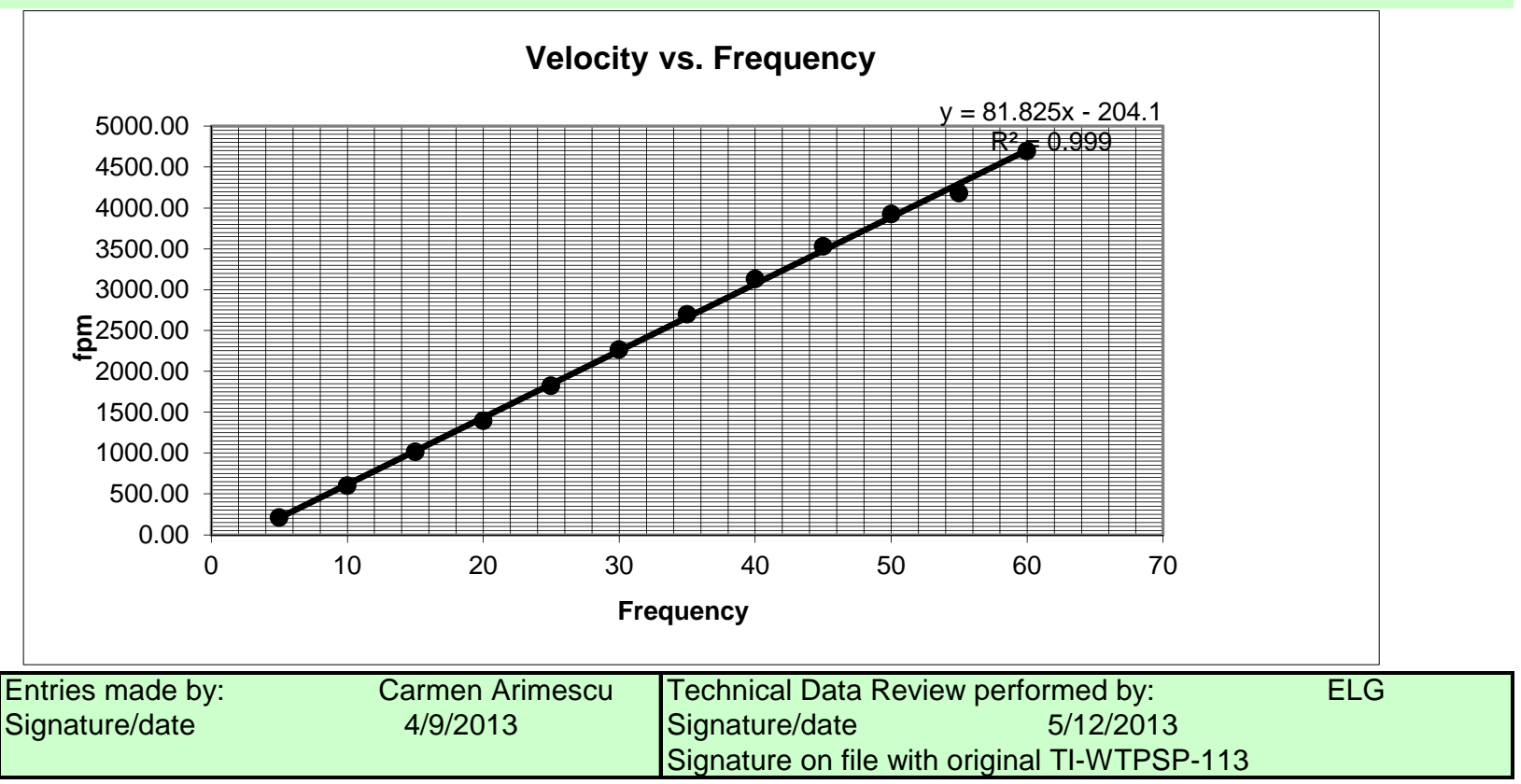

C.7 


\section{VELOCITY vS. FREQUENCY DATA FORM}

\begin{tabular}{rlr} 
Site & \multicolumn{1}{c}{ LV-S2 model } \\
\cline { 2 - 3 } Date & \multicolumn{2}{c}{$4 / 17 / 2013$} \\
\cline { 2 - 3 } Tester & \multicolumn{2}{c}{ EA, Ca } \\
\cline { 2 - 3 } Stack Dia. & 11.969 & in. \\
\cline { 2 - 2 } Stack X-Area & 112.5 & in2 \\
\cline { 2 - 3 } Test Port & 1 &
\end{tabular}

Dist. from disturbance $\overline{221.12}$

Velocity Readings, units =

inches
afpm

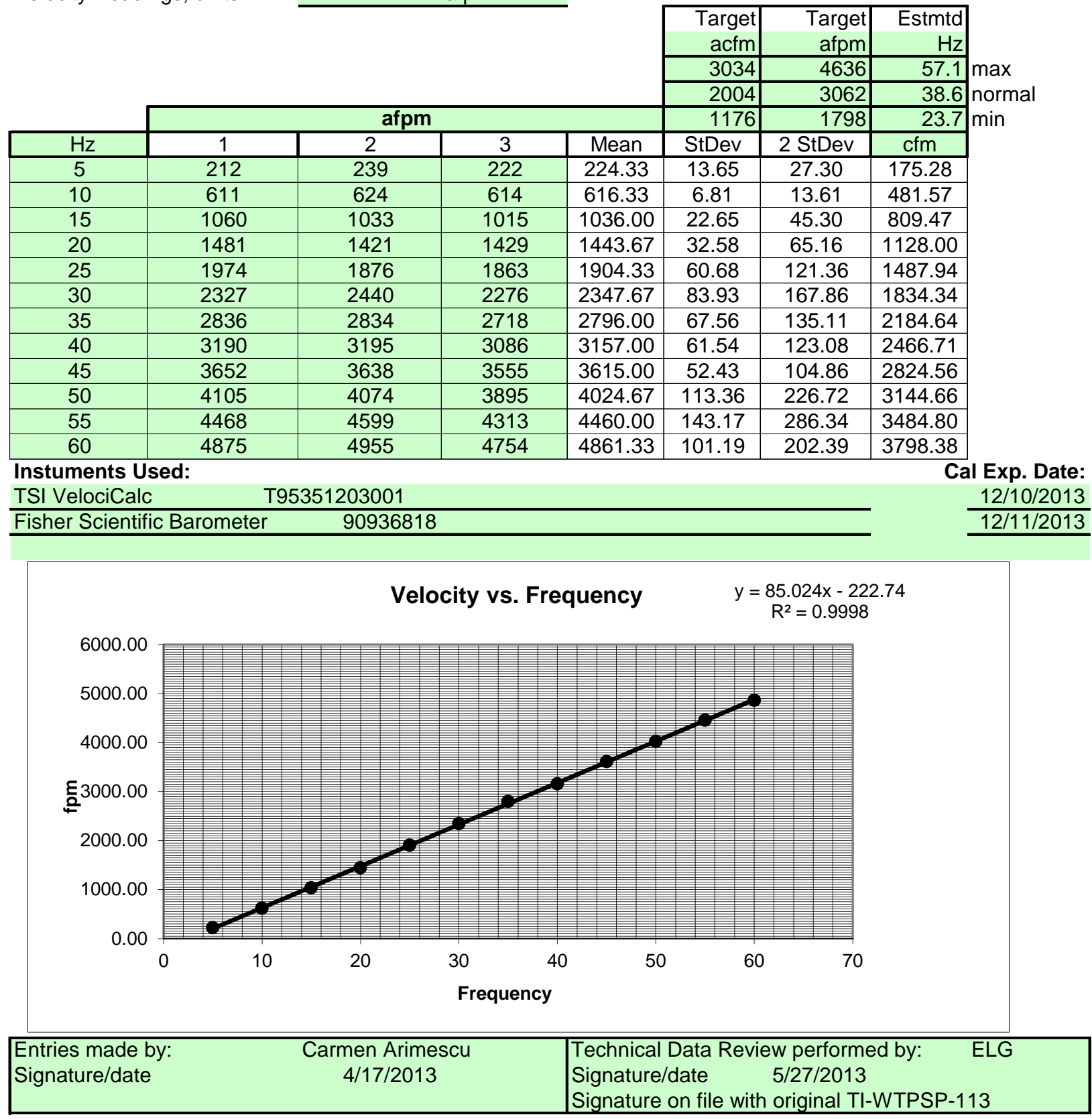




\section{C.2 LV-S2 Velocity Uniformity Data Sheets}

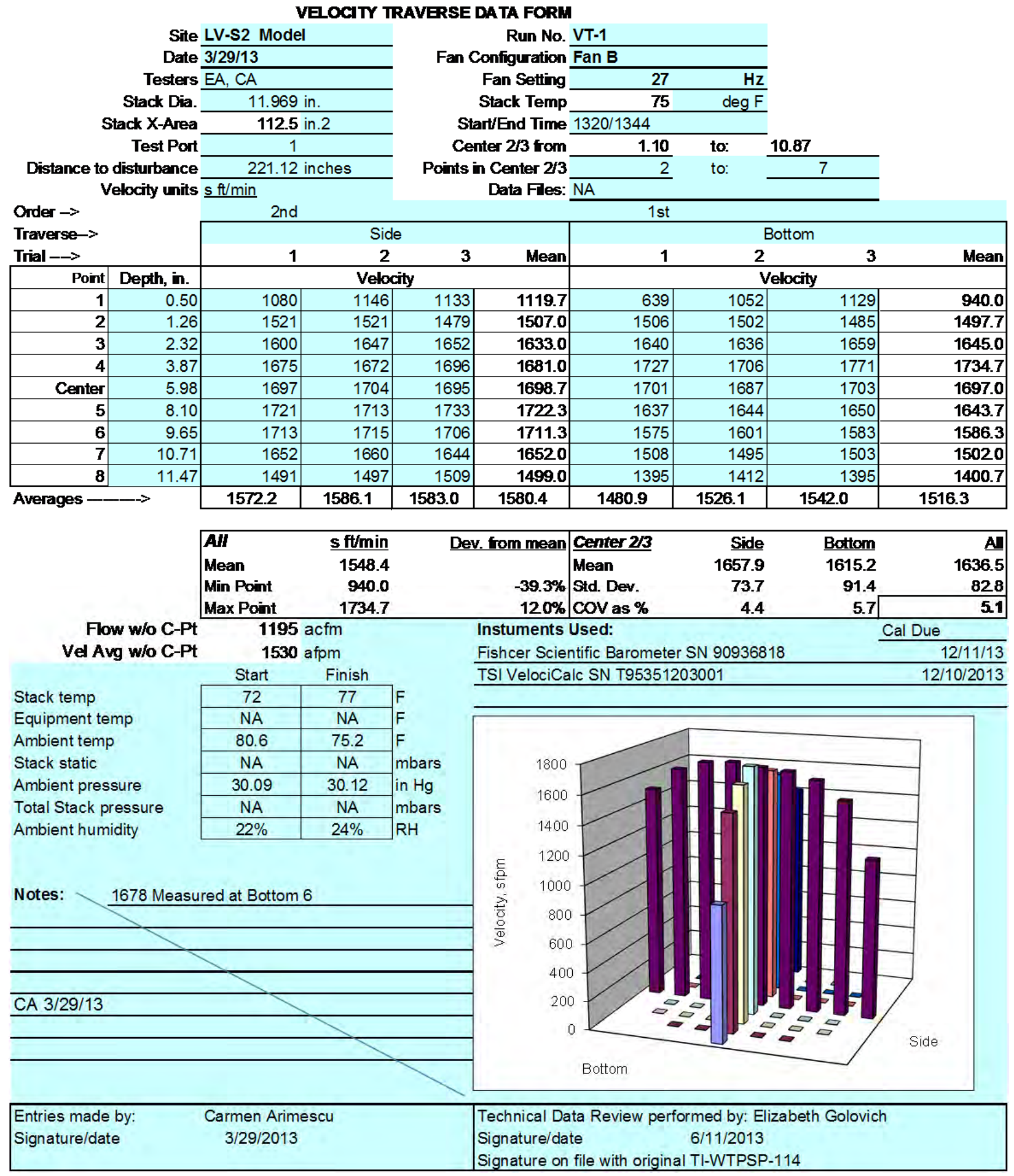




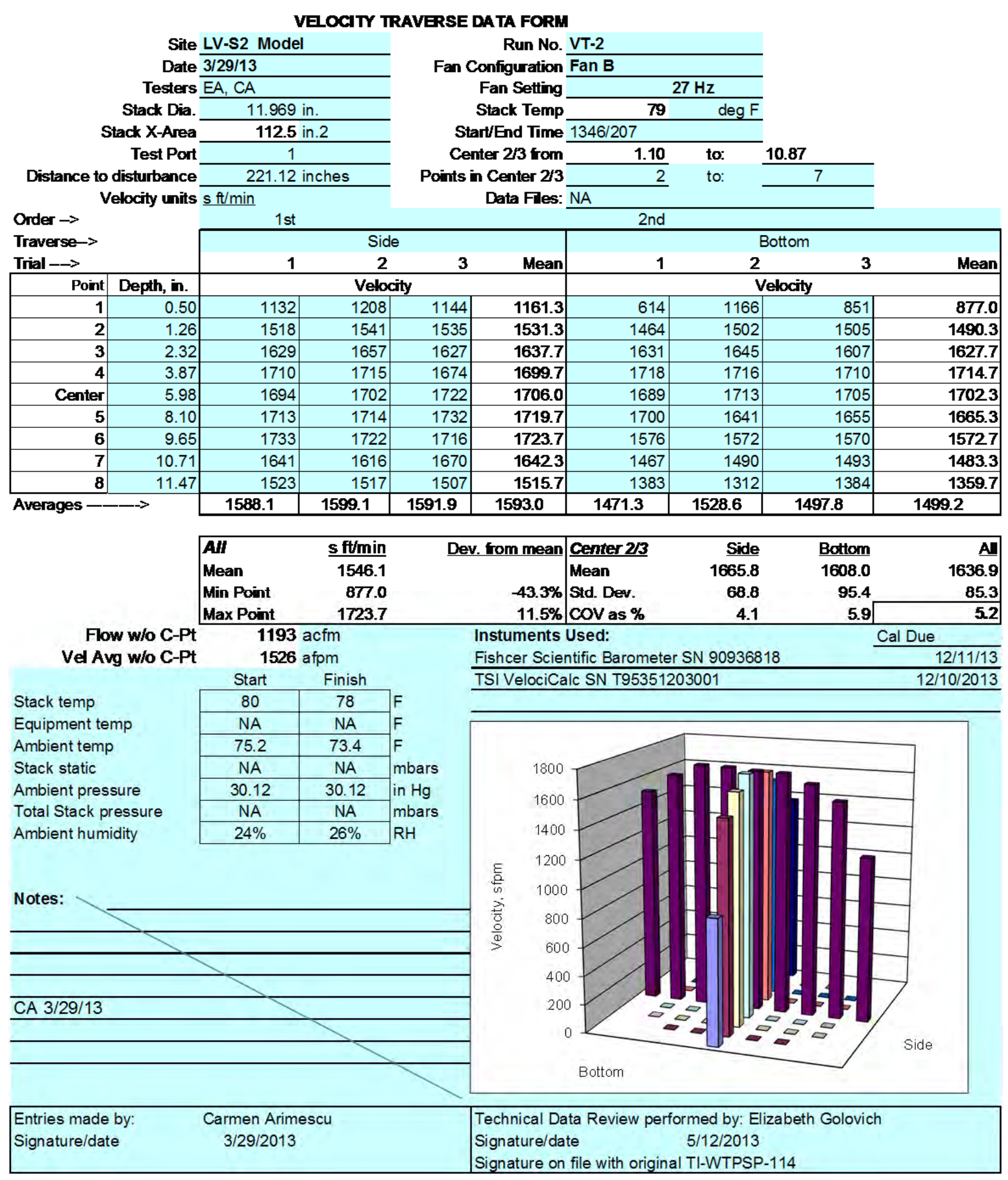




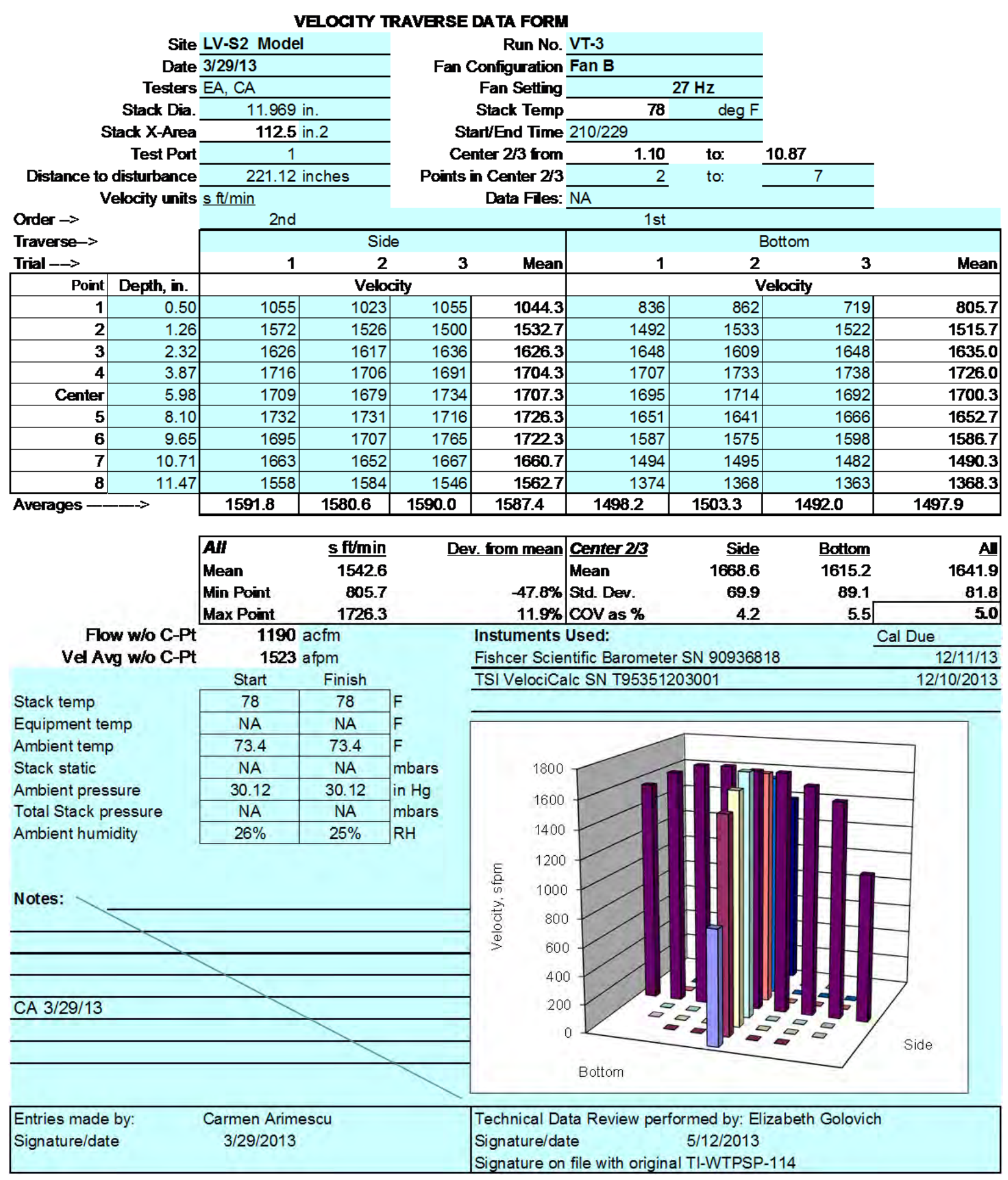




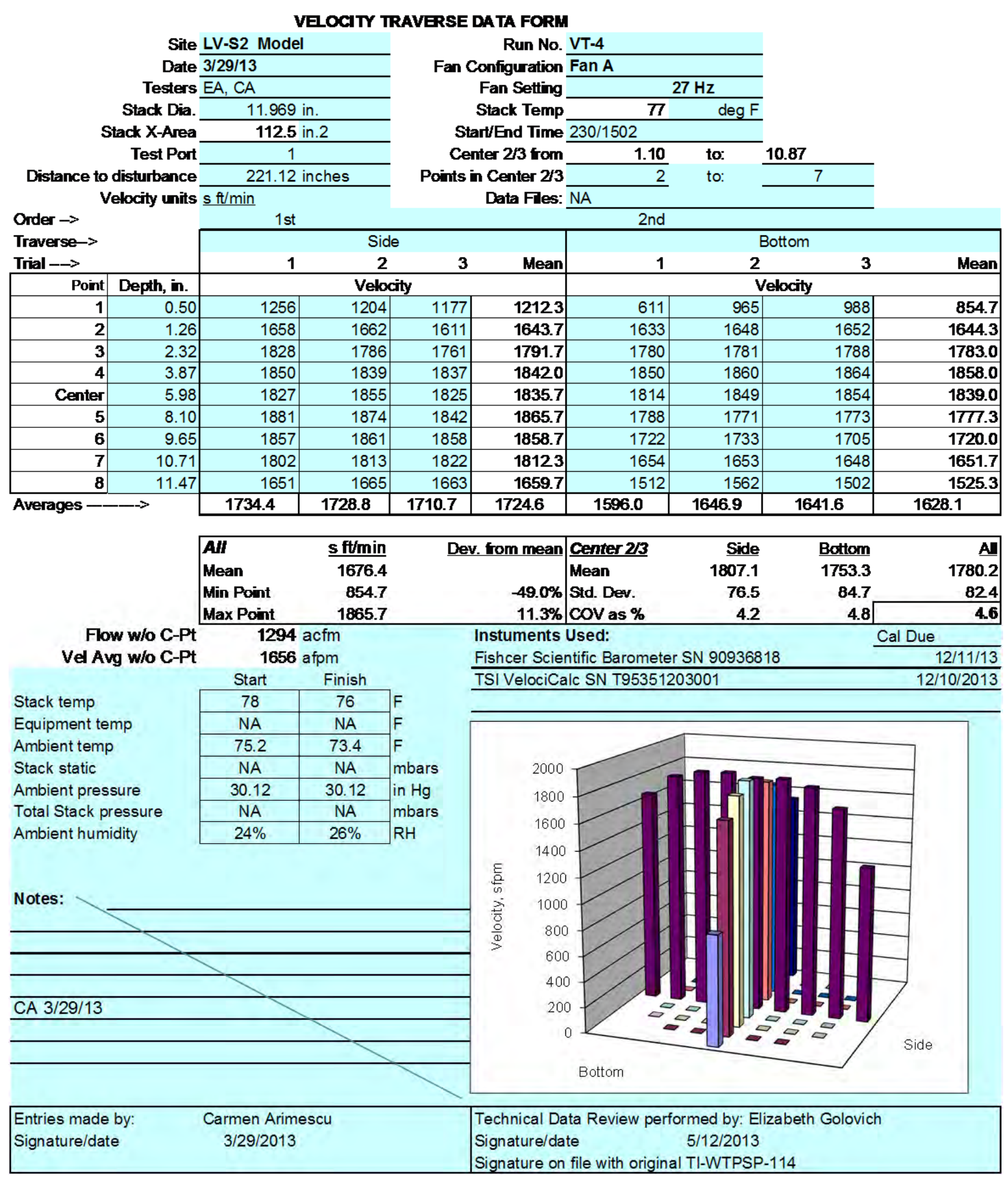




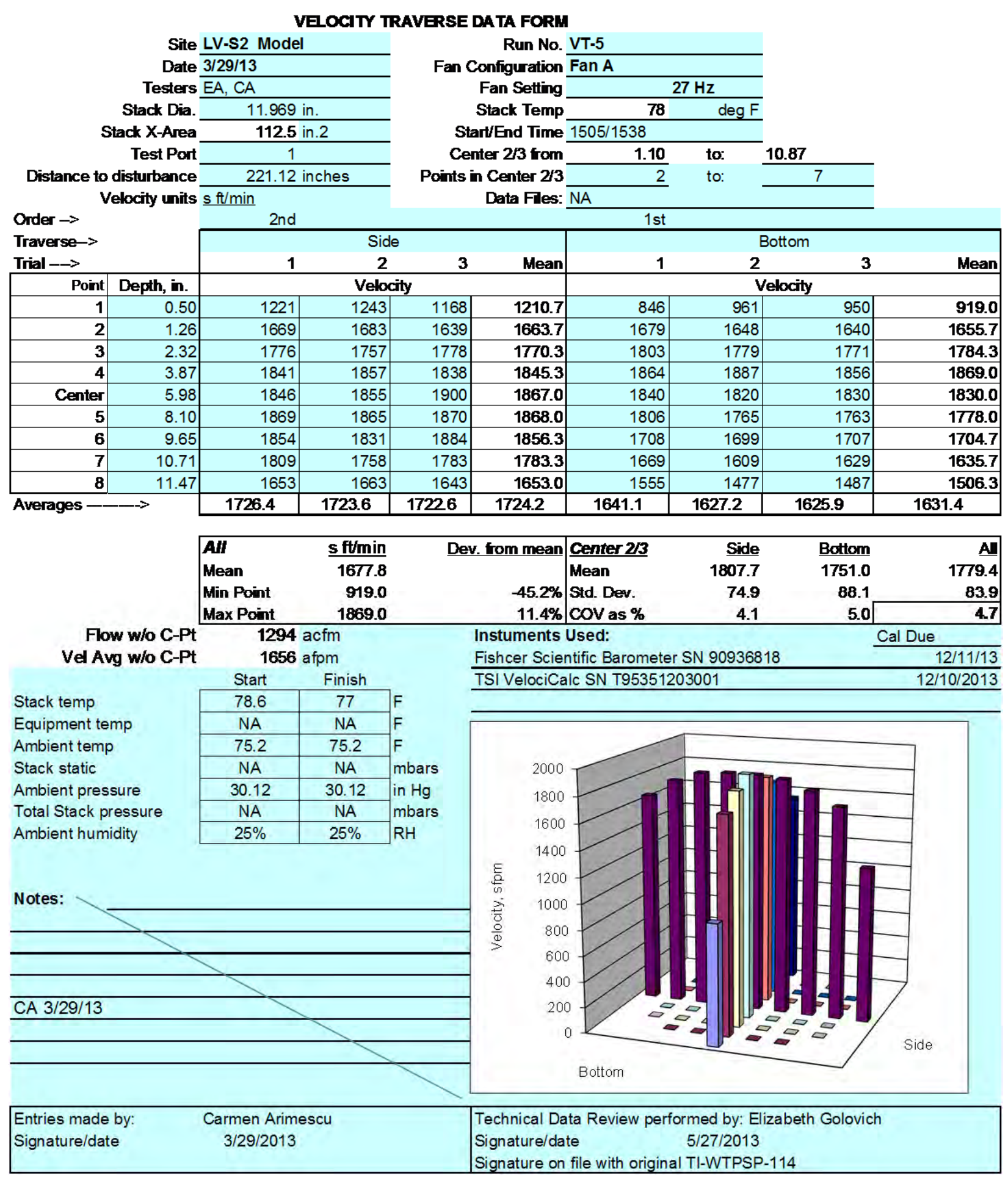




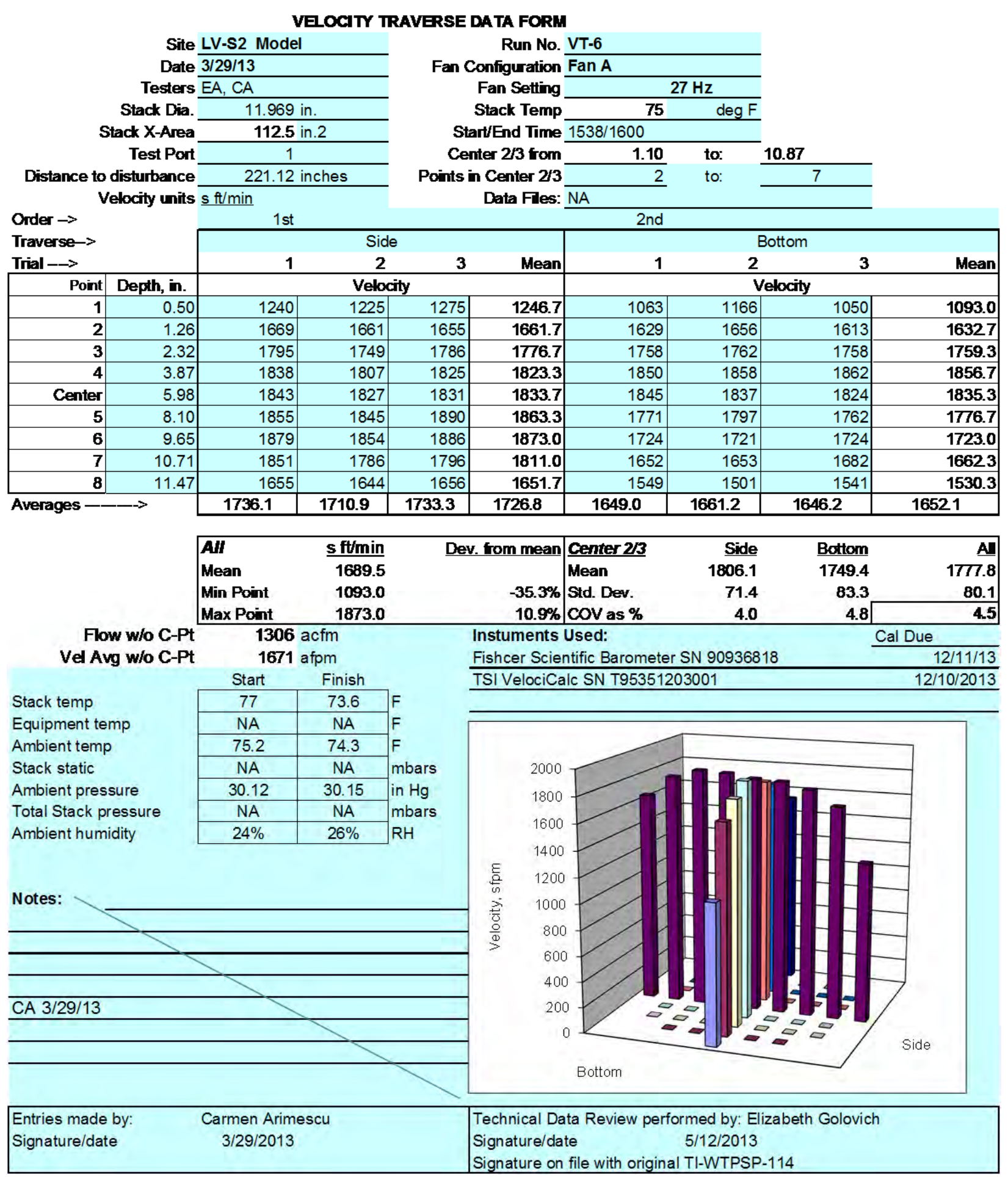




\section{VELCITY TRAVERSE DATA FORM}

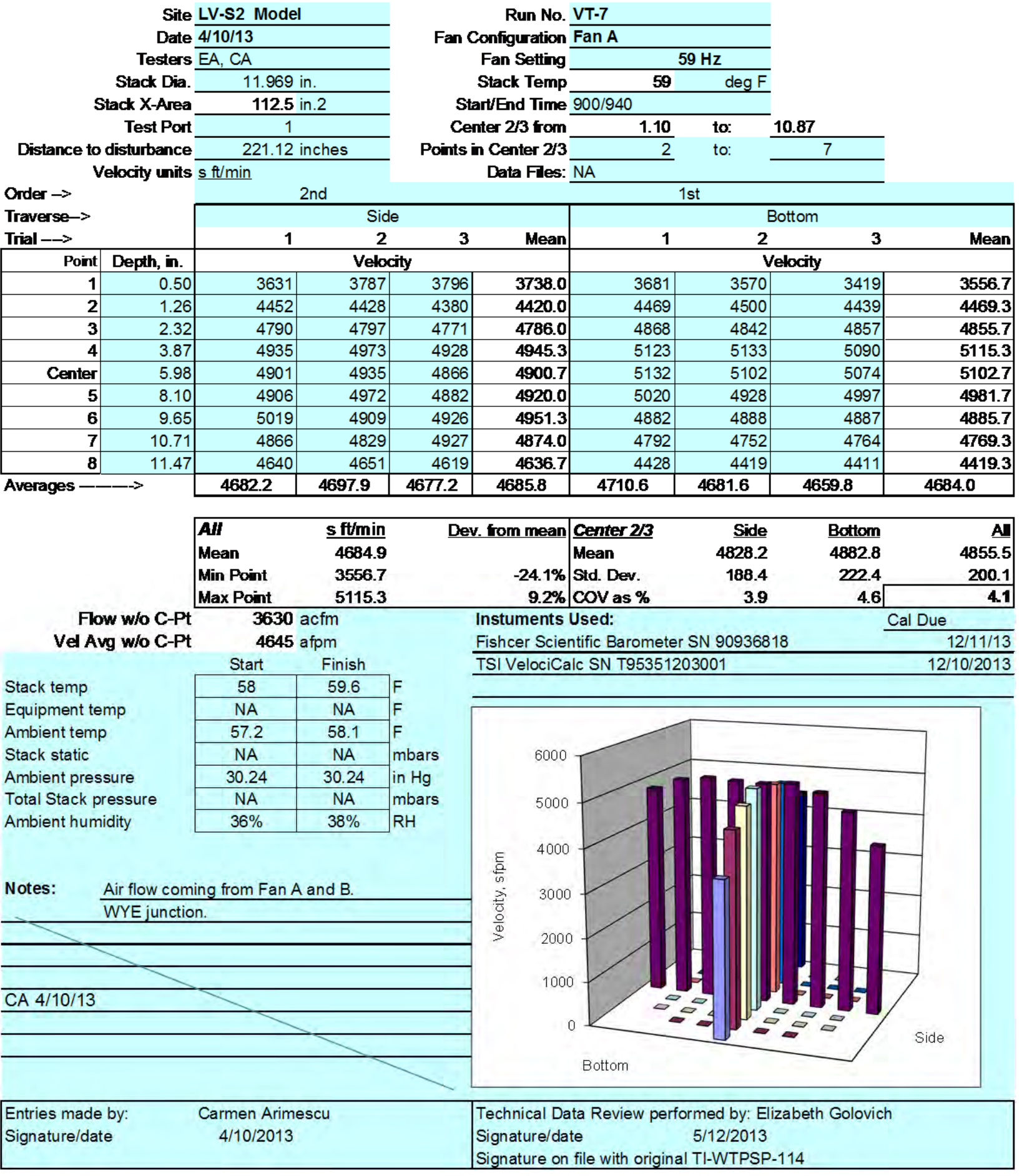




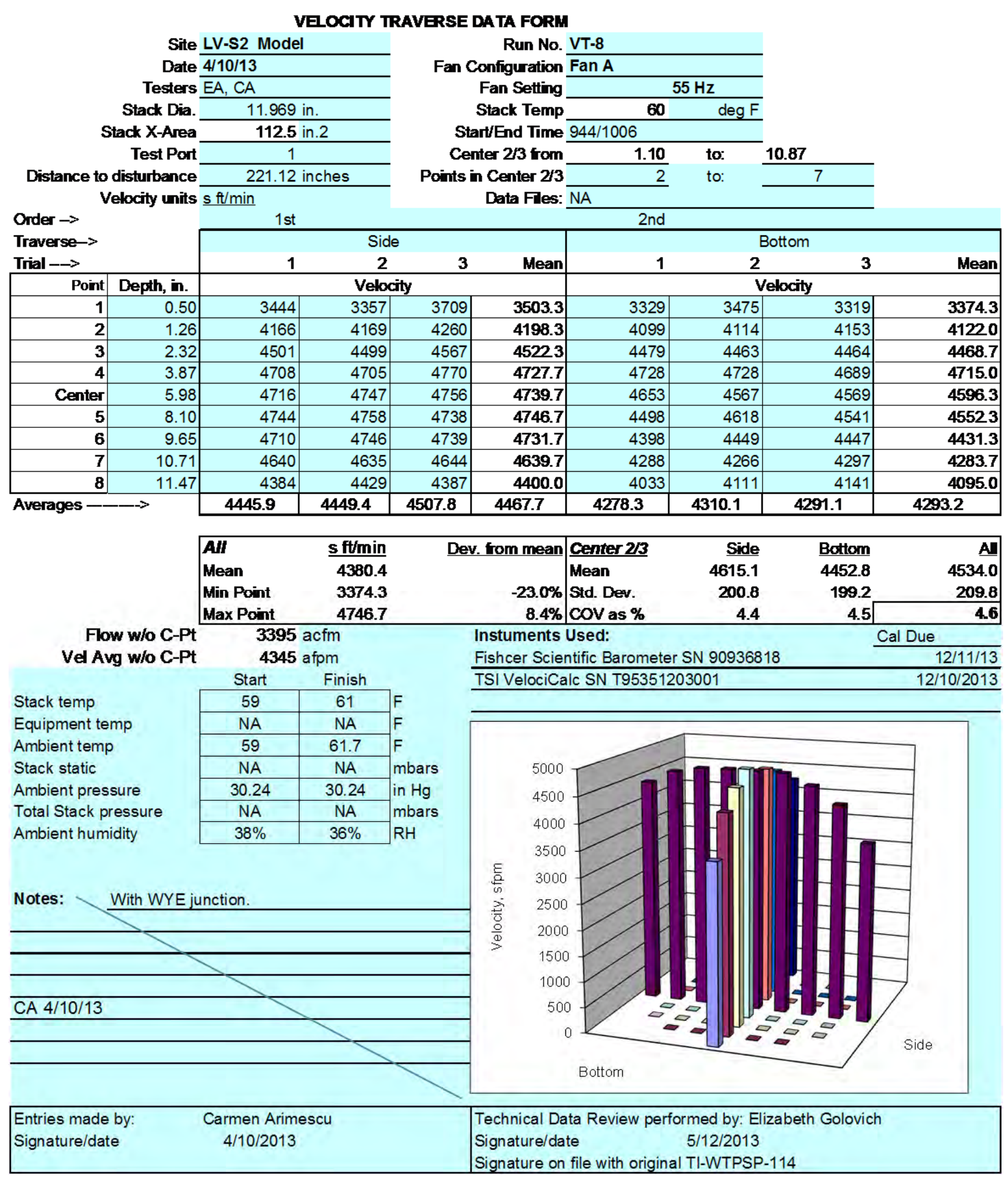




\section{VEOCITY TRAVERSE DATA FORM}

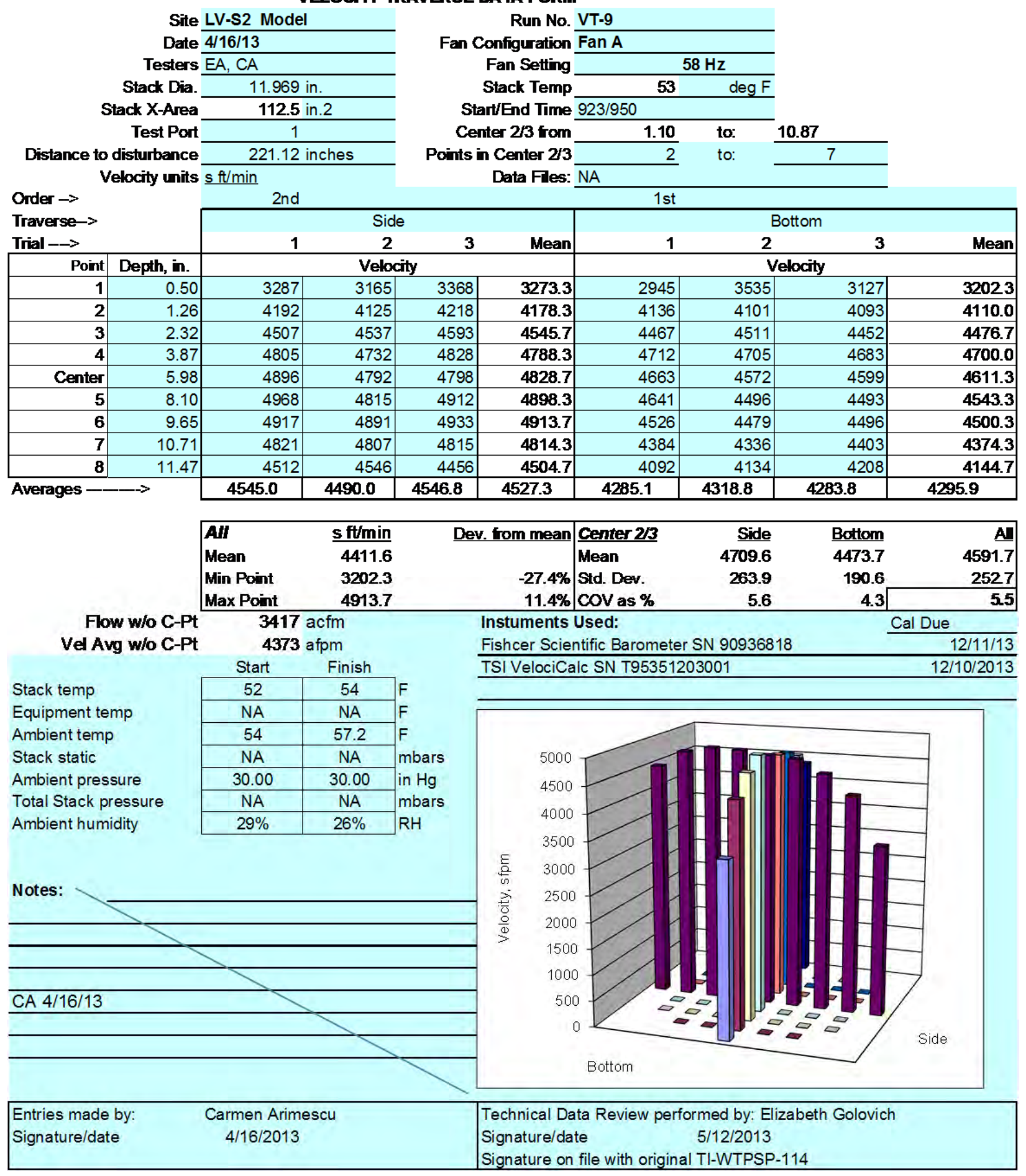




\section{VEOCITY TRAVERSE DATA FORM}

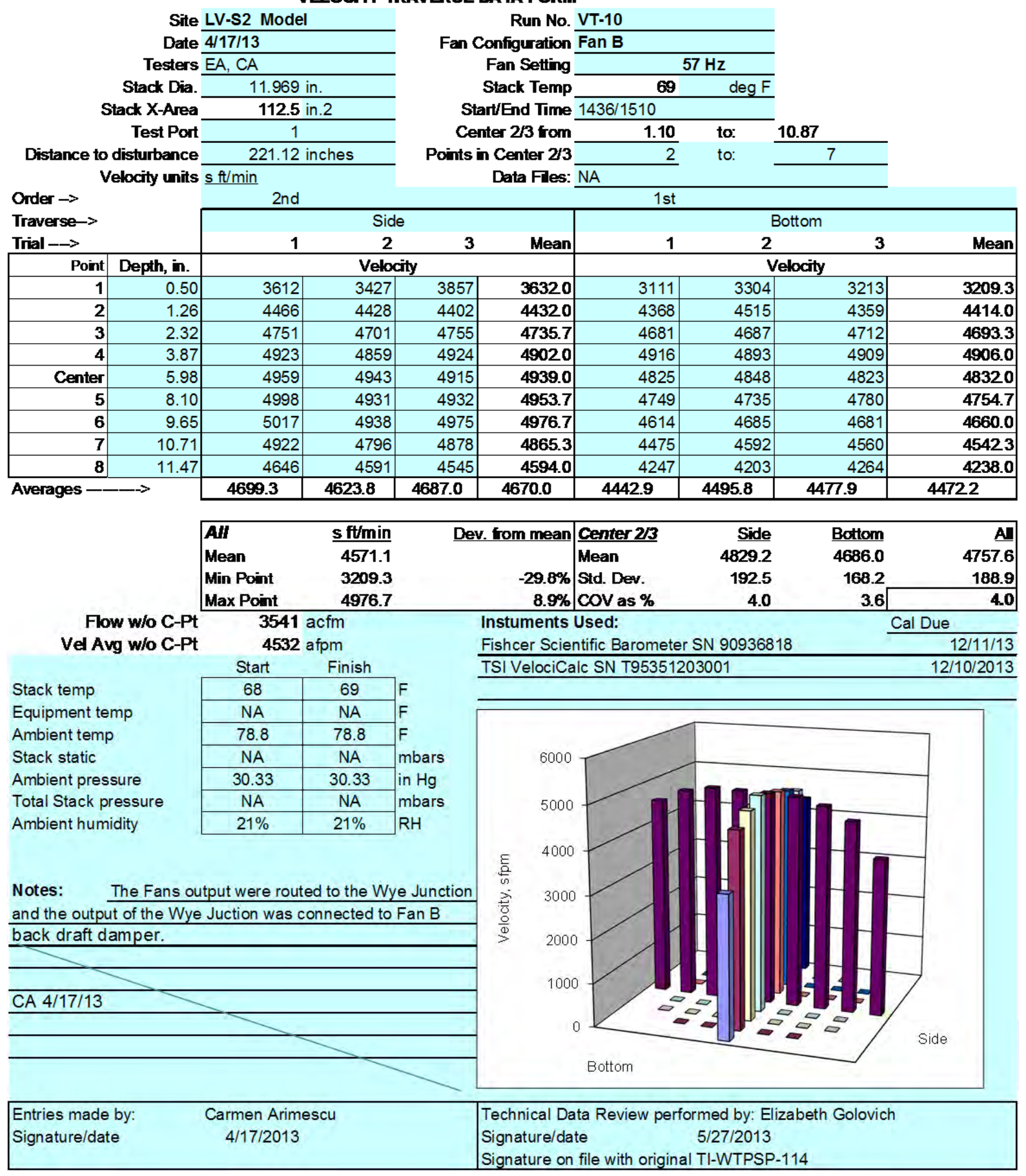




\section{C.3 LV-S2 Flow Angle Data Sheets}

FLOW ANGLE DATA FORM

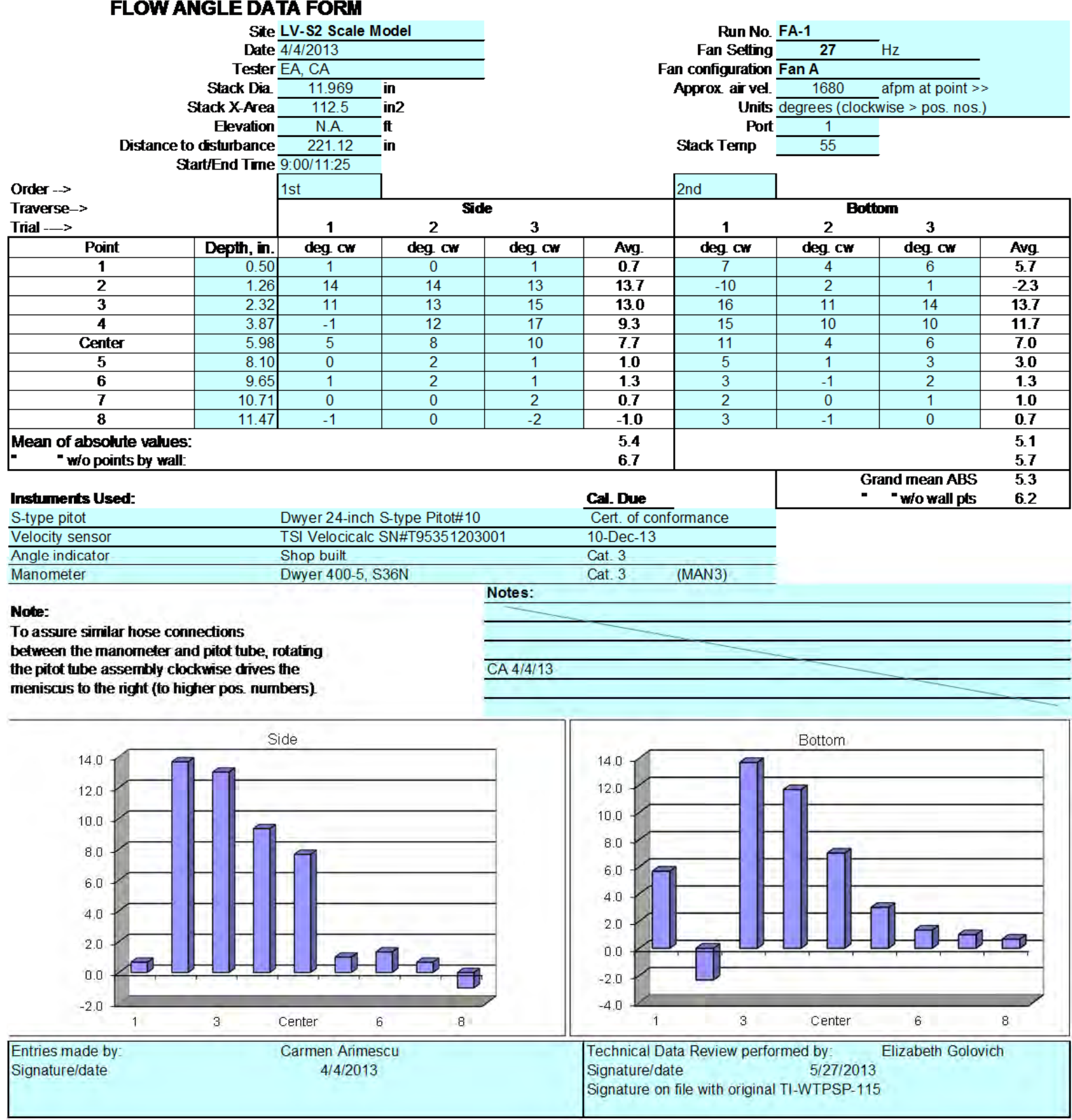




\section{FLOW ANGLE DATA FORM}

Site LV-S2 Scale Model

Date 4/4/2013

Tester EA, CA

Stack Dia. 11.969 in

Stack X-Area $\frac{112.5}{11}$ in2

Elevation N.A. $\mathrm{ft}$

Distance to disturbance $\frac{221.12}{11}$ in

Start/End Tme 1127/1200

Order $\rightarrow$

Traverse- $>$

Trial $\longrightarrow$

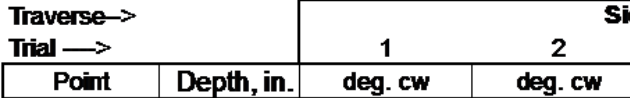

2nd
0.50

deg. cw 1 deg. cw
Run No. FA-2

Fan Setfing $27 \quad \mathrm{~Hz}$

Fan configuration Fan B

Approx. air vel. 1718 afpm at point $>>$

Units degrees (clockwise > pos. nos.)

Stack Temp

Port

$1 \mathrm{st}$

\section{\begin{tabular}{l|lll}
3 & 1 & 2 & 3
\end{tabular}}

3

\begin{tabular}{|c|r|}
\hline $\mathbf{1}$ & 0.5 \\
\hline $\mathbf{2}$ & 1.2 \\
\hline $\mathbf{3}$ & 2.3 \\
\hline $\mathbf{4}$ & 3.87 \\
\hline Center & 5.9 \\
\hline $\mathbf{5}$ & 8.1 \\
\hline $\mathbf{6}$ & 9.6 \\
\hline $\mathbf{7}$ & 10.7 \\
\hline $\mathbf{8}$ & 11.47 \\
\hline
\end{tabular}

Mean of absolute values:

- wo points by wall:

\begin{tabular}{c|c|c} 
deg. $\mathbf{c w}$ & deg. $\mathbf{~ w}$ & d \\
\hline 1 & 1 & \\
\hline 12 & 8 & \\
11 & 10 & \\
11 & 9 & \\
\hline 7 & 6 & \\
\hline 3 & 3 & \\
\hline 3 & 0 & \\
\hline 1 & -1 & \\
\hline 1 & 0 & \\
\hline
\end{tabular}

\begin{tabular}{c|c} 
deg. $\mathbf{c w}$ & \\
0 & \\
11 & \\
11 & \\
9 & \\
6 & \\
\hline 3 & \\
1 & \\
-1 & \\
\hline 2 & \\
\hline
\end{tabular}

ments Used:

S-type pitot

Velocity sensor

Angle indicator

Manometer

Sitych S-type Pitot 10

TSI Velocicalc SN\#T95351203001

Shop built

Dwyer 400-5, S36N

Note:

To assure similar hose connections

between the manometer and pitot tube, rotating

the pitot tube assembly clockwise dives the

meniscus to the right (to higher pos. numbers).

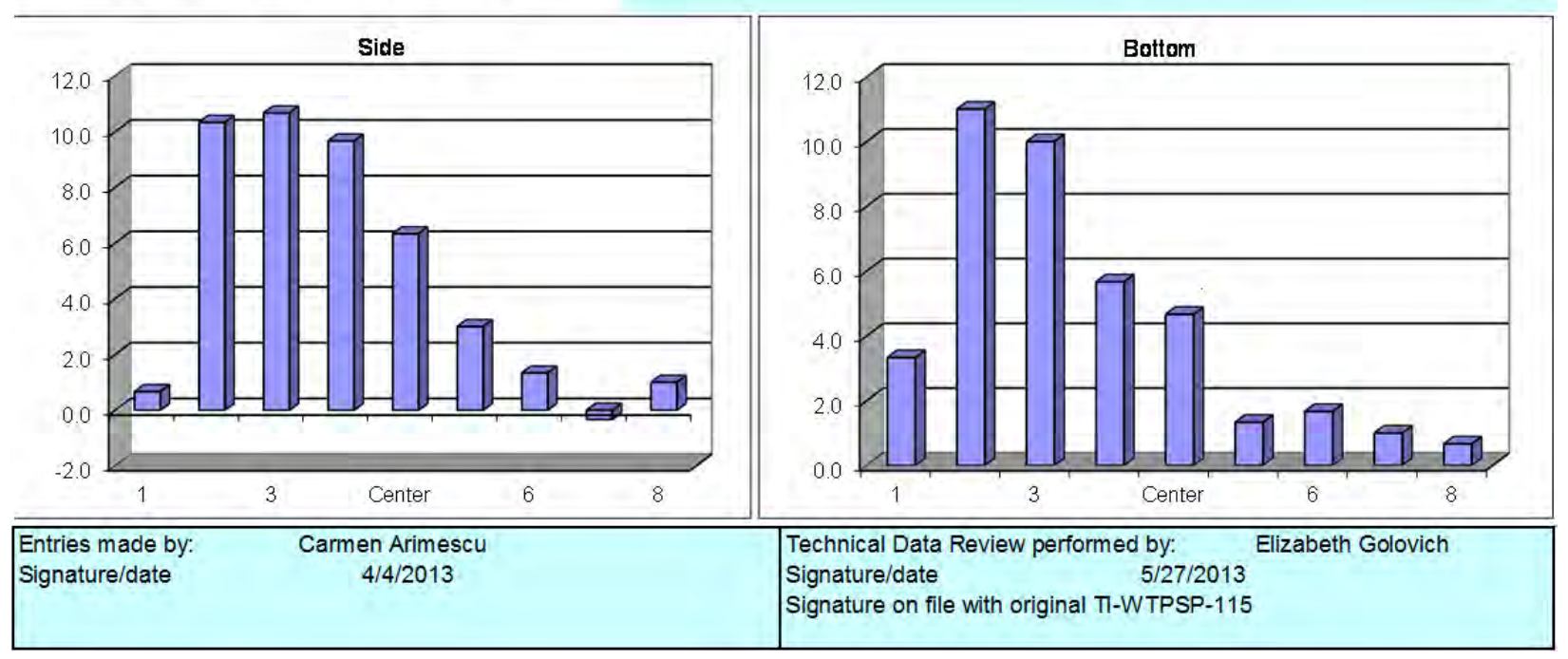




\section{FLOW ANGLE DATA FORM}

Site LV-S2 Scale Model

Date 4/4/2013

Tester EA, CA

Stack Dia. 11.969 in

Stack X-Area $\frac{112.5}{11}$ in2

Elevation N.A. $f$ t

Distance to disturbance 221.12 in

Start/End Tme 1200/1216

Order $\rightarrow$

Traverse $\rightarrow$

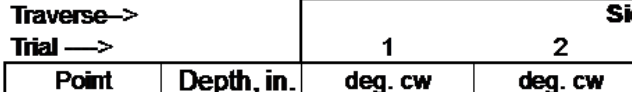

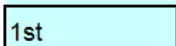

Run No. FA-3

Fan Setting $\mathrm{Hz}$

Fan configuration Fan B

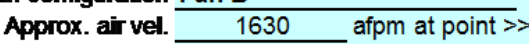

Units degrees (clockwise $>$ pos. nos.)

Por

Stack Temp 59

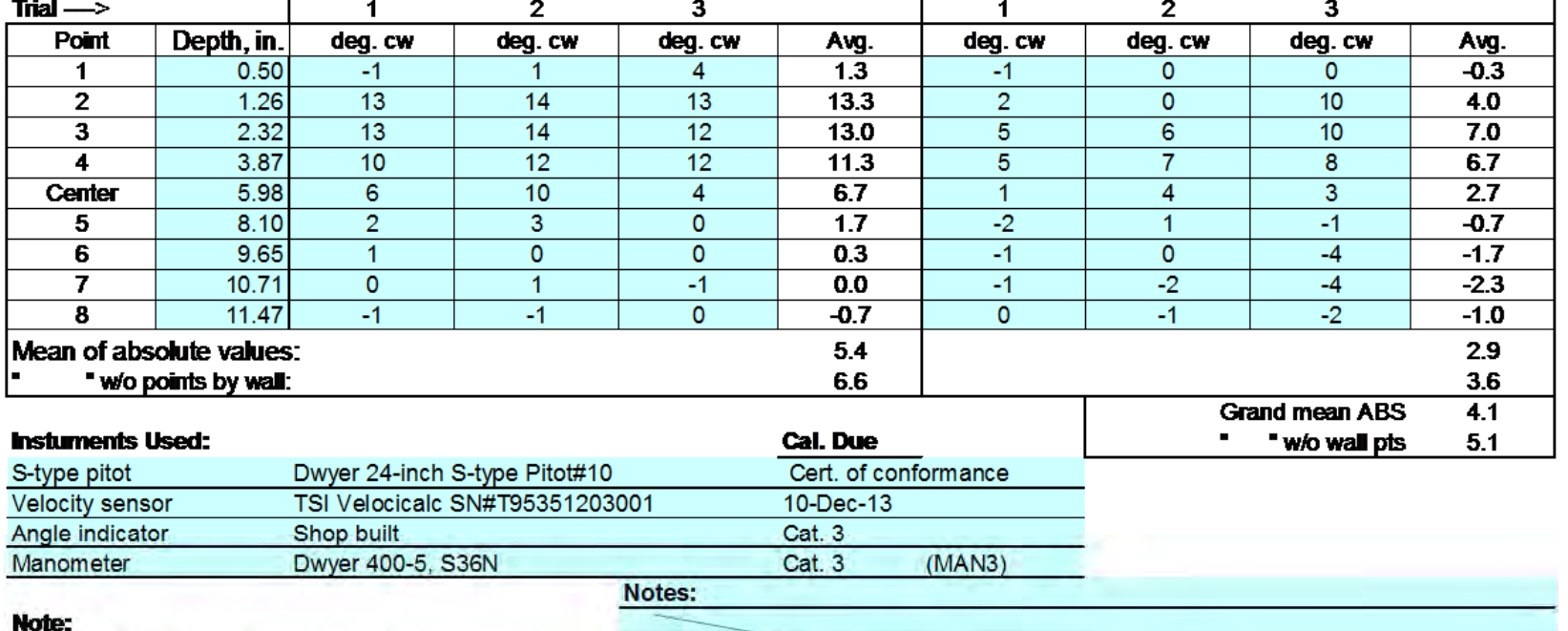

Note:

To assure similar hose connections

between the manometer and pitot tube, rotating

the pitot tube assembly clockwise dives the

meniscus to the right (to higher pos. numbers).

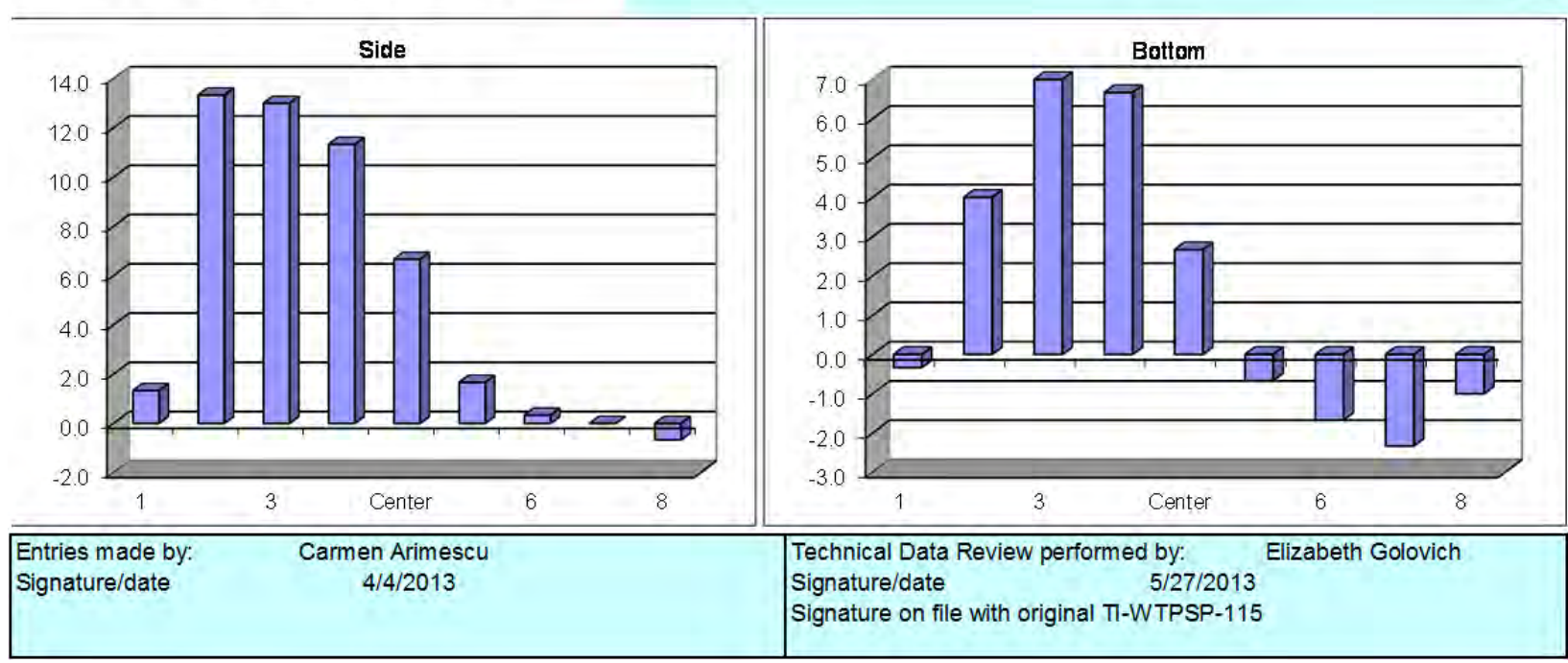




\section{FLOW ANGLE DATA FORM}

Site LV-S2 Scale Model

Date 4/4/2013

Tester EA, CA

Stack Dia. 11.969 in

Stack X-Area $\frac{112.5}{11}$ in2

Elevation N.A. $\mathrm{ft}$

Distance to disturbance 221.12 in

Start/End Tme $1222 / 1300$

Order $\rightarrow$

Traverse $\rightarrow$

Trial $\longrightarrow$

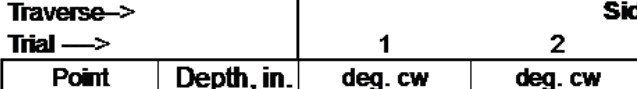

2nd
Run No. FA-4

Fan Setting $\mathrm{Hz}$

Fan configuration Fan B

Approx. air vel. 1594 afpm at point $>>$

Units degrees (clockwise > pos. nos.)

Port 1

Stack Temp 59.3

\begin{tabular}{|c|r|}
\hline $\mathbf{1}$ & 0.5 \\
\hline $\mathbf{2}$ & 1.2 \\
\hline $\mathbf{3}$ & 2.3 \\
\hline $\mathbf{4}$ & 3.87 \\
\hline Center & 5.9 \\
\hline $\mathbf{5}$ & 8.1 \\
\hline $\mathbf{6}$ & 9.6 \\
\hline $\mathbf{7}$ & 10.7 \\
\hline $\mathbf{8}$ & 11.47 \\
\hline
\end{tabular}

Mean of absolute values:

- wo points by wall:

\begin{tabular}{|c|c|}
\hline deg. cw & deg. cw \\
\hline 0 & 1 \\
\hline 12 & 12 \\
\hline 11 & 12 \\
\hline 12 & 10 \\
\hline 7 & 8 \\
\hline 5 & 4 \\
\hline 0 & 1 \\
\hline 0 & 1 \\
\hline 0 & 0 \\
\hline
\end{tabular}

Instuments Used:

S-type pitot

Velocity sensor

Angle indicator

Manometer

Dwyer 24-inch S-type Pitot\#10

TSI Velocicalc SN\#T95351203001

Shop built

Dwyer 400-5, S36N

1 st

\begin{tabular}{|c|c|c|c}
\hline . cw & Avg. & deg. cw & deg. \\
\hline 2 & $\mathbf{1 . 0}$ & 8 & \\
\hline 12 & $\mathbf{1 2 . 0}$ & 1 & \\
\hline 12 & $\mathbf{1 1 . 7}$ & 7 & \\
10 & $\mathbf{1 0 . 7}$ & 8 & \\
\hline 6 & $\mathbf{7 . 0}$ & 6 & \\
\hline 1 & $\mathbf{3 . 3}$ & 4 & \\
\hline 0 & $\mathbf{0 . 3}$ & 2 & \\
\hline 1 & $\mathbf{0 . 7}$ & 1 & \\
0 & $\mathbf{0 . 0}$ & 0 & \\
\hline
\end{tabular}

Bottom

3

Note:

To assure sinilar hose connections

between the manometer and pitot tube, rotating

the pitot tube assembly clockwise dives the

meniscus to the right (to higher pos. numbers).

6.5

\begin{tabular}{l|l} 
deg. $c w$ \\
\hline
\end{tabular}

deg. $c$

\begin{tabular}{l|l} 
Avg \\
\hline
\end{tabular}

\begin{tabular}{r|r|r}
\hline 1 & Avg \\
\hline
\end{tabular}

\begin{tabular}{c|c|c}
1 & -5 & -1.0 \\
\hline & 7 & 7.0
\end{tabular}

\begin{tabular}{l|c|c}
7 & 7 & 7.0 \\
\hline
\end{tabular}

\begin{tabular}{l|l|l}
\hline 8 & 6 & $\mathbf{7 . 3}$ \\
\hline 8 & 4 & $\mathbf{6 . 0}$ \\
\hline 6 & 2 & 4.0
\end{tabular}

\begin{tabular}{l|l|l}
6 & 2 & $\mathbf{4 . 0}$ \\
\hline 5 & 1 & $\mathbf{2 . 7}$ \\
\hline 0 & 1 & 0.7 \\
\hline
\end{tabular}

0

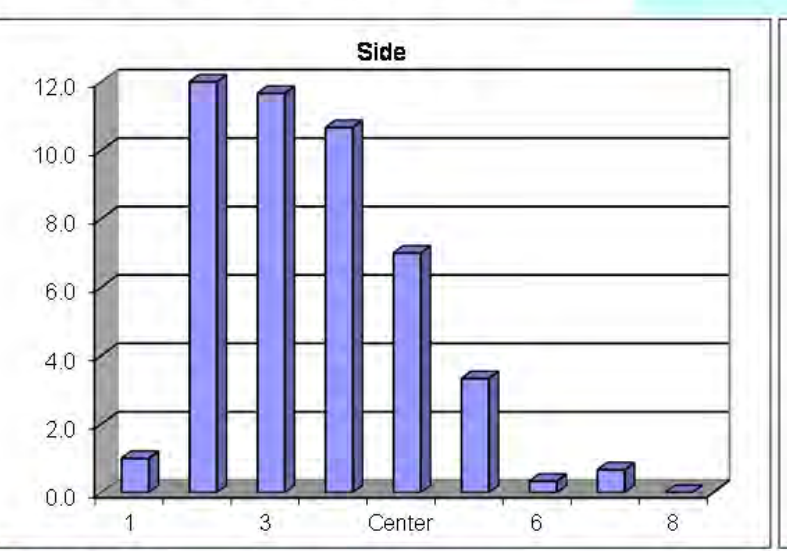

Cal. Due

Cert. of conformance

10-Dec-13

Cat. 3

Cat. 3

Notes:

MAN3)




\section{FLOW ANGLE DATA FORM}

Site LV-S2 Scale Model

Date 4/16/2013

Tester EA,CA

Stack Dia. 11.969 in

Stack X-Area $\frac{112.5}{11}$ in2

Elevation N.A. ft

Distance to disturbance 221.12 in

Start/End Tme 955/1055

Order $\rightarrow$

Traverse $\rightarrow$

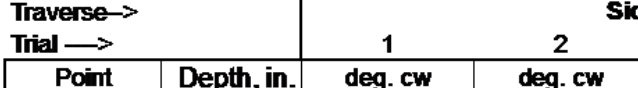

$$
1 \text { st }
$$

Run No. FA-5

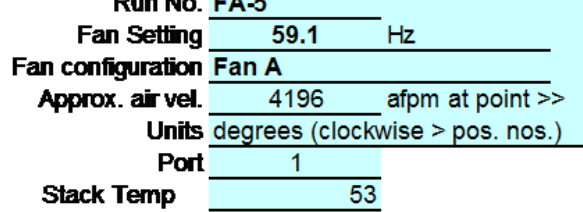

2nd

\begin{tabular}{|c|r|}
\hline $\mathbf{1}$ & 0.5 \\
\hline $\mathbf{2}$ & 1.26 \\
\hline $\mathbf{3}$ & 2.3 \\
\hline $\mathbf{4}$ & 3.87 \\
\hline Center & 5.9 \\
\hline $\mathbf{5}$ & 8.1 \\
\hline $\mathbf{6}$ & 9.6 \\
\hline $\mathbf{7}$ & 10.7 \\
\hline $\mathbf{8}$ & 11.4 \\
\hline
\end{tabular}

\begin{tabular}{l|}
\hline 50 \\
.26 \\
\hline .32 \\
.87 \\
.98 \\
\hline .10 \\
\hline .65 \\
\hline .71 \\
\hline 47
\end{tabular}

Mean of absolute values:

- wo points by wall:

\begin{tabular}{l|l|l} 
deg. cw & deg. cw & deg. cw
\end{tabular}

\begin{tabular}{c|c} 
deg. cw & deg. \\
\hline 0 & \\
\hline-2 & \\
\hline-2 & \\
\hline 0 & \\
\hline 0 & \\
\hline 3 & \\
\hline 1 & \\
\hline 0 & \\
\hline 0 & \\
\hline
\end{tabular}

\begin{tabular}{|l|l}
\hline 0 & \\
\hline 3 & \\
\hline 1 & \\
\hline 0 & \\
\hline 3 & \\
\hline 4 & \\
\hline 5 & \\
\hline 4 & \\
\hline 5 & \\
\hline
\end{tabular}

Instuments Used:

S-type pitot

Velocity sensor

Angle indicator

Manometer

\section{all:}

2.5

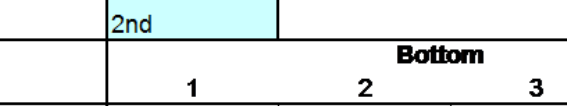

\begin{tabular}{|c|c|c|c|c|}
\hline & 1 & 2 & 3 & \\
\hline Avg. & deg. cw & deg. cw & deg. cw & Avg. \\
\hline-0.7 & 0 & -6 & -10 & -5.3 \\
\hline 3.7 & 0 & 6 & -2 & 1.3 \\
\hline 3.3 & -7 & 5 & -5 & -2.3 \\
\hline 2.0 & -6 & 3 & -5 & -2.7 \\
\hline 0.7 & -7 & -10 & -5 & -7.3 \\
\hline 3.0 & -4 & -5 & -4 & -4.3 \\
\hline 2.3 & -3 & -4 & -4 & -3.7 \\
\hline 2.3 & -4 & -4 & -4 & -4.0 \\
\hline 2.7 & -5 & -2 & -3 & -3.3 \\
\hline 2.3 & & & & 3.8 \\
\hline 2.5 & & & & 3.7 \\
\hline Due & & \multicolumn{2}{|c|}{$\begin{array}{l}\text { Grand mean ABS } \\
\text { - } \quad \text { - wo wall pt }\end{array}$} & $\begin{array}{l}3.1 \\
3.1 \\
\end{array}$ \\
\hline
\end{tabular}

Cert. of conformance

TSI Velocicalc SN\#T95351203001 10-Dec-13

Shop built $\quad$ Cat. 3

Cat. 3

\section{Note:}

To assure similar hose connections

between the manometer and pitot tube, rotating

the pilot tube assembly clockwise dives the

meniscus to the right (to higher pos. numbers).

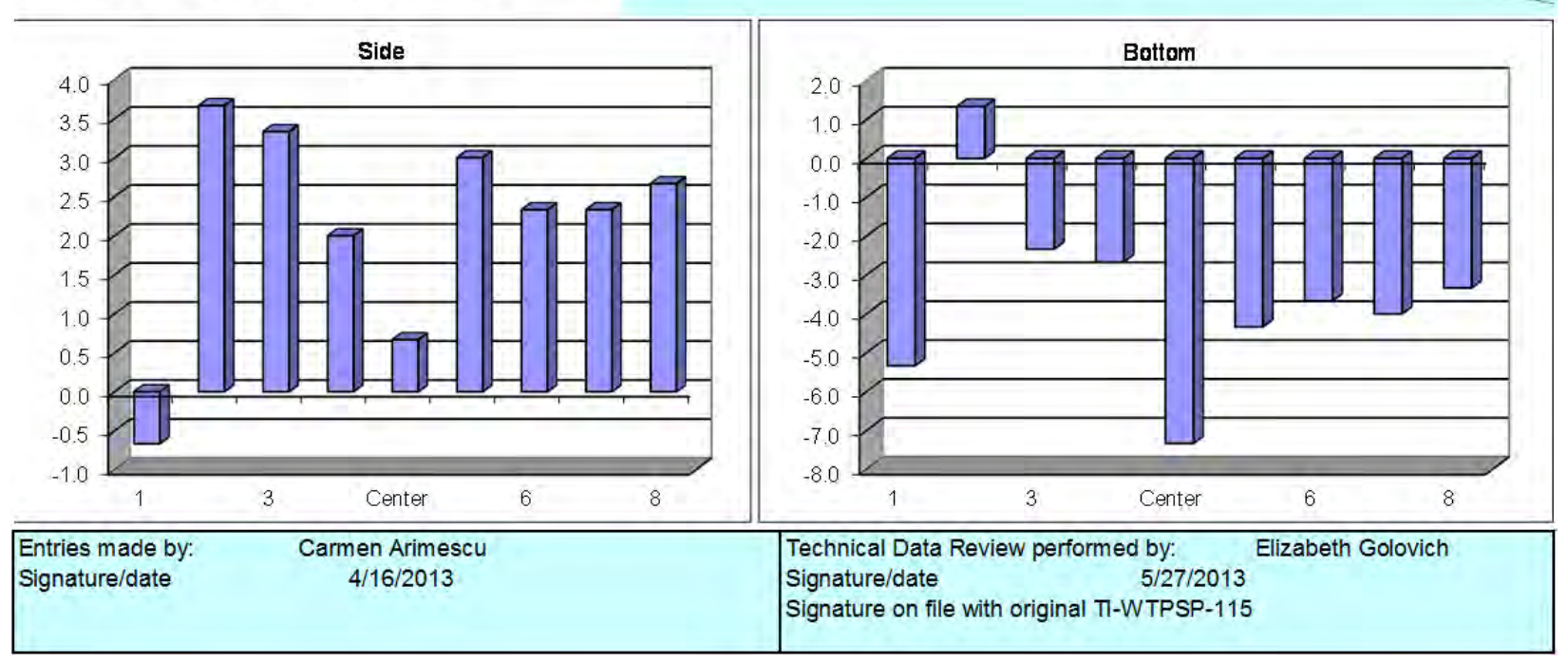




\section{FLOW ANGLE DATA FORM}

Site LV-S2 Scale Model

Date 4/17/2013

Tester EA, CA

Stack Dia. 11.969 in

Stack X-Area $\frac{112.5}{11}$ in2

Elevation N.A. ff

Distance to disturbance 221.12 in

Start/End Tme 910/952

Order $\rightarrow$

Traverse- $>$

Trial $\longrightarrow$

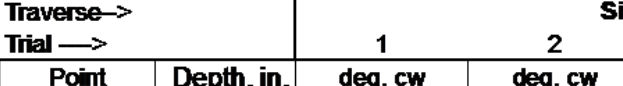
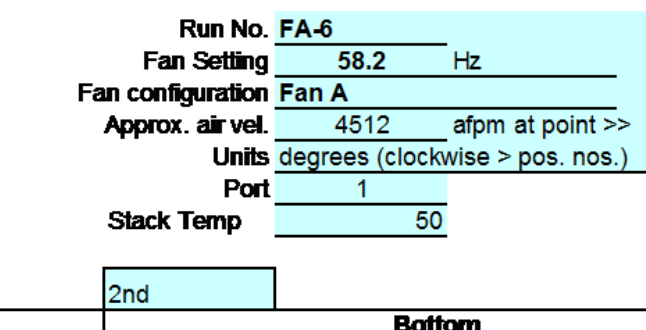

d

\begin{tabular}{|c|r|}
\hline Point & Depth, in \\
\hline $\mathbf{1}$ & 0.5 \\
\hline $\mathbf{2}$ & 1.26 \\
\hline $\mathbf{3}$ & 2.3 \\
\hline $\mathbf{4}$ & 3.87 \\
\hline Center & 5.9 \\
\hline $\mathbf{5}$ & 8.1 \\
\hline $\mathbf{6}$ & 9.6 \\
\hline $\mathbf{7}$ & 10.7 \\
\hline $\mathbf{8}$ & 11.47 \\
\hline
\end{tabular}

\begin{tabular}{l|}
0.50 \\
1.26 \\
2.32 \\
3.87 \\
5.98 \\
8.10 \\
9.65 \\
\hline 0.71 \\
\hline 1.47
\end{tabular}

Mean of absolute values:

- "wo points by wall:

\begin{tabular}{|c|c|c|}
\hline deg. cw & deg. cw & deg. cw \\
\hline
\end{tabular}

\begin{tabular}{c|c|c|} 
deg. $\mathbf{~ W ~}$ & deg. $\mathbf{~ w ~}$ & \\
\hline-3 & -1 & \\
10 & 10 & \\
\hline 8 & 7 & \\
\hline 7 & 6 & \\
\hline 0 & 0 & \\
\hline 0 & -1 & \\
0 & 0 & \\
0 & -1 & \\
\hline 0 & -1 & \\
\hline
\end{tabular}

\begin{tabular}{|c|c|}
\hline deg. cw & \\
\hline-1 & -1 \\
10 & \\
\hline 7 & \\
\hline 7 & \\
\hline 0 & \\
\hline 0 & \\
\hline 1 & \\
\hline 0 & \\
\hline 0 & -0 \\
\hline
\end{tabular}

\begin{tabular}{|c|c|} 
& \\
\hline Avg. & \\
\hline-1.7 & \\
\hline 10.0 & \\
\hline 7.3 & \\
\hline 6.7 & \\
\hline 0.0 & \\
\hline-0.3 & \\
\hline 0.3 & \\
\hline-0.3 & \\
\hline-0.3 & \\
\hline 3.0 & \\
3.6 & \\
\hline
\end{tabular}

Instuments Used:

S-type pitot

Velocity sensor

Angle indicator

Manometer

Dwyer 24-inch S-type Pitot\#10

Cal. Due

Cert. of conformance

$2^{\text {Bottom }} 3$

\begin{tabular}{c|c|c|c} 
deg. cw & deg. cw & deg. cw & Avg.
\end{tabular}

\begin{tabular}{l|c}
-6 & -5 \\
-17 & -15 \\
\hline-1
\end{tabular}

\begin{tabular}{l|l}
-17 & -15 \\
\hline-1 & -15 \\
\hline
\end{tabular}

\begin{tabular}{c|c|c}
-5 & -6 & $\mathbf{- 5 . 7}$ \\
\hline-15 & -14 & $\mathbf{- 1 5 . 3}$ \\
\hline-15 & 1 & $\mathbf{- 5 . 0}$
\end{tabular}

\begin{tabular}{l|l}
-1 & \\
0 & \\
\hline
\end{tabular}

\section{Note:}

To assure similar hose connections

between the manometer and pitot tube, rotating

the pilot tube assembly clockwise dives the

meniscus to the right (to higher pos. numbers).

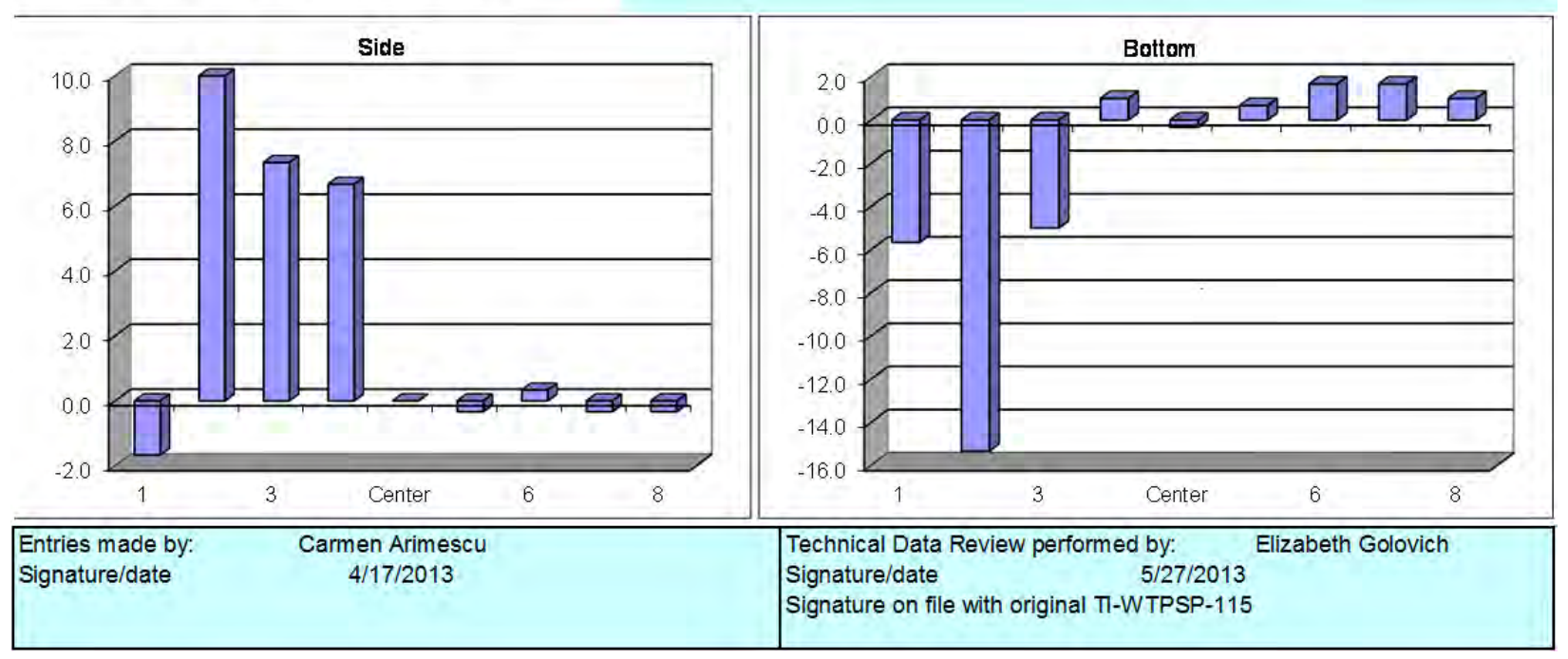




\section{FLOW ANGLE DATA FORM}

Site LV-S2 Scale Model

Date 4/17/2013

Tester EA, CA

Stack Dia. 11.969 in

Stack X-Area $\frac{112.5}{\text { in2 }}$

Elevation N.A. $\mathrm{ft}$

Distance to disturbance 221.12 in

Start/End Tme 957/1025

Order $\rightarrow$

Traverse $\rightarrow$

Trial $\longrightarrow$

\begin{tabular}{|c|c|}
\hline Point & Depth, in. \\
\hline
\end{tabular}

$$
1 \text { st }
$$

Run No. FA-7

$\begin{aligned} \text { Fan Setting } & \frac{58.2}{H z} \\ \text { Fan configuration } & \text { Fan A } \\ \text { Approx. air vel. } & 4504 \text { afpm at point }>>\end{aligned}$

Units degrees (clockwise > pos. nos.)

Port 1

Stack Temp

53

\begin{tabular}{|c|r|}
\hline Point & Depth, in \\
\hline $\mathbf{1}$ & 0.5 \\
\hline $\mathbf{2}$ & 1.26 \\
\hline $\mathbf{3}$ & 2.3 \\
\hline $\mathbf{4}$ & 3.87 \\
\hline Center & 5.98 \\
\hline $\mathbf{5}$ & 8.10 \\
\hline $\mathbf{6}$ & 9.6 \\
\hline $\mathbf{7}$ & 10.71 \\
\hline $\mathbf{8}$ & 11.47 \\
\hline
\end{tabular}

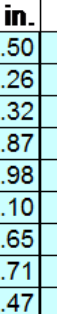

Mean of absolute values:

" wo points by wall:
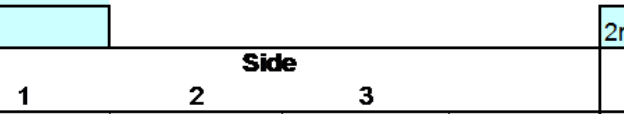

2nd

\begin{tabular}{c|c|c|c|}
\hline deg. $\mathbf{~ w ~}$ & deg. $\mathbf{~ w}$ & deg. $\mathbf{~ w}$ & \\
\hline-3 & -4 & -4 & \\
\hline 9 & 11 & 11 & \\
\hline 8 & 10 & 9 & \\
\hline 4 & 5 & 0 & \\
-1 & -4 & -5 & \\
\hline-2 & -1 & -2 & \\
-1 & -1 & -2 & \\
-1 & 1 & 0 & \\
\hline-1 & 0 & 0 & \\
\hline
\end{tabular}

\begin{tabular}{|c|c|c|c|c|}
\hline Avg. & deg. cw & deg. cw & deg. cw & Avg. \\
\hline-3.7 & -10 & -11 & -3 & -8.0 \\
\hline 10.3 & -20 & 6 & -21 & -11.7 \\
\hline 9.0 & 7 & 4 & 7 & 6.0 \\
\hline 3.0 & 6 & 3 & 5 & 4.7 \\
\hline-3.3 & -3 & -4 & -3 & -3.3 \\
\hline-1.7 & -2 & -3 & -3 & -2.7 \\
\hline-1.3 & -3 & -3 & -3 & -3.0 \\
\hline 0.0 & -3 & -4 & -3 & -3.3 \\
\hline-0.3 & -3 & -1 & -2 & -2.0 \\
\hline 3.6 & & & & 5.0 \\
\hline 4.1 & & & & 5.0 \\
\hline Due & & \multicolumn{2}{|c|}{$\begin{array}{l}\text { Grand mean AB } \\
\text { - } \quad \text { who wall pt }\end{array}$} & $\begin{array}{l}4.3 \\
4.5\end{array}$ \\
\hline
\end{tabular}

Instuments Used:

S-type pitot

Velocity sensor

Angle indicator

Dwyer 24-inch S-type Pitot\#10

Cert. of conformance

Manometer

Shop built

10-Dec-13

Note: Notes:

Cat. 3

Cat. 3

To assure similar hose connections

between the manometer and pitot tube, rotating

the pilot tube assembly clockwise dives the

meniscus to the right (to higher pos. numbers).

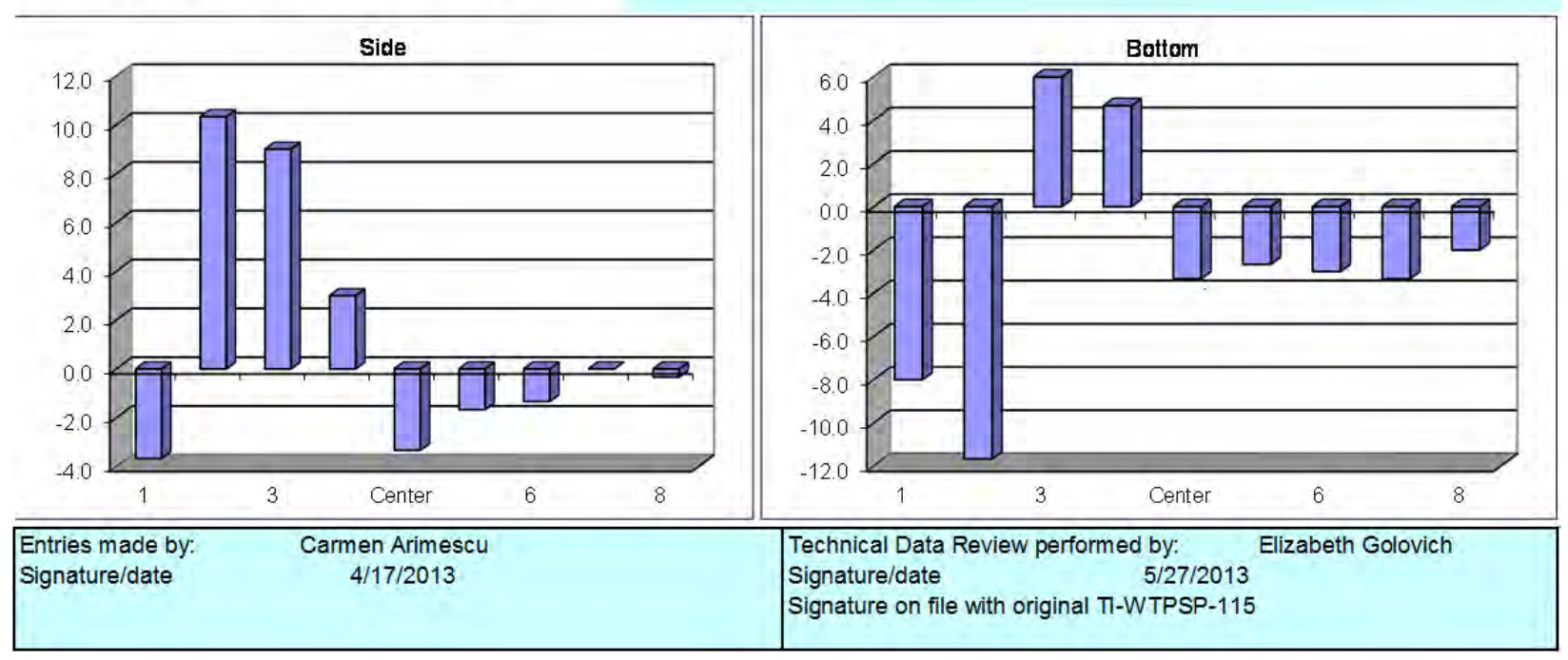




\section{FLOW ANGLE DATA FORM}

Site LV-S2 Scale Model

Date 4/17/2013

Tester EA, CA

Stack Dia. 11.969 in

Stack X-Area $\frac{112.5}{11}$ in2

Elevation N.A. ff

Distance to disturbance 221.12 in

Start/End Tme 300/330

Order $\rightarrow$

Traverse $\rightarrow$

Trial $\longrightarrow$

\begin{tabular}{l|ll} 
& 1 & 2 \\
\hline
\end{tabular}

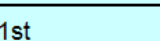

Run No. FA-

Fan Setting $\mathrm{Hz}$

Fan configuration Fan B

Approx. air vel. 4510 afpm at point $>>$

Units degrees (clockwise > pos. nos.)

Por

Stack Temp 69

\begin{tabular}{|c|r|}
\hline Point & Depth, in \\
\hline $\mathbf{1}$ & 0.5 \\
\hline $\mathbf{2}$ & 1.2 \\
\hline $\mathbf{3}$ & 2.3 \\
\hline $\mathbf{4}$ & 3.87 \\
\hline Center & 5.9 \\
\hline $\mathbf{5}$ & 8.1 \\
\hline $\mathbf{6}$ & 9.6 \\
\hline $\mathbf{7}$ & 10.7 \\
\hline $\mathbf{8}$ & 11.47 \\
\hline
\end{tabular}

Mean of absolute values:

- wo points by wall

\begin{tabular}{|c|c|}
\hline deg. cw & deg. cw \\
\hline-2 & -4 \\
\hline 11 & 11 \\
\hline 10 & 9 \\
\hline 7 & 6 \\
\hline-1 & -1 \\
\hline-1 & 0 \\
\hline-1 & 0 \\
\hline 0 & 1 \\
\hline 0 & 0 \\
\hline
\end{tabular}

\section{3}

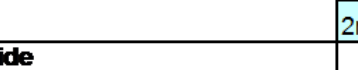

\begin{tabular}{|c|c|}
\hline deg. $\mathbf{~ w ~}$ & Avg \\
\hline-4 & -3 \\
\hline 11 & 11 \\
\hline 10 & \\
\hline 5 & \\
\hline-2 & -1 \\
\hline-1 & -0 \\
\hline-1 & -0 \\
\hline 0 & 0 \\
\hline-1 & -0. \\
\hline
\end{tabular}

$\begin{array}{llll} & 1 & 2 & 3\end{array}$

\begin{tabular}{c|c|r}
\hline Avg. & deg. $\mathbf{~ w ~}$ & deg. \\
\hline $\mathbf{- 3 . 3}$ & -10 & -10 \\
\hline $\mathbf{1 1 . 0}$ & -15 & -21 \\
$\mathbf{9 . 7}$ & 11 & \\
\hline $\mathbf{6 . 0}$ & 5 & \\
$\mathbf{- 1 . 3}$ & -4 & - \\
\hline $\mathbf{- 0 . 7}$ & -3 & -3 \\
\hline $\mathbf{- 0 . 7}$ & -2 & -3 \\
$\mathbf{0 . 3}$ & -1 & -1 \\
\hline $\mathbf{- 0 . 3}$ & -1 & -1 \\
\hline
\end{tabular}

3.7

4.2

\section{Instuments Used:}

S-type pitot

Velocity sensor

Angle indicator

Manometer

Note:

To assure similar hose connections

between the manometer and pitot tube, rotating

the pilot tube assembly clockwise dives the

meniscus to the right (to higher pos. numbers).
Cal. Due

Cert. of conformance

Dwyer 24-inch S-type Pitot\#10

TSI Velocicalc SN\#T95351203001

Shop built

10-Dec-13

Cat. 3

Cat. 3

2 cw

\begin{tabular}{|c|c|c}
\hline deg. cw & deg. cw & Avg.
\end{tabular}

\begin{tabular}{c|c|c}
-10 & -14 & -11.3 \\
-21 & -21 & -19.0 \\
\hline 7 & 8 & 8.7
\end{tabular}

\begin{tabular}{c|c|c}
7 & 8 & 8.7 \\
5 & 6 & 5.3 \\
-5 & -4 & -4.3 \\
\hline
\end{tabular}

\begin{tabular}{l|l|l}
-5 & -4 & -4.3 \\
-3 & -2 & -27
\end{tabular}

\begin{tabular}{l|l|l}
-3 & -2 & -2.7 \\
\hline-3 & -1 & -2.0 \\
\hline
\end{tabular}

-3
-1
-1

\begin{tabular}{l|l}
-1 & -1 \\
\hline-1 & -1
\end{tabular}

$-1.0$

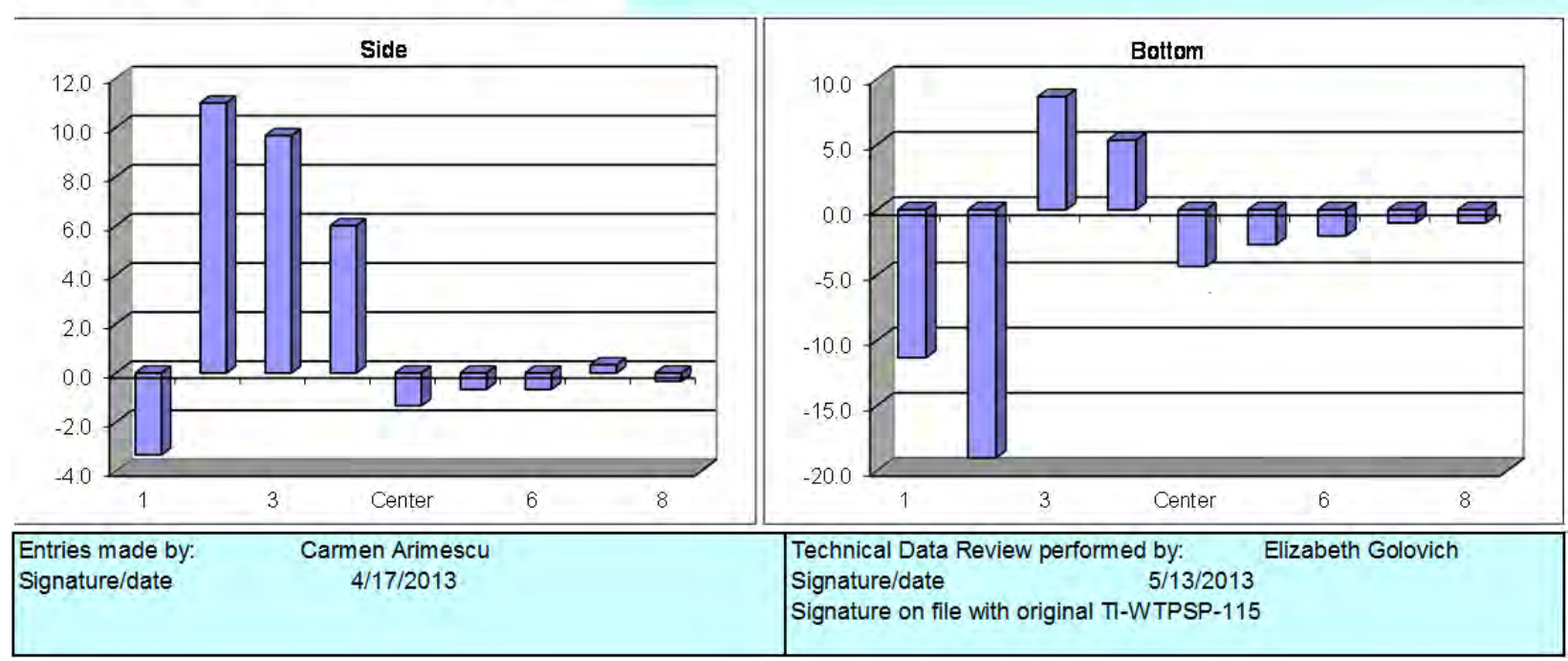




\section{FLOW ANGLE DATA FORM}

Site LV-S2 Scale Model

Date 4/18/2013

Tester EA, CA

Stack Dia. 11.969 in

Stack X-Area $\frac{112.5}{\text { in } 2}$

Elevation N.A. $f t$

Distance to disturbance 221.12 in

Start/End Tme 900/926

Order $\rightarrow$

Traverse- $>$

Trial $\longrightarrow$

\begin{tabular}{|c|c|}
\hline Point & Depth, in. \\
\hline 1
\end{tabular}

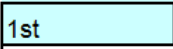

Run No. FA-9

Fan Setting $\mathrm{Hz}$

Fan configuration Fan B

Approx. air vel. 4520 afpm at point $>>$

Units degrees (clockwise > pos. nos.)

Stack Temp 62.7

$\begin{array}{ll}1 & \\ 62.7\end{array}$

\begin{tabular}{|c|r|}
\hline Point & Depth, in \\
\hline $\mathbf{1}$ & 0.5 \\
\hline $\mathbf{2}$ & 1.26 \\
\hline $\mathbf{3}$ & 2.3 \\
\hline $\mathbf{4}$ & 3.87 \\
\hline Center & 5.98 \\
\hline $\mathbf{5}$ & 8.10 \\
\hline $\mathbf{6}$ & 9.6 \\
\hline $\mathbf{7}$ & 10.71 \\
\hline $\mathbf{8}$ & 11.47 \\
\hline
\end{tabular}

Mean of absolute values:

- wo points by wall:

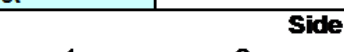

2nd

\section{Instuments Used:}

S-type pitot

Velocity sensor

Angle indicator

Manometer

\begin{tabular}{c|c}
1 & 2 \\
deg. cw & deg. cw
\end{tabular}

3

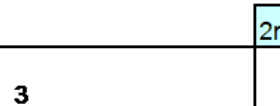

d

$\begin{array}{lll}1 & 2 & 3\end{array}$

\begin{tabular}{|c|c|c|c|c|}
\hline in. & deg. $\mathbf{c w}$ & deg. $\mathbf{c w}$ & deg. $\mathbf{~ w}$ & \\
\hline 0.50 & -2 & -3 & -3 & \\
\hline 1.26 & 12 & 12 & 11 & \\
\hline 2.32 & 10 & 10 & 10 & \\
\hline 3.87 & 8 & 6 & 6 & \\
\hline 5.98 & -1 & -1 & -1 & \\
\hline 8.10 & -1 & -1 & -1 & \\
\hline 9.65 & 0 & -1 & -1 & \\
\hline 0.71 & 1 & 1 & -1 & \\
11.47 & 1 & 0 & 1 & \\
\hline
\end{tabular}

\begin{tabular}{c|c|c} 
Avg. & deg. $\mathbf{c w}$ & deg. \\
\hline$-\mathbf{2 . 7}$ & -9 & \\
\hline $\mathbf{1 1 . 7}$ & 9 & \\
\hline $\mathbf{1 0 . 0}$ & 6 & \\
$\mathbf{6 . 7}$ & 3 & \\
$\mathbf{- 1 . 0}$ & -5 & \\
$\mathbf{- 1 . 0}$ & -4 & \\
\hline $\mathbf{0 . 7}$ & -4 & \\
$\mathbf{0 . 3}$ & -3 & \\
\hline $\mathbf{0 . 7}$ & -3 & \\
\hline
\end{tabular}

3.9

4.5

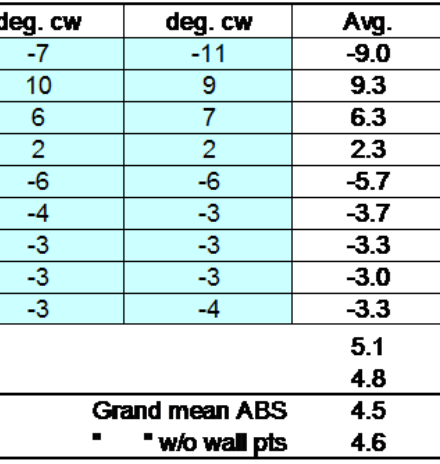

eg. cw

Cal. Due

Cert. of conformance

Dwyer 24-inch S-type Pitot\#10 10-Dec-13

Shop built $\quad$ Cat. 3

Cat. 3

Notes:

Note:

To assure similar hose connections

between the manometer and pitot tube, rotating

the pitot tube assembly clockwise dives the

meniscus to the right (to higher pos. numbers).

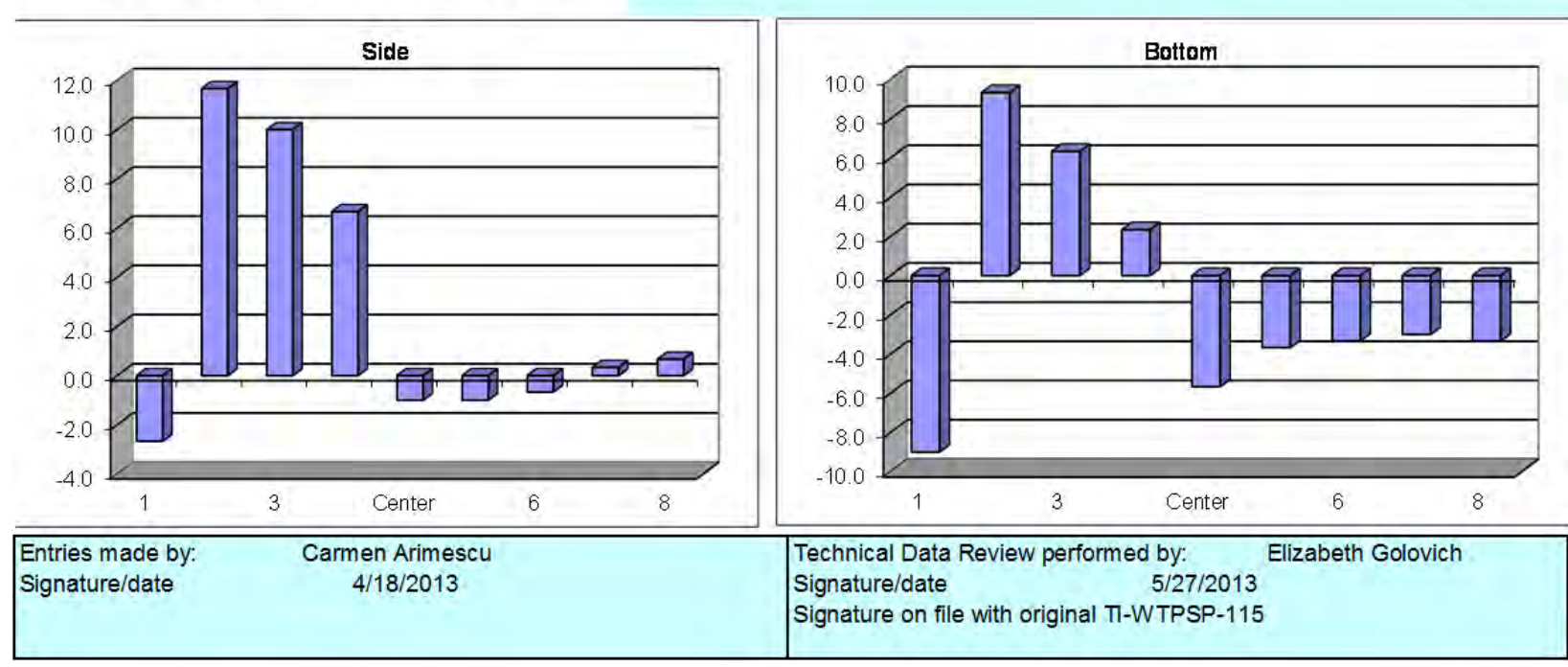




\section{FLOW ANGLE DATA FORM}

Site LV-S2 Scale Model

Date 4/18/2013

Tester $\mathrm{EA}, \mathrm{Ca}$

Stack Dia. 11.969

Stack X-Area $\frac{112.5}{11}$ in2

Elevation N.A. $f$ t

Distance to disturbance $\frac{221.12}{927 / 1000}$

Start/End Tme 927/1000

Order $\rightarrow$

Traverse $\rightarrow$

Tial $\longrightarrow$

\begin{tabular}{|c|c|}
\hline Point & Depth, in.
\end{tabular}

2nd

nd

Side

12

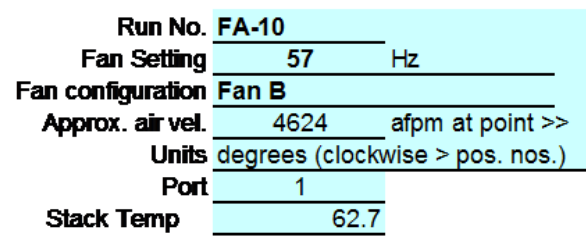

1 st

\begin{tabular}{|c|r|}
\hline $\mathbf{1}$ & 0.5 \\
\hline $\mathbf{2}$ & 1.2 \\
\hline $\mathbf{3}$ & 2.3 \\
\hline $\mathbf{4}$ & 3.87 \\
\hline Center & 5.9 \\
\hline $\mathbf{5}$ & 8.1 \\
\hline $\mathbf{6}$ & 9.6 \\
\hline $\mathbf{7}$ & 10.7 \\
\hline $\mathbf{8}$ & 11.47 \\
\hline
\end{tabular}

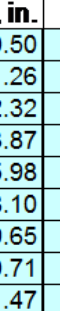

Mean of absolute values:

- wo points by wall: \begin{tabular}{l|l|l}
3 & & $2^{\text {Bottom }}$
\end{tabular} 3

\begin{tabular}{|c|c|c|}
\hline deg. $\mathbf{~ w ~}$ & deg. $\mathbf{c w}$ & \\
\hline-5 & -2 & \\
\hline 11 & 12 & \\
\hline 9 & 10 & \\
\hline 7 & 7 & \\
-2 & -2 & \\
\hline-1 & -1 & \\
\hline 0 & -1 & \\
\hline 1 & -1 & \\
\hline 0 & 0 & \\
\hline
\end{tabular}

\begin{tabular}{|c|c|}
\hline deg. $\mathbf{c w}$ & \\
-2 & \\
\hline 11 & \\
\hline 10 & \\
7 & \\
\hline-3 & \\
-1 & \\
-1 & \\
\hline 0 & \\
0 & \\
\hline
\end{tabular}

\begin{tabular}{|c|c|}
\hline Avg. & | \\
\hline-3.0 & \\
\hline 11.3 & \\
\hline 9.7 & \\
\hline 7.0 & \\
\hline-2.3 & \\
-1.0 & \\
\hline-0.7 & \\
\hline 0.0 & \\
\hline 0.0 & \\
\hline 3.9 & \\
4.6 & \\
\hline
\end{tabular}

Instuments Used:

S-type pitot

Velocity sensor

Angle indicator

Manometer

Dwyer 24-inch S-type Pitot\#10

Cal. Due

Cert. of conformance

\begin{tabular}{l|l|l} 
deg. cw & deg. $\mathbf{c w}$ & deg. $\mathrm{cw}$
\end{tabular}

\begin{tabular}{|l|l|l|l} 
deg. cw & deg. cw & deg. cw & Avg.
\end{tabular}

\begin{tabular}{c|c|c|c}
-5 & -9 & -5 & $\mathbf{- 6 . 3}$ \\
\hline 12 & 11 & 13 & $\mathbf{1 2 . 0}$ \\
\hline
\end{tabular}

\begin{tabular}{l|l|l|l}
12 & 11 & 13 & $\mathbf{1 2 . 0}$ \\
-2 & -2 & -3 & $-\mathbf{2 3}$
\end{tabular}

\begin{tabular}{c|c|c|c}
-2 & -2 & -3 & -2.3 \\
-2 & 7 & 6 & 3.7
\end{tabular}

\begin{tabular}{c|c|c|c}
-2 & 7 & 6 & 3.7 \\
-3 & -2 & -3 & -2.7 \\
\hline-1 & -1 & -2 & -1.3
\end{tabular}

-1
0

0
0
0

\begin{tabular}{l|l}
-1 \\
-1 \\
-1 \\
-1
\end{tabular}

\begin{tabular}{l|l}
-1 & \\
-1 & \\
-1 & \\
-1 &
\end{tabular}

-3
-1
-1

\begin{tabular}{l|l}
-2 & -1.3 \\
-1 & -0.7
\end{tabular}

Dwyer 400-5, S36N

\section{Note:}

To assure similar hose connections

between the manometer and pitot tube, rotating

the pilot tube assembly clockwise dives the

meniscus to the right (to higher pos. numbers).

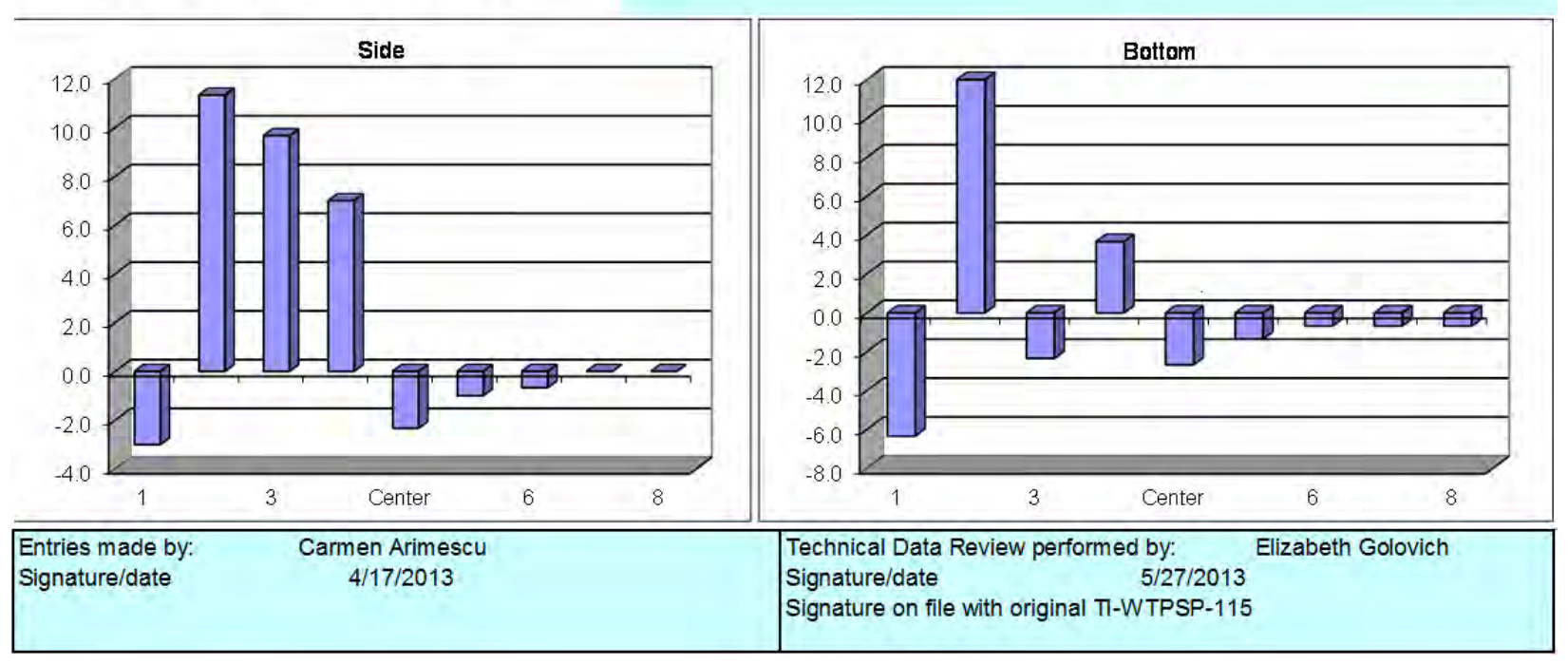




\section{C.4 LV-S2 Gas Tracer Calibration and Uniformity Data Sheets SULFUR HEXAFLUORIDE AND NITROUS OXIDE GAS INSTRUMENT CALIBRATION}

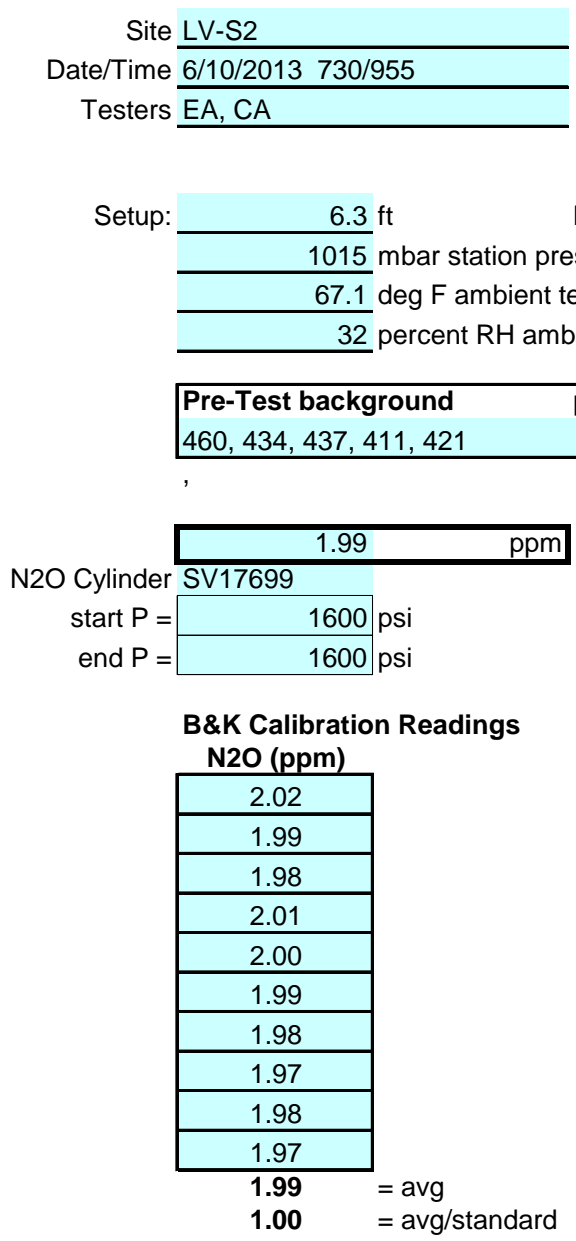

Standards Used:

Air Liquide 1.99 ppm N2O in air, SV17699

Air Liquide 59.6 ppm N2O in air, SV17805

Weather Station Used:

Fisher Scientific, S/N 90936818

B\&K sample inlet tube length

Instrument B\&K Model 1302

Serial No.

Property No.

1804888

WD54623

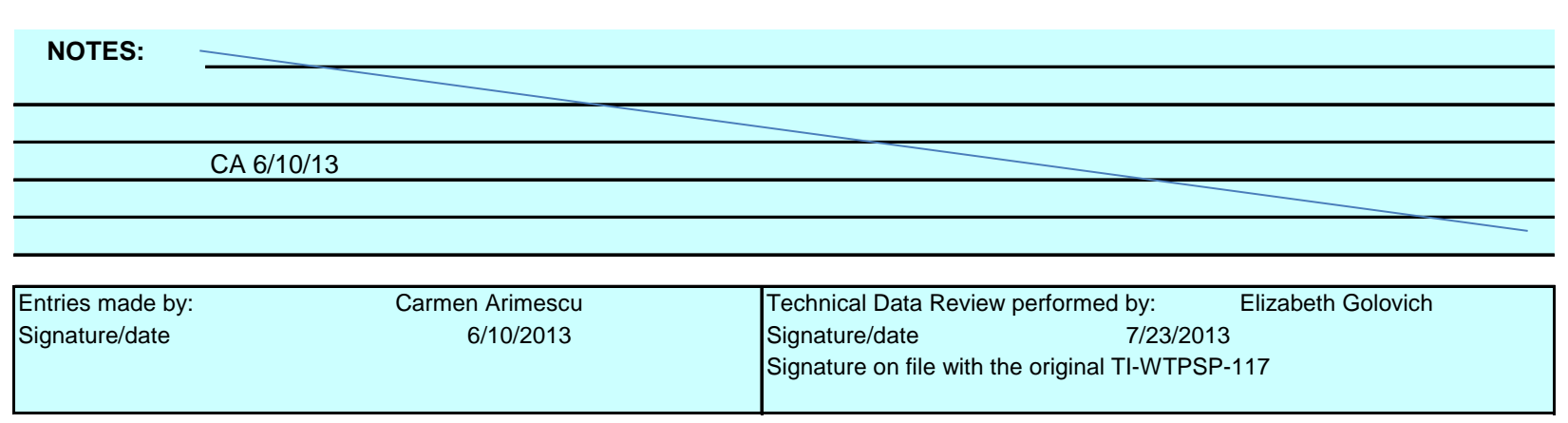

Expiration date:

$6 / 1 / 2014$

$6 / 1 / 2014$

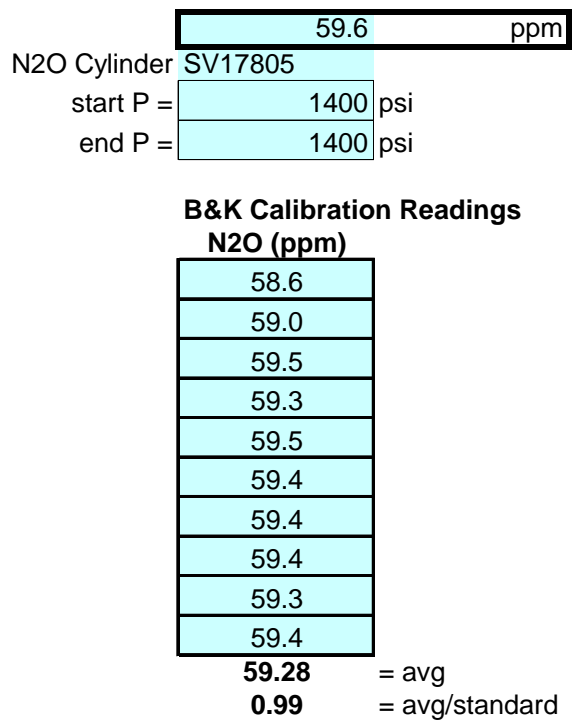




\section{SULFUR HEXAFLUORIDE AND NITROUS OXIDE GAS INSTRUMENT CALIBRATION}

Site LV-S2

Date/Time 6/17/2013 910/955

Testers $\mathrm{XYY}, \mathrm{CA}$
Instrument B\&K Model 1302

Serial No.

Property No.

WD54623

1016 mbar station pressure

68.9 deg F ambient temp analyzer corrects to 20 deg C

40 percent $\mathrm{RH}$ ambient humidity

\begin{tabular}{|lll|}
\hline $\begin{array}{l}\text { Pre-Test background } \\
432,409,397,393,393\end{array}$ & ppb N2O & compensating for water vapor \\
\hline
\end{tabular}

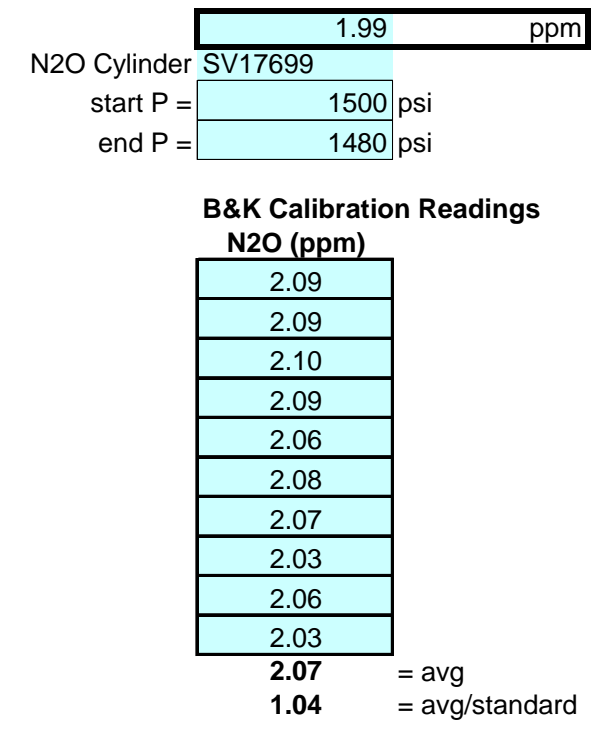

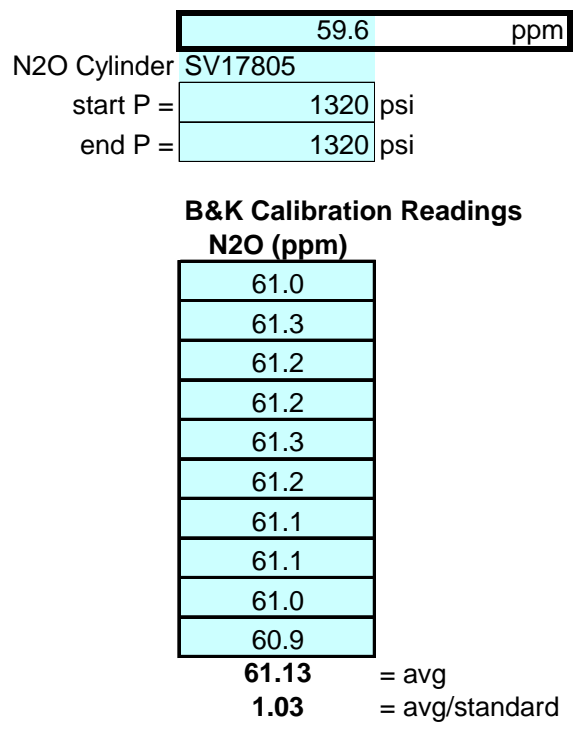

Standards Used:

Expiration date:

\begin{tabular}{llr}
\hline Air Liquide & 1.99 ppm N2O in air, SV17699 & $6 / 1 / 2014$ \\
\hline Air Liquide & 59.6 ppm N2O in ar, SV17805
\end{tabular}

Air Liquide 59.6 ppm N2O in air, SV17805 $6 / 1 / 2014$

Weather Station Used:

\begin{tabular}{ll}
\hline Fisher Scientific, S/N 90936818 & $12 / 11 / 2013$
\end{tabular}

\begin{tabular}{|c|c|c|}
\hline NOTES: & & \\
\hline \multicolumn{3}{|c|}{$\mathrm{XYY} 6 / 17 / 13$} \\
\hline Entries made by: & Xiao-Ying Yu & Technical Data Review performed by: \\
\hline Signature/date & $6 / 17 / 2013$ & $\begin{array}{l}\text { Signature/date } \\
\text { Signature on file with the original TI-WTPSP-117 }\end{array}$ \\
\hline
\end{tabular}




\section{SULFUR HEXAFLUORIDE AND NITROUS OXIDE GAS INSTRUMENT CALIBRATION}

Site LV-S2

Date/Time 6/24/2013 729/815

Testers CA
Instrument B\&K Model 1302

Serial No.

Property No.

Setup:

$6.3 \mathrm{ft}$

$B \& K$ sample inlet tube length

1004 mbar station pressure

67.1 deg F ambient temp

analyzer corrects to $20 \mathrm{deg} C$

46 percent $\mathrm{RH}$ ambient humidity

\begin{tabular}{|lll|}
\hline $\begin{array}{l}\text { Pre-Test background } \\
434,413,394,377,379\end{array}$ & ppb N2O & compensating for water vapor \\
\hline
\end{tabular}

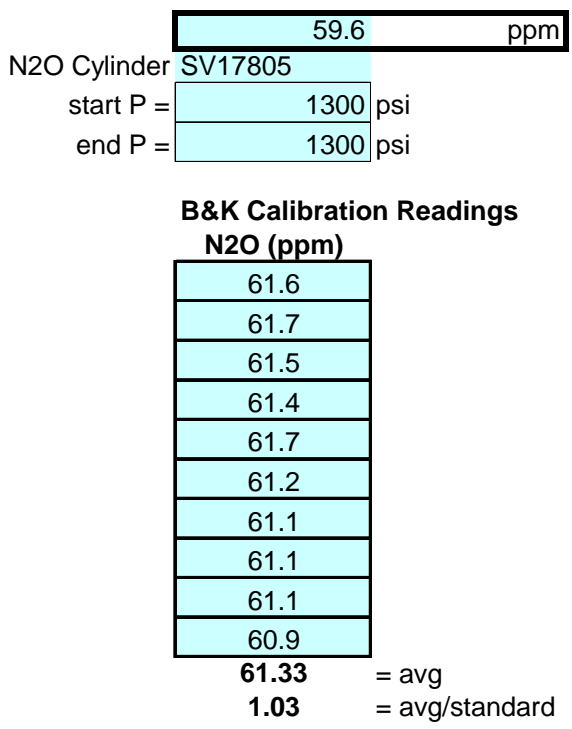

Standards Used:

Expiration date:

\begin{tabular}{llr}
\hline Air Liquide & 1.99 ppm N2O in air, SV17699 & $6 / 1 / 2014$ \\
\hline Air Liquide & 59.6 ppm N2O in ar, SV17805
\end{tabular}

Air Liquide 59.6 ppm N2O in air, SV17805 $6 / 1 / 2014$

Weather Station Used:

\begin{tabular}{ll}
\hline Fisher Scientific, S/N 90936818 & $12 / 11 / 2013$
\end{tabular}

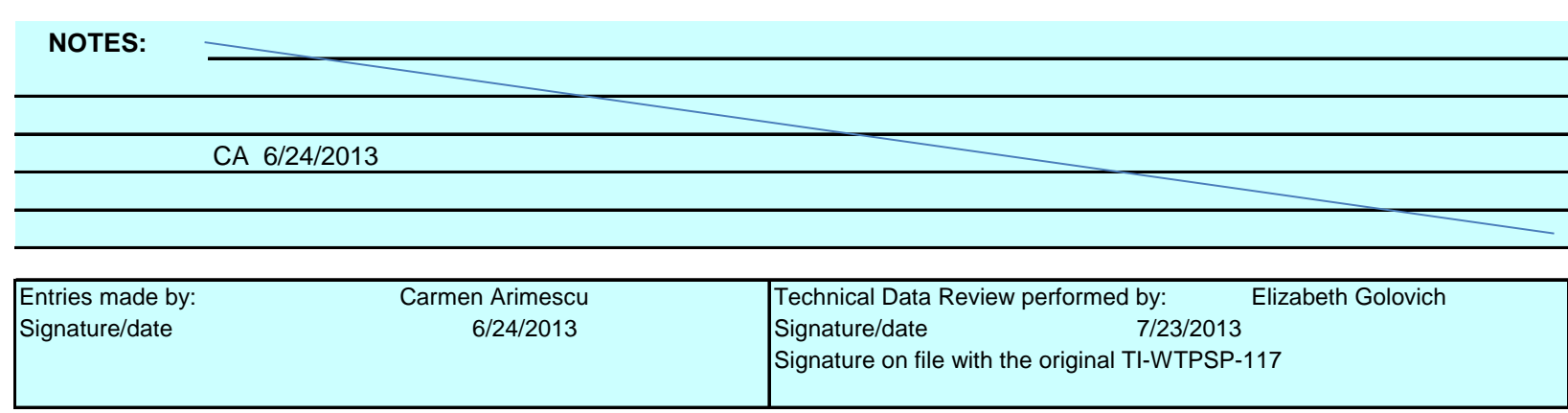




\section{SULFUR HEXAFLUORIDE AND NITROUS OXIDE GAS INSTRUMENT CALIBRATION}

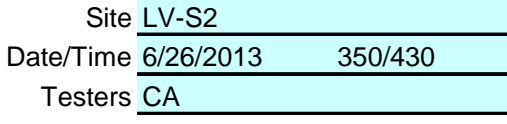

Instrument B\&K Model 1302
Serial No. $\frac{1804888}{2}$
Property No.

\begin{tabular}{|c|c|c|}
\hline \multirow[t]{4}{*}{ Setup: } & B\&K samp & ple inlet tube length \\
\hline & \multicolumn{2}{|l|}{1015 mbar station pressure } \\
\hline & $\overline{71.6}$ deg F ambient temp & analyzer corrects to $20 \mathrm{deg} C$ \\
\hline & \multicolumn{2}{|l|}{$\overline{42}$ percent $\mathrm{RH}$ ambient humidity } \\
\hline & Pre-Test background & compensating for water vapor \\
\hline & $467,430,436,414,367$ & \\
\hline
\end{tabular}

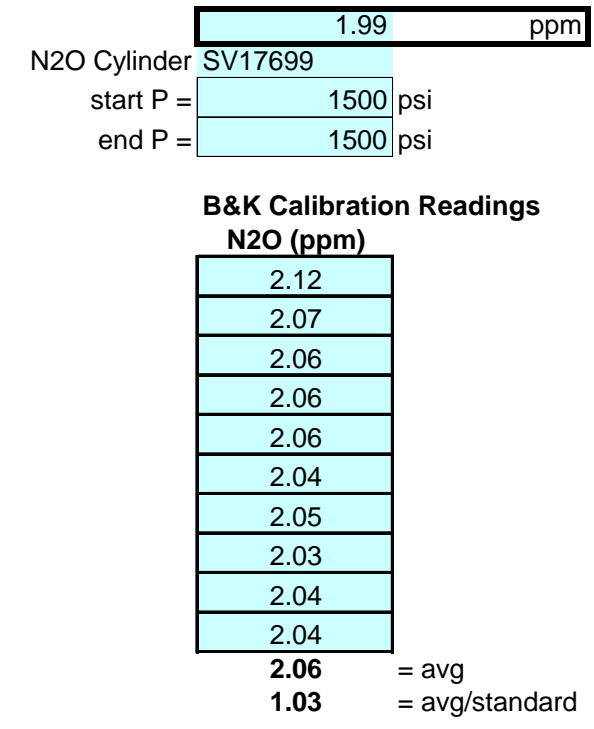

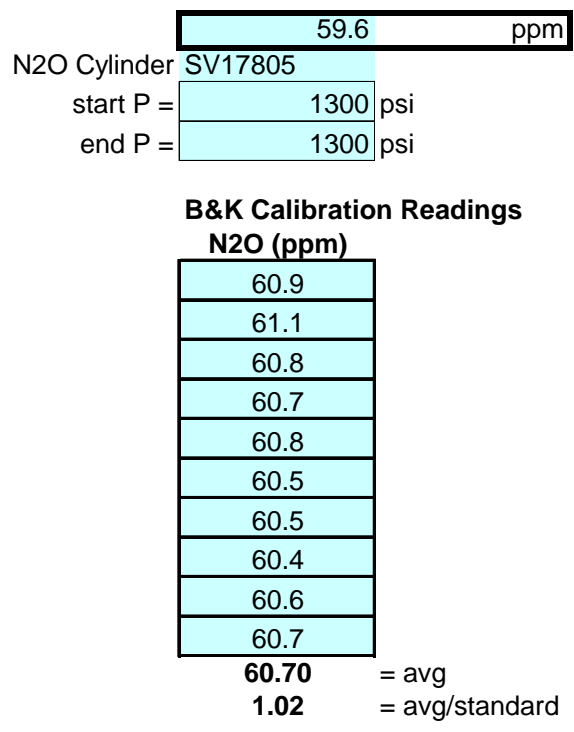

Standards Used:

Expiration date:

\begin{tabular}{llr}
\hline Air Liquide & 1.99 ppm N2O in air, SV17699 & $6 / 1 / 2014$ \\
\hline Air Liquide & 59.6 ppm N2O in ar, SV17805
\end{tabular}

Air Liquide 59.6 ppm N2O in air, SV17805 $6 / 1 / 2014$

Weather Station Used:

Fisher Scientific, S/N $90936818 \quad 12 / 11 / 2013$

\begin{tabular}{|c|c|c|}
\hline NOTES: & $=$ & \\
\hline & CA $6 / 26 / 2013$ & \\
\hline $\begin{array}{l}\text { Entries made by: } \\
\text { Signature/date }\end{array}$ & $\begin{array}{c}\text { Carmen Arimescu } \\
6 / 26 / 2013\end{array}$ & $\begin{array}{l}\text { Technical Data Review performed by: } \quad \text { Elizabeth Golovich } \\
\text { Signature/date } \quad 7 / 23 / 2013 \\
\text { Signature on file with the original TI-WTPSP-117 }\end{array}$ \\
\hline
\end{tabular}




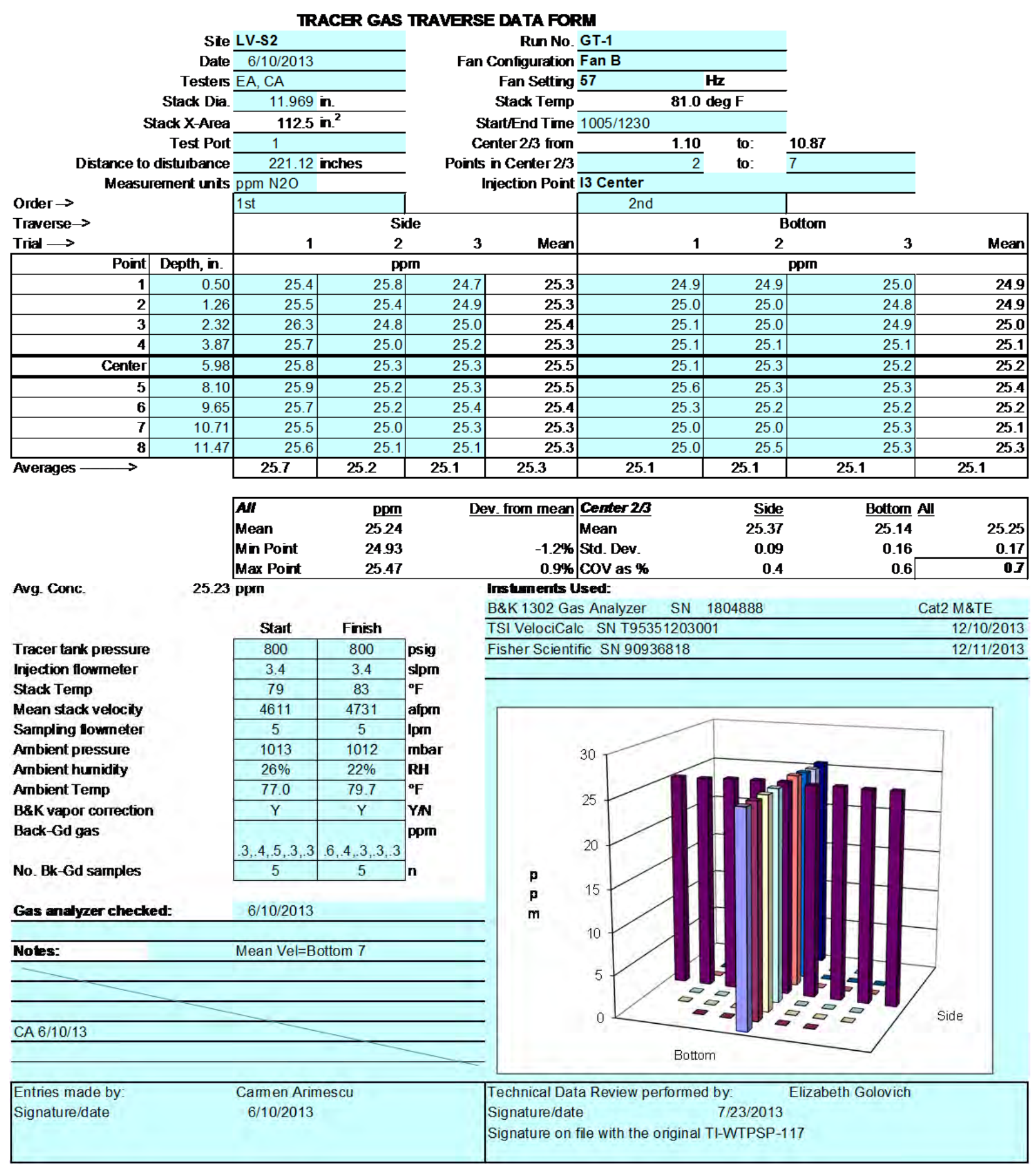




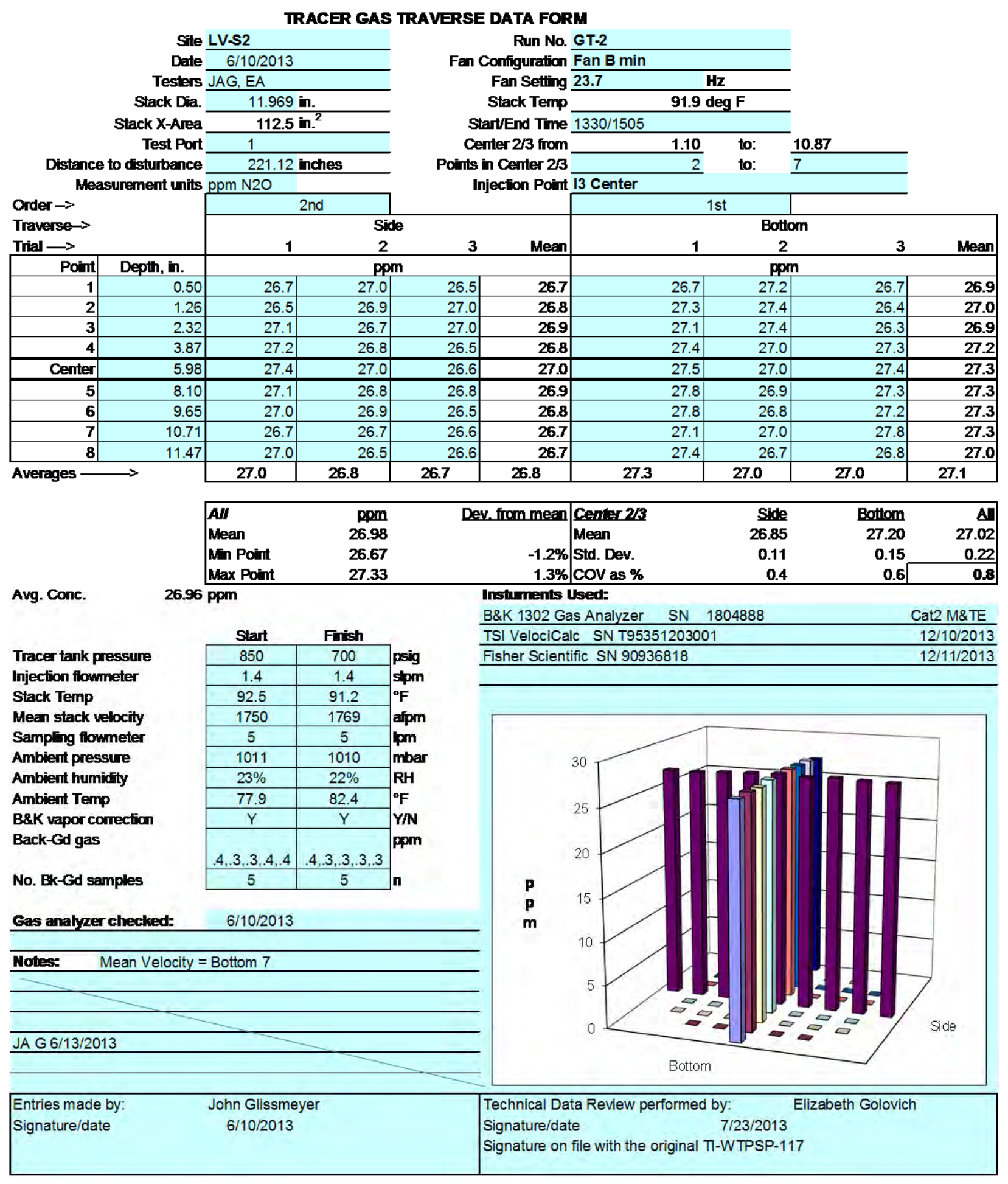




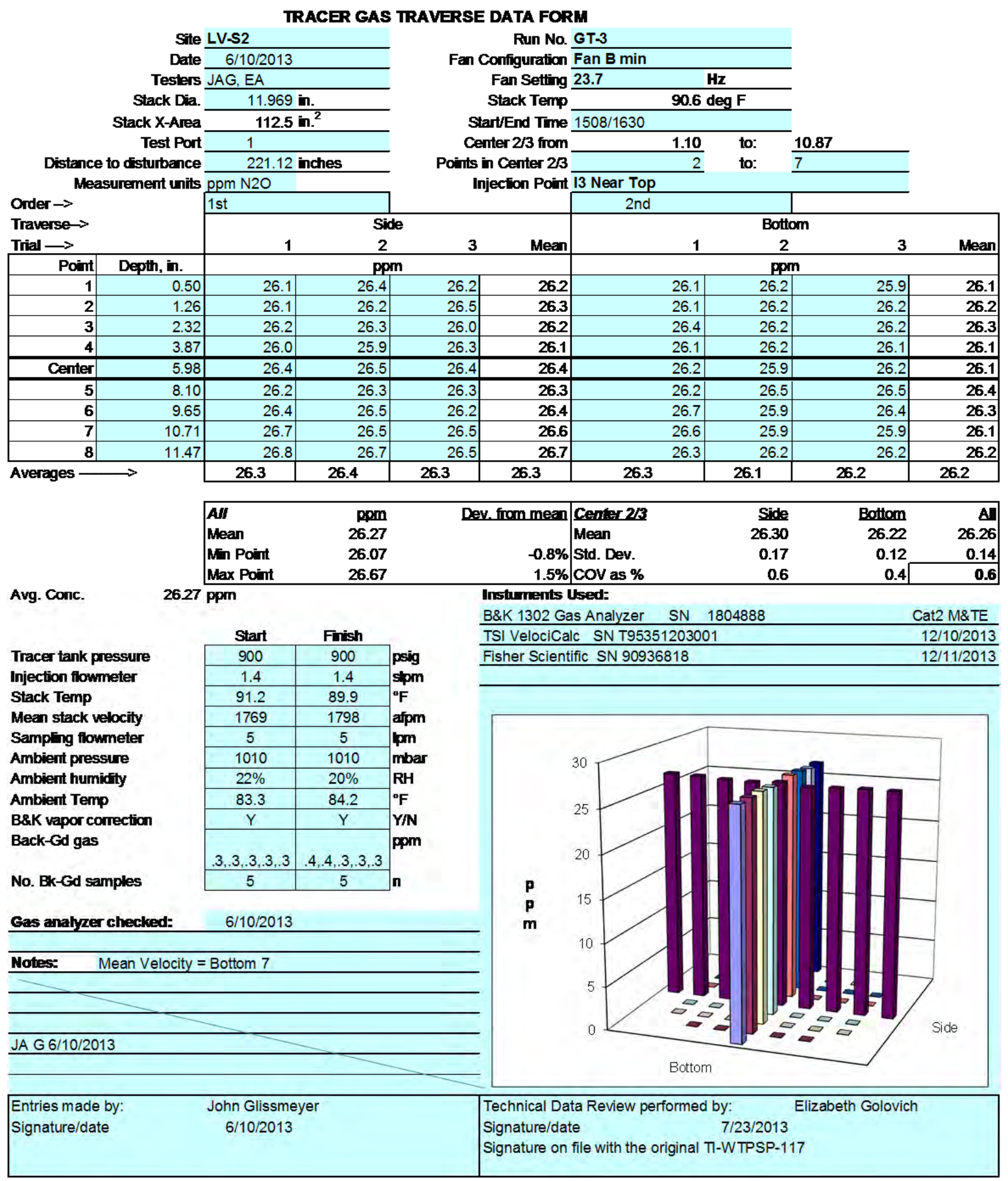




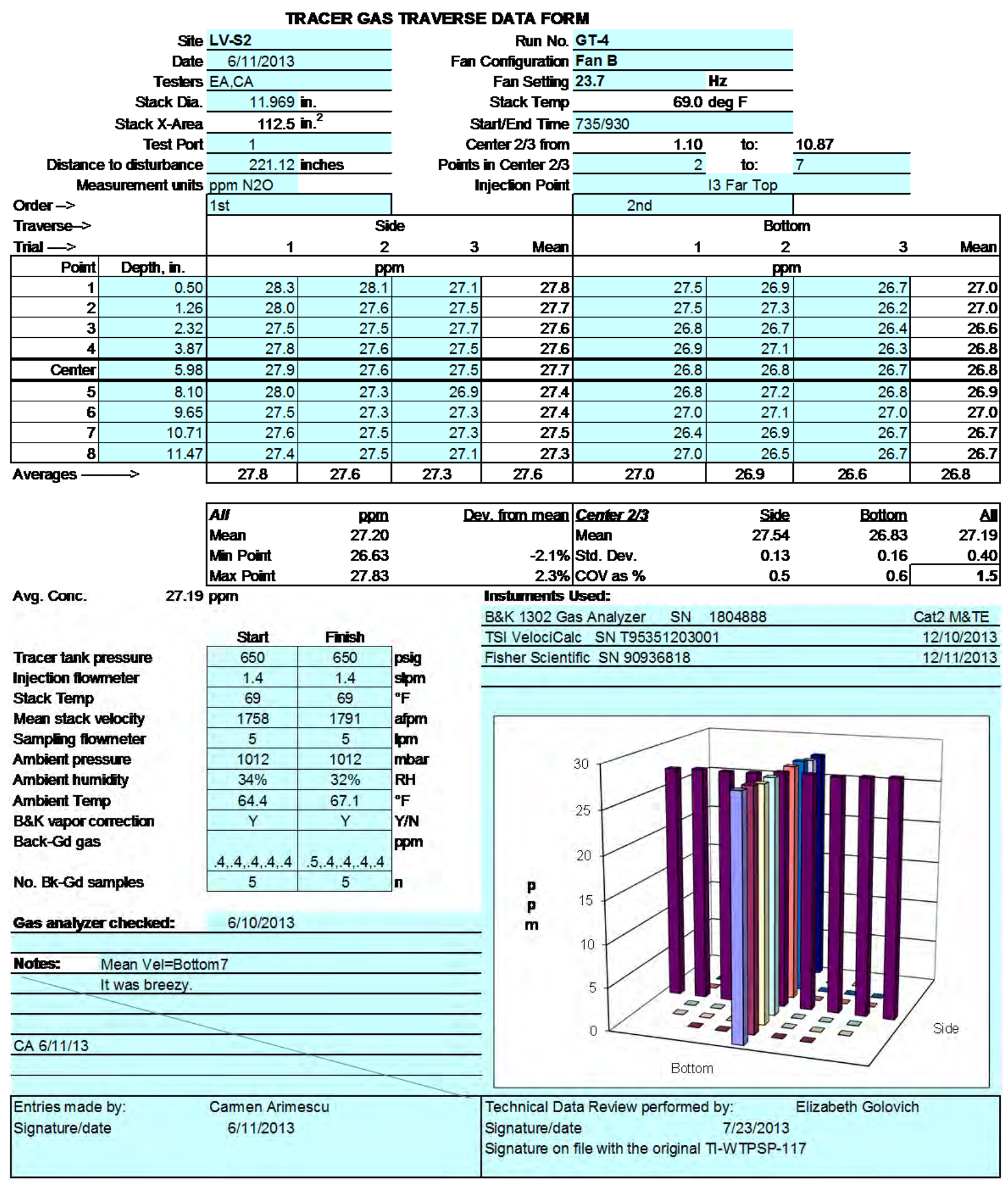




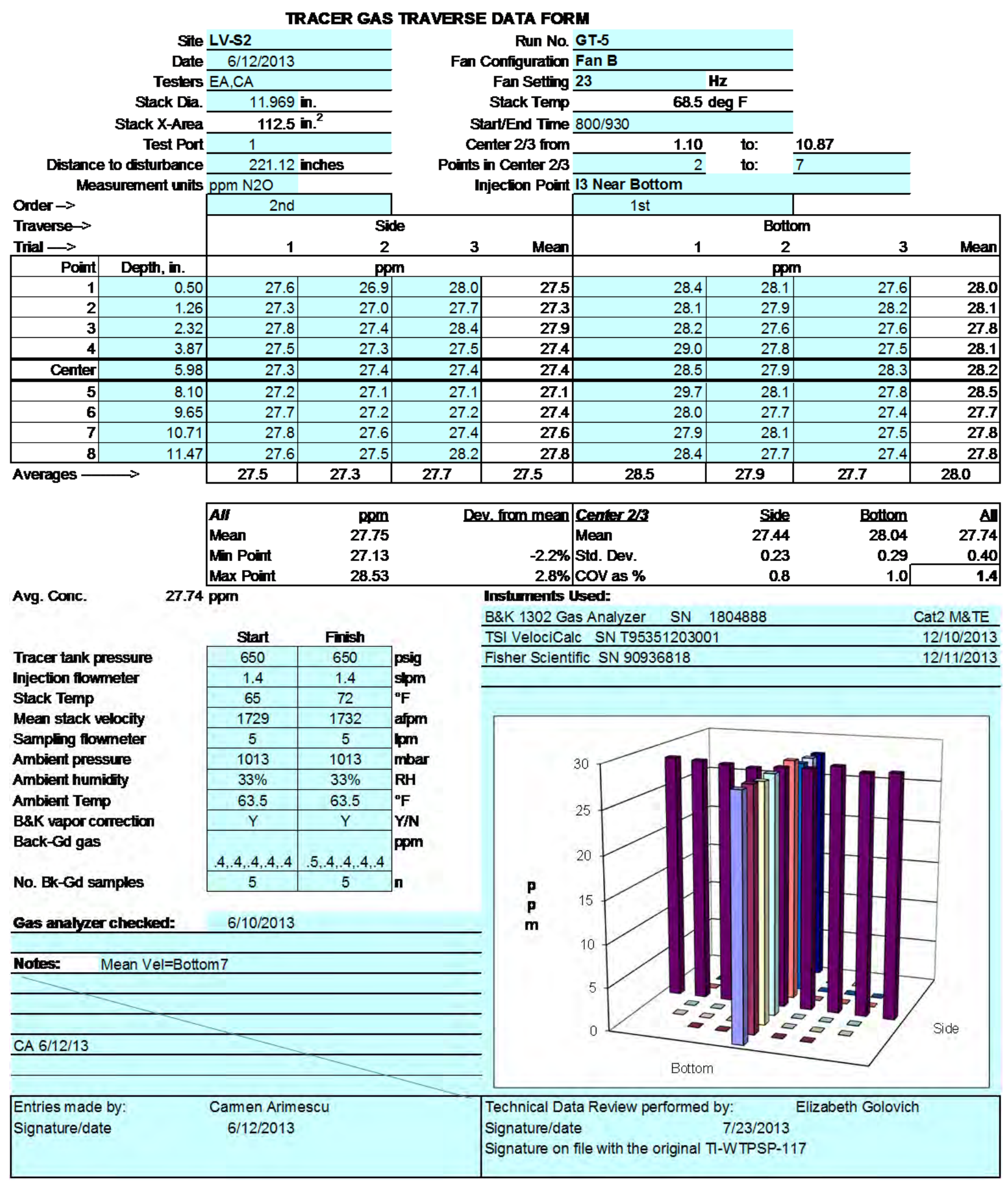




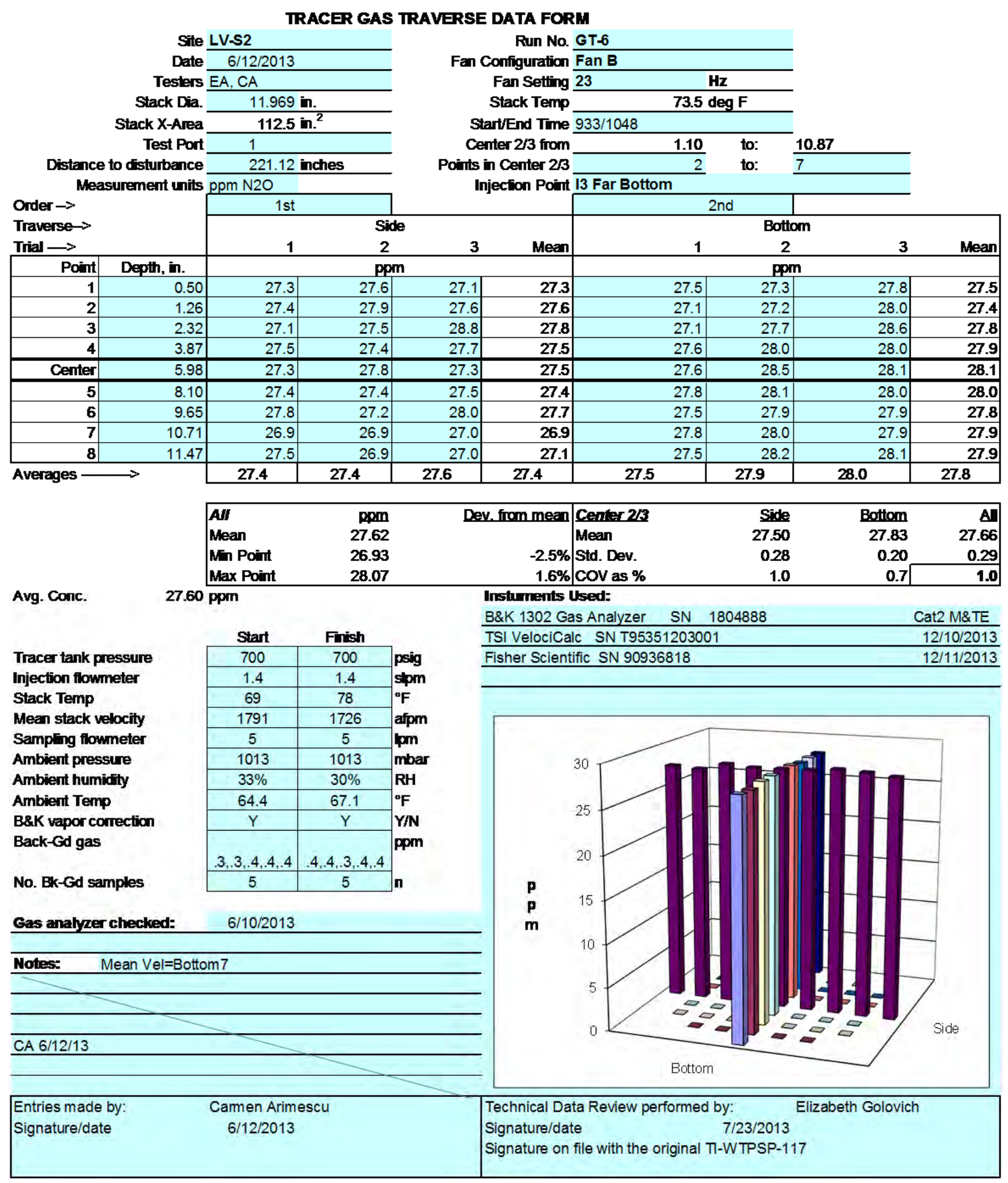




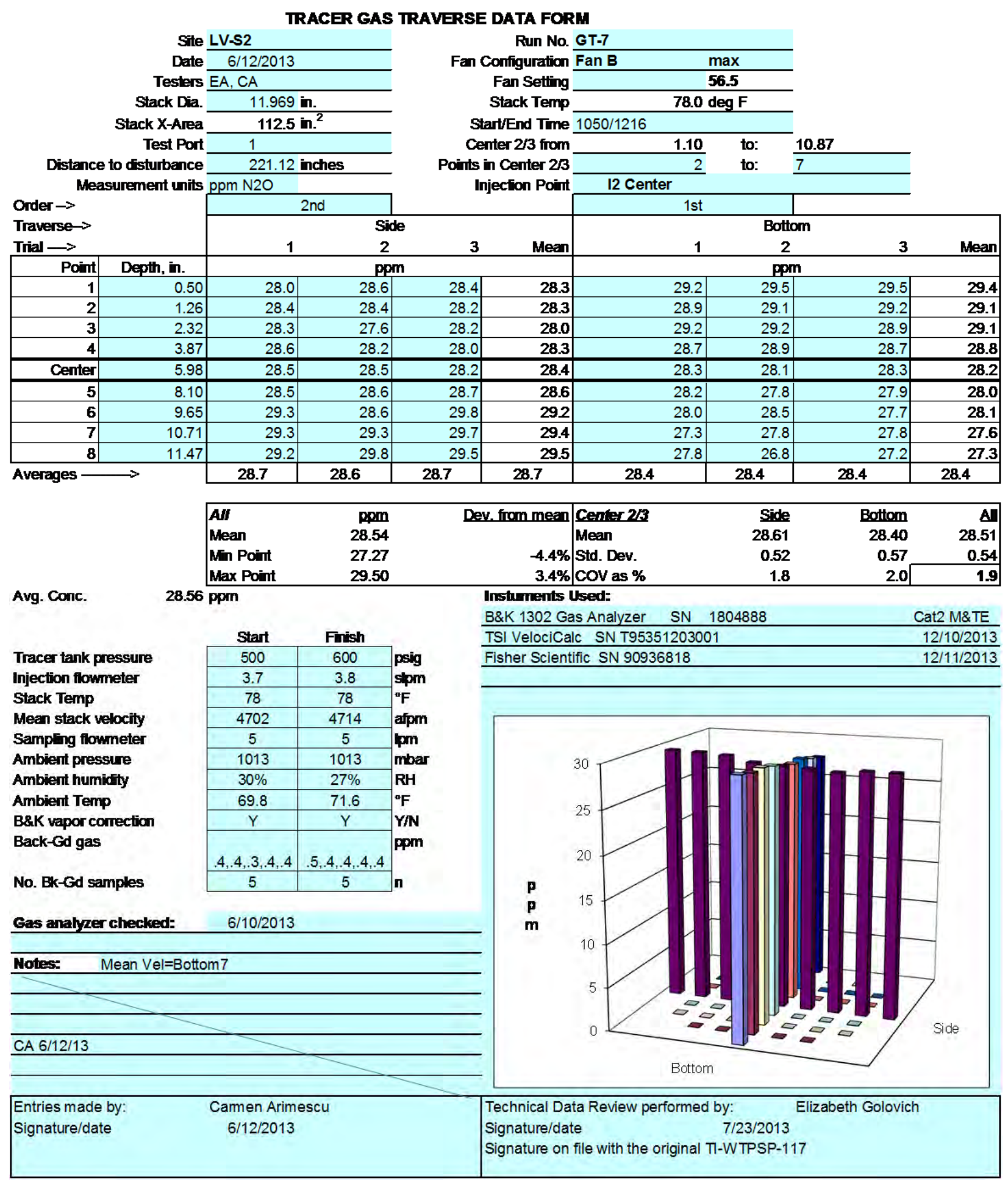




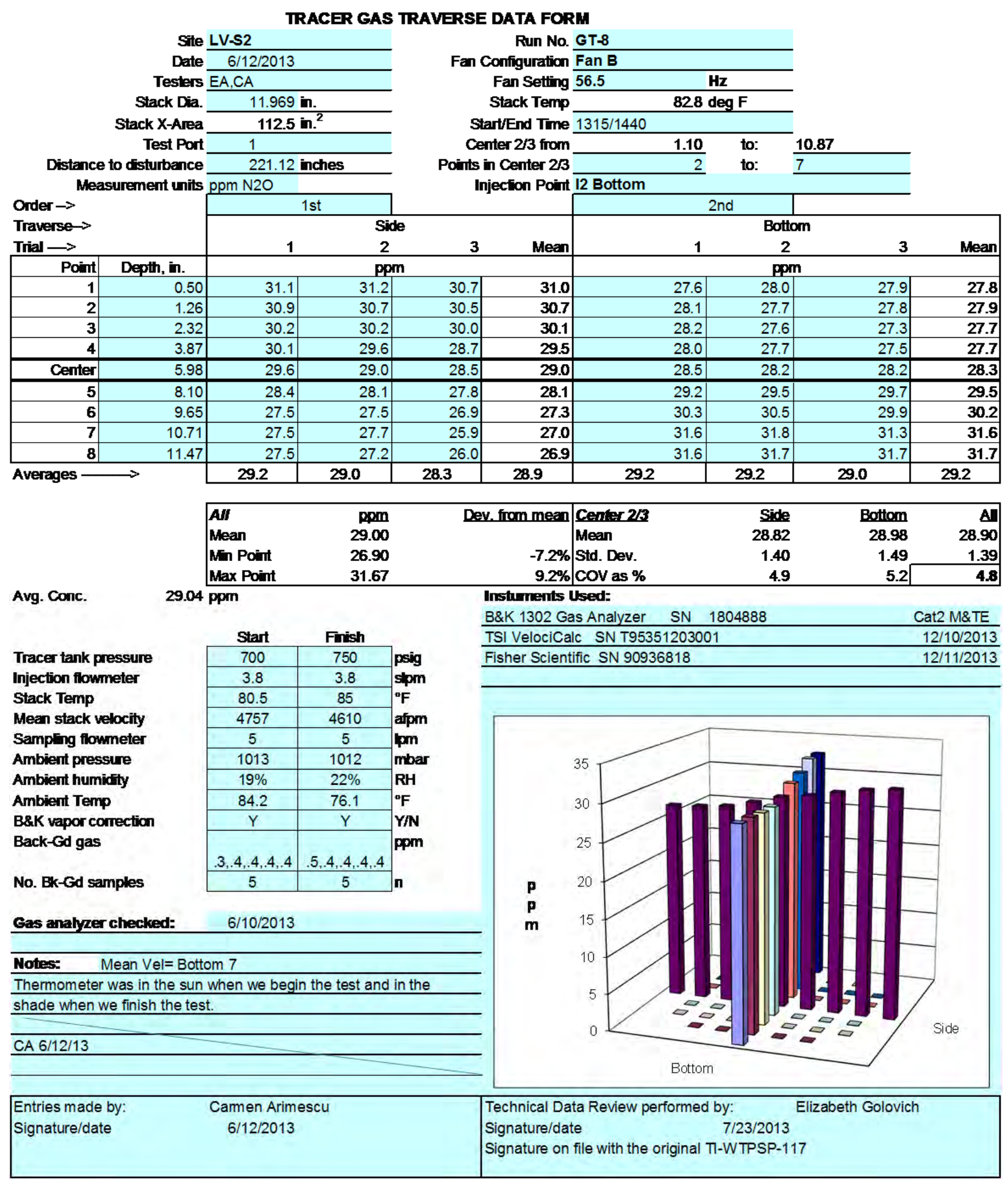




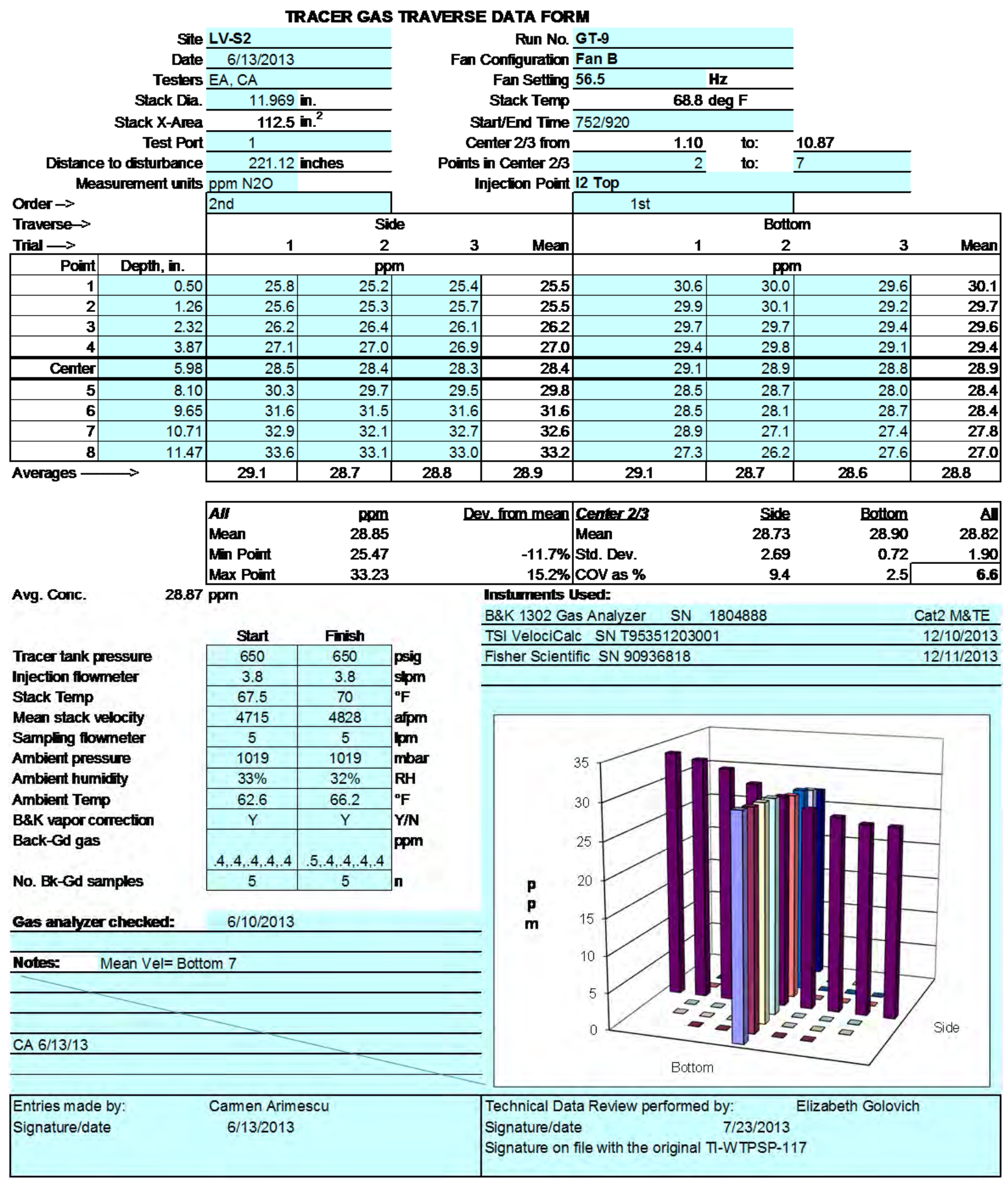




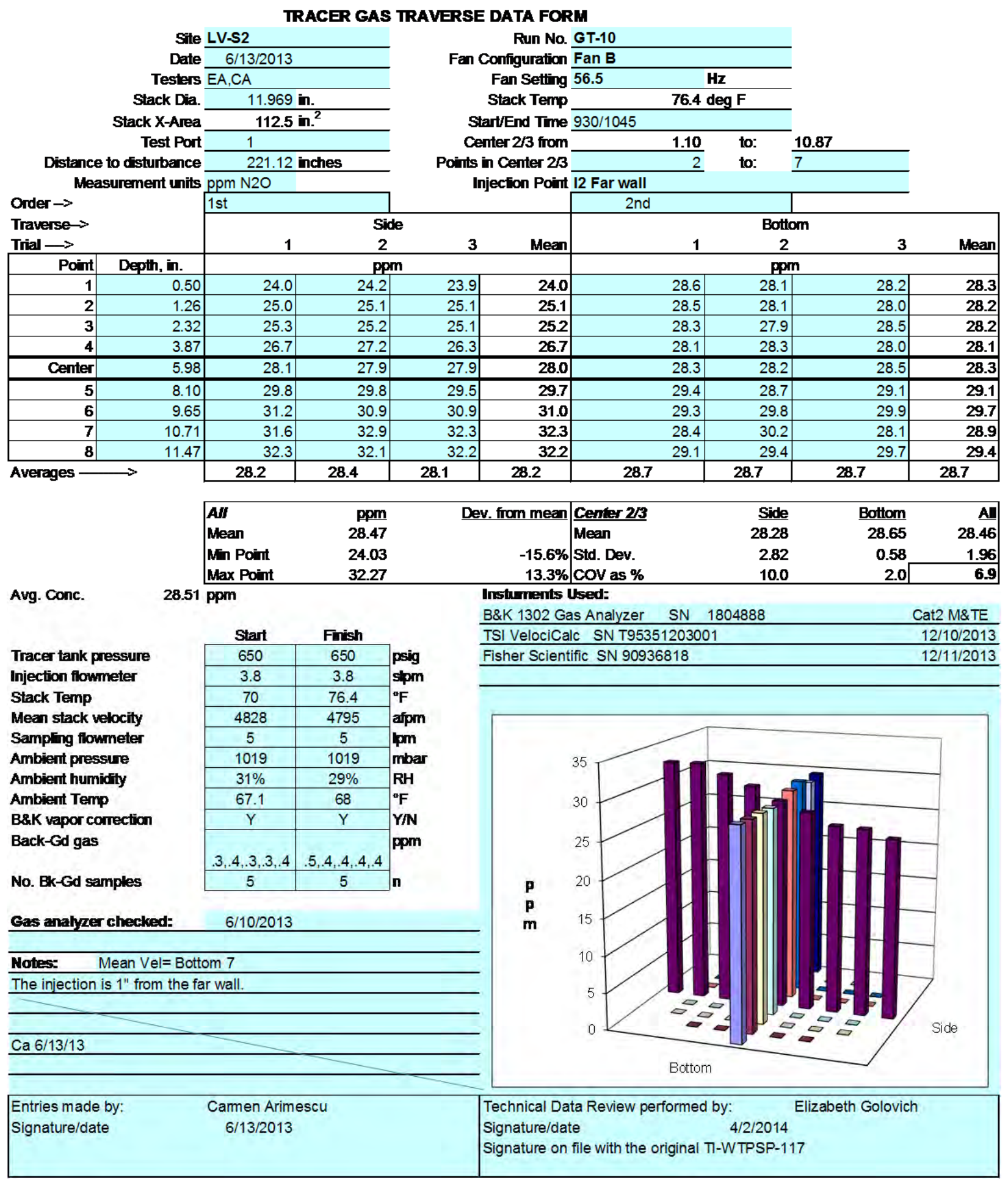




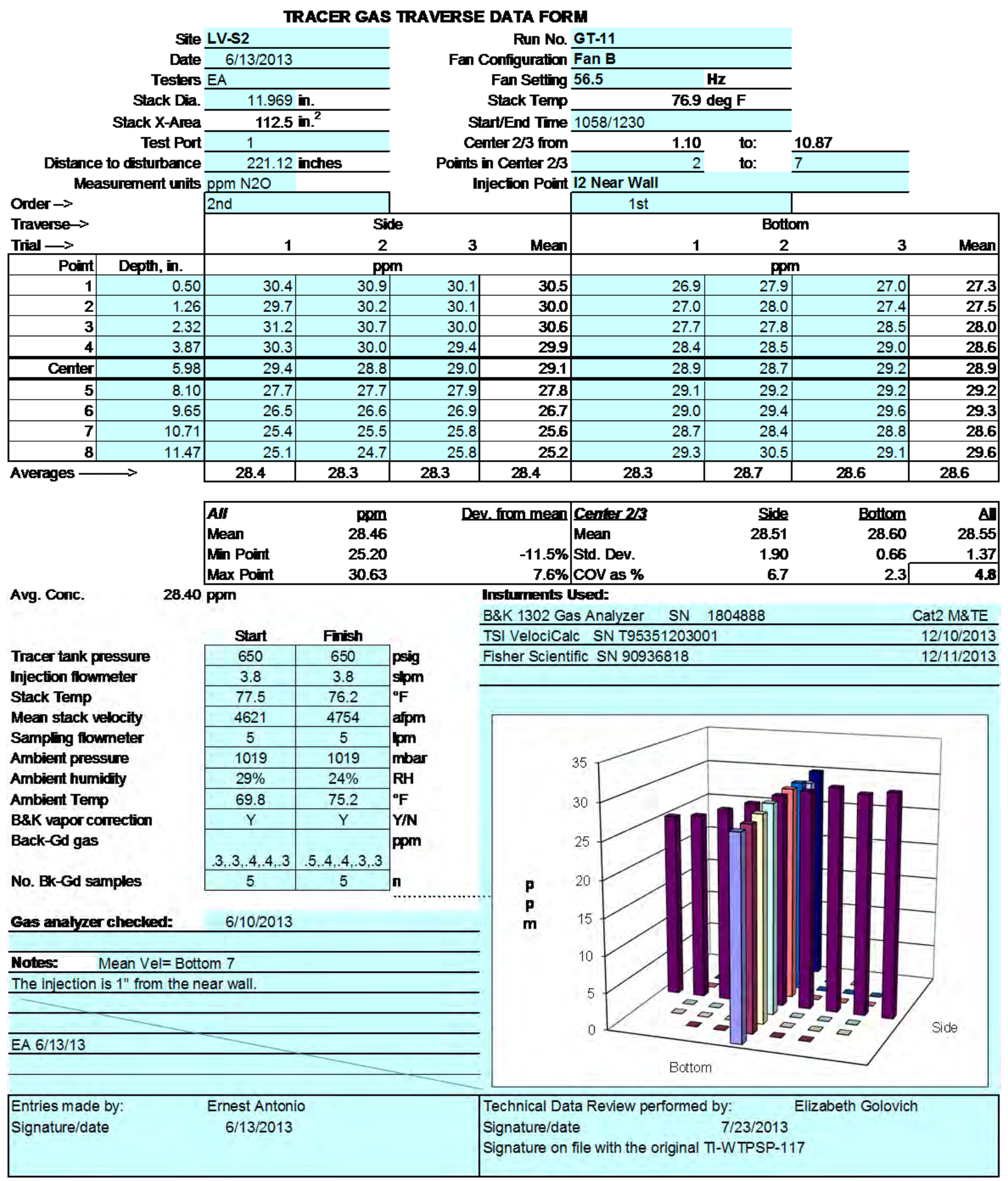




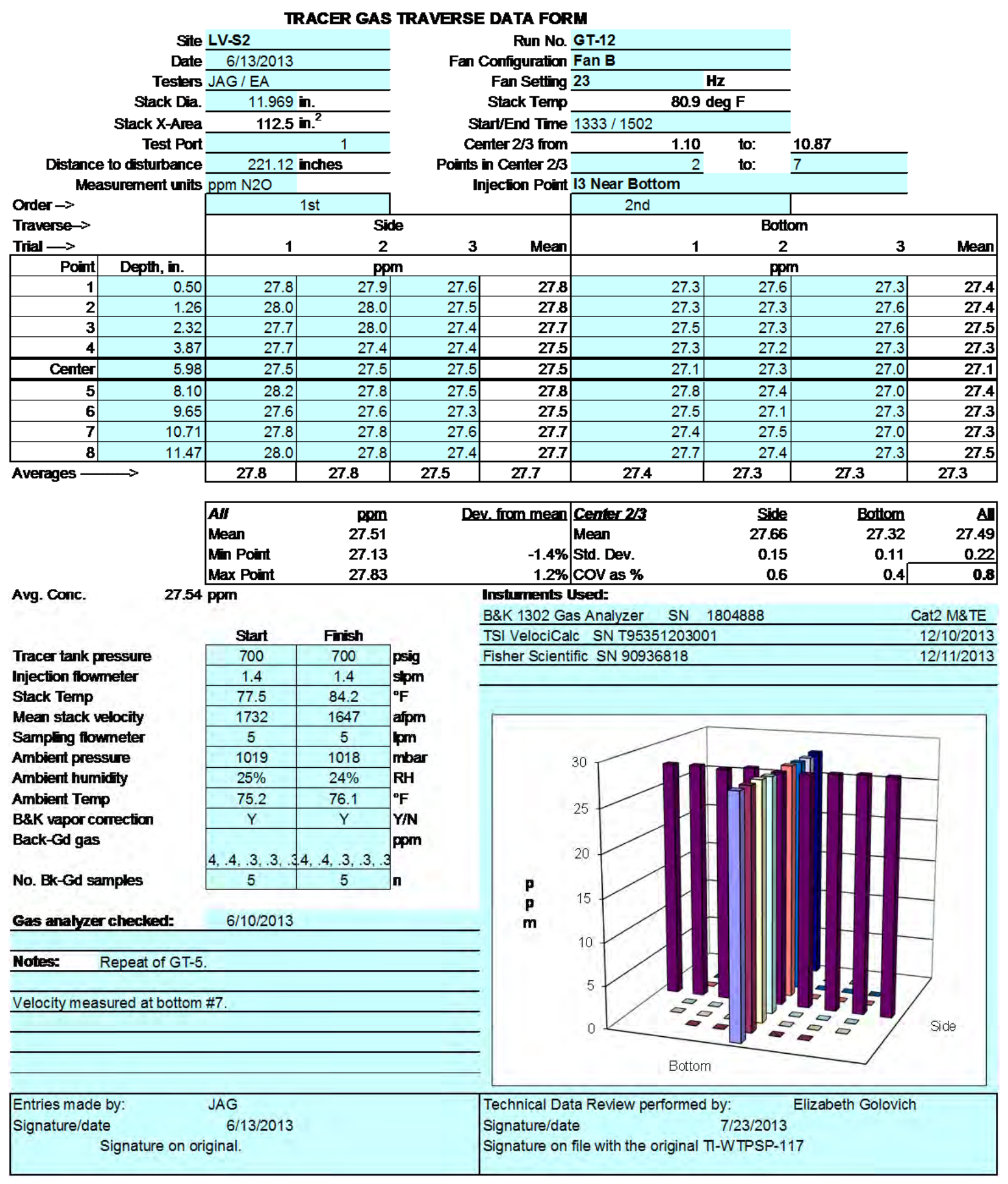




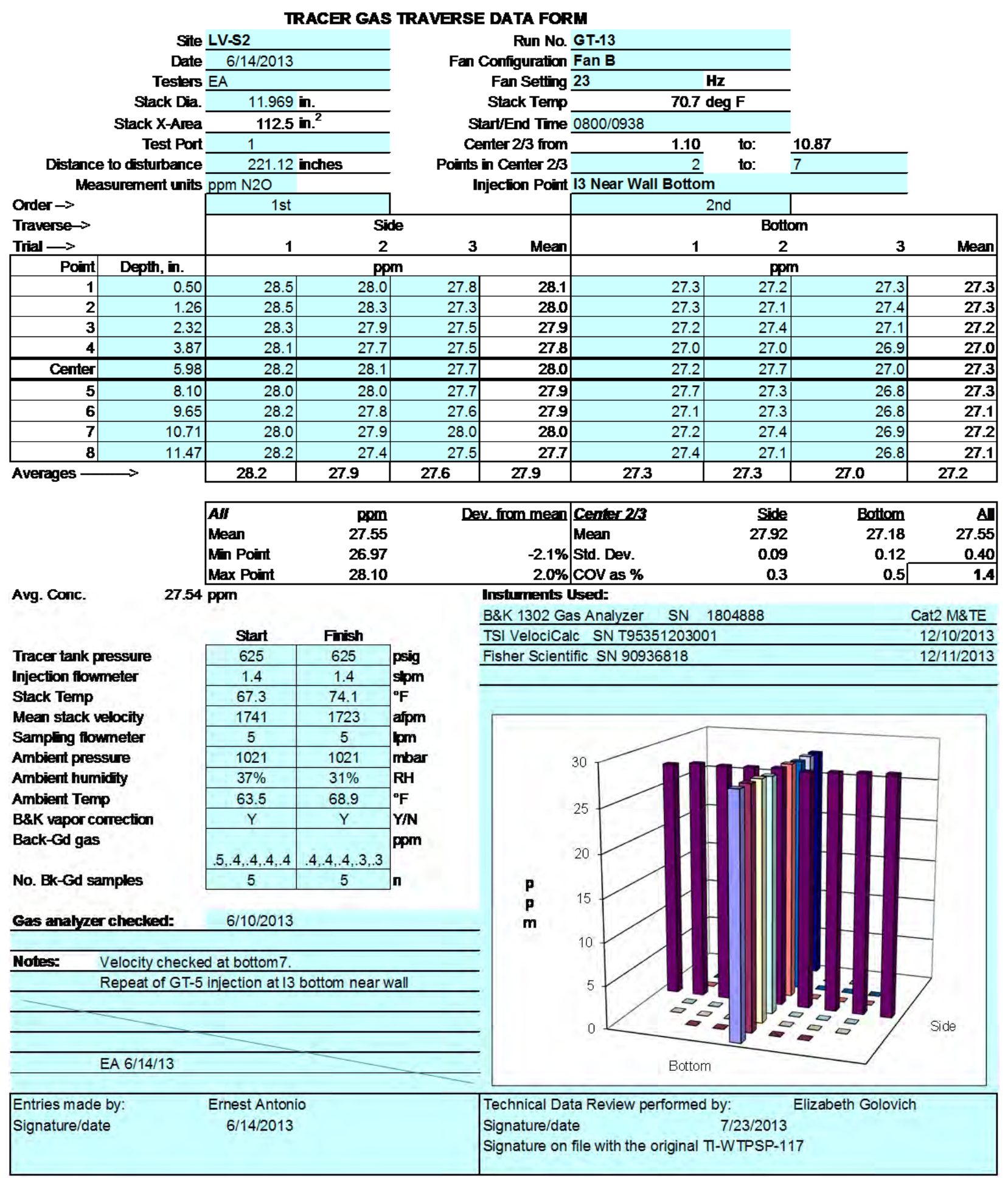




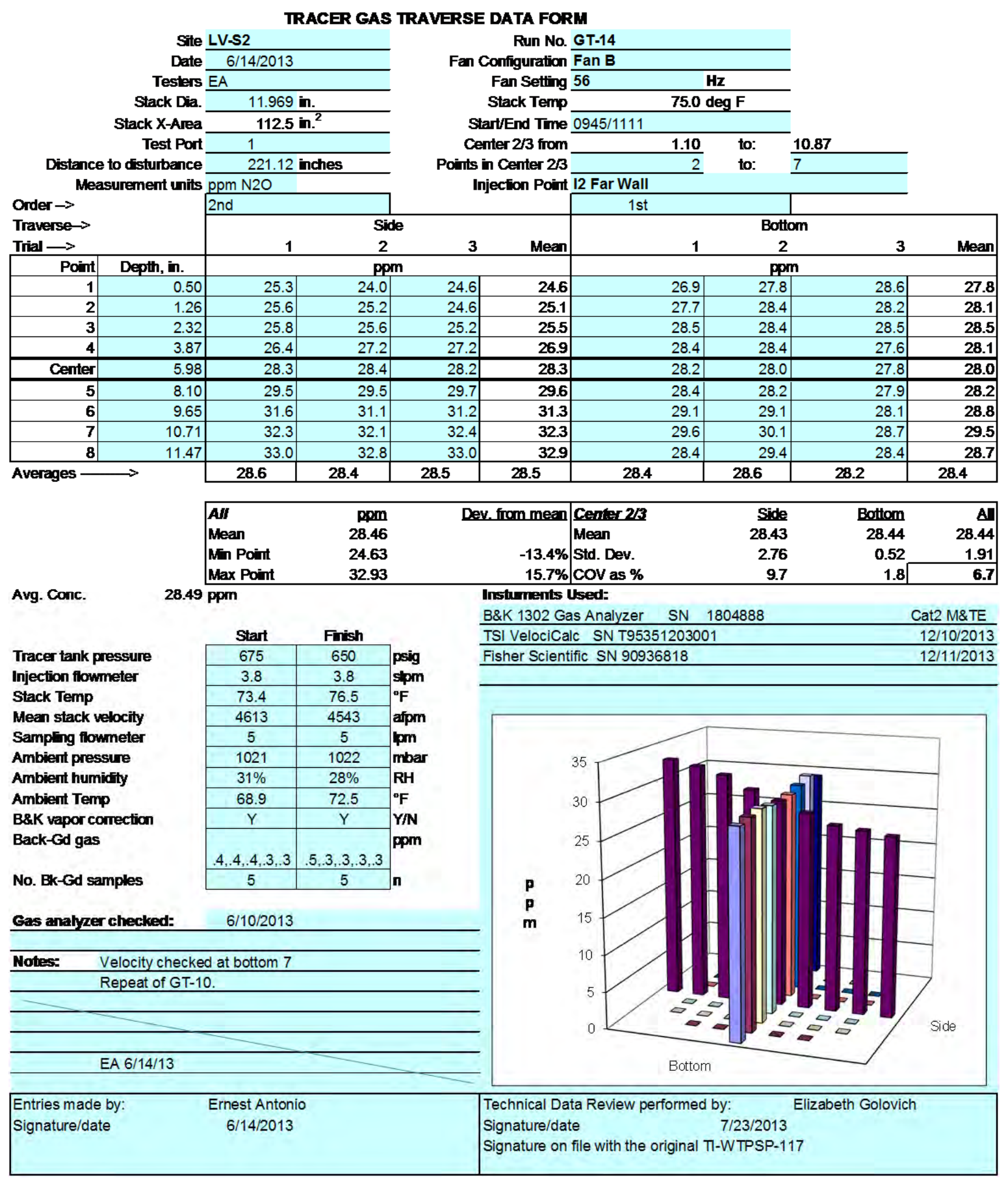




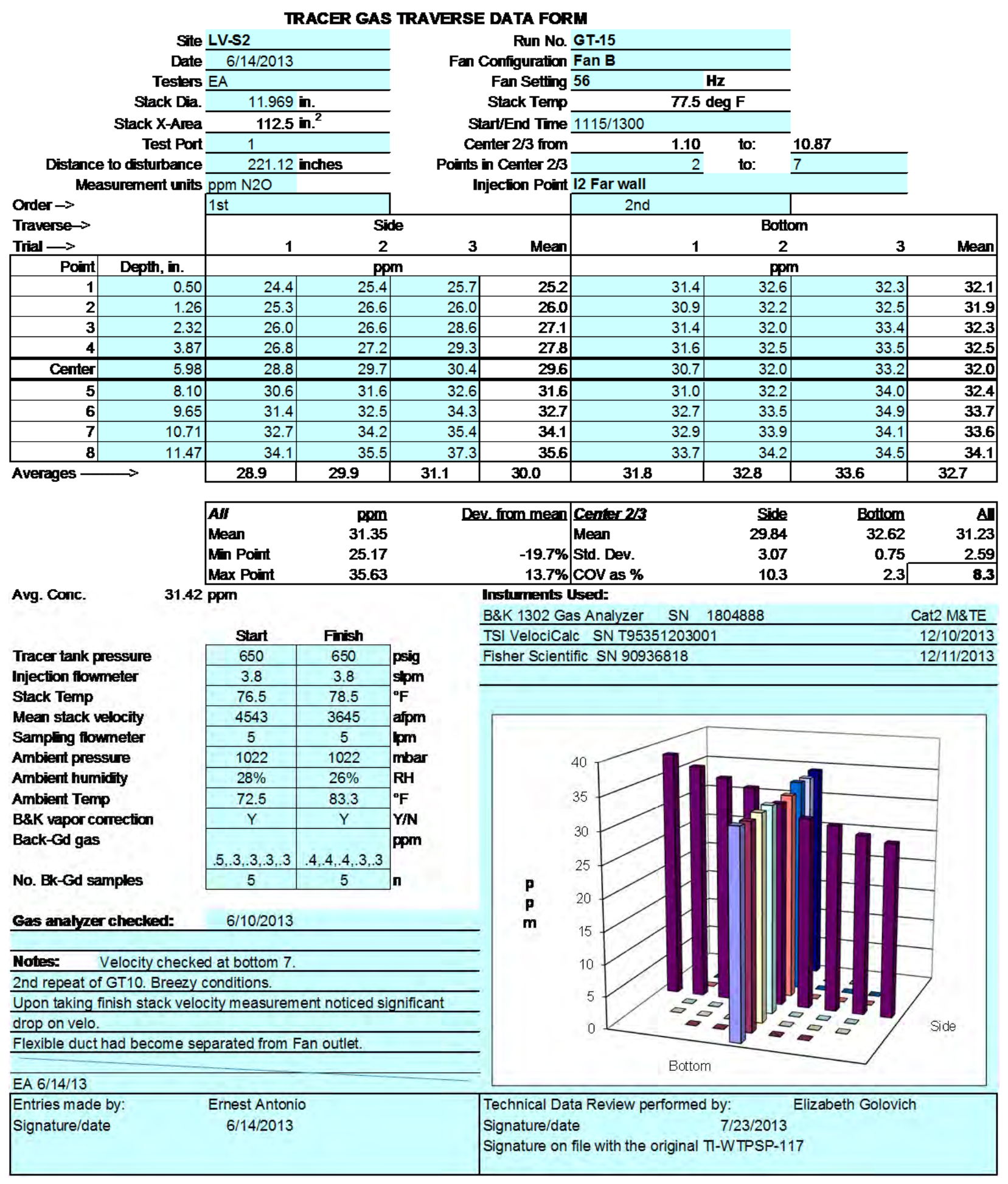




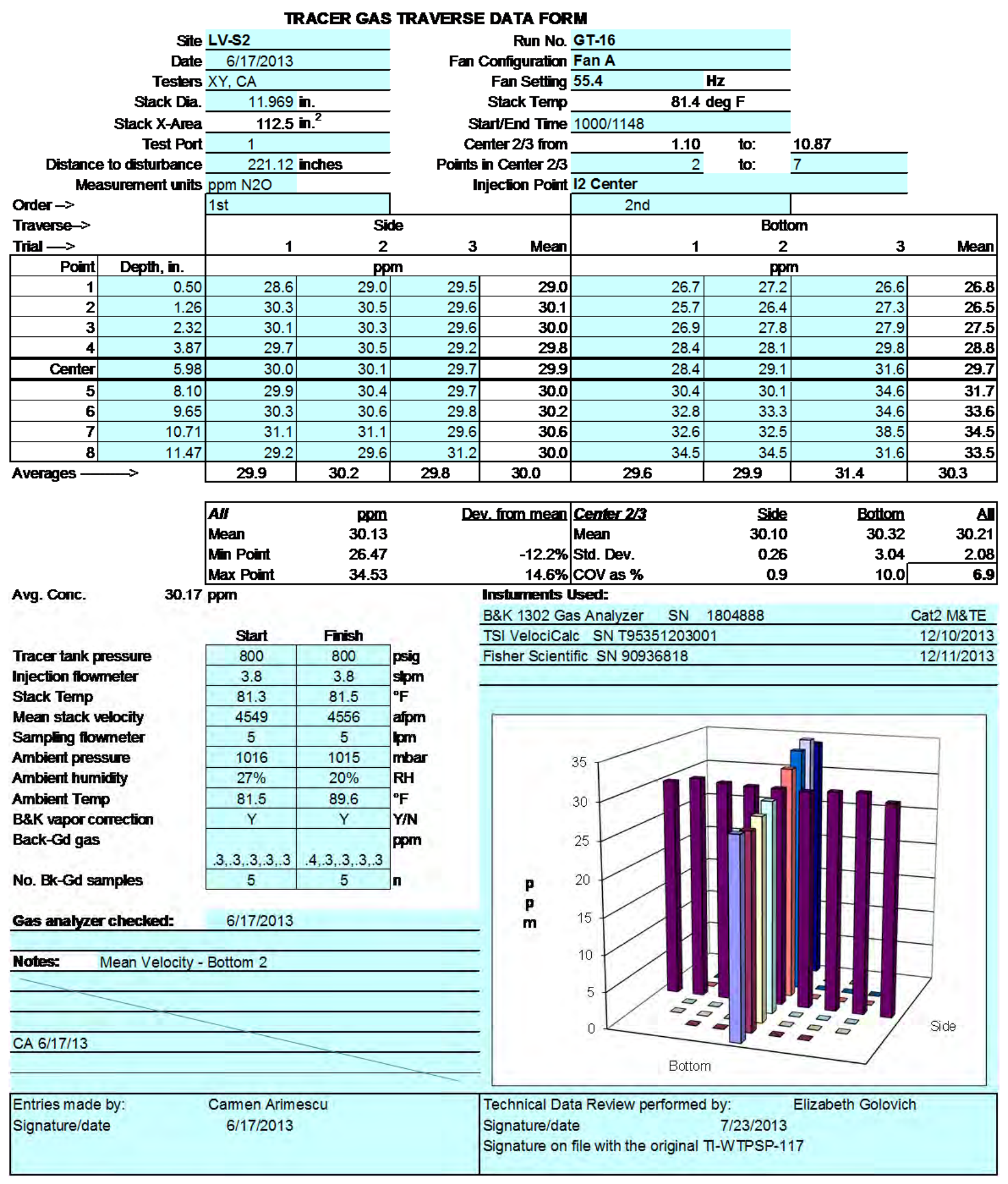




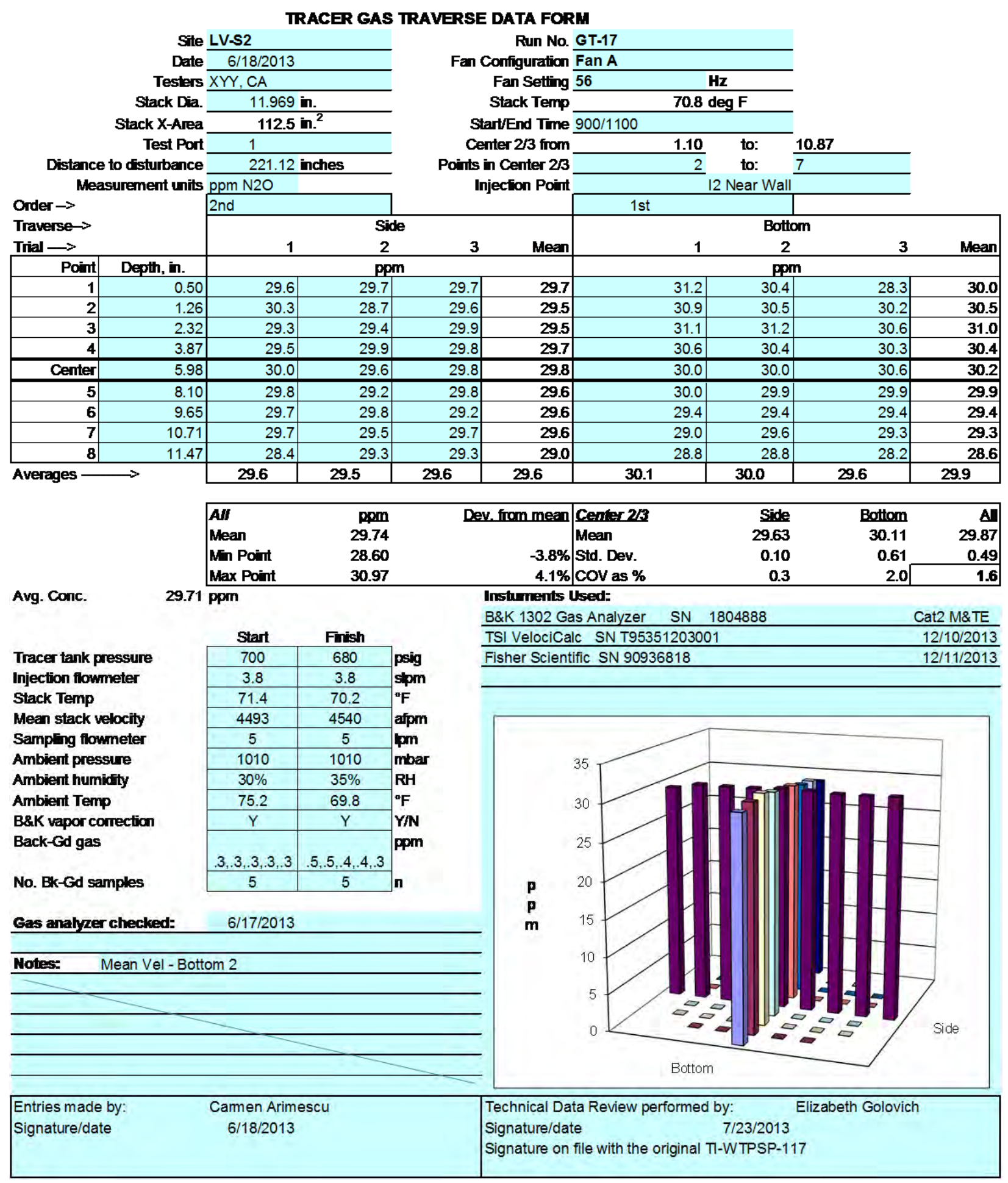




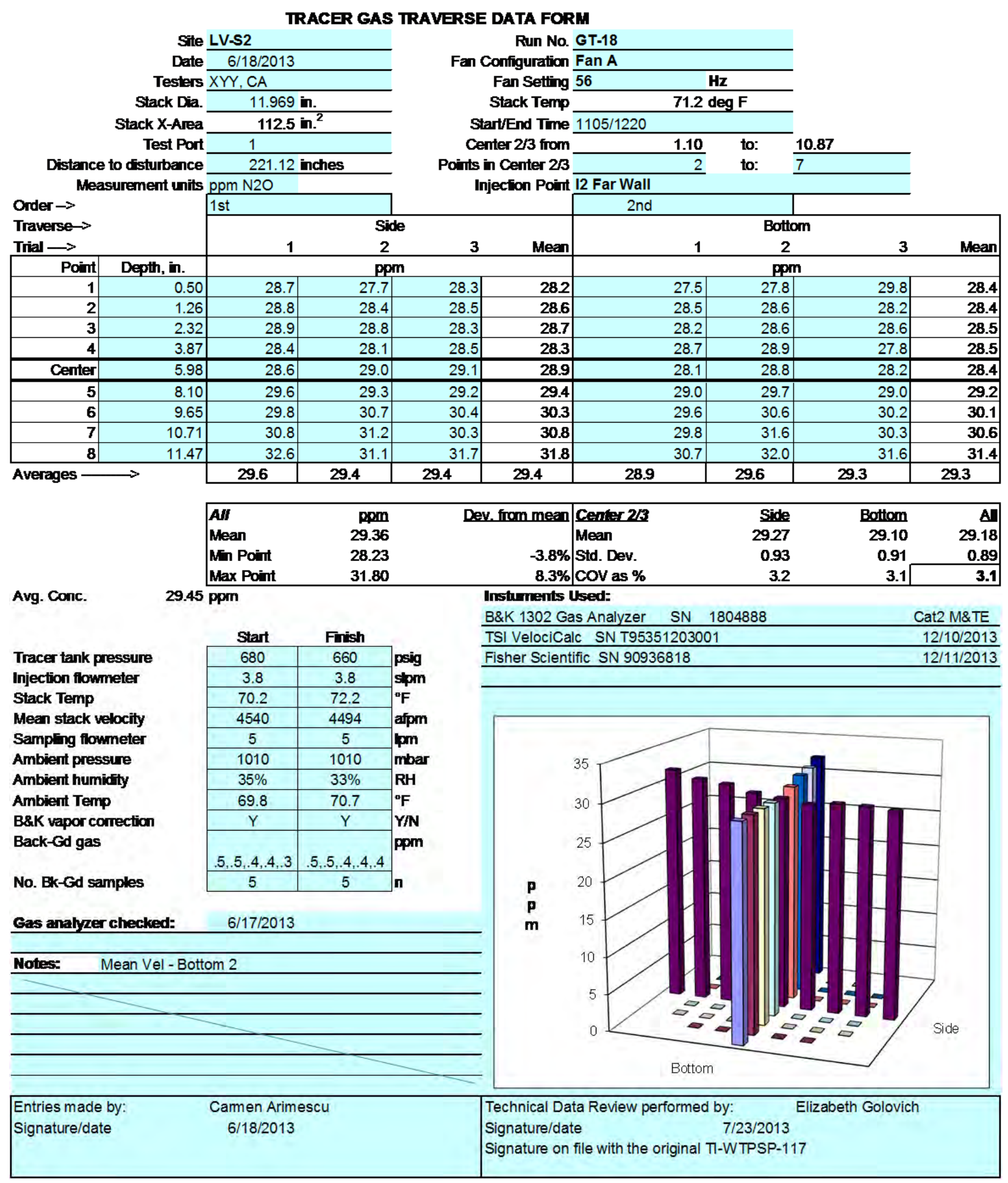




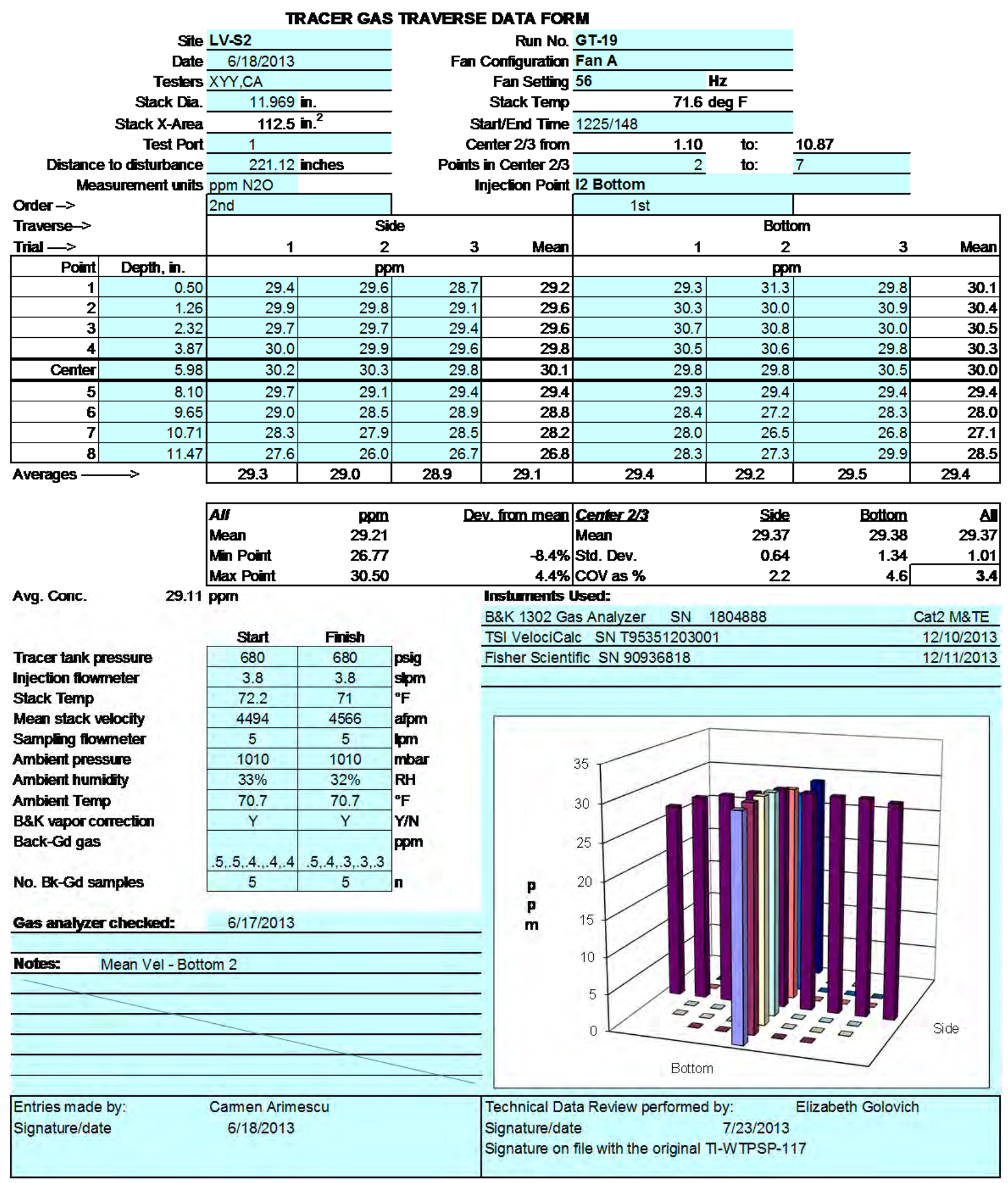




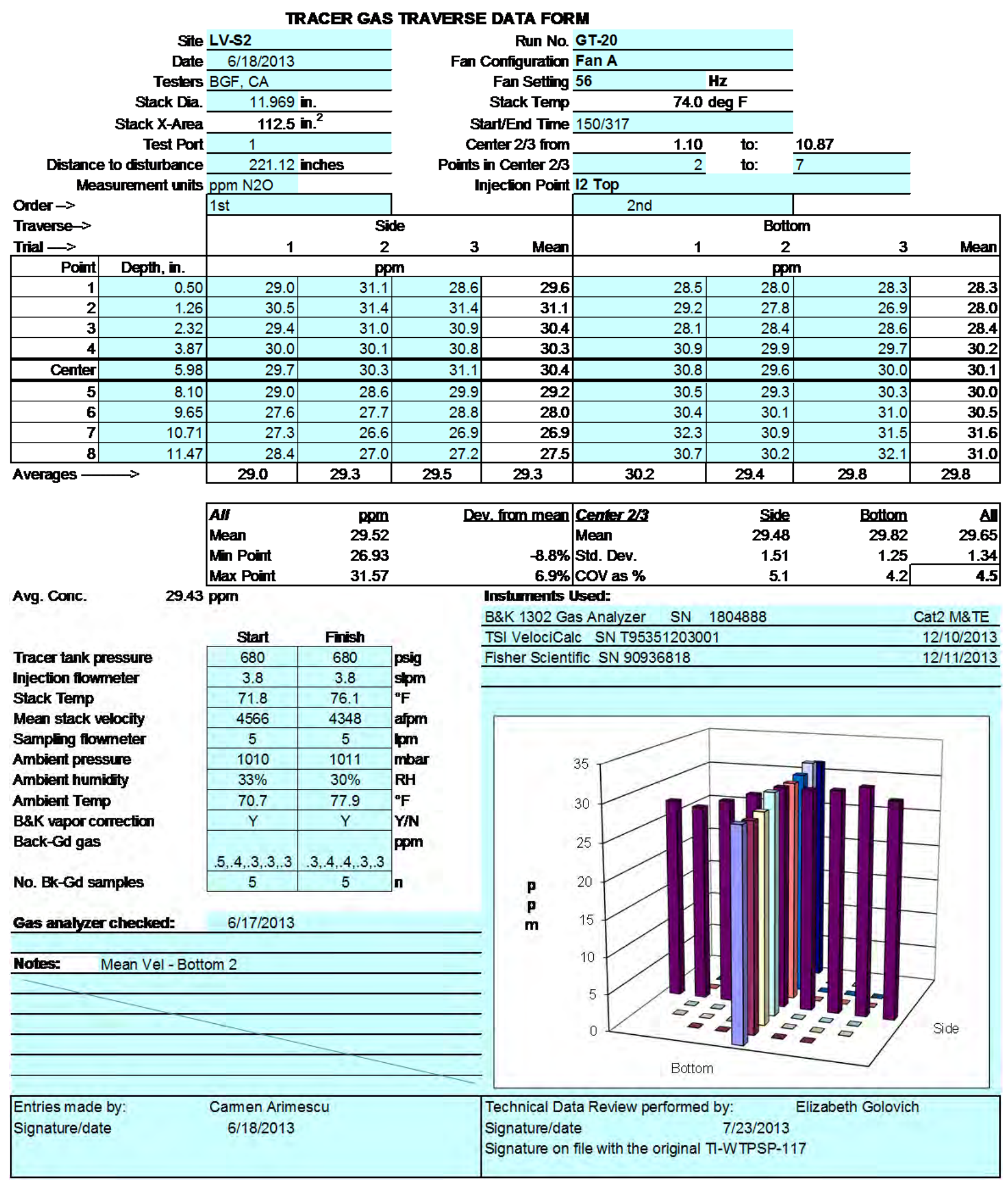




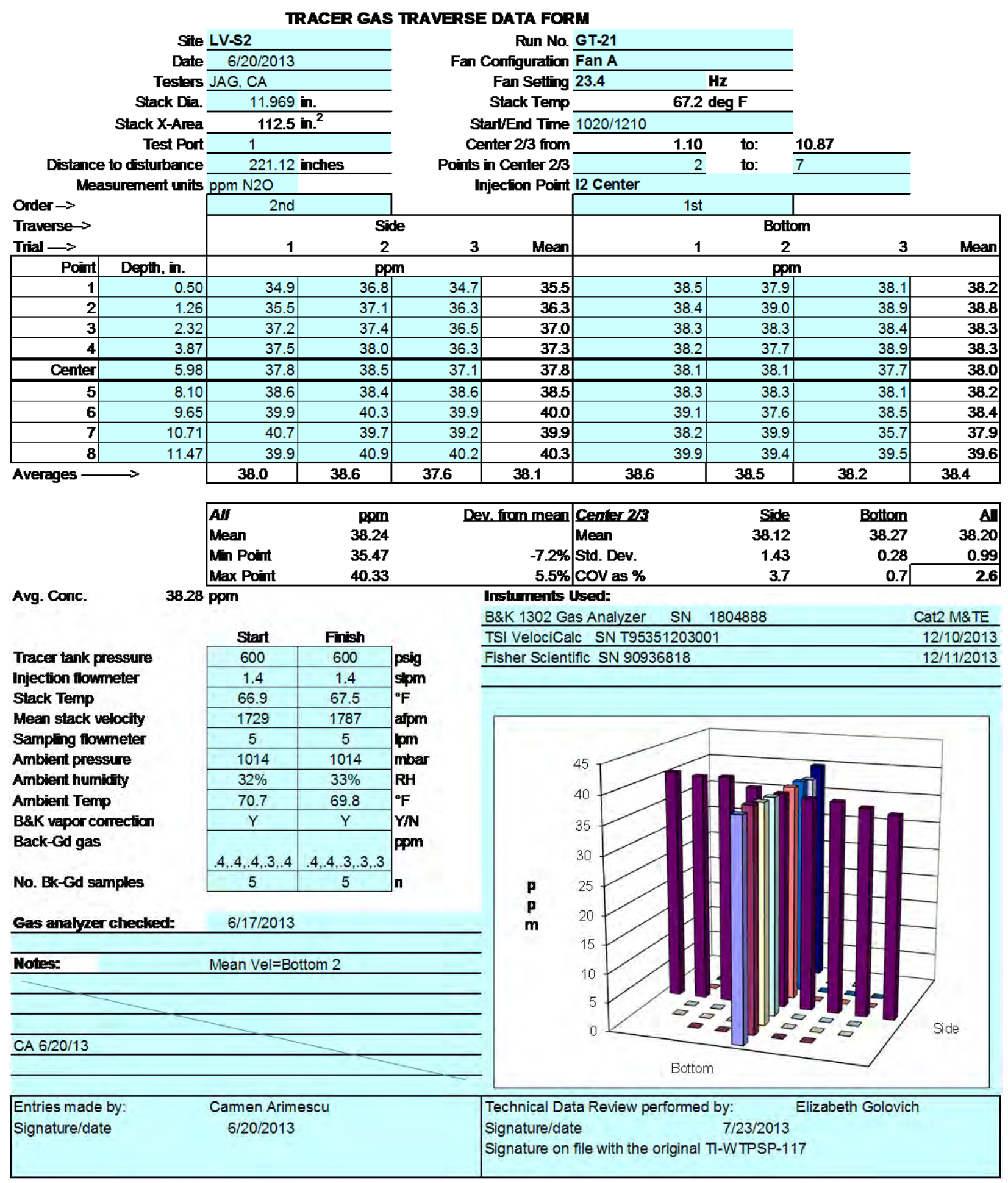




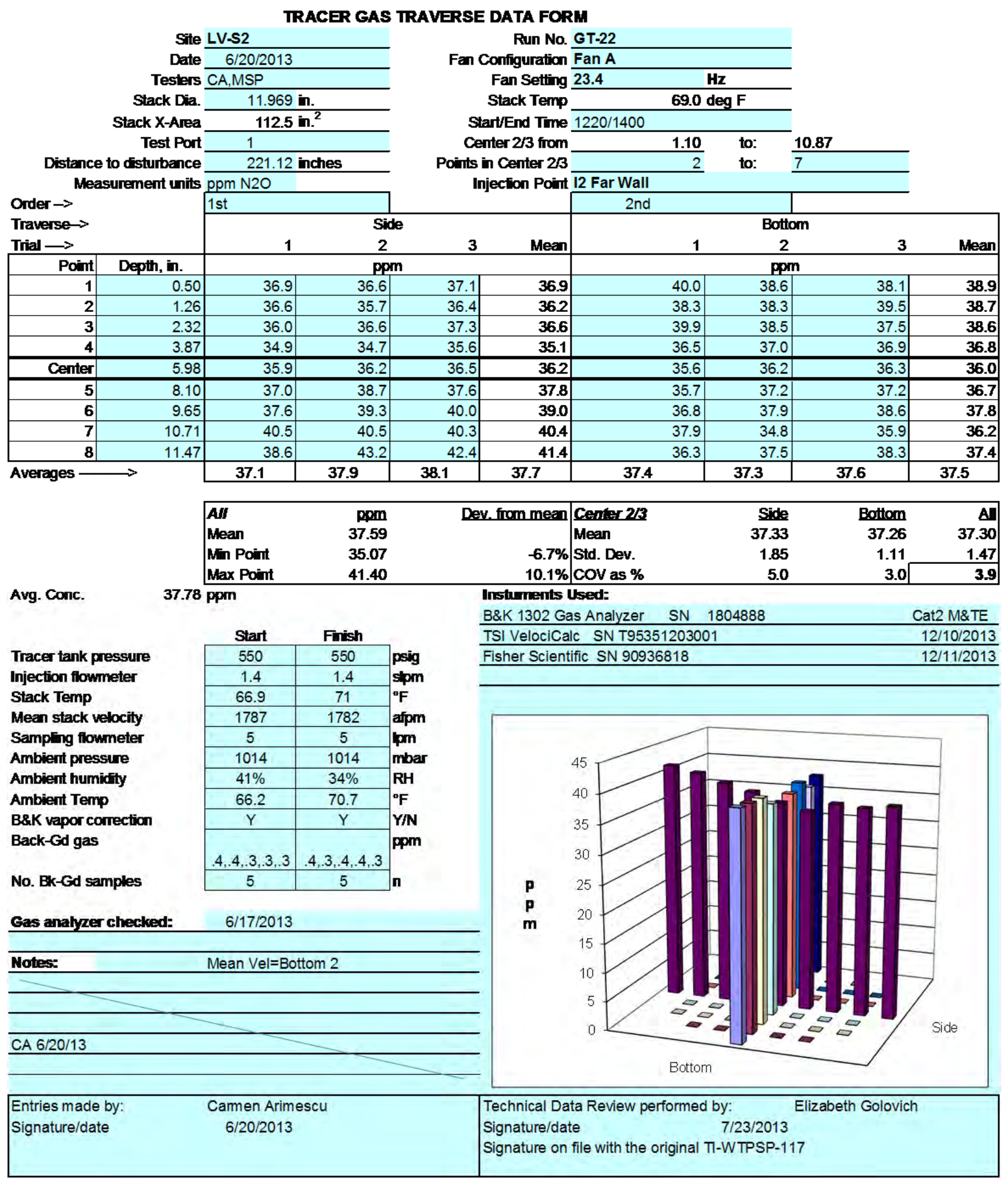




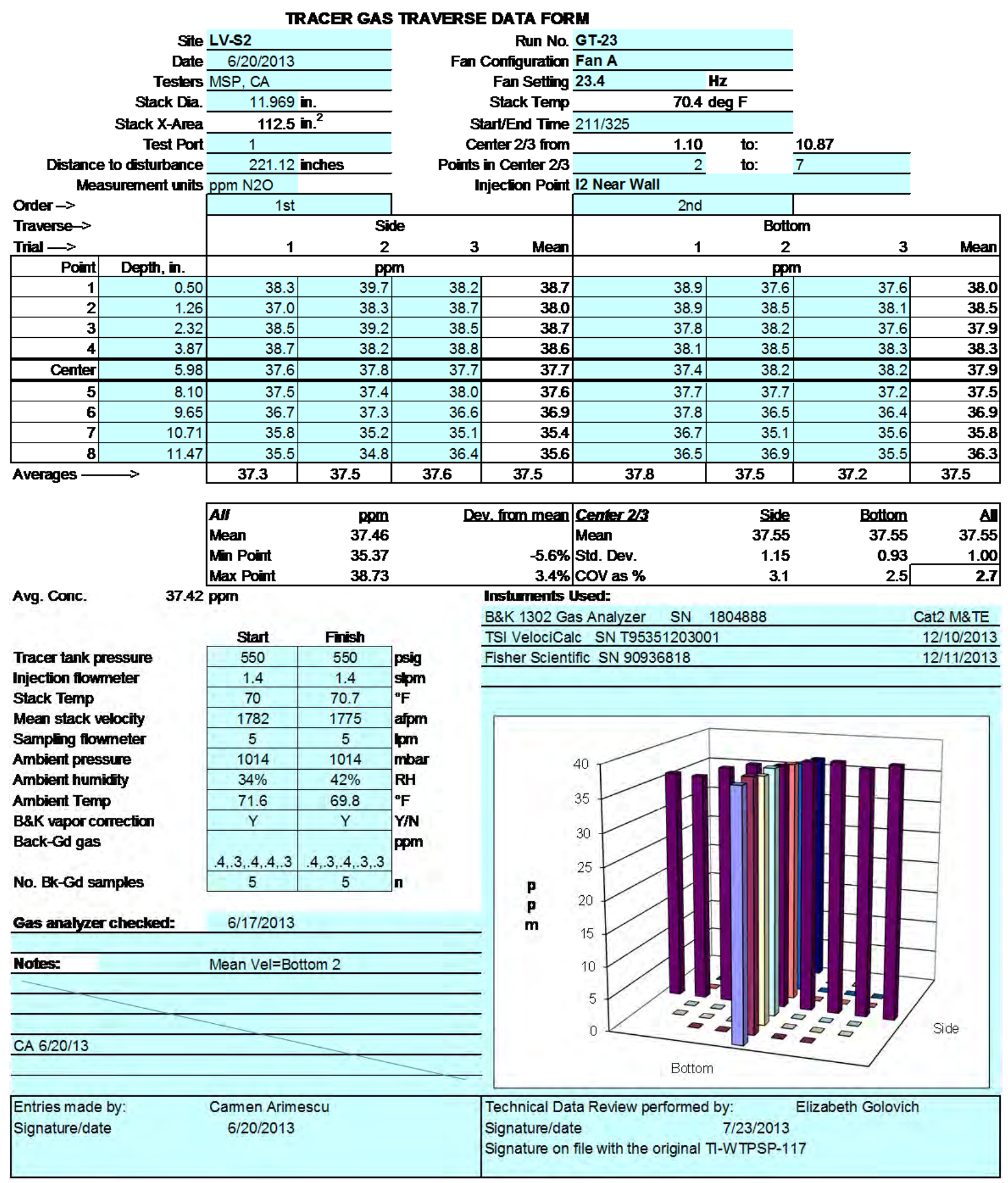




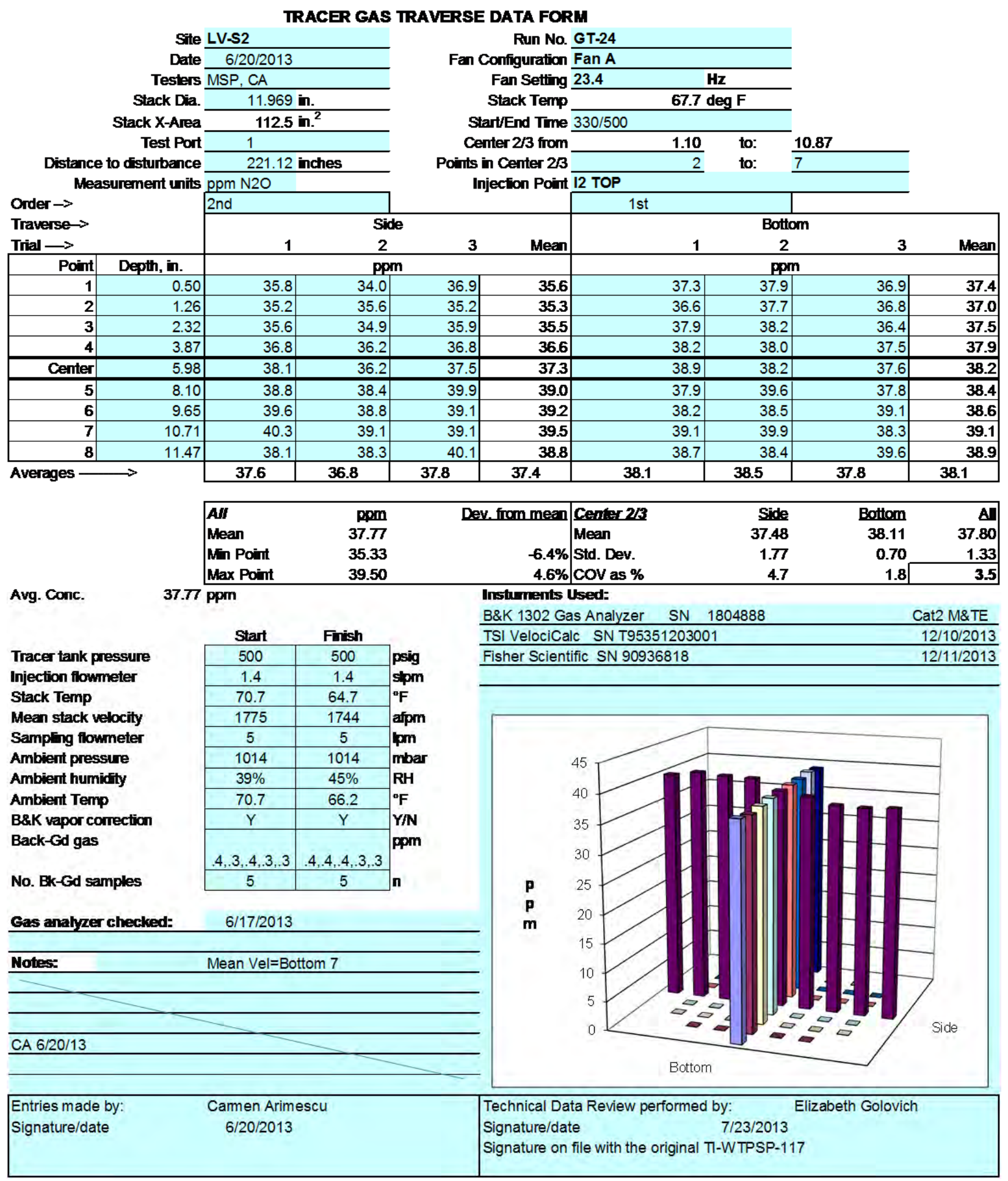




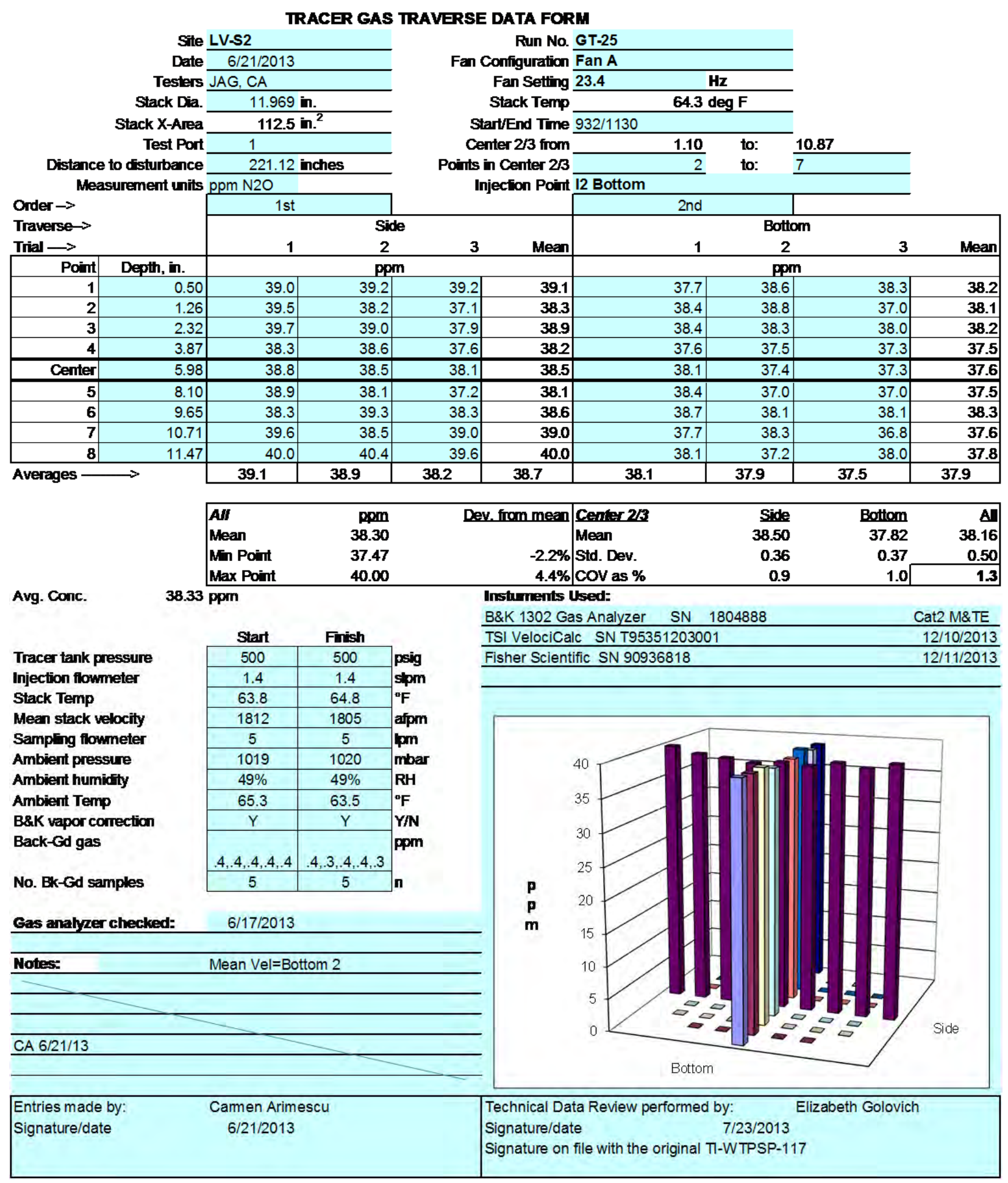




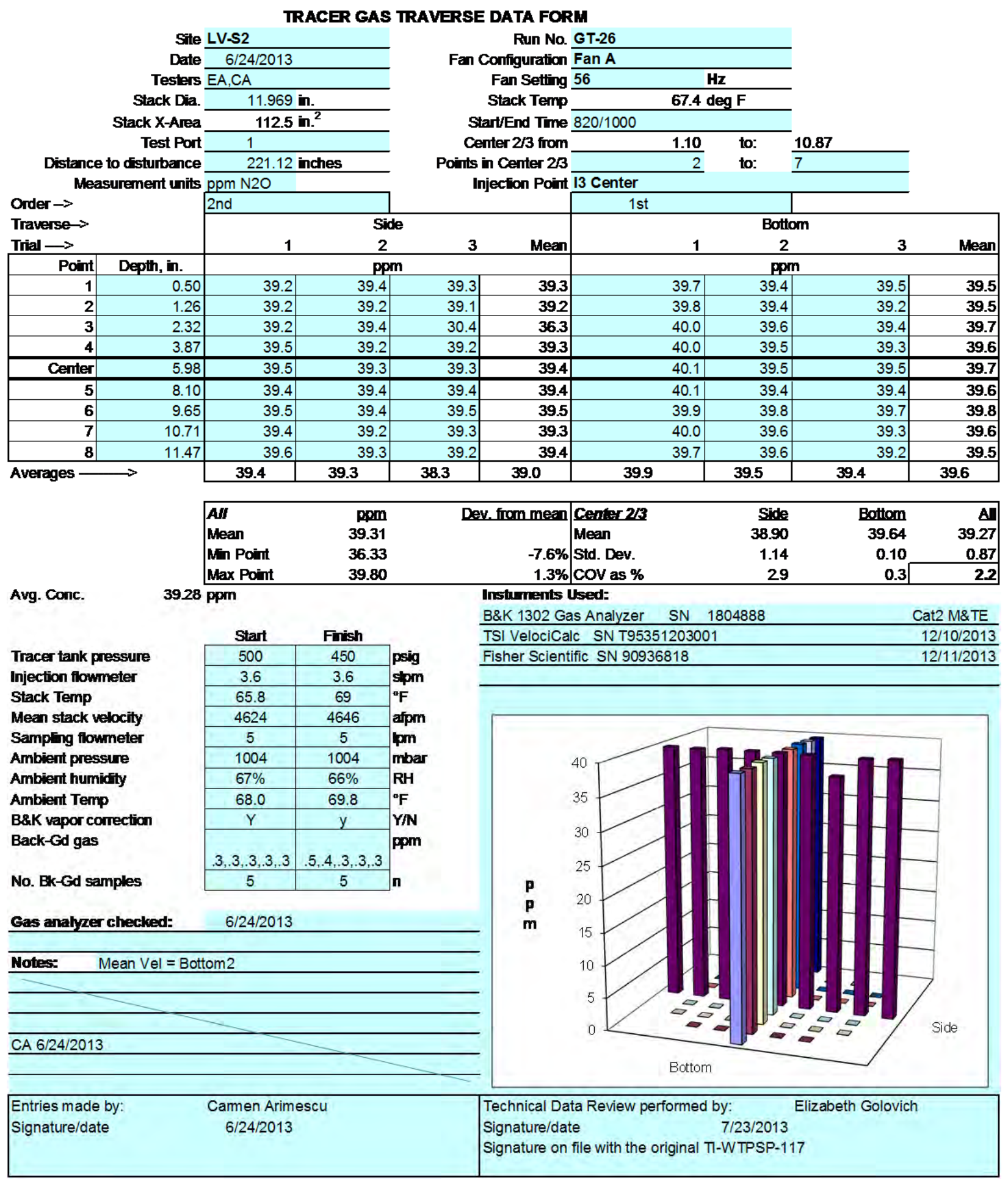




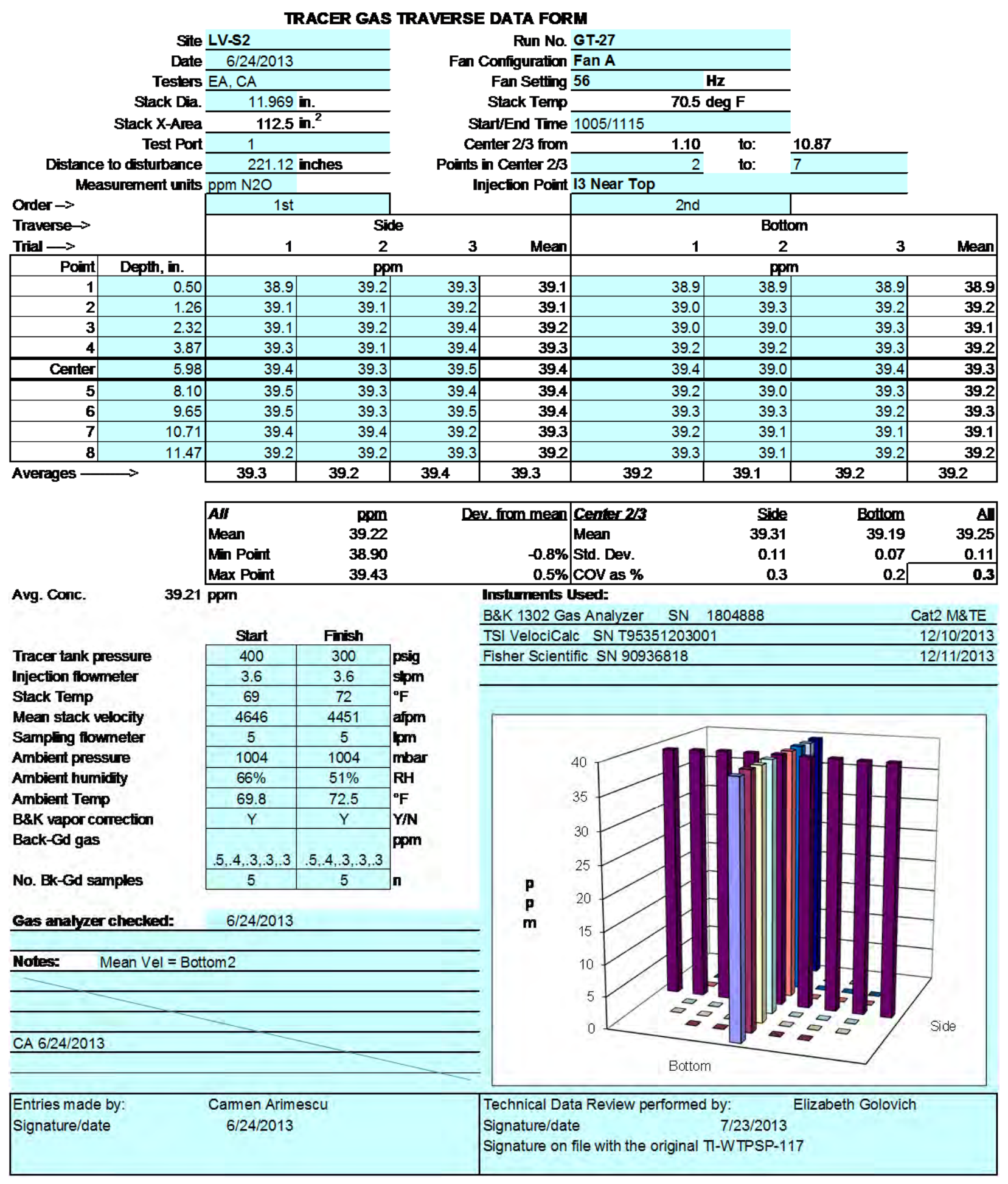




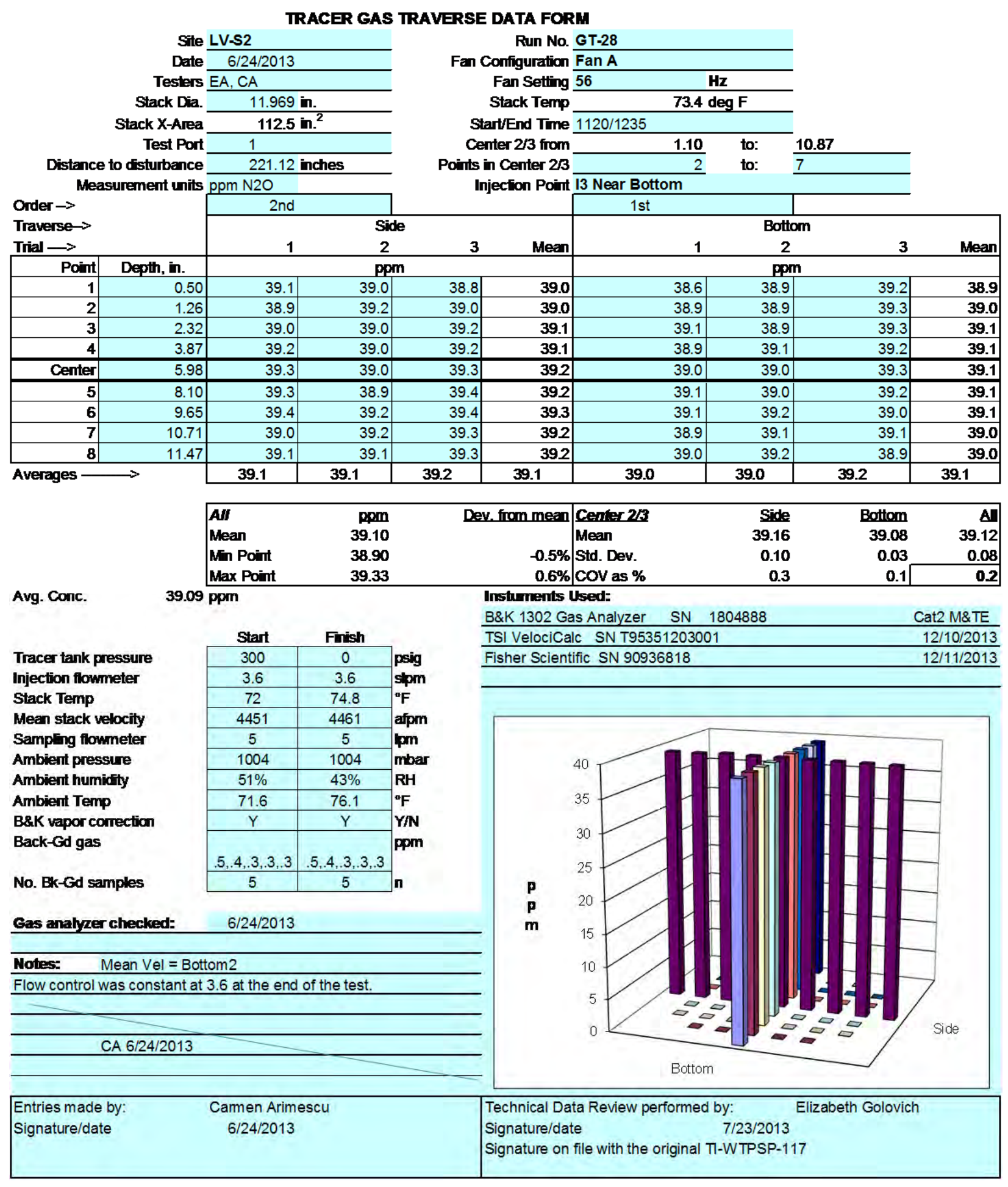




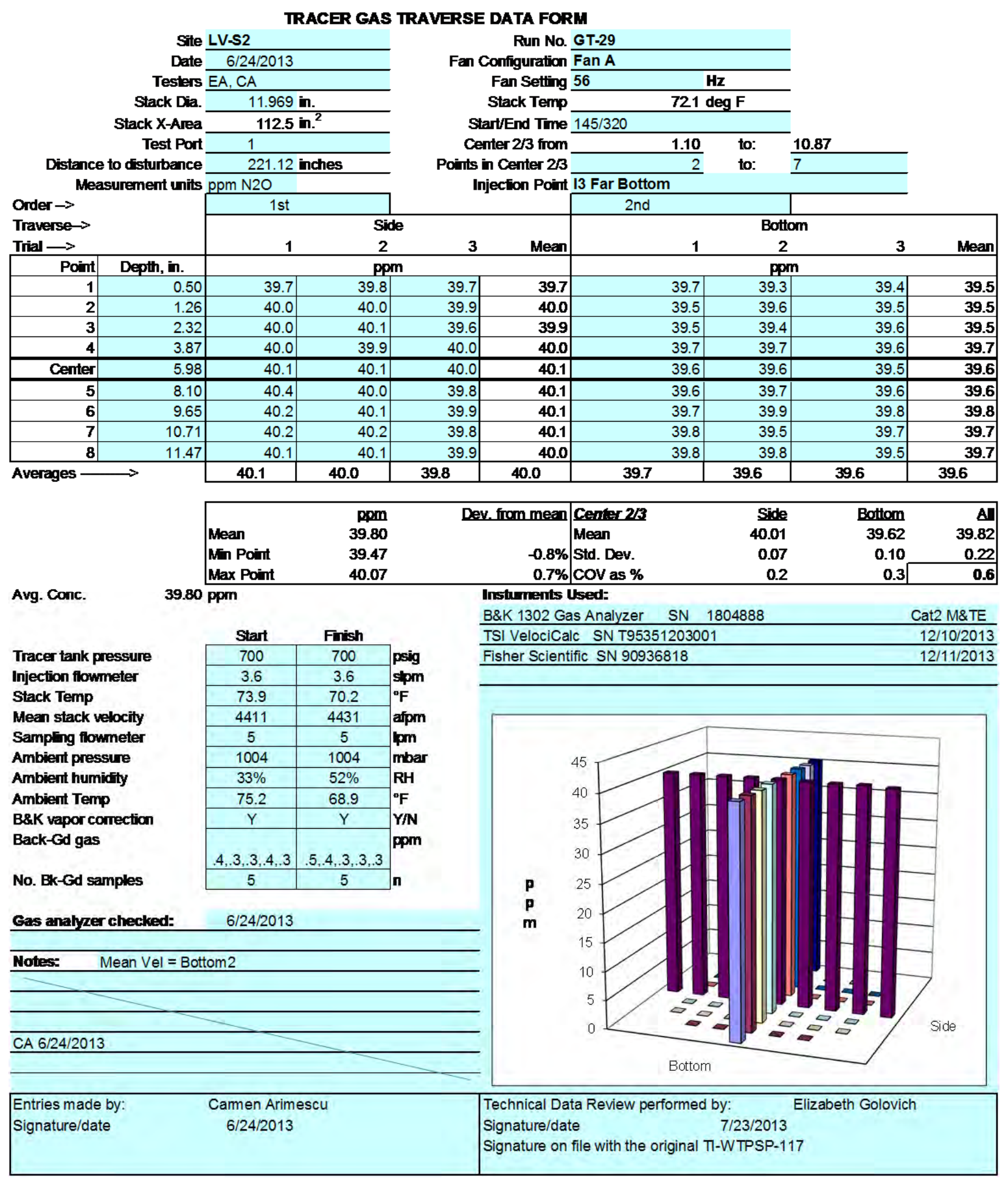




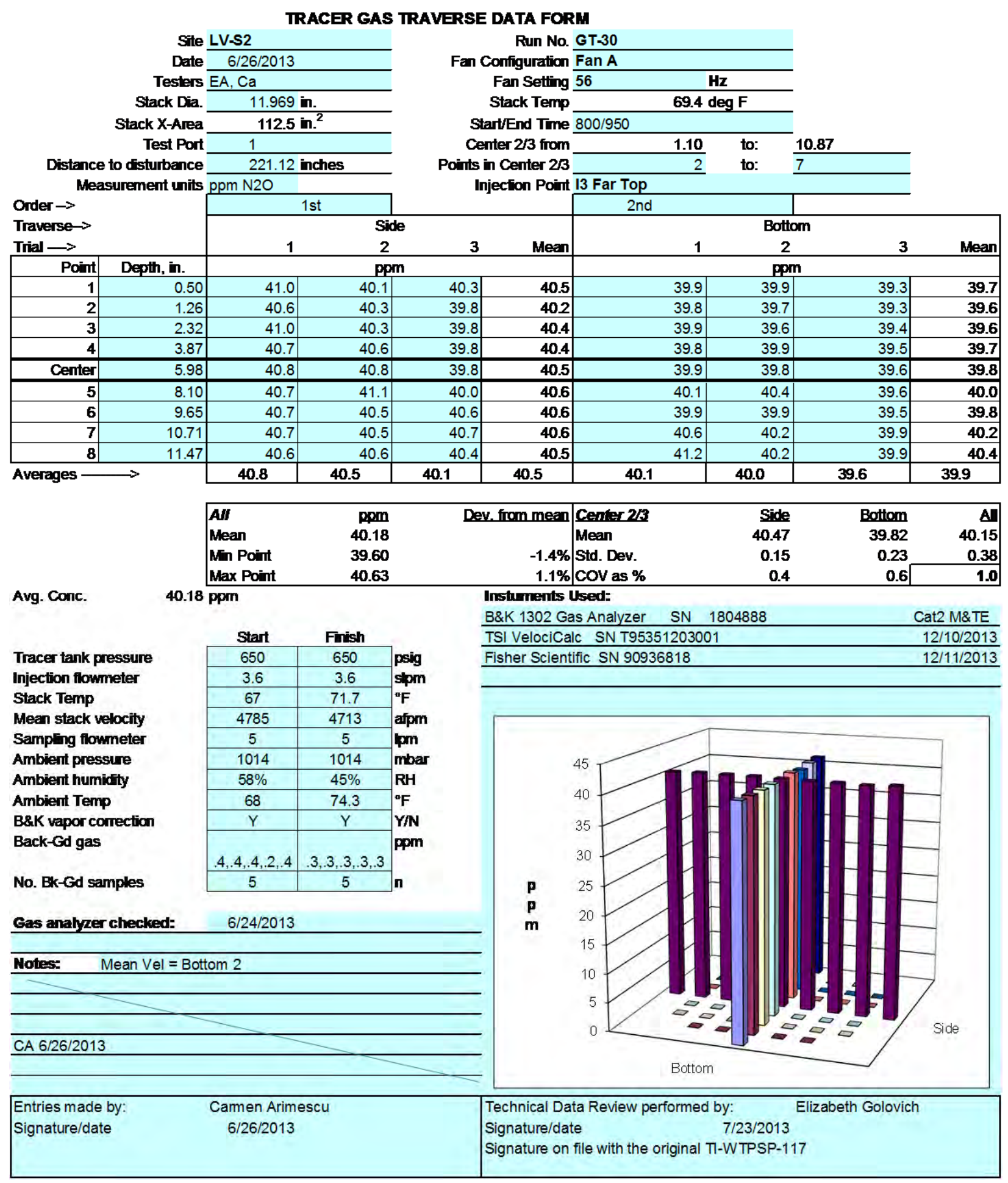




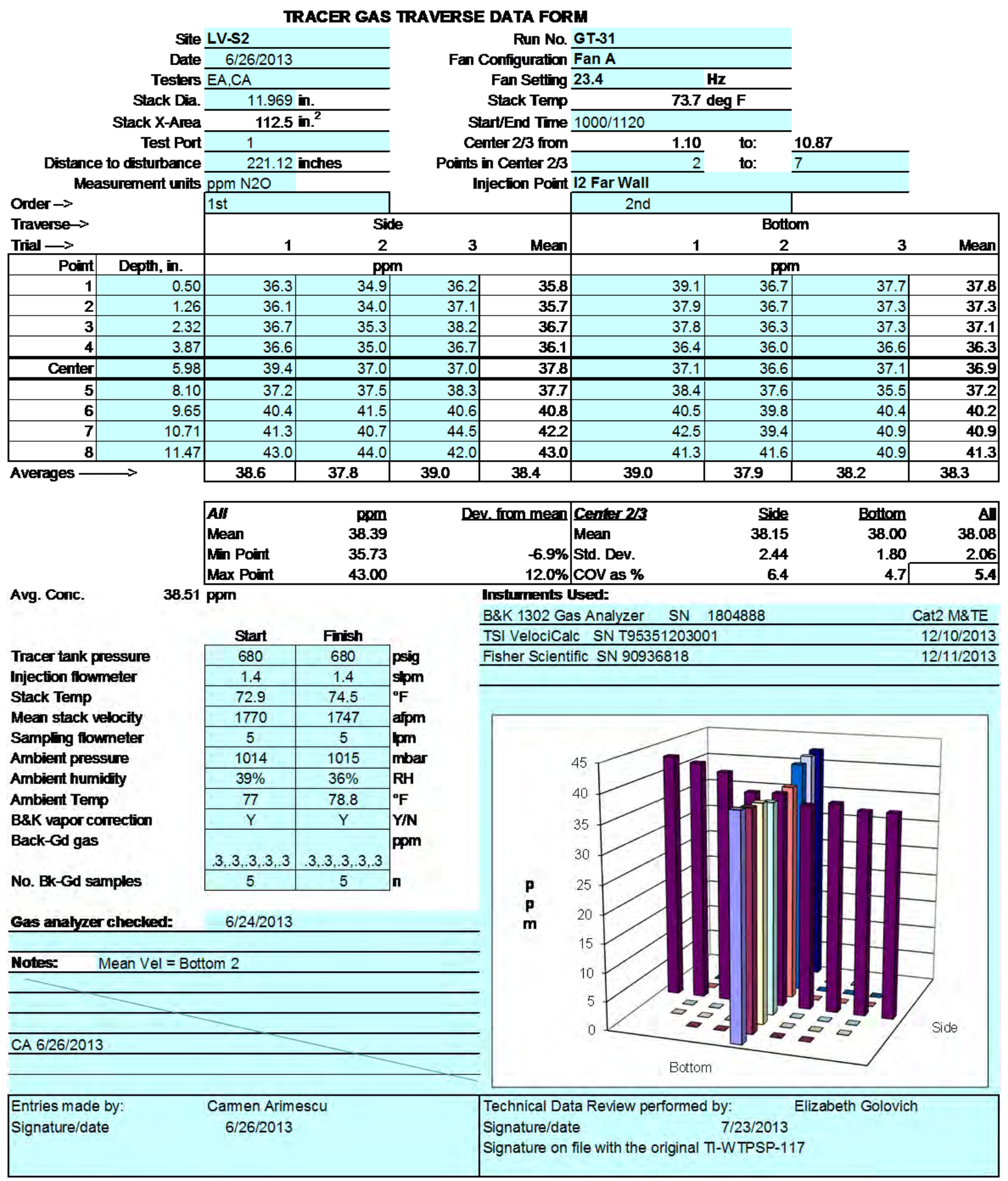




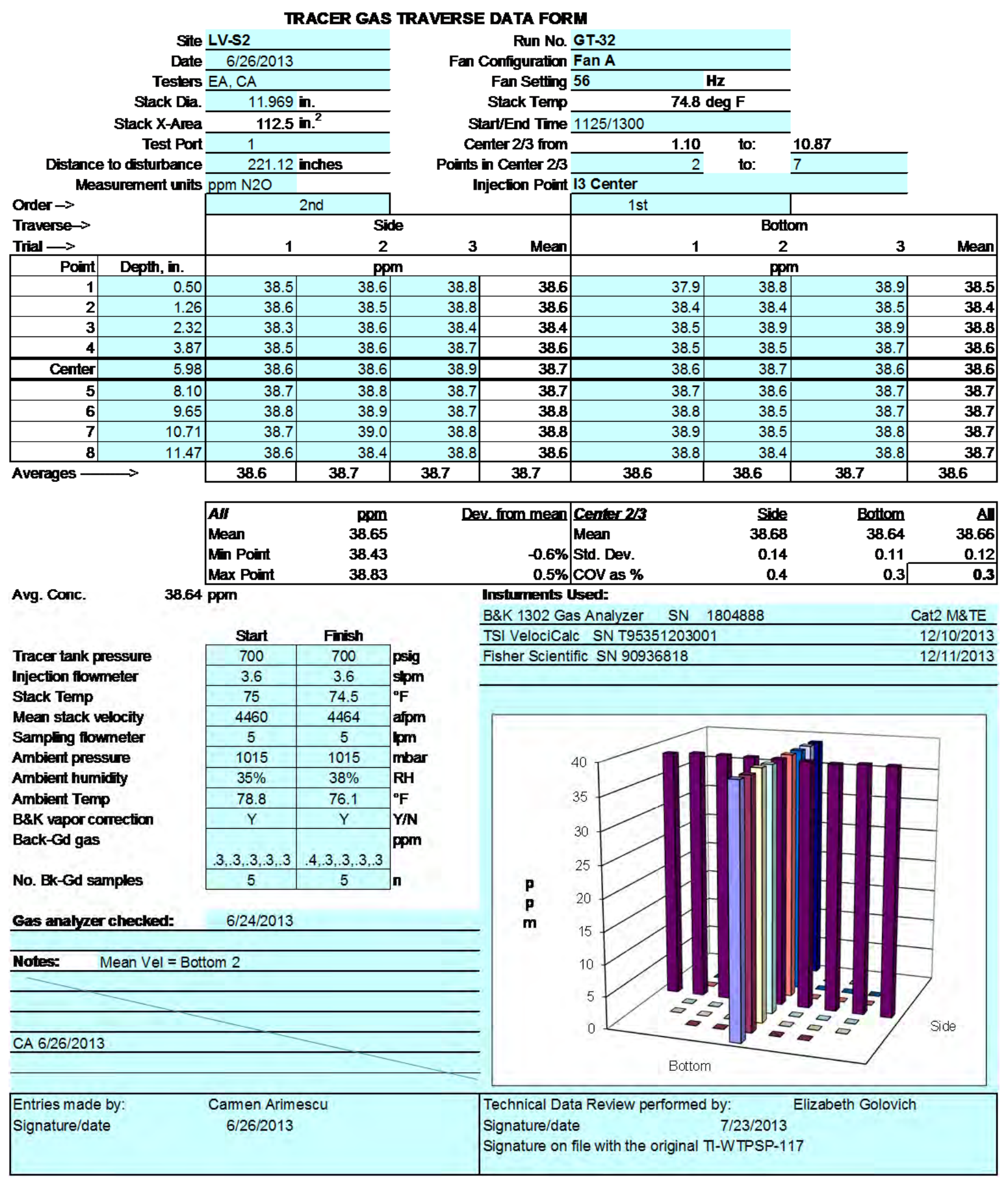




\section{C.5 LV-S2 Particle Tracer Uniformity Data Sheets}

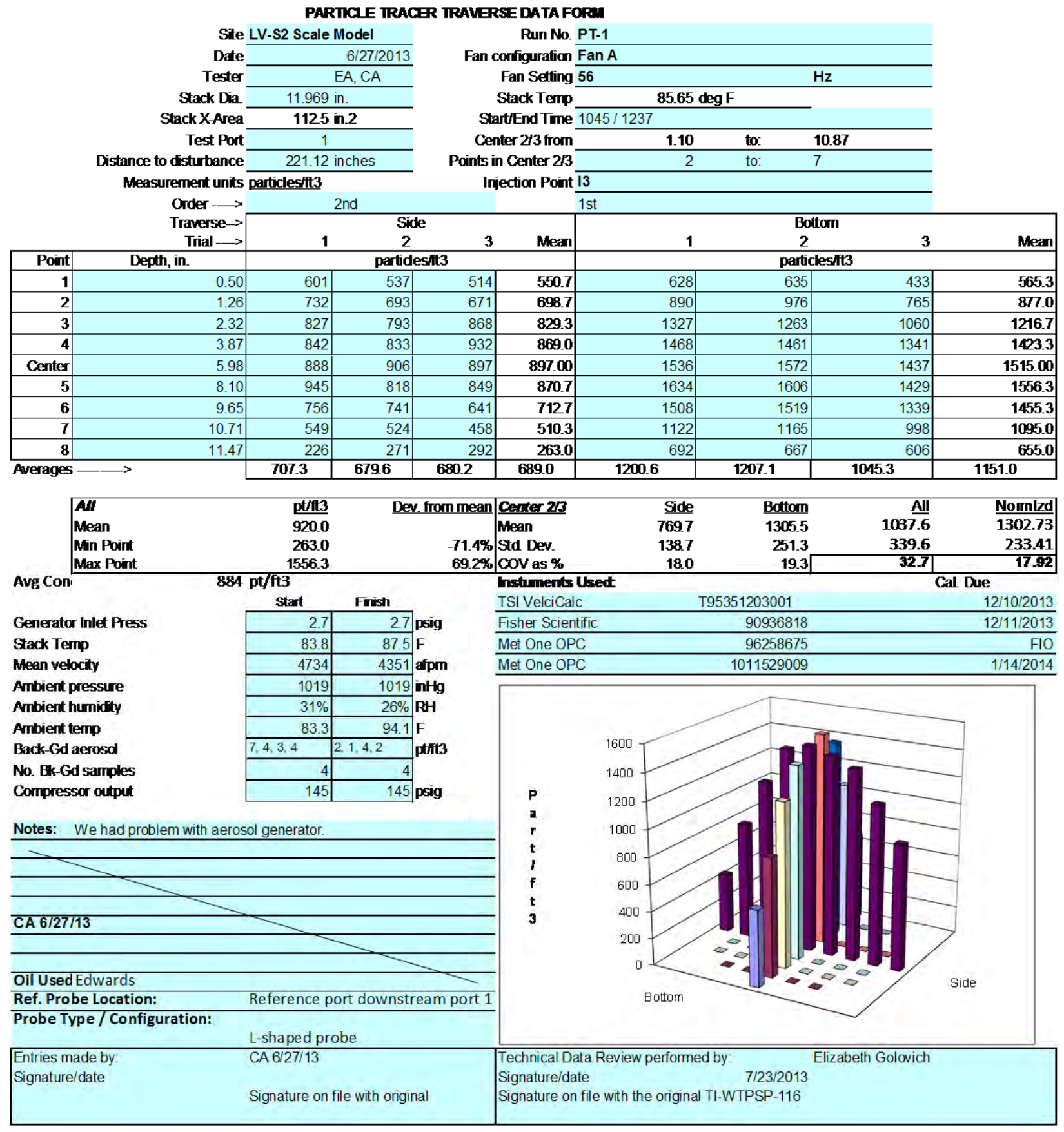


PARTICLE TRACER TRAVERSE DATA FORM

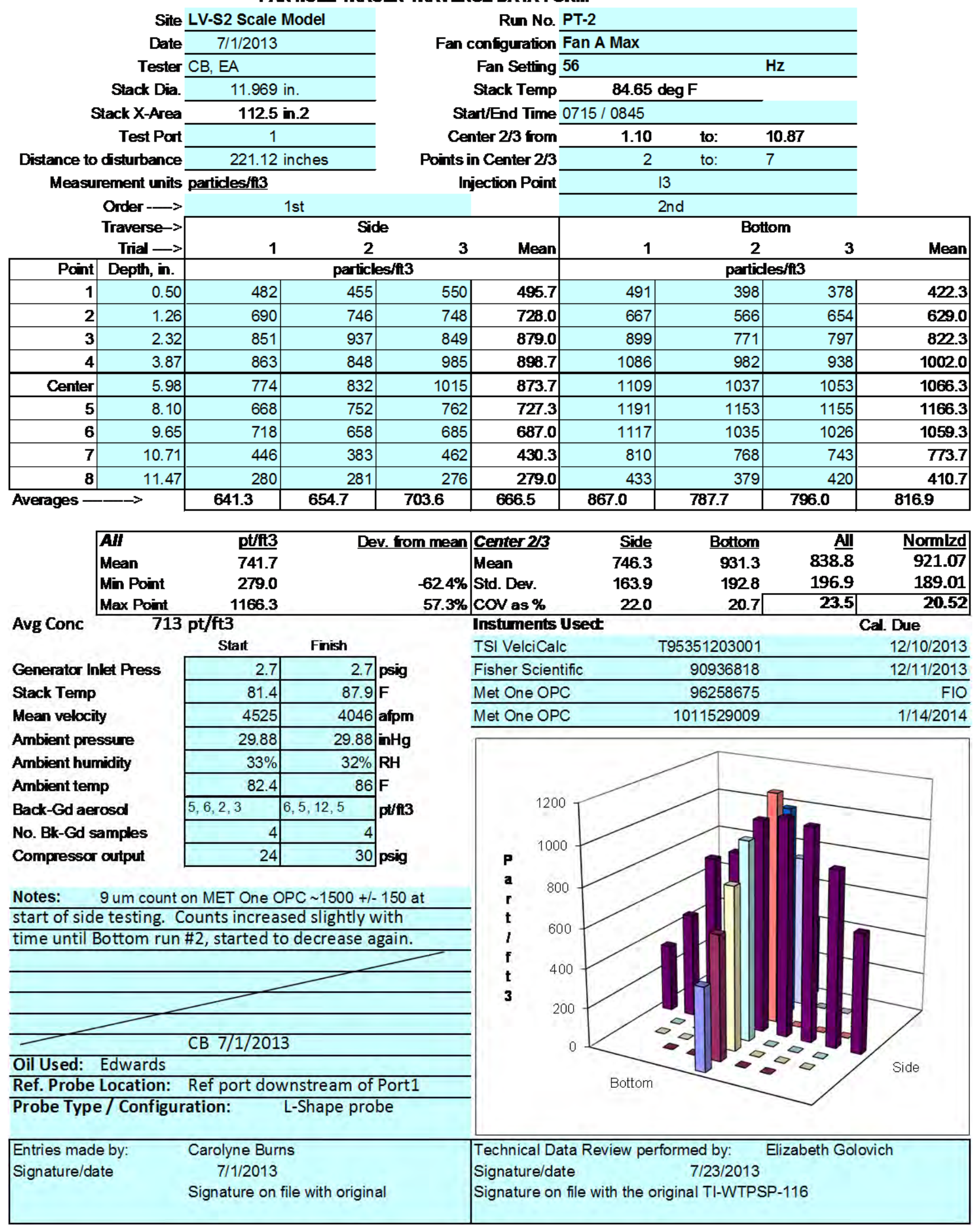


PARTICLE TRACER TRAVERSE DATA FORM

Site LV-S2 Scale Model

Date 7/1/2013

Tester CB, EA

Stack Dia.

Stack X-Area

Test Port

Distance to disturbance

Measurement units particles/fi3

Order $\longrightarrow$

Traverse->

Trial $\rightarrow$

Point Depth, in.

\begin{tabular}{|r|r|}
\hline Point & Depth, in. \\
\hline $\mathbf{1}$ & 0.5 \\
$\mathbf{2}$ & 1.2 \\
$\mathbf{3}$ & 2.3 \\
\hline $\mathbf{4}$ & 3.87 \\
\hline Center & 5.98 \\
\hline $\mathbf{5}$ & 8.10 \\
\hline $\mathbf{6}$ & 9.6 \\
\hline $\mathbf{7}$ & 10.7 \\
\hline $\mathbf{8}$ & 11.47 \\
\hline
\end{tabular}

\begin{tabular}{ll|l}
\hline Averages - $\longrightarrow$ & $\mathbf{7 6 1 . 3}$
\end{tabular}
Run No. PT-3

Fan confguration Fan A Max

Fan Setting 56

Stack Temp

Start/End Time $924 / 1115$

Center $2 / 3$ from

Points in Center 233

Injection Pcint

\begin{tabular}{rll}
\hline $\mathbf{1 . 1 0}$ & to: & $\mathbf{1 0 . 8 7}$ \\
\hline 2 & to: & 7 \\
\hline 13 & \\
\hline $2 n d$
\end{tabular}

\begin{tabular}{|c|c|c|c|c|c|c|c|}
\hline $\mathbf{A V}$ & pt/fi3 & Dey. from mean & Center $2 / 3$ & Side & Bettom & All & Nomlzd \\
\hline Mean & 8322 & & Mean & 855.7 & 1017.6 & 936.6 & 975.98 \\
\hline Min Point & 236.3 & $-71.6 \%$ & Std. Dev. & 199.6 & 174.6 & 198.8 & 194.58 \\
\hline Max Pcint & 1179.3 & $41.7 \%$ & COV as $\%$ & 23.3 & 17.2 & 21.2 & 19.94 \\
\hline
\end{tabular}

Avg Conc $796 \mathrm{pt} / \mathrm{ft3}$

Generator Inlet Press

Stack Temp

Mean velocity

Ambient pressure

Ambient humidity

Ambient temp

Back-Gd aerosol

No. Bk-Gd samples

Compressar output

\begin{tabular}{|c|c|c|}
\hline Stait & Finish & \\
\hline 2.6 & 2.7 & psig \\
\hline 91 & 99.7 & $F$ \\
\hline 4295 & 4212 & afpm \\
\hline 29.88 & 29.91 & $\mathrm{inHg}$ \\
\hline $33 \%$ & $27 \%$ & RH \\
\hline 85.1 & 94.1 & $F$ \\
\hline $6,22,8,8$ & $4,6,7,3$ & $p^{t / f t 3}$ \\
\hline 4 & 4 & \\
\hline 20 & 25 & psig \\
\hline
\end{tabular}

Notes: Ventillation duct disconnected at the wye at the beginning of the test. Retaped and started test $\sim 9: 50$.

Flow acceptable. Instuments Used

\begin{tabular}{lrr} 
TSI VelciCalc & T95351203001 & $12 / 10 / 2013$ \\
\hline Fisher Scientific & 90936818 & $12 / 11 / 2013$ \\
\hline Met One OPC & 96258675 & FIO \\
\hline Met One OPC & 1011529009 & $1 / 14 / 2014$ \\
\hline
\end{tabular}

\section{CB 7/1/2013}

Oil Used: Edwards

Ref. Probe Location: Ref port downstream of Port1

Probe Type/Configuration:

L-Shape probe

Entries made by

Signature/date

Carolyne Burns
$7 / 1 / 2013$
Signature on file with original

Signature on file with original

Technical Data Review performed by: Elizabeth Golovich

Signature/date

$7 / 23 / 2013$

Signature on file with the original TI-WTPSP-116 
PARTICLE TRACER TRAVERSE DATA FORM

Run No. PT-4

Fan configuration Fan A Min

Fan Setting 21

Stack Temp

Start/End Time $0616 / 0800$

Center $2 / 3$ from

Points in Center $2 / 3$

Injection Point

1st

Side

23

2nd

100

3 Mean 1

Site LV-S2 Scale Model

Date 7/2/2013

Tester SS, EA

Stack Dia. 11.969 in

Test Port

Distance to disturbance

Order $\longrightarrow$

Traverse--

Trial $\rightarrow$

Point Depth, in.

\begin{tabular}{|r|r|}
\hline Point & Depth, in \\
\hline $\mathbf{1}$ & 0.50 \\
\hline $\mathbf{2}$ & 1.2 \\
$\mathbf{3}$ & 2.3 \\
\hline $\mathbf{4}$ & 3.87 \\
\hline Center & 5.9 \\
\hline $\mathbf{5}$ & 8.10 \\
\hline $\mathbf{6}$ & 9.6 \\
\hline $\mathbf{7}$ & 10.71 \\
\hline $\mathbf{8}$ & 11.47 \\
\hline
\end{tabular}

\begin{tabular}{|l|r|}
\hline Averages $-\longrightarrow$ & 1326.1
\end{tabular}
Stack X-Area 1125 in.2

Measurement units particles/fi3

$83 \mathrm{deg} F$

\begin{tabular}{ccc}
1.10 & to: & 10.87 \\
\hline 2 & to: & 7 \\
\hline 13 & & \\
\hline
\end{tabular}

particles/ti3

\begin{tabular}{|c|c|}
\hline 1184 & 1276 \\
\hline 1263 & 1404 \\
\hline 1389 & 1398 \\
\hline 1419 & 1423 \\
\hline 1319 & 1522 \\
\hline 1443 & 1677 \\
\hline 1571 & 1579 \\
\hline 1344 & 1645 \\
\hline 1003 & 1316 \\
\hline 1326.1 & 1471.1 \\
\hline
\end{tabular}

\begin{tabular}{r|r}
1276 & \\
1404 & 13 \\
\hline & 1398 \\
\hline
\end{tabular}

319

\begin{tabular}{|l|}
1398 \\
\hline 423 \\
\hline 522
\end{tabular}

\begin{tabular}{l|l}
1522 & \\
\hline 677 &
\end{tabular}

\begin{tabular}{|l|l|}
\hline 79 & 166 \\
\hline 1645 & 1753 \\
\hline 16 & 1503 \\
\hline
\end{tabular}

\begin{tabular}{|lr}
\hline All & pt/fi3 \\
Mean & 1783.6 \\
Min Point & 1240.0 \\
Max Point & 2320.3 \\
\hline
\end{tabular}

Avg Conc $1779 \mathrm{pt} / \mathrm{ft} 3$

Generator Inlet Press

Stack Temp

Mean velocity

Ambient pressure

Ambient humidity

Ambient temp

Back-Gd aerosol

No. Bk-Gd samples

Compressar output

\begin{tabular}{|c|c|c|}
\hline Stat & Finish & \\
\hline 1.4 & 1.4 & psig \\
\hline 75.8 & 90.2 & $F$ \\
\hline 1581 & 1513 & afpm \\
\hline 29.85 & 29.88 & $\mathrm{inHg}$ \\
\hline $63 \%$ & $37 \%$ & RH \\
\hline 74.3 & 89.6 & $F$ \\
\hline $8,10,12,4$ & $5,12,6,3$ & $p_{p} / \mathrm{ft}^{3}$ \\
\hline 4 & 4 & \\
\hline 28 & 28 & psig \\
\hline
\end{tabular}

Notes: Ref data started at 1705 and ended at 2225 (end of runs.)

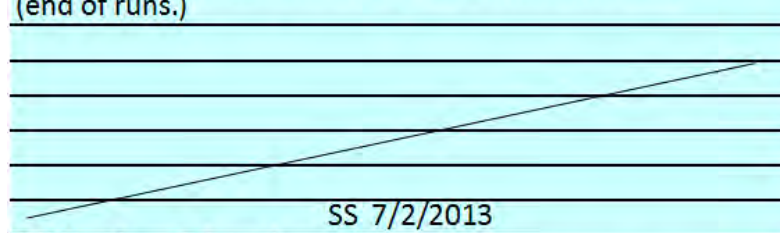

Oil Used: Edwards

Ref. Probe Location: Ref port downstream of Port1

Probe Type/Configuration:

L-Shape probe

Entries made by

Signature/date

Suan Sande
$\quad 7 / 2 / 2013$
Signature on file with original

Suan Sande

ignature on file with original

1503

\begin{tabular}{r|lrrrr|} 
& & & & \\
$-30.5 \%$ & Side & Bottom & All & Normlzd \\
$\mathbf{3 0 . 1 \%}$ & Std. Dev. & 1468.7 & 2221.1 & 1844.9 & 2249.55 \\
COV as \% & 105.3 & 91.8 & 401.8 & 130.69 \\
\hline
\end{tabular}

\begin{tabular}{|r|r|r|r|r|r|}
\hline 260 & $\mathbf{1 2 4 0 . 0}$ & 1517 & 1509 & 1970 & $\mathbf{1 6 6 5 . 3}$ \\
\cline { 2 - 6 } & $\mathbf{1 3 2 8 . 7}$ & 1941 & 1933 & 2241 & $\mathbf{2 0 3 8 . 3}$ \\
\hline 319 & $\mathbf{1 3 8 7 . 7}$ & 1975 & 2166 & 2422 & $\mathbf{2 1 8 7 . 7}$ \\
\hline 1464 & $\mathbf{1 4 1 5 . 3}$ & 2071 & 2304 & 2343 & $\mathbf{2 2 3 9 . 3}$ \\
\hline 438 & $\mathbf{1 4 2 6 . 3}$ & 2164 & 2177 & 2296 & $\mathbf{2 2 1 2 . 3}$ \\
\hline 492 & $\mathbf{1 5 3 7 . 3}$ & 2193 & 2258 & 2361 & $\mathbf{2 2 7 0 . 7}$ \\
\hline 664 & $\mathbf{1 6 0 4 . 7}$ & 2237 & 2336 & 2388 & $\mathbf{2 3 2 0 . 3}$ \\
\hline 753 & $\mathbf{1 5 8 0 . 7}$ & 2086 & 2416 & 2335 & $\mathbf{2 2 7 9 . 0}$ \\
\hline 1503 & $\mathbf{1 2 7 4 . 0}$ & 2005 & 2205 & 2080 & $\mathbf{2 0 9 6 . 7}$ \\
\hline 7 & $\mathbf{1 4 2 1 . 6}$ & $\mathbf{2 0 2 1 . 0}$ & $\mathbf{2 1 4 4 . 9}$ & $\mathbf{2 2 7 0 . 7}$ & $\mathbf{2 1 4 5 . 5}$ \\
\hline
\end{tabular}

\begin{tabular}{|c|c|c|c|c|}
\hline \multirow[b]{2}{*}{ Mean } & \multicolumn{4}{|c|}{ Bottom } \\
\hline & 1 & 2 & 3 & Mean \\
\hline & \multicolumn{4}{|c|}{ particles/fi3 } \\
\hline 1240.0 & 1517 & 1509 & 1970 & 1665.3 \\
\hline 1328.7 & 1941 & 1933 & 2241 & 2038.3 \\
\hline 1387.7 & 1975 & 2166 & 2422 & 2187.7 \\
\hline 1415.3 & 2071 & 2304 & 2343 & 2239.3 \\
\hline 1426.3 & 2164 & 2177 & 2296 & 2212.3 \\
\hline 1537.3 & 2193 & 2258 & 2361 & 2270.7 \\
\hline 1604.7 & 2237 & 2336 & 2388 & 2320.3 \\
\hline 1580.7 & 2086 & 2416 & 2335 & 2279.0 \\
\hline 1274.0 & 2005 & 2205 & 2080 & 2096.7 \\
\hline 1421.6 & 2021.0 & 2144.9 & 2270.7 & 2145.5 \\
\hline
\end{tabular}

\begin{tabular}{lrr}
\hline Instuments Usect & T95351203001 & \multicolumn{1}{c}{ Cal. Due } \\
TSI VelciCalc & 90936818 & $12 / 10 / 2013$ \\
\hline Fisher Scientific & 96258675 & $12 / 11 / 2013$ \\
\hline Met One OPC & 1011529009 & FIO \\
\hline Met One OPC & $1 / 14 / 2014$ \\
\hline
\end{tabular}

Signature on file with the original TI-WTPSP-116


PARTICLE TRACER TRAVERSE DATA FORM

Site LV-S2 Scale Model

Date $7 / 2 / 2013$

Tester SS, EA

Stack Dia. 11.969 in

Stack X-Area 1125 in.2

Test Port

Distance to disturbance

Measurement units particles/fi3

Order $\longrightarrow$

Traverse--

Trial $\rightarrow$

Point Depth, in.

\begin{tabular}{|r|r|}
\hline Point & Depth, in \\
\hline $\mathbf{1}$ & 0.50 \\
\hline $\mathbf{2}$ & 1.26 \\
\hline $\mathbf{3}$ & 2.32 \\
\hline $\mathbf{4}$ & 3.87 \\
\hline Center & 5.98 \\
\hline $\mathbf{5}$ & 8.10 \\
\hline $\mathbf{6}$ & 9.65 \\
\hline $\mathbf{7}$ & 10.71 \\
\hline $\mathbf{8}$ & 11.47 \\
\hline
\end{tabular}

\begin{tabular}{ll|l}
\hline Averages $-\longrightarrow$ & 1099.2
\end{tabular}

Run No. PT-5

Fan confguration Fan B Min

Fan Setting 22

Stack Temp

Start/End Time $0850 / 1040$

Center $2 / 3$ from

Points in Center 233

Injection Pcint

\begin{tabular}{ccc}
1.10 & to: & $\mathbf{1 0 . 8 7}$ \\
\hline 2 & to: & 7 \\
\hline 13 & & \\
\hline 1 st &
\end{tabular}

Bottom

2

23

3 Mean 1

particles/fi3

\begin{tabular}{|r|r|r|r|}
\hline 50 & 994 & 893 & 938 \\
\hline .26 & 1088 & 938 & 9 \\
\hline 32 & 1037 & 966 & 1012 \\
\hline 87 & 1144 & 1016 & 907 \\
\hline .98 & 1141 & 1123 & 1001 \\
\hline 10 & 1264 & 1092 & 11 \\
\hline 65 & 1361 & 1227 & 11 \\
\hline .71 & 1109 & 1014 & 11 \\
\hline 47 & 755 & 828 & 731 \\
\hline & $\mathbf{1 0 9 . 2}$ & $\mathbf{1 0 1 0 . 8}$ & $\mathbf{9 9 4 . 4}$ \\
\hline
\end{tabular}

\begin{tabular}{l|l|r|r}
\hline 938 & 941.7 & 1302
\end{tabular}

particles/fit

905

907

977

1005.0

1088.3

\begin{tabular}{l|l}
1162 & 1172 \\
1176 & 125 \\
\hline
\end{tabular}

1118 1080

\begin{tabular}{l|lr}
\hline All & pt/ft3 \\
Mean & 1231.3 \\
Min Point & 771.3 \\
Max Pcint & 1549.7 \\
\cline { 2 - 3 } & \multicolumn{2}{|c|}{$1220 \mathrm{pt} / \mathrm{ft3}$}
\end{tabular}

\section{Generator Inlet Press \\ Stack Temp \\ Mean velocity \\ Ambient pressure \\ Ambient humicity \\ Ambient temp \\ Back-Gd aerosol \\ No. Bik-Gd samples \\ Compressar output}

\begin{tabular}{|c|c|c|}
\hline Stait & Finish & \\
\hline 1.1 & 1.1 & 1) psig \\
\hline 99.6 & 104.1 & 1] $F$ \\
\hline 1575 & 1610 & afpm \\
\hline 29.91 & 29.91 & in \\
\hline $33 \%$ & $24 \%$ & $\mathrm{RH}$ \\
\hline 92.3 & 99.5 & $F$ \\
\hline $10,17,20,10$ & $3,4,6,8$ & $p t / f 3$ \\
\hline 4 & 4 & \\
\hline 22 & 20 & psig \\
\hline
\end{tabular}

Notes:

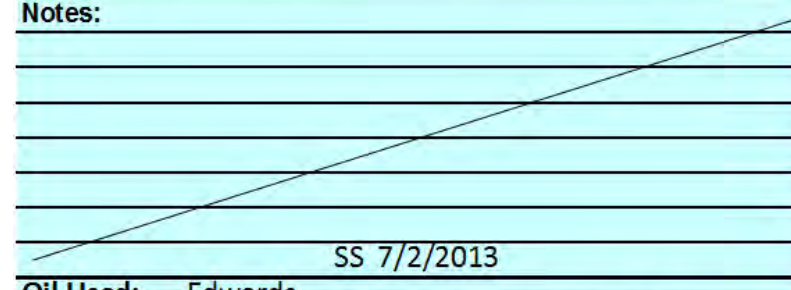

Oil Used: Edwards

Ref. Probe Location: Ref port downstream of Port1

Probe Type/Configuration:

L-Shape probe

Entries made by:

Signature/date

Suan Sande
$\quad 7 / 2 / 2013$
Signature on file with original

73

\begin{tabular}{r|r}
\cline { 2 - 2 } 17 & \\
\hline
\end{tabular}

Dev. from

\begin{tabular}{r|lrrrr|}
\hline$m$ mean & Center 2/3 & Side & Bottom & $\underline{\text { All }}$ & Nomlzd \\
\cline { 2 - 6 }$-37.4 \%$ & Mean & 1085.8 & 1476.3 & 1281.0 & 1511.15 \\
$25.9 \%$ & Std. Dev. & 98.7 & 41.8 & 215.3 & 106.01 \\
\hline COV as \% & 9.1 & 28 & 16.8 & 7.02 \\
\hline
\end{tabular}

\begin{tabular}{|c|c|c|}
\hline COV as \% & 28 & 16.8 \\
\hline Instuments Used & & Cal. Due \\
\hline TSI VelciCalc & T95351203001 & $12 / 10 / 2013$ \\
\hline Fisher Scientific & 90936818 & $12 / 11 / 2013$ \\
\hline Met One OPC & 96258675 & $\mathrm{FIO}$ \\
\hline Met One OPC & 1011529009 & $1 / 14 / 2014$ \\
\hline
\end{tabular}

- Met One OPC


PARTICLE TRACER TRAVERSE DATA FORM

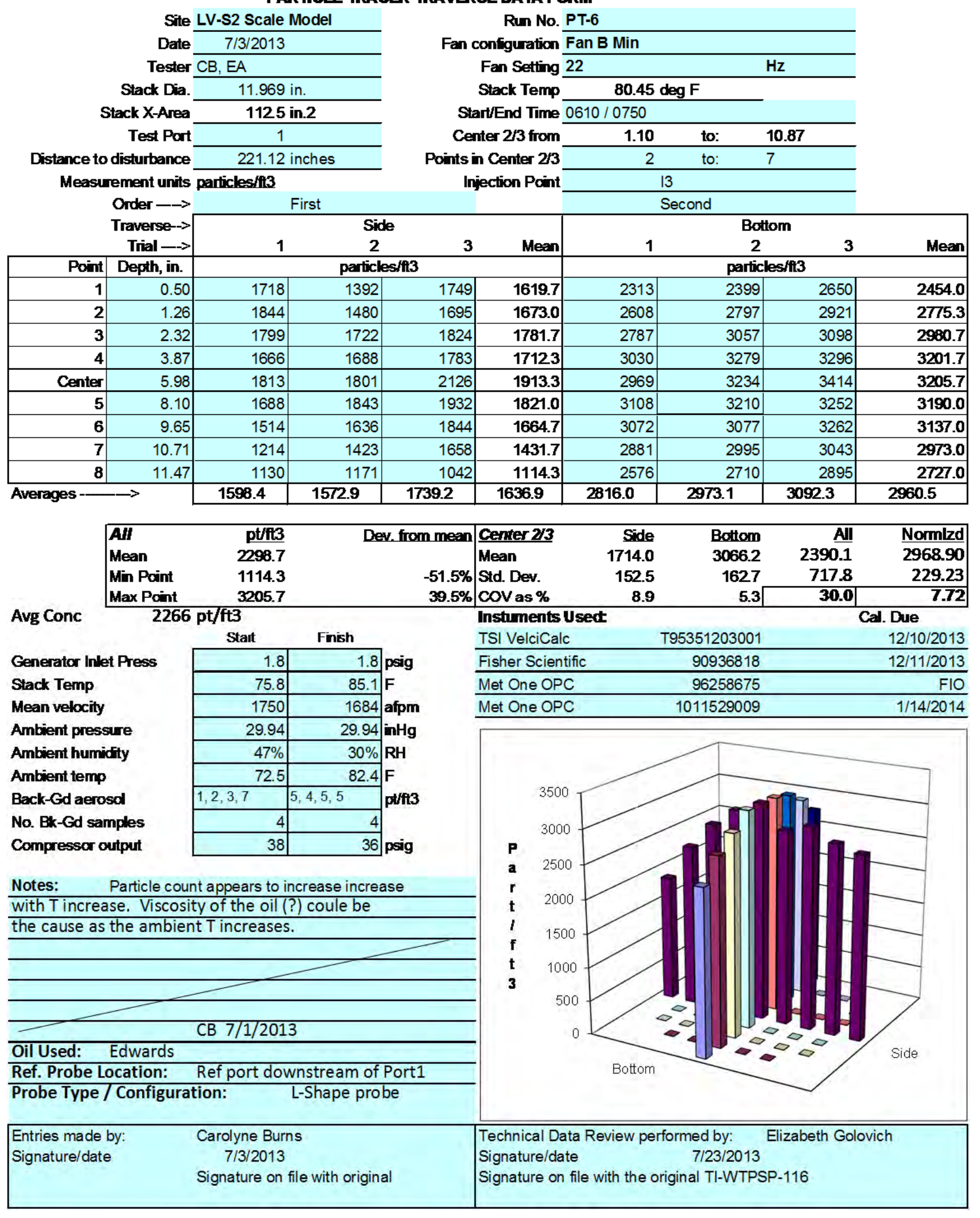


PARTICLE TRACER TRAVERSE DATA FORM

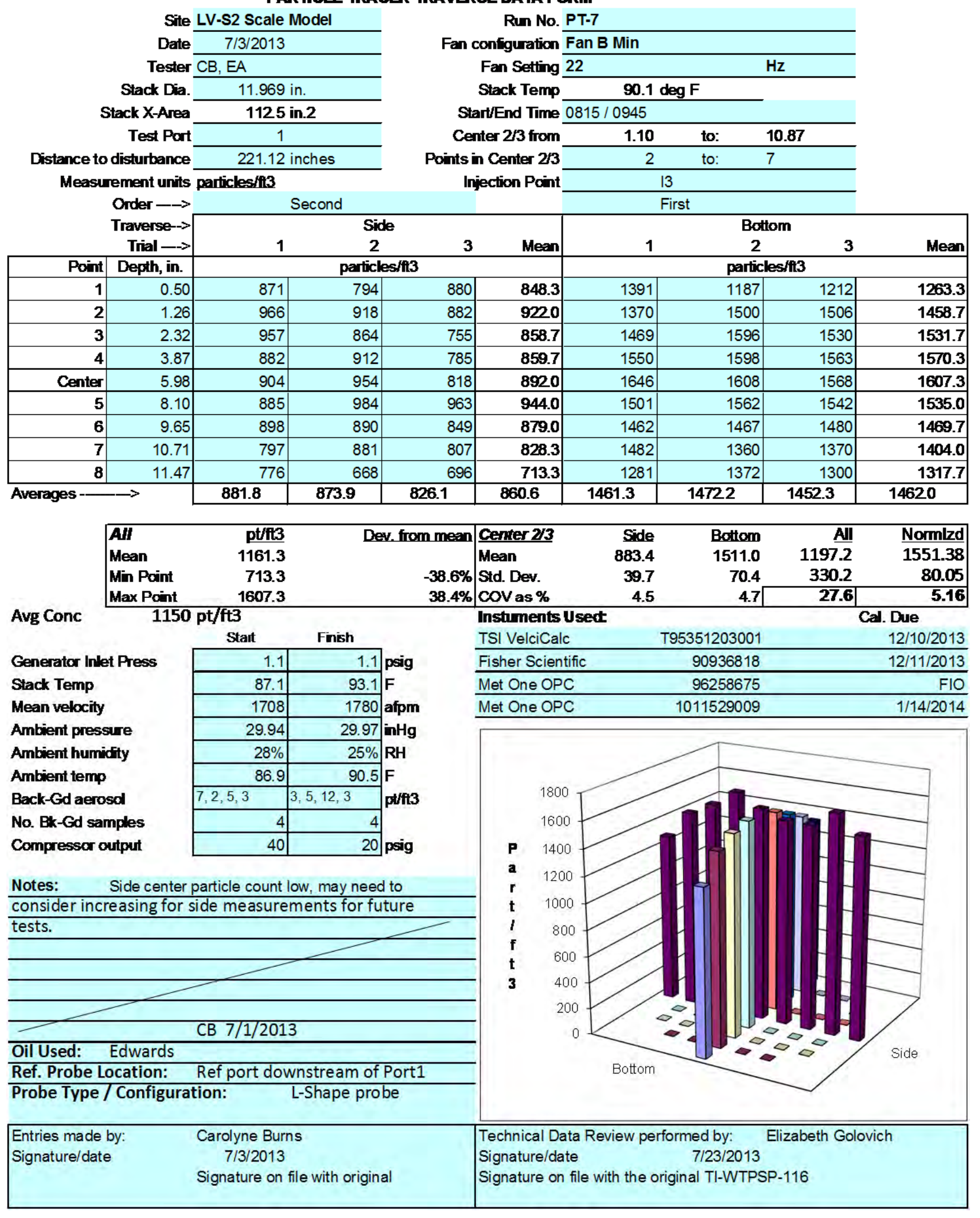


PARTICLE TRACER TRAVERSE DATA FORM

Site LV-S2 Scale Model

Date $7 / 3 / 2013$

Tester CB, EA

Stack Dia. 11.969 in

Test Port

Distance to disturbance

Measurement units particles/fi3

Order $\longrightarrow \quad$ First

Traverse-

Trial $\rightarrow$

Point Depth, in.

\begin{tabular}{|r|r|}
\hline Point & Depth, in. \\
\hline $\mathbf{1}$ & 0.50 \\
$\mathbf{2}$ & 1.26 \\
$\mathbf{3}$ & 2.32 \\
\hline $\mathbf{4}$ & 3.87 \\
\hline Center & 5.98 \\
\hline $\mathbf{5}$ & 8.10 \\
\hline $\mathbf{6}$ & 9.65 \\
\hline $\mathbf{7}$ & 10.7 \\
\hline $\mathbf{8}$ & 11.47 \\
\hline
\end{tabular}

Stack X-Area 1125 in.2

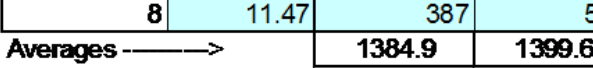

Run No. PT-8

Fan configuration Fan B MAX

Fan Setting 48

Stack Temp

Start/End Time 1015/1140

Center 233 from

Points in Center 283

Injection Pcint

\begin{tabular}{ccc}
1.10 & to: & $\mathbf{1 0 . 8 7}$ \\
\hline 2 & to: & 7 \\
\hline 13 & & \\
\hline
\end{tabular}

\begin{tabular}{|c|c|c|c|c|}
\hline \multirow[b]{3}{*}{ Mean } & \multicolumn{3}{|c|}{ Second } & \multirow[b]{3}{*}{ Mean } \\
\hline & \multicolumn{3}{|c|}{ Bottom } & \\
\hline & \multicolumn{2}{|l|}{1} & 2 & \\
\hline & \multicolumn{4}{|c|}{ particles/fi3 } \\
\hline 1061.3 & 1698 & 1577 & 1698 & 1657.7 \\
\hline 1550.3 & 2354 & 2253 & 2279 & 2295.3 \\
\hline 1684.7 & 2859 & 2691 & 2710 & 2753.3 \\
\hline 1667.7 & 3139 & 3048 & 2974 & 3053.7 \\
\hline 1807.0 & 3070 & 3037 & 3035 & 3047.3 \\
\hline 1706.7 & 2703 & 2594 & 2570 & 2622.3 \\
\hline 1500.0 & 2322 & 2107 & 2102 & 2177.0 \\
\hline 1114.0 & 1605 & 1654 & 1562 & 1607.0 \\
\hline 5123 & 897 & 911 & 865 & 891.0 \\
\hline 1400.4 & 2294.1 & 2208.0 & 2199.4 & 2233.9 \\
\hline
\end{tabular}

\begin{tabular}{llr}
\hline All & pt/ft3 \\
Mean & 1817.1 \\
Min Point & 5123 \\
Max Pcint & 3053.7 \\
\cline { 2 - 3 } & \multicolumn{1}{|l|}{$1741 \mathrm{pt} / \mathrm{ft3}$}
\end{tabular}

\section{Generator Inlet Press \\ Stack Temp \\ Mean velocity \\ Ambient pressure \\ Ambient humicity \\ Ambient temp \\ Back-Gd aerosol \\ No. Bk-Gd samples \\ Compressar output}

\begin{tabular}{|c|c|c|}
\hline Stat & Finish & \\
\hline 2.5 & 2.6 & psig \\
\hline 93.1 & 97.2 & $F$ \\
\hline 4099 & 4018 & afpm \\
\hline 29.97 & 30 & $\mathrm{inHg}$ \\
\hline $25 \%$ & $21 \%$ & $\mathrm{RH}$ \\
\hline 89.6 & 95 & $F$ \\
\hline $5,6,4,5$ & $1,6,4,8$ & $p^{t / f t 3}$ \\
\hline 4 & 4 & \\
\hline 29 & 36 & psig \\
\hline
\end{tabular}

Notes:

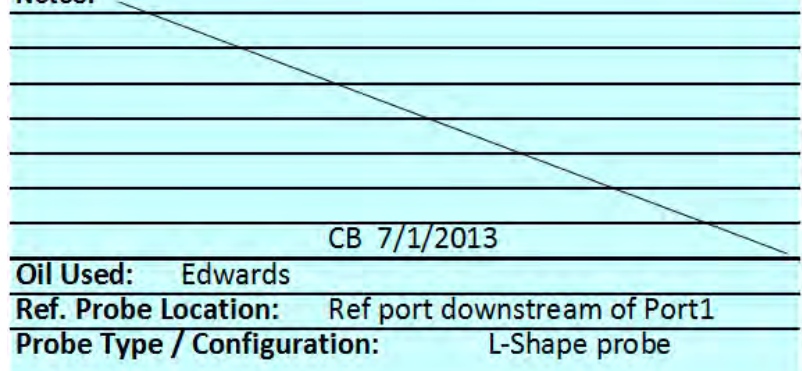

Entries made by:

Signature/date

Carolyne Burns
$7 / 3 / 2013$
Signature on file with original

\begin{tabular}{rrrr|} 
Side & Bottom & All & Normlzd \\
1575.8 & 2508.0 & 2041.9 & 2582.69 \\
227.5 & 521.0 & 619.0 & 446.33 \\
14.4 & 20.8 & 30.3 & 17.28 \\
\hline
\end{tabular}

\begin{tabular}{l|ll}
$68.0 \%$ & COV as $\%$ & 14.4
\end{tabular}

Cal. Due

\begin{tabular}{lrr} 
Instuments Used & T95351203001 & Cal. Due \\
TSI VelciCalc & 90936818 & $12 / 10 / 2013$ \\
\hline Fisher Scientific & 96258675 & $12 / 11 / 2013$ \\
\hline Met One OPC & 1011529009 & FIO \\
\hline Met One OPC & $1 / 14 / 2014$ \\
\hline
\end{tabular}

Signature on file with the original TI-WTPS-116
Signature/date
$\mathbf{3}$
$\mathbf{I}$


PARTICLE TRACER TRAVERSE DATA FORM

Site LV-S2 Scale Model

Date $7 / 5 / 2013$

Tester SS, EA

Stack Dia. 11.969 in

Stack X-Area

Test Port

Distance to disturbance

Measurement units particles/fi3

Order $\longrightarrow$

Traverse--

Trial $\rightarrow$

Point Depth, in.

\begin{tabular}{|r|r|}
\hline Point & Depth, in. \\
\hline $\mathbf{1}$ & 0.5 \\
$\mathbf{2}$ & 1.2 \\
$\mathbf{3}$ & 2.3 \\
\hline $\mathbf{4}$ & 3.87 \\
\hline Center & 5.98 \\
\hline $\mathbf{5}$ & 8.10 \\
\hline $\mathbf{6}$ & 9.6 \\
\hline $\mathbf{7}$ & 10.7 \\
\hline $\mathbf{8}$ & 11.47 \\
\hline
\end{tabular}

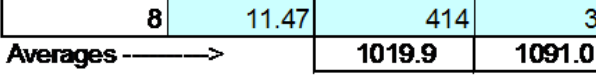

Run No. PT-9

Fan confguration Fan B Max

Fan Setting 45

Stack Temp

Start/End Time $0603 / 0740$

Center $2 / 3$ from

Points in Center 23

Injection Pcint I3
$68.35 \mathrm{deg} F$

\begin{tabular}{rll}
1.10 & to: & $\mathbf{1 0 . 8 7}$ \\
\hline 2 & to: & 7 \\
\hline
\end{tabular}

2nd

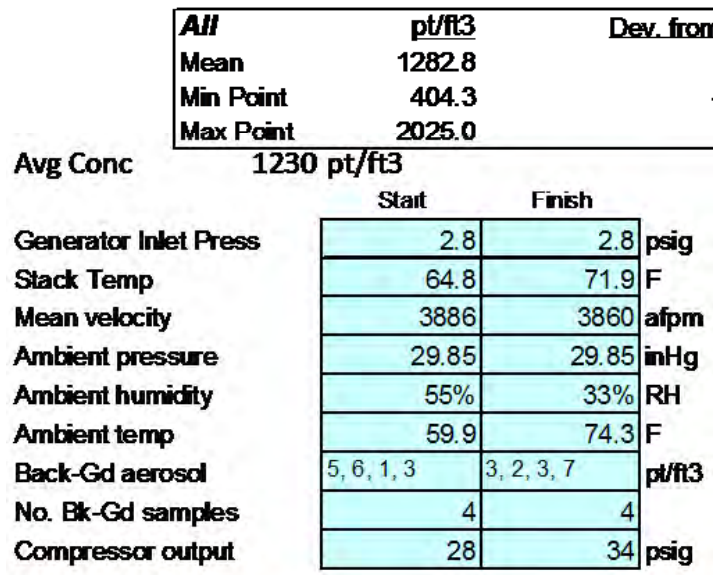

Notes:

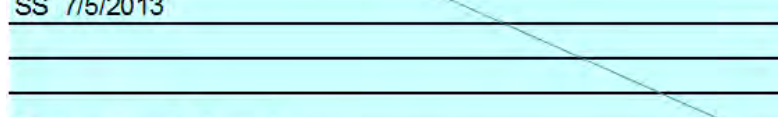

Oil Used: Edwards

Ref. Probe Location: Ref port downstream of Port1 Probe Type / Configuration:

L-Shape probe

Entries made by:

Signature/date

Susan Sande
$\quad 7 / 5 / 2013$
Signature on file with original

Susan Sande

Signature on file with original

\begin{tabular}{|c|c|c|c|c|}
\hline \multirow[b]{3}{*}{ Mean } & \multicolumn{3}{|c|}{ 2nd } & \multirow[b]{3}{*}{ Mean } \\
\hline & \multicolumn{3}{|c|}{ Bottom } & \\
\hline & 1 & 2 & 3 & \\
\hline & \multicolumn{4}{|c|}{ particles/fi3 } \\
\hline 830.3 & 852 & 935 & 890 & 892.3 \\
\hline 1084.7 & 1275 & 1462 & 1382 & 1373.0 \\
\hline 1276.3 & 1538 & 1751 & 1652 & 1647.0 \\
\hline 1313.3 & 1815 & 1995 & 2081 & 1963.7 \\
\hline 1386.3 & 1883 & 2124 & 2068 & 2025.0 \\
\hline 1385.0 & 1713 & 1939 & 1939 & 1863.7 \\
\hline 1199.3 & 1553 & 1618 & 1637 & 1602.7 \\
\hline 7823 & 1276 & 1316 & 1324 & 1305.3 \\
\hline 404.3 & 744 & 734 & 787 & 755.0 \\
\hline 1073.6 & 1405.4 & 1541.6 & 1528.9 & 14920 \\
\hline
\end{tabular}

109.8

\begin{tabular}{r|lrrrr|}
\hline mean & Certer 2/3 & Side & Bottom & $\underline{\text { All }}$ & Normlzd \\
\cline { 2 - 5 } & Mean & 1203.9 & 16829 & 1443.4 & 1720.72 \\
$57.5 \%$ & Std. Dev. & 214.2 & 281.3 & 345.6 & 288.52 \\
\hline COV as $\%$ & 17.8 & 16.7 & 23.9 & 16.77 \\
\hline
\end{tabular}

\section{Instuments Used}

TSI VelciCalc

\begin{tabular}{lrr}
\hline Fisher Scientific & T95351203001 & $12 / 10 / 2013$ \\
\hline Met One OPC & 90936818 & $12 / 11 / 2013$ \\
\hline Met One OPC & 1011529009675 & FIO \\
\hline
\end{tabular}

Met One OPC
$\mathbf{3}$
$\mathbf{3}$
$\mathbf{f}$
$\mathbf{f}$


PARTICLE TRACER TRAVERSE DATA FORM

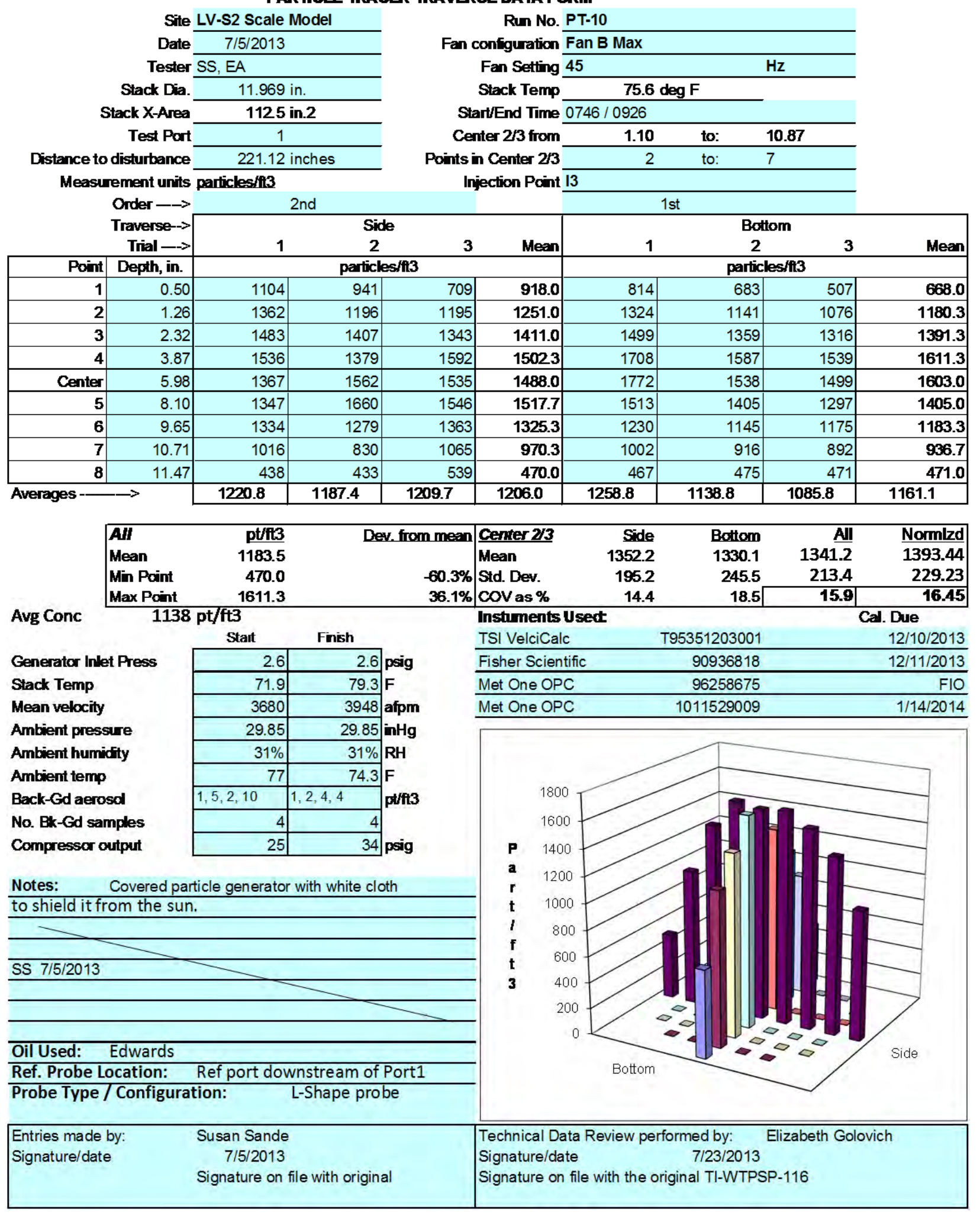


PARTICLE TRACER TRAVERSE DATA FORM

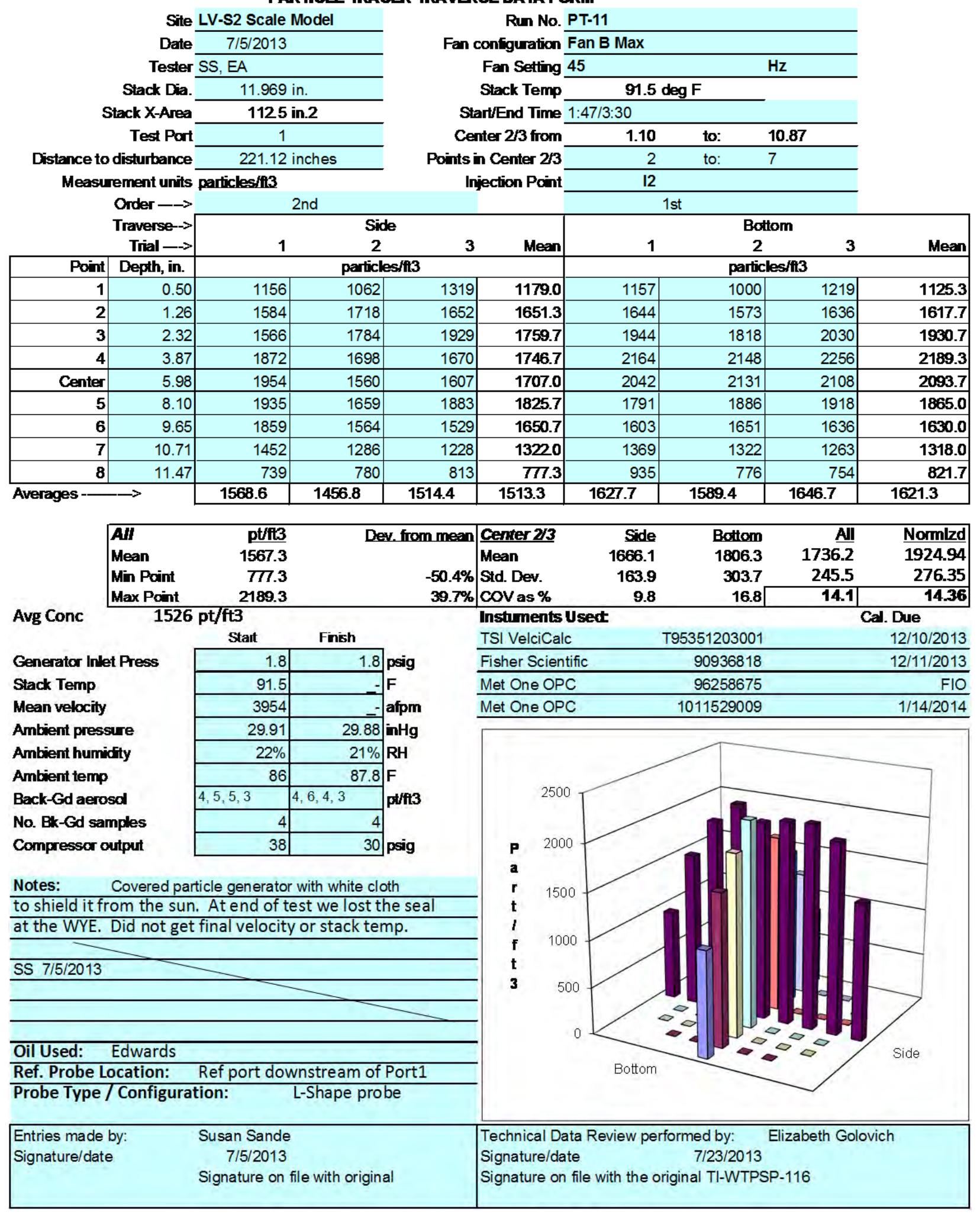


PARTICLE TRACER TRAVERSE DATA FORM

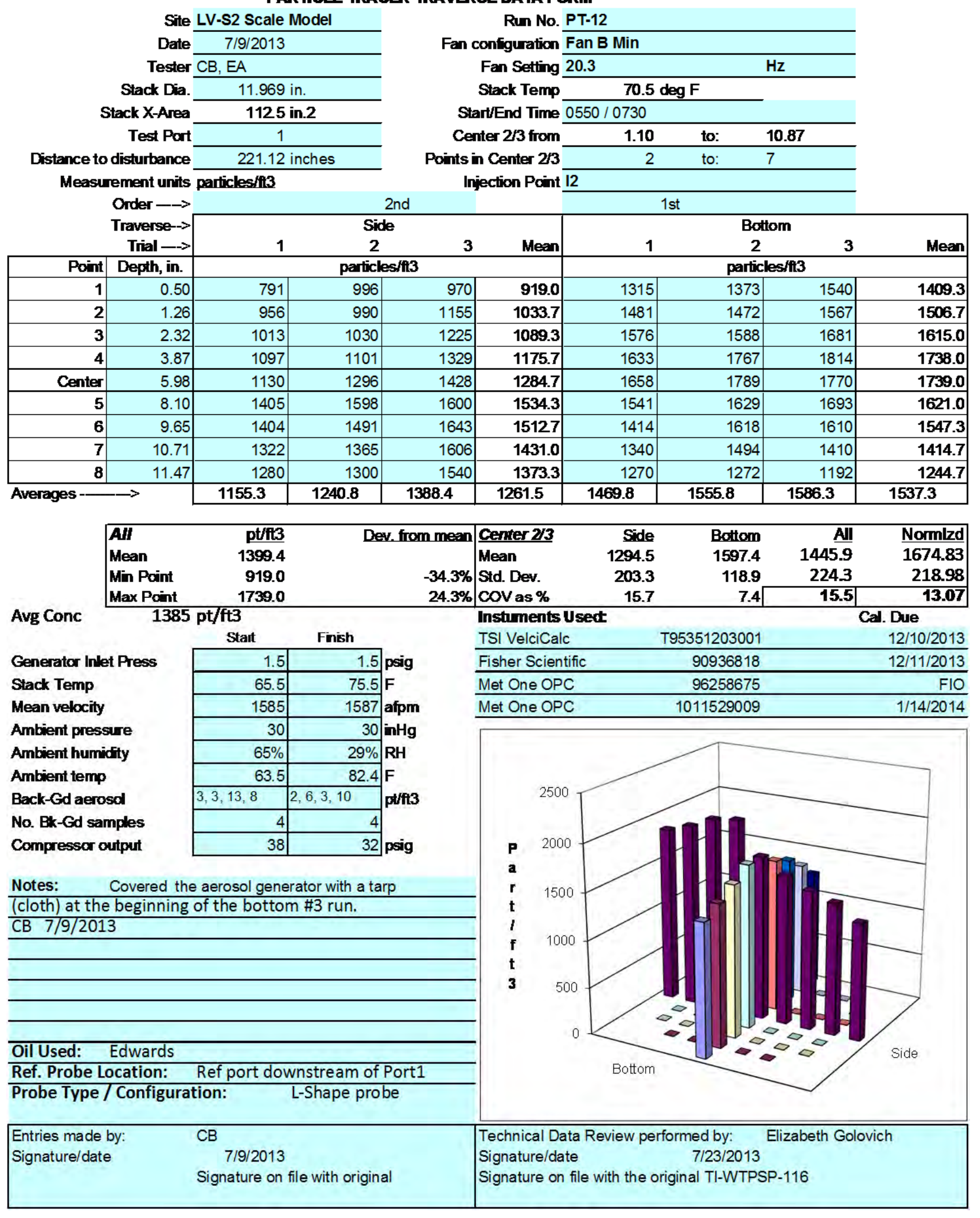


PARTICLE TRACER TRAVERSE DATA FORM

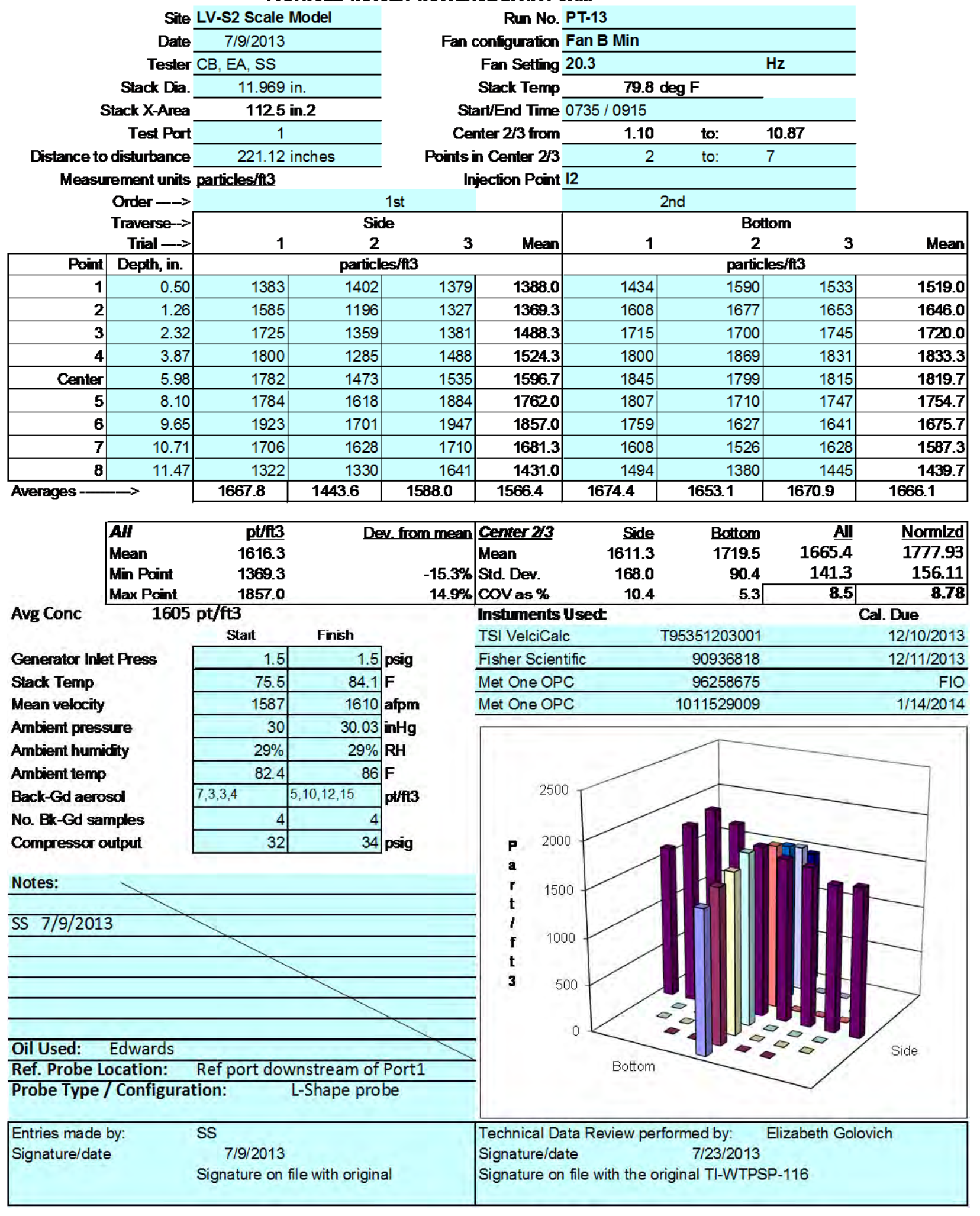


PARTICLE TRACER TRAVERSE DATA FORM

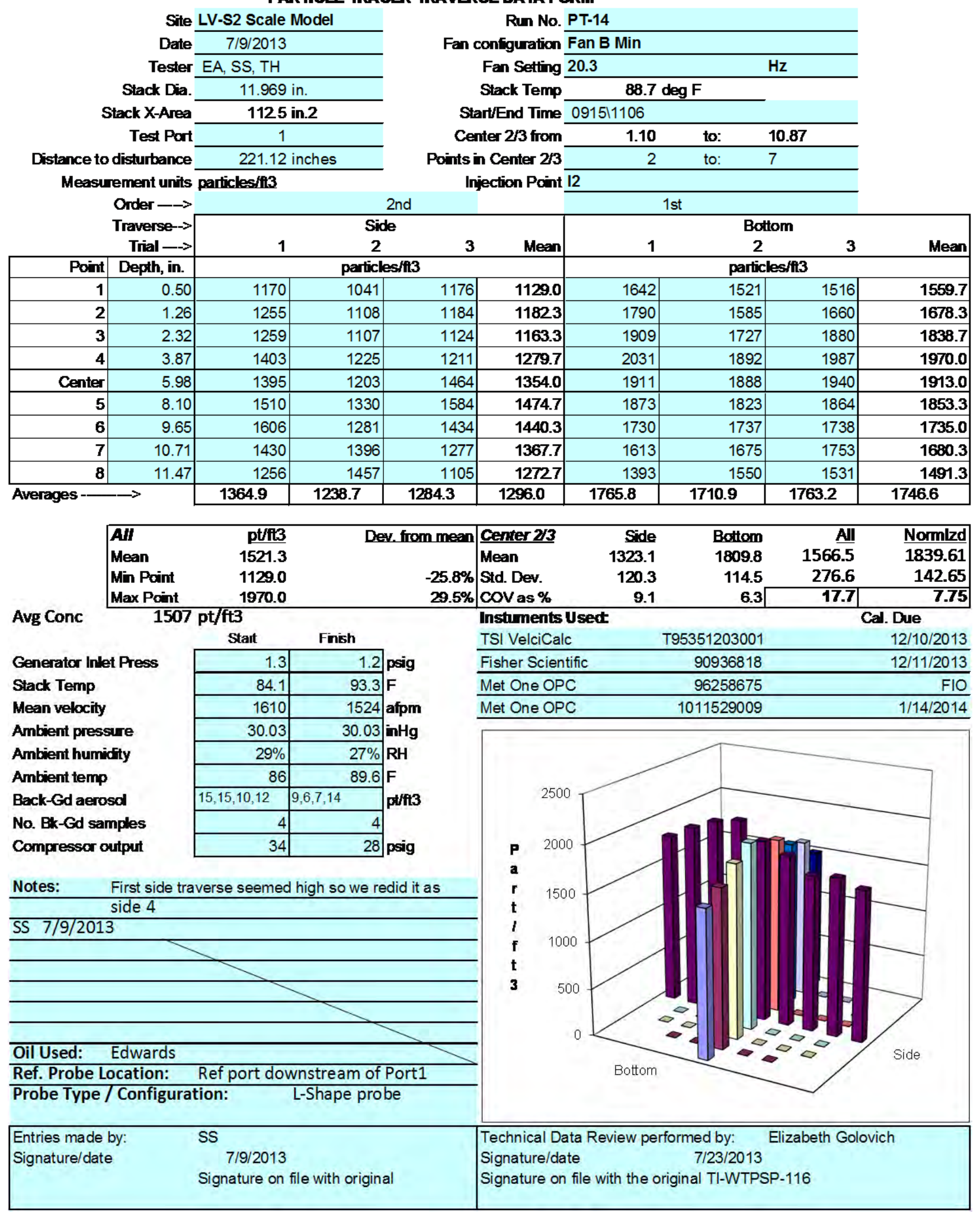


PARTICLE TRACER TRAVERSE DATA FORM

Site LV-S2 Scale Model

Date 7/10/2013

Tester EA, SS

Stack Dia.

Stack X-Area

Test Port

Distance to disturbance

Measurement units particles/fi3

Order $\longrightarrow$

Traverse--

Trial $\rightarrow$

\begin{tabular}{|r|r|}
\hline Point & Depth, in. \\
\hline $\mathbf{1}$ & 0.50 \\
$\mathbf{2}$ & 1.26 \\
$\mathbf{3}$ & 2.32 \\
\hline $\mathbf{4}$ & 3.87 \\
\hline Center & 5.98 \\
\hline $\mathbf{5}$ & 8.10 \\
\hline $\mathbf{6}$ & 9.65 \\
\hline $\mathbf{7}$ & 10.7 \\
\hline $\mathbf{8}$ & 11.47 \\
\hline
\end{tabular}

Averages
1.969 in. 1125 in. 2

221.12 inches

2nd
Run No. PT-15

Fan confguration Fan A Min

Fan Setting 20.3

Stack Temp

Start/End Time 0606/0745

Center $2 / 3$ from

Points in Center 283

Injection Pcint
$75.7 \mathrm{deg} F$

$\mathrm{Hz}$

\author{
44
}

\begin{tabular}{ccc}
1.10 & to: & $\mathbf{1 0 . 8 7}$ \\
\hline 2 & to: & 7 \\
\hline 12 & & \\
\hline
\end{tabular}

1 st

Botom

Side

\begin{tabular}{|c|c|}
$\mathbf{1}$ & $\mathbf{2}$ \\
\hline & \multicolumn{2}{c}{ particles/t3 } \\
\hline 1139 & 1265
\end{tabular}

3 Mean 1

1

2

\begin{tabular}{|l}
134 \\
15 \\
15 \\
1617 \\
16 \\
\hline 1634 \\
1690 \\
16 \\
1571 \\
\hline $\mathbf{5 8 4 . 7}$
\end{tabular}

\begin{tabular}{|c|c|c|c|c|}
\hline & \\
\hline 1248.3 & 1104 & 1174 & 1244 & 1174.0 \\
\hline 1404.3 & 1247 & 1368 & 1337 & 1317.3 \\
\hline 14523 & 1422 & 1463 & 1442 & 1442.3 \\
\hline 1505.7 & 1460 & 1564 & 1563 & 1529.0 \\
\hline 1536.0 & 1537 & 1496 & 1489 & 1507.3 \\
\hline 16027 & 1444 & 1420 & 1510 & 1458.0 \\
\hline 1680.0 & 1430 & 1408 & 1476 & 1438.0 \\
\hline 1575.0 & 1402 & 1246 & 1289 & 1312.3 \\
\hline 14720 & 1202 & 1217 & 1230 & 1216.3 \\
\hline 1497.4 & 1360.9 & 13729 & 1397.8 & 1377.2 \\
\hline
\end{tabular}

\begin{tabular}{|c|c|c|c|c|}
\hline \multirow{3}{*}{ Avg Conc } & $\begin{array}{l}\text { AlI } \\
\text { Mean } \\
\text { Min Point } \\
\text { Max Point }\end{array}$ & $\begin{array}{r}\text { pt/fi3 } \\
1437.3 \\
1174.0 \\
1680.0\end{array}$ & & ev. from \\
\hline & \multicolumn{2}{|c|}{$1427 \mathrm{pt} / \mathrm{ft} 3$} & \multirow[b]{2}{*}{ Finish } & \\
\hline & & Stait & & \\
\hline \multicolumn{2}{|c|}{ Generator Inlet Press } & 1.5 & 1.5 & psig \\
\hline \multicolumn{2}{|c|}{ Stack Temp } & 70 & 81.4 & $F$ \\
\hline \multicolumn{2}{|c|}{ Mean velocity } & 1570 & 1616 & afpm \\
\hline \multicolumn{2}{|c|}{ Ambient pressure } & 29.91 & 29.88 & $\mathrm{inHg}$ \\
\hline \multicolumn{2}{|c|}{ Ambient humidity } & $50 \%$ & $31 \%$ & $\mathrm{RH}$ \\
\hline \multicolumn{2}{|c|}{ Ambient temp } & 67.1 & 81.5 & $F$ \\
\hline \multicolumn{2}{|c|}{ Back-Gd aerosd } & $8,8,12,13$ & $8,8,8,9$ & $\mathrm{pt} / \mathrm{ft} 3$ \\
\hline \multicolumn{2}{|c|}{ No. Bk-Gd samples } & 4 & 4 & \\
\hline \multicolumn{2}{|c|}{ Compressar output } & 29 & 32 & psig \\
\hline
\end{tabular}

Notes:

\section{Oil Used: Edwards}

Ref. Probe Location: Ref port downstream of Port1 Probe Type/Configuration:

L-Shape probe

-18
16
3

\begin{tabular}{l|l|l}
\hline $18.3 \%$ & Center 2/3 \\
\hline Mean & Std. Dev. \\
$16.9 \%$ & COV as \\
\hline
\end{tabular}

S

\begin{tabular}{lrr}
\hline Instuments Usect & T95351203001 & \multicolumn{1}{c}{ Cal. Due } \\
TSI VelciCalc & 90936818 & $12 / 10 / 2013$ \\
\hline Fisher Scientific & 96258675 & $12 / 11 / 2013$ \\
\hline Met One OPC & 1011529009 & FIO \\
\hline Met One OPC & $1 / 14 / 2014$ \\
\hline
\end{tabular}

Technical Data Review performed by: Elizabeth Golovich


PARTICLE TRACER TRAVERSE DATA FORM

Site LV-S2 Scale Model

Date 7/10/2013

Tester EA, SS, TH

Stack Dia. 11.969 in

Stack X-Are 1125 in. 2

Test Port

Distance to disturbance

Measurement units particles/fi3

Order $\longrightarrow$

Traverse--

Trial $\rightarrow$ Point Depth, in.

\begin{tabular}{|r|r|}
\hline Point & Depth, in. \\
\hline $\mathbf{1}$ & 0.50 \\
$\mathbf{2}$ & 1.26 \\
$\mathbf{3}$ & 2.32 \\
\hline $\mathbf{4}$ & 3.87 \\
\hline Center & 5.98 \\
\hline $\mathbf{5}$ & 8.10 \\
\hline $\mathbf{6}$ & 9.65 \\
\hline $\mathbf{7}$ & 10.7 \\
\hline $\mathbf{8}$ & \multicolumn{2}{|c}{11.47} \\
\hline
\end{tabular}

\begin{tabular}{|l|}
\hline \\
\hline \\
\hline
\end{tabular}

$1 \mathrm{st}$

1

\begin{tabular}{|r|r|r|}
850 & 799 & 769 \\
\hline 1088 & 987 & 921 \\
1158 & 1177 & 1216 \\
\hline 1353 & 1325 & 1169 \\
\hline 1367 & 1249 & 1165 \\
\hline 1432 & 1248 & 1251 \\
1083 & 1193 & 1145 \\
\hline 533 & 774 & 744 \\
\hline 340 & 199 & 396 \\
\hline $\mathbf{2 0} .7$ & $\mathbf{9 9 4 . 6}$ & $\mathbf{9 7 5 . 1}$ \\
\hline
\end{tabular}

particles/ $/ \mathrm{t} 3$

Run No. PT-16

Fan confguration Fan A Max

Fan Setting 49

Stack Temp

Start/End Time $0752 / 0935$

Center $2 / 3$ from

Points in Center $2 / 3$

Injection Point

\begin{tabular}{ccl}
\hline 1.10 & to: & $\mathbf{1 0 . 8 7}$ \\
\hline 2 & to: & 7 \\
\hline 12 & & \\
2nd & & \\
\end{tabular}

Bottom

3 Mean 1

1

\begin{tabular}{|r|r|}
\hline $\mathbf{8 0 6}$ \\
\hline $\mathbf{9 9 8}$ \\
\hline
\end{tabular}

116

\begin{tabular}{|r|r|}
12823 & \\
\hline 165 & 1260.3 \\
\hline
\end{tabular}

1310.3

1140.3

\begin{tabular}{r}
683. \\
\hline 311 \\
997.4
\end{tabular}

\begin{tabular}{r|lrrrr|}
\hline Dev. from mean & Center 2/3 & Side & Bottom & All & Normlzd \\
\cline { 2 - 6 }$-73.3 \%$ & Mean & 1122.8 & 1527.7 & 1325.2 & 1586.53 \\
$58.0 \%$ & Std. Dev. & 220.5 & 273.5 & 318.0 & 294.03 \\
\hline COV as \% & 19.6 & 17.9 & 24.0 & 18.53 \\
\hline
\end{tabular}

Avg Conc

\begin{tabular}{|lr|}
\hline$A I I$ & $\mathrm{pt} / \mathrm{tt} 3$ \\
Mean & 1169.3 \\
Min Point & 311.7 \\
Max Point & 1847.0 \\
\hline
\end{tabular}
$58.0 \%$ COV as \% 19.6 Instuments Used

\begin{tabular}{lrr} 
TSI VelciCalc & T95351203001 & $12 / 10 / 2013$ \\
\hline Fisher Scientific & 90936818 & $12 / 11 / 2013$ \\
\hline Met One OPC & 96258675 & FIO \\
\hline Met One OPC & 1011529009 & $1 / 14 / 2014$ \\
\hline
\end{tabular}

\section{Stack Temp}

Mean velocity

Ambient pressure

Ambient humicity

Ambient temp

Back-Gd aerosol

No. Bk-Gd samples

Compressor output

\begin{tabular}{|c|c|c|}
\hline Statt & Finish & \\
\hline 2.0 & 2.0 & psig \\
\hline 81.9 & 89.4 & $\mathrm{~F}$ \\
\hline 3973 & 3795 & afpm \\
\hline 29.88 & 29.88 & $\mathrm{inHg}$ \\
\hline $31 \%$ & $29 \%$ & $\mathrm{RH}$ \\
\hline 81.5 & 86 & $F$ \\
\hline $12,14,11,6$ & $7,11,7,8$ & $\mathrm{pt} / \mathrm{fl}$ \\
\hline 4 & 4 & \\
\hline 32 & 22 & psig \\
\hline
\end{tabular}

Notes:

SS $7 / 10 / 2013$

Oil Used: Edwards

Ref. Probe Location: Ref port downstream of Port1 Probe Type/Configuration:

L-Shape probe

Entries made by:

Signature/date

Susan Sande
$7 / 10 / 2013$
Signature on file with original

Signature on file with original 
PARTICLE TRACER TRAVERSE DATA FORM

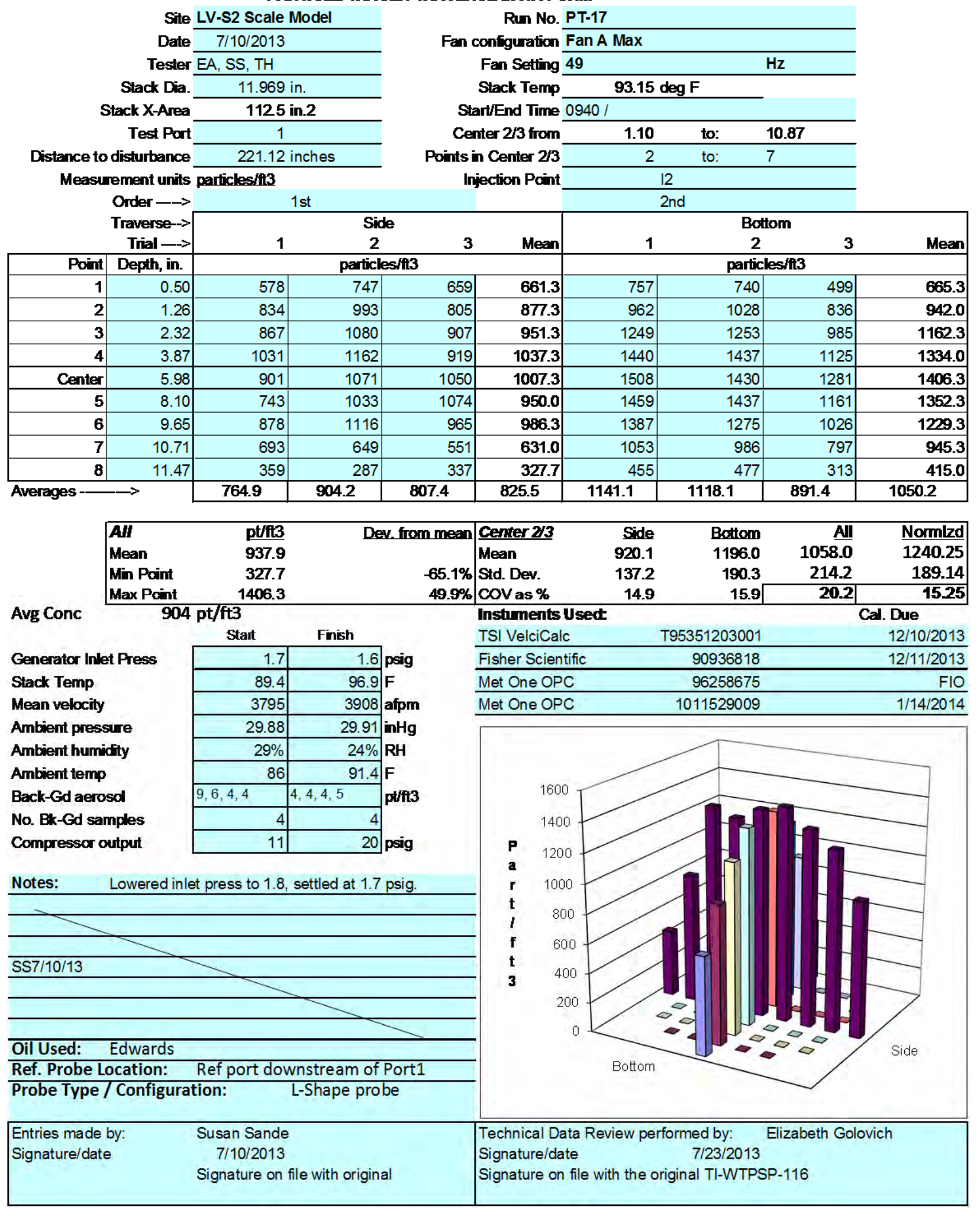


PARTICLE TRACER TRAVERSE DATA FORM

Site LV-S2 Scale Model

Date 7/10/2013

Tester TH, SS

Stack Dia.

Stack X-Area

Test Port

Distance to disturbance

Measurement units particles/fi3

Order $\longrightarrow$

Traverse-->

Trial $\rightarrow$

Point Depth, in.

\begin{tabular}{|r|r|}
\hline Point & Depth, in. \\
\hline $\mathbf{1}$ & 0.5 \\
$\mathbf{2}$ & 1.2 \\
$\mathbf{3}$ & 2.3 \\
\hline $\mathbf{4}$ & 3.87 \\
\hline Center & 5.9 \\
\hline $\mathbf{5}$ & 8.10 \\
\hline $\mathbf{6}$ & 9.6 \\
\hline $\mathbf{7}$ & 10.7 \\
\hline $\mathbf{8}$ & 11.4 \\
\hline
\end{tabular}

Averages -

1st

32

\begin{tabular}{r|}
87 \\
\hline 98
\end{tabular}

1560

Run No. PT-18

Fan configuration Fan A Max

Fan Setting 56

Stack Temp

Start/End Time $1314 / 1515$

Center $2 / 3$ from

Points in Center $2 / 3$

Injection Point

\begin{tabular}{ccc}
\hline 1.10 & to: & 10.87 \\
\hline 2 & to: & 7 \\
\hline 12 & & \\
\hline 2 nd &
\end{tabular}

Side

\begin{tabular}{rr}
1 & $\mathbf{2}$ \\
\hline & particles/ti3
\end{tabular}

3 Mean 1

2nd

\begin{tabular}{l|l|l|}
\hline 1.26 & 1358 & 1194 \\
\hline
\end{tabular}

\begin{tabular}{l}
1648 \\
\hline 60
\end{tabular}

\begin{tabular}{|l|l|}
\hline 40 & \\
\hline 00 & \\
\hline
\end{tabular}

122

\begin{tabular}{|l|l|}
\hline 23 & 1172 \\
\hline 94 & 1500 \\
\hline 06 & 1702 \\
\hline 37 & 1740 \\
\hline 85 & 1787 \\
\hline 42 & 1851 \\
\hline 65 & 1794 \\
\hline 68 & 1602 \\
\hline 09 & 1159 \\
\hline & 1589.9 \\
\hline
\end{tabular}

\begin{tabular}{|c|c|}
\hline $\mathbf{2 0}$ & 163 \\
\hline $\mathbf{2 0}$ & 177 \\
\hline $\mathbf{0 . 0}$ & 191 \\
\hline $\mathbf{7 7 . 7}$ & 222 \\
\hline $\mathbf{2 1 . 7}$ & 239 \\
\hline $\mathbf{2 7}$ & 220 \\
\hline $\mathbf{2 9}$ & 205 \\
\hline $\mathbf{9}$ & 180 \\
\hline
\end{tabular}

1

\begin{tabular}{|c|r|r|}
\hline $\begin{array}{c}\text { Bottom } \\
\mathbf{2}\end{array}$ & $\mathbf{3}$ & Mean \\
\hline \multicolumn{2}{|c|}{ particles/fi3 } \\
\hline 1494 & 1562 & $\mathbf{1 5 6 4 . 0}$ \\
\hline 1744 & 1870 & $\mathbf{1 7 9 7 . 7}$ \\
\hline 2006 & 2114 & $\mathbf{2 0 1 1 . 7}$ \\
\hline 2297 & 2288 & $\mathbf{2 2 7 1 . 3}$ \\
\hline 2267 & 2244 & $\mathbf{2 3 0 0 . 3}$ \\
\hline 2172 & 2288 & $\mathbf{2 2 2 1 . 7}$ \\
\hline 2067 & 2108 & $\mathbf{2 0 7 7 . 3}$ \\
\hline 1753 & 1895 & $\mathbf{1 8 1 6 . 3}$ \\
\hline 1374 & 1279 & $\mathbf{1 3 2 7 . 0}$ \\
\hline $\mathbf{0 8 . 2}$ & $\mathbf{1 9 6 0 . 9}$ & $\mathbf{1 9 3 1 . 9}$ \\
\hline
\end{tabular}

\begin{tabular}{llr}
\hline Avg & pt/fl3 \\
Mean & 1760.9 \\
Min Point & 1159.0 \\
Max Point & 2300.3 \\
\cline { 2 - 3 } & \multicolumn{2}{|c|}{$1726 \mathrm{pt} / \mathrm{ft} 3$}
\end{tabular}

Avg Conc $1726 \mathrm{pt} / \mathrm{ft} 3$

Generator Inlet Press

Stack Temp

Mean velocity

Ambient pressure

Ambient humicity

Ambient temp

Back-Gd aerosol

No. Bk-Gd samples

Compressar output

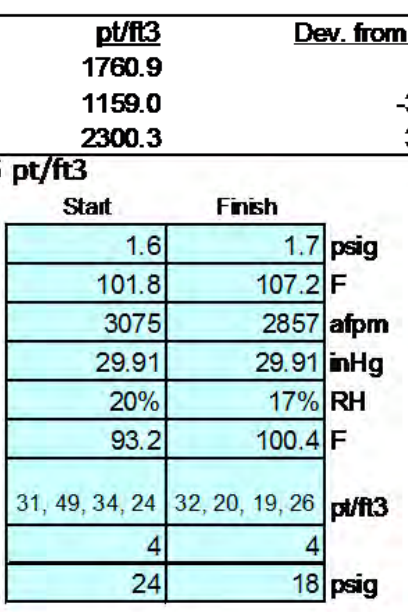

Notes:

\begin{tabular}{l}
\hline SS7/10/13 \\
\hline Oil Used: Edwards \\
\hline Pef. Probe Location: Ref port downstream of Port1
\end{tabular}

Entries made by:

Signature/date

Susan Sande
$7 / 10 / 2013$
Signature on file with original

\begin{tabular}{r|lrrrr|}
\hline & & & & \\
$-34.2 \%$ & Center 2/3 & Side & Bottom & All & Normlzd \\
$30.6 \%$ & Mean & 1711.1 & 2070.9 & 1891.0 & 2136.38 \\
Std. Dev. & 122.3 & 207.6 & 248.3 & 189.57 \\
\hline
\end{tabular}

$$
\text { Instuments Usedt }
$$

\begin{tabular}{lrr} 
TSI VelciCalc & T95351203001 & $12 / 10 / 2013$ \\
\hline Fisher Scientific & 90936818 & $12 / 11 / 2013$ \\
\hline Met One OPC & 96258675 & FIO \\
\hline Met One OPC & 1011529009 & $1 / 14 / 2014$ \\
\hline
\end{tabular}

Sechnical Data Review performed by: Elizabeth Golovich


PARTICLE TRACER TRAVERSE DATA FORM

Site LV-S2 Scale Model

Date 7/11/2013

Tester EA, CB, SS

Stack Dia. 11.969 in.

Stack X-Aree 1125 in. 2

Test Port

Measurement units particles/fi3

221.12 inches

Order $\longrightarrow$

Traverse--

Trial $\rightarrow$

\begin{tabular}{|r|r|}
\hline Point & Depth, in. \\
\hline $\mathbf{1}$ & 0.5 \\
$\mathbf{2}$ & 1.2 \\
\hline $\mathbf{3}$ & 2.3 \\
\hline $\mathbf{4}$ & 3.8 \\
\hline Center & 5.9 \\
\hline $\mathbf{5}$ & 8.1 \\
\hline $\mathbf{6}$ & 9.6 \\
\hline $\mathbf{7}$ & 10.7 \\
\hline $\mathbf{8}$ & \multicolumn{1}{|c}{11.4} \\
\hline
\end{tabular}

\begin{tabular}{|r|r|}
\hline Point & Depth, in \\
\hline $\mathbf{1}$ & 0.50 \\
\hline $\mathbf{2}$ & 1.2 \\
\hline $\mathbf{3}$ & 2.3 \\
\hline $\mathbf{4}$ & 3.87 \\
\hline Center & 5.98 \\
\hline $\mathbf{5}$ & 8.10 \\
\hline $\mathbf{6}$ & 9.6 \\
\hline $\mathbf{7}$ & 10.71 \\
\hline $\mathbf{8}$ & \multicolumn{2}{|c}{11.47} \\
\hline Averages - $\longrightarrow$ \\
\hline
\end{tabular}

\begin{tabular}{|l|}
\hline \\
\hline \\
\hline
\end{tabular}

Distance to disturbance 2nd 2nd

12

2

particles/fi3

\begin{tabular}{r|r|}
693 & 931 \\
1098 & 1032 \\
\hline
\end{tabular}

1377

1706

1545

1582

1116

474

\author{
(1)
}

F

Run No. PT-19

Fan configuration Fan A Max

Fan Setting 55.5

Stack Temp

Start/End Time $0550 / 0808$

Center $2 / 3$ from

Points in Center $2 / 3$

Injection Point 12

\begin{tabular}{rll}
1.10 & to: & 10.87 \\
\hline 2 & to: & 7 \\
\hline
\end{tabular}

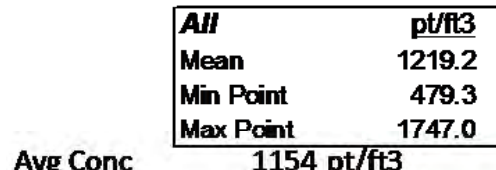

Avg Conc $1154 \mathrm{pt} / \mathrm{ft} 3$

Generator Inlet Press

Stack Temp

Mean velocity

Ambient pressure

Ambient humicity

Ambient temp

Back-Gd aerosol

No. Bk-Gd samples

Compressar output

\begin{tabular}{|c|c|c|}
\hline Stat & Finish & \\
\hline 2.6 & 2.6 & 6 psig \\
\hline 66.4 & 76.9 & $9 \mathrm{~F}$ \\
\hline 4583 & 4633 & 3 afpm \\
\hline 29.83 & 29.85 & 5 inHg \\
\hline $35 \%$ & $33 \%$ & to $\mathrm{RH}$ \\
\hline 64.4 & 76.1 & $1 \mathrm{~F}$ \\
\hline $28,19,13,18$ & $7,4,4,4$ & $\mathrm{p}_{\mathrm{p}} / \mathrm{ft}$ \\
\hline 4 & 4 & \\
\hline 29 & 34 & 4]psig \\
\hline
\end{tabular}

Notes: High backgrount counts

SS $7 / 11 / 13$

Oil Used: Edwards

Ref. Probe Location: Ref port downstream of Port1

Probe Type/Configuration:

L-Shape probe

Entries made by: Susan Sande

Signature/date $\quad 7 / 11 / 2013$

\begin{tabular}{|r|lrrrr|} 
Dev. from mean & Center 2/3 & Side & Bottom & All & Normlzd \\
\cline { 2 - 6 } & Mean & 1401.4 & 14027 & 1402.1 & 1405.03 \\
$-60.7 \%$ & Std. Dev. & 283.2 & 276.4 & 268.8 & 269.41 \\
$43.3 \%$ & COV as \% & 20.2 & 19.7 & 19.2 & 19.17 \\
\hline
\end{tabular}

Instuments Usect

\begin{tabular}{lrr} 
TSI VelciCalc & T95351203001 & $12 / 10 / 2013$ \\
\hline Fisher Scientific & 90936818 & $12 / 11 / 2013$ \\
\hline Met One OPC & 96258675 & FIO \\
\hline Met One OPC & 1011529009 & $1 / 14 / 2014$ \\
\hline
\end{tabular}

Technical Data Review performed by: Elizabeth Golovich


PARTICLE TRACER TRAVERSE DATA FORM

Site LV-S2 Scale Model

Date $7 / 11 / 2013$

Tester EA, CB, SS, TH

Stack $\mathrm{Da}$. $11.969 \mathrm{in}$

Stack X-Area 1125 in.2

Test Port

Distance to disturbance

Measurement units particles/fi3

221.12 inches

Order $\longrightarrow$

Traverse-->

Trial $\rightarrow$

\begin{tabular}{|r|r|}
\hline Point & Depth, in. \\
\hline $\mathbf{1}$ & 0.50 \\
$\mathbf{2}$ & 1.26 \\
\hline $\mathbf{3}$ & 2.32 \\
\hline $\mathbf{4}$ & 3.87 \\
\hline Center & 5.98 \\
\hline $\mathbf{5}$ & 8.10 \\
\hline $\mathbf{6}$ & 9.65 \\
\hline $\mathbf{7}$ & 10.7 \\
\hline $\mathbf{8}$ & 11.47 \\
\hline
\end{tabular}

\begin{tabular}{l|l|}
\hline Averages $-\longrightarrow$ & $\mathbf{8 9 8 . 1}$ \\
\hline
\end{tabular}
Run No. PT-20

Fan configuration Fan A Max

Fan Setting 46.5

Stack Temp

Start/End Time $0820 / 1010$

Center $2 / 3$ from

Points in Center $2 / 3$

Injection Point 13
$81.85 \operatorname{deg} F$

\begin{tabular}{rll}
1.10 & to: & $\mathbf{1 0 . 8 7}$ \\
\hline 2 & to: & 7 \\
\hline
\end{tabular}

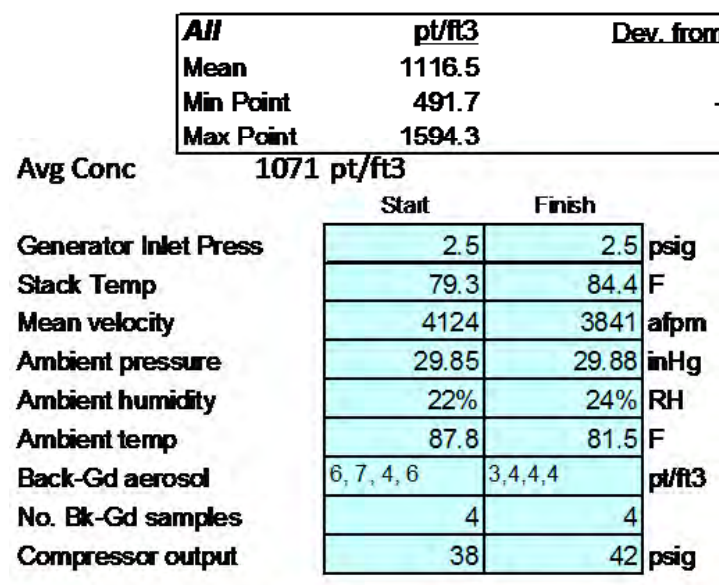

Notes:

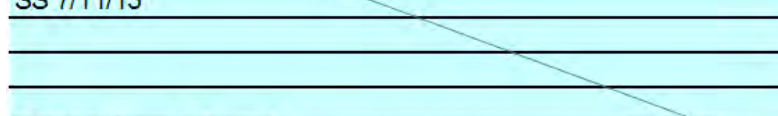

Oil Used: Edwards

Ref. Probe Location: Ref port downstream of Port1 Probe Type/Configuration:

L-Shape probe

\begin{tabular}{r|lrrrr|} 
& & & & \\
$-56.0 \%$ & Side & Bottom & All & Normlzd \\
$42.8 \%$ & Std. Dev. & 1166.3 & 1379.4 & 1272.8 & 1350.65 \\
\cline { 2 - 5 } & 200.3 & 227.7 & 233.8 & 220.47 \\
\hline
\end{tabular}

\section{Instuments Usect}

\begin{tabular}{|c|c|c|}
\hline TSI VelciCalc & T95351203001 & $12 / 10 / 2013$ \\
\hline Fisher Scientific & 90936818 & $12 / 11 / 2013$ \\
\hline Met One OPC & 96258675 & $\mathrm{FIO}$ \\
\hline Met One OP & 1011529009 & $1 / 14 / 2014$ \\
\hline
\end{tabular}

\begin{tabular}{lr}
\hline Entries made by: & Susan Sande \\
Signature/date & $7 / 11 / 2013$
\end{tabular}

Technical Data Review performed by: Elizabeth Golovich 
PARTICLE TRACER TRAVERSE DATA FORM

Site LV-S2 Scale Model

Date $7 / 11 / 2013$

Tester EA, CB, SS, TH

Stack $\mathrm{Da} \quad 11969 \mathrm{in}$

Stack X-Are 1125 in. 2

Test Port

Distance to disturbance

Measurement units particles/fi3

221.12 inches

Order $\longrightarrow$

Traverse- $\rightarrow$

Trial $\rightarrow$

\begin{tabular}{|r|r|}
\hline Point & Depth, in. \\
\hline $\mathbf{1}$ & 0.50 \\
$\mathbf{2}$ & 367.00 \\
\hline $\mathbf{3}$ & 2.32 \\
\hline $\mathbf{4}$ & 3.87 \\
\hline Center & 5.98 \\
\hline $\mathbf{5}$ & 8.10 \\
\hline $\mathbf{6}$ & 9.65 \\
\hline $\mathbf{7}$ & 10.7 \\
\hline $\mathbf{8}$ & 11.47 \\
\hline &
\end{tabular}

\begin{tabular}{|r|r|}
\hline Point & Depth, in \\
\hline $\mathbf{1}$ & 0.50 \\
\hline $\mathbf{2}$ & 367.00 \\
\hline $\mathbf{3}$ & 2.3 \\
\hline $\mathbf{4}$ & 3.87 \\
\hline Center & 5.9 \\
\hline $\mathbf{5}$ & 8.10 \\
\hline $\mathbf{6}$ & 9.6 \\
\hline $\mathbf{7}$ & 10.7 \\
\hline $\mathbf{8}$ & 11.4 \\
\hline
\end{tabular}

\begin{tabular}{|l|}
\hline \\
\hline \\
\hline
\end{tabular}

2nd
Run No. PT-21

Fan configuration Fan A Max

Fan Setting 46.5

Stack Temp

Start/End Time $1020 / 1200$

Center $2 / 3$ from

Points in Center $2 / 3$

Injection Point 13

\begin{tabular}{rll}
1.10 & to: & $\mathbf{1 0 . 8 7}$ \\
\hline 2 & to: & 7 \\
\hline
\end{tabular}

\begin{tabular}{|c|c|c|c|}
\hline \multirow{3}{*}{ Avg Conc } & $\begin{array}{r}\mathrm{pt} / \mathrm{ft} 3 \\
1243.7 \\
569.0 \\
1707.7\end{array}$ & & Dev. fron \\
\hline & $1194 \mathrm{pt} / \mathrm{ft3}$ & & \\
\hline & Statt & Finsh & \\
\hline Generator Inlet Press & 2.4 & 2.5 & 5 psig \\
\hline Słack Temp & 84.4 & 89.9 & $F$ \\
\hline Mean velocity & 3841 & 3653 & afpm \\
\hline Ambient pressure & 29.88 & 29.91 & inHg \\
\hline Ambient humidity & $24 \%$ & $22 \%$ & $\mathrm{RH}$ \\
\hline Ambient temp & 81.5 & 84.2 & $F$ \\
\hline Back-Gd aerosol & $4,5,7,9$ & $8,6,2,2$ & $\mathrm{p} / \mathrm{ft} 3$ \\
\hline No. Bk-Gd samples & 4 & 4 & \\
\hline Compressar output & 42 & 30 & psig \\
\hline
\end{tabular}

Notes:

SS $7 / 11 / 13$

Oil Used: Edwards

Ref. Probe Location: Ref port downstream of Port1 Probe Type/Configuration:

L-Shape probe

Entries made by: Susan Sande



Appendix D

LV-S3 Data Sheets 



\section{D.1 LV-S3 Calibration of Ventilation Flow Controller}

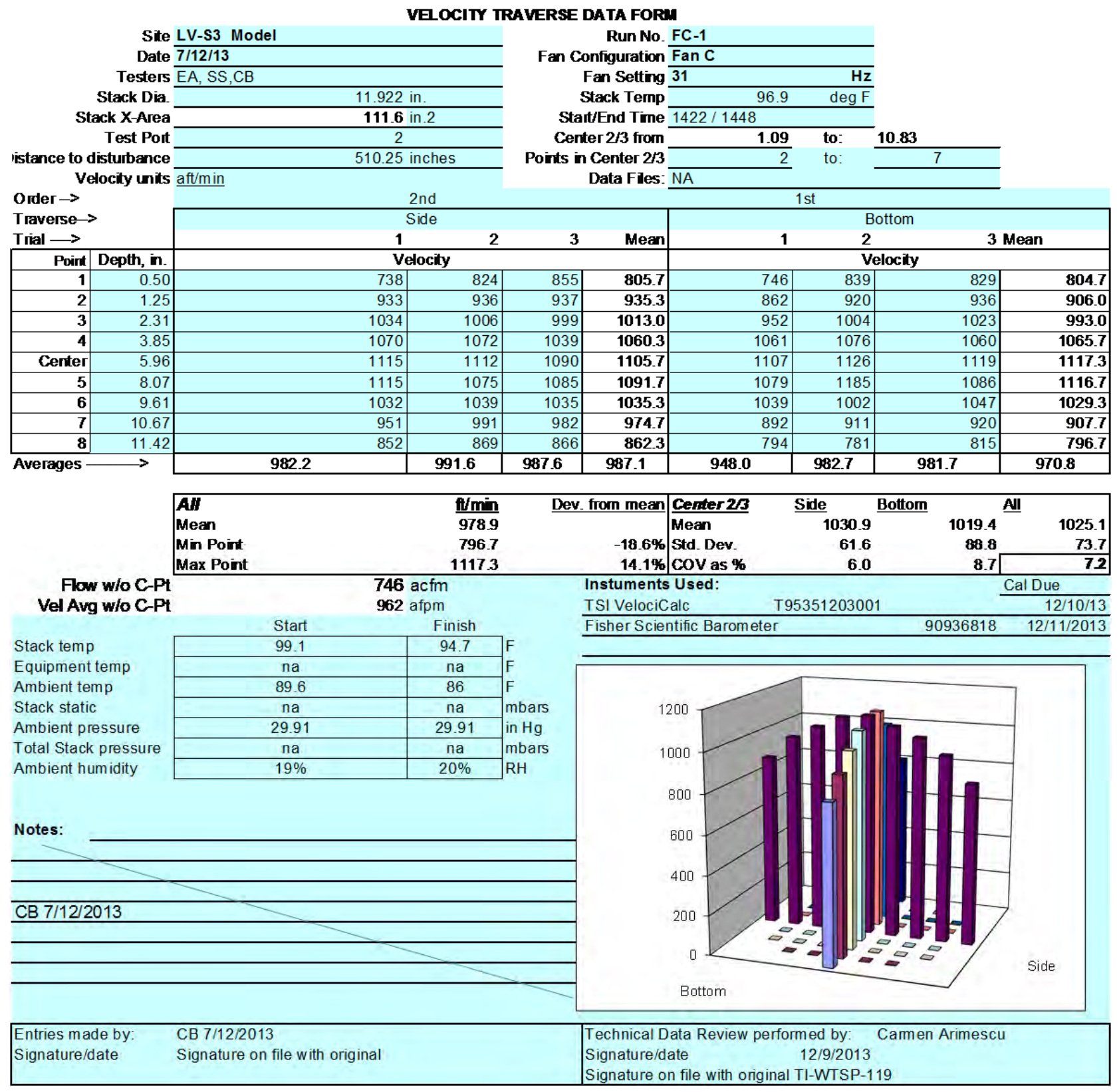


VELOCITY TRAVERSE DATA FORM

Site LV-S3 Model

Date 7/15/13

Testers EA, SS

Stack Dia

Stack X-Area

Test Port

\begin{tabular}{c}
\hline 11.922 in. \\
\hline $\mathbf{1 1 1 . 6}$ in. 2 \\
\hline 2 \\
\hline 510.25 inches
\end{tabular}

Distance to disturbance

Velocity units aft/min
Run No. FC-2

Fan Configuration Fan B Only

Fan Setting 32

Stack Temp

Start/End Time $0546 / 0610$

Center $2 / 3$ from

Points in Center $2 / 3$

Data Files: NA

$\begin{aligned} & \text { Order } \rightarrow \\
& \text { Traverse } \rightarrow\end{aligned}$
Trial $\longrightarrow$.
\begin{tabular}{|r|r|}
\hline Point & Depth, in. \\
\hline $\mathbf{1}$ & 0.50 \\
\hline $\mathbf{2}$ & 1.25 \\
\hline $\mathbf{3}$ & 2.31 \\
\hline $\mathbf{4}$ & 3.85 \\
\hline Center & 5.96 \\
\hline $\mathbf{5}$ & 8.07 \\
\hline $\mathbf{6}$ & 9.61 \\
\hline $\mathbf{7}$ & 10.67 \\
\hline $\mathbf{8}$ & 11.42 \\
\hline
\end{tabular}

\begin{tabular}{|c|}
\hline 1 \\
\hline $\mathrm{Ve}$ \\
\hline 67 \\
\hline 80 \\
\hline 89 \\
\hline 97 \\
\hline 100 \\
\hline 96 \\
\hline 91 \\
\hline 87 \\
\hline 75 \\
\hline 73.1 \\
\hline
\end{tabular}

Side
2

\author{
$2 n d$
}

$\frac{1.09}{2}$

$\frac{\mathrm{Hz}}{\operatorname{deg} \mathrm{F}}$

elocity

\begin{tabular}{|c|c|c|c|c|}
\hline \multirow[t]{2}{*}{ Mean } & 1 & 2 & 3 & Mean \\
\hline & \multicolumn{4}{|c|}{ Velocity } \\
\hline 700.0 & 761 & 809 & 809 & 793.0 \\
\hline 822.7 & 823 & 834 & 870 & 8423 \\
\hline 902.0 & 920 & 927 & 960 & 935.7 \\
\hline 966.7 & 1002 & 970 & 1018 & 996.7 \\
\hline 1004.0 & 1042 & 1017 & 1063 & 1040.7 \\
\hline 978.0 & 1041 & 1055 & 1044 & 1046.7 \\
\hline 929.7 & 977 & 992 & 983 & 984.0 \\
\hline 878.0 & 902 & 921 & 890 & 904.3 \\
\hline 773.7 & 761 & 802 & 776 & 779.7 \\
\hline 883.9 & 914.3 & 925.2 & 934.8 & 924.8 \\
\hline
\end{tabular}

$1 \quad$ Bottom

to: 10.83

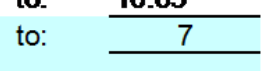

\begin{tabular}{|lr}
\hline $\boldsymbol{A} I$ & $\underline{\mathrm{ft} / \mathrm{min}}$ \\
Mean & $\mathbf{9 0 4 . 3}$ \\
Min Point & 700.0 \\
Max Point & 1046.7 \\
\hline
\end{tabular}

Flow w/o C-Pt Vel Avg w/o C-Pt

$690 \mathrm{acfm}$

890 afpm

Stack temp

Equipment temp Ambient temp

Stack static

Ambient pressure

Total Stack pressure

Ambient humidity

\begin{tabular}{|c|c|c|}
\hline \multicolumn{1}{|c|}{ Start } & Finish & \multicolumn{1}{c}{} \\
\hline 66.8 & 72.5 & $\mathrm{~F}$ \\
\cline { 1 - 2 } na & na & $\mathrm{F}$ \\
\hline 64.4 & 67.1 & $\mathrm{~F}$ \\
\hline na & na & mbars \\
\cline { 1 - 2 } 29.88 & 29.91 & in Hg \\
\cline { 1 - 2 } na & na & mbars \\
\cline { 1 - 2 } $33 \%$ & $32 \%$ & $\mathrm{RH}$ \\
\hline
\end{tabular}

Notes:

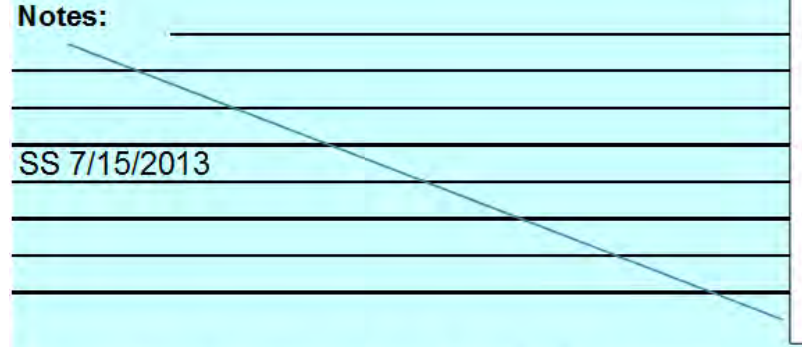

Entries made by:

Susan Sande 7/15/2013

Signature/date

Signature on file with original

\begin{tabular}{|c|c|c|c|c|}
\hline Dev. from mean & Center 2/3 & Side & Bottom & All \\
\hline & Mean & $\overline{925.9}$ & 964.3 & 945.1 \\
\hline$-22.6 \%$ & Std. Dev. & 63.3 & 74.5 & 69.4 \\
\hline $15.7 \%$ & COV as $\%$ & 6.8 & 7.7 & 7.3 \\
\hline
\end{tabular}

Instuments Used:

TSI VelociCalc $\quad$ T95351203001 $\quad 12 / 10 / 13$

Cal Due

Fisher Scientific Barometer $\quad 90936818 \quad 12 / 11 / 2013$

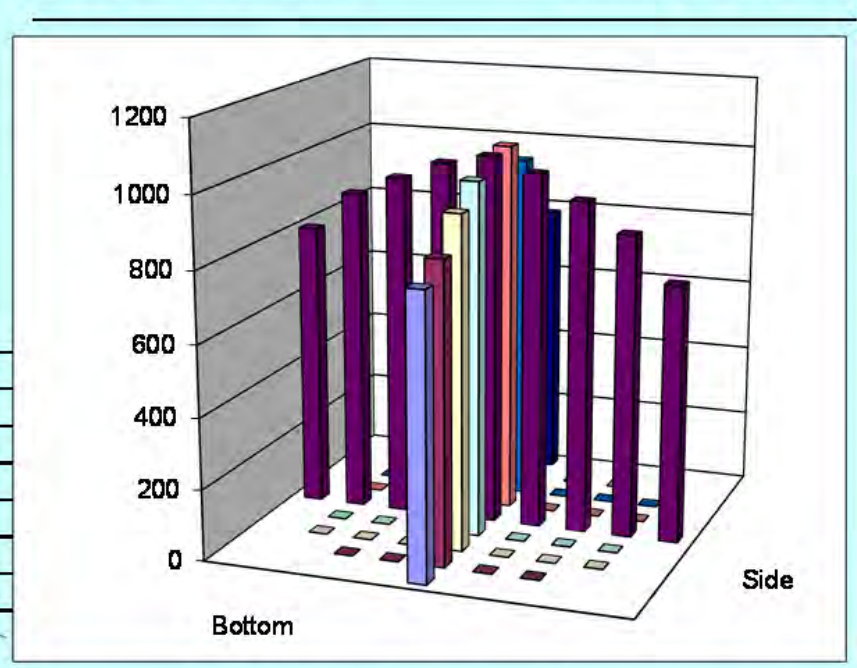

Technical Data Review performed by: Carmen Arimescu Signature/date $12 / 9 / 2013$

Signature on file with original TI-WTSP-119 


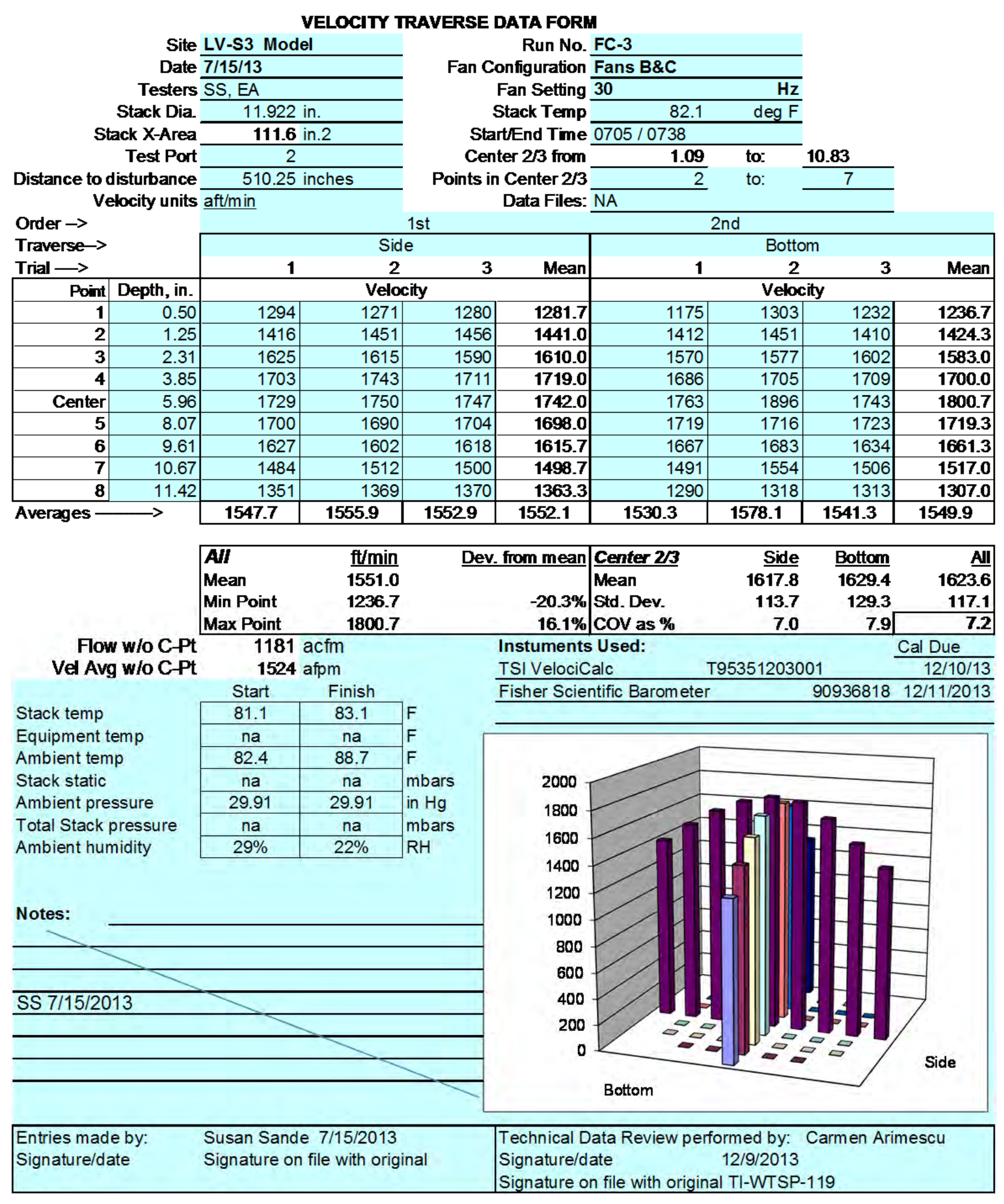


VELOCITY TRAVERSE DATA FORM

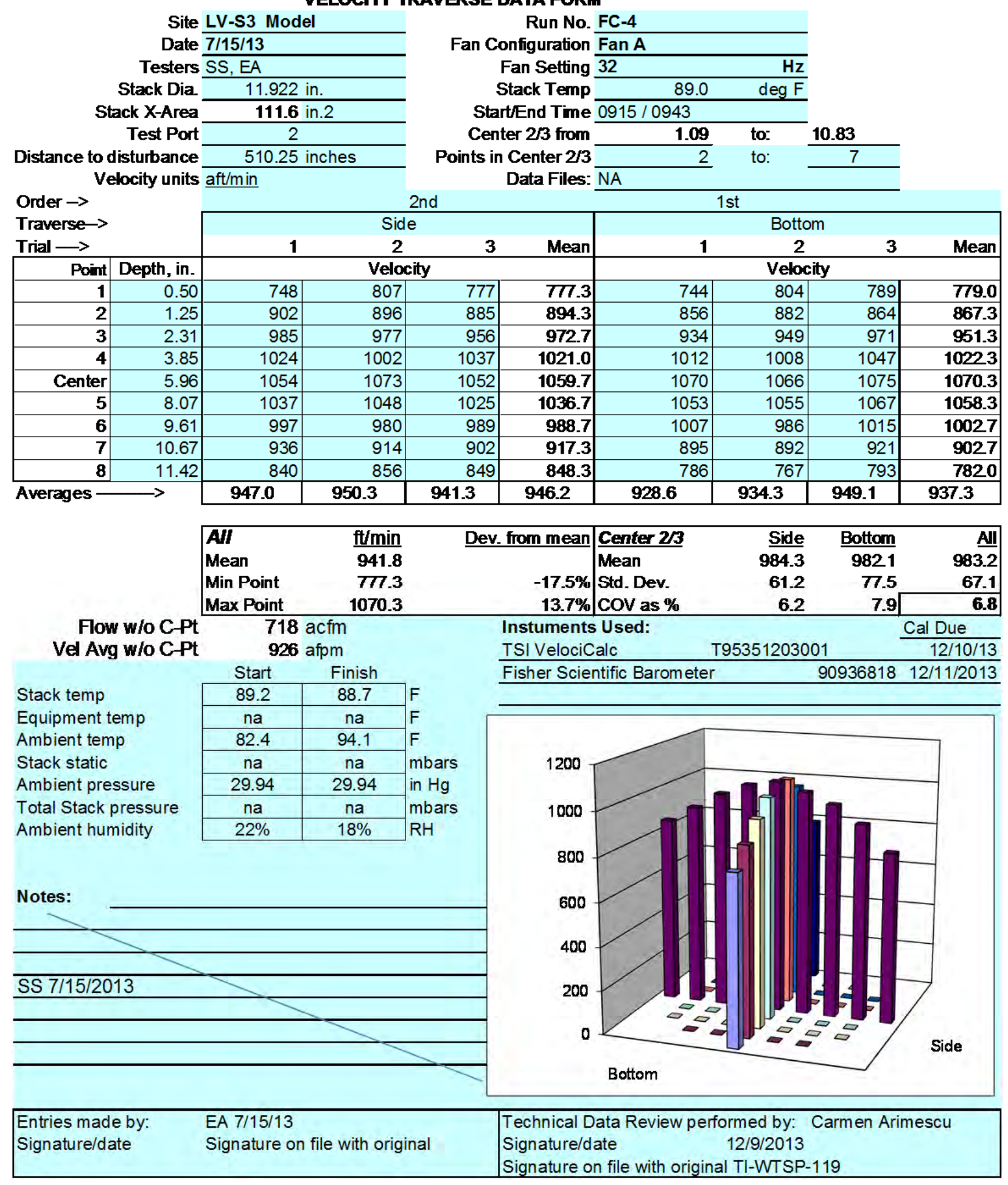




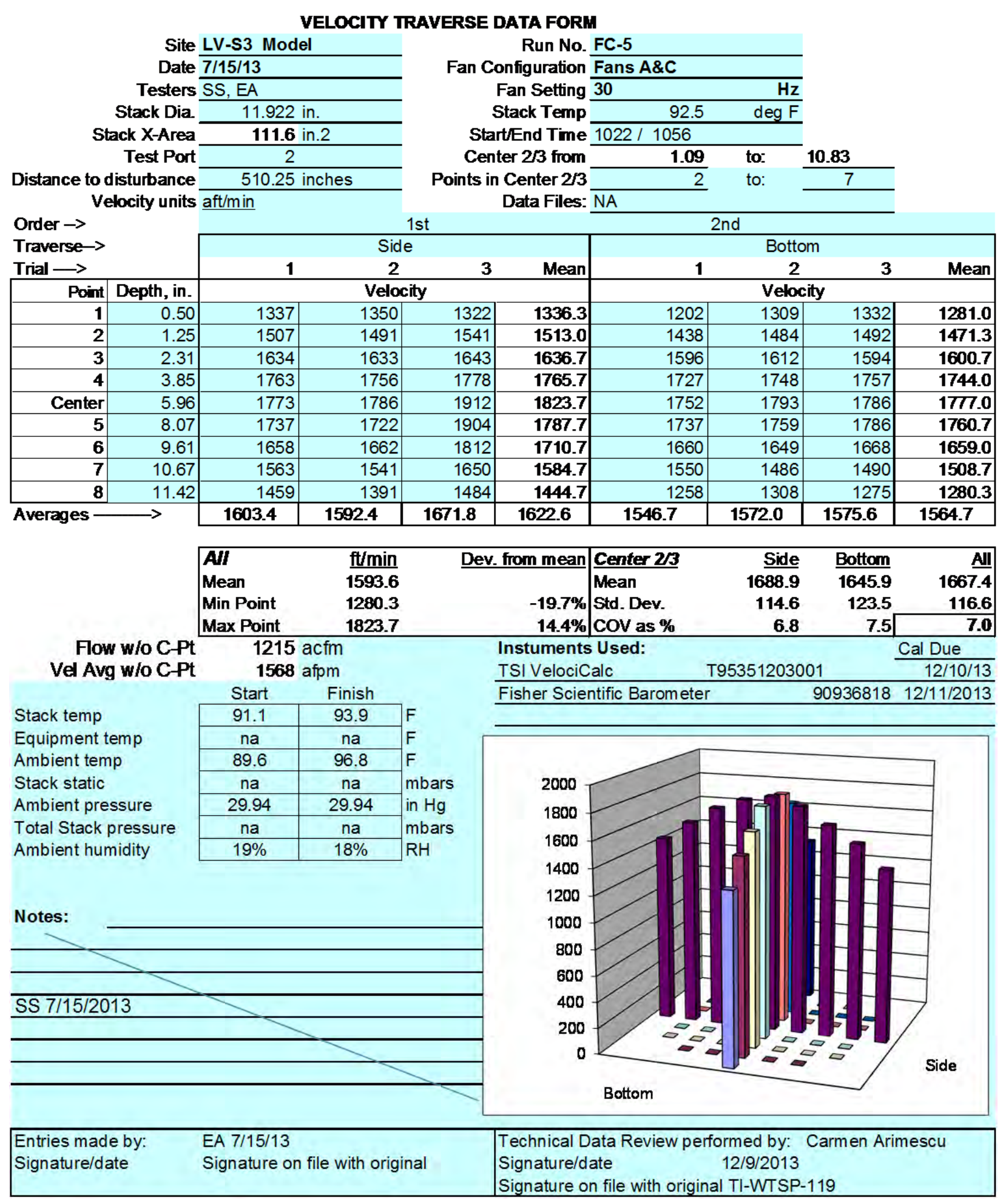




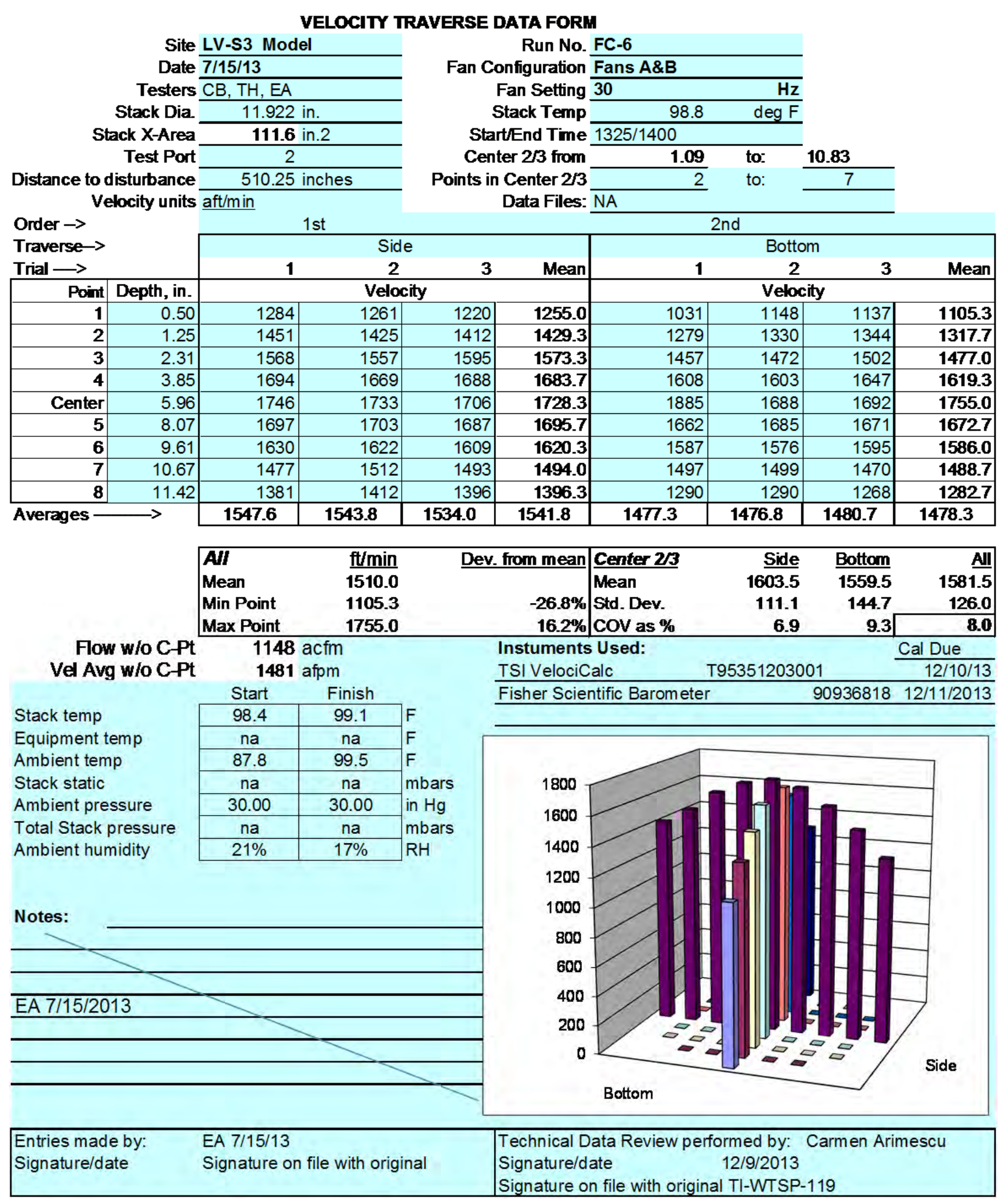




\section{VELOCITY vS. FREQUENCY DATA FORM}

\begin{tabular}{|c|c|c|}
\hline Site & \multicolumn{2}{|c|}{$\frac{\text { LV-S3 model }}{7 / 12 / 2013}$} \\
\hline $\begin{array}{l}\text { Date } \\
\text { Tester }\end{array}$ & \multicolumn{2}{|c|}{$\mathrm{CB}, \mathrm{EA}$} \\
\hline Stack Dia & 11.922 & in. \\
\hline tack X-Area & 111.6 & in2 \\
\hline
\end{tabular}

\begin{tabular}{rc} 
Run No. VF-1 \\
\cline { 2 - 2 } Stack Temp \\
\cline { 2 - 2 } Stack RH\% \\
Baro Press & 104.4 \\
\cline { 2 - 2 } Fan Configuration & $20 \%$ \\
Start/End Time & Fan C \\
\cline { 2 - 2 }
\end{tabular}

Dist. from disturbance $\underline{510.25 \text { inches }}$ Reference point from velocity test VC : Velocity Readings, units $=\frac{\text { afpm }}{2}$

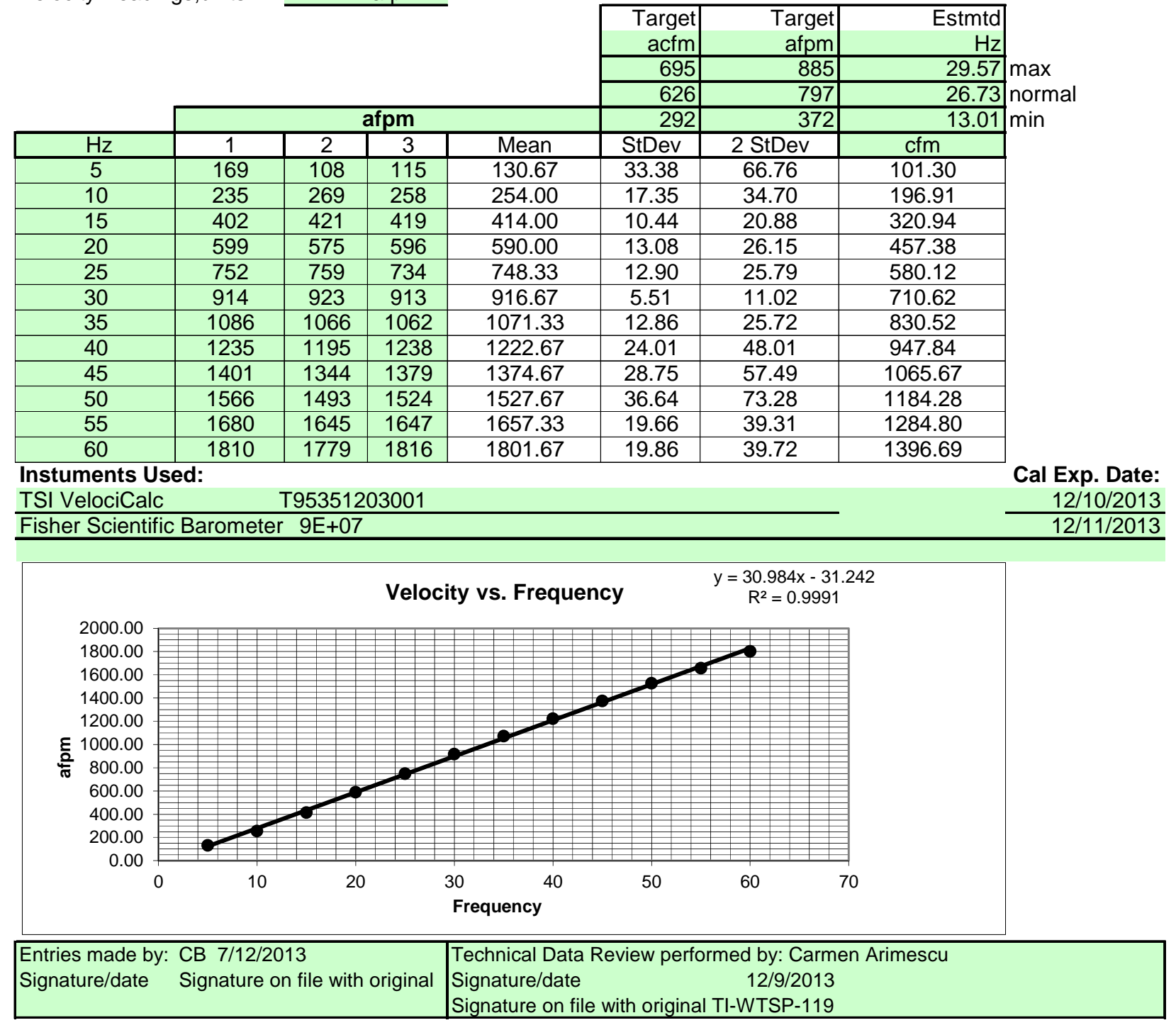


VELOCITY vS. FREQUENCY DATA FORM

\begin{tabular}{|c|c|c|c|c|}
\hline Site & \multicolumn{2}{|c|}{ LV-S3 model } & \multicolumn{2}{|c|}{ Run No. VF-2 } \\
\hline Date & & & Stack Temp & 78.1 \\
\hline Tester & & & Stack $\mathrm{RH}^{\circ}$ & $30 \%$ \\
\hline Stack Dia. & 11.922 & in. & Baro Press & 29.91 \\
\hline ack X-Area & 111.6 & in2 & Fan Configuration & Fan B Only \\
\hline Test Port & 2 & & Start/End Time & $0630 / 0655$ \\
\hline
\end{tabular}

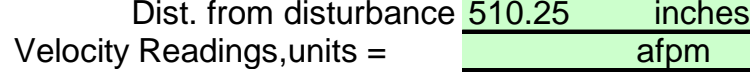

Start/End Time $0630 / 0655$

Side 7

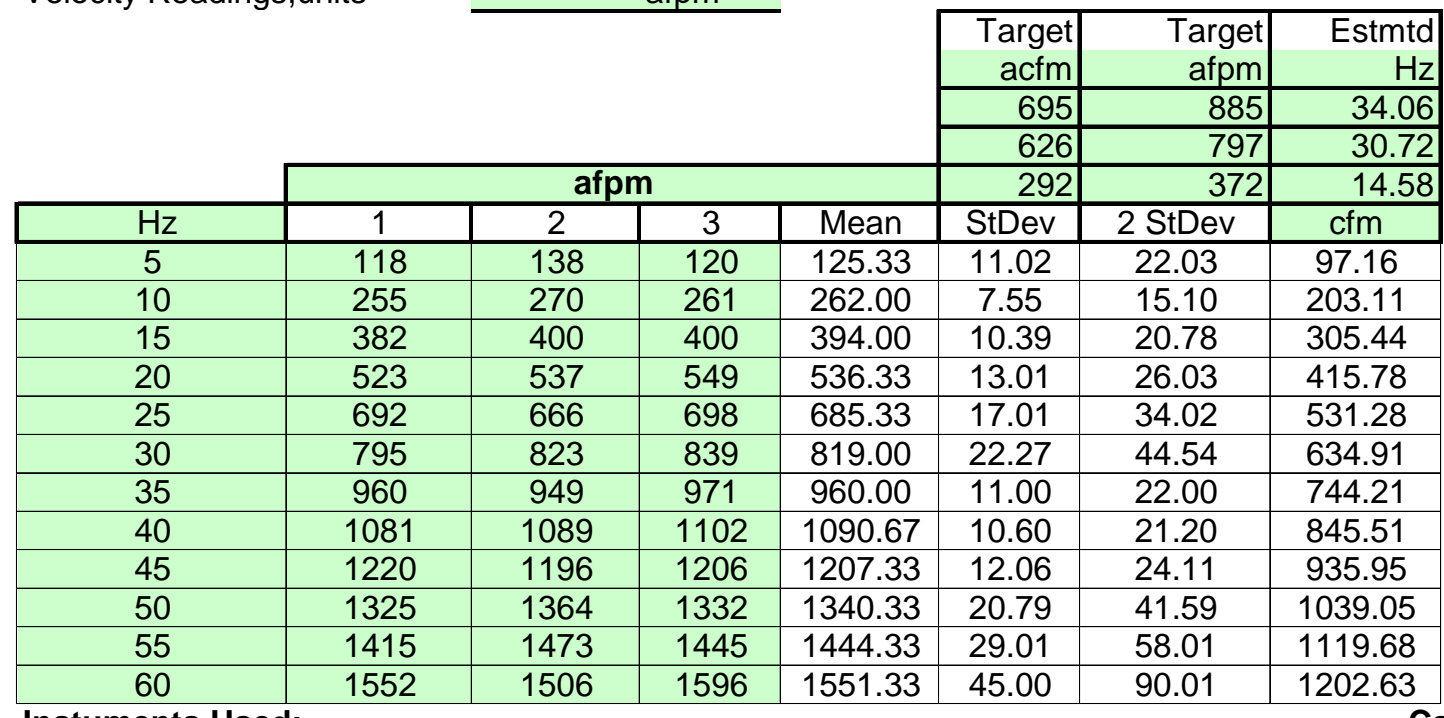

Instuments Used:

TSI VelociCalc T95351203001

Cal Exp. Date:

Fisher Scientific Barometer 90936818

$12 / 10 / 2013$

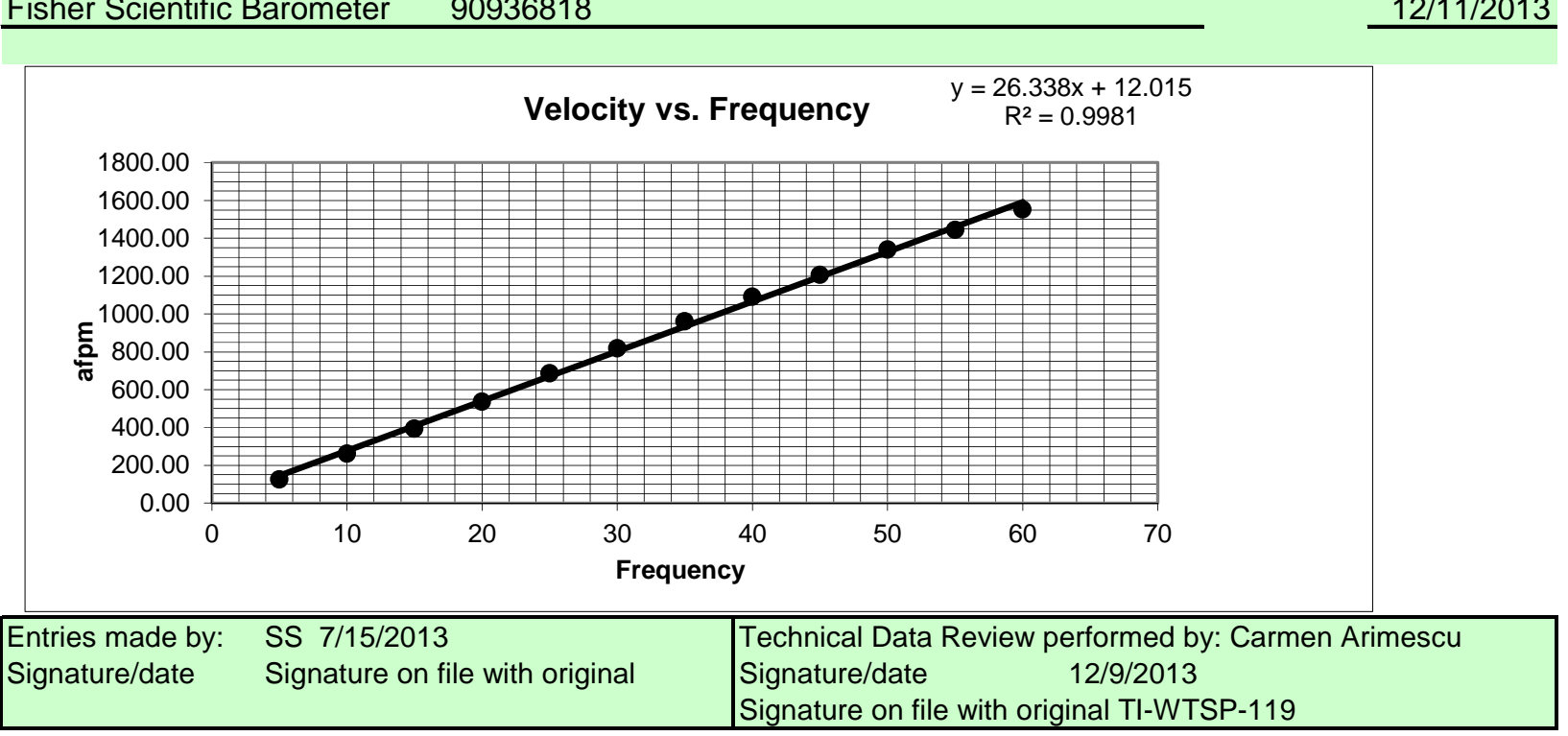

D.8 
VELOCITY vS. FREQUENCY DATA FORM

\begin{tabular}{|c|c|c|c|c|c|c|c|c|c|}
\hline & Site & LV-S & nodel & & Run No. & F-3 & & & \\
\hline & Date & $7 / 1$ & 013 & & ck Temp & & 84. & & \\
\hline & Tester & & & & Ick RH\% & & 210 & & \\
\hline & Stack Dia. & 11.922 & in. & & ro Press & & 29.9 & & \\
\hline & Stack X-Area & 111.6 & in2 & Fan Con & iguration & & $\overline{\text { Fans }}$ & $3 \& C$ & \\
\hline & Test Port & 2 & & Start/ & nd Time & & 74421 & 0816 & \\
\hline Dist. fro & om disturbance & 510.25 & inches & Reference & point from & veloc & ity test & VC: & Bottom 7 \\
\hline Velocity Readir & רgs,units = & & afpm & & & & & & \\
\hline & & & & & Target & & arget & Estmtd & \\
\hline & & & & & acfm & & afpm & $\mathrm{Hz}$ & \\
\hline & & & & & 695 & & 885 & 15.89 & $\max$ \\
\hline & & & & & 626 & & 797 & 14.63 & normal \\
\hline & & & & & 292 & & 372 & 8.53 & $\min$ \\
\hline $\mathrm{Hz}$ & 1 & 2 & 3 & Mean & StDev & $2 \mathrm{Stt}$ & Dev & $\mathrm{cfm}$ & \\
\hline 5 & 191 & 201 & 189 & 193.67 & 6.43 & 12. & & 150.13 & \\
\hline 10 & 495 & 522 & 507 & 508.00 & 13.53 & 27. & & 393.81 & \\
\hline 15 & 827 & 841 & 822 & 830.00 & 9.85 & 19. & & 643.43 & \\
\hline 20 & 1138 & 1149 & 1151 & 1146.00 & 7.00 & 14. & & 888.40 & \\
\hline 25 & 1416 & 1487 & 1453 & 1452.00 & 35.51 & 71. & & 1125.62 & \\
\hline 30 & 1803 & 1883 & 1839 & 1841.67 & 40.07 & 80. & & 1427.70 & \\
\hline 35 & 2168 & 2210 & 2193 & 2190.33 & 21.13 & 42. & & 1697.99 & \\
\hline 40 & 2499 & 2543 & 2623 & 2555.00 & 62.86 & 125 & & 1980.69 & \\
\hline 45 & 2897 & 2944 & 2910 & 2917.00 & 24.27 & 48. & & 2261.32 & \\
\hline 50 & 3307 & 3169 & 3203 & 3226.33 & 71.90 & 143 & & 2501.12 & \\
\hline 55 & 3664 & 3666 & 3551 & 3627.00 & 65.83 & 131 & & 2811.73 & \\
\hline 60 & 4076 & 4067 & 3963 & 4035.33 & 62.80 & 125 & & 3128.28 & \\
\hline Instuments Us & sed: & & & & & & & & al Exp. Date: \\
\hline TSI VelociCalc & T9 & 5351203 & & & & & & & $12 / 10 / 2013$ \\
\hline Fisher Scientifi & ic Barometer & 909368 & & & & & & & $12 / 11 / 2013$ \\
\hline & & & ocity vs & requenc & & $\begin{aligned}= & 69.66 \\
& \mathrm{R}^{2}=\end{aligned}$ & 0.9989 & & \\
\hline 4500.00 & & & & & & & & & \\
\hline 4000.00 & & & & & & E & & & \\
\hline 3500.00 & & & & & & & 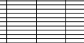 & & \\
\hline 3000.00 & & & & & & & 楱 & & \\
\hline$\varepsilon^{2500.00}$ & & & & & & & 輷基 & & \\
\hline$\frac{0}{\sigma} 2000.00$ & & & & & 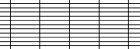 & & $\bar{E}$ & & \\
\hline 1500.00 & & & 击 & 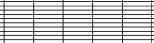 & 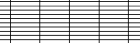 & 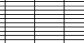 & 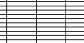 & & \\
\hline 1000.00 & 㐁 & & & 軍 & 青其楱 & & 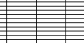 & & \\
\hline 500.00 & 11 & & 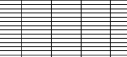 & 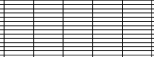 & 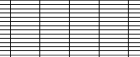 & 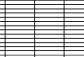 & 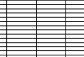 & & \\
\hline $0.00-$ & 10 & 20 & 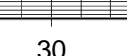 & 40 & 50 & 60 & & 70 & \\
\hline & & & Frequ & ncy & & & & & \\
\hline $\begin{array}{l}\text { Entries made by } \\
\text { Signature/date }\end{array}$ & $\begin{array}{r}\text { EA 7/15/201 } \\
\text { Signature }\end{array}$ & $\begin{array}{l}13 \\
\text { on file with }\end{array}$ & & $\begin{array}{l}\text { Technical } \\
\text { Signature/c } \\
\text { Signature }\end{array}$ & $\begin{array}{l}\text { ata Revien } \\
\text { ate } \\
\text { file with o }\end{array}$ & $\begin{array}{r}\text { perfor } \\
12 / 9 \\
\text { iginal }\end{array}$ & $\begin{array}{l}\text { med b) } \\
/ 2013 \\
\text { TI-WTs }\end{array}$ & $\begin{array}{l}\text { Carmen Al } \\
\text { P-119 }\end{array}$ & rimescu \\
\hline
\end{tabular}

D.9 


\section{VELOCITY vS. FREQUENCY DATA FORM}

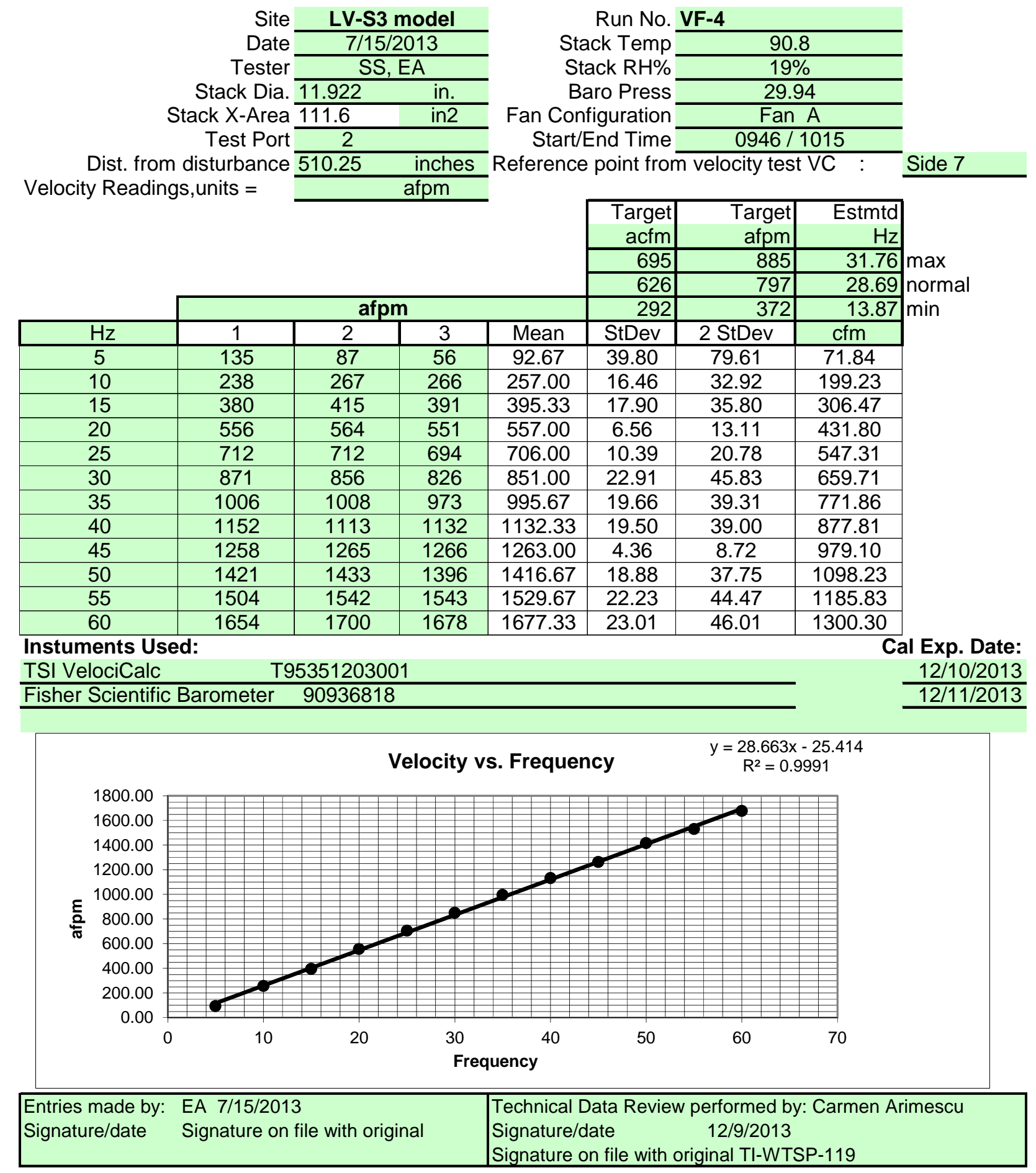




\section{VELOCITY vS. FREQUENCY DATA FORM}

\begin{tabular}{|c|c|c|}
\hline Site & \multicolumn{2}{|c|}{ LV-S3 model } \\
\hline Date & & \\
\hline Tester & \multicolumn{2}{|c|}{ SS, EA } \\
\hline Stack Dia. & 11.922 & in. \\
\hline Stack X-Area & 111.6 & in2 \\
\hline Test Port & 2 & \\
\hline
\end{tabular}

\begin{tabular}{rc} 
Run No. VF-5 \\
\cline { 2 - 2 } Stack Temp \\
\cline { 2 - 2 } Stack RH\% & 94.6 \\
\cline { 2 - 2 } Baro Press & $17 \%$ \\
\cline { 2 - 2 } Fan Configuration & Fans A\&C \\
Start/End Time & $1058 / 1131$
\end{tabular}

Dist. from disturbance $\overline{510.25 \quad \text { inches }}$ Reference point from velocity test VC : $\quad$ Side 7 Velocity Readings, units = afpm

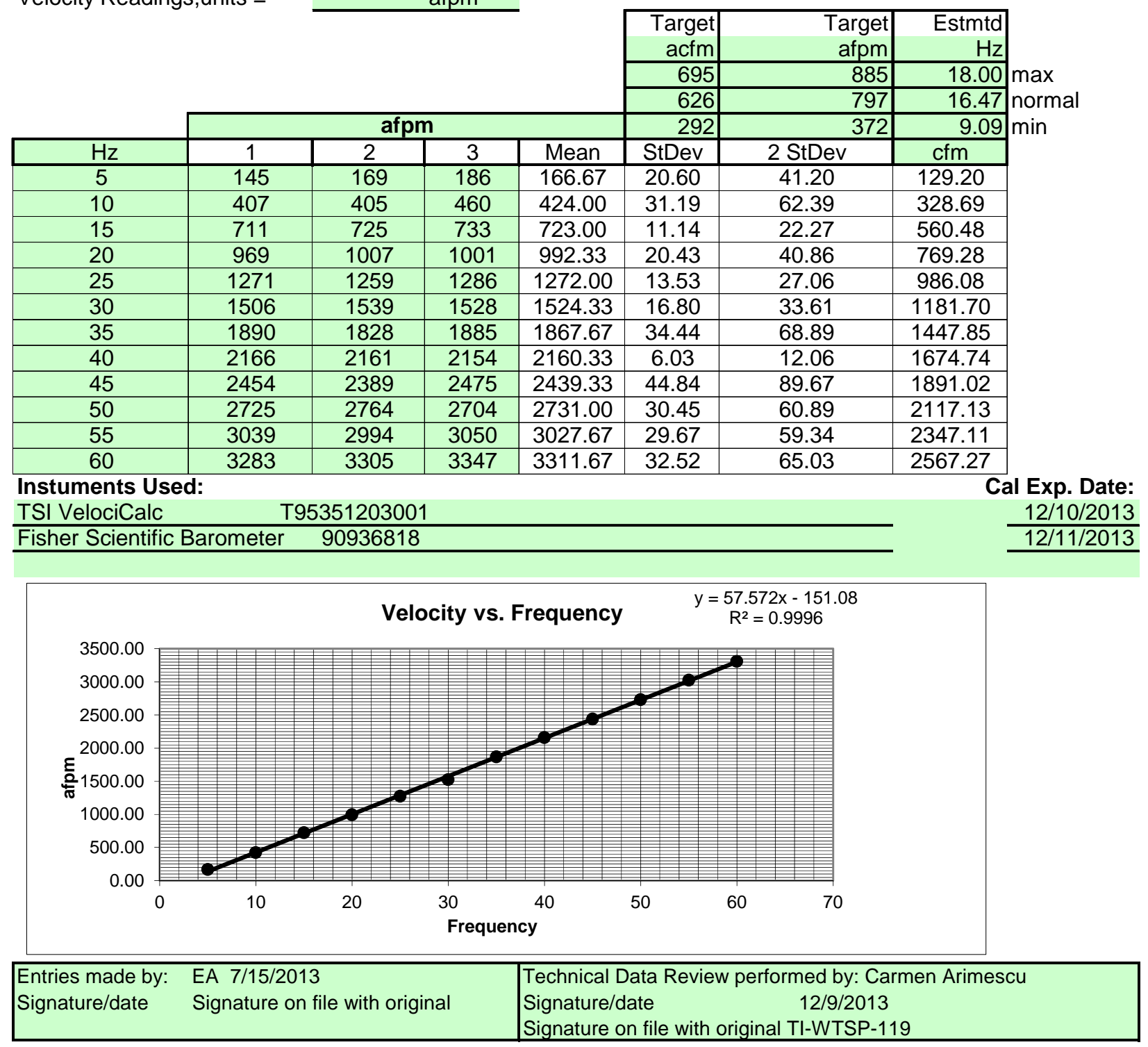




\section{VELOCITY vS. FREQUENCY DATA FORM}

\begin{tabular}{rcc} 
Site & \multicolumn{1}{c}{ LV-S3 model } \\
\cline { 2 - 2 } Date & \multicolumn{2}{c}{$7 / 15 / 2013$} \\
\cline { 2 - 2 } Tester & SS, TH. EA \\
\cline { 2 - 2 } Stack Dia. & 11.922 & in. \\
Stack X-Area & $\frac{111.6}{2}$ in2 \\
Test Port &
\end{tabular}

\begin{tabular}{rc} 
Run No. VF-6 \\
\cline { 2 - 2 } Stack Temp & 99.1 \\
Stack RH\% & $17 \%$ \\
Baro Press & 30 \\
\cline { 2 - 2 } Configuration & Fans A\&B \\
\cline { 2 - 2 } Start/End Time & $1405 / 1438$
\end{tabular}

Dist. from disturbance $\overline{510.25 \quad \text { inches }}$

Velocity Readings, units = afpm

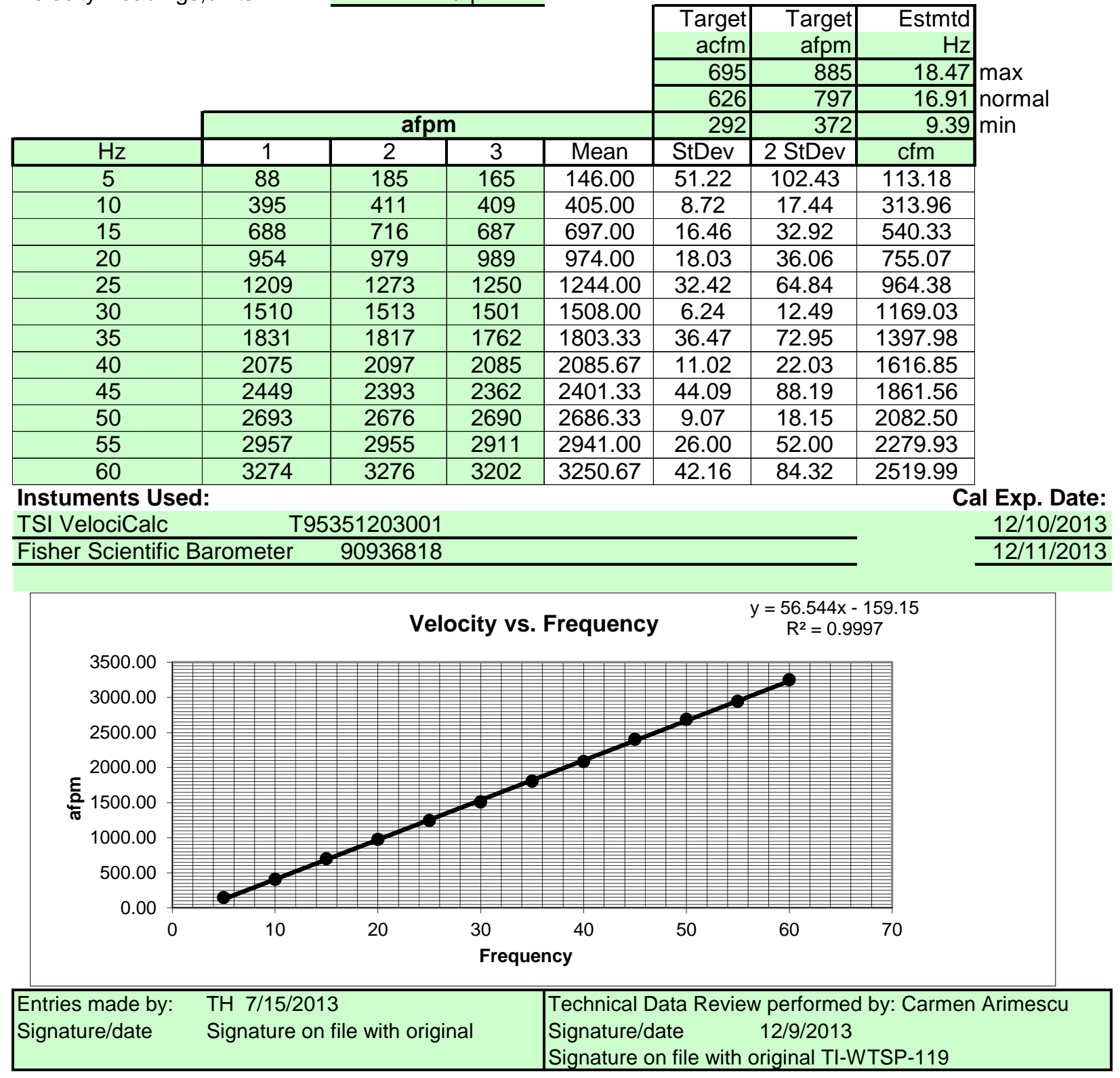




\section{D.2 LV-S3 Velocity Uniformity Data Sheets}

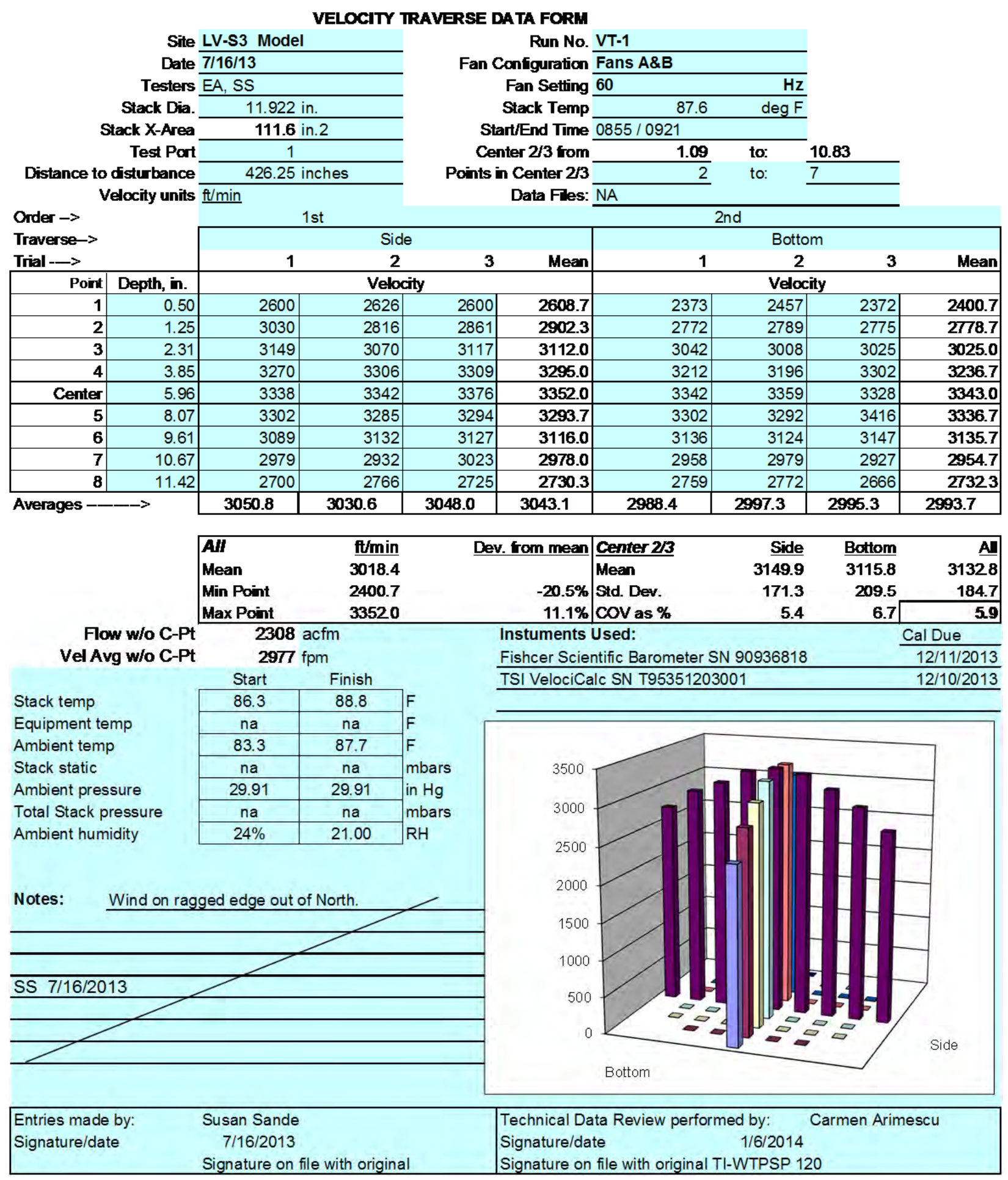


VELOCITY TRAVERSE DATA FORM

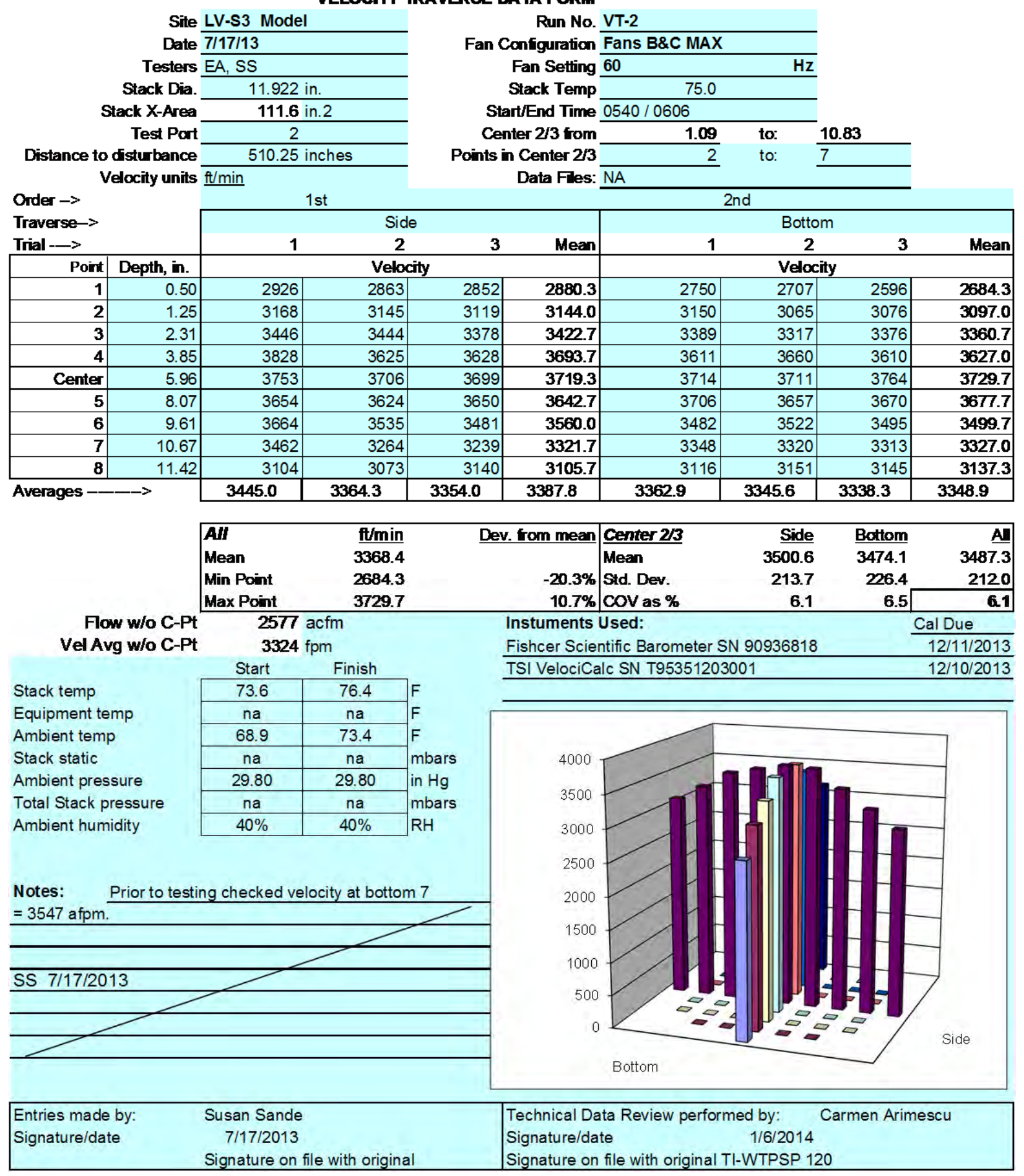


VELOCITY TRAVERSE DATA FORM

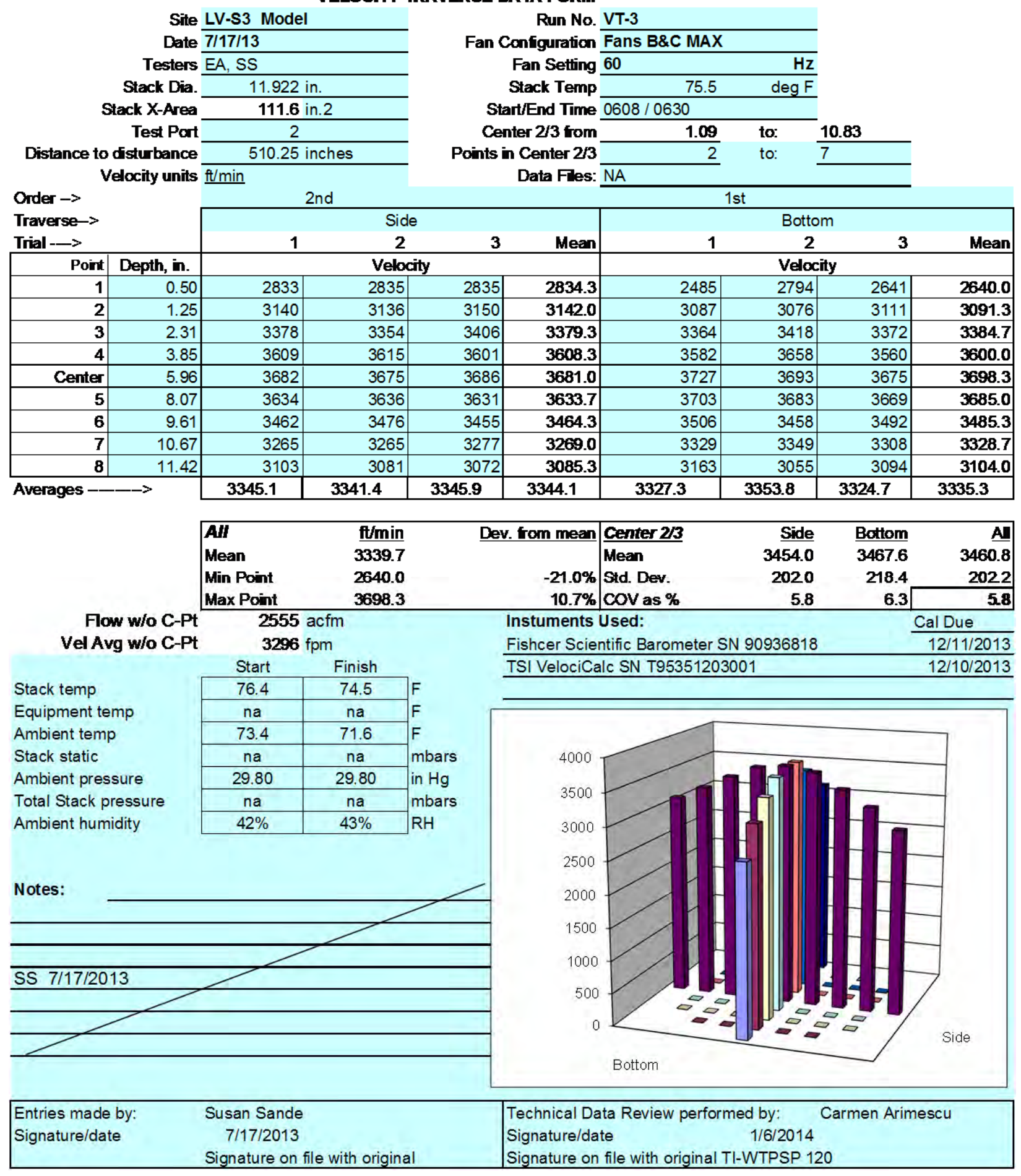


VELOCITY TRAVERSE DATA FORM

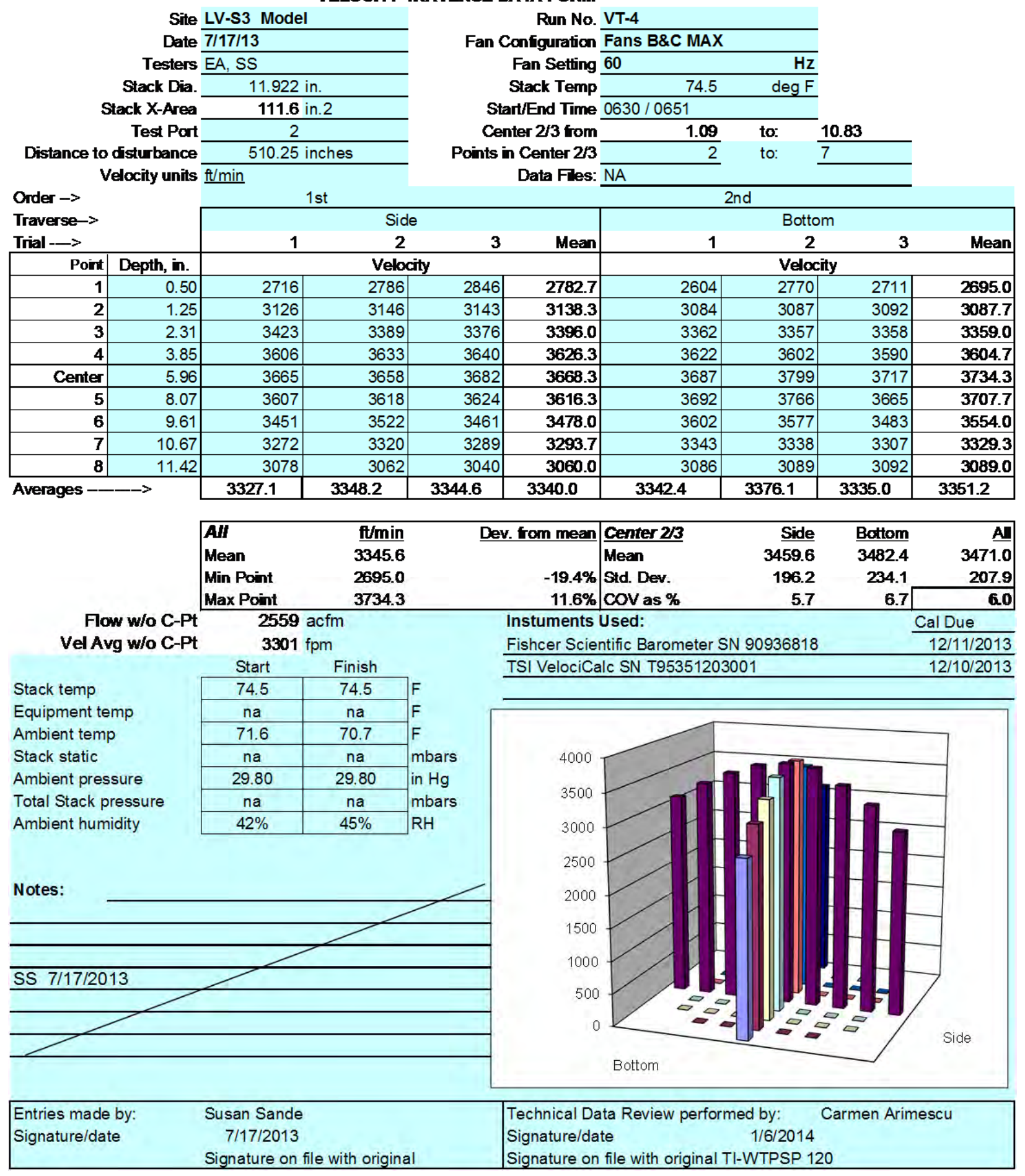


VELOCITY TRAVERSE DATA FORM

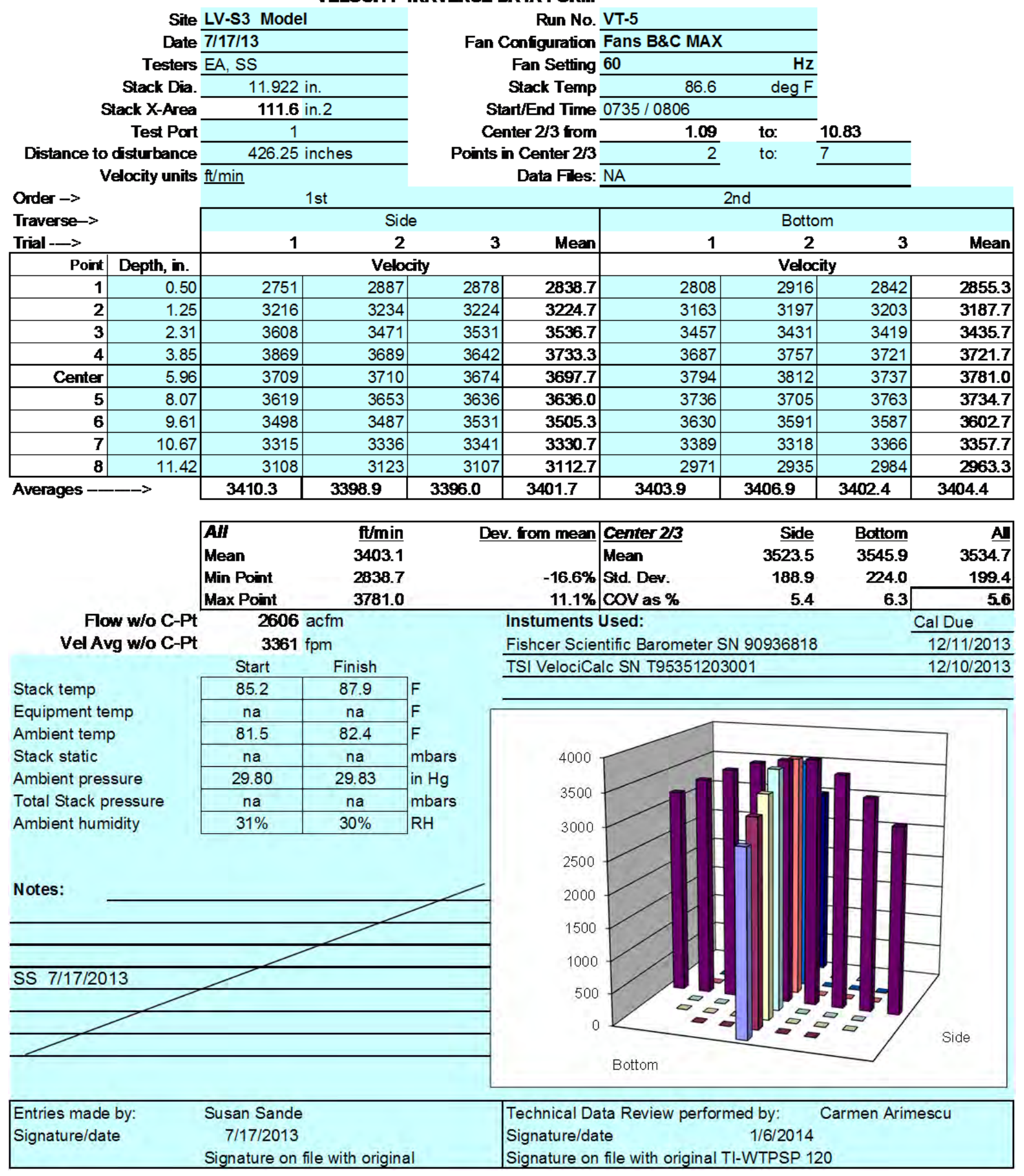


VELOCITY TRAVERSE DATA FORM

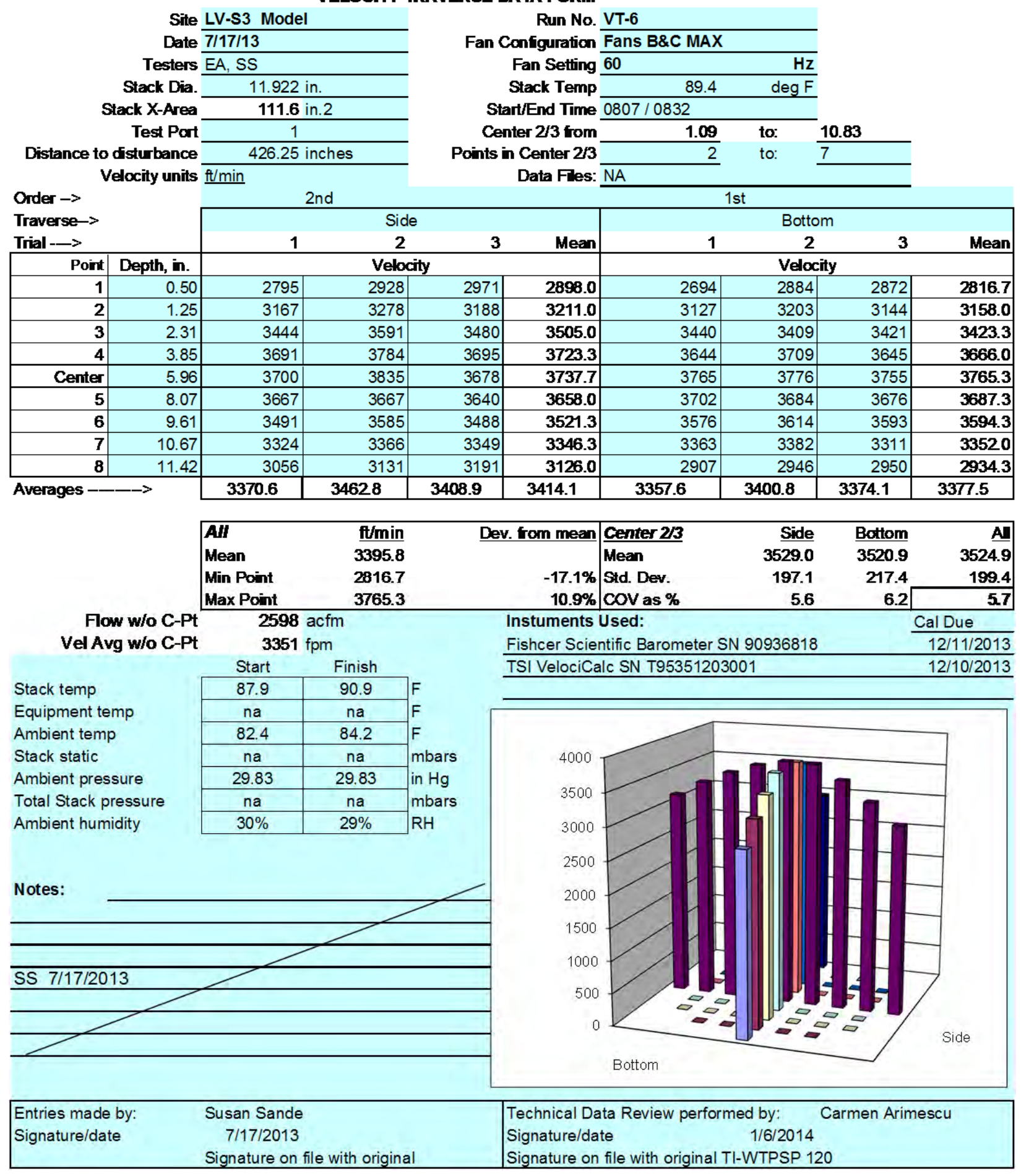


VELOCITY TRAVERSE DATA FORM

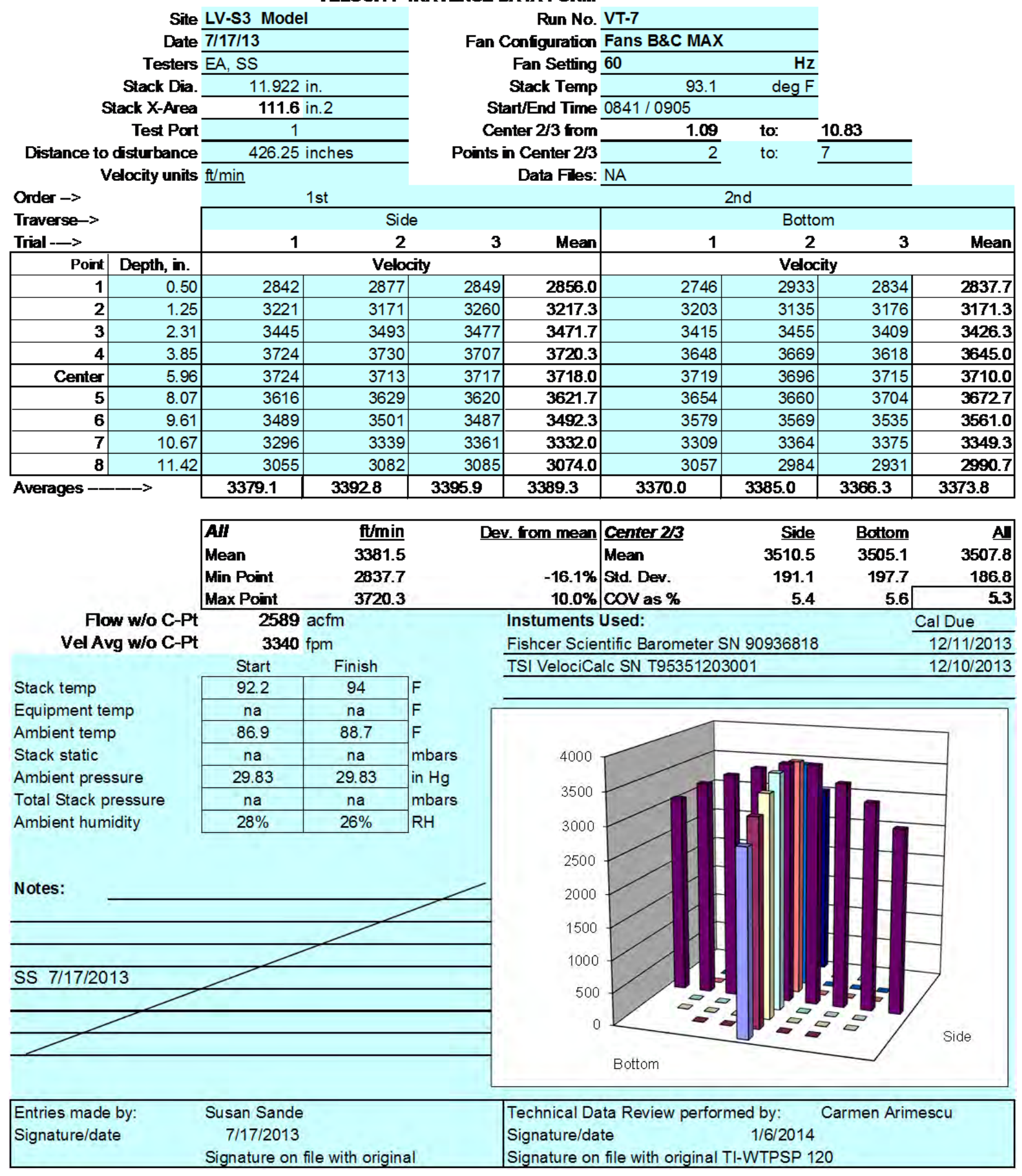


VELOCITY TRAVERSE DATA FORM

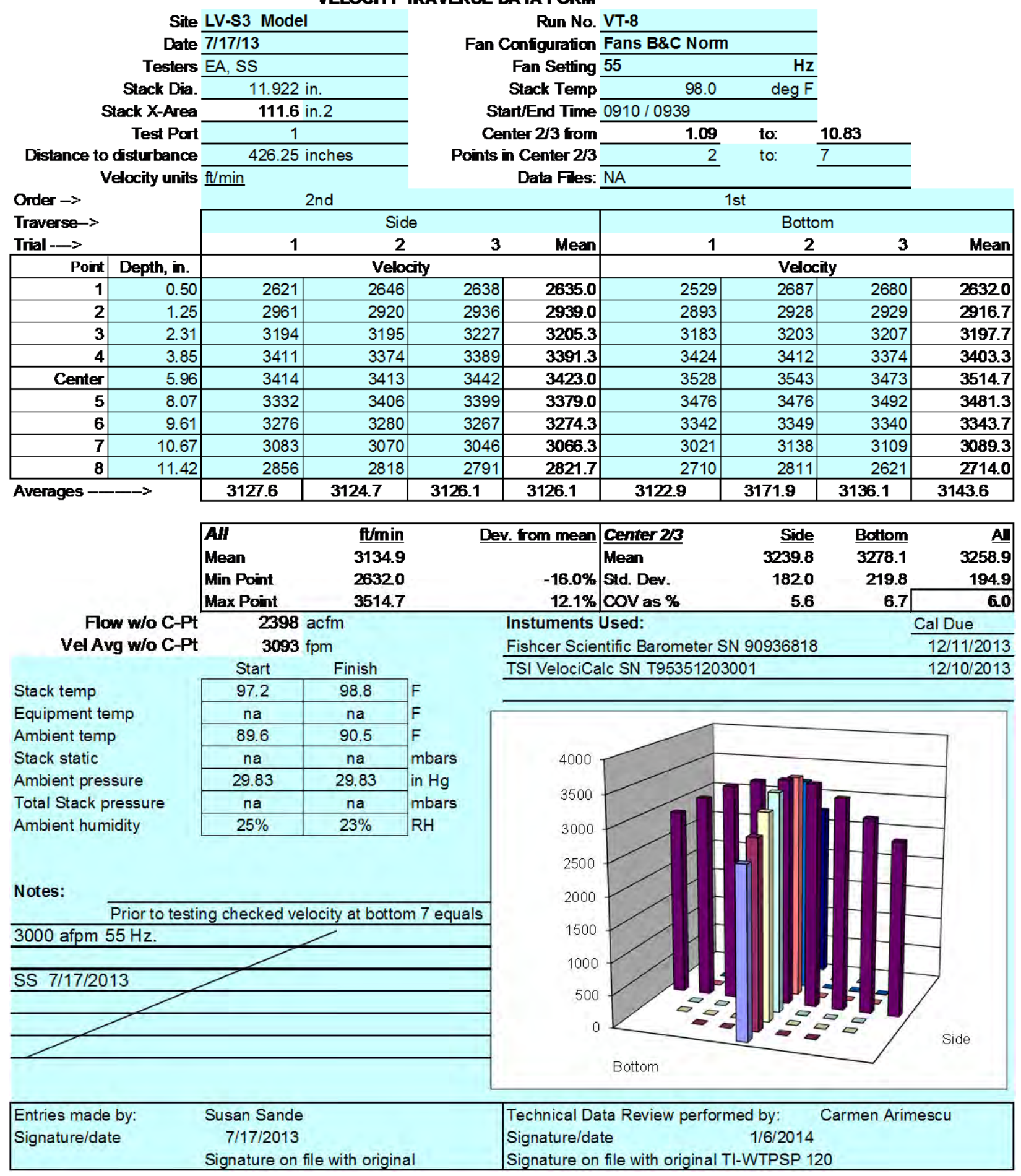


VELOCITY TRAVERSE DATA FORM

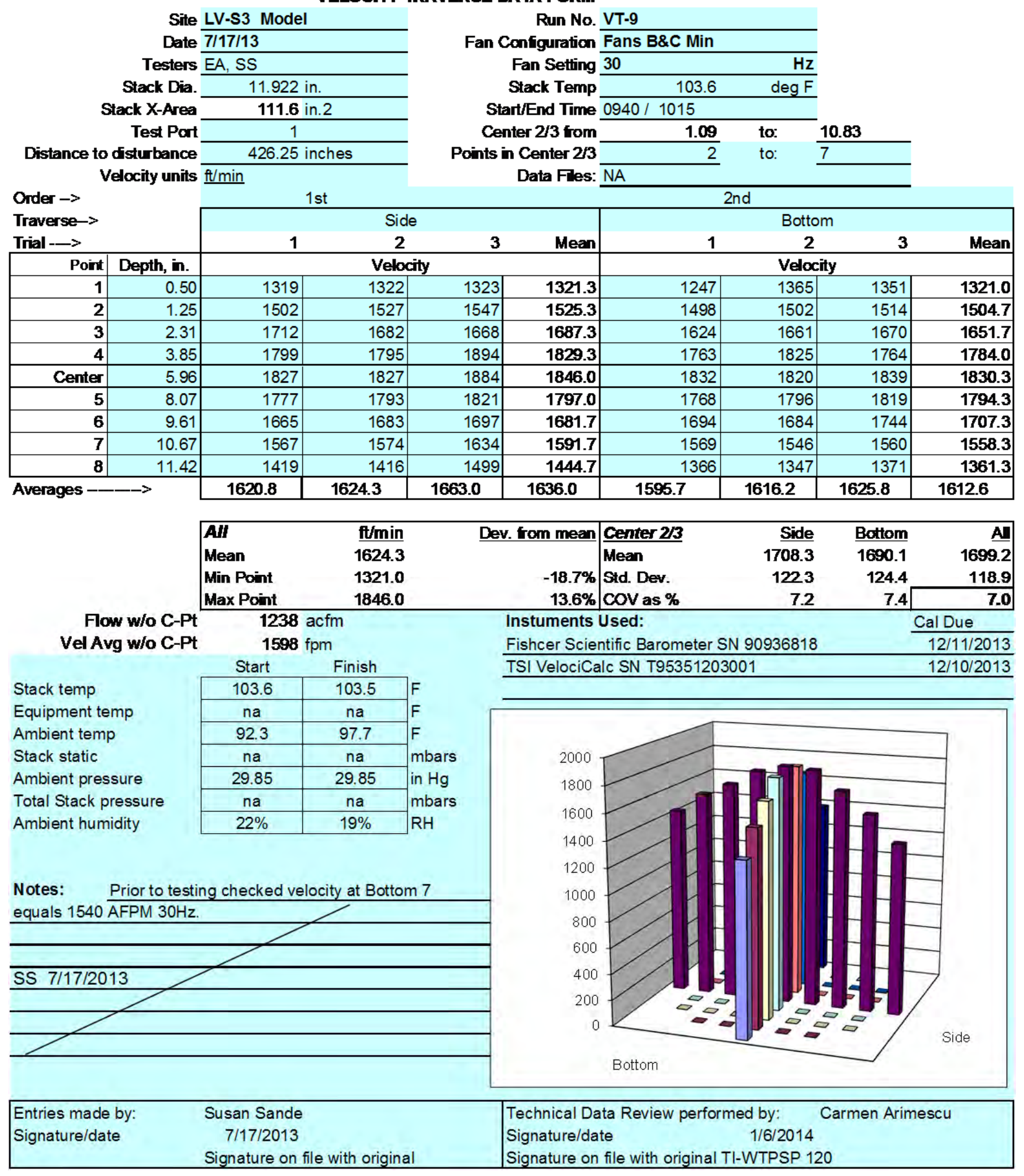


VELOCITY TRAVERSE DATA FORM

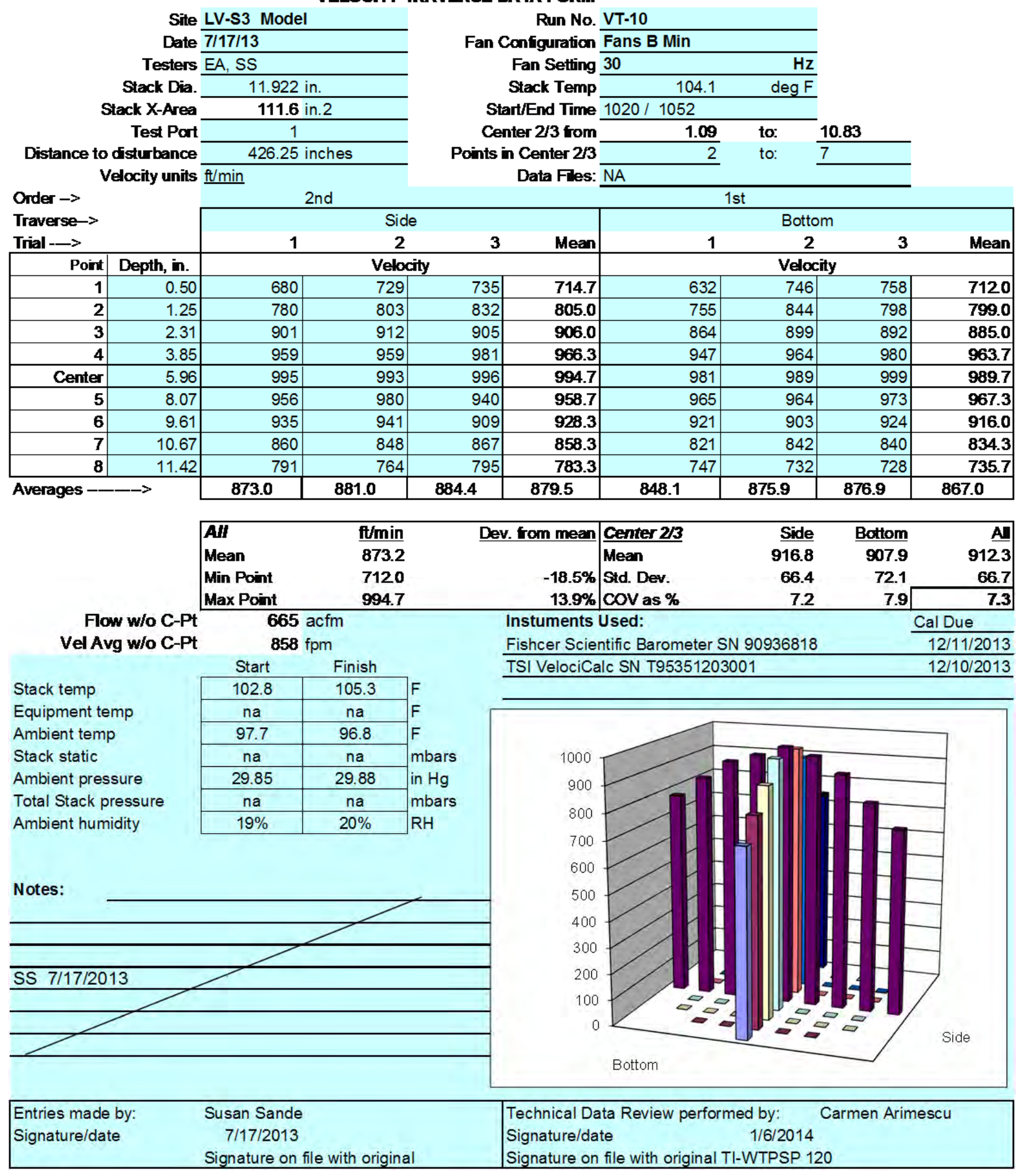


VELOCITY TRAVERSE DATA FORM

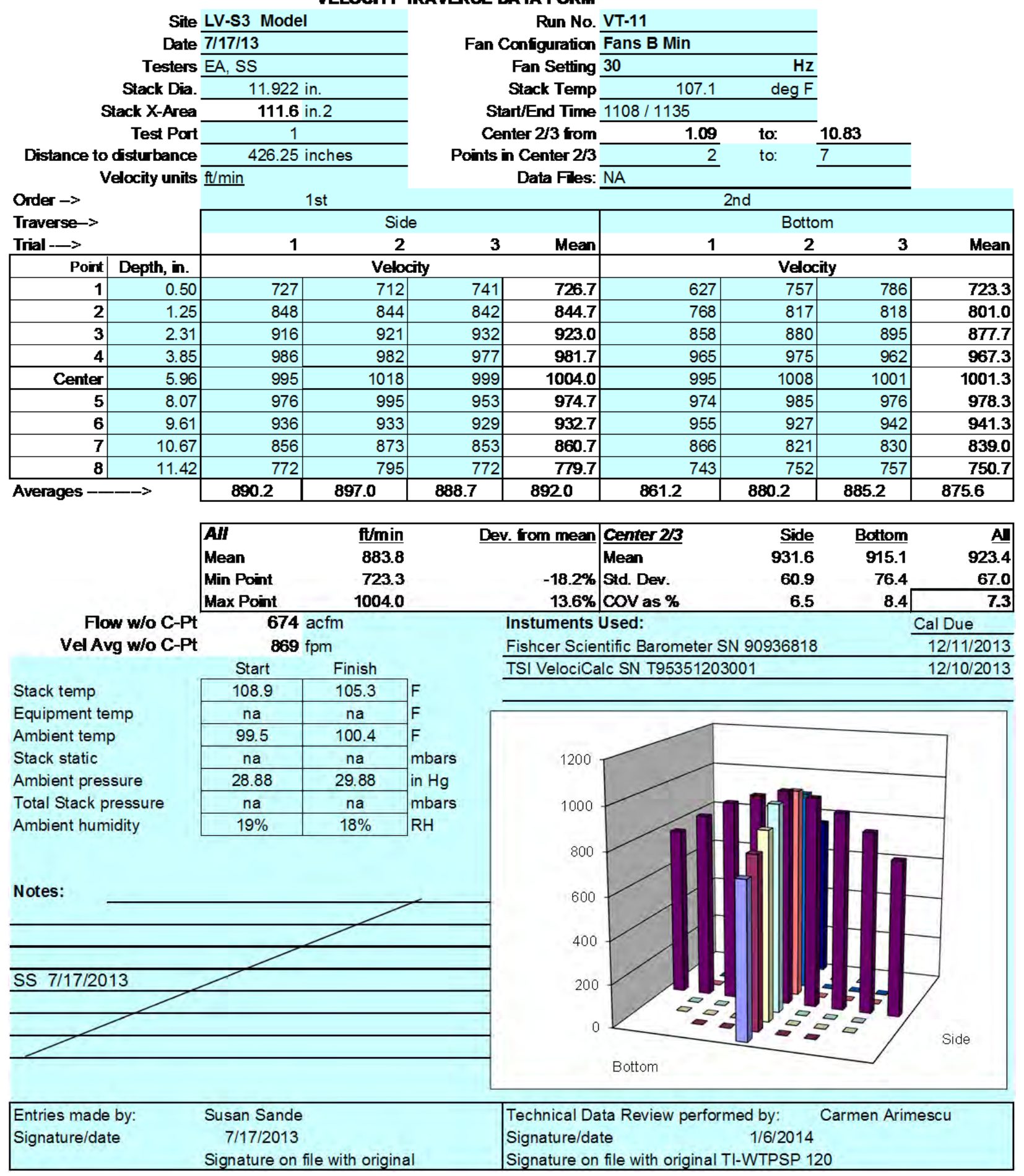


VELOCITY TRAVERSE DATA FORM

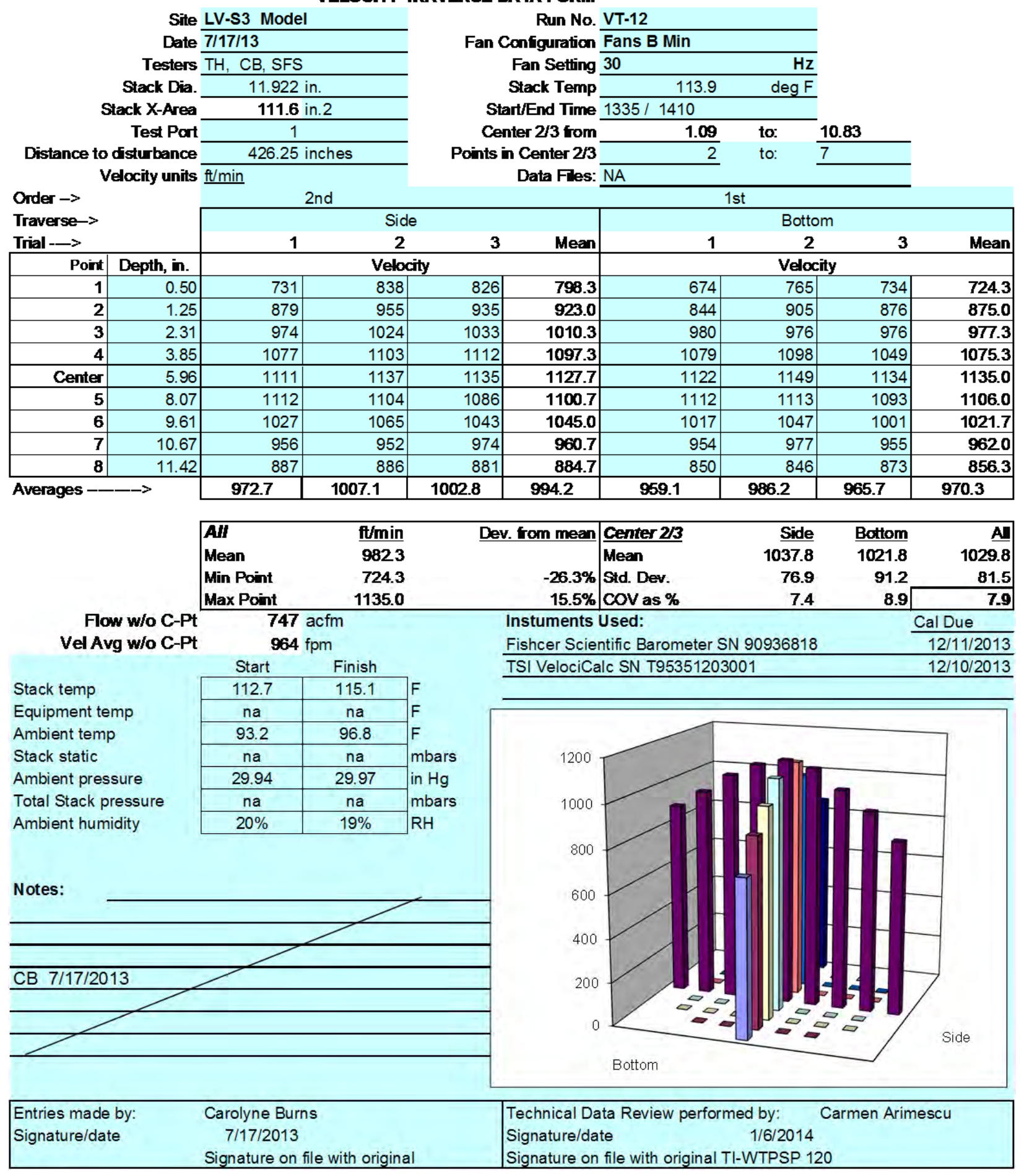


VELOCITY TRAVERSE DATA FORM

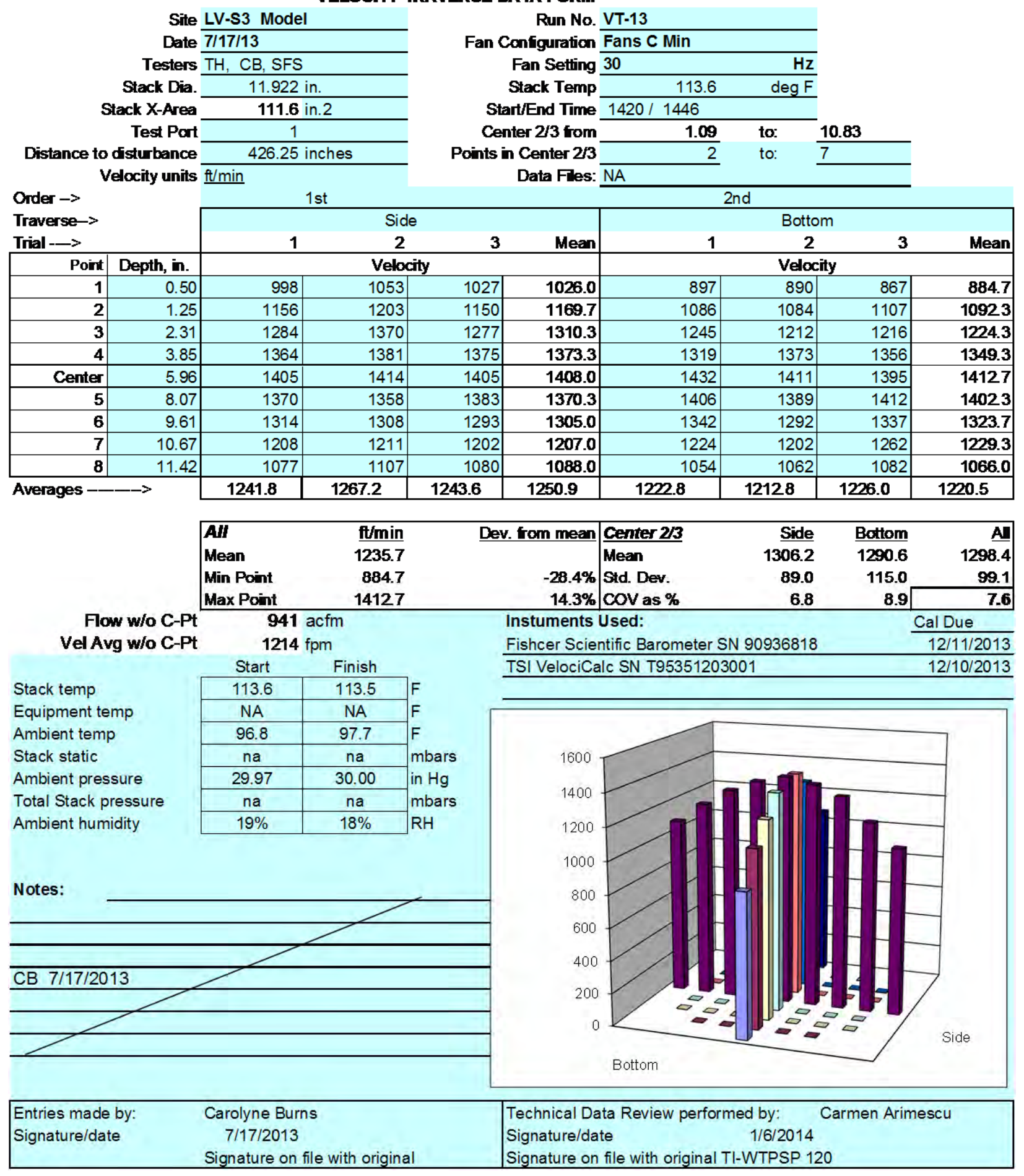


VELOCITY TRAVERSE DATA FORM

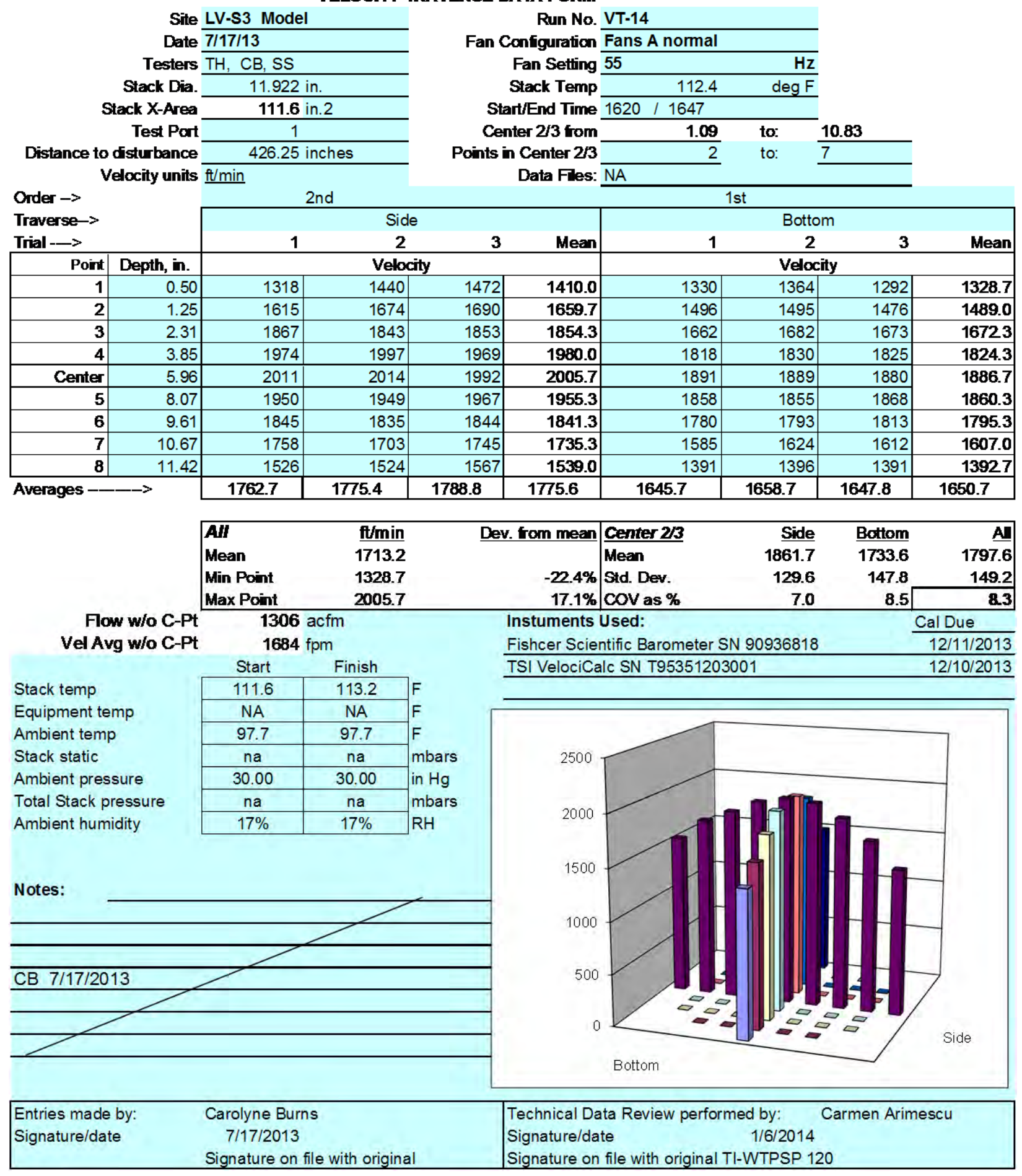


VELOCITY TRAVERSE DATA FORM

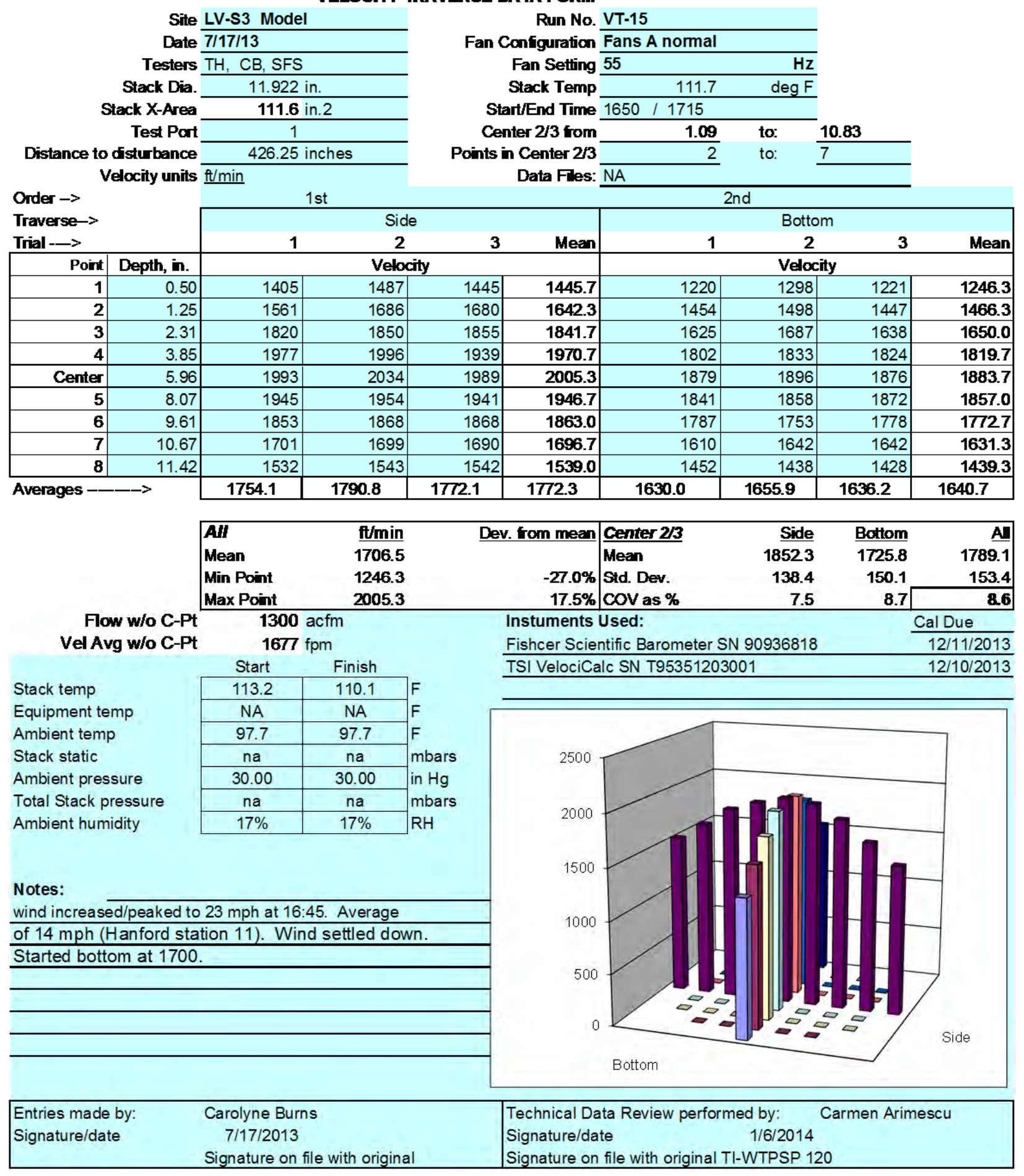




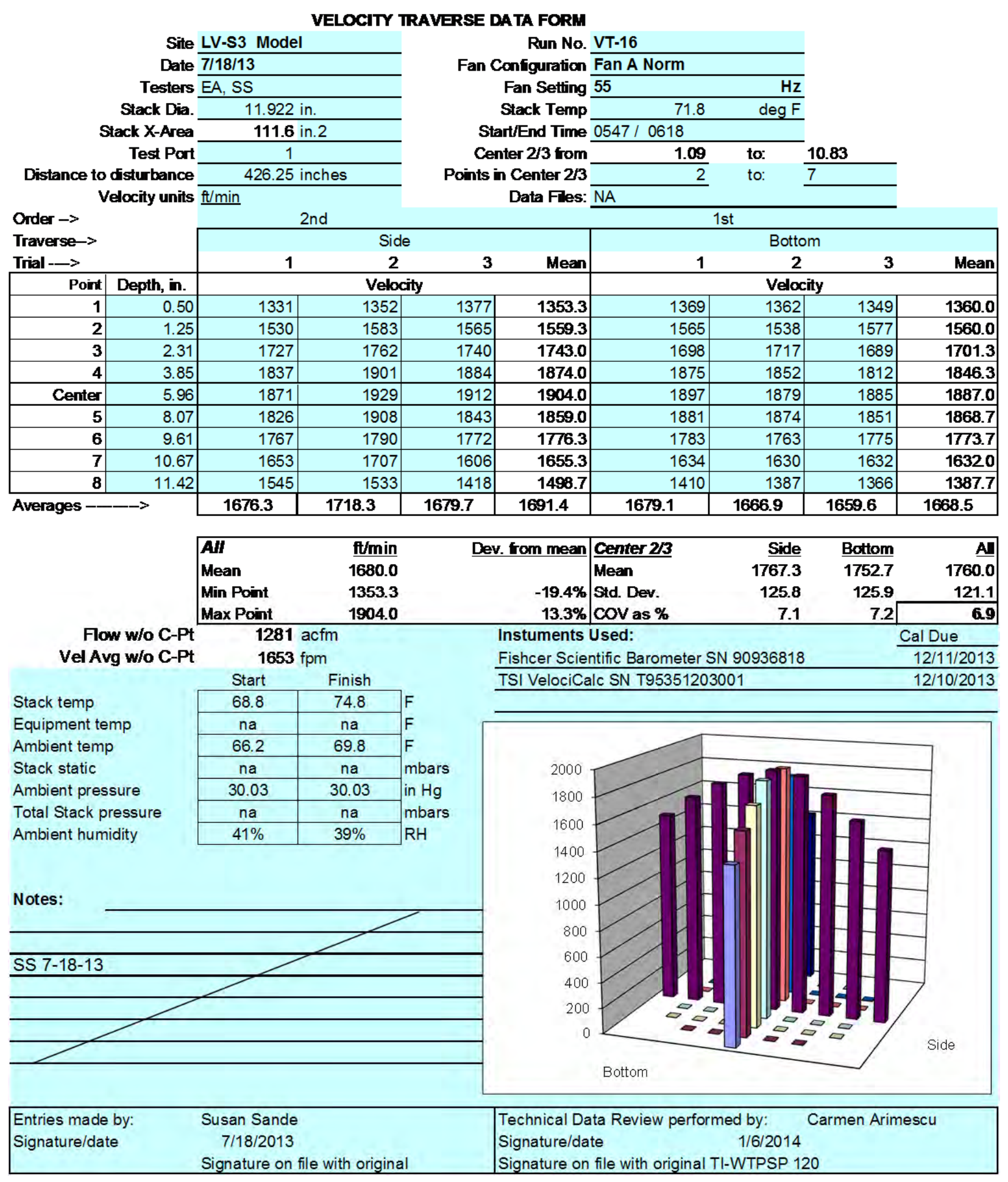




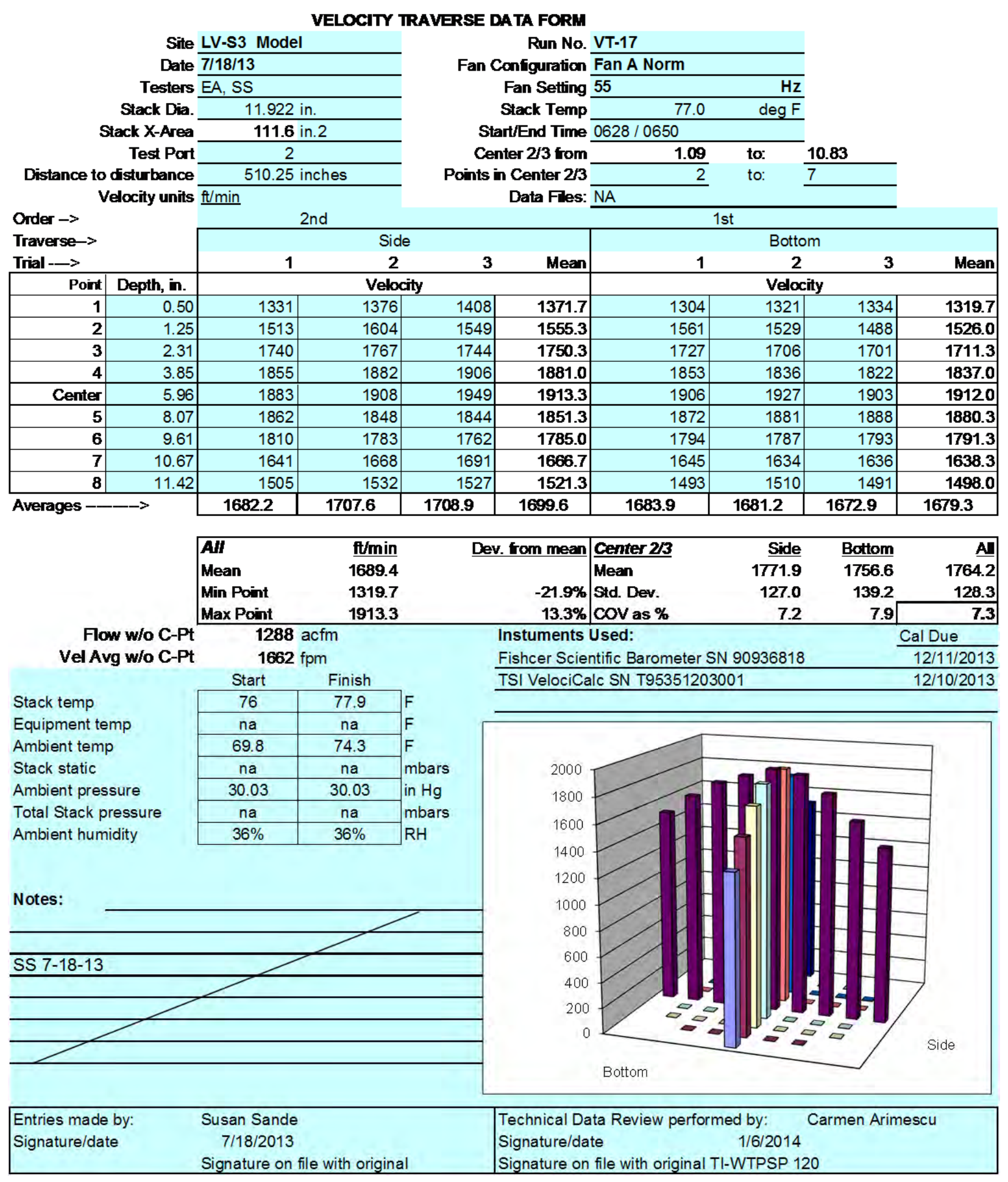


VELOCITY TRAVERSE DATA FORM

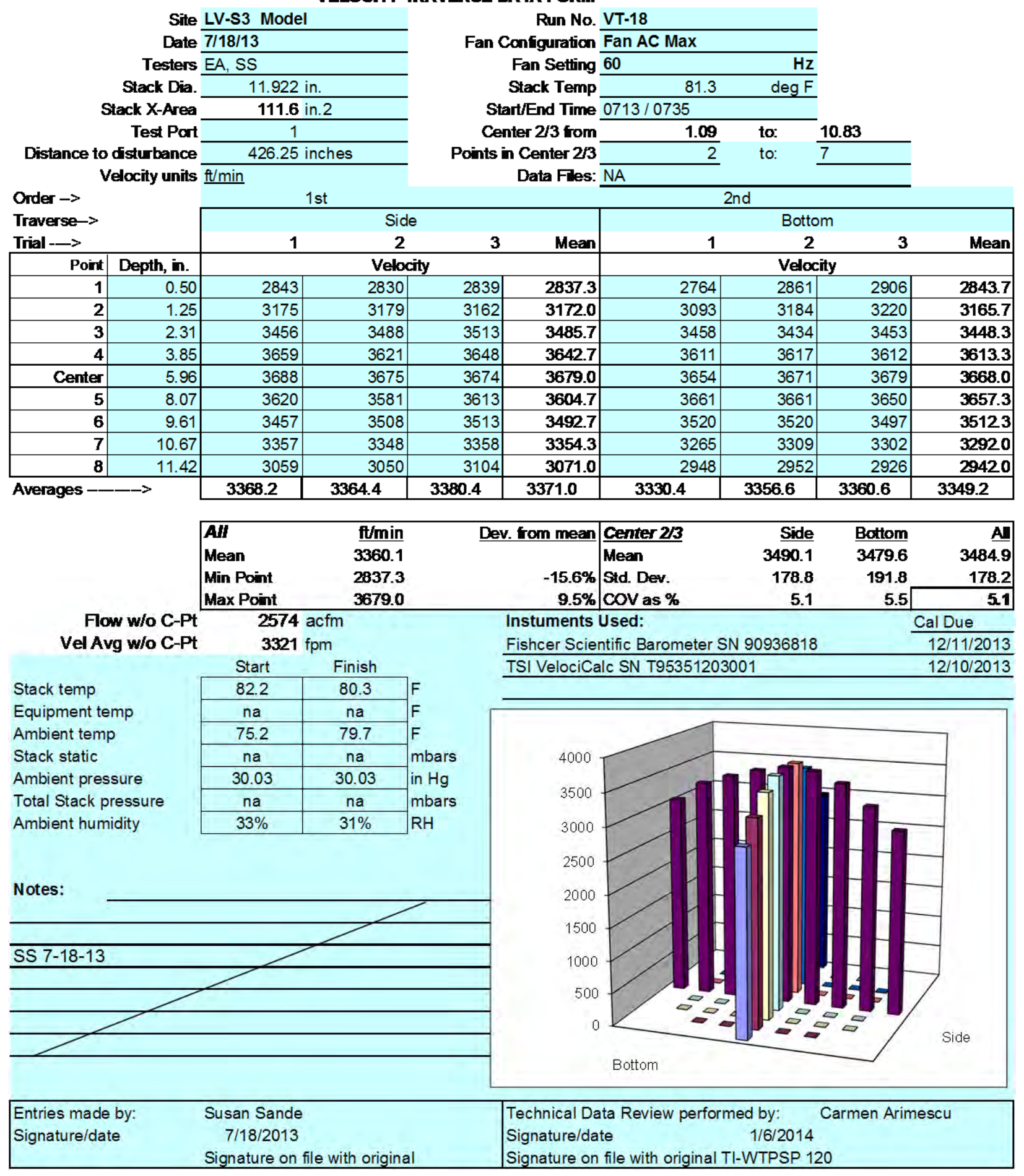




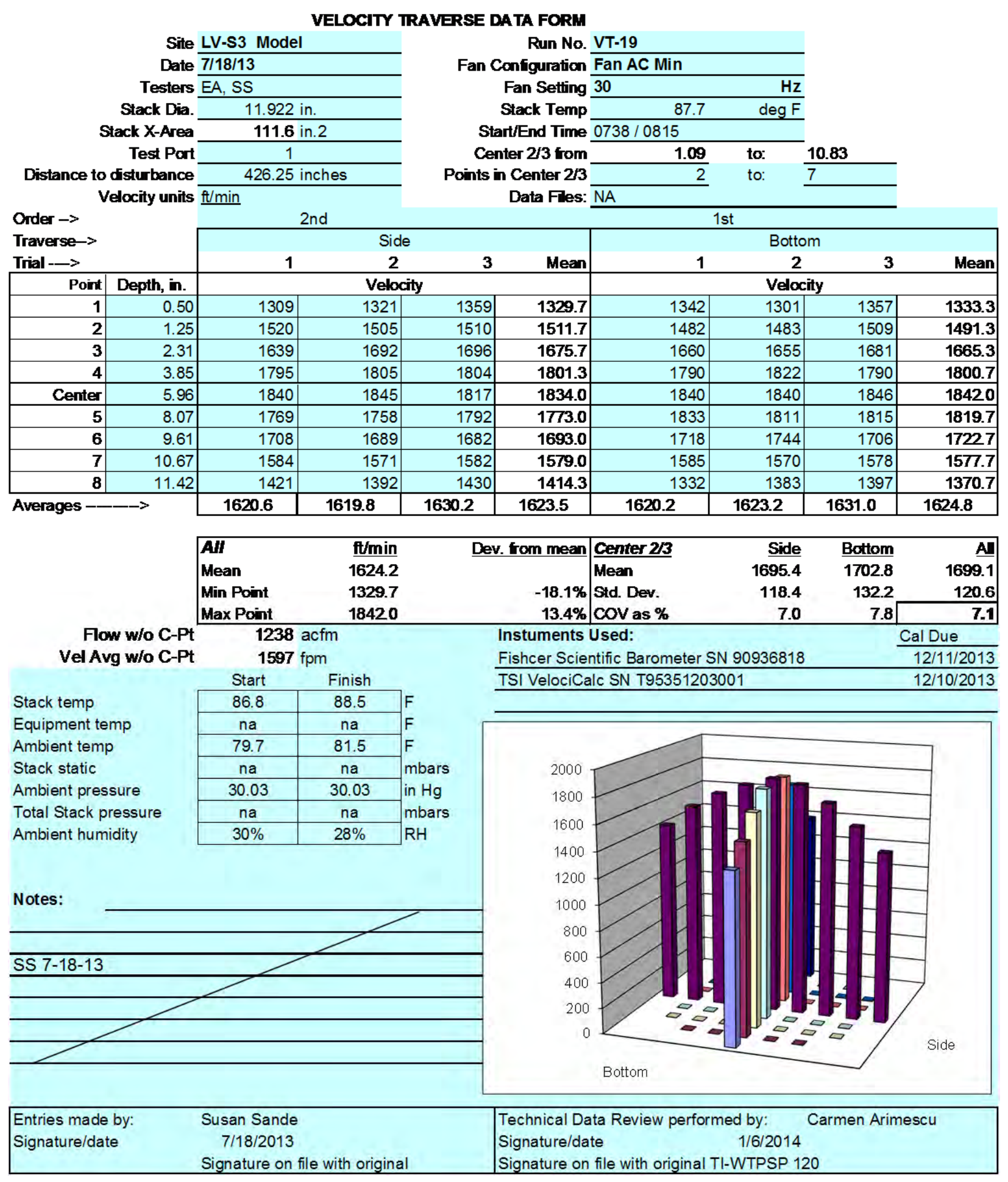


VELOCITY TRAVERSE DATA FORM

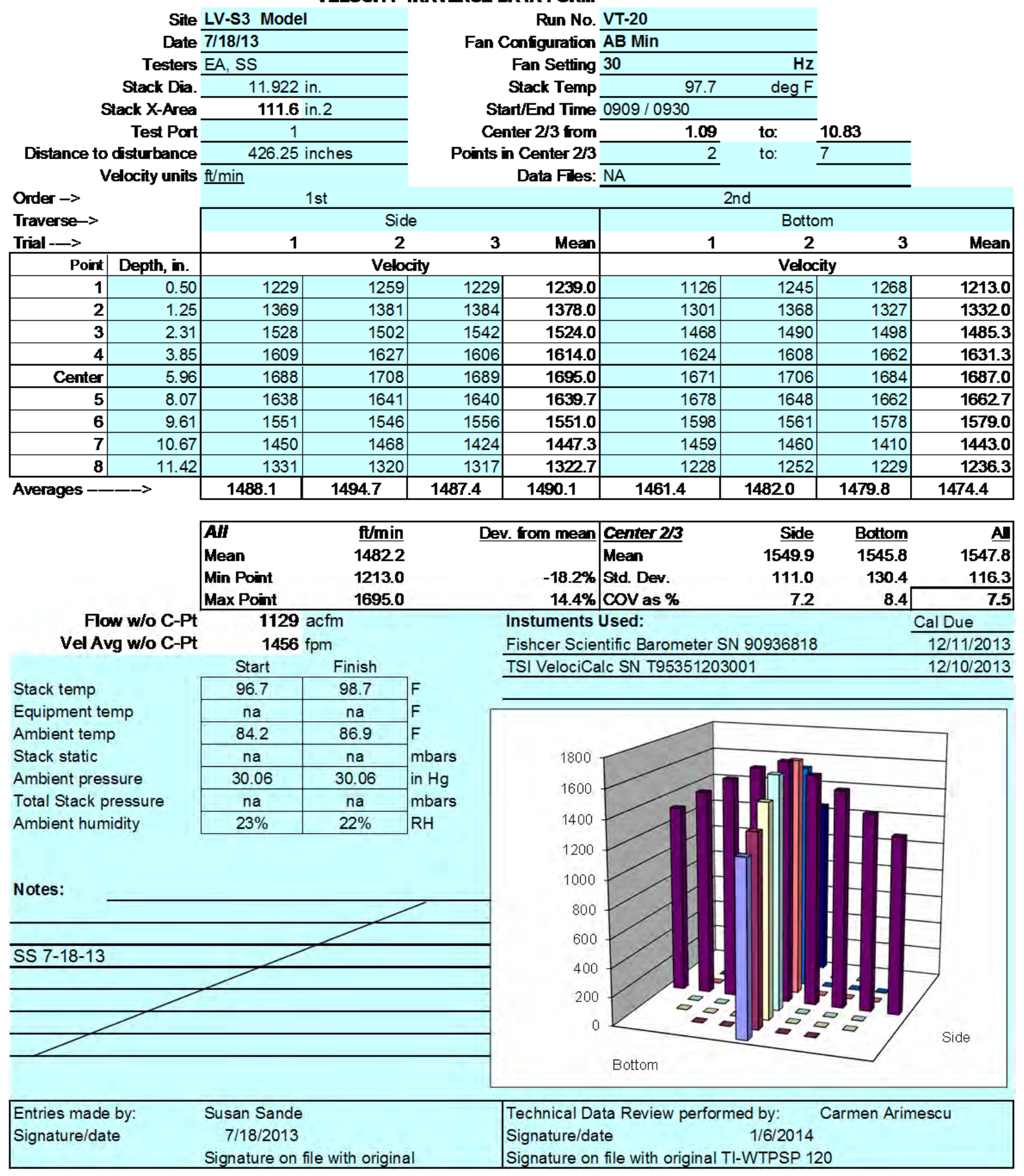


VELOCITY TRAVERSE DATA FORM

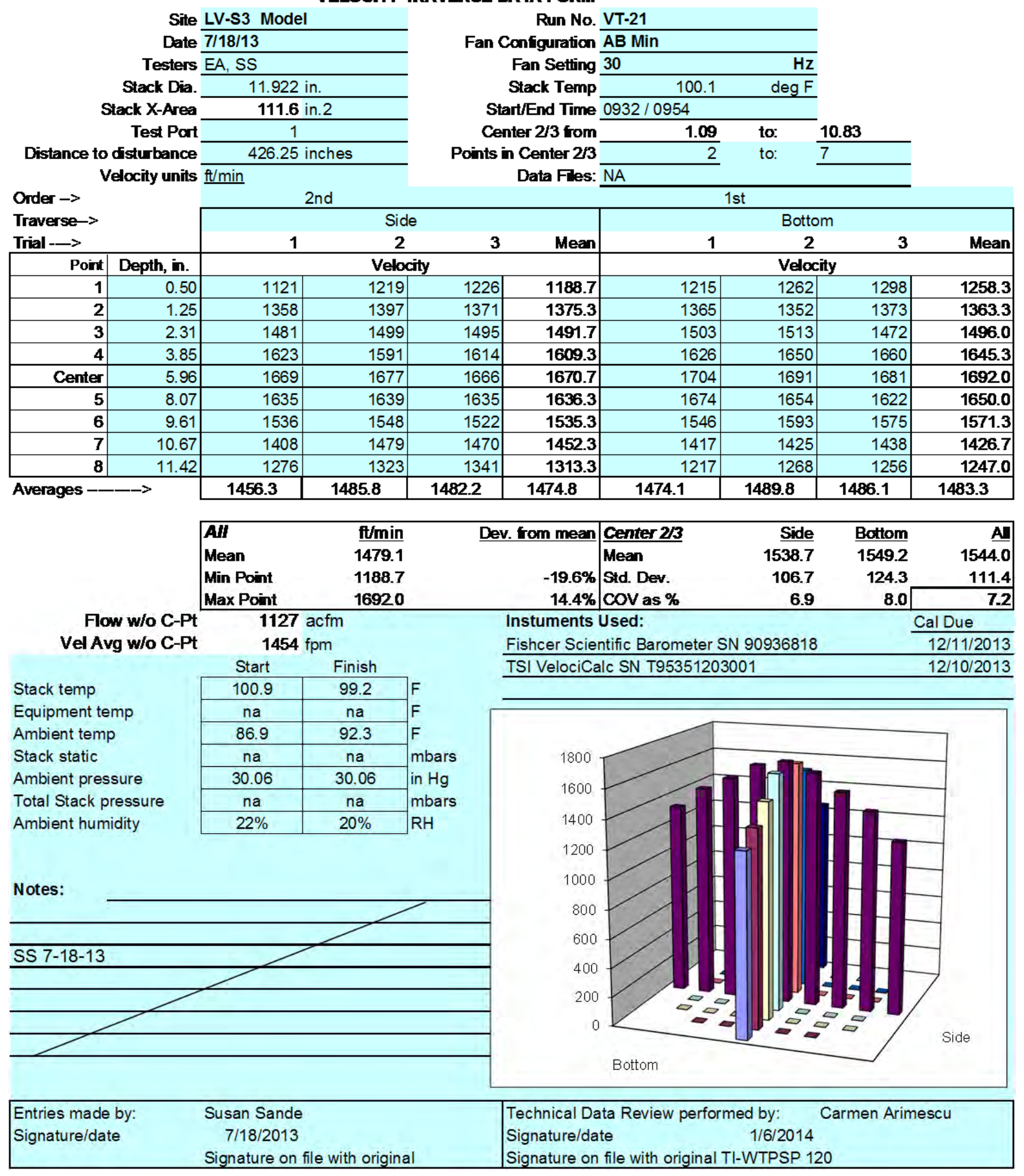


VELOCITY TRAVERSE DATA FORM

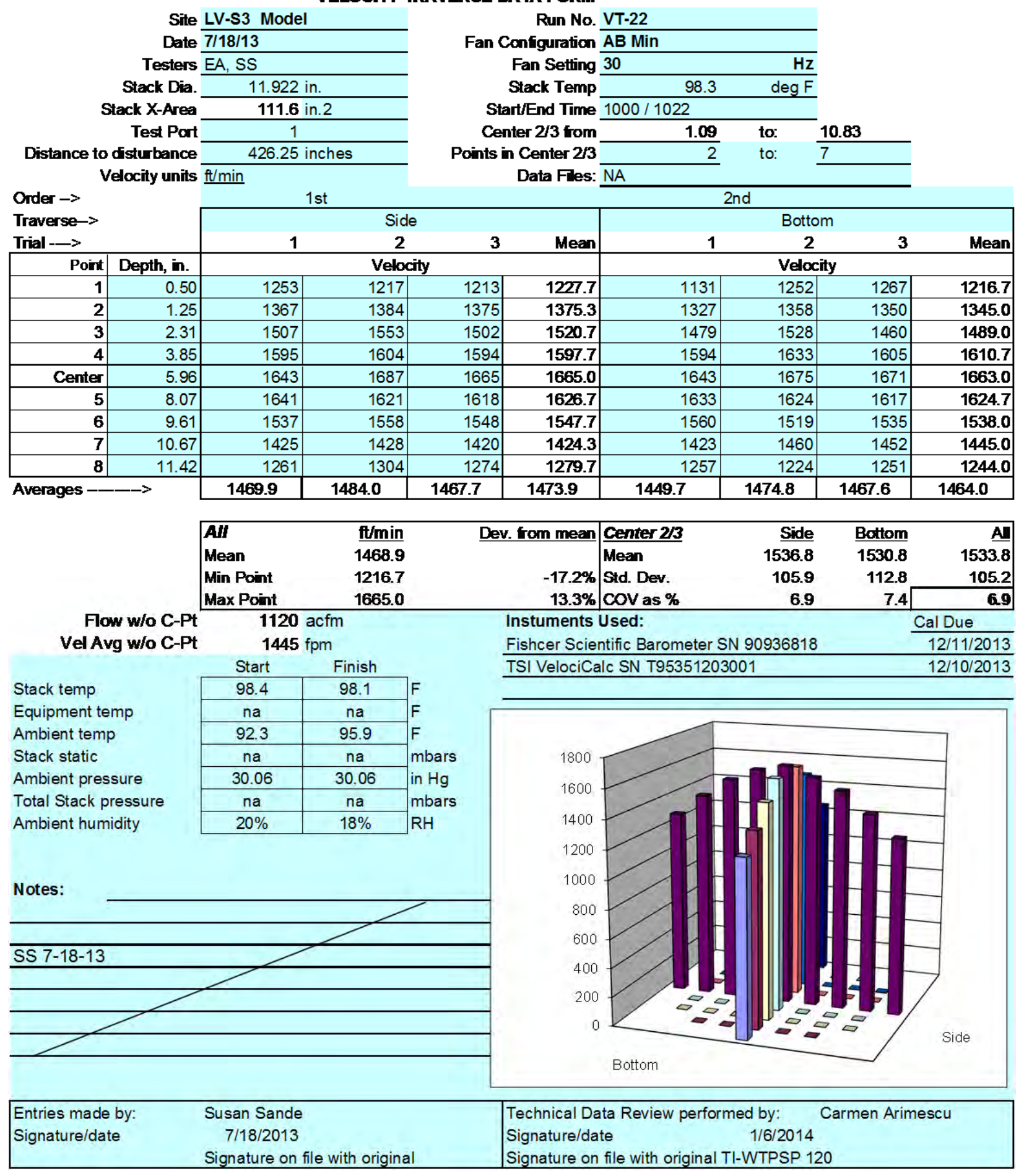




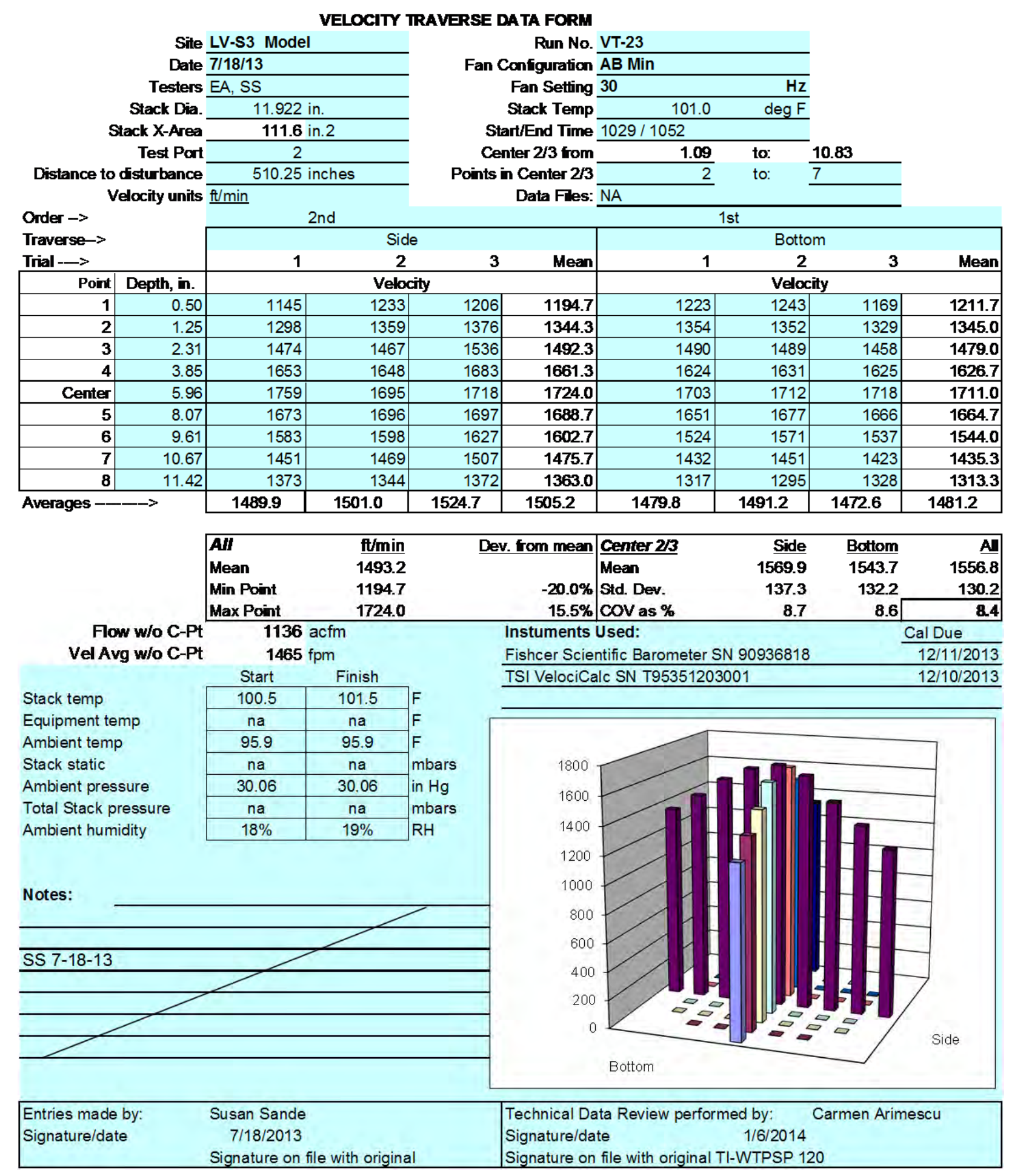


VELOCITY TRAVERSE DATA FORM

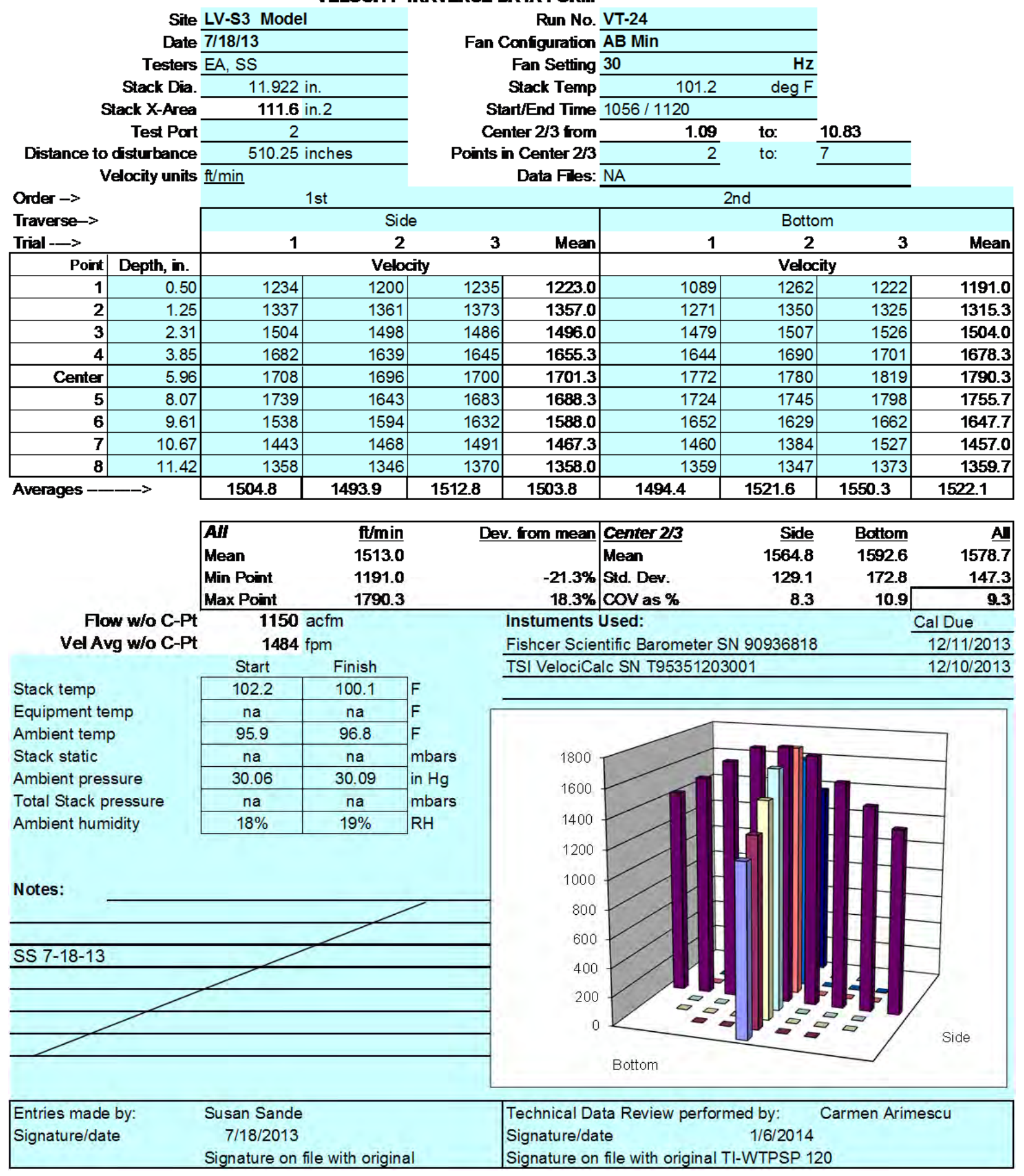


VELOCITY TRAVERSE DATA FORM

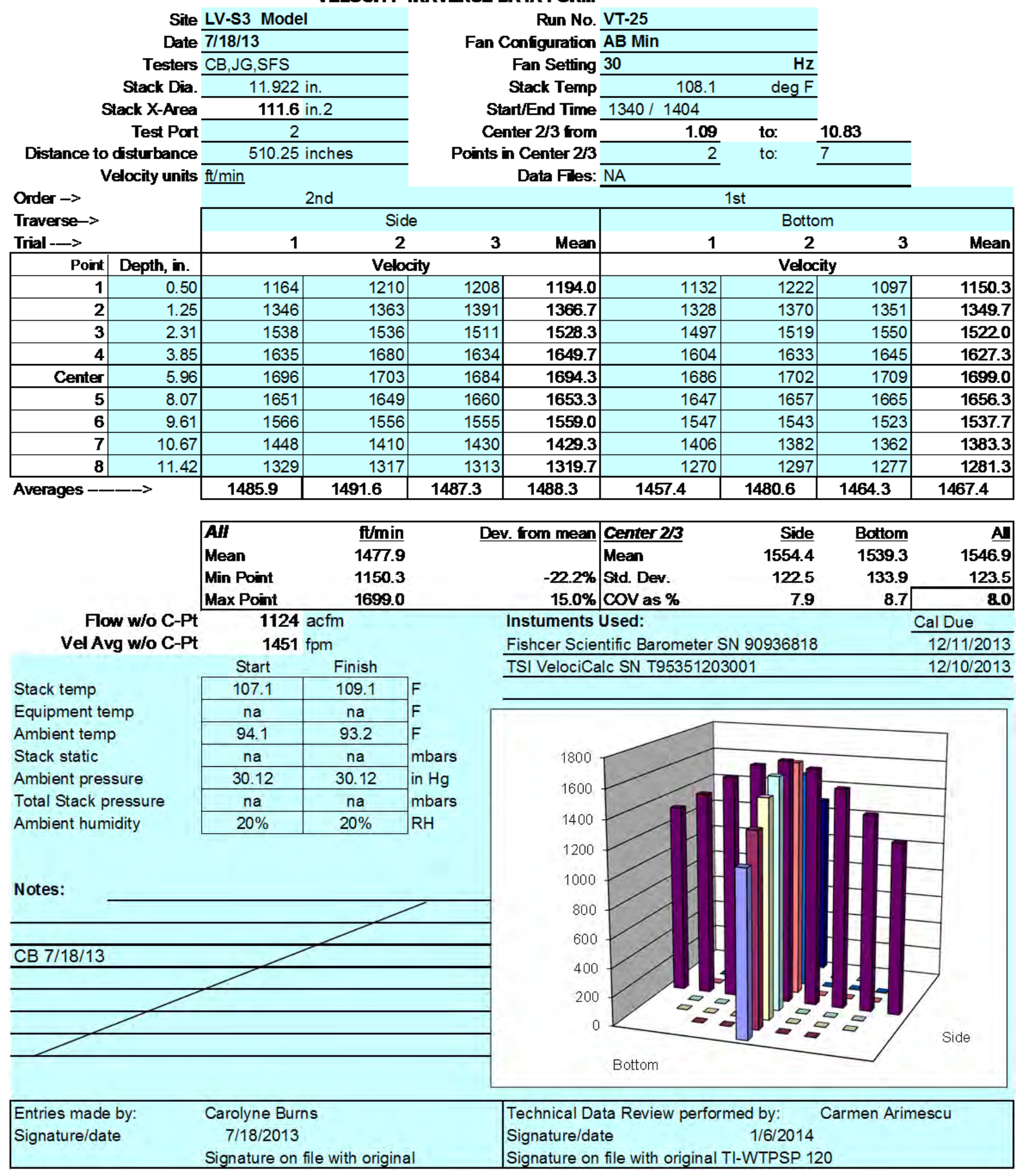


VELOCITY TRAVERSE DATA FORM

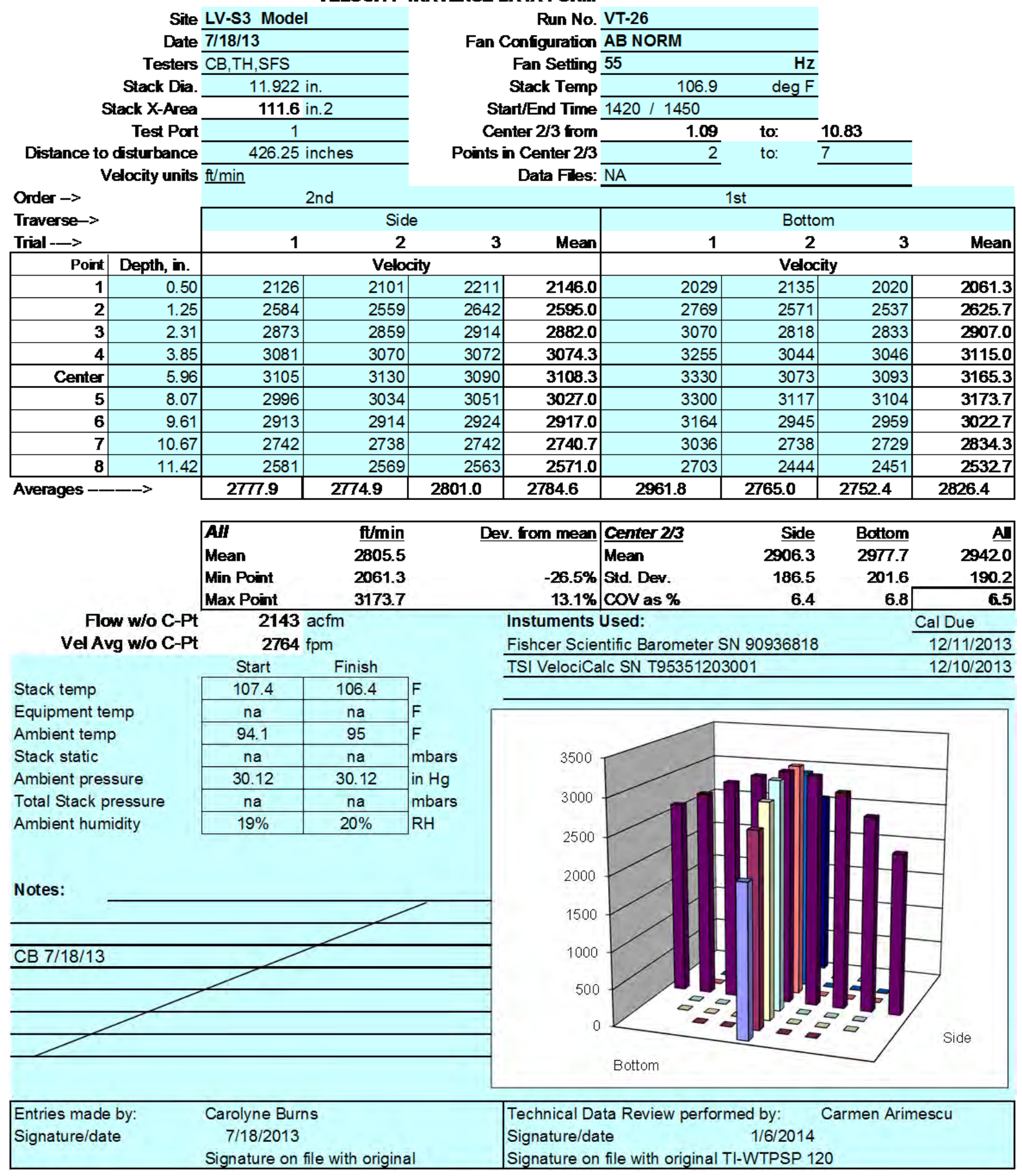




\section{D.3 LV-S3 Flow Angle Data Sheets}

TI-WTPSP-121

Page

\section{FLOW ANGLE DATA FORM}

Site LV-S3 Scale Model

Date 7/18/2013

Tester cb,th, jg, sfs

Stack Dia. 11.922

Stack X-Area $\frac{111.6}{\text { in } 2}$

Elevation N.A. $\mathrm{ft}$

Distance to distubance 426.25 in

Start/End Tme $1605 / 1700$
LV-S3_FlowAngle.xkx$$
1 \text { st }
$$

CCP-WTPSP-\#\#

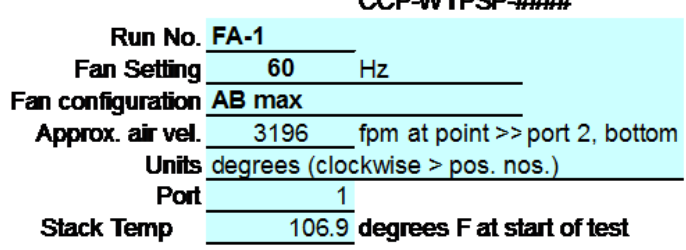

Run No. FA-1

Fan Setting

Fan configuration AB max

Units degrees (clockwise > pos. nos.)

Port 1

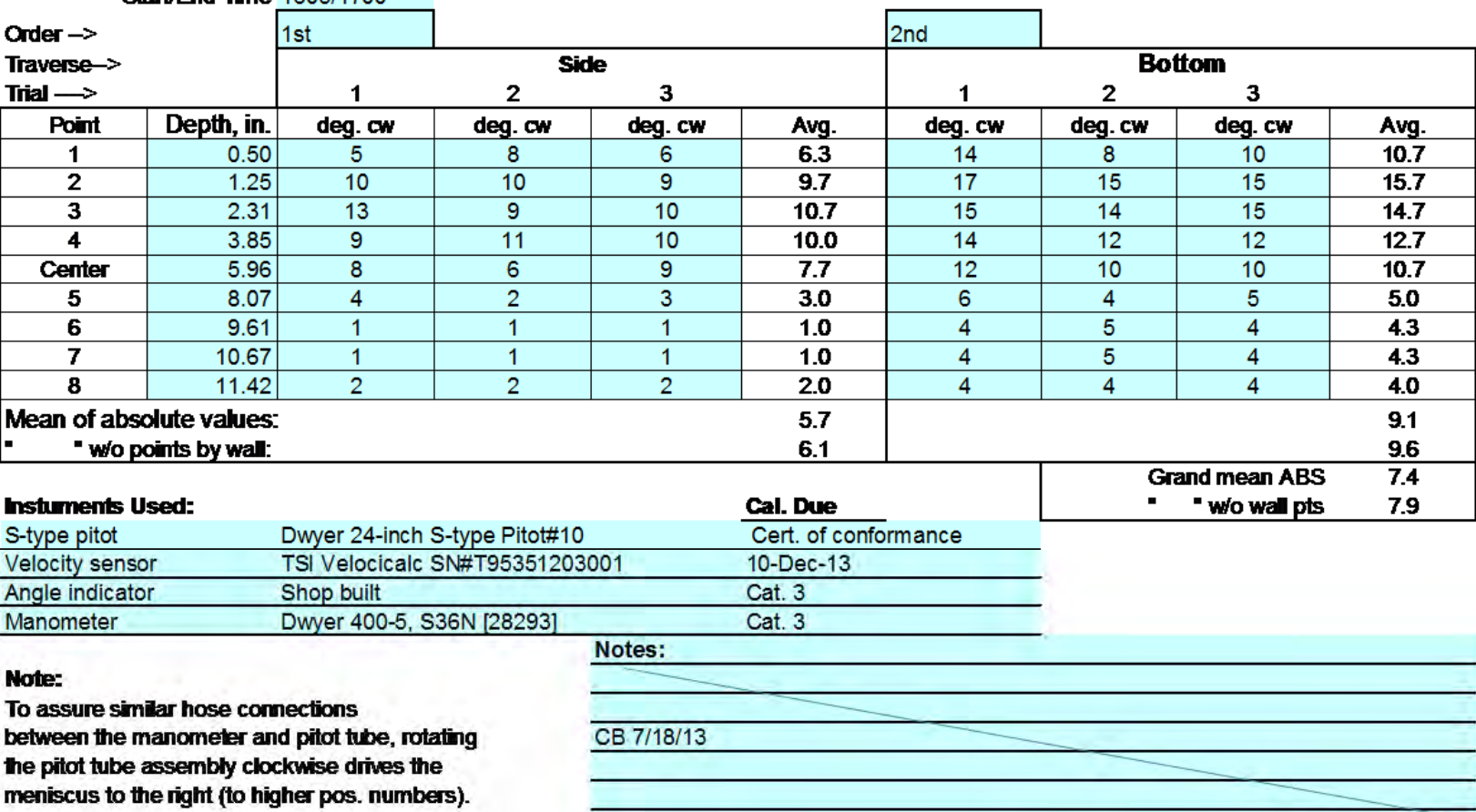


TI-WTPSP-121

Page

FLOW ANGLE DATA FORM

Site LV-S3 Scale Model

Date $7 / 19 / 2013$

Tester CB, SS

$\begin{array}{rlr}\text { Stack Dia. } & 11.922 & \text { in } \\ \text { Stack X-Area } & 111.6 & \text { in2 } \\ \text { Elevation } & \text { N.A. } & \text { ft } \\ & 426.25 & \text { disturbance }\end{array}$

Distance to disturbance 426.25 in

Start/End Trme $0700 / 0730$
LV-S3_FlowAngle.xlsx

Run No. FA-2

Fan Setting

Fan configuration $\mathrm{AB}$ Norm

Approx. air vel. 3104 fpm at point $\gg$

Units degrees (clockwise > pos. nos.)

Port 1

Stack Temp $\quad 76.6$ degrees F

\section{(n)}

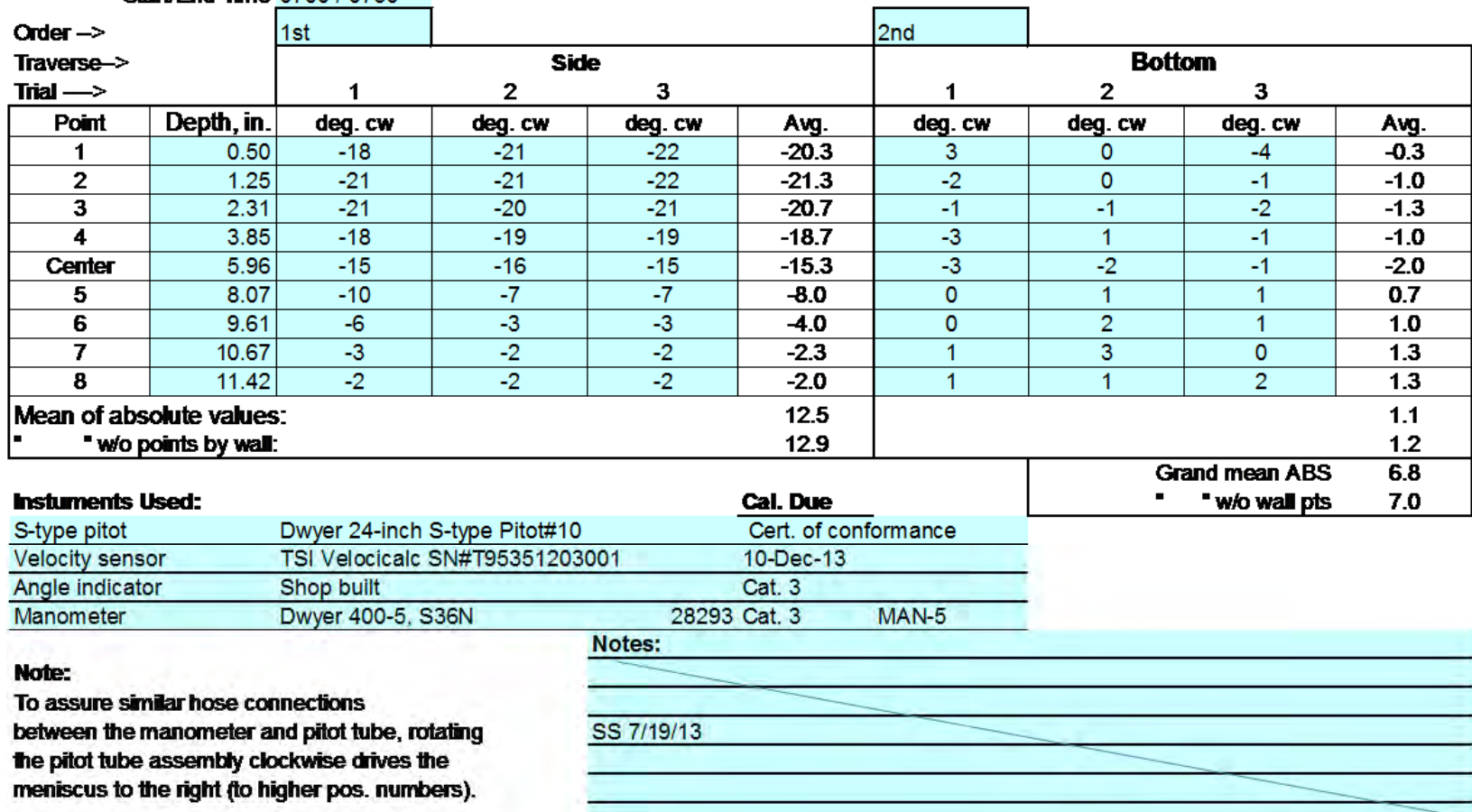

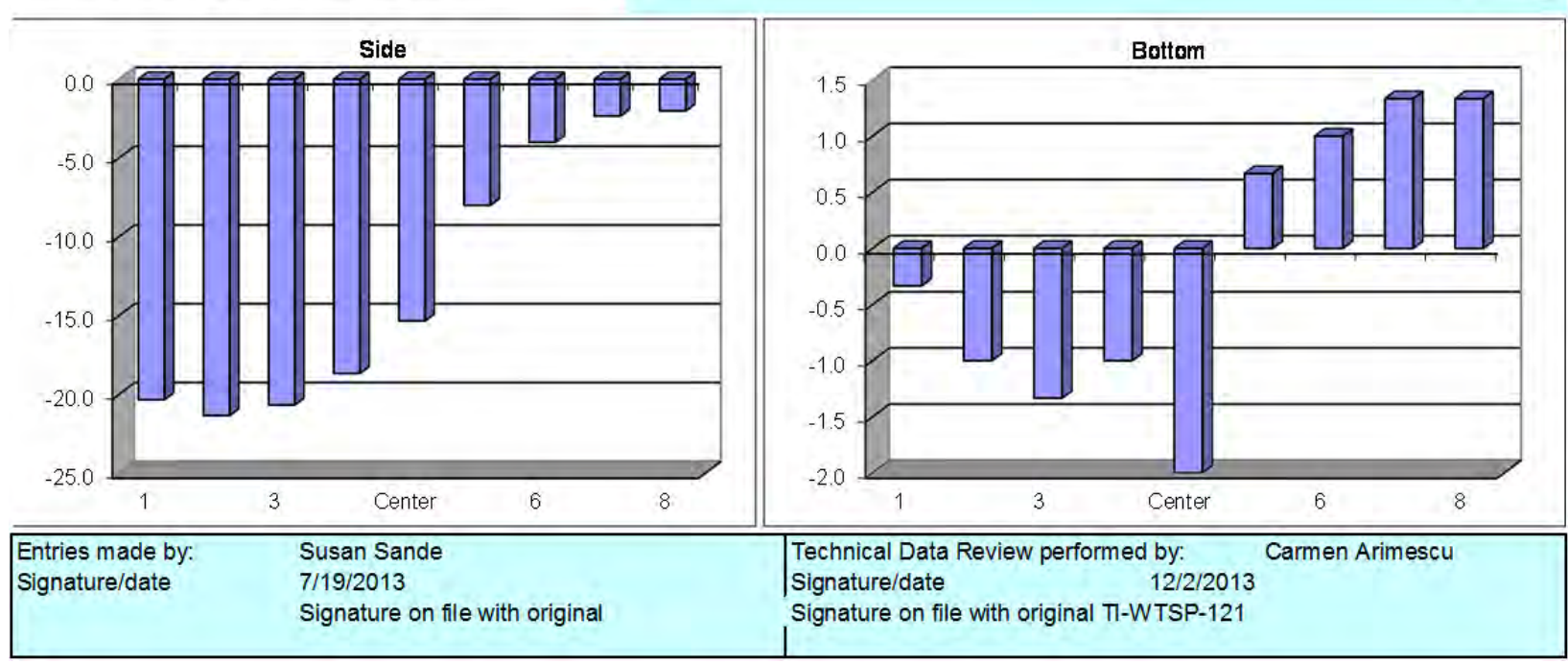


Th-WTPSP-121

Page

FLOW ANGLE DATA FORM

LV-S3_FlowAngle.xlsx

Site LV-S3 Scale Model

Date $7 / 19 / 2013$

Tester CB, SS

Stack Dia. $\frac{11.922 \text { in }}{111.6}$

Stack X-Area 111.6 in2

Elevation N.A. $\mathrm{ft}$

Distance to disturbance 426.25 in

Start/End Trme $0733 / 0754$

CCP-WTPSP-\#\#

Run No. FA-3

Fan Setting $\mathrm{Hz}$

Fan configuration AB Min

Approx. air vel. 1517 fpm at point $\gg$

Units degrees (clockwise > pos. nos.)

Port 1

Stack Temp 78.8 degrees F

Order $\rightarrow$

Traverse->

Trial $\longrightarrow$

Point Depth, in

2nd

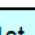

\begin{tabular}{|c|r|c|c|c|c|}
\hline Point & Depth, in. & deg. cw & deg. cw & deg. cw & \\
\hline $\mathbf{1}$ & 0.50 & -11 & 7 & 8 & \\
\hline $\mathbf{2}$ & 1.25 & -2 & 10 & -7 & \\
\hline $\mathbf{3}$ & 2.31 & -11 & 9 & -10 & \\
\hline $\mathbf{4}$ & 3.85 & -10 & 11 & 7 & \\
\hline Center & 5.96 & 4 & 10 & 9 & \\
\hline $\mathbf{5}$ & 8.07 & 1 & 4 & 5 & -1 \\
\hline $\mathbf{6}$ & 9.61 & -2 & 2 & -2 & 1 \\
\hline $\mathbf{7}$ & 10.67 & -2 & 2 & 1 & \\
\hline $\mathbf{8}$ & 11.42 & 2 & 1 & \\
\hline $\begin{array}{l}\text { Mean of absolute values: } \\
\text { - wo points by wal: }\end{array}$
\end{tabular}

\section{Instuments Used:}

S-type pitot

Velocity sensor

Dwyer 24-inch S-type Pitot\#10

Angle indicato

TSI Velocicalc SN\#T95351203001

Shop built

Manometer Dwyer 400-5, S36N

Note:

To assure simiar hose connections

between the manometer and pitot tube, rotating

the pitot tube assembly clockwise dives the

meniscus to the right (to higher pos. numbers).

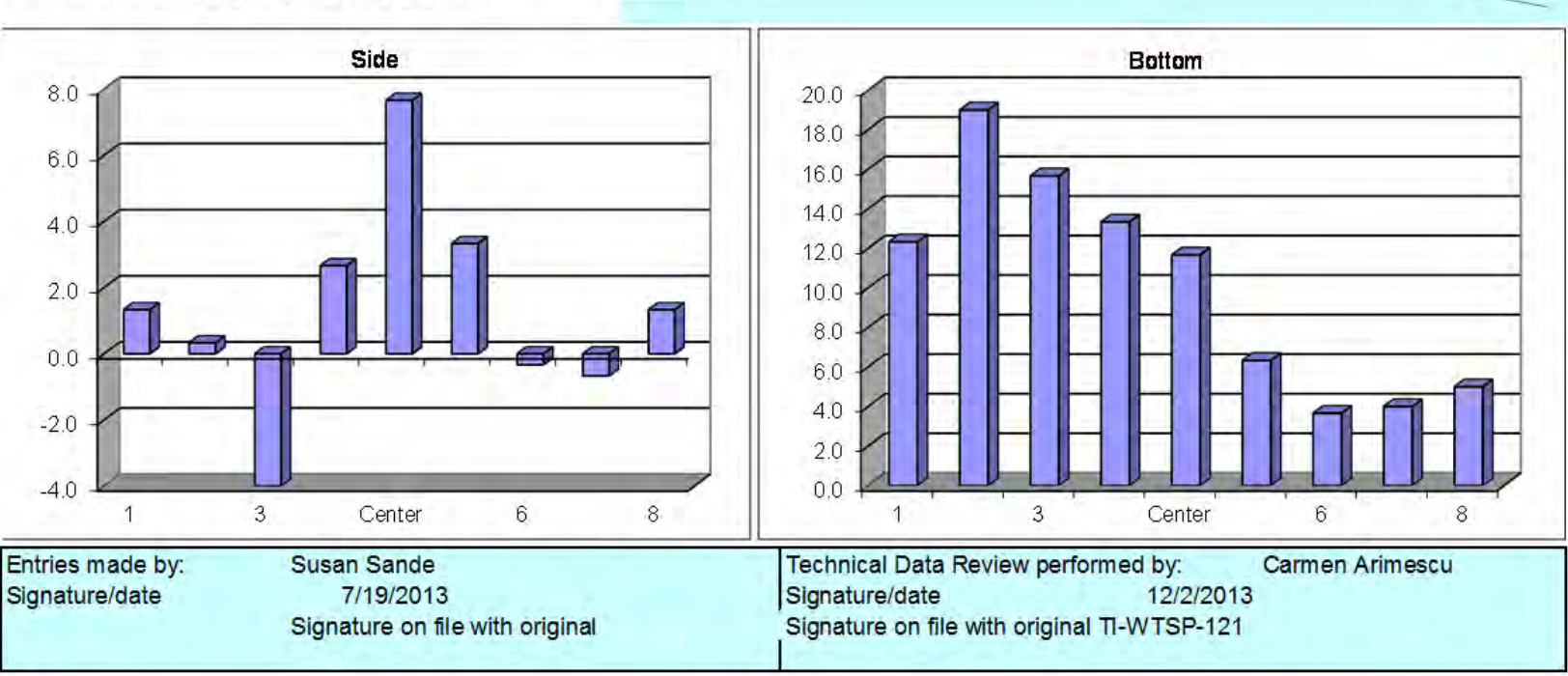


TI-WTPSP-121

Page

FLOW ANGLE DATA FORM

Site LV-S3 Scale Model

Date $7 / 19 / 2013$

Tester CB, SS

Stack Dia. 11.922 in

Stack X-Area 111.6 in2

Elevation N.A. $\mathrm{ft}$

Distance to disturbance 426.25 in

Start/End Tme $0756 / 0810$

LV-S3_FlowAngle.xlsx

Run No. FA-4

Fan Setting

Fan configuration AB Min

Approx. air vel. 1533 fpm at point $\gg$

Units degrees (clockwise > pos. nos.)

Port 1

Stack Temp

82.2 degrees F

Onder $\rightarrow$

Traverse $\rightarrow$

Trial $\longrightarrow$

Point Depth, in

$$
1 \text { st }
$$

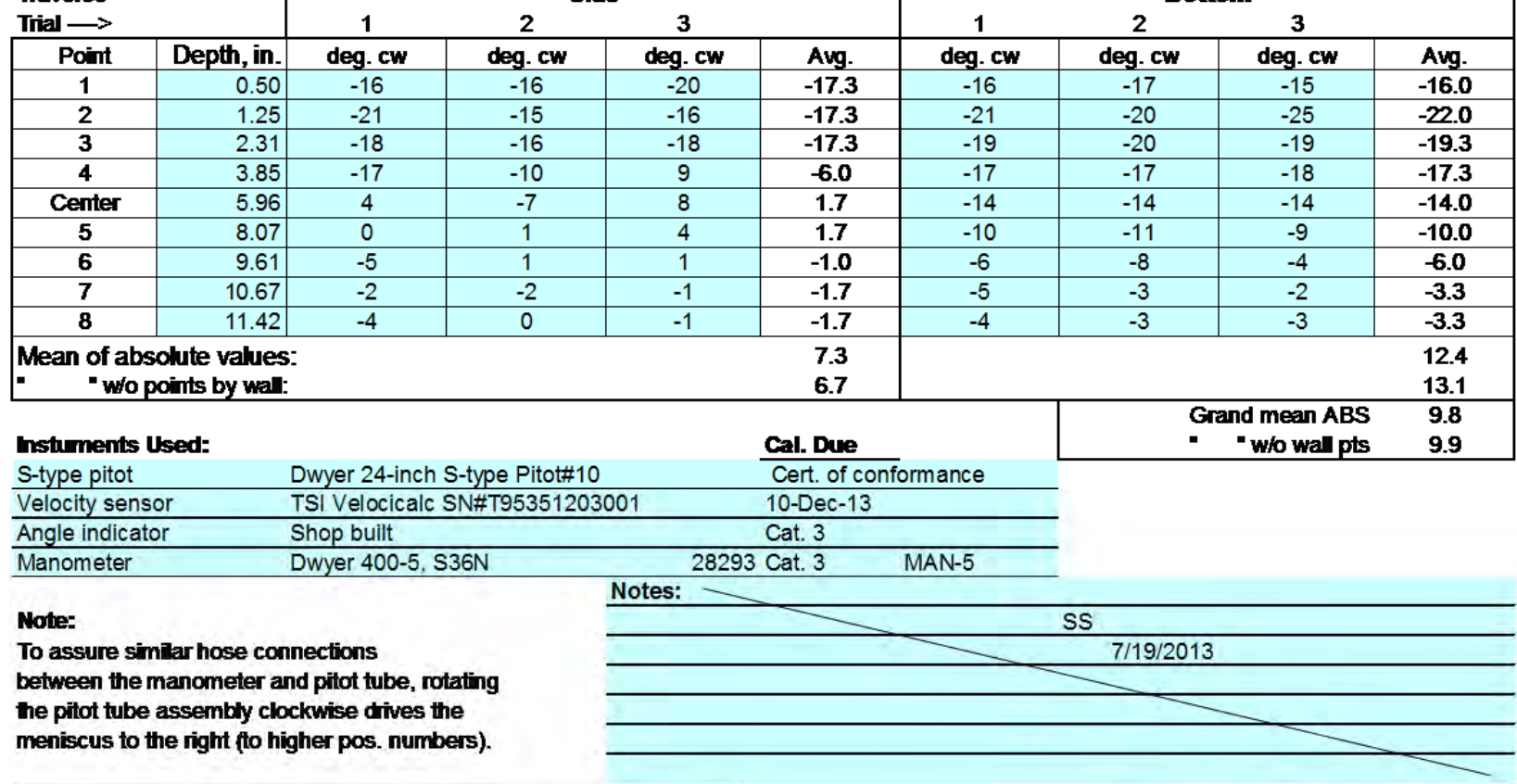

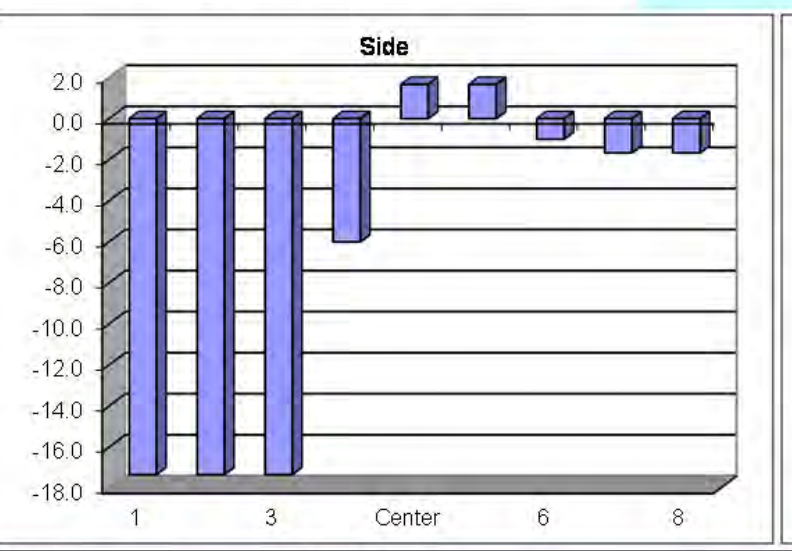

Entries made by: Signature/date
Susan Sande

$7 / 19 / 2013$

Signature on file with original

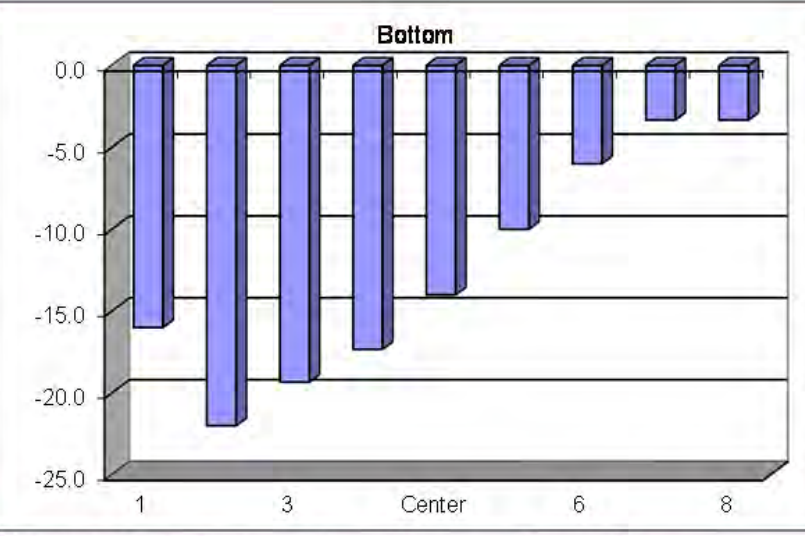

Technical Data Review performed by: Carmen Arimescu

Signature/date

$12 / 2 / 2013$

Signature on file with original TI-WTSP-121 
TT-WIPSP-121

Page

FLOW ANGLE DATA FORM

Site LV-S3 Scale Model

Date 7/19/2013

Tester CB, SS

Stack Dia. 11.922 in

Stack X-Area 111.6 in2

Elevation N.A ft

Distance to disturbance 426.25 in

Start/End Time 0825/ 0848
LV-S3 HowAngle.xdsx

2nd

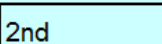

Order $\rightarrow$

Traverse-->

Trial $\longrightarrow$

\begin{tabular}{|c|r|}
\hline Point & Depth, in \\
\hline $\mathbf{1}$ & 0.50 \\
\hline $\mathbf{2}$ & 1.2 \\
\hline $\mathbf{3}$ & 2.31 \\
\hline $\mathbf{4}$ & 3.8 \\
\hline Center & 5.9 \\
\hline $\mathbf{5}$ & 8.07 \\
\hline $\mathbf{6}$ & 9.61 \\
\hline $\mathbf{7}$ & 10. \\
\hline $\mathbf{8}$ & 11. \\
\hline
\end{tabular}

Mean of absolute velues:

" "w/o points by wall:

Instuments Used

S-type pitot

Velocity sensor

Angle indicator

Manometer

\begin{tabular}{|c|c|}
\hline & 1 \\
\hline 50 & deg. cw \\
\hline 25 & 3 \\
\hline 31 & -5 \\
\hline 85 & -8 \\
\hline 96 & -8 \\
\hline 07 & 3 \\
\hline 61 & -1 \\
\hline 67 & 1 \\
\hline 42 & 1 \\
\hline
\end{tabular}

\begin{tabular}{l|l}
-1 \\
1 & 1
\end{tabular}

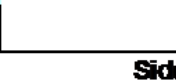

23

\begin{tabular}{|c|c|}
\hline deg. Cw & deg. Cw \\
\hline 3 & 0 \\
\hline-6 & -4 \\
\hline-4 & -7 \\
\hline-8 & -5 \\
\hline 8 & 9 \\
\hline 3 & 2 \\
\hline 2 & 1 \\
\hline 1 & 2 \\
\hline 1 & -2 \\
\hline
\end{tabular}

3

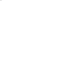

(2)

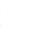

Run No. FA-5

Fan Seting $\mathrm{Hz}$

Fan configuration $A B$ Min

Approx air vel. 1533 fpm at point $\gg$

Units degrees (clockwise > pos. nos.)

Port 1

Stack Temp 82.2 degrees F

CCP-WIPSP\#\#\#

$1 \mathrm{st}$

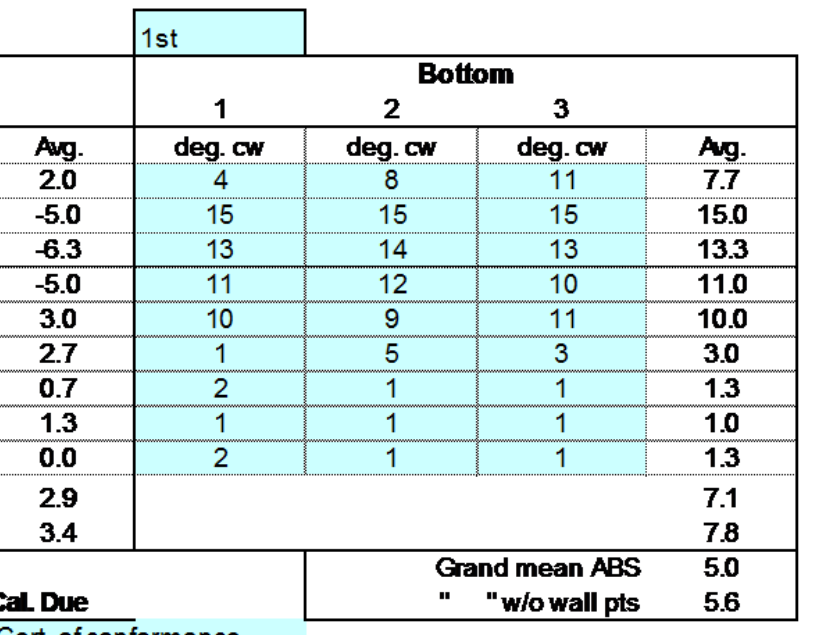

Note:

To assure similar hose connections

between the manometer and pitot tube, rotafing

the pitot tube assembly clockwise drives the

meniscus to the right (to higher pos. numbers).

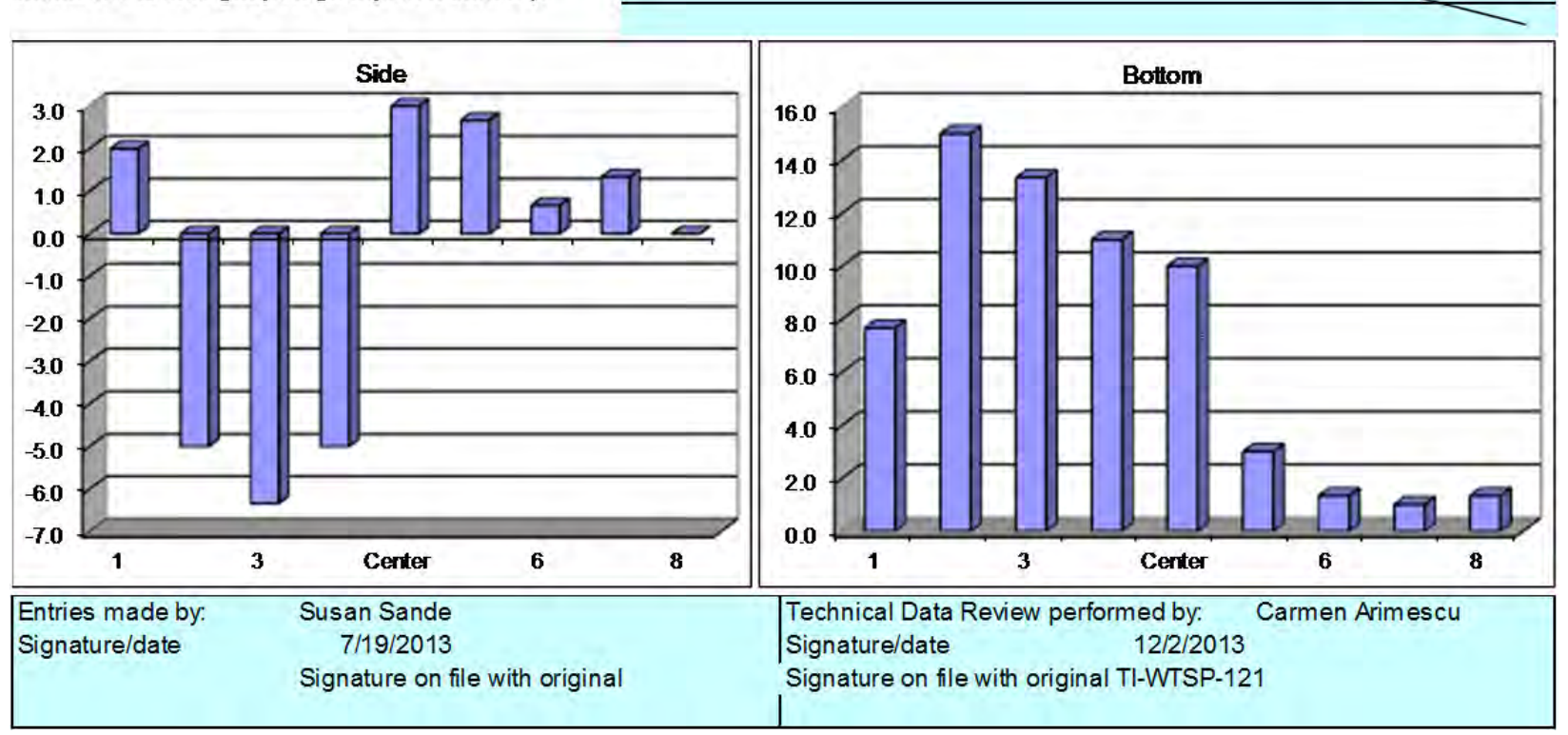

D.43 
TI-WTPSP-121

Page

FLOW ANGLE DATA FORM

Site LV-S3 Scale Model

Date $7 / 19 / 2013$

Tester CB, SS

Stack Dia. 11.922 in

Stack X-Area 111.6 in2

Elevation N.A. $\mathrm{ft}$

Distance to disturbance 426.25 in

Start/End Time $0900 / 0952$
LV-S3_FlowAngle.xlsx

Run No. FA-6

Fan Seting

Fan configuration $\mathbf{B}$ Min

Approx. air vel. $797 \quad$ fipm at point $\gg$

Units degrees (clockwise > pos. nos.)

Port 1

Stack Temp

96.8 degrees $F$

Order $\rightarrow$

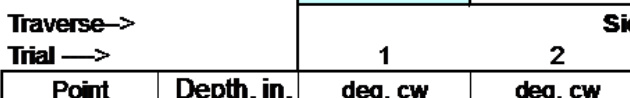

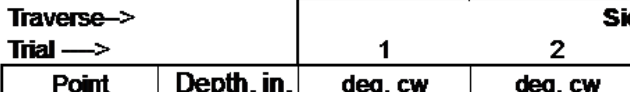

CCP-WTPSP-\#\#

2nd

\begin{tabular}{|c|c|c|c|}
\hline \multirow[b]{3}{*}{3} & 2nd & & \\
\hline & \multicolumn{3}{|c|}{ Bottom } \\
\hline & 1 & 2 & 3 \\
\hline
\end{tabular}

\begin{tabular}{|l|l|l|l|l|l} 
deg. cw & Avg. & deg. cw & deg. cw & deg. cw & Avg.
\end{tabular}

\begin{tabular}{|r|c|c|c|}
\hline th, in. & deg. cw & deg. cw & c \\
\hline 0.50 & 16 & 14 & \\
\hline 1.25 & 14 & 12 & \\
\hline 2.31 & 18 & 17 & \\
\hline 3.85 & 16 & 12 & \\
\hline 5.96 & 14 & 11 & \\
\hline 8.07 & 14 & 3 & \\
\hline 9.61 & 15 & 1 & \\
10.67 & 13 & 5 & \\
11.42 & 2 & -2 & \\
\hline
\end{tabular}

11.42

\section{Mean of absolute values: \\ - wo points by wal:}

Lstuments Used:

S-type pitot

Velocity sensor

Angle indicator

Manometer

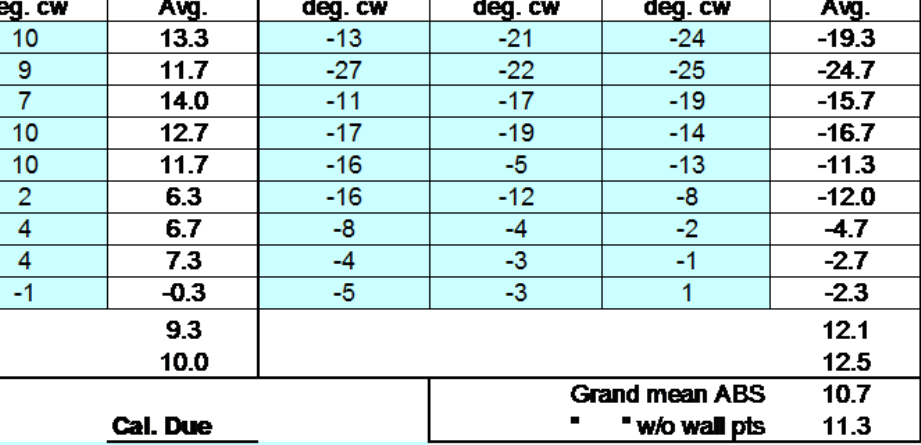

Note:

To assure simiar hose connections

between the manometer and pitot tube, rotating

the pitot tube assembly clockwise dives the

meniscus to the right (to higher pos. numbers).

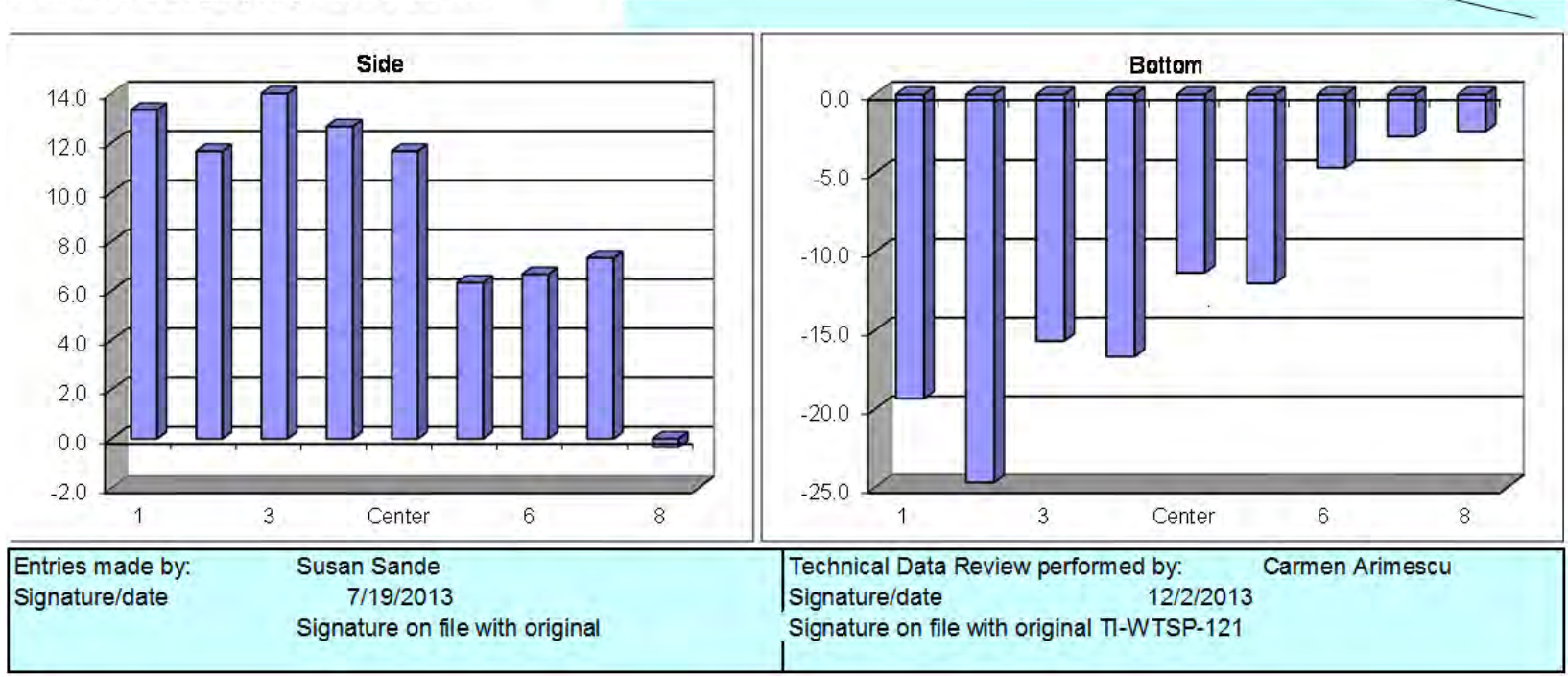


T1-WTPSP-121

Page

FLOW ANGLE DATA FORM

Site LV-S3 Scale Model

Date $7 / 19 / 2013$

Tester CB, SS

Stack Dia. 11.922 in

Stack X-Area 111.6 in2

Elevation N.A. $\mathrm{ft}$

Distance to disturbance 426.25 in

Start/End Tre $1030 / 1100$
LV-S3_FlowAngle.xlsx

Run No. FA-7

Fan Setting 55

Fan configuration Fan A Norm

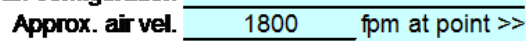

Units degrees (clockwise > pos. nos.)

Port 1

Stack Temp 105.9 degrees F

$1 \mathrm{st}$

Order $\rightarrow$

Traverse $\rightarrow$

Trial $\longrightarrow$

Point Depth, in

$$
\text { 2nd }
$$

\section{1}

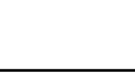

\begin{tabular}{|c|c|c|c|c|}
\hline & 1st & & & \\
\hline & \multicolumn{4}{|c|}{ Bottom } \\
\hline & 1 & 2 & 3 & \\
\hline Avg. & deg. cw & deg. cw & deg. cw & Avg. \\
\hline-22.7 & -4 & -10 & -9 & -7.7 \\
\hline-19.7 & -2 & -6 & -4 & -4.0 \\
\hline-20.3 & -4 & -5 & -4 & -4.3 \\
\hline-18.0 & -3 & -3 & -2 & -2.7 \\
\hline-10.7 & -3 & -2 & -1 & -2.0 \\
\hline-8.0 & -6 & -7 & -7 & -6.7 \\
\hline-2.7 & -3 & -3 & -3 & -3.0 \\
\hline-1.7 & -2 & -3 & -3 & -2.7 \\
\hline-1.3 & -1 & -2 & -2 & -1.7 \\
\hline 11.7 & & & & 3.9 \\
\hline 11.6 & & & & 3.6 \\
\hline & & & I mean ABS & 7.8 \\
\hline Due & & & who wall pts & 7.6 \\
\hline
\end{tabular}

S-type pitot

Velocity sensor

Cert. of conformance

Angle indicator

Manometer Dwyer 400-5, S36N

TSI Velocicalc SN\#T95351203001

10-Dec-13

Note:

To assure simiar hose connections

between the manometer and pitot tube, rotating

the pitot tube assembly clockwise dives the

meniscus to the right (to higher pos. numbers).

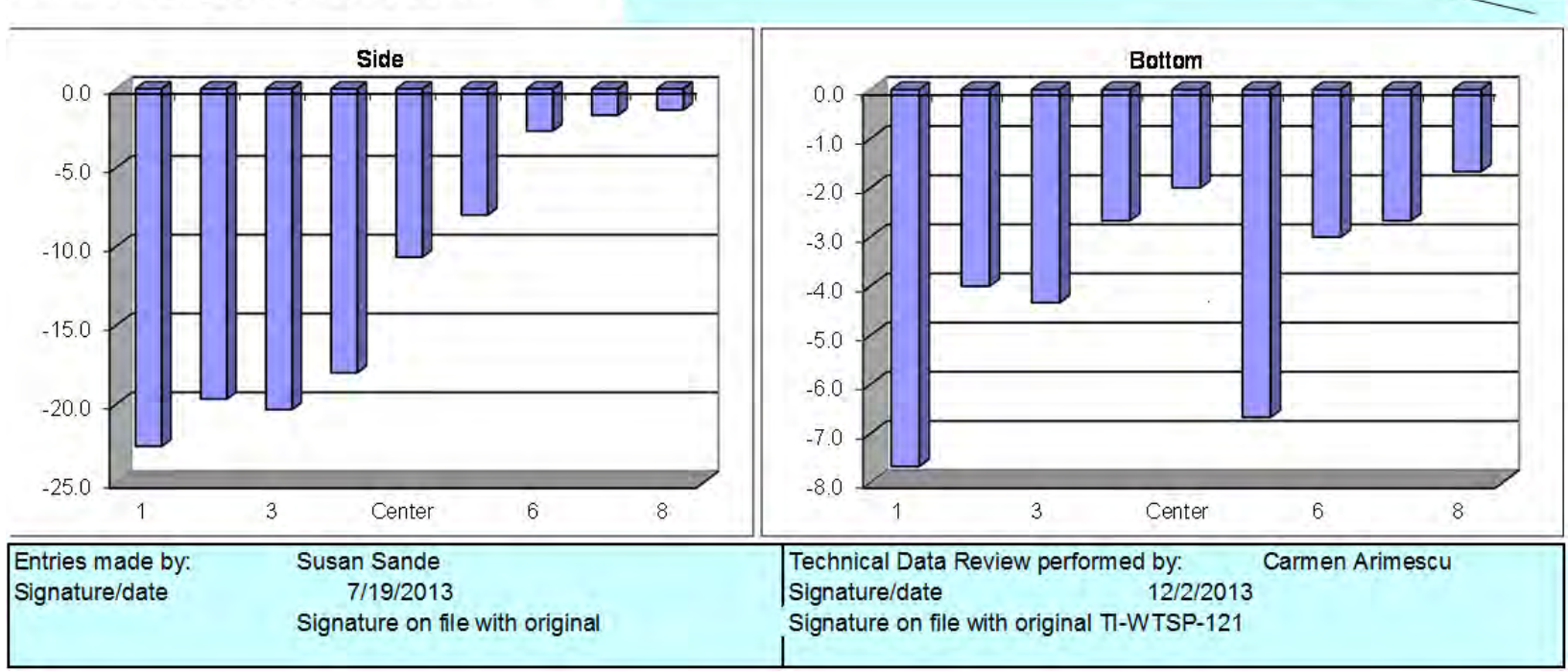


T1-WTPSP-121

Page

FLOW ANGLE DATA FORM

Site LV-S3 Scale Model

Date $7 / 19 / 2013$

Tester CB, SS

Stack Dia. 11.922 in

Stack X-Area 111.6 in2

Elevation N.A. $f t$

Distance to disturbance 510.25 in

Start/End Tme $1116 / 1145$

LV-S3_FlowAngle.xlsx

Run No. FA-8

Fan Seting 55

Fan configuration Fan A Norm

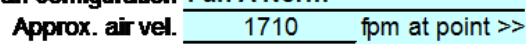

Units degrees (clockwise > pos. nos.)

Port 2

Stack Temp

07.6 degrees F

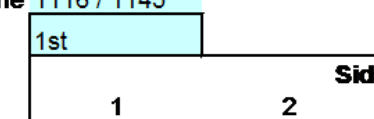

3

in. deg. cw deg. cw

\begin{tabular}{|c|c|c|}
\hline $\operatorname{leg} . \mathrm{cw}$ & Avg. & deg. $c w$ \\
\hline-4 & -2.0 & 4 \\
\hline-14 & -4.3 & 13 \\
\hline 6 & 7.7 & 14 \\
\hline 5 & 6.3 & 14 \\
\hline 4 & 4.0 & 8 \\
\hline-1 & 0.0 & 5 \\
\hline-1 & -1.0 & 4 \\
\hline-2 & -1.7 & 3 \\
\hline-1 & -0.7 & 3 \\
\hline
\end{tabular}

3.1

3.6

- wo points by wall:

Instuments Used:

S-type pitot

1.25

2.31

\begin{tabular}{l|l}
-7 & \\
-8 & \\
-8 & \\
\hline
\end{tabular}

\begin{tabular}{l|l}
\hline 5 & -4 \\
\hline
\end{tabular}

Velocity sensor

Angle indicator

Manometer

TSI Velocicalc SN\#T95351203001

Cert. of conformance

10-Dec-13

wyer 400-5, S36N

Note:

To assure similar hose connections

between the manometer and pitot tube, rotating

the pitot tube assembly clockwise dives the

meniscus to the right (to higher pos. numbers)

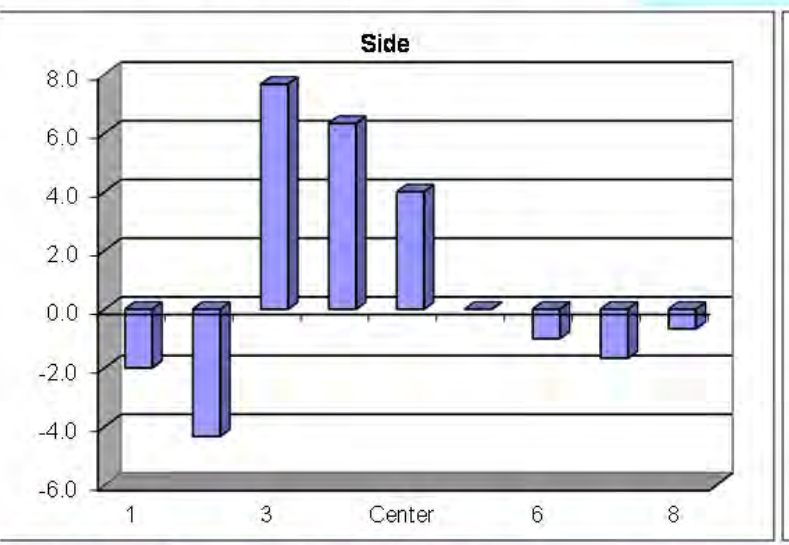

Entries made by:

Carolyne Burns

$7 / 18 / 2013$

Cat. 3

2nd

\section{車}

CCP-WTPSP-\#\# 
TI-WTPSP-121

Page

FLOW ANGLE DATA FORM

Site LV-S3 Scale Model

Date $7 / 22 / 2013$

Tester SS, EA

Stack Dia. $\frac{11.922}{111.6}$

Stack X-Area 111.6 in2

Elevation N.A. fi

Distance to disturbance 510.25

Start/End Trme $0719 / 0745$
LV-S3_FlowAngle.xlsx

$$
\text { 2nd }
$$

Run No. FA-9

CCP-WTPSP-\#\#

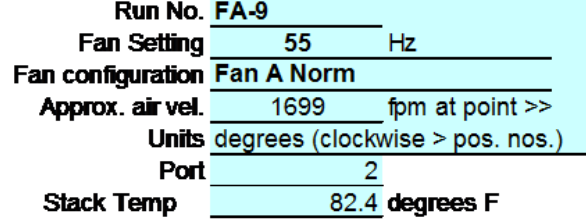

Stack Temp 82.4 degrees F

\begin{tabular}{|c|c|c|c|}
\hline \multicolumn{2}{|c|}{ 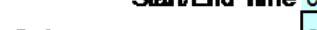 } & \multirow{2}{*}{\multicolumn{2}{|c|}{ 2nd }} \\
\hline Order $\rightarrow$ & & & \\
\hline $\begin{array}{l}\text { Traverse- } \\
\text { Trial } \longrightarrow\end{array}$ & & 1 & \\
\hline Point & Depth, in. & deg. cw & deg \\
\hline 1 & 0.50 & -19 & -2 \\
\hline 2 & 1.25 & 7 & \\
\hline 3 & 2.31 & 8 & 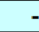 \\
\hline 4 & 3.85 & 5 & \\
\hline Center & 5.96 & -1 & \\
\hline 5 & 8.07 & -5 & - \\
\hline 6 & 9.61 & -4 & \\
\hline 7 & 10.67 & -4 & \\
\hline 8 & 11.42 & -3 & \\
\hline
\end{tabular}
- wo points by wall:

Instuments Used:

S-type pitot

Velocity sensor

Angle indicator

Manometer

Dwyer 24-inch S-type Pitot\#10

TSI Velocicalc SN\#T95351203001

Shop built

Dwyer 400-5, S36N 28293

2 Side 3

deg. cw

\begin{tabular}{c|c|c}
\hline- CW & deg. Cw & Avg. \\
\hline 22 & -23 & $-\mathbf{2 1 . 3}$ \\
0 & 0 & $\mathbf{2 . 3}$ \\
-3 & 3 & $\mathbf{2 . 7}$ \\
\hline 3 & 0 & $\mathbf{2 . 7}$ \\
\hline-9 & -15 & $-\mathbf{8 . 3}$ \\
\hline-8 & -8 & $-\mathbf{7 . 0}$ \\
\hline-6 & -6 & $-\mathbf{5 . 3}$ \\
\hline-5 & -4 & $-\mathbf{4 . 3}$ \\
\hline-4 & -4 & $-\mathbf{3 . 7}$ \\
\hline
\end{tabular}
$1 \mathrm{st}$

\begin{tabular}{l|l|l} 
& \multicolumn{3}{|c|}{ Bottom } & 3 \\
& 2 &
\end{tabular}

\begin{tabular}{|l|l|l|l} 
deg. cw & deg. cw & deg. cw & Avg.
\end{tabular}

\begin{tabular}{|c|c|c|c|}
\hline deg. cw & deg. cw & deg. cw & Avg. \\
\hline-10 & -7 & -9 & -8.7 \\
\hline-5 & -4 & -6 & -5.0 \\
\hline-7 & -2 & -1 & -3.3 \\
\hline-6 & -2 & -1 & -3.0 \\
\hline-1 & -1 & -9 & -3.7 \\
\hline-2 & -2 & -3 & -2.3 \\
\hline-1 & -2 & -2 & -1.7 \\
\hline 0 & -2 & -2 & -1.3 \\
\hline-2 & -2 & -2 & -2.0 \\
\hline & & & $\begin{array}{l}3.4 \\
2.9\end{array}$ \\
\hline & \multicolumn{2}{|c|}{$\begin{array}{l}\text { Grand mean ABS } \\
\text { - " w/o wall pts }\end{array}$} & $\begin{array}{l}4.9 \\
3.8 \\
\end{array}$ \\
\hline
\end{tabular}

Note:

To assure simiar hose connections

between the manometer and pitot tube, rotating

the pitot tube assembly clockwise dives the

meniscus to the right (to higher pos. numbers).

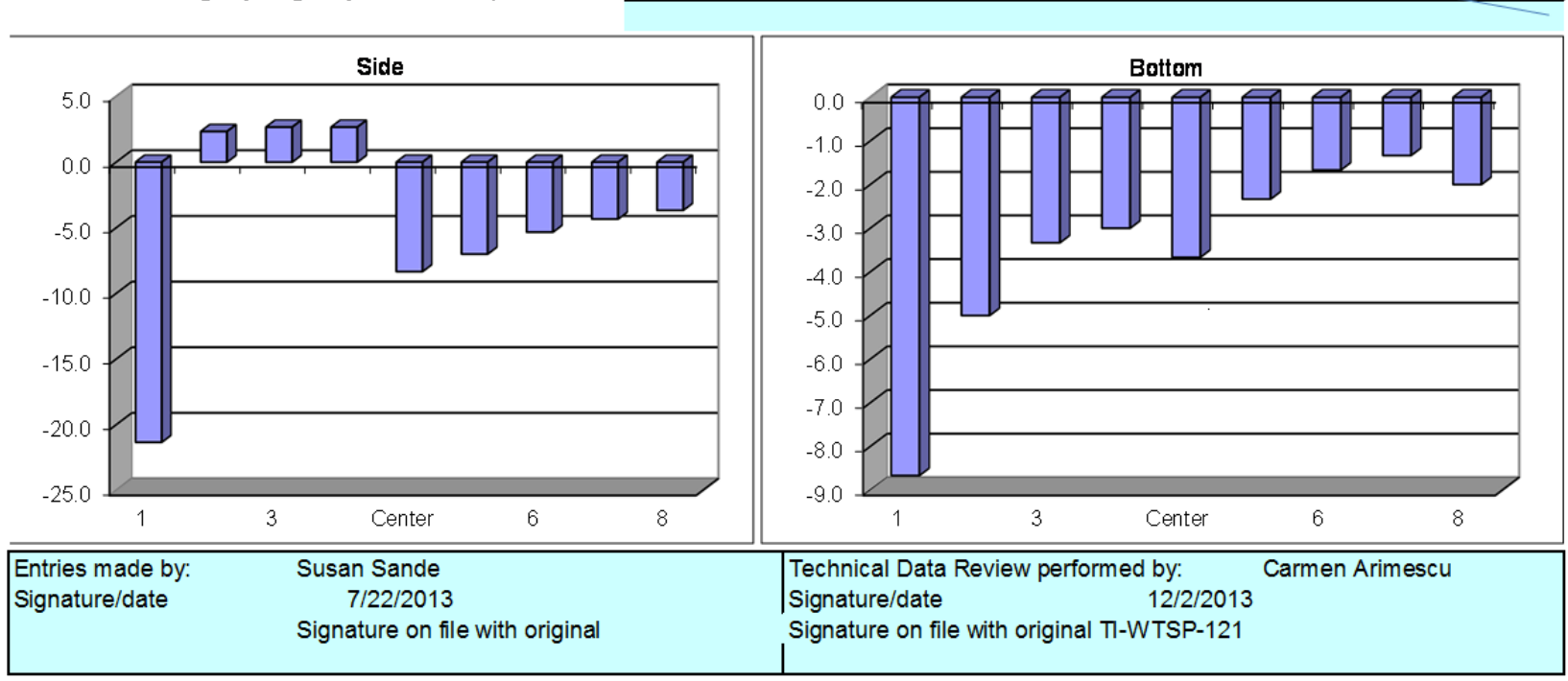


T1-WTPSP-121

Page

FLOW ANGLE DATA FORM

Site LV-S3 Scale Model

Date $7 / 22 / 2013$

Tester SS, EA

Stack X-Area 111.6

Elevation N.A. $\mathrm{ft}$

Distance to disturbance 510.25

Start/End Time $0750 / 0823$

Stack Dia. $\frac{11.922}{111.6}$ in

LV-S3_FlowAngle.xlsx

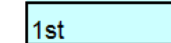

Run No. FA-10

CCP-WTPSP-\#\#

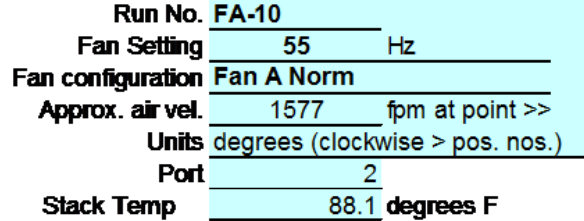

Stack Temp 88.1 degrees $F$

\begin{tabular}{|c|c|c|c|}
\hline \multirow{3}{*}{\multicolumn{2}{|c|}{$\begin{array}{l}\text { Order } \rightarrow \\
\text { Traverse } \rightarrow \\
\text { Tial } \rightarrow\end{array}$}} & \multirow{2}{*}{\multicolumn{2}{|c|}{1 st }} \\
\hline & & & \\
\hline & & 1 & \\
\hline Point & Depth, in. & deg. cw & deg \\
\hline 1 & 0.50 & -21 & -2 \\
\hline 2 & 1.25 & -2 & \\
\hline 3 & 2.31 & 7 & \\
\hline 4 & 3.85 & 5 & \\
\hline Center & 5.96 & 0 & \\
\hline 5 & 8.07 & -6 & \\
\hline 6 & 9.61 & -4 & \\
\hline 7 & 10.67 & -4 & \\
\hline 8 & 11.42 & -3 & \\
\hline
\end{tabular}
- "wo points by wall:

Instuments Used:

S-type pitot

Velocity sensor

Angle indicator

Manometer

Dwyer 24-inch S-type Pitot\#10

TSI Velocicalc SN\#T95351203001

Shop built

Dwyer 400-5, S36N 28293

2 Side 3

\begin{tabular}{|c|c|c|}
\hline . cw & deg. cw & Avg. \\
\hline 20 & -21 & -20.7 \\
\hline-1 & -1 & -1.3 \\
-2 & -3 & $\mathbf{0 . 7}$ \\
\hline 4 & -4 & $-\mathbf{1 . 0}$ \\
\hline 0 & 0 & $\mathbf{0 . 0}$ \\
\hline 5 & -5 & $-\mathbf{5 . 3}$ \\
\hline 5 & -4 & $-\mathbf{4 . 3}$ \\
\hline 3 & -3 & $-\mathbf{3 . 3}$ \\
\hline
\end{tabular}

2nd

Note:

To assure simiar hose connections

between the manometer and pitot tube, rotating

the pitot tube assembly clockwise dives the

meniscus to the right (to higher pos. numbers).

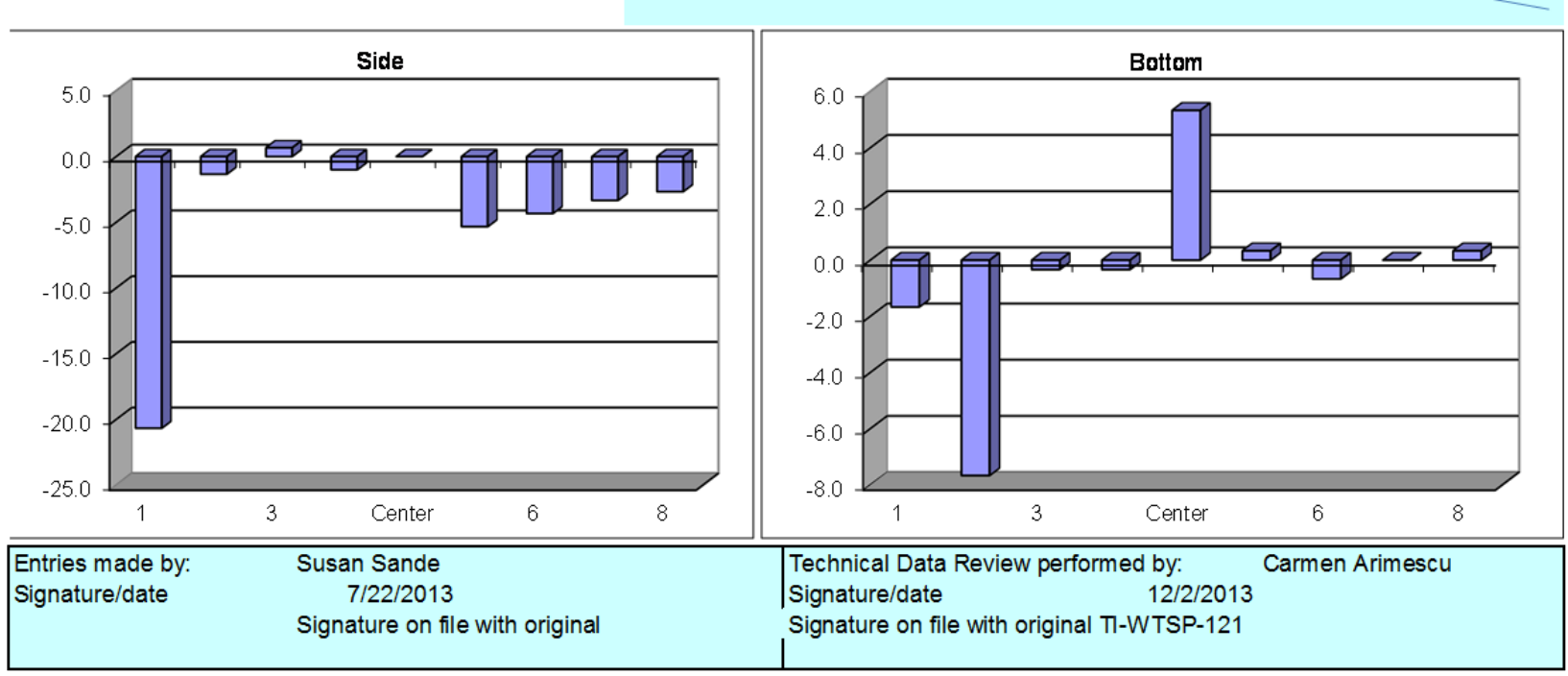


TI-WTPSP-121

Page

FLOW ANGLE DATA FORM

Site LV-S3 Scale Model

Date $7 / 22 / 2013$

Tester SS, EA

\begin{tabular}{|c|c|}
\hline Stack Dia. & 11.922 \\
\hline Stac & 111.6 \\
\hline & $\begin{array}{c}\text { N.A. } \\
510.25\end{array}$ \\
\hline
\end{tabular}

Distance to disturbance 510.25

Start/End Tme $0826 / 0913$
LV-S3_FlowAngle.xlsx

$$
\text { 2nd }
$$

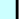

Run No. FA-11

CCP-WTPSP-\#\#

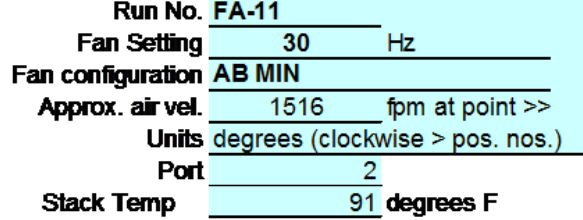

Stack Temp

91 degrees F

Oder $\rightarrow$

Traverse->

\begin{tabular}{|r|r} 
Trial $\longrightarrow$ Point & Depth, in.
\end{tabular} 1 st

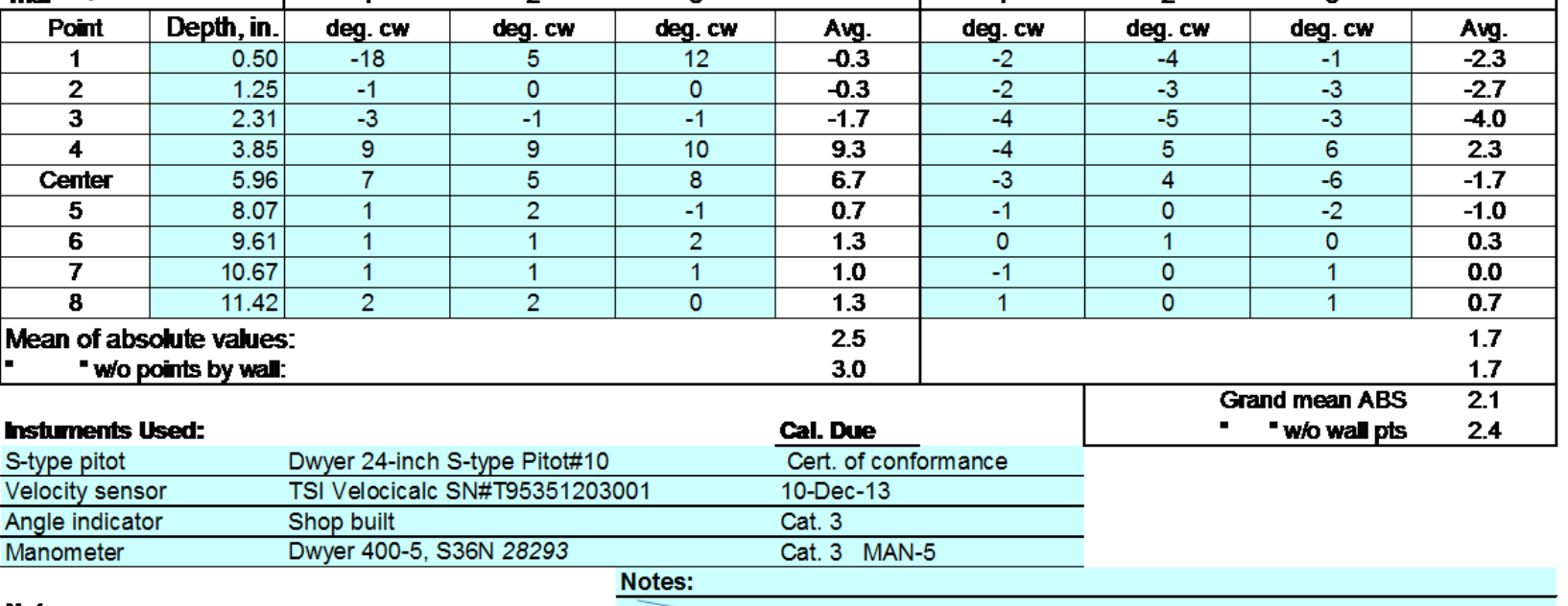

Note:

To assure similar hose connections

between the manometer and pitot tube, rotating

the pitot tube assembly clockwise dives the

meniscus to the right (to higher pos. numbers).
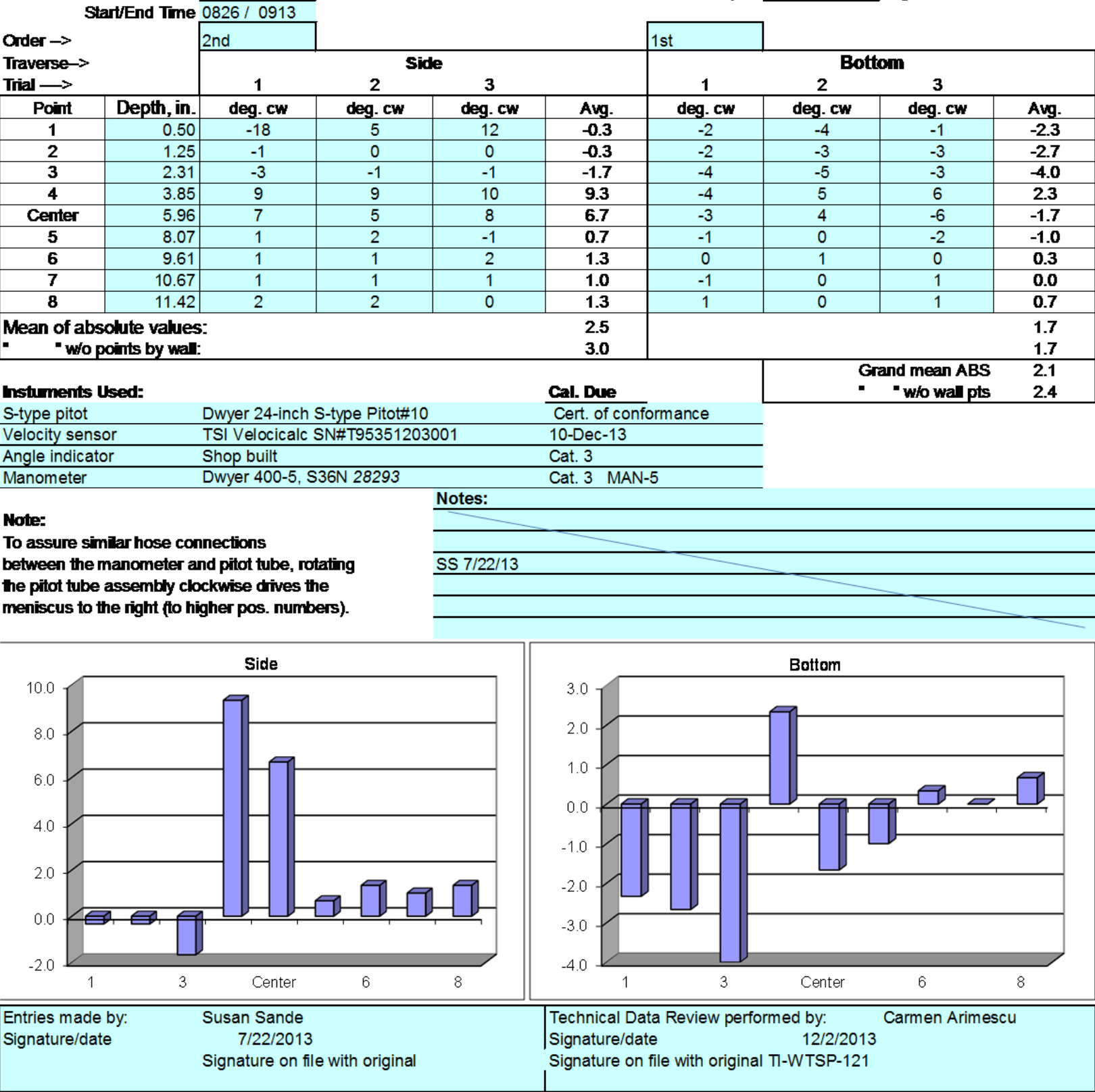
T1-WTPSP-121

Page

FLOW ANGLE DATA FORM

Site LV-S3 Scale Model

Date $7 / 22 / 2013$

Tester SS, EA

Stack Dia. 11.922 in

Stack X-Area 111.6 in2

Elevation N.A. fi

Distance to disturbance 510.25 in

Start/End Tme 0915/0943
LV-S3_FlowAngle.xlsx$$
1 \text { st }
$$

st

1

(nide
Run No. FA-12

CCP-WTPSP-\#\#

Fan Setting 30

Fan configuration AB MIN

Approx. air vel. NA fipm at point $>>$

Units degrees (clockwise > pos. nos.)

Stack Temp $\begin{array}{ll}\text { Port } & 2 \\ \text { degrees } F\end{array}$

2nd

(amp

NA degrees F

Order $\rightarrow$

Traverse $\rightarrow$

\begin{tabular}{|c|c|} 
Trial $\rightarrow$ Point & Depth, in.
\end{tabular}

\begin{tabular}{|c|c|c|c|c|}
\hline rial $\longrightarrow$ & & 1 & 2 & 3 \\
\hline Point & Depth, in. & deg. cw & deg. cw & deg. cw \\
\hline 1 & 0.50 & -1 & 7 & -11 \\
\hline 2 & 1.25 & 13 & -2 & 10 \\
\hline 3 & 2.31 & -4 & 10 & 9 \\
\hline 4 & 3.85 & -6 & 8 & 8 \\
\hline Center & 5.96 & 7 & 6 & 6 \\
\hline 5 & 8.07 & 2 & 0 & 2 \\
\hline 6 & 9.61 & 0 & 1 & -1 \\
\hline 7 & 10.67 & 0 & 0 & 1 \\
\hline 8 & 11.42 & -1 & 1 & 1 \\
\hline
\end{tabular}

Mean of absolute values:

- wo points by wall:

Instuments Used:

S-type pitot

Velocity sensor

Angle indicator

Manometer

Dwyer 24-inch S-type Pitot\#10

TSI Velocicalc SN\#T95351203001

\begin{tabular}{ll}
1 & 0.3 \\
\hline
\end{tabular}

2.8

3.3

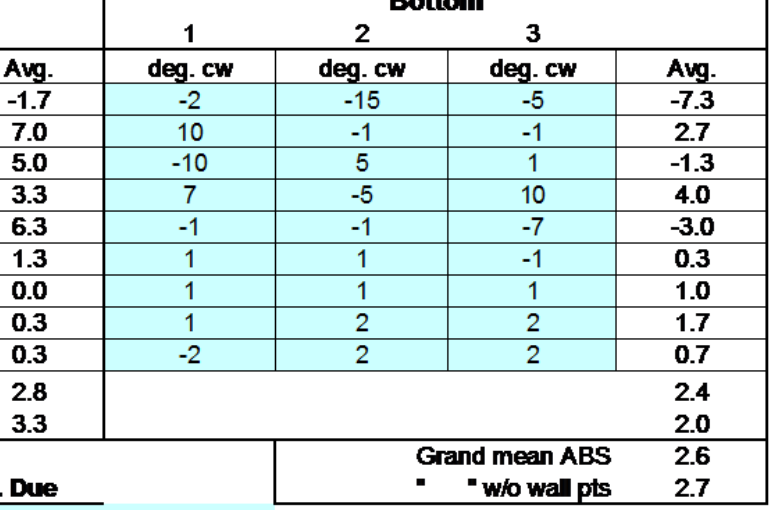

Note:

To assure similar hose connections

between the manometer and pitot tube, rotating

the pitot tube assembly clockwise dives the

meniscus to the right (to higher pos. numbers).

Cert. of conformance

10-Dec-13

Cat. 3

Dwyer 400-5, S36N $28293 \quad$ Cat. 3 MAN-5

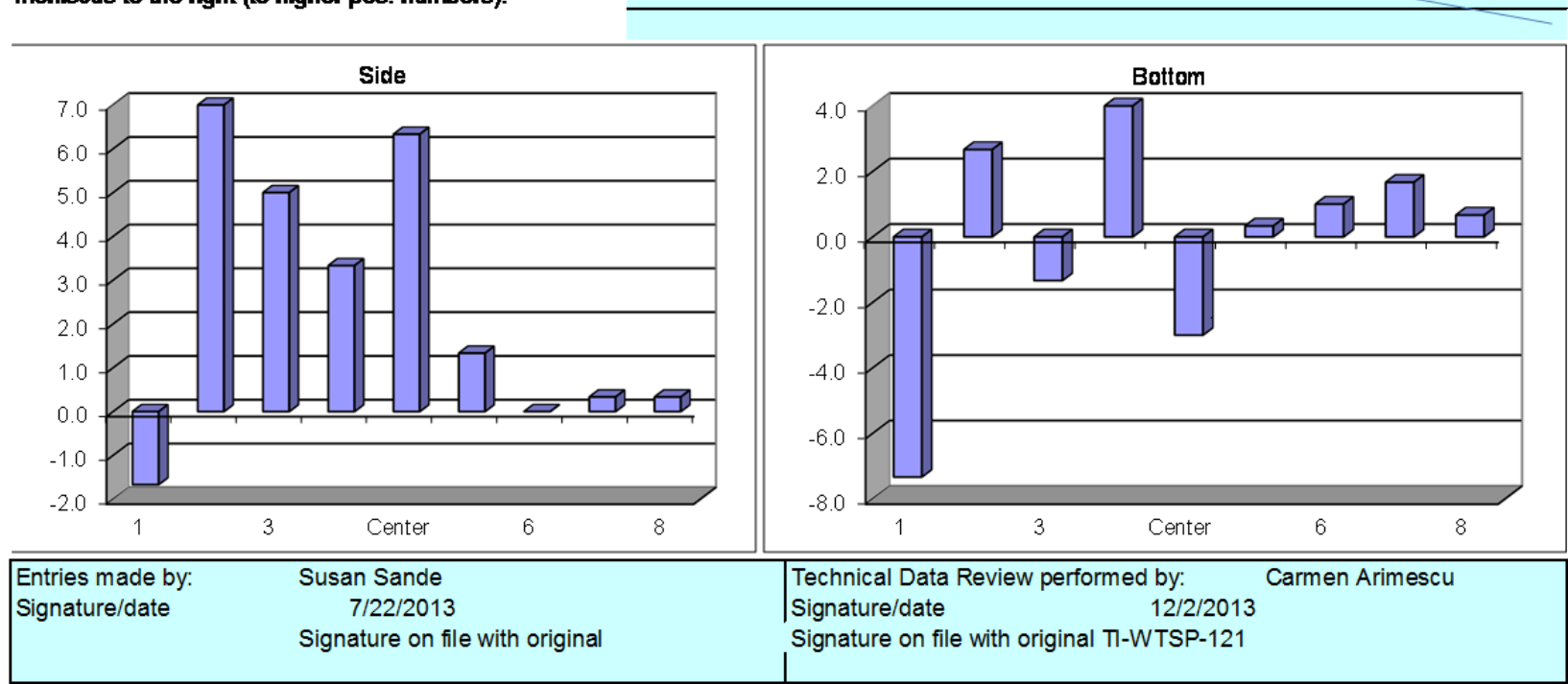


T1-WTPSP-121

Page

FLOW ANGLE DATA FORM

Site LV-S3 Scale Model

Date $7 / 22 / 2013$

Tester SS, EA

Stack Dia. 11.922 in

Stack X-Area 111.6 in2

Elevation N.A. fi

Distance to disturbance 510.25

Start/End Trme $0945 / 1020$
LV-S3_FlowAngle.xlsx

$$
\text { 2nd }
$$

Run No. FA-13

CCP-WTPSP-\#\#

Fan Setting $\frac{30}{30}$

Fan configuration AB MIN

Approx. air vel. 1544 fipm at point $\gg$

Units degrees (clockwise > pos. nos.)

Port $\frac{2}{97.1}$

Stack Temp $\quad 97.1$ degrees F

1 st

Order $\rightarrow$

Traverse $\rightarrow$

Trial $\longrightarrow$

\begin{tabular}{|c|c|}
\hline Point & Depth, in.
\end{tabular}

side

\begin{tabular}{l|l}
\cline { 2 - 2 } & 1 st \\
\hline
\end{tabular}

3

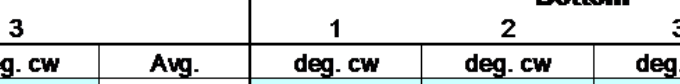

\begin{tabular}{l|c|c|c|c|c}
\hline Avg. & deg. $\mathbf{~ w ~}$ & deg. cw & deg. cw & Avg. \\
\hline-11 & -6.7 & -1 & & & -0.7
\end{tabular}

\begin{tabular}{|c|r|c|c|r|}
\hline & \multicolumn{1}{c}{$\mathbf{1}$} \\
\hline Point & Depth, in. & deg. cw & deg. cw & deg. \\
\hline $\mathbf{1}$ & 0.50 & -14 & 5 & -11 \\
\hline $\mathbf{2}$ & 1.25 & -16 & 0 & \\
\hline $\mathbf{3}$ & 2.31 & 0 & 0 & \\
\hline $\mathbf{4}$ & 3.85 & 9 & 7 & \\
\hline Center & 5.96 & 0 & 7 & \\
\hline $\mathbf{5}$ & 8.07 & 1 & -2 & \\
\hline $\mathbf{6}$ & 9.61 & 0 & -1 & -1 \\
\hline $\mathbf{7}$ & 10.67 & 0 & 1 & -1 \\
\hline $\mathbf{8}$ & 11.42 & 1 & 2 & \\
\hline
\end{tabular}

Mean of absolute values:

- wo points by wall:

\begin{tabular}{c|c|c|c|c|c}
-11 & $-\mathbf{6 . 7}$ & -1 & 0 & -1 & -0.7 \\
\hline 1 & -5.0 & -1 & -1 & 0 & -0.7 \\
\hline
\end{tabular}

\begin{tabular}{l|r}
-5 & -1.7 \\
\hline & 8
\end{tabular}

\begin{tabular}{l|l}
9 & 8.3 \\
\hline 9 & 5.3
\end{tabular}

\begin{tabular}{l|l}
2 & 0.3 \\
-1 & -0.7
\end{tabular}

\begin{tabular}{l|r}
-1 & -0.7 \\
\hline-1 & 0.0
\end{tabular}

0.0

3.3

Instuments Used:

S-type pitot

Velocity sensor

Angle indicator

Manometer

Dwyer 24-inch S-type Pitot\#10

TSI Velocicalc SN\#T95351203001

Shop built

Dwyer 400-5, S36N 28293

3.0

Note:

To assure similar hose connections

between the manometer and pitot tube, rotating

the pitot tube assembly clockwise dives the

meniscus to the right (to higher pos. numbers).

Cal. Due

Cert. of conformance

10-Dec-13

Cat. 3

Cat. 3 MAN-5

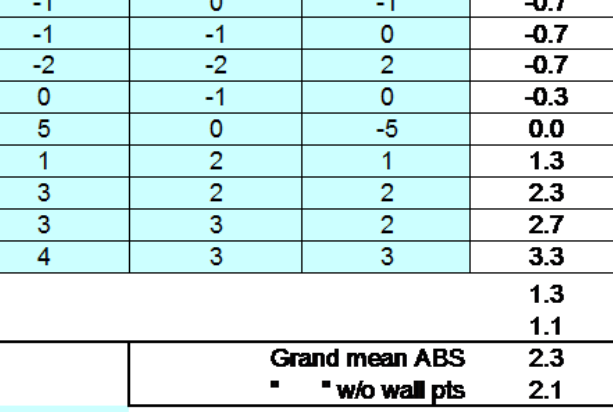

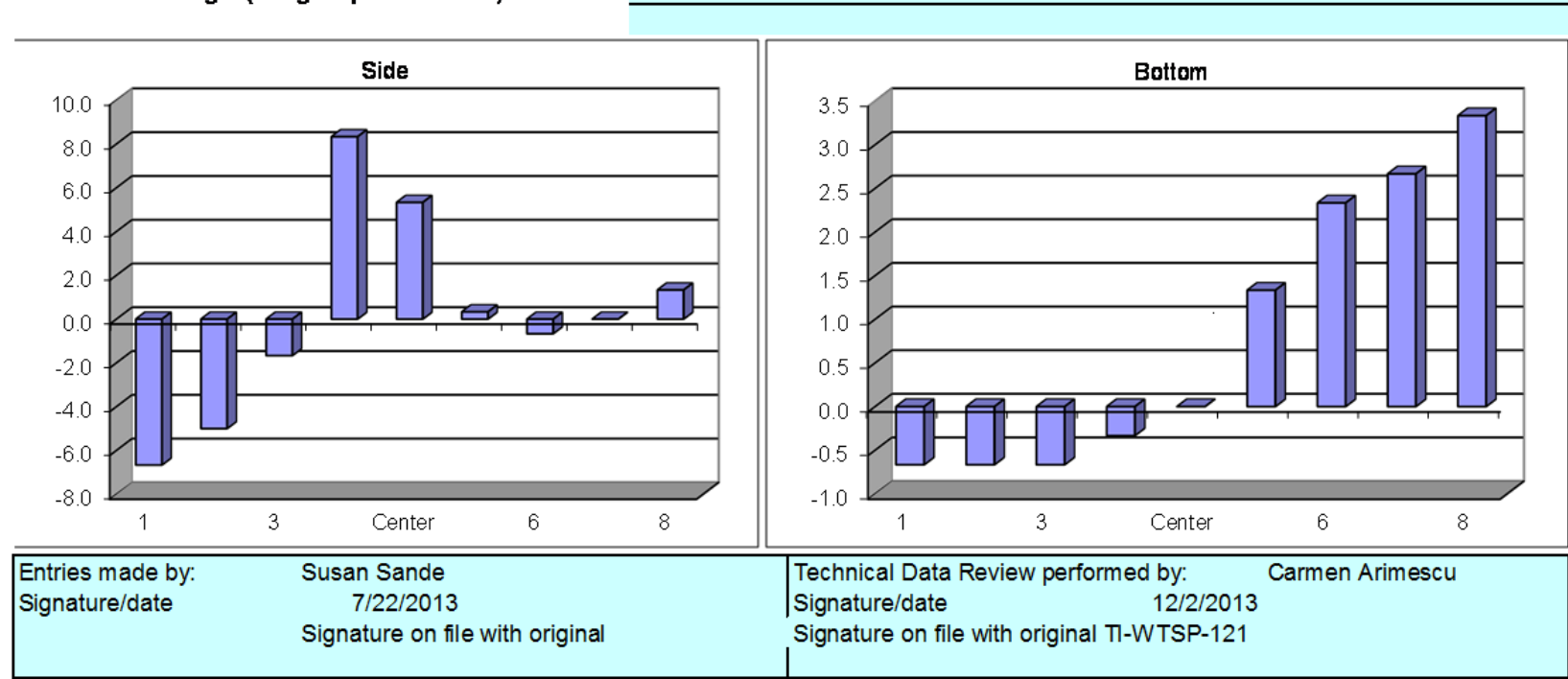


T1-WTPSP-121

Page

FLOW ANGLE DATA FORM

Site LV-S3 Scale Model

Date $7 / 22 / 2013$

Tester EA, SFS

Stack Dia. 11.922 in

Stack X-Area 111.6 in2

Elevation N.A. fi

Distance to disturbance 510.25

Start/End Tme $1105 / 1140$
LV-S3_FlowAngle.xlsx

1 st

st

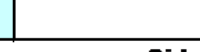

Run No. FA-14

CCP-WTPSP-\#\#

Fan Setting 30

Fan configuration AC MIN

Approx. air vel. 1744 fipm at point $>>$

Units degrees (clockwise > pos. nos.)

Port $\frac{2}{108.7}$

Stack Temp 108.7 degrees F

Order $\rightarrow$

Traverse $\rightarrow$

\begin{tabular}{|c|c|} 
Trial $\longrightarrow$ Point & Depth, in.
\end{tabular}

2nd

Bottom

\begin{tabular}{|c|r|c|c|c|c|}
\hline Point & Depth, in. & deg. cw & deg. cw & deg. cw & Av \\
\hline $\mathbf{1}$ & 0.50 & -20 & -21 & -20 & -20 \\
\hline $\mathbf{2}$ & 1.25 & -1 & 0 & 1 & \\
\hline $\mathbf{3}$ & 2.31 & -3 & -3 & -1 & \\
\hline $\mathbf{4}$ & 3.85 & -4 & -4 & -1 & \\
\hline Center & 5.96 & 2 & -3 & 0 & \\
\hline $\mathbf{5}$ & 8.07 & -4 & 0 & -2 & -2 \\
\hline $\mathbf{6}$ & 9.61 & -2 & -2 & -1 & -1 \\
\hline $\mathbf{7}$ & 10.67 & -2 & -1 & -2 & -1 \\
\hline $\mathbf{8}$ & 11.42 & -1 & -1 & -1 & -1 \\
\hline
\end{tabular}

Mean of absolute values:

- wo points by wall:

Instuments Used:

S-type pitot

Velocity sensor

Angle indicator

Manometer

Note:

To assure similar hose connections

between the manometer and pitot tube, rotating

the pitot tube assembly clockwise dives the

meniscus to the right (to higher pos. numbers).
Dwyer 24-inch S-type Pitot\#10

TSI Velocicalc SN\#T95351203001

Shop built

Dwyer 400-5, S36N 28293

\section{Notes:}

$1 \quad 3 \quad 2 \quad 3$

\begin{tabular}{l|l|l|l|l} 
Avg. & deg. cw & deg. cw & deg. cw & Avg
\end{tabular}

$-20.3$

0.0

$-2.3$

$-0.3$

$-2.0$

$-1.7$

$-1.0$

3.6
1.6

\begin{tabular}{c|c} 
deg. $\mathbf{c w}$ & deg. \\
\hline-9 & \\
\hline 3 & \\
\hline-1 & \\
\hline-1 & \\
\hline 1 & \\
\hline-5 & \\
\hline-2 & \\
\hline-1 & \\
\hline-1 & \\
\hline
\end{tabular}

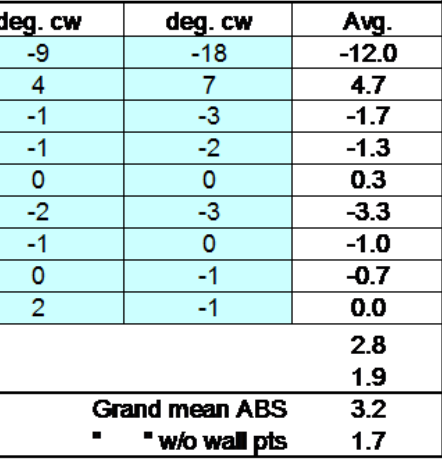

Cert. of conformance

10-Dec-13

Cat. 3

Cat. 3 MAN-5

Ambient T 95.0 F

Ambient $P 29.83$ in. $\mathrm{Hg}$

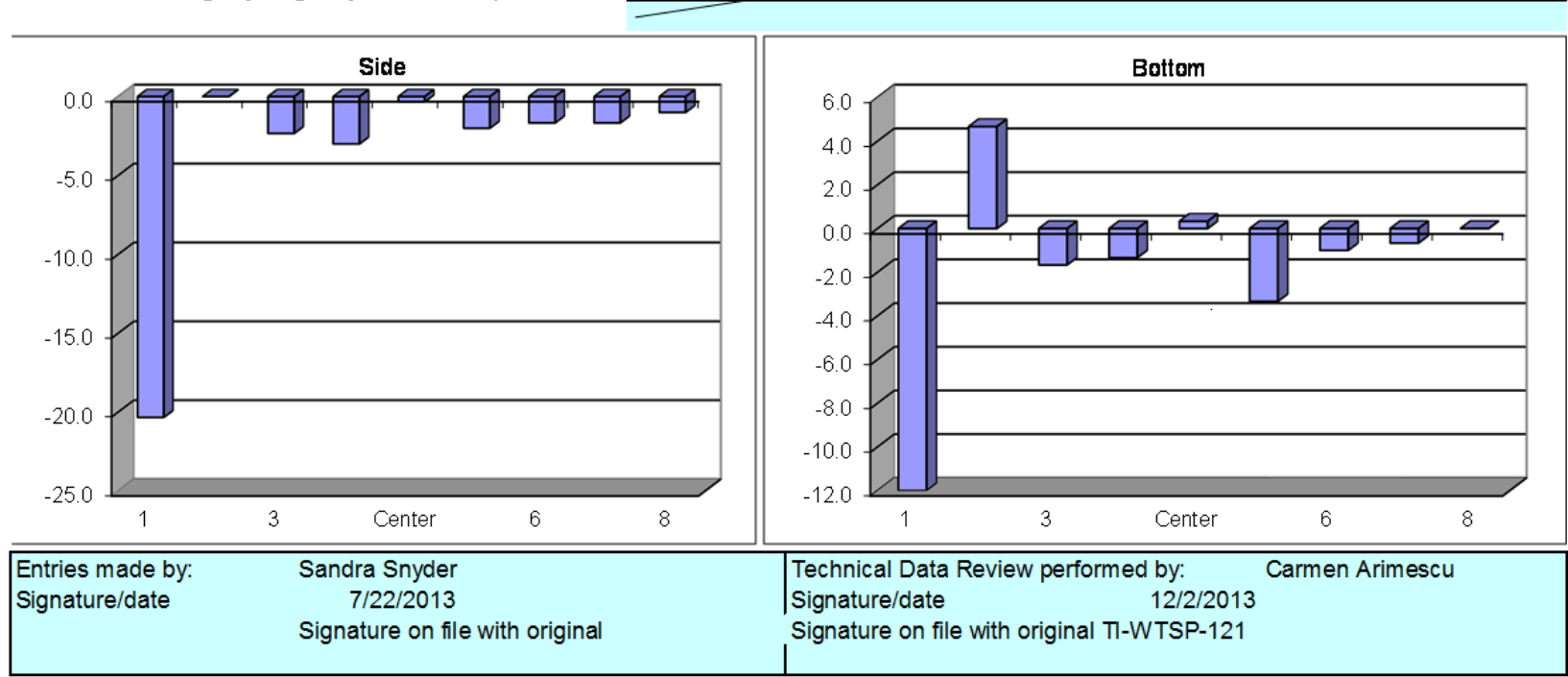


T1-WTPSP-121

Page

FLOW ANGLE DATA FORM

Site LV-S3 Scale Model

Date $7 / 22 / 2013$

Tester CB,SFS

Stack Dia. 11.922 in

Stack X-Area 111.6 in2

Elevation N.A. fi

Distance to disturbance 510.25 in

Start/End Trme $1209 / 1242$
LV-S3_FlowAngle.xlsx

$$
\text { 2nd }
$$

d

1
Run No. FA-15

CCP-WTPSP-\#\#

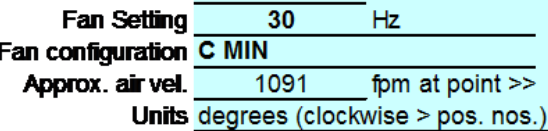

Stack Temp 111.1 degrees F

Order $\rightarrow$

Traverse $\rightarrow$

\begin{tabular}{|r|r|}
\multicolumn{1}{|c|}{ Trial $\longrightarrow$ Point } & Depth, in.
\end{tabular}

1 st

\begin{tabular}{|c|r|c|c|r|}
\hline Point & Depth, in. & deg. $\mathbf{~ w ~}$ & deg. $\mathbf{~ w ~}$ & deg. \\
\hline $\mathbf{1}$ & 0.50 & 6 & -17 & -20 \\
\hline $\mathbf{2}$ & 1.25 & -4 & -19 & -17 \\
\hline $\mathbf{3}$ & 2.31 & 7 & -5 & -19 \\
\hline $\mathbf{4}$ & 3.85 & 6 & -16 & \\
\hline Center & 5.96 & 5 & -11 & \\
\hline $\mathbf{5}$ & 8.07 & -1 & -4 & \\
\hline $\mathbf{6}$ & 9.61 & -3 & -4 & \\
\hline $\mathbf{7}$ & 10.67 & -4 & -4 & \\
\hline $\mathbf{8}$ & 11.42 & -3 & -3 & \\
\hline
\end{tabular}

Mean of absolute values:

- "wo points by wall:

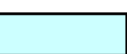

Bottom

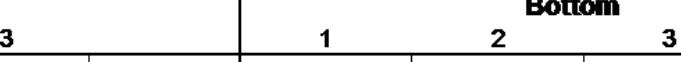

\begin{tabular}{|l|l|l|l|l|l}
\hline Avg. cw & Avg. & deg. cw & deg. cw & deg. cw & Avg
\end{tabular}

\begin{tabular}{l|l}
-20 & -10.3 \\
-17 & -13.3
\end{tabular}

\begin{tabular}{l|r}
-19 & -5.7 \\
\hline-9 & -6.3
\end{tabular}

\begin{tabular}{l|l}
-5 & -6.3 \\
\hline & -3.7
\end{tabular}

\begin{tabular}{|r|r|}
\hline-5 & -3.7 \\
\hline 1 & -1.3 \\
\hline-2 & -3.0 \\
\hline-4 & -4.0 \\
\hline
\end{tabular}

\begin{tabular}{|r|r}
-4 & -4.0 \\
\hline 1 & -1.7 \\
\hline
\end{tabular}

$-1.7$

5.3

Instuments Used:

S-type pitot

Velocity sensor

Angle indicator

Manometer

Cal. Due

\begin{tabular}{c|c|c|c} 
deg. Cw & deg. $\mathbf{~ w ~}$ & deg. $\mathbf{~ w ~}$ & Avg. \\
-12 & -19 & -20 & -17.0
\end{tabular}

\begin{tabular}{l|l|l|l}
-12 & -19 & -20 & -17.0 \\
-22 & -22 & -22 & -22
\end{tabular}

\begin{tabular}{|c|c|}
\hline 3 & -22 \\
\hline
\end{tabular}

\begin{tabular}{|c|c|c}
\hline .7 & -5 & -20 \\
\hline-3 & -16 & -20
\end{tabular}

$-16$

$-20$

$-11$

\begin{tabular}{l|l|l}
\hline 3 & -9 & -14 \\
\hline
\end{tabular}

|

$-21$

\begin{tabular}{l|r}
-17 & -15.3 \\
-14 & -17.7
\end{tabular}

\begin{tabular}{|c|c|c|c|}
\hline-9 & -9 & -12 & -13.0 \\
\hline-4 & -4 & -6 & -10.0 \\
\hline
\end{tabular}

\begin{tabular}{|l|l|l|l|}
\hline-2 & -5 & -6 & -4.7 \\
\hline-2 & -3 & -3 & -3.7 \\
\hline
\end{tabular}

Note:

To assure similar hose connections

between the manometer and pitot tube, rotating

the pitot tube assembly clockwise dives the

meniscus to the right (to higher pos. numbers).

Cert. of conformance

TSI Velocicalc SN\#T95351203001

10-Dec-13

Cat. 3

Cat. 3 MAN-5

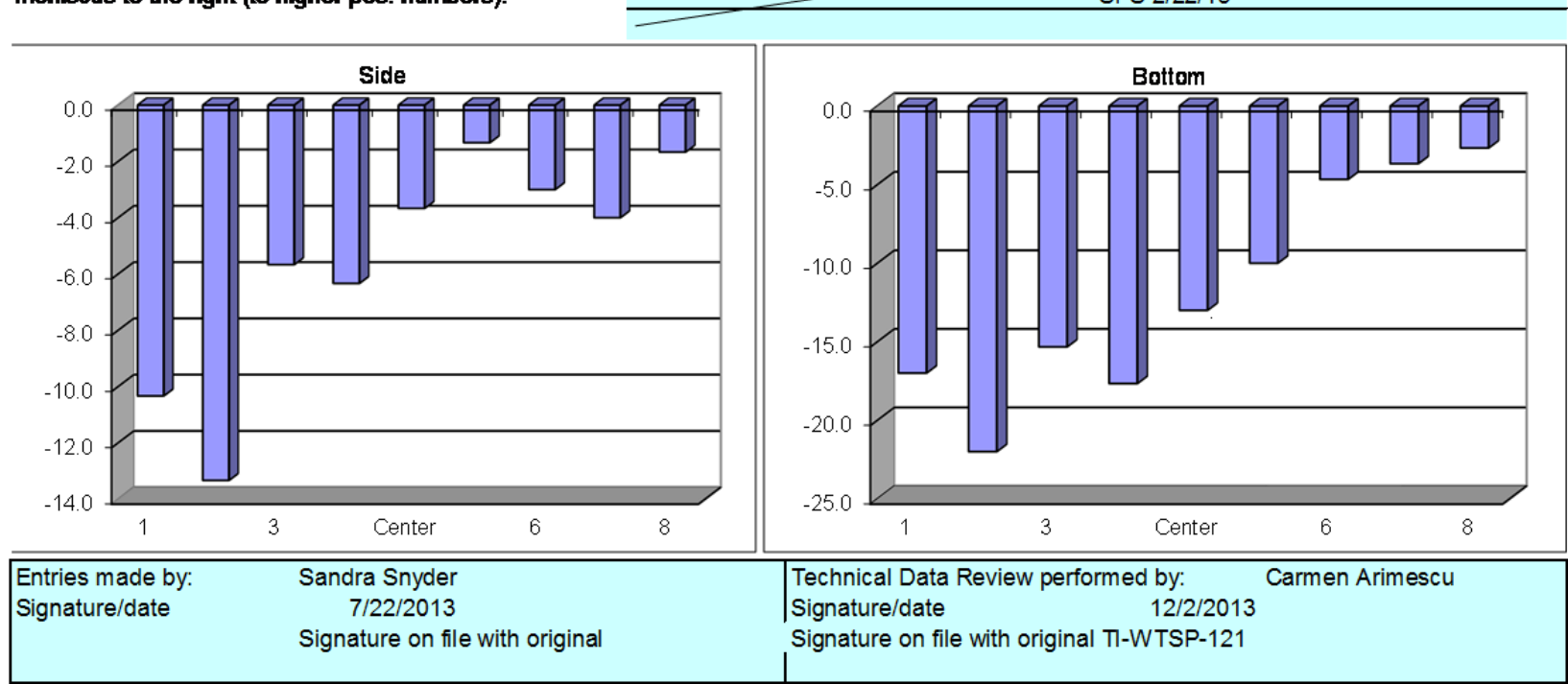


TI-WTPSP-121

Page

FLOW ANGLE DATA FORM

Site LV-S3 Scale Model

Date $7 / 22 / 2013$

Tester $\mathrm{CB}, \mathrm{SFS}, \mathrm{TH}$

Stack Dia. $\frac{11.922 \text { in }}{111.6}$

Stack X-Area 111.6 in2

Elevation N.A. fi

Distance to disturbance $\frac{510.25}{12.43 / 13.35}$

Start/End Trme 12;43 / 13:35
LV-S3_FlowAngle.xlsx

$$
1 \text { st }
$$

Run No. FA- 16

CCP-WTPSP-\#\#

Fan Setting $\mathrm{Hz}$

Fan configuration $\mathrm{C}$ MIN

Approx. air vel. 1184 fpm at point $>>$

Units degrees (clockwise > pos. nos.)

Port 2

Stack Temp 116.5 degrees F

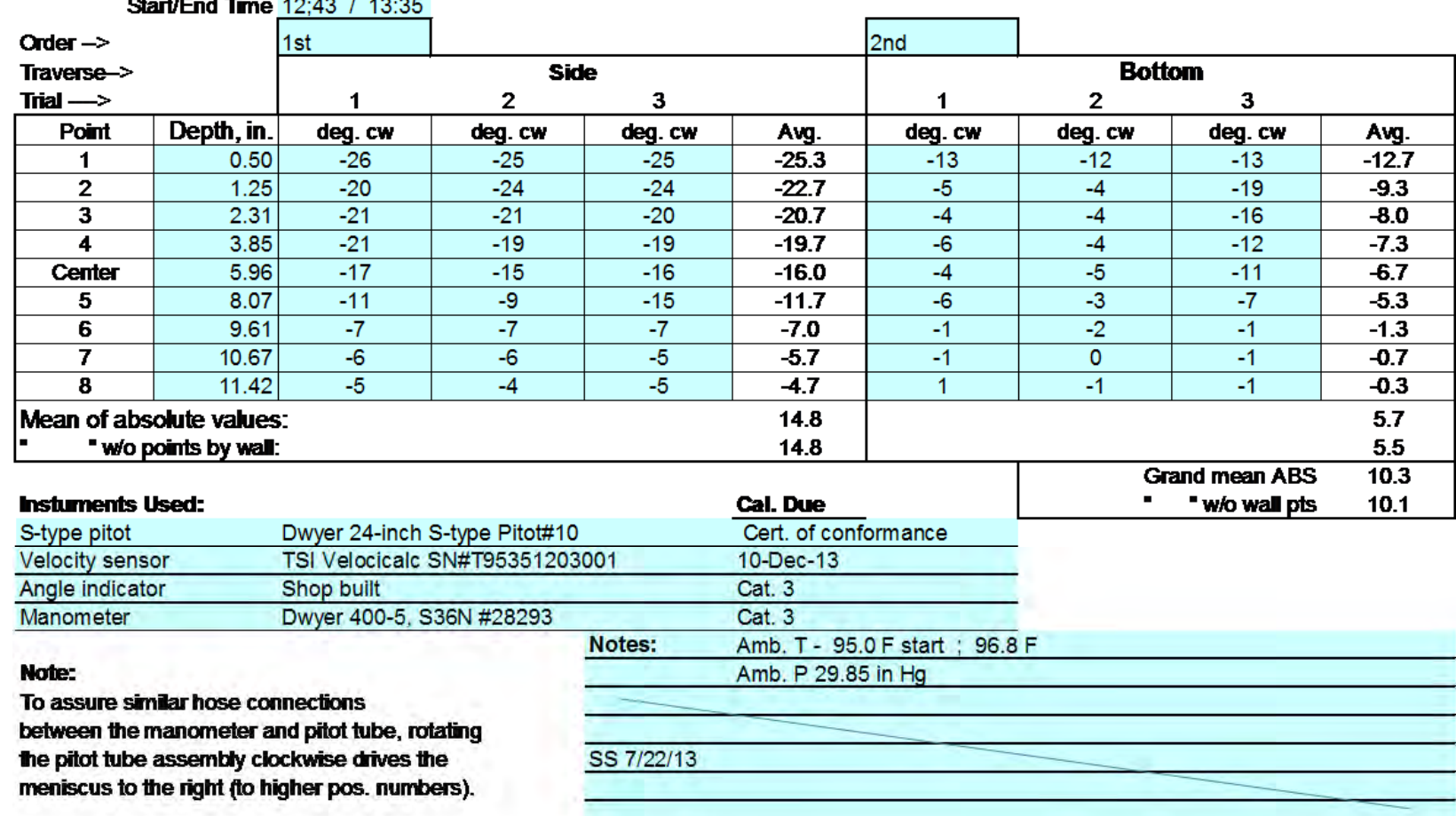

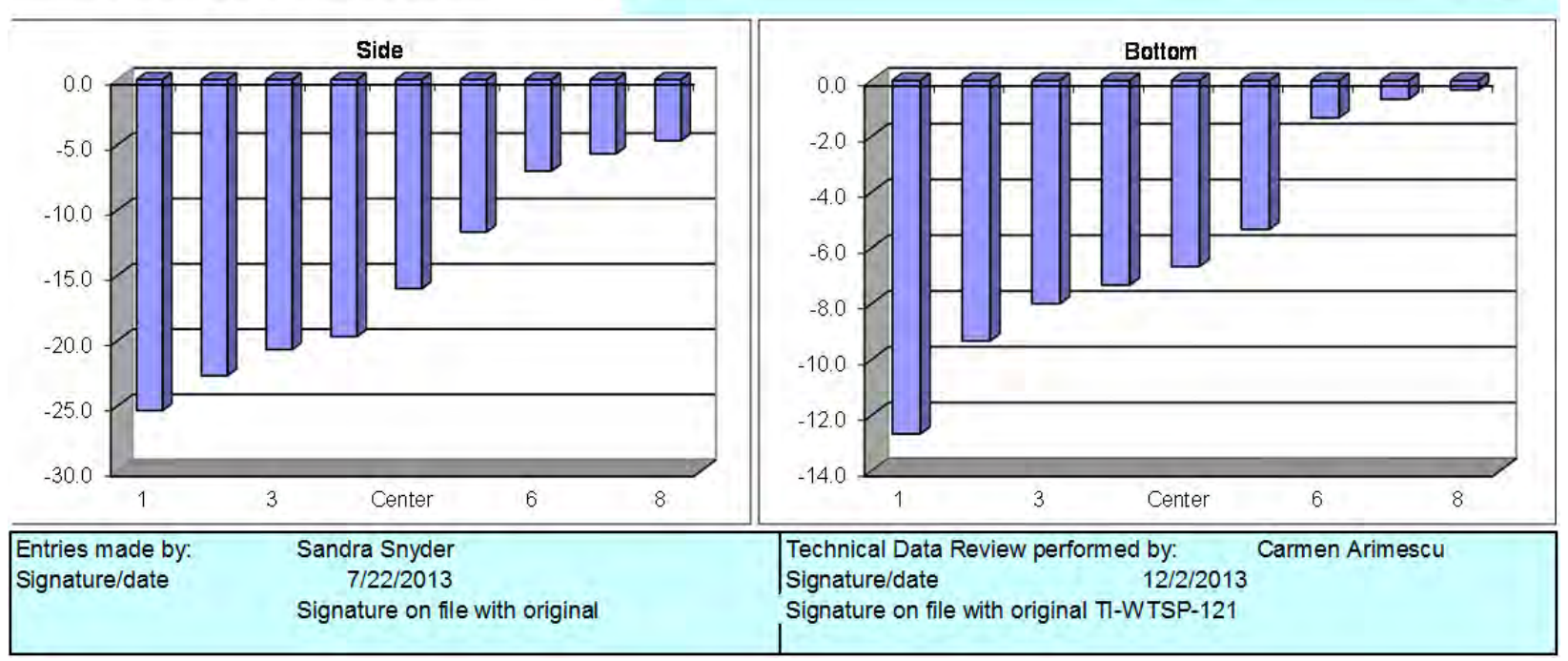


T1-WTPSP-121

Page

FLOW ANGLE DATA FORM

Site LV-S3 Scale Model

Date $7 / 22 / 2013$

Tester TH, SFS

\begin{tabular}{|c|c|}
\hline Stack Dia. & 11.922 \\
\hline Stack X-Area & 111.6 \\
\hline Elevation & N.A. \\
\hline
\end{tabular}

Distance to disturbance 426.25
Start/End Trme $1344 / 1407$
LV-S3_FlowAngle.xlsx

$$
\text { 2nd }
$$

Run No. FA-17

CCP-WTPSP-\#\#

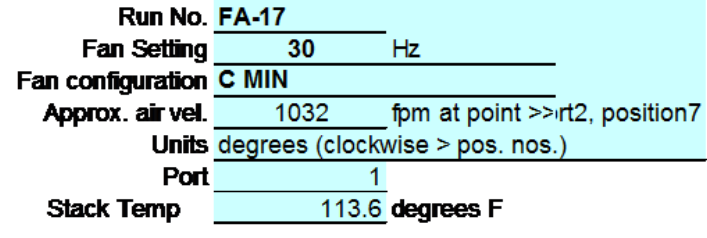
1 st

Order $\rightarrow$

Traverse-

Trial $\longrightarrow$

\begin{tabular}{|c|c|}
\hline Point & Depth, in
\end{tabular}

side

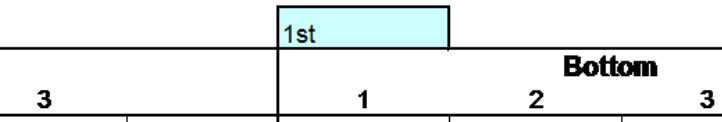

\begin{tabular}{|c|c|c|c|c|}
\hline Point & Depth, in. & deg. cw & deg. cw & deg. cw \\
\hline 1 & 0.50 & 7 & 4 & 6 \\
\hline 2 & 1.25 & 9 & 10 & 11 \\
\hline 3 & 2.31 & 7 & 9 & 8 \\
\hline 4 & 3.85 & 8 & 10 & 9 \\
\hline Center & 5.96 & 6 & 6 & 7 \\
\hline 5 & 8.07 & 4 & 4 & 3 \\
\hline 6 & 9.61 & 4 & 1 & 0 \\
\hline 7 & 10.67 & 8 & -1 & -1 \\
\hline 8 & 11.42 & 6 & -2 & -2 \\
\hline
\end{tabular}

Mean of absolute values:

- wo points by wall:

\begin{tabular}{|c|c|c|c|c|}
\hline Avg. & deg. cw & deg. cw & deg. cw & Avg. \\
\hline 5.7 & 9 & 11 & 8 & 9.3 \\
\hline 10.0 & 11 & 7 & 12 & 10.0 \\
\hline 8.0 & 12 & 7 & 11 & 10.0 \\
\hline 9.0 & 10 & 7 & 8 & 8.3 \\
\hline 6.3 & 6 & 6 & 5 & 5.7 \\
\hline 3.7 & 3 & 4 & 4 & 3.7 \\
\hline 1.7 & 0 & 0 & -1 & -0.3 \\
\hline 2.0 & -2 & -1 & -1 & -1.3 \\
\hline 0.7 & -1 & -2 & -2 & -1.7 \\
\hline $\begin{array}{l}5.2 \\
5.8\end{array}$ & & & & $\begin{array}{l}5.6 \\
5.6\end{array}$ \\
\hline Due & & \multicolumn{2}{|c|}{$\begin{array}{l}\text { Grand mean ABS } \\
\text { - " wo wall pt }\end{array}$} & $\begin{array}{l}5.4 \\
5.7\end{array}$ \\
\hline
\end{tabular}

Instuments Used:

S-type pitot

Velocity sensor

Dwyer 24-inch S-type Pitot\#10

Angle indicator

TSI Velocicalc SN\#T95351203001

Cert. of conformance

Manometer

Dwyer 400-5, S36N \#28293

10-Dec-13

Cat. 3

Cat. 3

Note:

To assure similar hose connections

between the manometer and pitot tube, rotating

the pitot tube assembly clockwise dives the

meniscus to the right (to higher pos. numbers).

\section{Notes:}

(1)

101.3F amb T at start; $98.6 \mathrm{~F}$ at end

Amb P 29.88 in $\mathrm{Hg}$

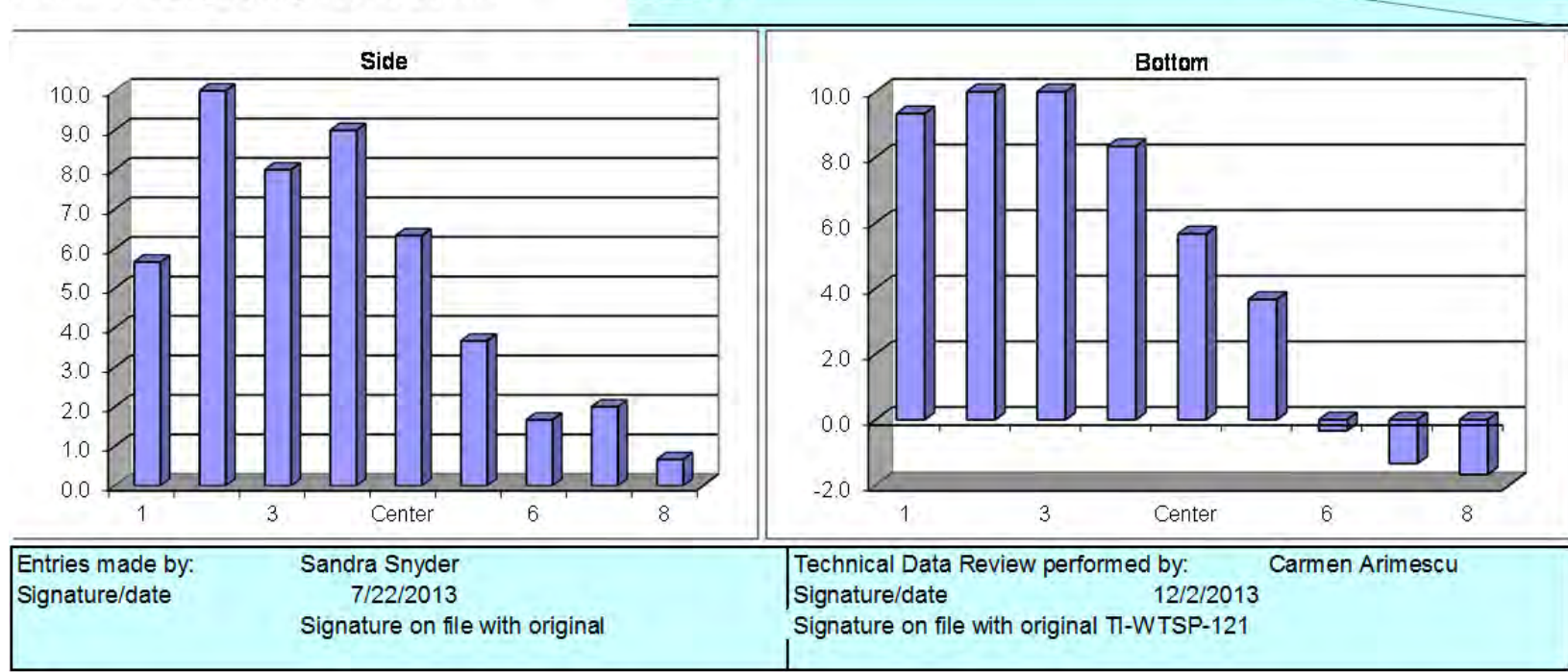


TI-WTPSP-121

Page

FLOW ANGLE DATA FORM

Site LV-S3 Scale Model

Date $7 / 22 / 2013$

Tester $\mathrm{cb}, \mathrm{TH}$

Stack Dia. $\frac{11.922 \text { in }}{111.6}$

Stack X-Area 111.6 in2

Elevation N.A. fi

Distance to disturbance 426.25 in

Start/End Trme 14:20/14:35
LV-S3_FlowAngle.xlsx

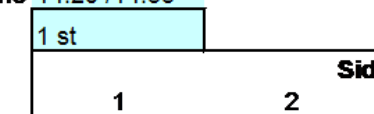

$12{ }^{\text {side }} 3$

Run No. FA-18

CCP-WTPSP-\#\#

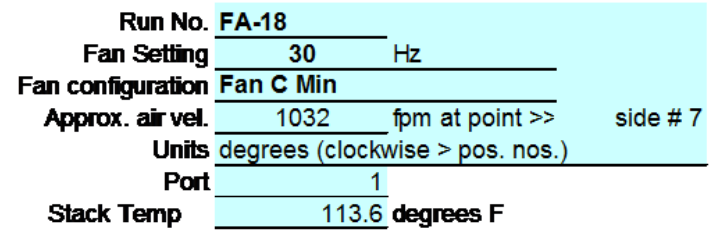

Order $\rightarrow$

Traverse->

Trial $\longrightarrow$

Point Depth, in

\begin{tabular}{c|c|c}
1 & 2 & 3 \\
\hline deg. cw & deg. cw & deg. cw
\end{tabular}

\begin{tabular}{c|c|c} 
& & 1 \\
cw & Avg. & deg. cw
\end{tabular}

2

Bottom

\begin{tabular}{|c|r|r|r|r|r|}
\hline $\mathbf{1}$ & 0.50 & 2 & -5 & 1 & $-\mathbf{7}$ \\
\hline $\mathbf{2}$ & 1.25 & 6 & -5 & 7 & $\mathbf{2}$ \\
\hline $\mathbf{3}$ & 2.31 & 8 & 4 & 6 & $\mathbf{6 . 0}$ \\
\hline $\mathbf{4}$ & 3.85 & 8 & 6 & 9 & $\mathbf{7}$ \\
\hline Center & 5.96 & 5 & 8 & 5 & $\mathbf{6}$ \\
\hline $\mathbf{5}$ & 8.07 & 2 & 6 & 4 & $\mathbf{4}$ \\
\hline $\mathbf{6}$ & 9.61 & -2 & 2 & -3 & -1.0 \\
\hline $\mathbf{7}$ & 10.67 & -4 & -2 & -3 & -3.0 \\
\hline $\mathbf{8}$ & 11.42 & -4 & -3 & -4 & $-\mathbf{7}$ \\
\hline
\end{tabular}

Mean of absolute values:

- wo points by wall:

Instuments Used:

S-type pitot

Velocity sensor

Angle indicator

Manometer

TSI Velocicalc SN\#T95351203001

Shop built

Dwyer 400-5, S36N \#28293

Note:

To assure similar hose connections

between the manometer and pitot tube, rotating

the pitot tube assembly clockwise dives the

meniscus to the right (to higher pos. numbers).

\begin{tabular}{|c|c|c|c|}
\hline Av & deg. cw & deg. cw & deg. cw \\
\hline
\end{tabular}

\begin{tabular}{l|c|c|c|c}
\hline $\mathbf{0 . 7}$ & 10 & 13 & 16 & $\mathbf{1 3 . 0}$ \\
\hline $\mathbf{2 7}$ & 12 & 14 & 15 & $\mathbf{1 3 . 7}$
\end{tabular}

6.0

6.0

$-1.0$

$-3.7$

3.9
4.3

\begin{tabular}{c|c}
10 & \\
12 & \\
13 & \\
10 & \\
10 & \\
\hline 5 & \\
\hline 3 & \\
\hline 0 & \\
\hline 1 & \\
\hline
\end{tabular}

\begin{tabular}{|c|l}
\hline 14 & \\
13 & \\
13 & \\
11 & \\
\hline 9 & \\
\hline 4 & \\
\hline 3 & \\
\hline 3 & \\
\hline
\end{tabular}

\begin{tabular}{|c|c|}
\hline 16 & $\mathbf{1 3 . 0}$ \\
\hline 15 & $\mathbf{1 3 . 7}$ \\
\hline 14 & $\mathbf{1 3 . 3}$ \\
\hline 10 & $\mathbf{1 2 . 3}$ \\
\hline 8 & $\mathbf{1 0 . 3}$ \\
\hline 5 & $\mathbf{7 . 3}$ \\
\hline 3 & $\mathbf{4 . 0}$ \\
\hline 2 & $\mathbf{2 . 0}$ \\
\hline & $\mathbf{2 . 0}$ \\
\hline & $\mathbf{8 . 7}$ \\
mean ABS & $\mathbf{9 . 0}$ \\
\hline wo wal pts & $\mathbf{6 . 3}$ \\
\hline
\end{tabular}

Cert. of conformance

10-Dec-13

Cat. 3

Cat. 3

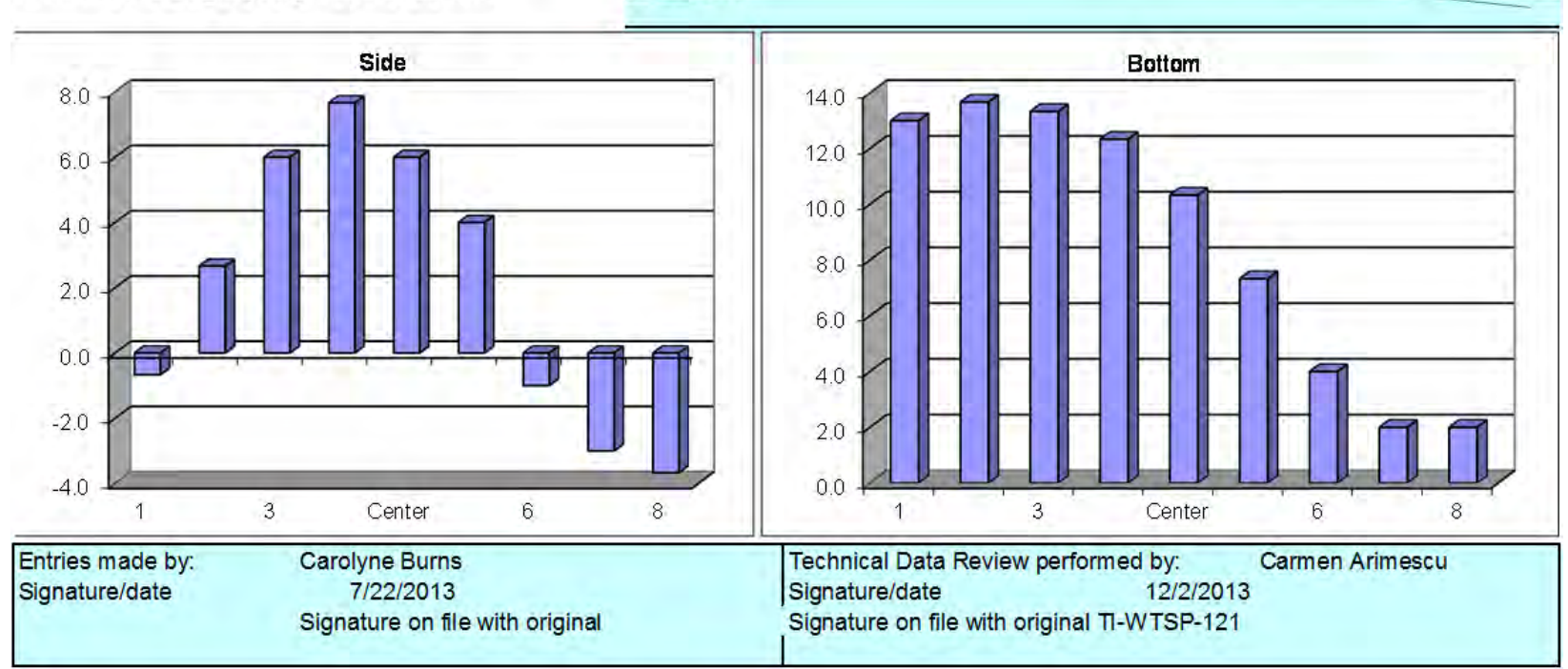


TI-WTPSP-121

Page

FLOW ANGLE DATA FORM
LV-S3_FlowAngle.xlsx

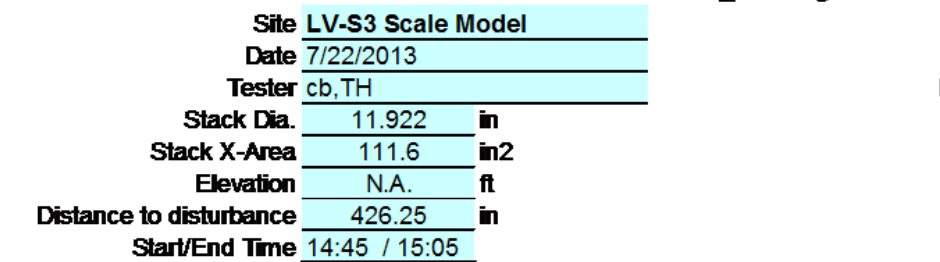

\begin{tabular}{|c|c|c|c|c|c|}
\hline $\mathbf{2}$ & 1.25 & -21 & -20 & -19 & $\mathbf{- 2 0 . 0}$ \\
\hline $\mathbf{3}$ & 2.31 & -18 & -18 & -18 & $\mathbf{- 1 8 . 0}$ \\
\hline $\mathbf{4}$ & 3.85 & -7 & -9 & -7 & $\mathbf{- 7 . 7}$ \\
\hline Center & 5.96 & -2 & -7 & -2 & $-\mathbf{3 . 7}$ \\
\hline $\mathbf{5}$ & 8.07 & -4 & -2 & -1 & $\mathbf{- 2 . 3}$ \\
\hline $\mathbf{6}$ & 9.61 & -3 & -2 & -2 & $\mathbf{- 2 . 3}$ \\
\hline $\mathbf{7}$ & 10.67 & -1 & -3 & -1 & $-\mathbf{1 . 7}$ \\
\hline $\mathbf{8}$ & 11.42 & -4 & -5 & -2 & $\mathbf{- 3 . 7}$ \\
\hline
\end{tabular}

\begin{tabular}{|c|c|c|c|}
\hline & 2 & 3 \\
\hline deg. cw & deg. cw & deg. cw & Avg
\end{tabular}

\begin{tabular}{l|c|c|c}
10 & 11 & 11 & 10.7 \\
\hline 7 & 9 & 7 & 7.7 \\
\hline
\end{tabular}

\begin{tabular}{l|l|l|l|l|}
-2.3 & 3 & 4 & 5 & 4.0 \\
\hline-1.7 & 2 & 3 & 4 & 3.0 \\
\hline-3.7 & 1 & 1 & 3 & 1.7
\end{tabular}

8.7

8.0

Instuments Used:

S-type pitot

Velocity sensor

Cal. Due

Cert. of conformance

TSI Velocicalc SN\#T95351203001

10-Dec-13

Angle indicato

Shop built

Cat. 3

Manometer

Dwyer 400-5, S36N \#28293

Cat. 3

Note:

To assure similar hose connections

between the manometer and pitot tube, rotating

the pitot tube assembly clockwise dives the

meniscus to the right (to higher pos. numbers).

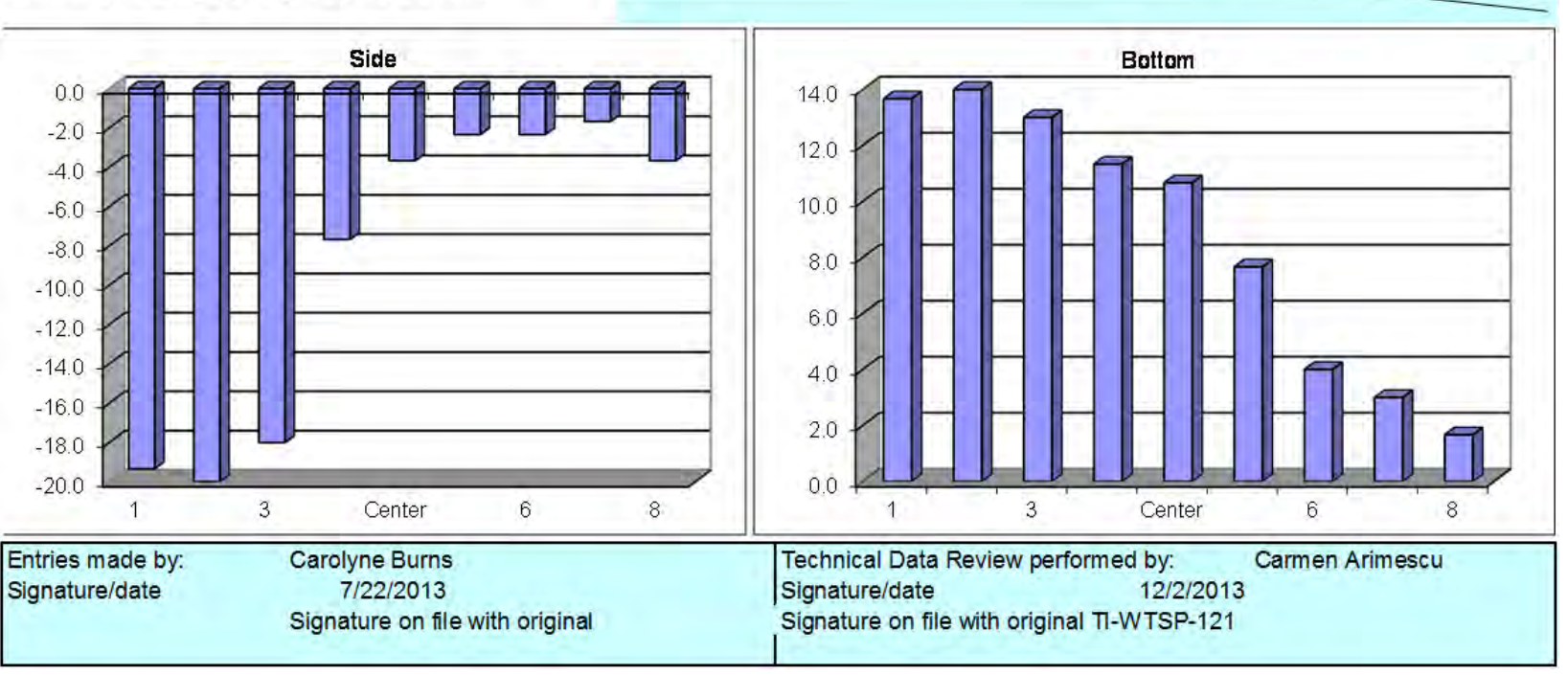


TI-WTPSP-121

Page

FLOW ANGLE DATA FORM

Site LV-S3 Scale Model

Date $7 / 22 / 2013$

Tester $\mathrm{cb}, \mathrm{TH}$

Stack Dia. $\frac{11.922 \text { in }}{111.6}$

Stack X-Area 111.6 in2

Elevation N.A. fi

Distance to disturbance 426.25

Start/End Tme 3:10/3:40 pm
LV-S3_FlowAngle.xlsx

$$
1 \mathrm{st}
$$

Run No. FA-20

CCP-WTPSP-\#\#

Setting

Approx. air vel. $\frac{1636 \quad \text { fpm at point } \gg>}{}$

Units degrees (clockwise > pos. nos.)

7 side Port 1

Stack Temp 117.3 degrees F

Order $\rightarrow$

Traverse $\rightarrow$

\begin{tabular}{r|r} 
Trial $\rightarrow$ Point & Depth, in.
\end{tabular}

Side

2 nd

3

\begin{tabular}{l|l|l|l} 
deg. cw & deg. cw & deg. cw & Avg.
\end{tabular}

3 Avg.

\begin{tabular}{l|c|c} 
g. Cw & deg. Cw & Avg. \\
\hline-16 & -20 & -18.7 \\
-19 & -18 & -18.3
\end{tabular}

\begin{tabular}{|c|c|c|c|c|c|}
\hline $\mathbf{2}$ & 1.25 & -18 & -19 & -18 & $\mathbf{- 1 8 . 3}$ \\
\hline $\mathbf{3}$ & 2.31 & -17 & -18 & -16 & $\mathbf{- 1 7 . 0}$ \\
\hline $\mathbf{4}$ & 3.85 & -16 & -17 & -15 & $\mathbf{- 1 6 . 0}$ \\
\hline Center & 5.96 & -10 & -11 & 4 & $-\mathbf{5 . 7}$ \\
\hline $\mathbf{5}$ & 8.07 & -5 & -2 & 1 & $-\mathbf{2 . 0}$ \\
\hline $\mathbf{6}$ & 9.61 & 0 & -3 & -2 & $-\mathbf{1 . 7}$ \\
\hline $\mathbf{7}$ & 10.67 & -3 & -2 & -2 & $\mathbf{- 2 . 3}$ \\
\hline $\mathbf{8}$ & 11.42 & -3 & -3 & -1 & $\mathbf{- 2 . 3}$ \\
\hline
\end{tabular}

Mean of absolute values:

- wo points by wall:

9.3

9.0

Instuments Used:

S-type pitot

Velocity sensor

Dwyer 24-inch S-type Pitot\#10

TSI Velocicalc SN\#T95351203001

Shop built

Dwyer 400-5, S36N \#28293

Manometer

Notes:

Note:

To assure similar hose connections

between the manometer and pitot tube, rotating

the pitot tube assembly clockwise dives the

meniscus to the right (to higher pos. numbers).

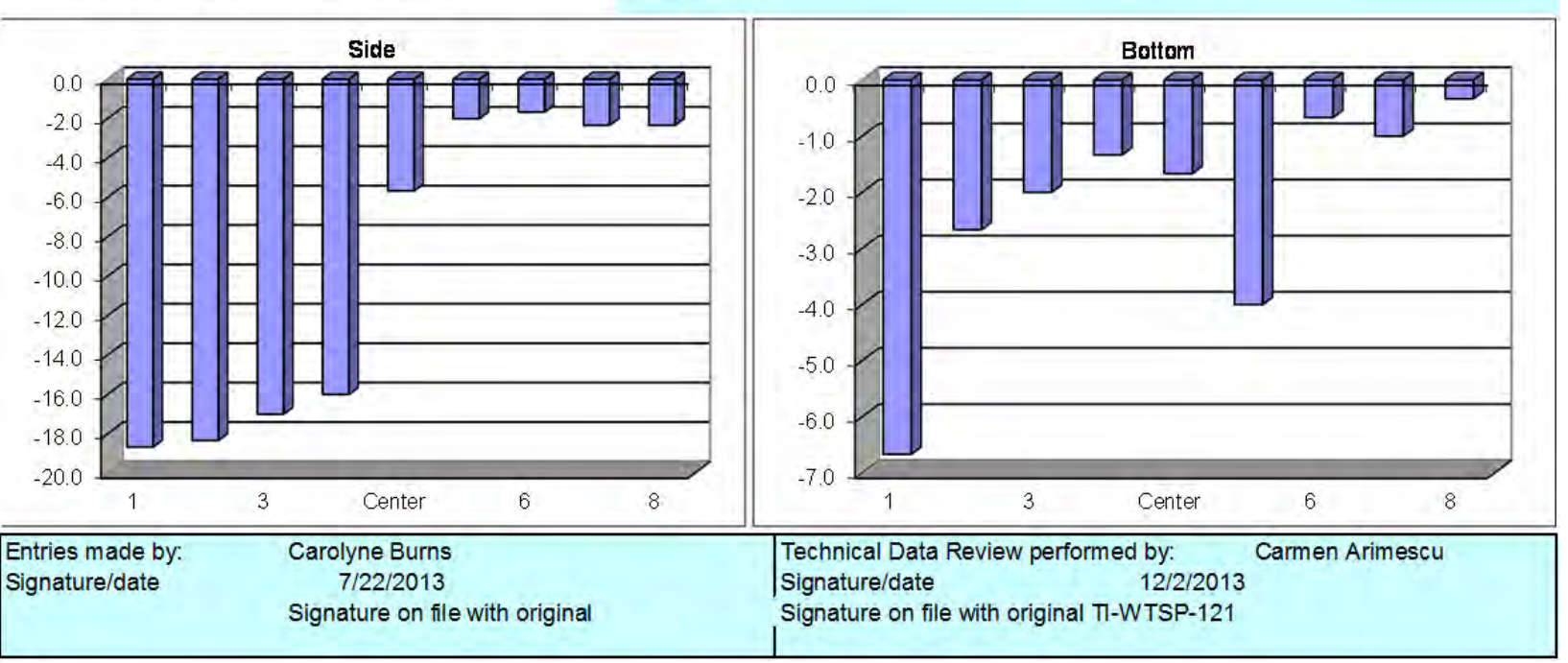


TI-WTPSP-121

Page

FLOW ANGLE DATA FORM

Site LV-S3 Scale Model

Date $7 / 22 / 2013$

Tester $\mathrm{cb}, \mathrm{TH}$

Stack Dia. $\frac{11.922 \text { in }}{111.6}$

Stack X-Area 111.6 in2

Elevation N.A. fi

Distance to disturbance 426.25 in

Start/End Tme 15:55/17:05
LV-S3_FlowAngle.xlsx

$$
2 \text { nd }
$$

Run No. FA-21

CCP-WTPSP-\#\#

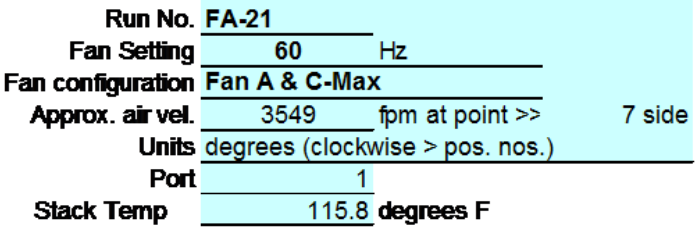

Oder $\rightarrow$

Traverse->

\begin{tabular}{|r|r|}
\multicolumn{1}{|c|}{ Trial $\longrightarrow$} \\
\hline Point & Depth, in.
\end{tabular}

Side
$1 \mathrm{st}$

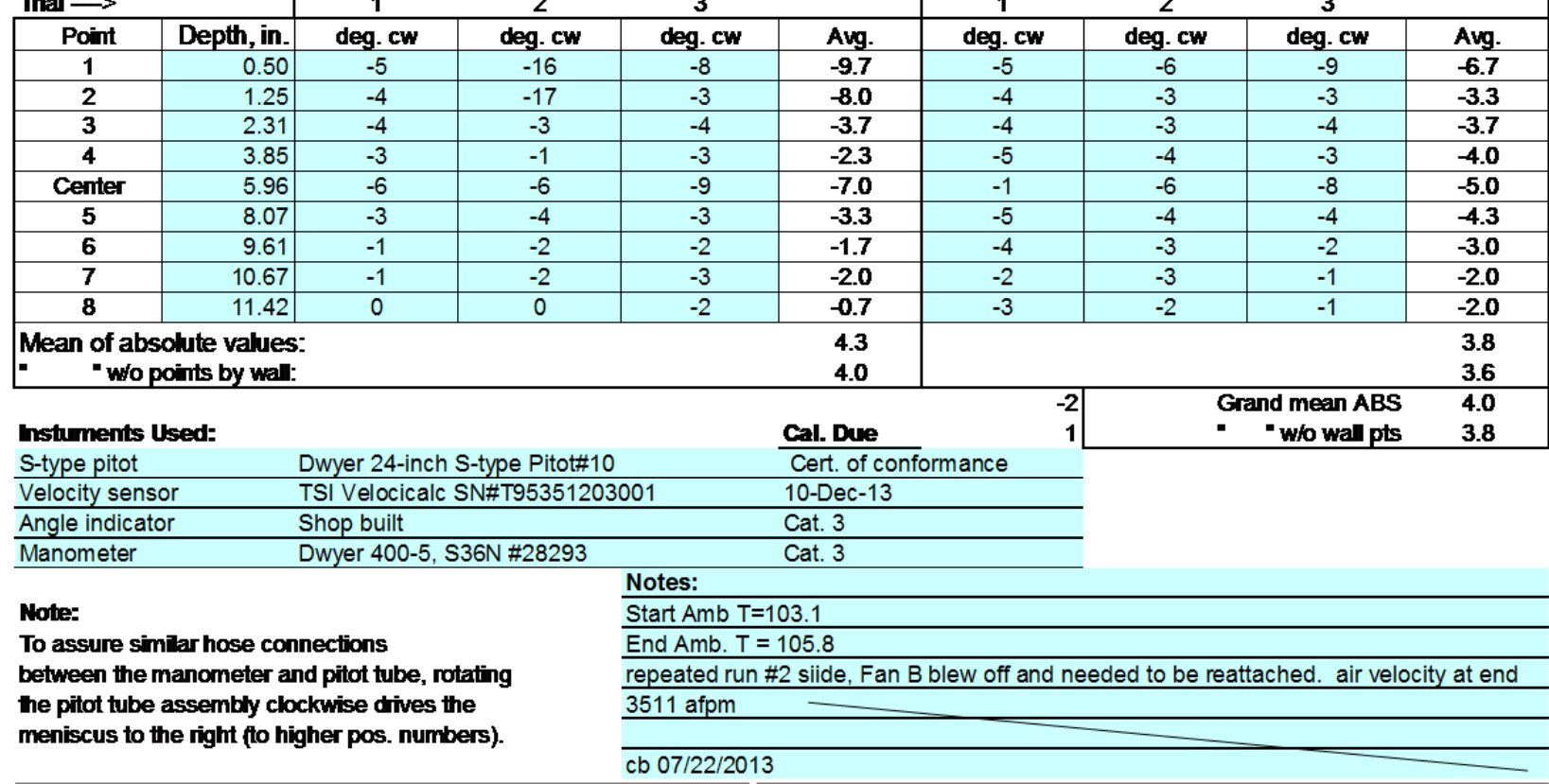

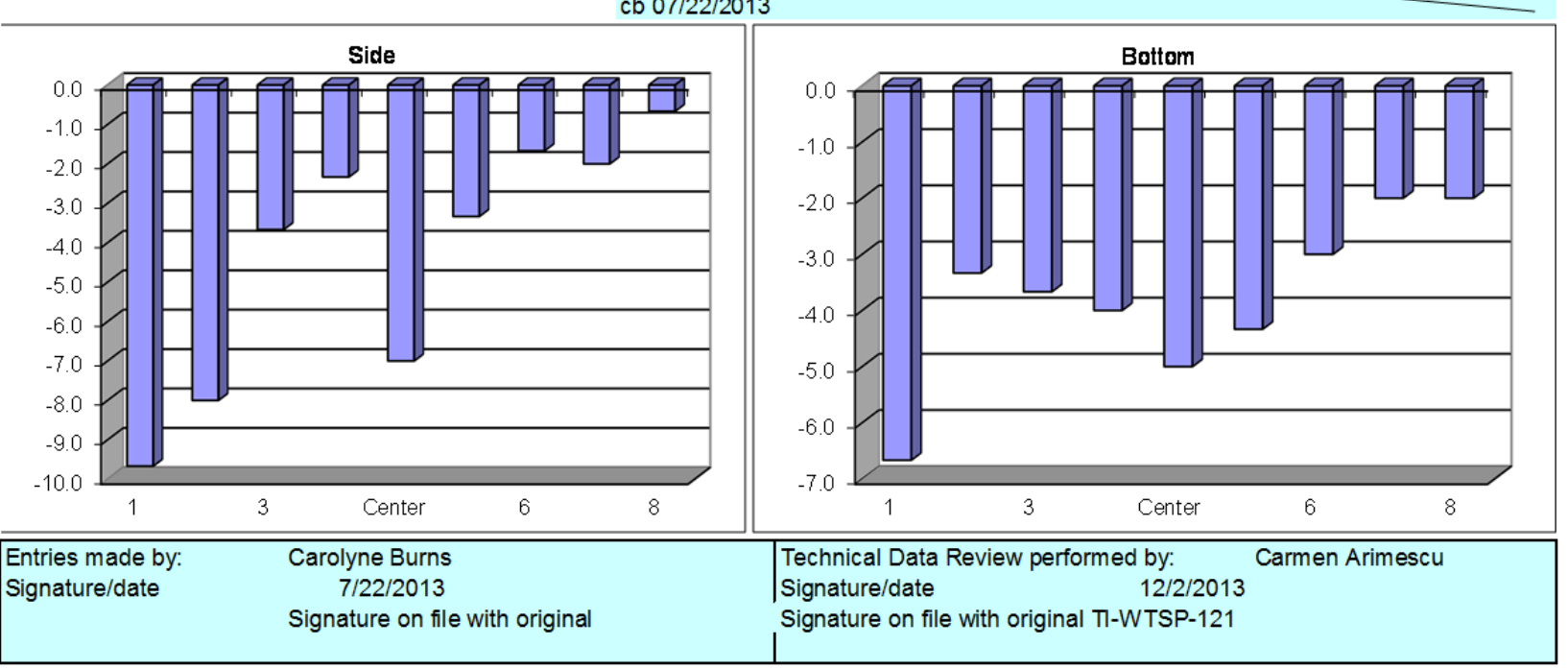


TI-WTPSP-121

Page

FLOW ANGLE DATA FORM

Site LV-S3 Scale Model

Date $7 / 23 / 2013$

Tester SS,SFS, EA

Stack Dia. 11.922

Stack X-Area 111.6 in2

Elevation N.A. fi

Distance to disturbance 426.25

Start/End Tme $0650 / 0728$
LV-S3_FlowAngle.xlsx

$$
2 \text { nd }
$$

Run No. FA-22

CCP-WTPSP-\#\#

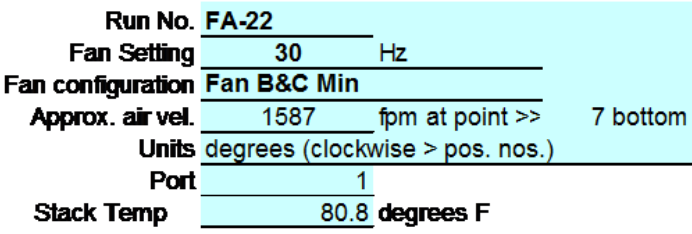

Oder $\rightarrow$

Traverse->

\begin{tabular}{|r|r|}
\multicolumn{1}{|c|}{ Trial $\longrightarrow$ Point } & Depth, in.
\end{tabular}
$1 \mathrm{st}$
LI

(amp

80.8 degrees $F$

\begin{tabular}{|c|c|c|c|}
\hline Point & Denth in & dea cw & dog cw \\
\hline 1 & 0.50 & -7 & -11 \\
\hline 2 & 1.25 & -2 & 1 \\
\hline 3 & 2.31 & -2 & -2 \\
\hline 4 & 3.85 & -1 & -2 \\
\hline Center & 5.96 & -4 & -2 \\
\hline 5 & 8.07 & -2 & 1 \\
\hline 6 & 9.61 & 0 & 2 \\
\hline 7 & 10.67 & 2 & 1 \\
\hline 8 & 11.42 & 1 & 2 \\
\hline
\end{tabular}

3

Mean of absolute values:

- wo points by wall:

Instuments Used:

S-type pitot

Velocity sensor

Angle indicator

Manometer

\section{al:}

1.5

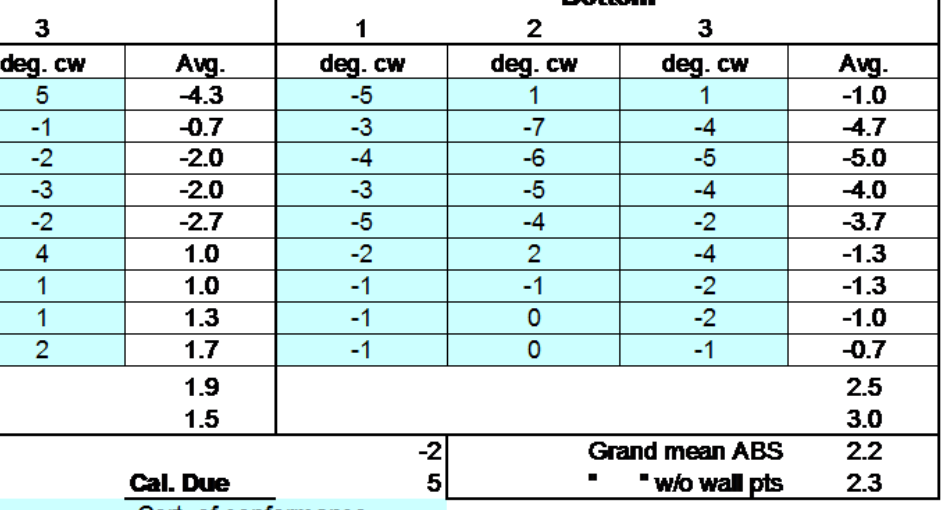

\section{Note:}

To assure similar hose connections

between the manometer and pitot tube, rotating

the pitot tube assembly clockwise dives the

meniscus to the right (to higher pos. numbers).

Cert. of conformance

TSI Velocicalc SN\#T95351203001 10-Dec-13

Shop built $\quad$ Cat. 3

Dwyer 400-5, S36N \#28293 Cat. 3 MAN-5

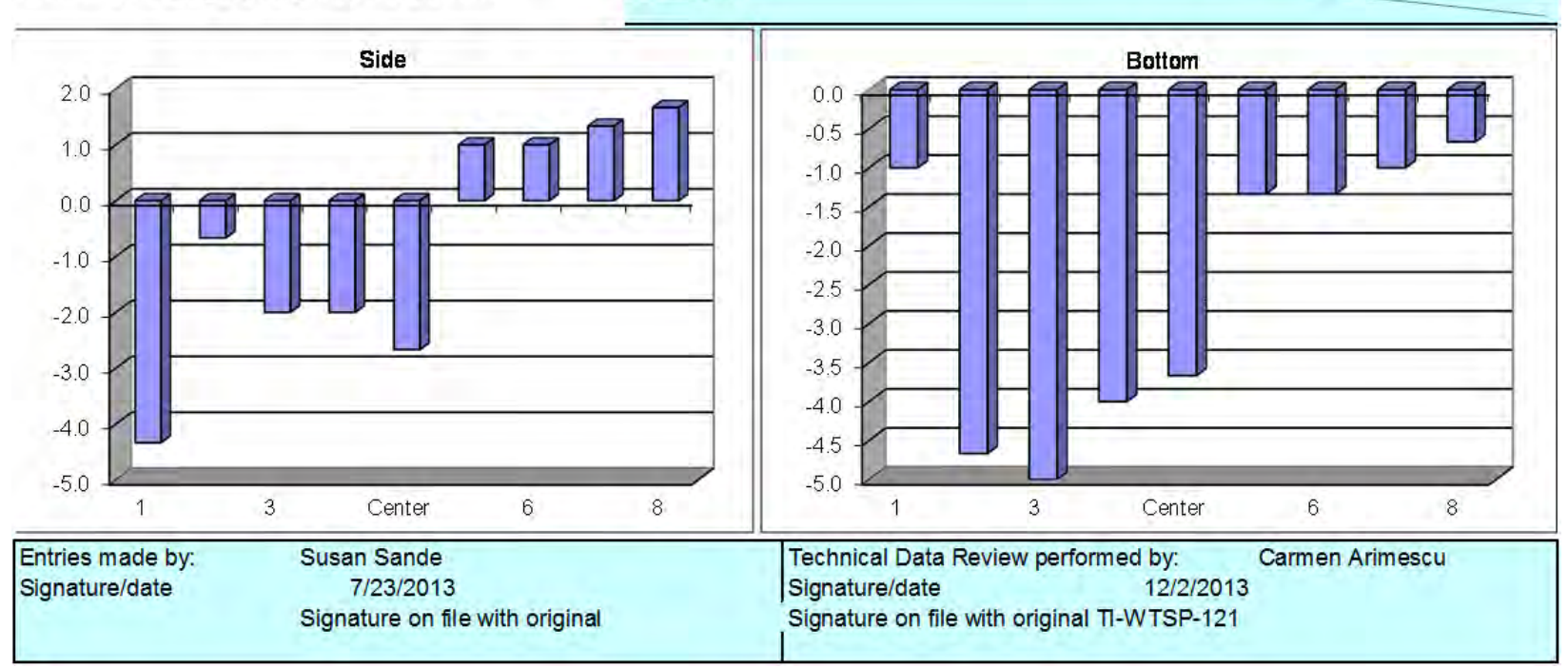


TI-WTPSP-121

Page

FLOW ANGLE DATA FORM

Site LV-S3 Scale Model

Date $7 / 23 / 2013$

Tester SS,SFS, EA

Stack Dia. 11.922

Stack X-Area 111.6 in2

Elevation N.A. fi

Distance to disturbance 426.25 in

Start/End Tme $0730 / 0805$
LV-S3_FlowAngle.xlsx

$$
1 \text { st }
$$

Run No. FA-23

CCP-WTPSP-\#\#

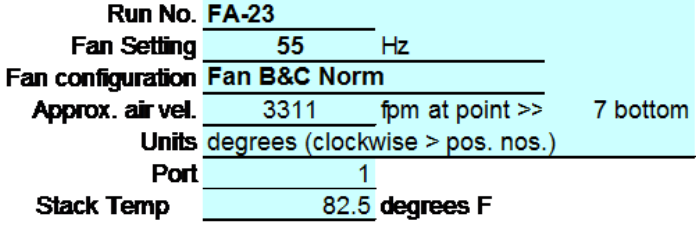

Order $\rightarrow$

Traverse $\rightarrow$

\begin{tabular}{|c|c|} 
Trial $\rightarrow$ Point & Depth, in.
\end{tabular}

2nd

\begin{tabular}{|c|r|c|c|r|}
\hline Point & Depth, in. & deg. cw & deg. cw & deg. \\
\hline $\mathbf{1}$ & 0.50 & -9 & -16 & -17 \\
\hline $\mathbf{2}$ & 1.25 & -1 & -2 & \\
\hline $\mathbf{3}$ & 2.31 & -3 & -3 & \\
\hline $\mathbf{4}$ & 3.85 & -3 & -3 & \\
\hline Center & 5.96 & 2 & -5 & \\
\hline $\mathbf{5}$ & 8.07 & -1 & 0 & \\
\hline $\mathbf{6}$ & 9.61 & 0 & -1 & \\
\hline $\mathbf{7}$ & 10.67 & -1 & 0 & \\
\hline $\mathbf{8}$ & 11.42 & -1 & -2 & \\
\hline
\end{tabular}

Mean of absolute values:

- "wo points by wall:

Instuments Used:

S-type pitot

Velocity sensor

Angle indicator

Manometer

Dwyer 24-inch S-type Pitot\#10

TSI Velocicalc SN\#T95351203001

Shop built

Dwyer 400-5, S36N \#28293

Note:

To assure similar hose connections

between the manometer and pitot tube, rotating

the pitot tube assembly clockwise dives the

meniscus to the right (to higher pos. numbers).

Notes:

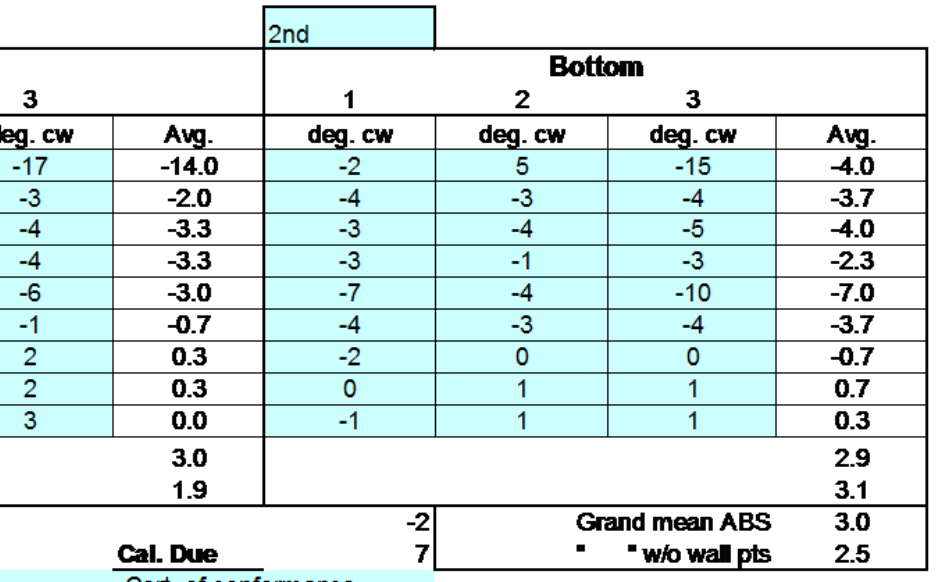

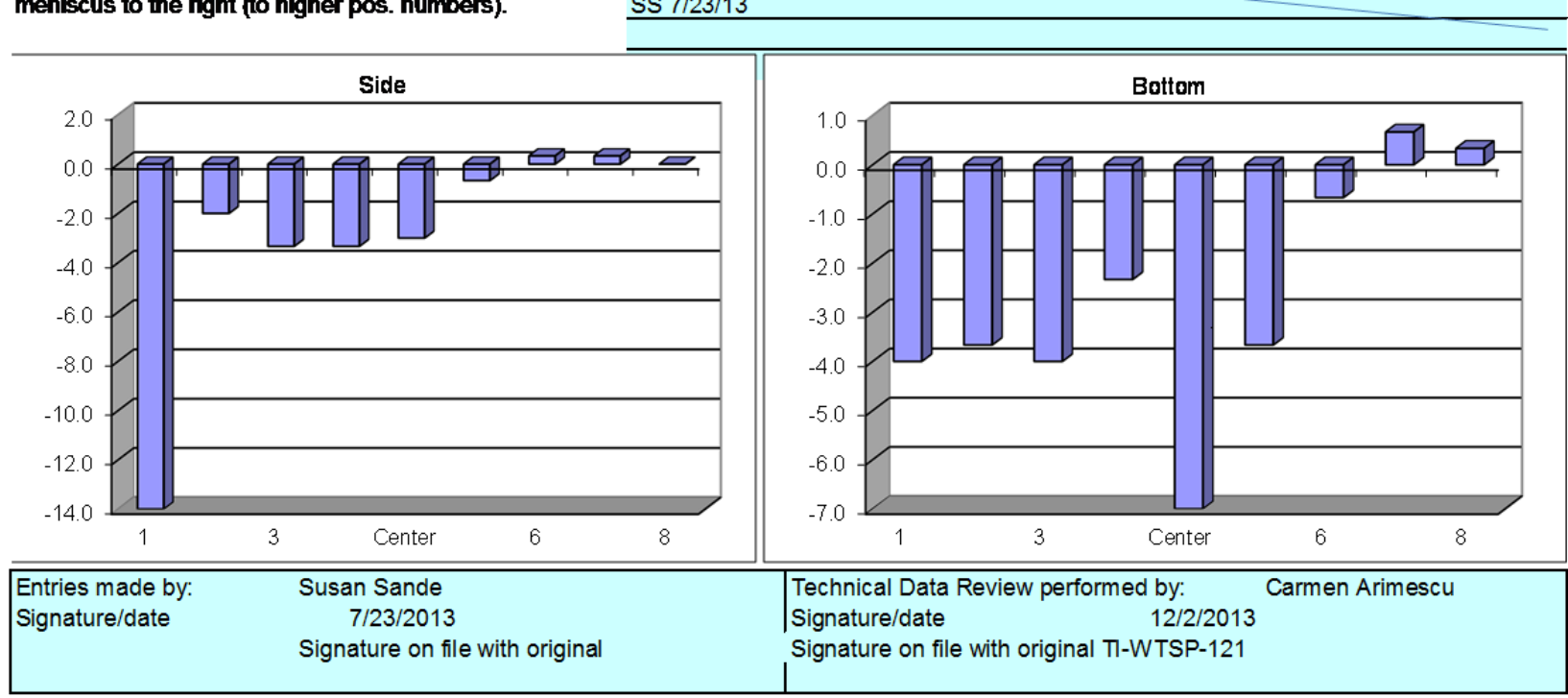


T1-WTPSP-121

Page

FLOW ANGLE DATA FORM

Site LV-S3 Scale Model

Date $7 / 23 / 2013$

Tester SS,SFS, EA

Stack Dia. $\frac{11.922 \text { in }}{111.6}$

Stack X-Area 111.6 in2

Elevation N.A. fi

Distance to disturbance 426.25 in

Start/End Trme $0810 / 0901$
LV-S3_FlowAngle.xlsx

$$
\text { 2nd }
$$

Run No. FA-24

CCP-WTPSP-\#\#

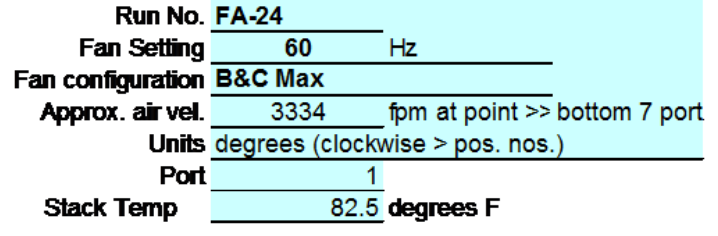

1 st

Onder $\rightarrow$

Traverse $\rightarrow$

Trial $\longrightarrow$

\begin{tabular}{|c|c|}
\hline Point & Depth, in.
\end{tabular}

rende

Side

$122^{\text {Side }} 3$

\begin{tabular}{|c|r|c|c|}
\hline $\mathbf{1}$ & Depth, in. & deg. $\mathbf{c w}$ & deg \\
\hline $\mathbf{2}$ & 0.50 & -2 & \\
\hline $\mathbf{3}$ & 1.25 & -1 & \\
\hline $\mathbf{4}$ & 2.31 & 0 & \\
\hline Center & 3.85 & -2 & \\
\hline $\mathbf{5}$ & 5.96 & -4 & \\
\hline $\mathbf{6}$ & 8.07 & -1 & \\
\hline $\mathbf{7}$ & 9.61 & 0 & \\
\hline $\mathbf{8}$ & 10.67 & 1 & \\
\hline
\end{tabular}

Mean of absolute values:

- wo points by wall:

Instuments Used:

S-type pitot

Velocity sensor

Angle indicator

Manometer

Dwyer 24-inch S-type Pitot\#10

TSI Velocicalc SN\#T95351203001

Shop built

Dwyer 400-5, S36N \#28293

Note:

To assure sinilar hose connections

between the manometer and pitot tube, rotating

the pitot tube assembly clockwise dives the

meniscus to the right (to higher pos. numbers).

\begin{tabular}{|c|c|c|}
\hline eg. $\mathbf{~ w ~}$ & deg. $\mathbf{~ w}$ & Avg. \\
\hline-15 & -16 & $-\mathbf{1 1 . 0}$ \\
\hline-2 & -20 & $\mathbf{- 7 . 7}$ \\
\hline-3 & -6 & $\mathbf{- 3 . 0}$ \\
\hline-4 & -6 & $-\mathbf{4 . 0}$ \\
\hline 5 & 3 & $\mathbf{1 . 3}$ \\
\hline-1 & -1 & $-\mathbf{1 . 0}$ \\
\hline 0 & 0 & $\mathbf{0 . 0}$ \\
\hline 0 & 0 & $\mathbf{0 . 3}$ \\
\hline 1 & -1 & $\mathbf{0 . 3}$ \\
\hline
\end{tabular}

3.2

2.5

\begin{tabular}{c|c|c|c|}
\cline { 2 - 3 } & 1 st & \multicolumn{2}{c}{ Bottom } \\
& 1 & $2^{3}$ & 3 \\
\hline
\end{tabular}

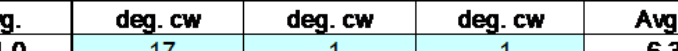

$-7.7$

$-4.0$

1.0

$-1$

\begin{tabular}{c|c|c|c}
-17 & -1 & -1 & -6.3 \\
\hline-3 & -3 & -3 & -3.0
\end{tabular}

\begin{tabular}{l|c|c|c}
-3 & -3 & -3 & -3.0 \\
\hline
\end{tabular}

\begin{tabular}{c|c|c|c}
-4 & -3 & -2 & -3.0 \\
-4 & 0 & -2 & -2.0
\end{tabular}

\begin{tabular}{l|l}
-4 & \\
0 &
\end{tabular}

0
-1
2
2

\begin{tabular}{l|l|l|l}
2 & 1 & 2 & $\mathbf{1 . 7}$ \\
2 & 2 & 1 & $\mathbf{1 . 7}$ \\
2 & 1 & 1 & $\mathbf{1 . 3}$ \\
\hline
\end{tabular}

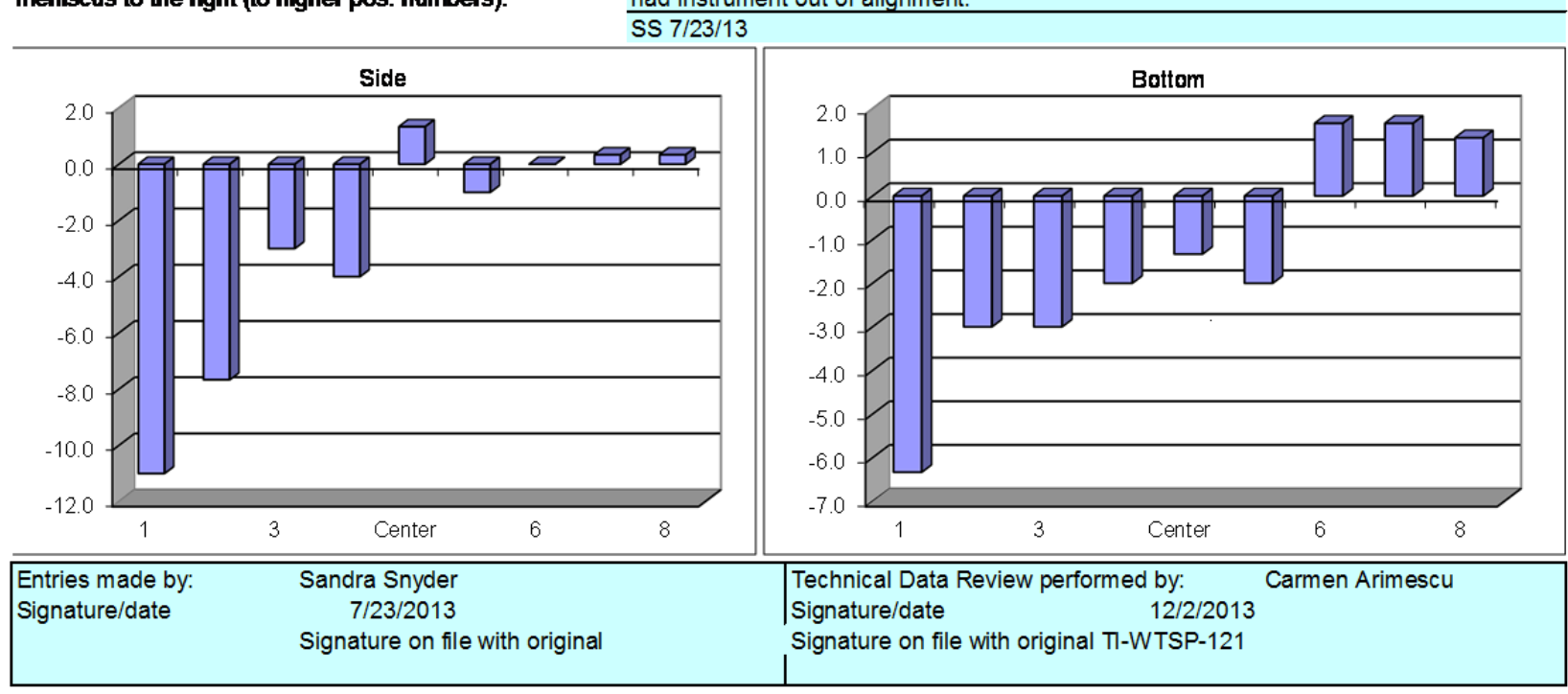


T1-WTPSP-121

Page

FLOW ANGLE DATA FORM

Site LV-S3 Scale Model

Date $7 / 23 / 2013$

Tester SS, SFS

Stack Dia. 11.922 in

Stack X-Area 111.6 in2

Elevation N.A. fi

Distance to disturbance 426.25 in

Start/End Trme 911/933
LV-S3_FlowAngle.xlsx$$
1 \text { st }
$$

st
Run No. FA-25

Fan Setting 60

Fan configuration B\&C MAX

Approx. air vel. 3296 fipm at point $\gg 7$ bottom, por Units degrees (clockwise > pos. nos.)

Port 1

Stack Temp 92.2 degrees F

2nd

Order $\rightarrow$

Traverse $\rightarrow$

Trial $\longrightarrow$

\begin{tabular}{c|c}
\hline Point & Depth, in
\end{tabular}

CCP-WTPSP-\#\#

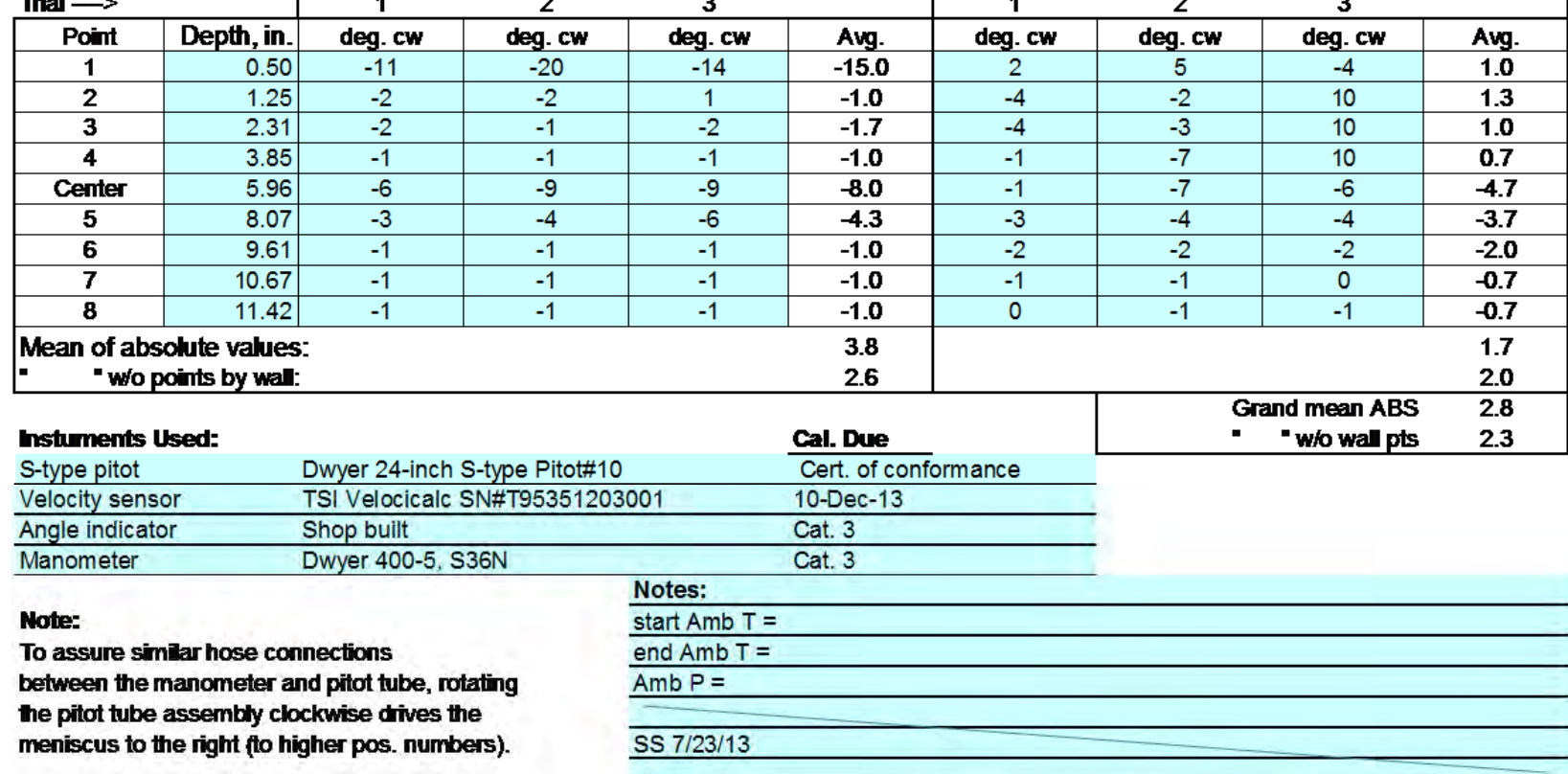

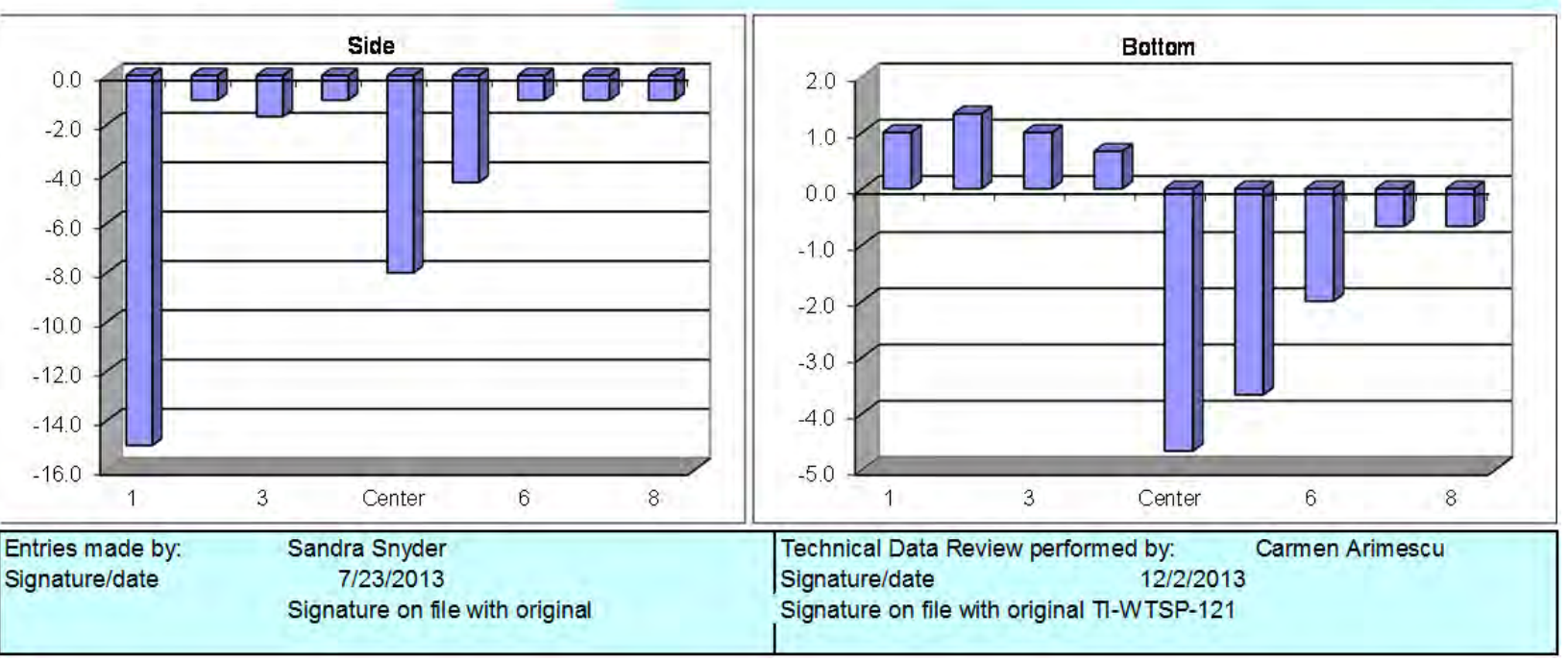


T1-WTPSP-121

Page

FLOW ANGLE DATA FORM

Site LV-S3 Scale Model

Date $7 / 23 / 2013$

Tester SS, SFS

Stack Dia. 11.922 in

Stack X-Area 111.6 in2

Elevation N.A. fi

Distance to disturbance 426.25

Start/End Trme $0936 / 1012$
LV-S3_FlowAngle.xlsx

$$
\text { 2nd }
$$

Run No. FA-26

Fan Setting 60

Fan configuration B\&C MAX

Approx. air vel. 3577 fipm at point $\gg 7$, bottom Por Units degrees (clockwise > pos. nos.)

Port $\frac{1}{95.6}$

Stack Temp 95.6 degrees F

$1 \mathrm{st}$

Order $\rightarrow$

Traverse $\rightarrow$

\begin{tabular}{|l|l|} 
Trial $\rightarrow$ Point & Depth, in.
\end{tabular}

1 Side

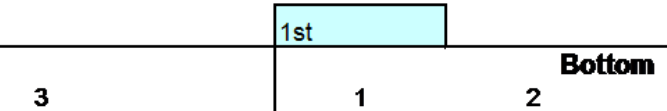

\begin{tabular}{|c|c|c|c|c|c|}
\hline rial $\rightarrow$ & & 1 & 2 & 3 & \\
\hline Point & Depth, in. & deg. cw & deg. cw & deg. cw & A \\
\hline 1 & 0.50 & -14 & -14 & -13 & -1 \\
\hline 2 & 1.25 & -2 & -2 & -2 & 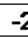 \\
\hline 3 & 2.31 & -3 & -3 & -3 & -3 \\
\hline 4 & 3.85 & -3 & -3 & -2 & -2 \\
\hline Center & 5.96 & -6 & 0 & -7 & - \\
\hline 5 & 8.07 & -4 & -1 & -2 & -2 \\
\hline 6 & 9.61 & -2 & 0 & 0 & -0 \\
\hline 7 & 10.67 & 0 & 1 & 1 & 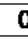 \\
\hline 8 & 11.42 & 1 & 1 & 1 & \\
\hline
\end{tabular}

Mean of absolute values:

- wo points by wall:

Instuments Used:

S-type pitot

Velocity sensor

Angle indicator

Manometer

Dwyer 24-inch S-type Pitot\#10

TSI Velocicalc SN\#T95351203001

\begin{tabular}{rr}
1.0 \\
\hline
\end{tabular}

\begin{tabular}{l|l|l|l|l} 
Avg. & deg. cw & deg. cw & deg. cw & Avg
\end{tabular}

\begin{tabular}{c|c|c|c|c}
-13.7 & -11 & -7 & -11 & -9.7 \\
\hline-2.0 & -4 & -5 & -5 & -4.7
\end{tabular}

$-3.0$

$-2.7$

$-4$

\begin{tabular}{l|l|l|l}
-2 & -4 & -5 & -4.7 \\
\hline
\end{tabular}

$-4.3$

$-2$

0.7

3.4
2.2

$-1$

\begin{tabular}{l|l}
\hline & \\
\hline & \\
\hline & \\
\hline & \\
\hline
\end{tabular}

\begin{tabular}{l|l|l}
-4 & -4 & -3.3 \\
-4 & -3 & -3.7 \\
\hline
\end{tabular}

\begin{tabular}{l|l}
0 & -3 \\
-3 & -2 \\
-1 & -1
\end{tabular}

Note:

To assure similar hose connections

between the manometer and pitot tube, rotating

the pitot tube assembly clockwise dives the

meniscus to the right (to higher pos. numbers).

Cal. Due

Cert. of conformance

10-Dec-13

Cat. 3

Cat. 3 MAN-5

$\begin{array}{rrr}0 & 0 & -0.3 \\ -1 & 3.2\end{array}$

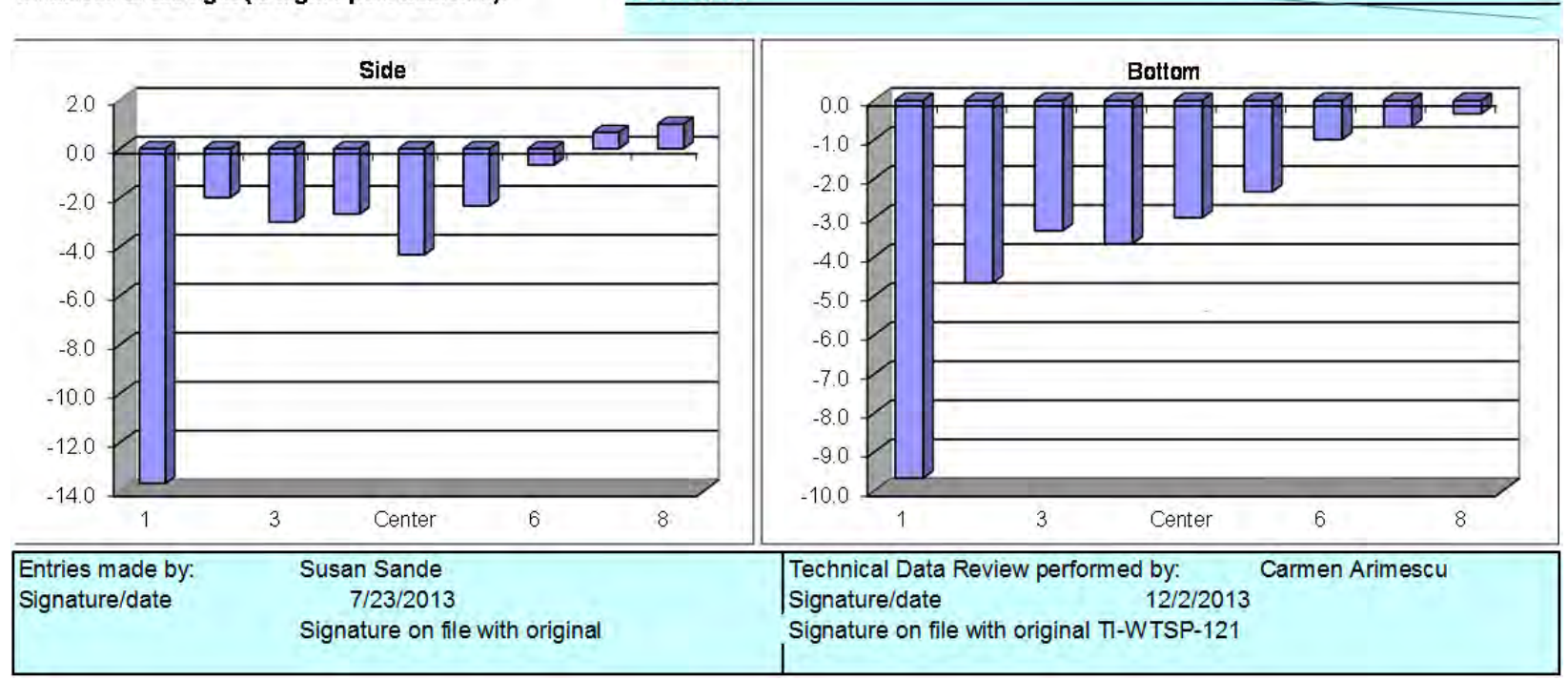


T1-WTPSP-121

Page

FLOW ANGLE DATA FORM

Site LV-S3 Scale Model

Date $7 / 23 / 2013$

Tester SS, SFS

Stack Dia. 11.922 in

Stack X-Area 111.6 in2

Elevation N.A. fi

Distance to disturbance 510.25

Start/End Tme $1020 / 1105$
LV-S3_FlowAngle.xlsx

$$
\text { 2ND }
$$

Order $\rightarrow$

Traverse $\rightarrow$

\begin{tabular}{r} 
Trial $\rightarrow$ Point \\
\multicolumn{1}{c}{ Depth, in. }
\end{tabular}

1 Side

CCP-WTPSP-\#\#

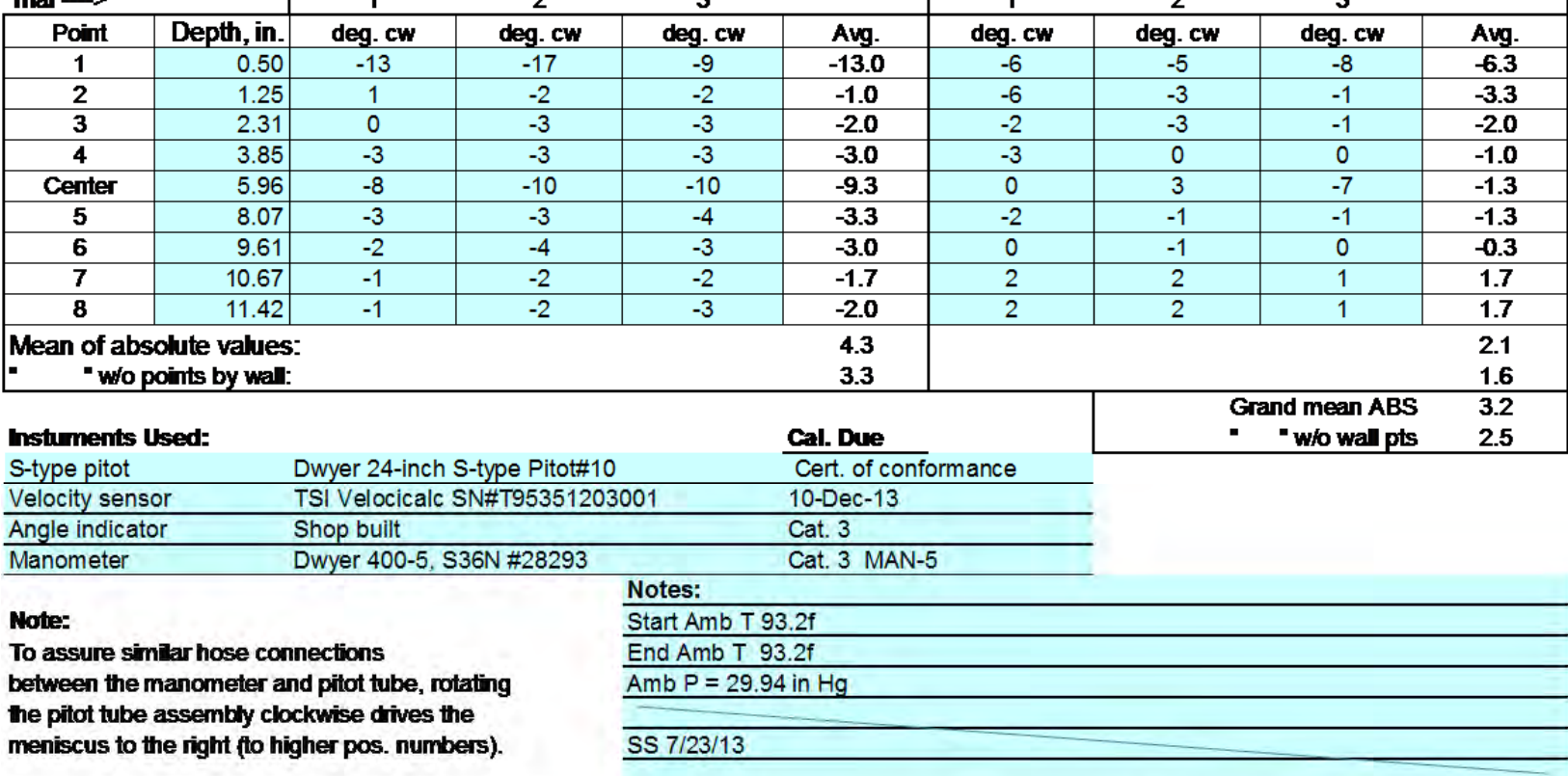

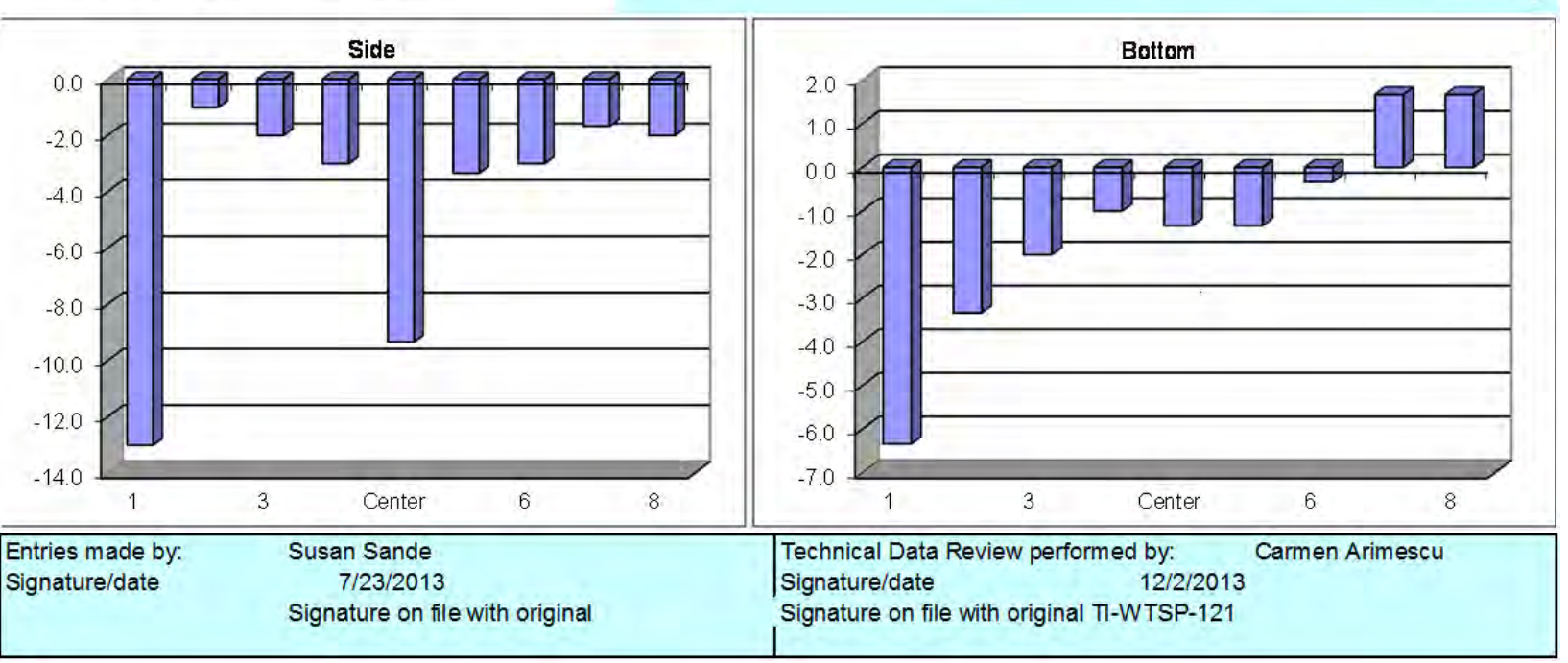




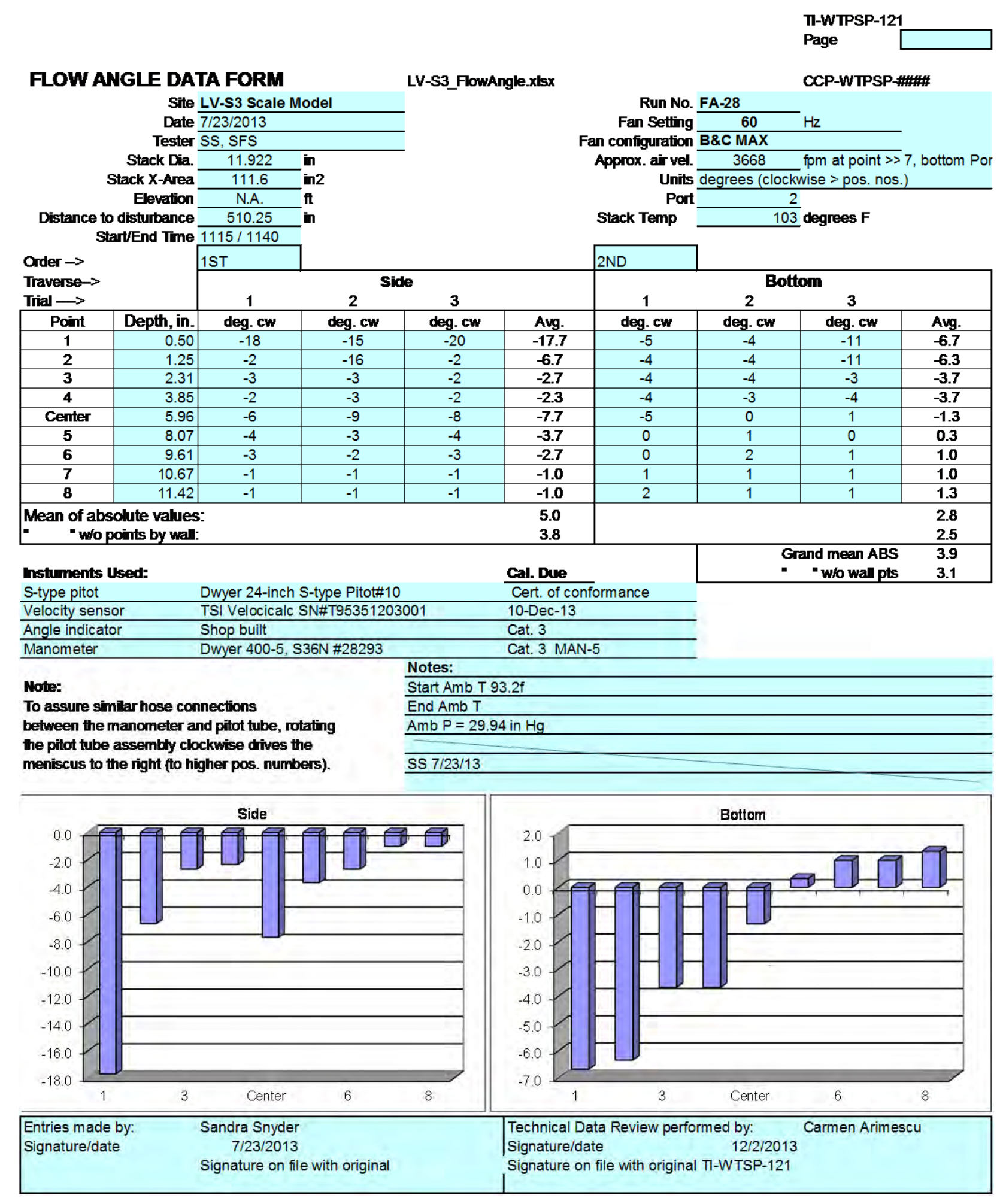




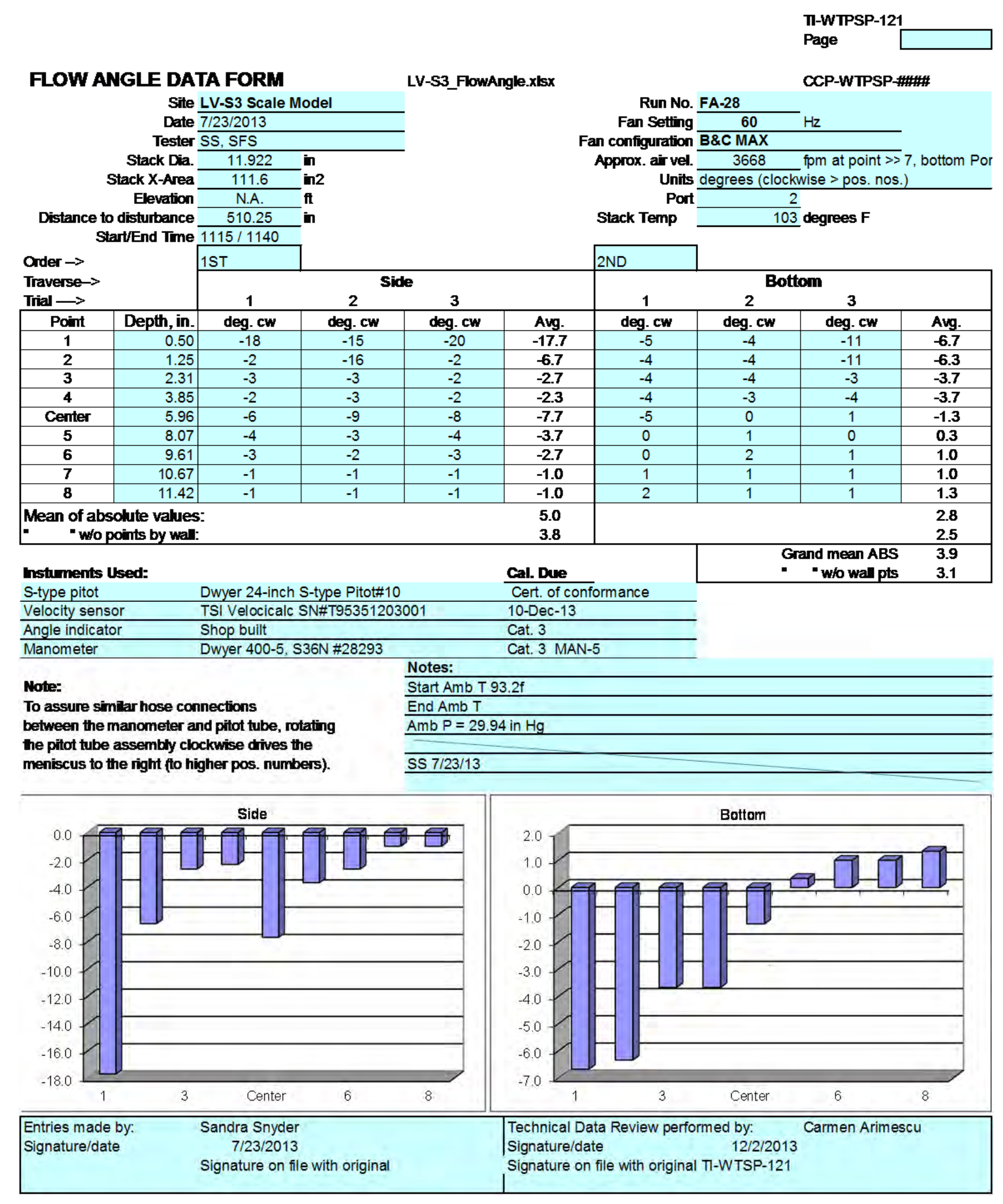


T1-WTPSP-121

Page

FLOW ANGLE DATA FORM

Site LV-S3 Scale Model

Date $7 / 23 / 2013$

Tester SS, SFS

Stack Dia. 11.922 in

Stack X-Area 111.6 in2

Elevation N.A. fi

Distance to disturbance 510.25 in

Start/End Tme $1148 / 1210$
LV-S3_FlowAngle.xlsx

$$
\text { 2nd }
$$

Run No. FA-29

Fan Setting 60

Fan configuration B\&C MAX

Approx. air vel. 3569 fipm at point $\gg$

Units degrees (clockwise > pos. nos.)

Port

Stack Temp

$$
2
$$

1 st

Traverse-

Trial $\longrightarrow$

\begin{tabular}{r|r} 
Point & Depth, in
\end{tabular}

side

Side

123

\begin{tabular}{l|l|l} 
deg. cw & deg. cw & deg. cw
\end{tabular}

3

\begin{tabular}{l|l|l|l} 
& & \multicolumn{1}{c}{$\mathbf{2}$} \\
\hline
\end{tabular}

\begin{tabular}{|c|r|c|r|}
\hline $\mathbf{1}$ & 0.50 & 2 & - \\
\hline $\mathbf{2}$ & 1.25 & -2 & -10 \\
\hline $\mathbf{3}$ & 2.31 & 6 & -1 \\
\hline $\mathbf{4}$ & 3.85 & -2 & 5 \\
\hline Center & 5.96 & -1 & \\
\hline $\mathbf{5}$ & 8.07 & -3 & -2 \\
\hline $\mathbf{6}$ & 9.61 & -2 & -2 \\
\hline $\mathbf{7}$ & 10.67 & -1 & -2 \\
\hline $\mathbf{8}$ & 11.42 & -1 & -2 \\
\hline
\end{tabular}

Mean of absolute values:

- wo points by wal.

Instuments Used:

S-type pitot

Velocity sensor

Angle indicator

Manometer

g. CW

\begin{tabular}{ll|l|l|l|l}
\hline-12 & Avg. & deg. CW & deg. Cw & deg. cw & Avg.
\end{tabular}

\begin{tabular}{l|l|l}
-9 & -12 & -6.3 \\
-10 & -12 & -8.0
\end{tabular}

\begin{tabular}{l|l|l}
-10 & -12 & -8.0 \\
\hline & -4 & -3.7
\end{tabular}

\begin{tabular}{l|l}
5 & -3
\end{tabular}

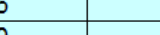

\begin{tabular}{c|c}
0 & -5 \\
-2 & -2 \\
\hline
\end{tabular}

-5
-2
-2

$-2$

\begin{tabular}{l|l}
-2 & -2.3 \\
-2 & -2.0 \\
\hline-1 & -1.7 \\
\hline
\end{tabular}

$-1.3$

2.8

\begin{tabular}{l|c|c|c|c}
\hline .3 & -4 & -6 & -3 & -4.3 \\
\hline .0 & -18 & -19 & -3 & -13.3
\end{tabular}

0.0

\begin{tabular}{l|l}
-4 \\
\hline
\end{tabular}

\begin{tabular}{l|r}
-4 & -3 \\
\hline-4 & -2 \\
\hline & -2
\end{tabular}

\begin{tabular}{c|c|c} 
& 12 & 1.7 \\
\hline-2 & -2.7 \\
\hline & 0 & -0.7
\end{tabular}

(3)

\begin{tabular}{c|c}
\hline-1 & \\
0 & \\
0 & \\
\hline 2 & \\
\hline
\end{tabular}

\begin{tabular}{|l|l}
\hline 2 & -1 \\
\hline & -1 \\
\hline & \\
\hline
\end{tabular}

\begin{tabular}{c|c}
0 & -0.7 \\
-1 & 0.0 \\
-1 & 0.0 \\
-1 & 0.0
\end{tabular}

Note:

To assure similar hose connections

between the manometer and pitot tube, rotating

the pitot tube assembly clockwise dives the

meniscus to the right (to higher pos. numbers).

Cal. Due

Grand mean ABS

- w/o wall pts

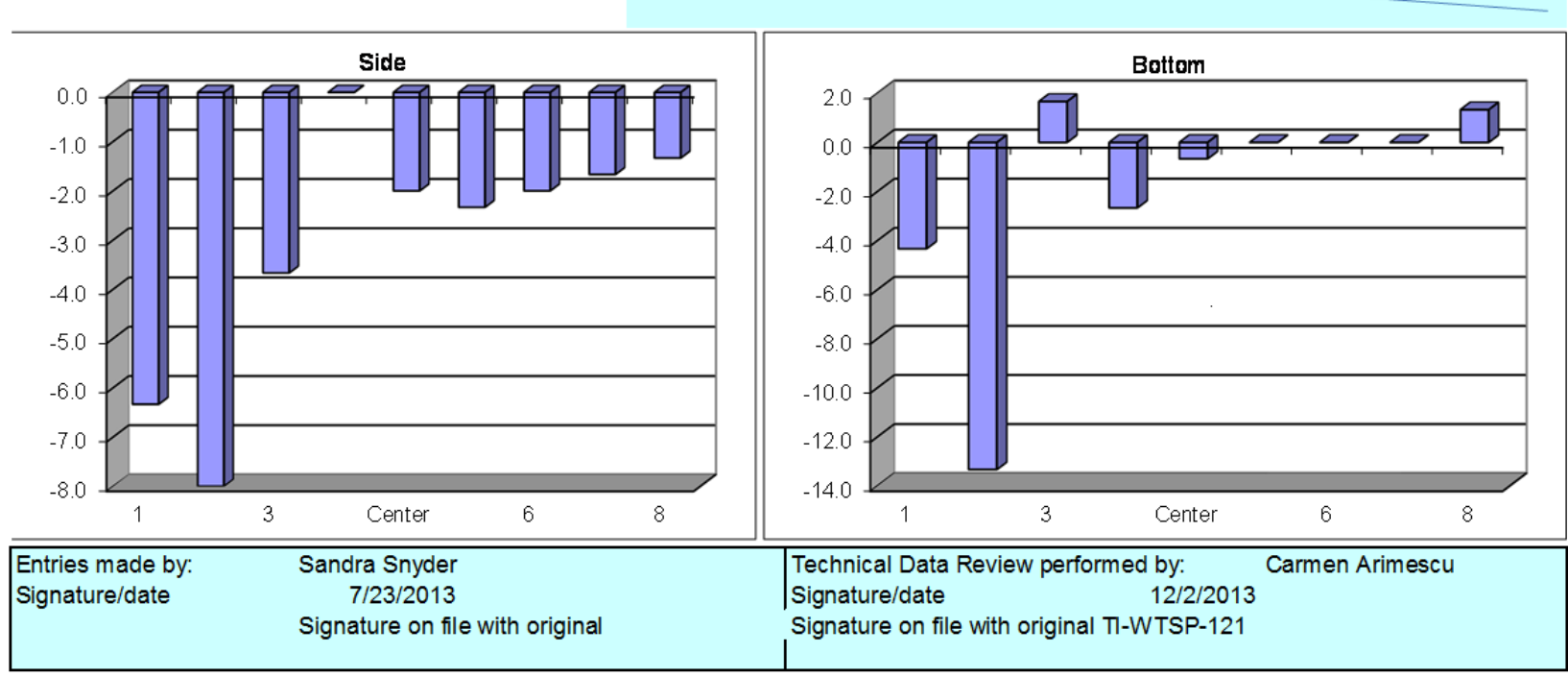




\section{D.4 LV-S3 Gas Tracer Calibration and Uniformity Data Sheets}

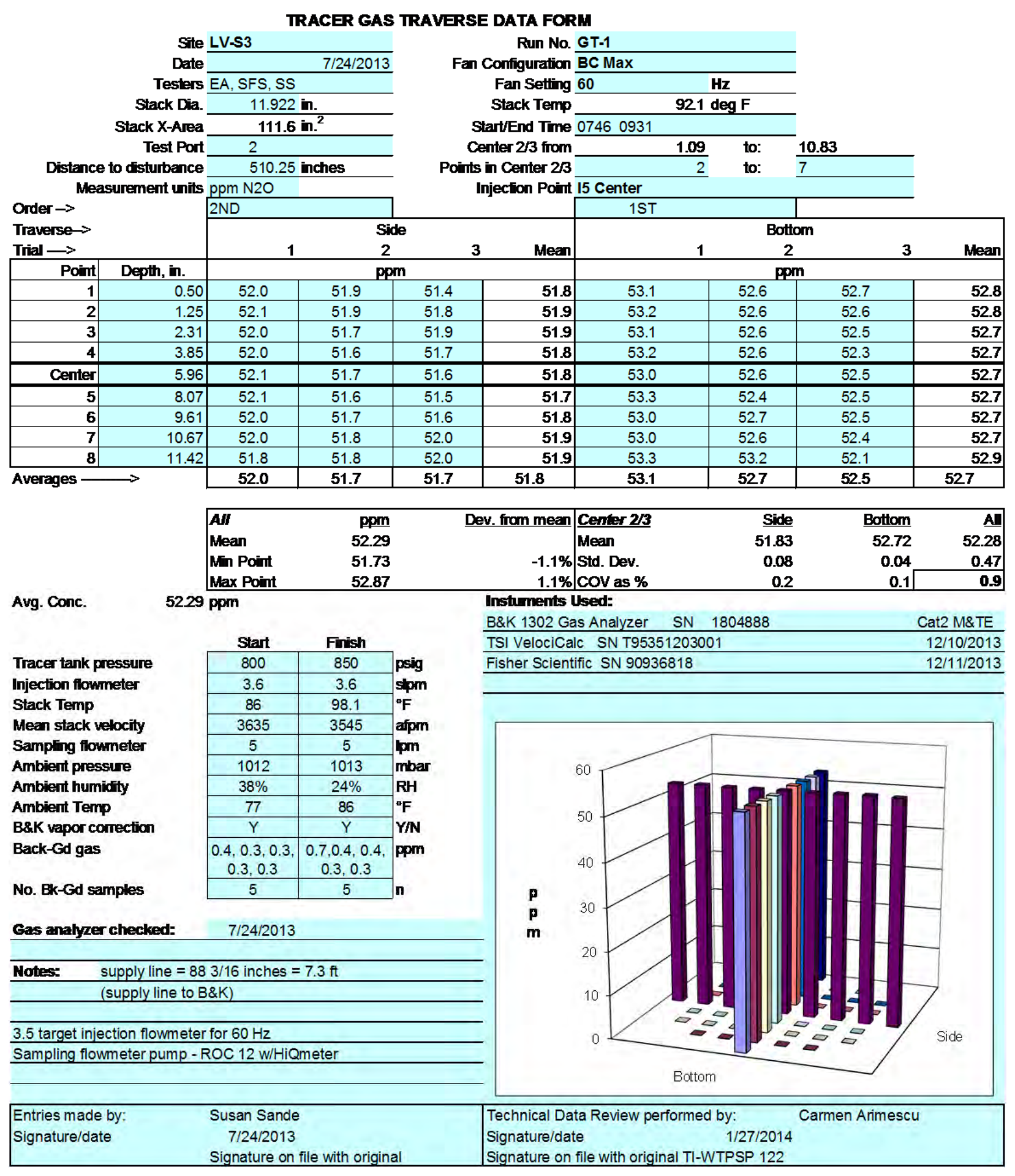




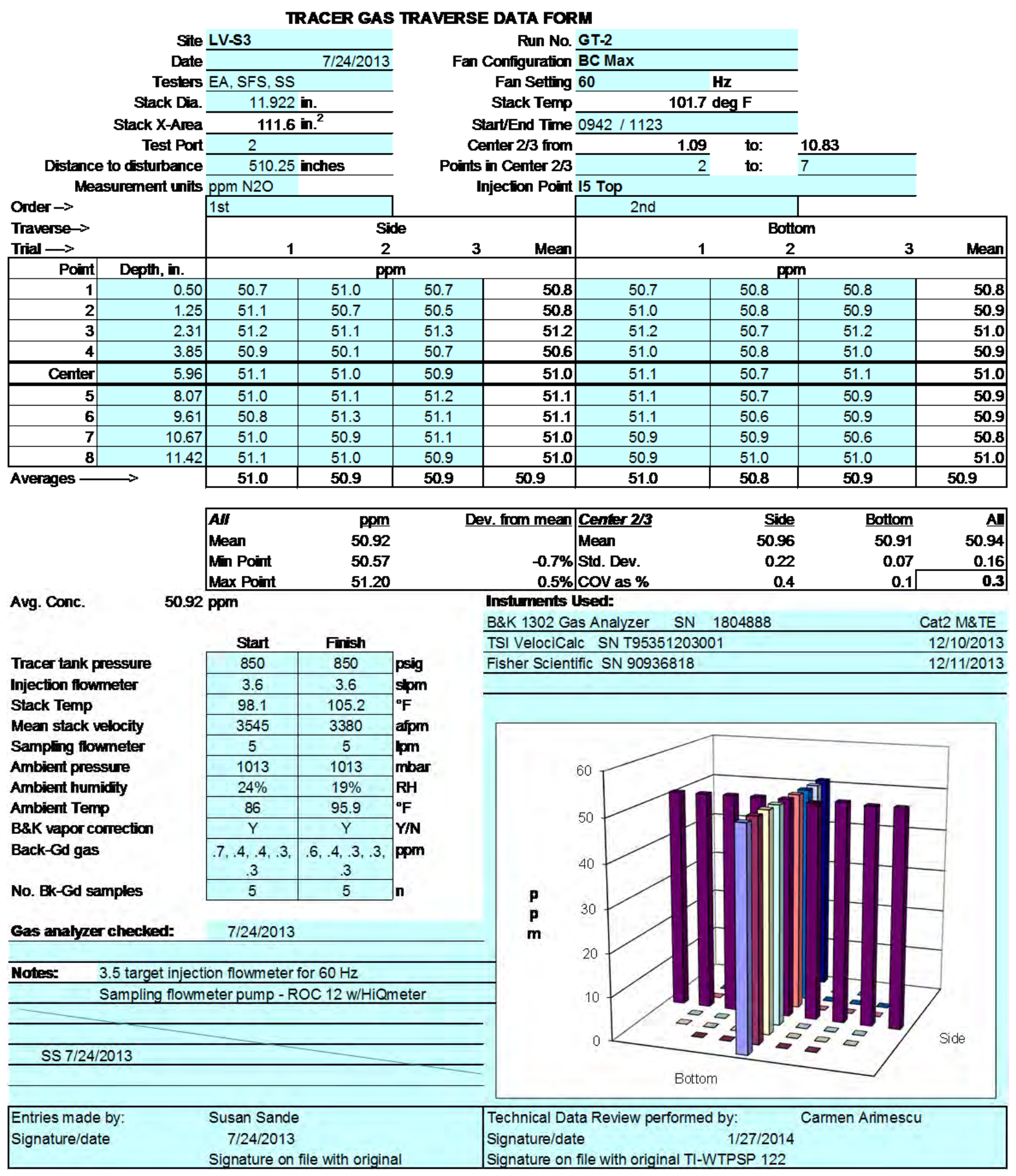




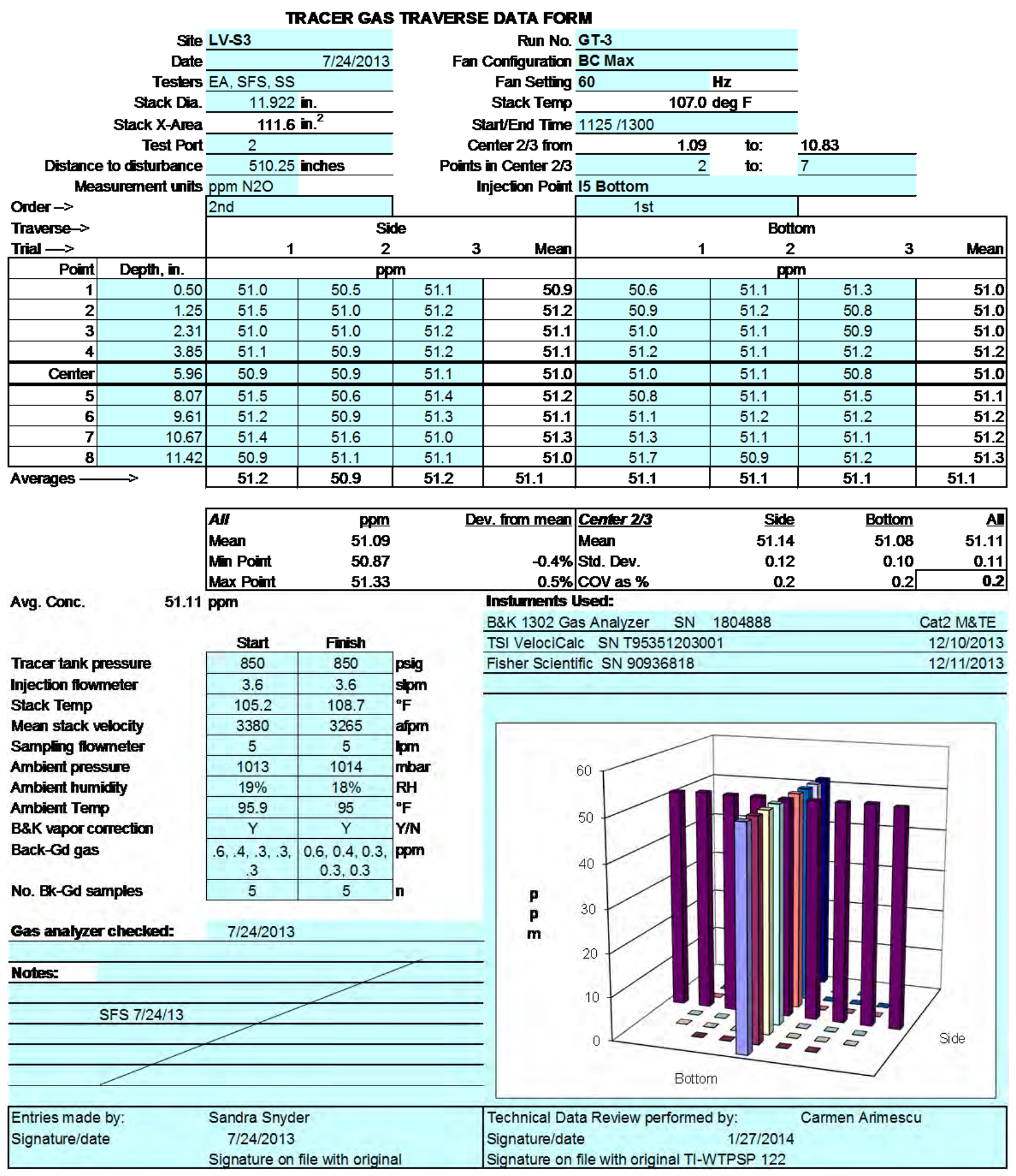




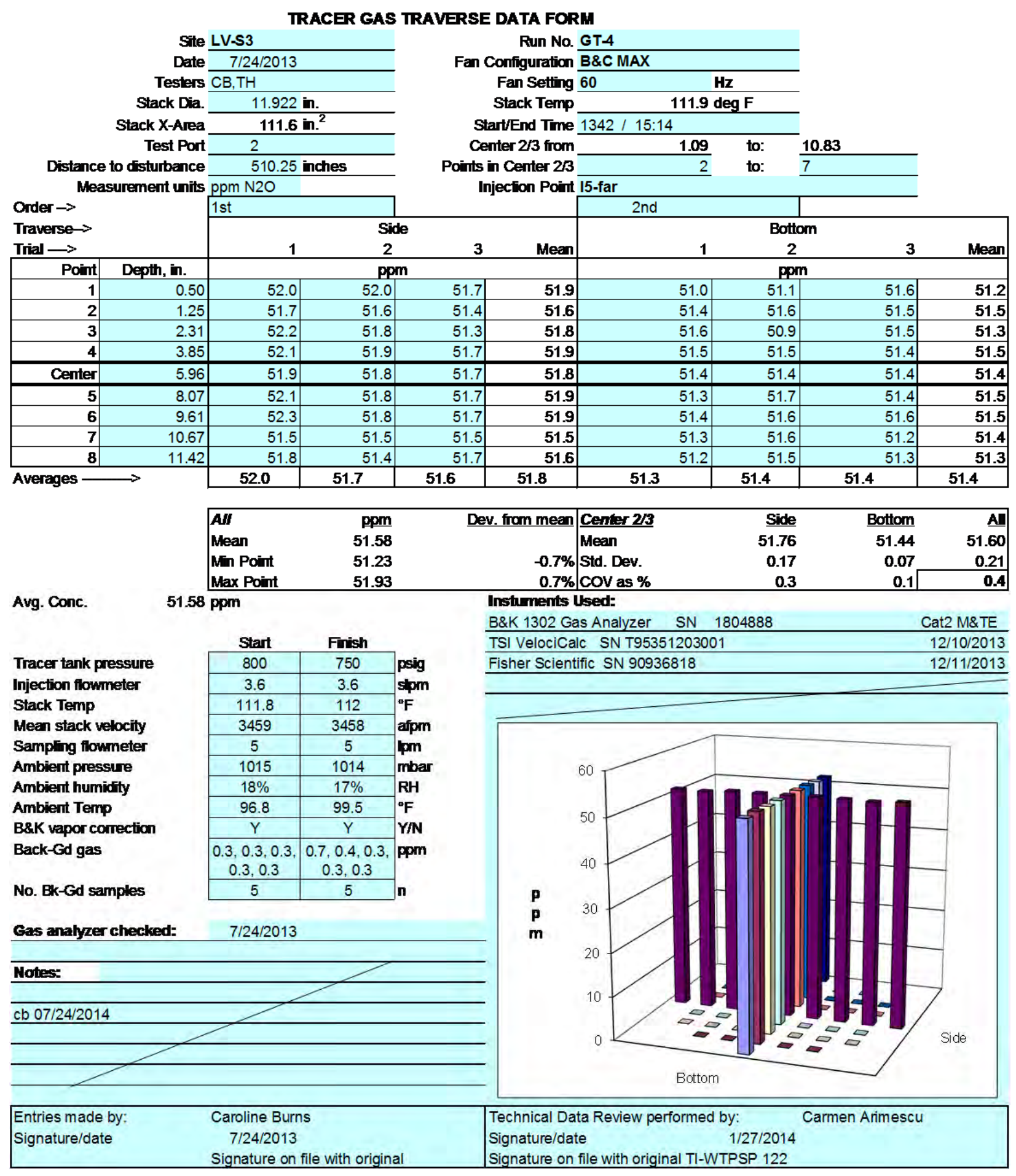




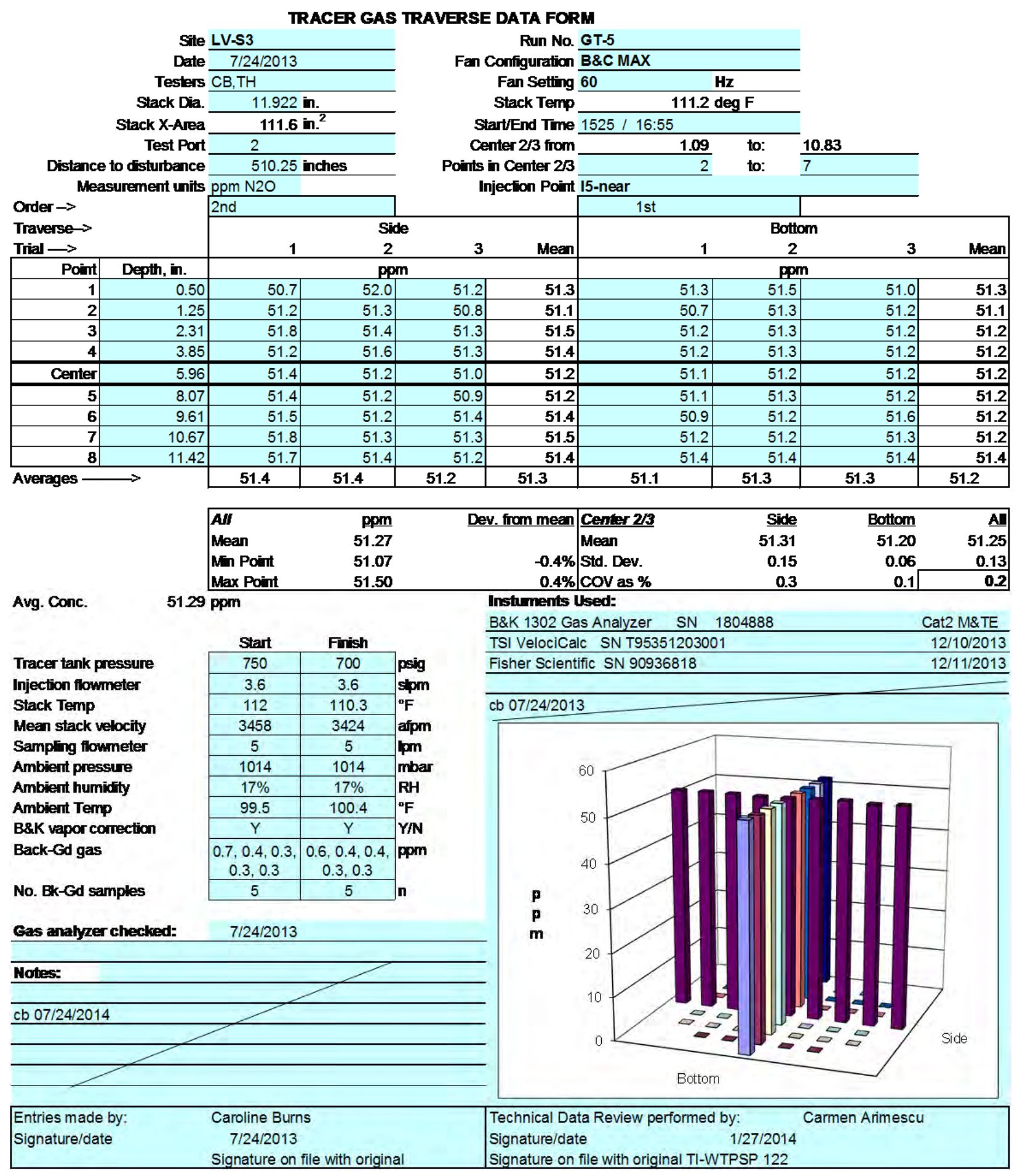




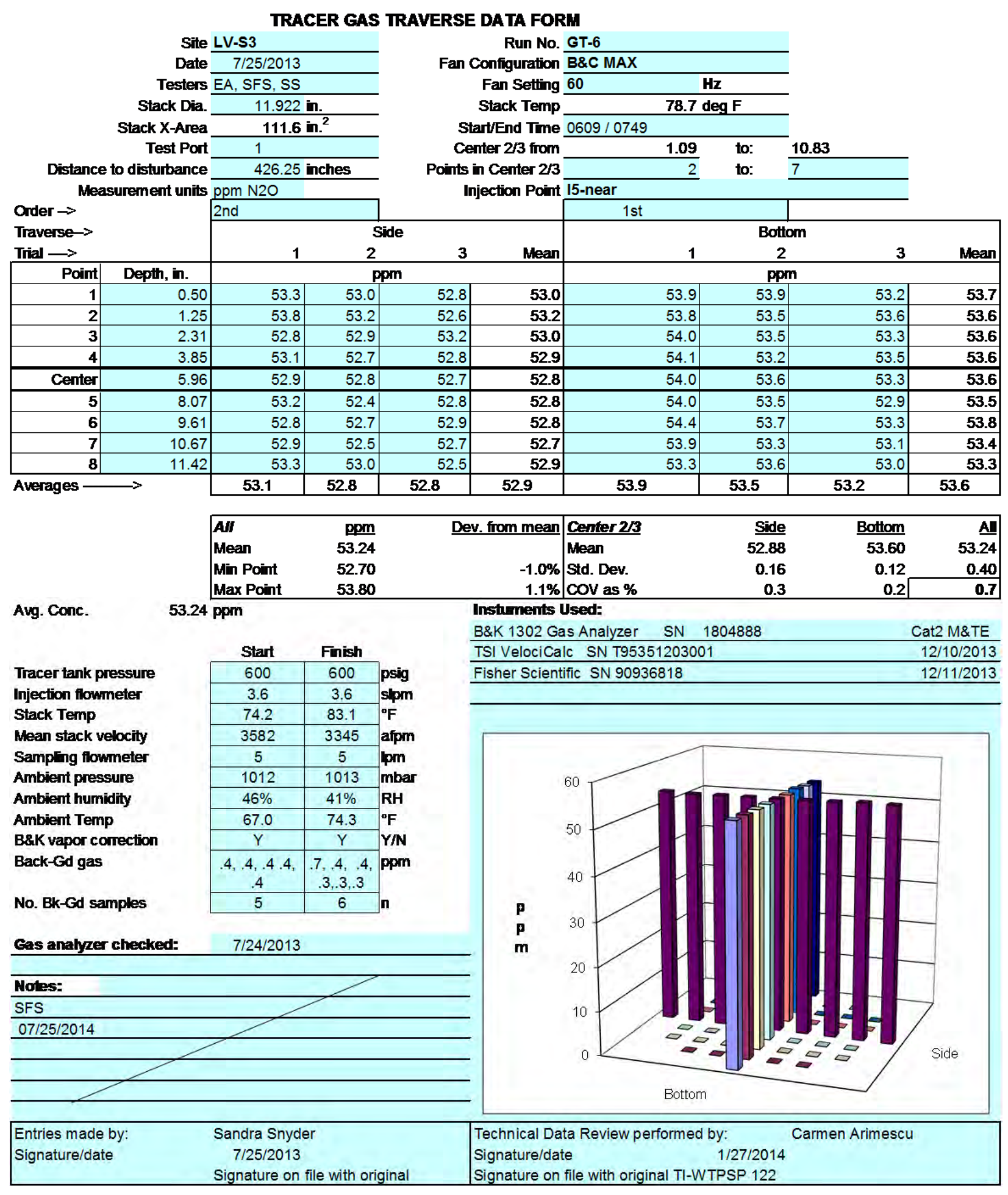




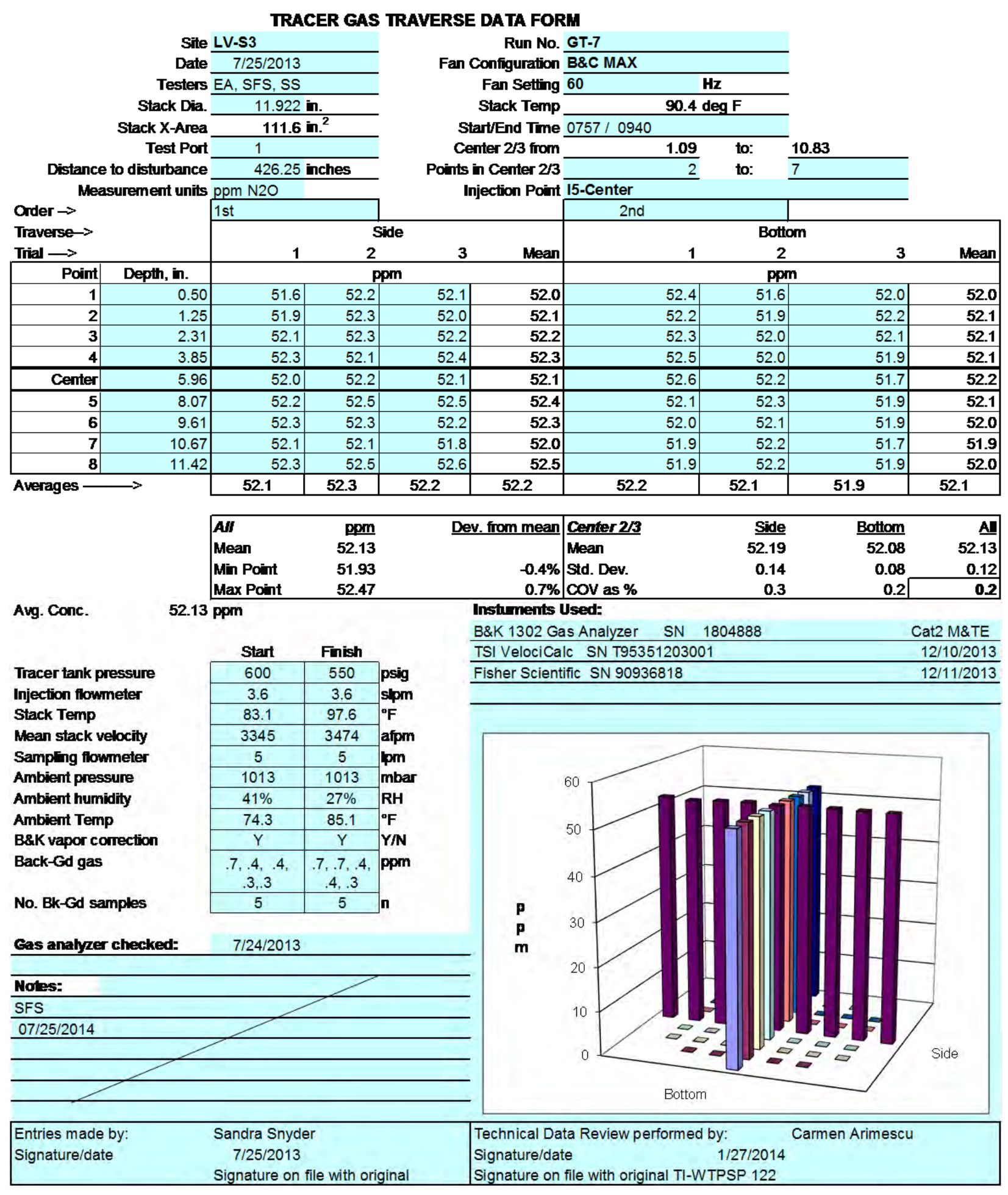




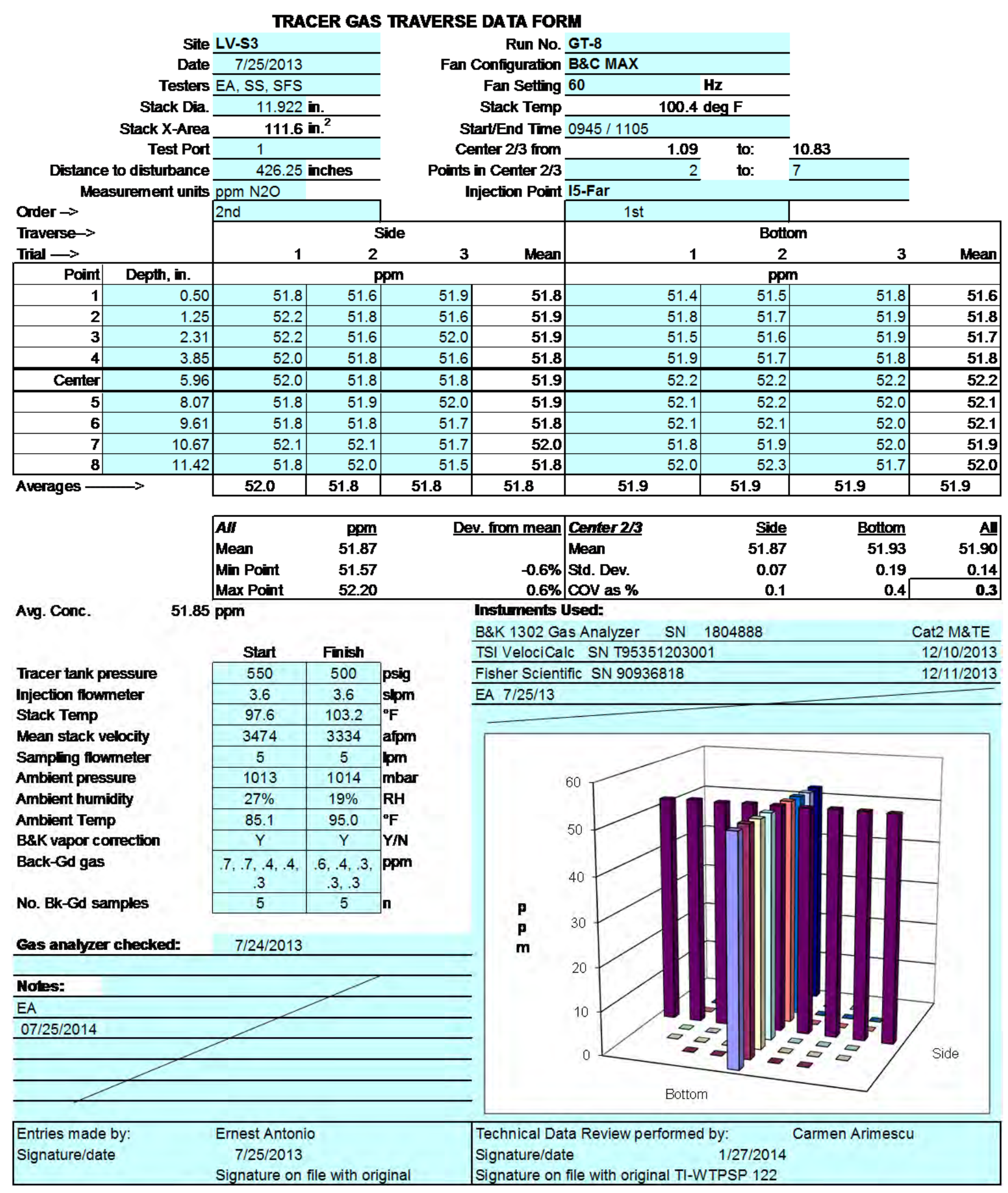




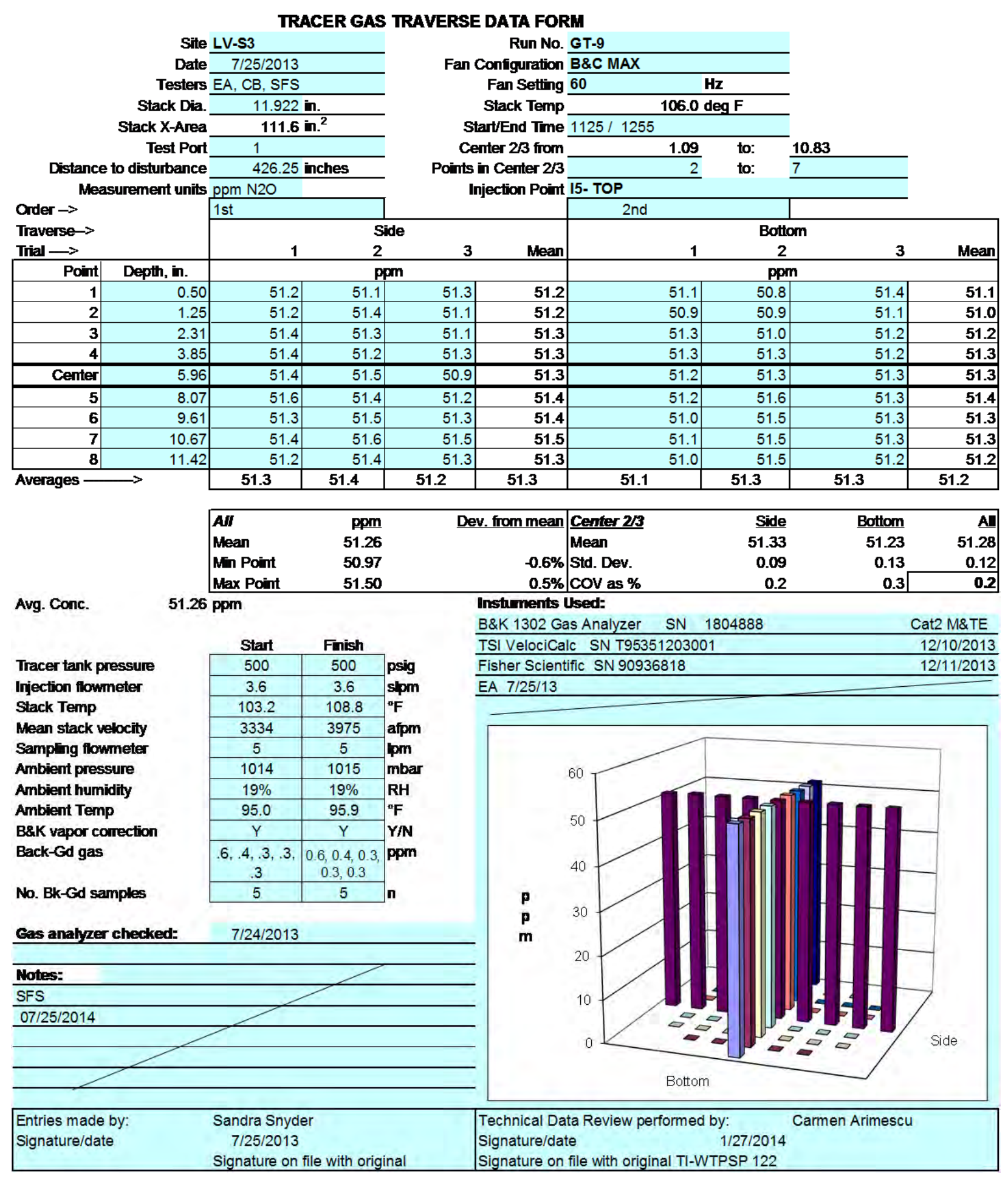




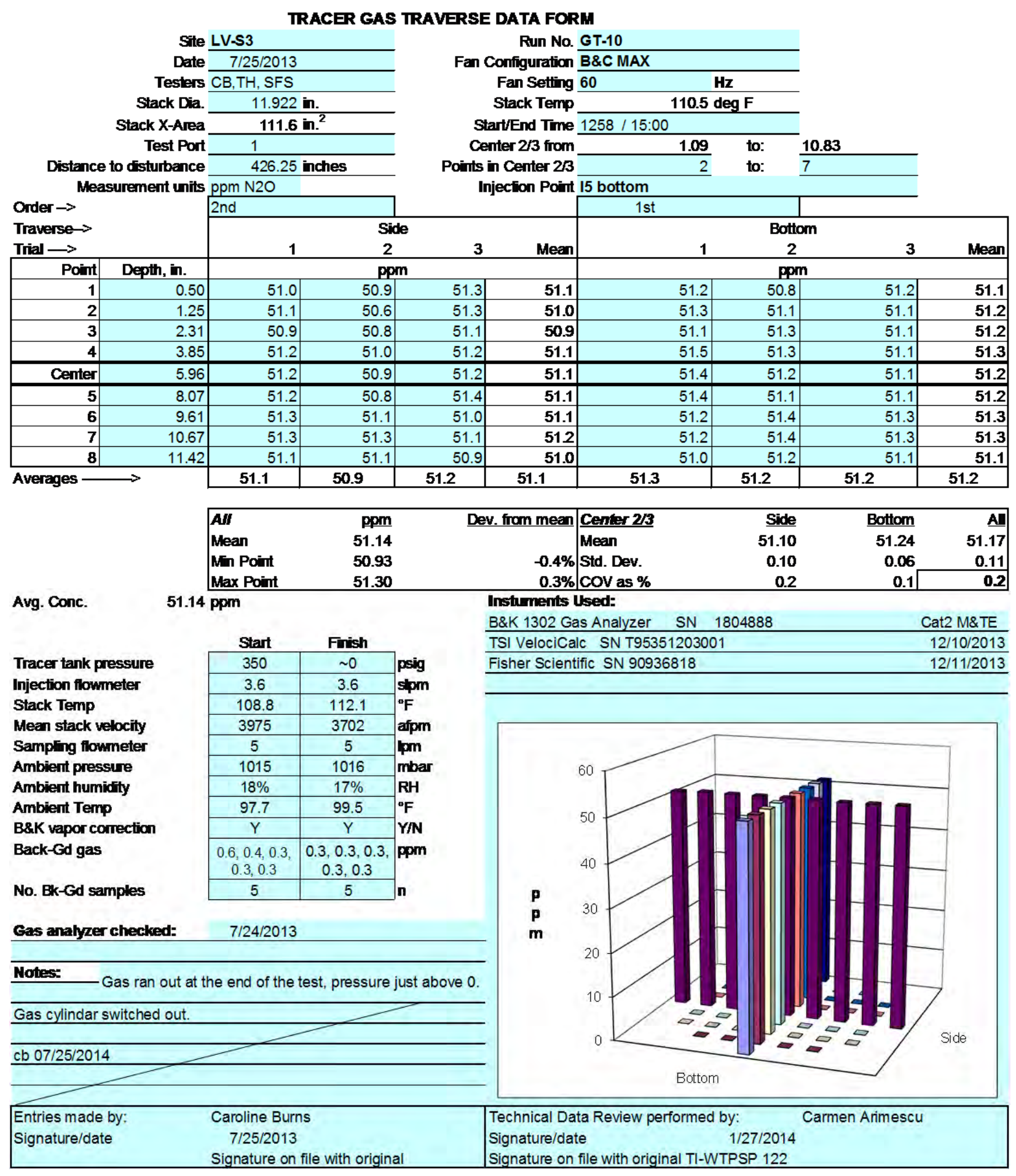




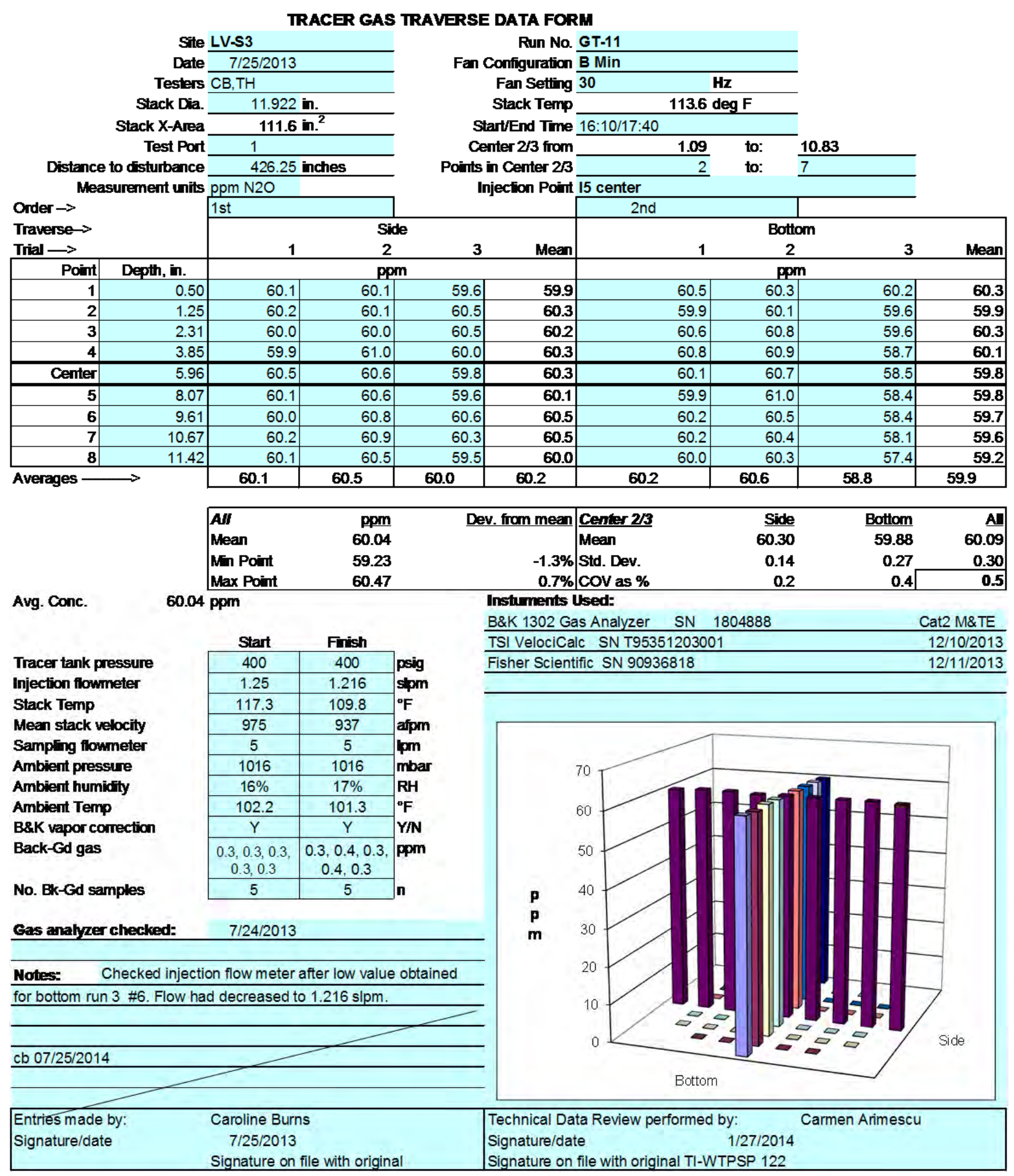




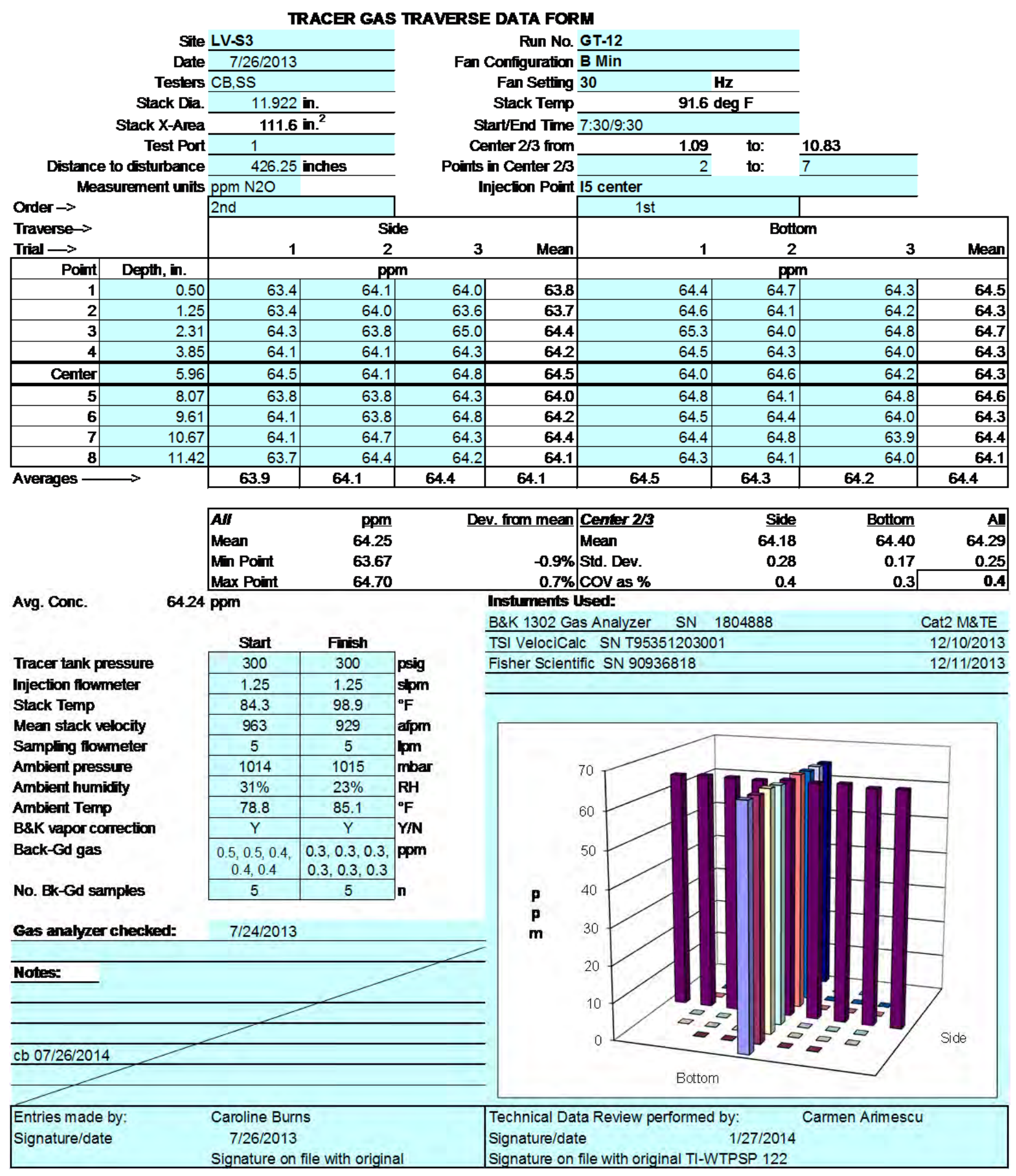




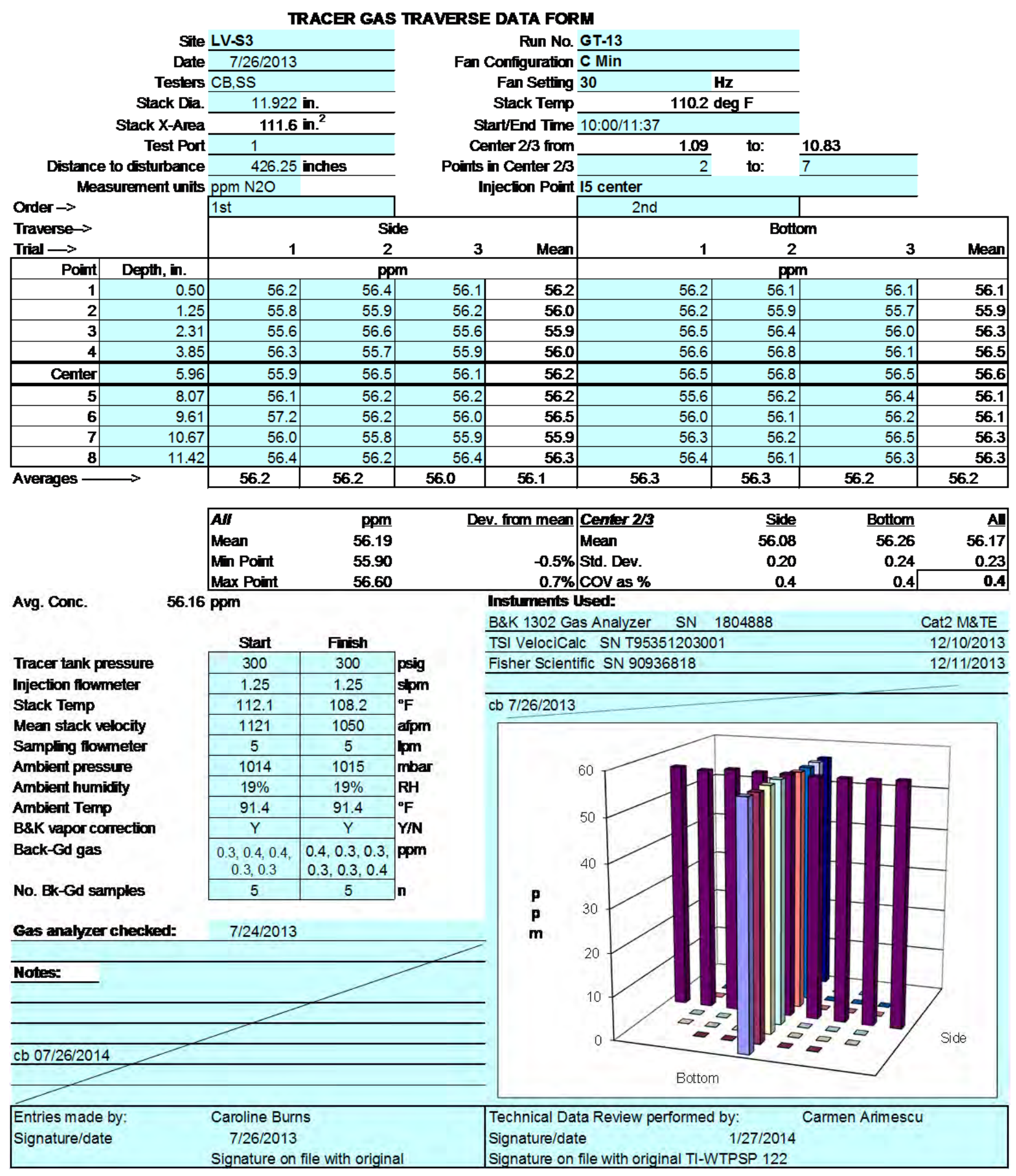




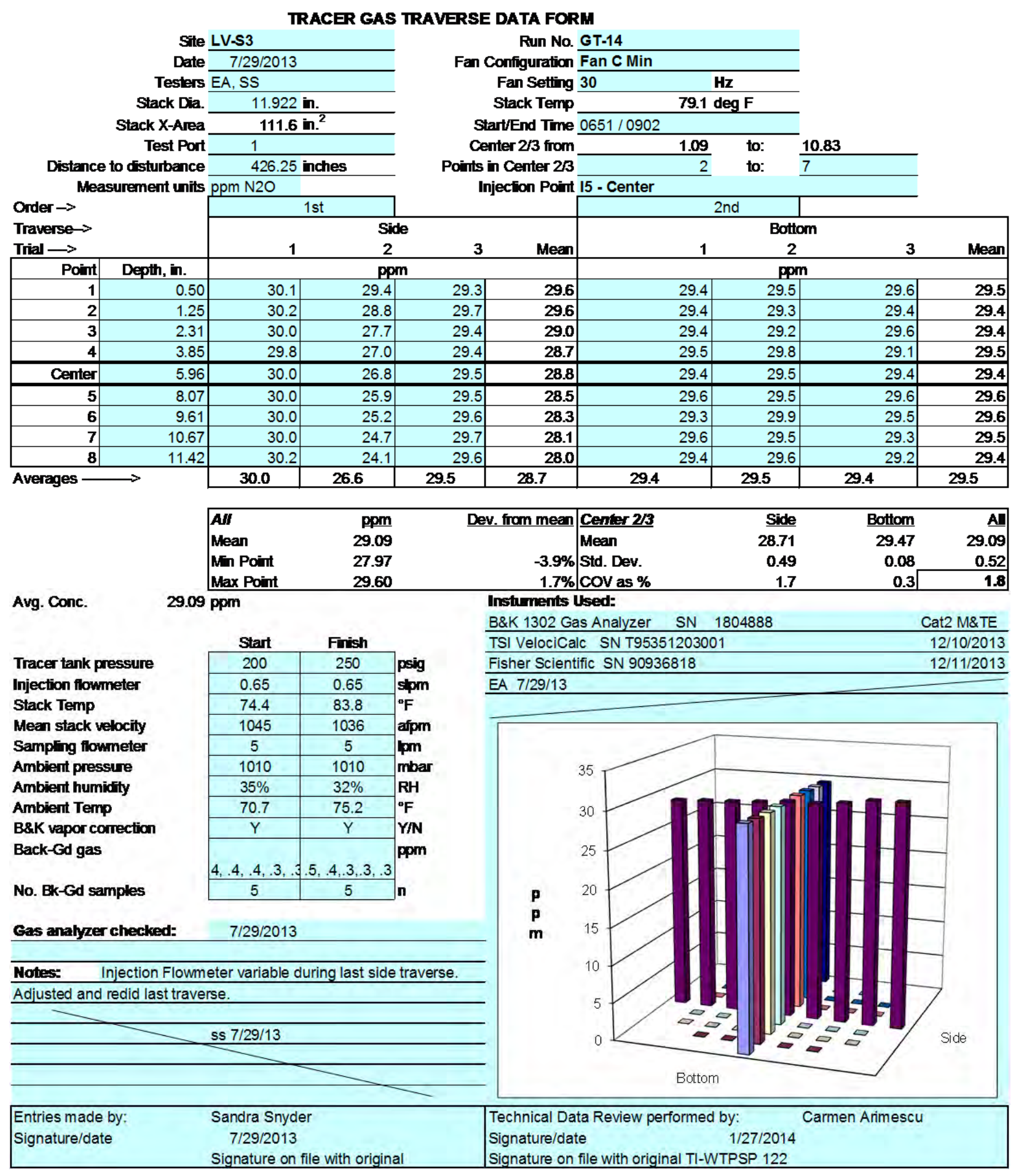




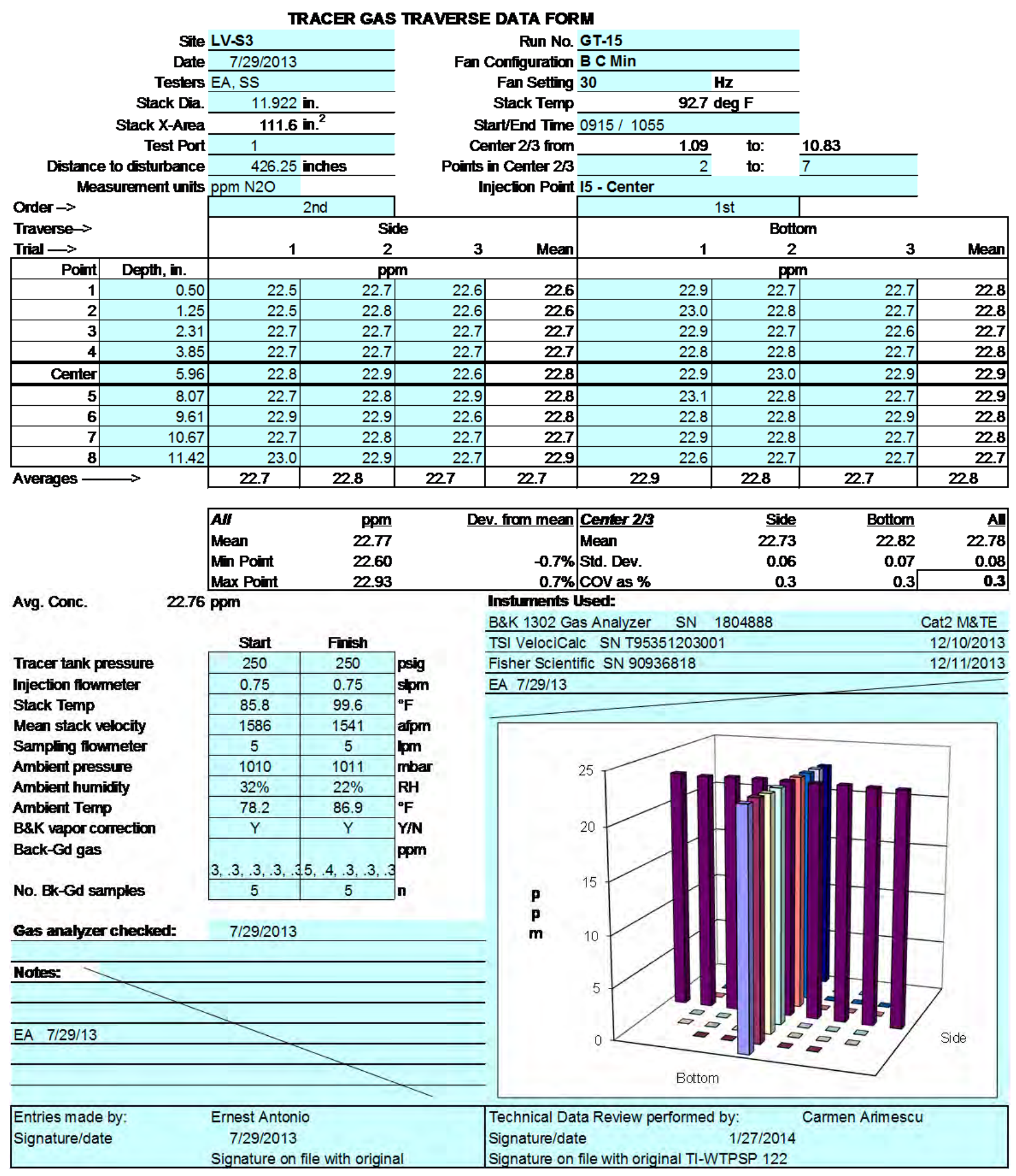




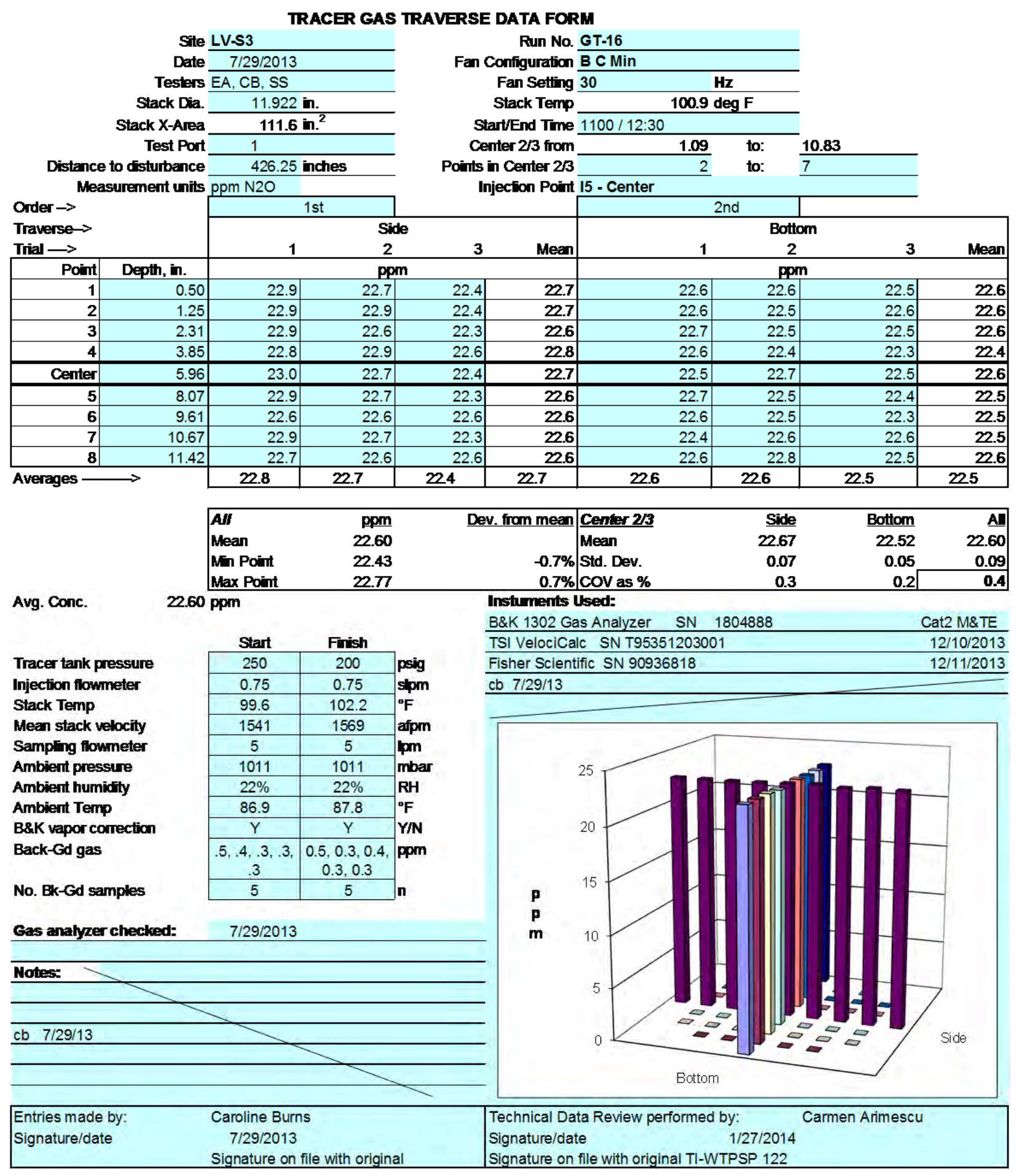




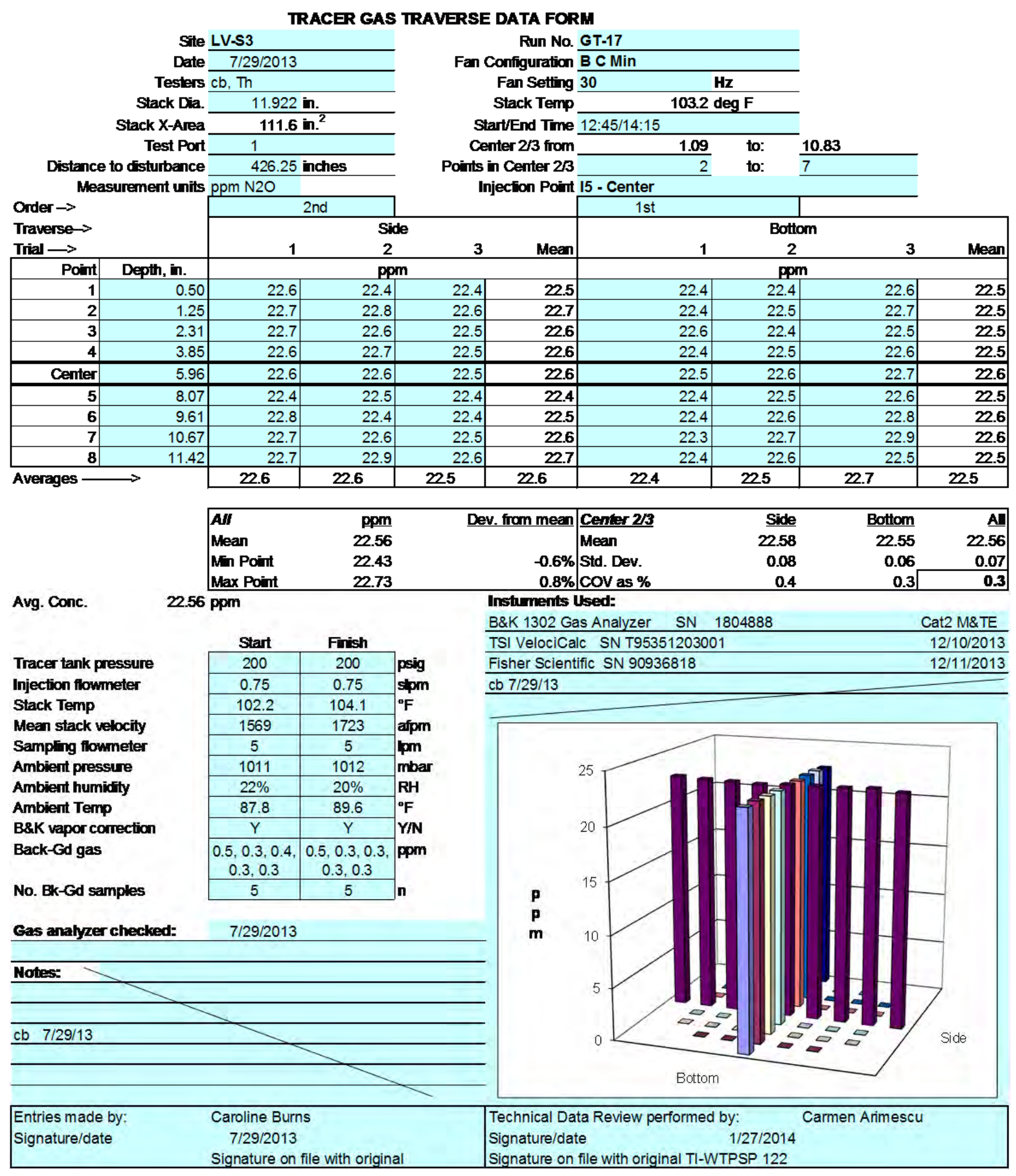




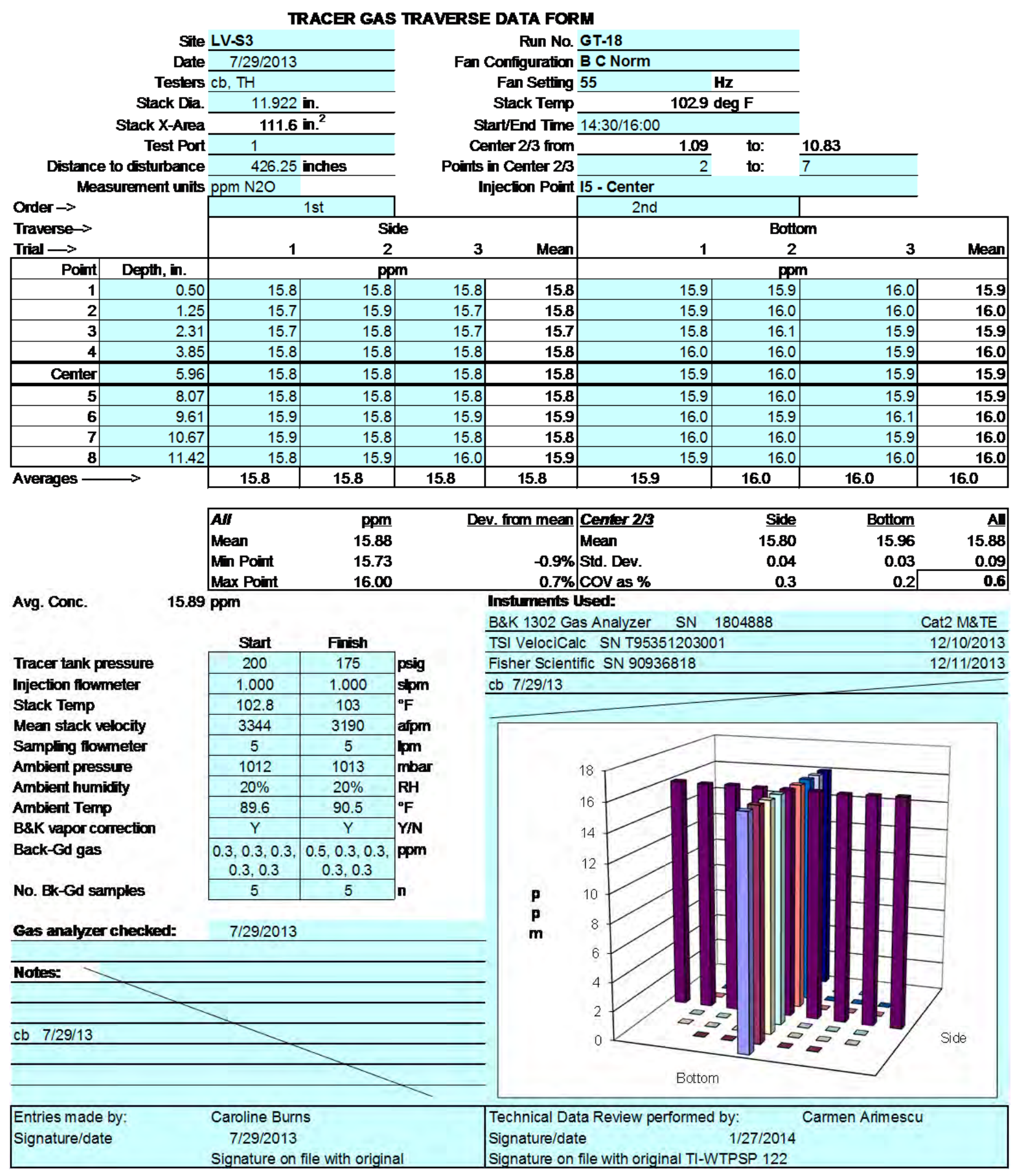




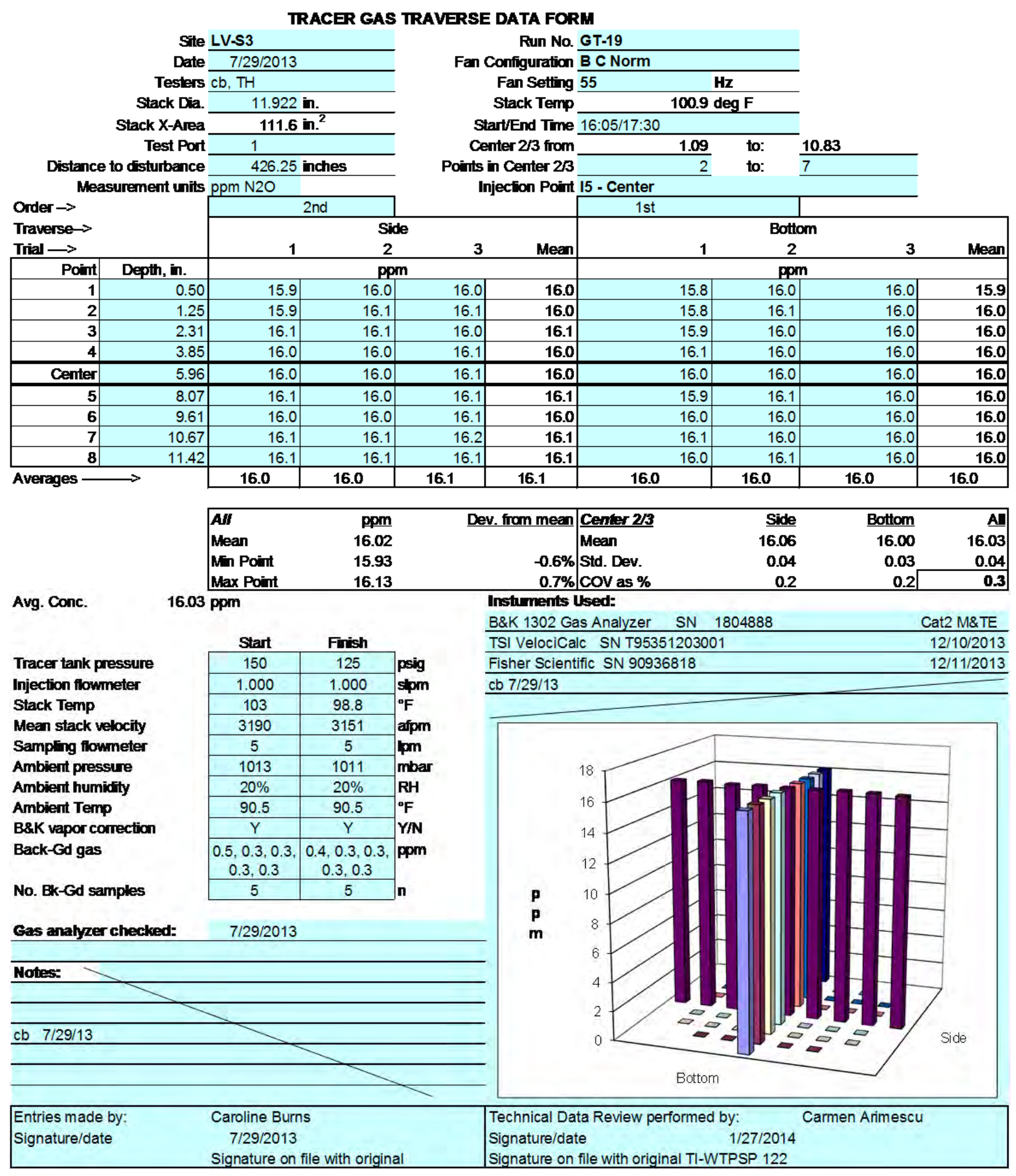




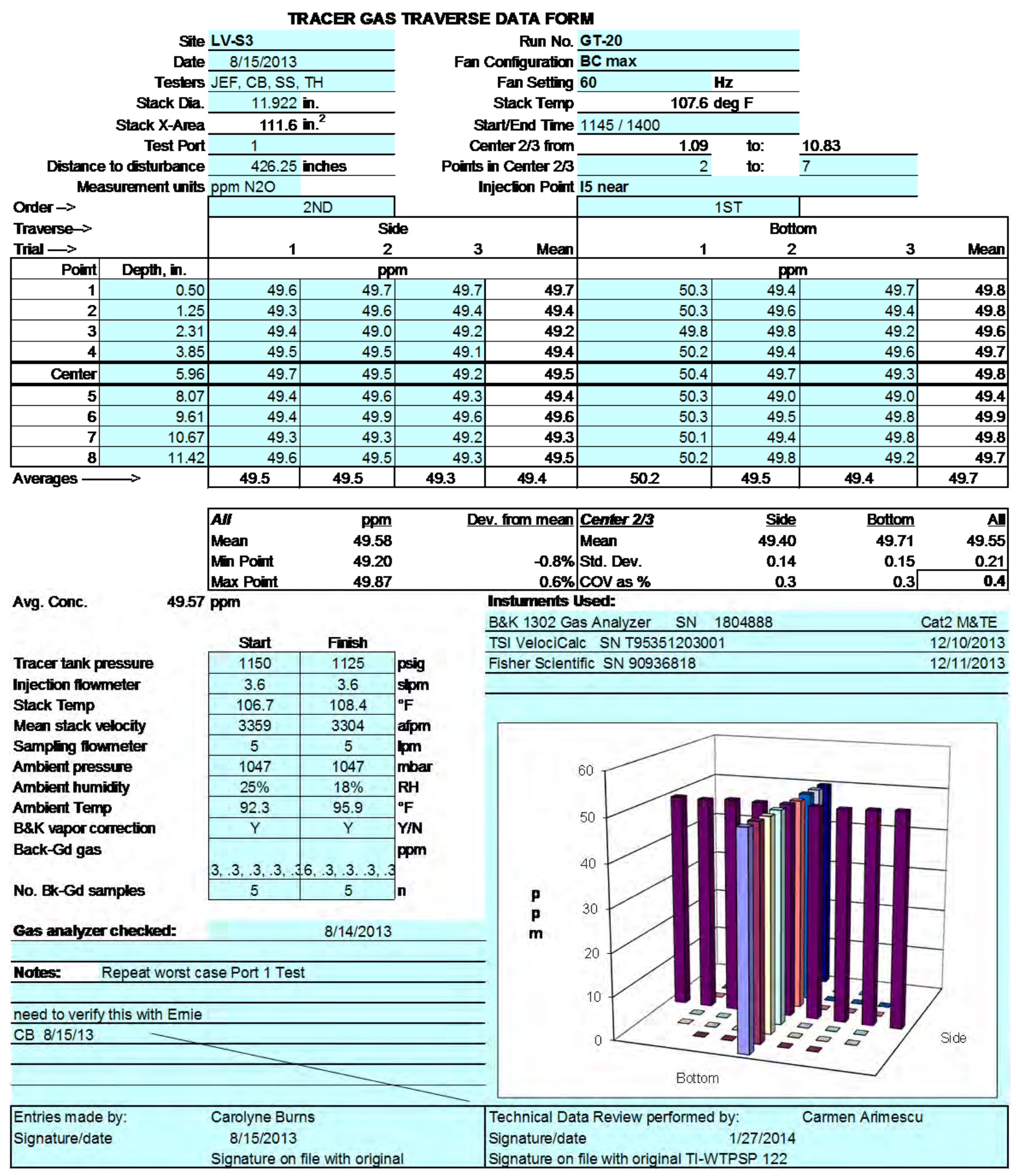




\begin{tabular}{|c|c|c|c|c|c|c|c|c|c|}
\hline & & & ACER GAS & TRAVERSE & DATA FOR & & & & \\
\hline & Site & LV.S3 & & & Run No. & GT-21 & & & \\
\hline & Date & $8 / 16 / 2013$ & & Fan C & Configuration & BC MAX & & & \\
\hline & Testers & $E A, S S$ & & & Fan Setting & 60 & & & \\
\hline & Stack Dia. & 11.922 & & & Stack Temp. & $75.2 \mathrm{c}$ & $g F$ & & \\
\hline & Stack X-Area & 111.6 & & & art/End Tme & $0636 / 0815$ & & & \\
\hline & Test Port & 1 & & & nter $2 / 3$ from & 1.09 & to: & 10.83 & \\
\hline Distance & to disturbence & 426.25 & nches & Points i & in Center 23 & 2 & to: & 7 & \\
\hline Mee & surement units & ppm N2O & & & njection Point & 15 Near & & & \\
\hline Order $\rightarrow$ & & $1 \mathrm{ST}$ & & & & $2 \mathrm{ND}$ & & & \\
\hline Traverse $\rightarrow$ & & & Sid & de & & & Botto & om & \\
\hline Trial $\longrightarrow$ & & 1 & 2 & 3 & Mean & 1 & 2 & 3 & Mean \\
\hline Point & Depth, in. & & ppn & $m$ & & & pprn & & \\
\hline 1 & 0.50 & 54.3 & 53.4 & 52.9 & 53.5 & 52.4 & 51.9 & 51.8 & 52.0 \\
\hline 2 & 1.25 & 54.1 & 53.4 & 52.9 & 53.5 & 52.3 & 51.9 & 51.9 & 52.0 \\
\hline 3 & 2.31 & 54.3 & 53.5 & 52.8 & 53.5 & 52.4 & 51.9 & 51.6 & 52.0 \\
\hline 4 & 3.85 & 54.2 & 53.6 & 53.0 & 53.6 & 52.4 & 52.0 & 51.5 & 52.0 \\
\hline Center & 5.96 & 54.1 & 53.4 & 52.7 & 53.4 & 52.2 & 52.0 & 51.7 & 52.0 \\
\hline 5 & 8.07 & 53.9 & 53.5 & 52.7 & 53.4 & 52.2 & 52.2 & 51.5 & 52.0 \\
\hline 6 & 9.61 & 54.0 & 53.5 & 52.8 & 53.4 & 52.4 & 52.0 & 51.8 & 52.1 \\
\hline 7 & $\mid 10.67$ & 53.7 & 53.3 & 52.5 & 532 & 52.1 & 52.0 & 51.8 & 52.0 \\
\hline 8 & 11.42 & 53.6 & 53.2 & 52.6 & 53.1 & 51.9 & 52.1 & 51.5 & 51.8 \\
\hline Averages - & $\longrightarrow$ & 54.0 & 53.4 & 528 & 53.4 & 52.3 & 520 & 51.7 & 520 \\
\hline & & $A V$ & ppm & & v. from mean & Center 2/3 & Side & Bottom & \\
\hline & & Mean & 52.69 & & & Mean & $\overline{53.42}$ & 51.99 & 52.71 \\
\hline & & Mn Point & 51.83 & & $-1.6 \%$ & Std. Dev. & 0.14 & 0.04 & 0.75 \\
\hline & & Max Point & 53.60 & & $1.7 \%$ & Cov as $\%$ & 0.3 & 0.1 & 1.4 \\
\hline Avg. Conc. & 52.69 & ppm & & & Instuments U & Used: & & & \\
\hline & & & & & $\mathrm{B} \& \mathrm{~K} 1302 \mathrm{Ga}$ & as Analyzer SN & 804888 & & Cat2 M\&TE \\
\hline & & Start & Finish & & TSI VelociCal & alc SN T9535120300 & & & $12 / 10 / 2013$ \\
\hline Tracer tank $p$ & essure & 750 & 750 & psig & Fisher Scienti & tific SN 90936818 & & & $12 / 11 / 2013$ \\
\hline Injection flow & neter & 3.7 & 3.7 & stpm & & & & & \\
\hline Stack Temp & & 71.9 & 78.4 & ${ }^{\circ} \mathrm{F}$ & & & & & \\
\hline Mean stack v & *ocity & 3378 & 3323 & afpm & & & & & \\
\hline Sampling flor & meter & 5 & 5 & pm & & & & & \\
\hline Ambient pres & sure & 1047 & 1047 & mbar & & 60 & & & \\
\hline Ambient hum & & $68 \%$ & $52 \%$ & RH & & & & & \\
\hline Ambient Tem & & 67.1 & 75.2 & ${ }^{\circ} \mathrm{F}$ & & 50 & & & \\
\hline B\&K vapor cc & mection & $Y$ & $\mathrm{Y}$ & Y/N & & 50 & & & \\
\hline Back-Gd gas & & $.4, .4, .4, .4,4$ & $7,4,4,3,3$ & ppm & & 40 & & & \\
\hline No. Bk-Gd se & mples & 5 & 5 & n & $\mathbf{p}$ & & & & \\
\hline Gas analyze & checked: & $8 / 14 / 2013$ & & & $\begin{array}{l}\mathbf{p} \\
\mathbf{m}\end{array}$ & & & & \\
\hline Notes: & & & & 2 & & & & & \\
\hline & & & 1 & & & 10 & & & \\
\hline & & 2 & SS 8-16-13 & & & & & & de \\
\hline 7 & & & & & & Bottom & & & \\
\hline Entries made & & Susan Sande & & & Technical Dat & ta Review performed & & Carmen Arimescu & \\
\hline Signature/da & & $\begin{array}{r}8 / 16 / 2013 \\
\text { Signature on }\end{array}$ & le with origina & & $\begin{array}{l}\text { Signature/dat } \\
\text { Signature on }\end{array}$ & $\begin{array}{l}\text { te } \\
\text { file with original TI-W }\end{array}$ & $\begin{array}{l}1 / 27 / 2014 \\
\text { PSP } 122\end{array}$ & & \\
\hline
\end{tabular}




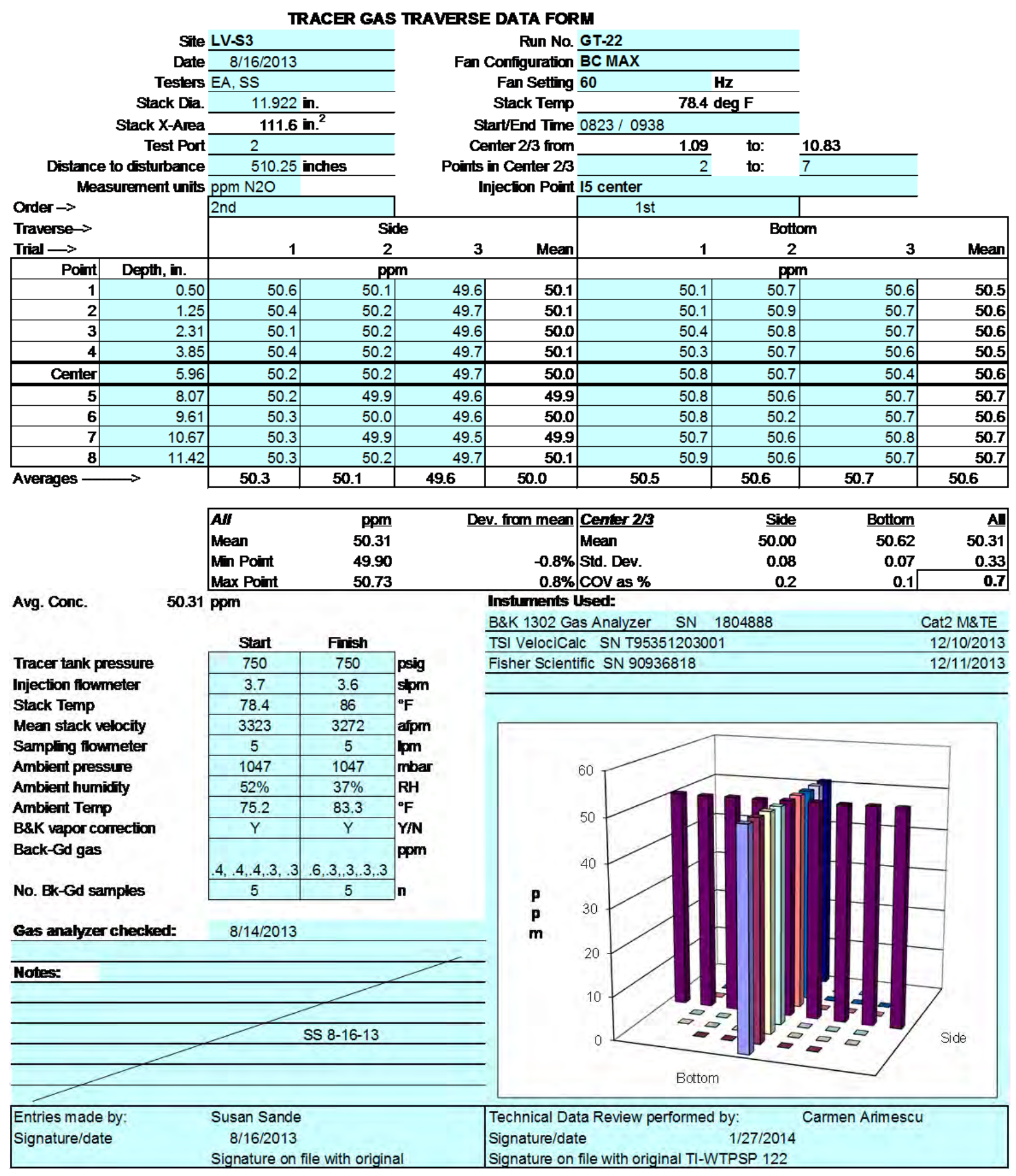




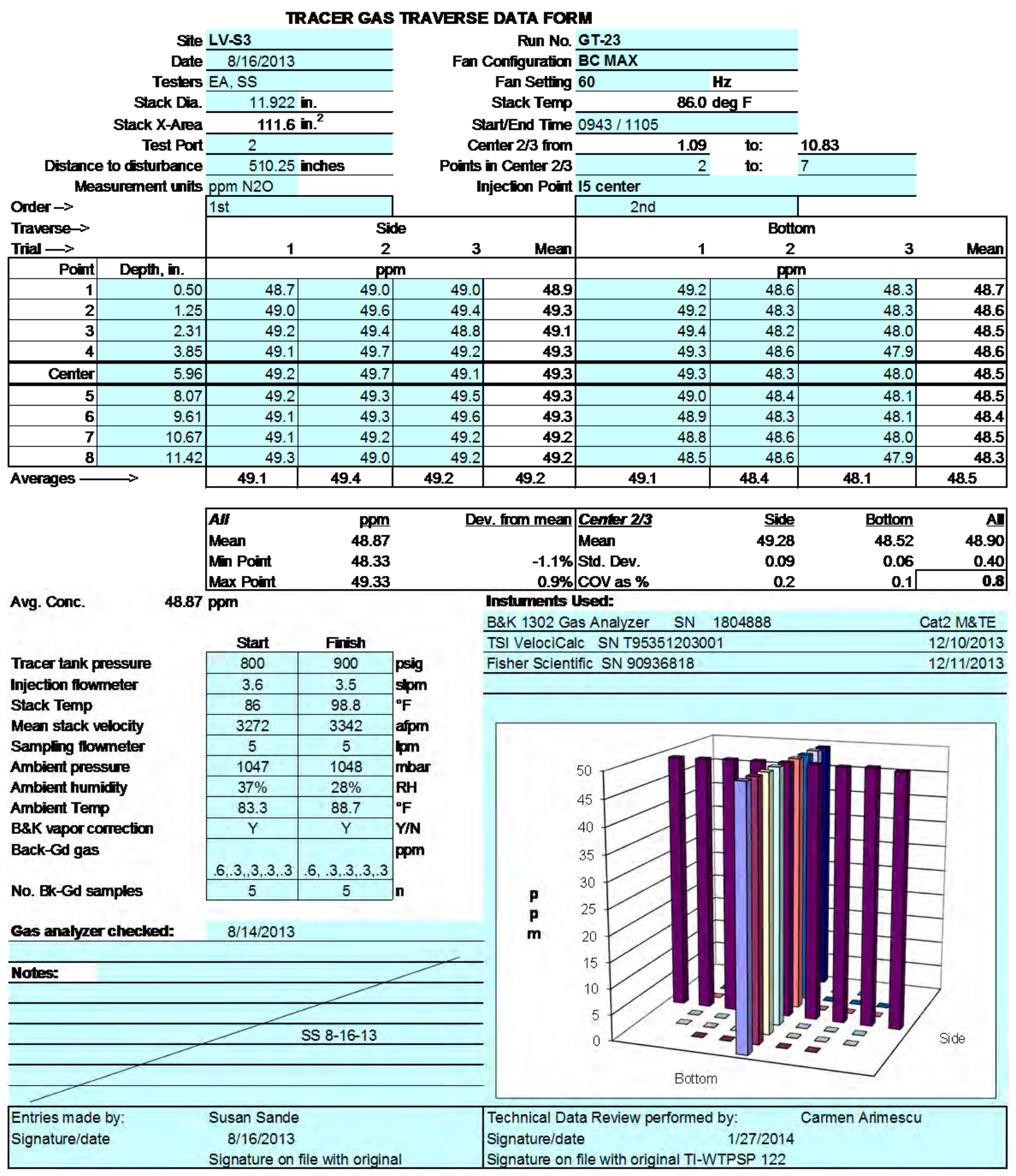




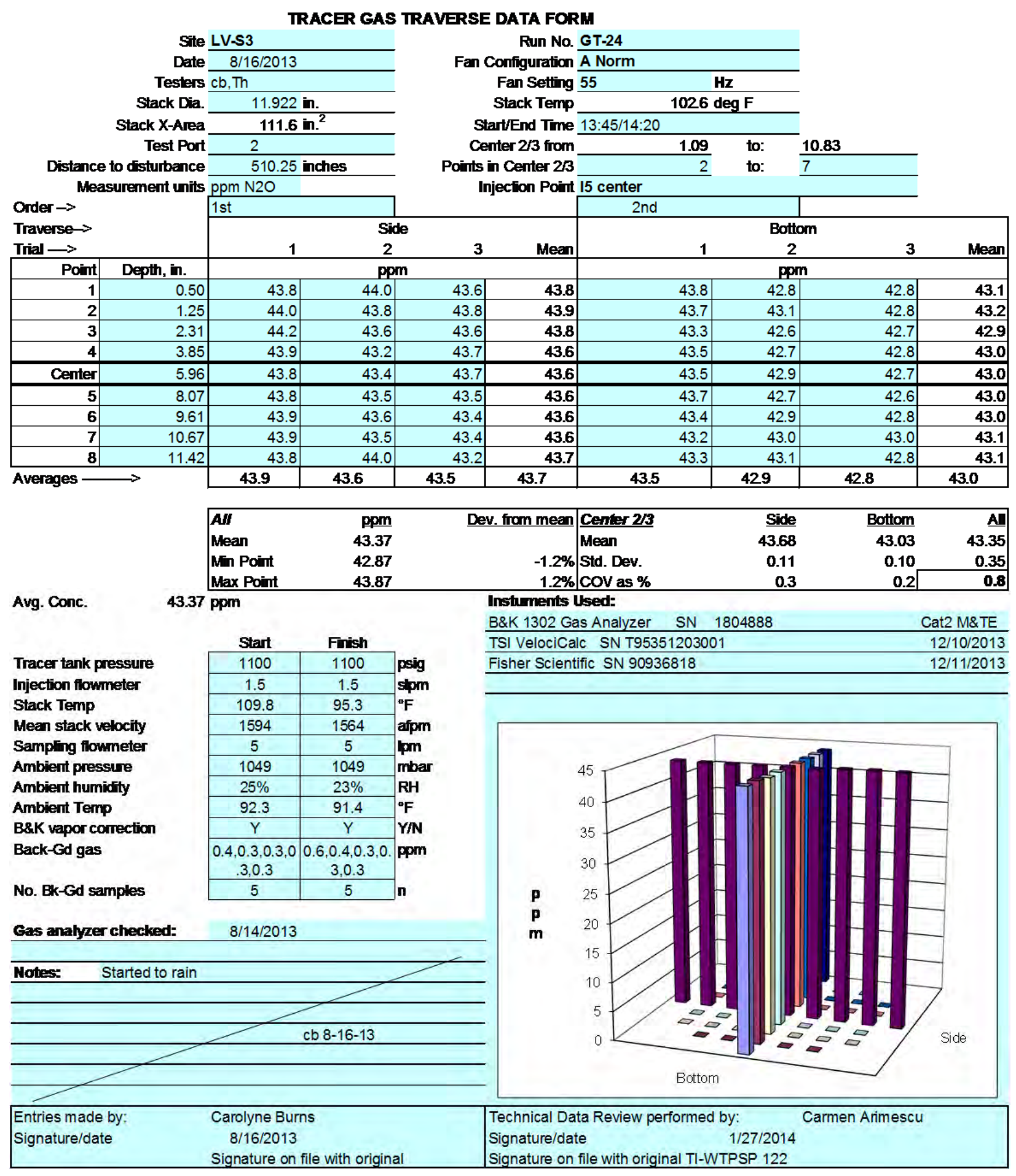




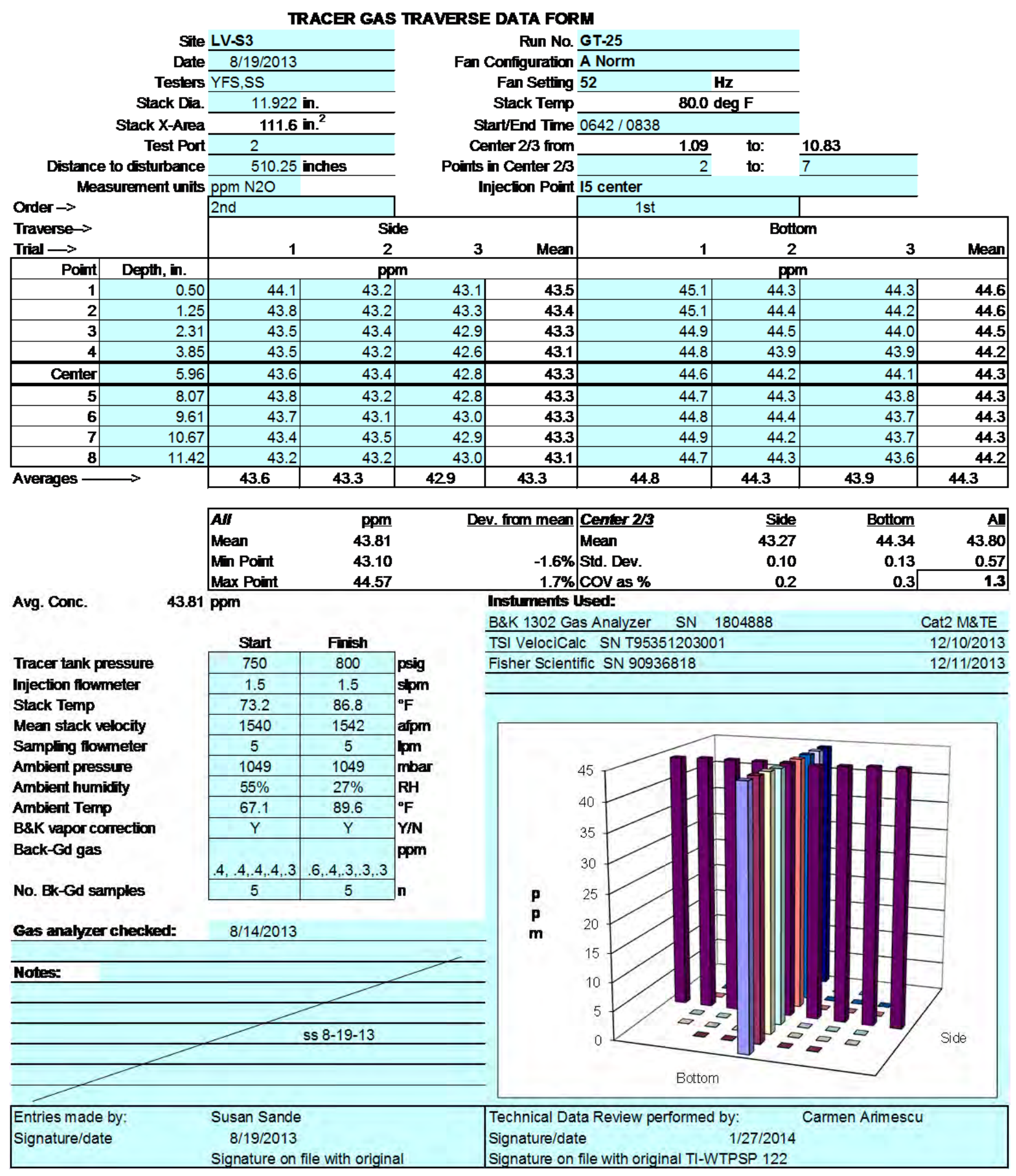




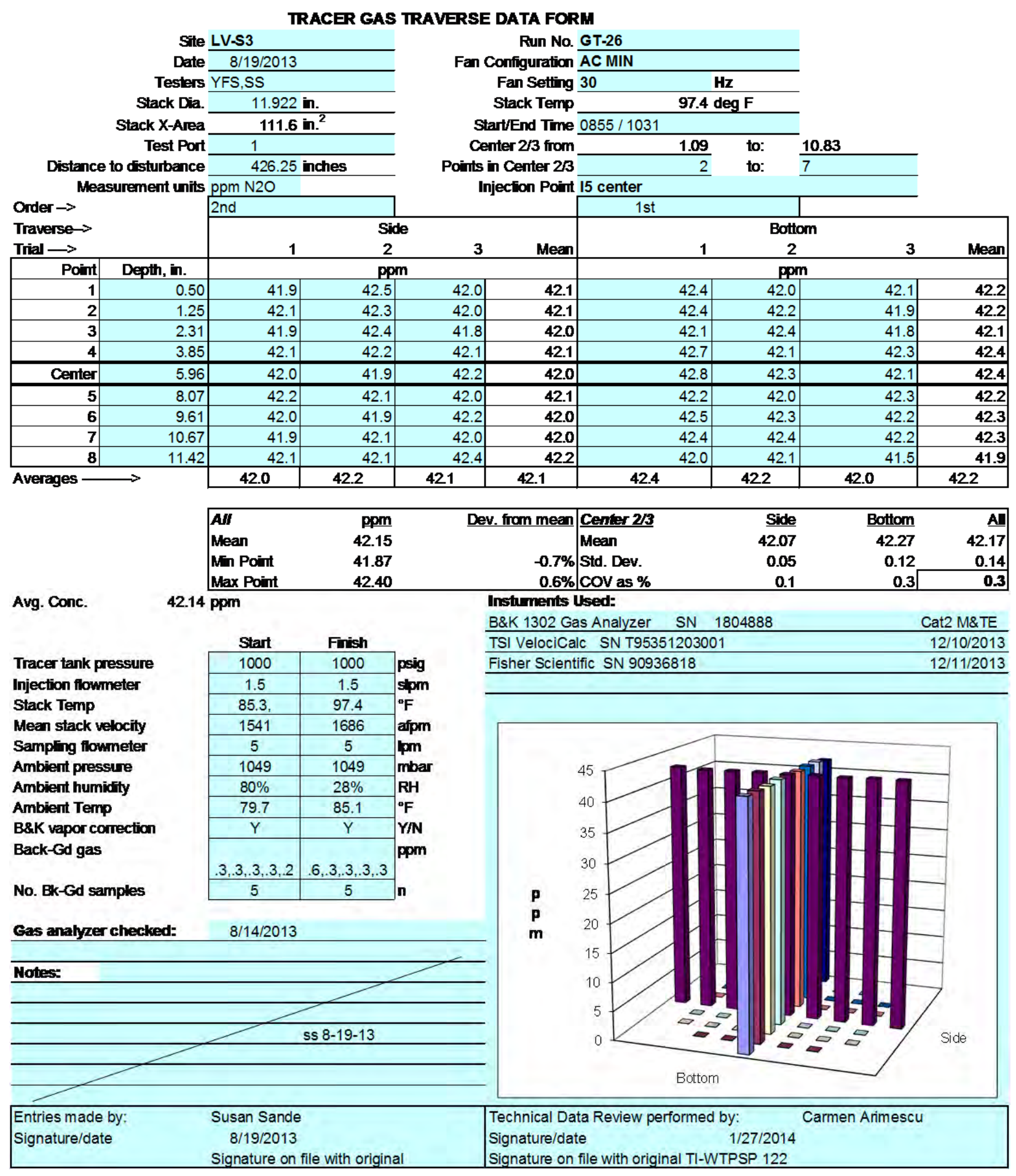




\begin{tabular}{|c|c|c|c|c|c|c|c|c|c|}
\hline & & & ACER GAS & TRAVERSE & DATA FOR & RM & & & \\
\hline & Site & LV-S3 & & & Run No. & GT-27 & & & \\
\hline & Date & $8 / 19 / 2013$ & & Fan $C$ & Configuration & AC MIN & & & \\
\hline & Testers & YFS,SS & & & Fan Setting & 30 & & & \\
\hline & Stack Dia. & 11.922 & & & Stack Temp & 100.7 & $g F$ & & \\
\hline & Stack X-Area & 111.6 & & & art/End Tme & $1040 / 1215$ & & & \\
\hline & Test Port & 1 & & & nter $2 / 3$ from & 1.09 & to: & 10.83 & \\
\hline Distance & to disturbance & 426.25 & nches & Points i & in Center 23 & 2 & to: & 7 & \\
\hline Mea & ssurement units & ppm N2O & & & njection Point & I5 NEAR TOP & & & \\
\hline Order $\rightarrow$ & & $1 \mathrm{ST}$ & & & & $2 \mathrm{ND}$ & & & \\
\hline Traverse $\rightarrow$ & & & Sid & & & & Botto & om & \\
\hline Trial $\longrightarrow$ & & 1 & 2 & 3 & Mean & 1 & 2 & 3 & Mean \\
\hline Point & Depth, in. & & ppn & & & & ppm & & \\
\hline 1 & 0.50 & 41.3 & \begin{tabular}{l|l|}
41.9 \\
\end{tabular} & \begin{tabular}{l|l}
41.9 \\
\end{tabular} & 41.7 & \begin{tabular}{l|l}
41.7 \\
\end{tabular} & 42.0 & 41.7 & 41.8 \\
\hline 2 & 1.25 & 41.6 & 41.5 & 41.3 & 41.5 & 42.1 & 42.1 & 42.2 & 42.1 \\
\hline 3 & 2.31 & 41.8 & 42.0 & 42.1 & 42.0 & 42.3 & 42.0 & 42.1 & 42.1 \\
\hline 4 & 3.85 & 41.5 & 41.9 & 41.8 & 41.7 & 41.7 & 41.7 & 42.1 & 41.8 \\
\hline Center & 5.96 & 42.0 & 42.0 & 41.9 & 42.0 & 41.8 & 42.1 & 41.9 & 41.9 \\
\hline 5 & 8.07 & 41.2 & \begin{tabular}{l|}
41.7 \\
\end{tabular} & \begin{tabular}{l|}
42.2 \\
\end{tabular} & 41.7 & 42.0 & 42.5 & 41.9 & 42.1 \\
\hline 6 & 9.61 & 42.4 & 41.9 & 42.3 & 422 & 42.1 & 42.0 & 42.0 & 42.0 \\
\hline 7 & 10.67 & 42.2 & 42.0 & 42.2 & 42.1 & 42.0 & 42.3 & 42.1 & 42.1 \\
\hline 8 & 11.42 & 42.2 & 41.9 & 42.1 & 42.1 & 41.9 & 41.9 & 41.8 & 41.9 \\
\hline Averages - & $\longrightarrow$ & 41.8 & 41.9 & 420 & 41.9 & 42.0 & 421 & 42.0 & 420 \\
\hline & & $A \boldsymbol{A}$ & ppm & & v. from mean & Center 2/3 & Side & Bottom & A \\
\hline & & Mean & 41.94 & & & Mean & 41.88 & 42.05 & 41.96 \\
\hline & & Mn Point & 41.47 & & $-1.1 \%$ & Std. Dev. & 0.26 & 0.12 & 0.21 \\
\hline & & Max Point & 42.20 & & $0.6 \%$ & Cov as $\%$ & 0.6 & 0.3 & 0.5 \\
\hline Avg. Conc. & 41.94 & ppm & & & Instuments U & Used: & & & \\
\hline & & & & & B\&K $1302 \mathrm{Ga}$ & as Analyzer SN & 304888 & & Cat2 M\&TE \\
\hline & & Start & Finish & & TSI VelociCal & alc SN T9535120300 & & & $12 / 10 / 2013$ \\
\hline Tracer tank $p$ & ressure & 1000 & 1100 & psig & Fisher Scienti & tific SN 90936818 & & & $12 / 11 / 2013$ \\
\hline Injection flow & meter & 1.5 & 1.5 & stpm & & & & & \\
\hline Stack Temp & & 97.4 & 103.9 & ${ }^{\circ} \mathrm{F}$ & & & & & \\
\hline Mean stack $v$ & elocity & 1686 & 1545 & afpm & & & & & \\
\hline Sampling flou & meter & 5 & 5 & pm & & & & & \\
\hline Ambient pres & sure & 1049 & 1050 & mbar & & 45 & & & \\
\hline Ambient hum & idity & $28 \%$ & $22 \%$ & $\mathbf{R H}$ & & & & & \\
\hline Ambient Tem & & 85.1 & 89.6 & ${ }^{\circ} \mathrm{F}$ & & 40 & & & \\
\hline B\&K vapor co & mrection & $Y$ & $y$ & YN & & 35 & & & \\
\hline Back-Gd gas & & 33333 & 63333 & ppm & & 30 & & & \\
\hline No. Bk-Gd sa & mples & $\frac{.3,3,3,3,3,3}{5}$ & $\frac{6, .3,3, .3, .3}{5}$ & $\mathbf{n}$ & p & 25 & & & \\
\hline Gas analyze & r checked: & $8 / 14 / 2013$ & & & $\begin{array}{l}\mathbf{p} \\
\mathbf{m}\end{array}$ & 20 & & & \\
\hline Notes: & & & & & & & & & \\
\hline & At the end of te & est GT-28 it we & $s$ discovered $t$ & that the gas & & & & & \\
\hline & probe & $\begin{array}{r}\text { as pointing ups } \\
\mathrm{cb} 08 / 19\end{array}$ & $\begin{array}{l}\text { ream of the } \\
2013\end{array}$ & & & & & & \\
\hline & 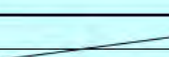 & 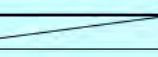 & 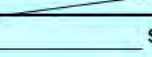 & ss $8-19-13$ & & Bottom & & & \\
\hline Entries made & & Susan Sande & & & Technical Dat & ta Review performed & & Carmen Arimescu & \\
\hline Signature/dat & & $\begin{array}{r}8 / 19 / 2013 \\
\text { Signature on }\end{array}$ & le with origina & & $\begin{array}{l}\text { Signature/dat } \\
\text { Signature on }\end{array}$ & $\begin{array}{l}\text { ite } \\
\text { file with original TI-W }\end{array}$ & $\begin{array}{l}1 / 27 / 2014 \\
\text { PSP } 122\end{array}$ & & \\
\hline
\end{tabular}




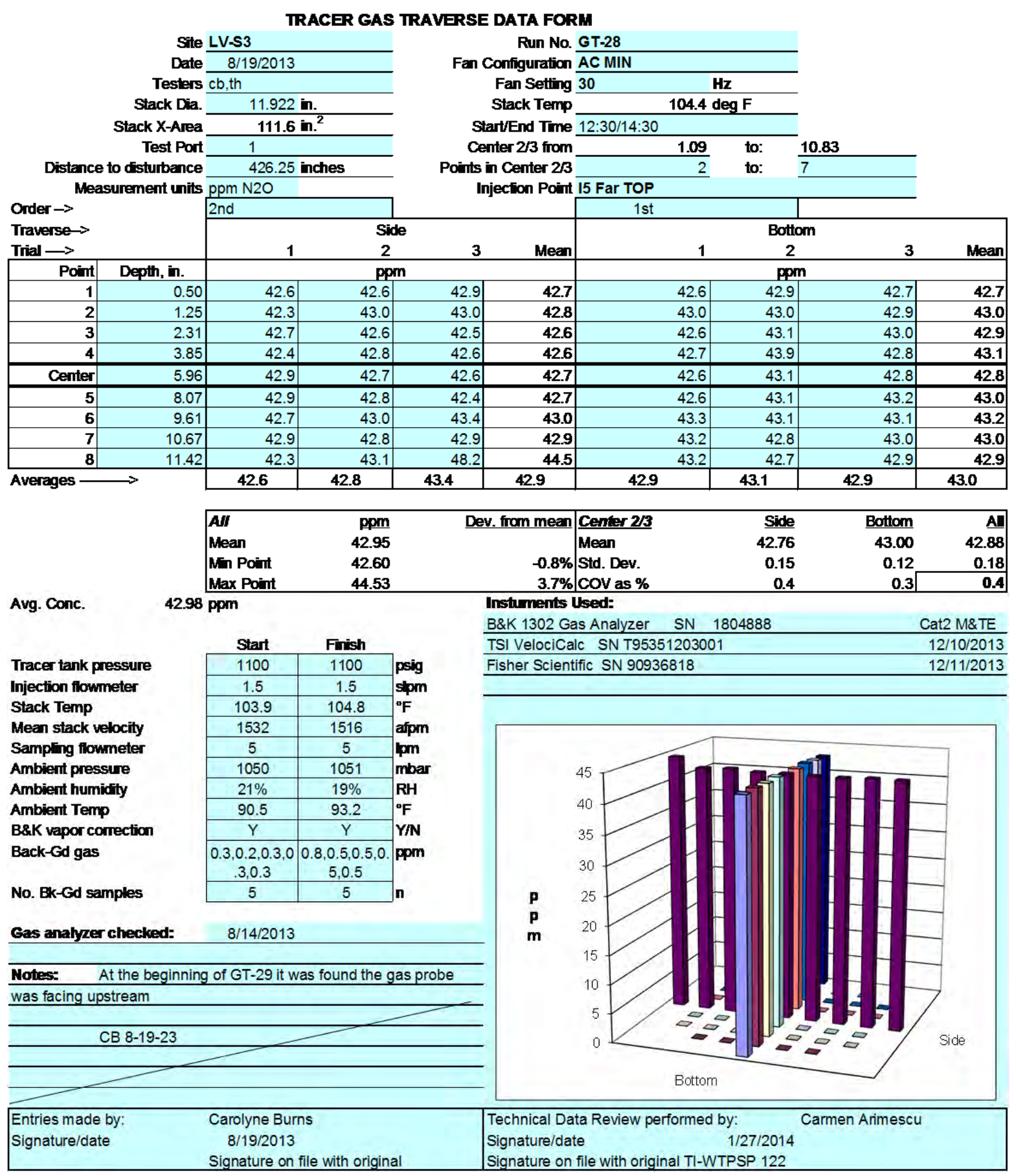




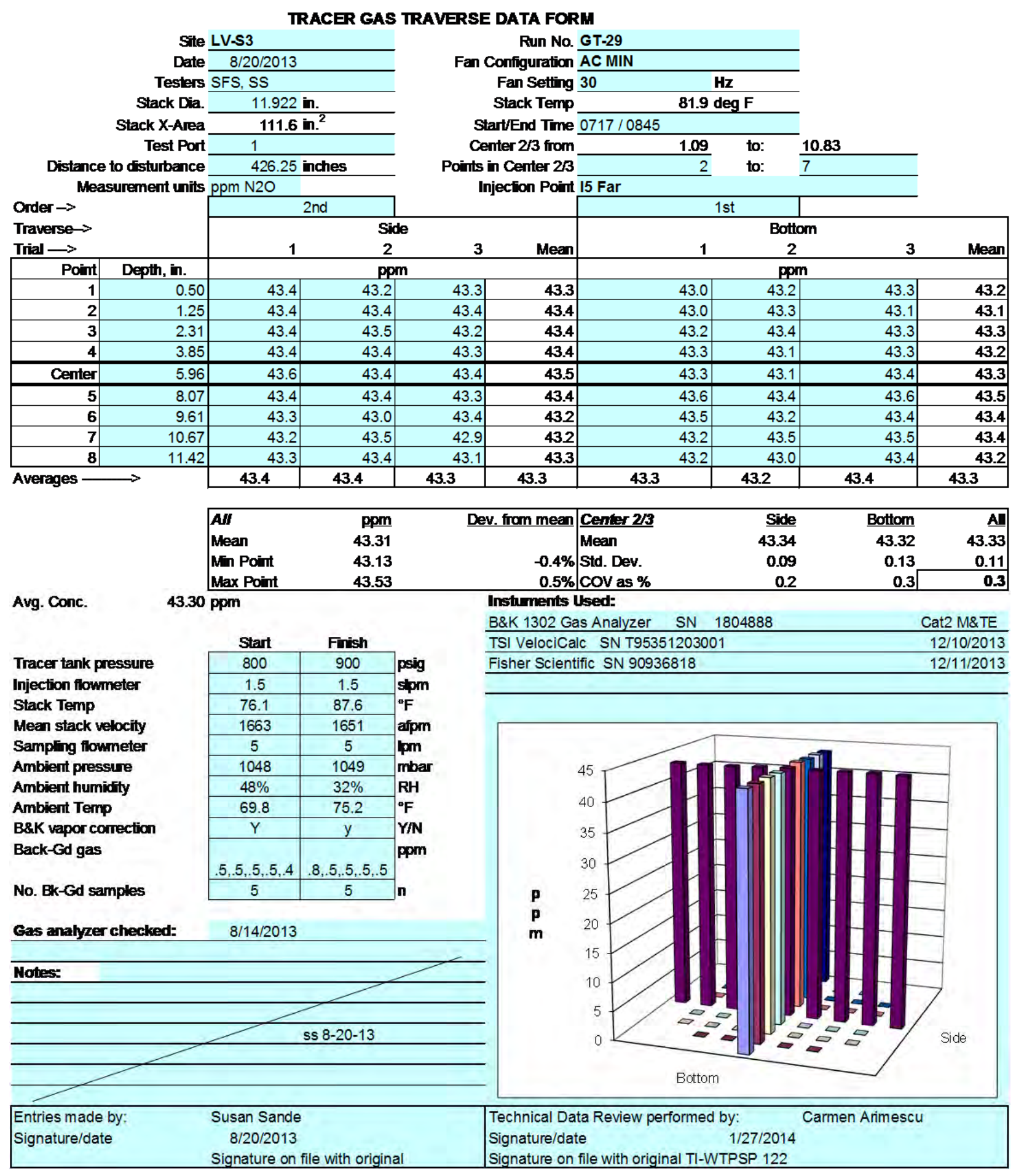




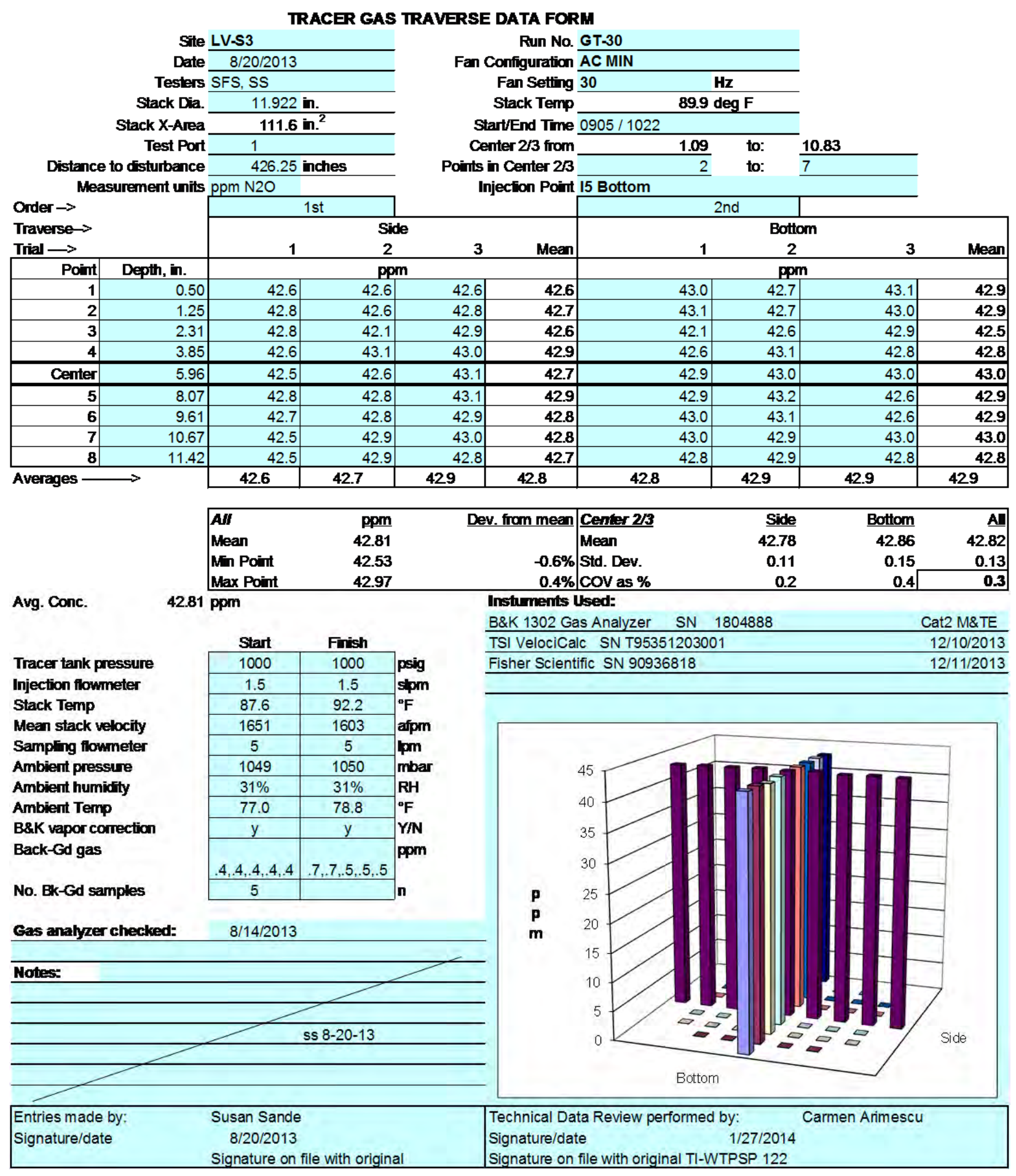




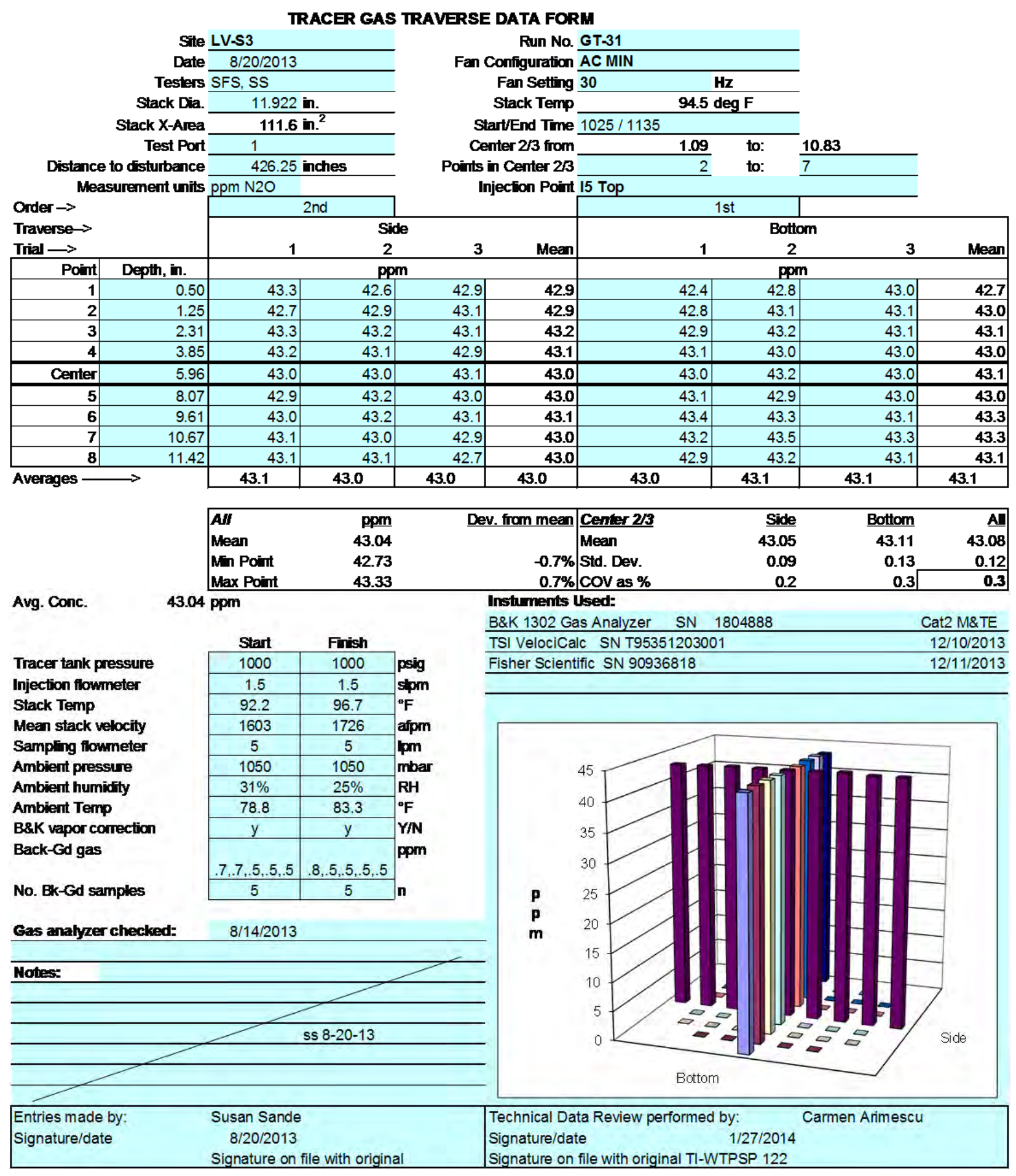




\begin{tabular}{|c|c|c|c|c|c|c|c|c|c|}
\hline & & & RACER GAS & TRAVERSE & DATA FOR & $\mathbf{R M}$ & & & \\
\hline & Site & LV-S3 & & & Run No. & GT-32 & & & \\
\hline & Date & $8 / 21 / 2013$ & & Fan $C$ & Configuration & AC MIN & & & \\
\hline & Testers & SFS, SS & & & Fan Setting & 30 & & & \\
\hline & Stack Dia. & 11.922 & & & Stack Temp. & $723 c$ & $g F$ & & \\
\hline & Stack X-Area & 111.6 & & & art/End Tme & $0655 / 0825$ & & & \\
\hline & Test Port & 1 & & & nter $2 / 3$ from & 1.09 & to: & 10.83 & \\
\hline Distance & to disturbance & 426.25 & inches & Points i & in Center 23 & 2 & to: & 7 & \\
\hline Mee & surement units & ppm N2O & & & njection Point & 15 Near & & & \\
\hline Order $\rightarrow$ & & $1 \mathrm{st}$ & & & & 2nd & & & \\
\hline Traverse $\rightarrow$ & & & Sid & de & & & Botto & om & \\
\hline Trial $\longrightarrow$ & & 1 & 2 & 3 & Mean & 1 & 2 & 3 & Mean \\
\hline Point & Depth, in. & & ppn & $m$ & & & ppm & & \\
\hline 1 & 0.50 & 43.8 & 43.8 & 43.5 & 43.7 & 43.6 & 43.4 & 42.8 & 43.3 \\
\hline 2 & 1.25 & 43.6 & 43.5 & 43.6 & 43.6 & 43.4 & 43.4 & 43.3 & 43.4 \\
\hline 3 & 2.31 & 43.9 & 43.6 & 43.4 & 43.6 & 43.4 & 43.0 & 42.9 & 43.1 \\
\hline 4 & 3.85 & 43.9 & 43.8 & 43.6 & 43.8 & 43.2 & 43.1 & 43.4 & 43.2 \\
\hline Center & 5.96 & 44.1 & 43.7 & 43.3 & 43.7 & 43.4 & 43.2 & 43.3 & 43.3 \\
\hline 5 & 8.07 & 43.7 & 43.6 & 43.9 & 43.7 & 43.4 & 43.3 & 43.6 & 43.4 \\
\hline 6 & 9.61 & 43.9 & 44.0 & 43.6 & 43.8 & \begin{tabular}{l|l}
43.2 \\
\end{tabular} & 43.5 & 43.4 & 43.4 \\
\hline 7 & 10.67 & 44.0 & 43.4 & 43.4 & 43.6 & 43.5 & 43.2 & 43.2 & 43.3 \\
\hline 8 & 11.42 & 44.1 & 43.4 & 43.5 & 43.7 & 43.3 & 43.4 & 43.3 & 43.3 \\
\hline Averages - & $\longrightarrow$ & 43.9 & 43.6 & 43.5 & 43.7 & 43.4 & 43.3 & 43.2 & 43.3 \\
\hline & & $A \boldsymbol{A}$ & ppm & & v. from mean & Center 2/3 & Side & Bottom & A \\
\hline & & Mean & 43.49 & & & Mean & 43.69 & 43.30 & 43.50 \\
\hline & & Min Point & 43.10 & & $-0.9 \%$ & Std. Dev. & 0.10 & 0.11 & 0.23 \\
\hline & & Max Point & 43.83 & & $0.8 \%$ & COV as $\%$ & 0.2 & 0.3 & 0.5 \\
\hline Avg. Conc. & 43.49 & ppm & & & Instuments U & Used: & & & \\
\hline & & & & & $\mathrm{B} \& \mathrm{~K} 1302 \mathrm{Ga}$ & as Analyzer SN & 304888 & & Cat2 M\&TE \\
\hline & & Start & Finish & & TSI VelociCal & alc SN T9535120300 & & & $12 / 10 / 2013$ \\
\hline Tracer tank $p$ & essure & 725 & 800 & psig & Fisher Scienti & tific SN 90936818 & & & $12 / 11 / 2013$ \\
\hline Injection flow & neter & 1.5 & 1.5 & stpm & & & & & \\
\hline Stack Temp & & 66 & 78.5 & ${ }^{\circ} \mathrm{F}$ & & & & & \\
\hline Mean stack v & *ocity & 1580 & 1606 & afpm & & & & & \\
\hline Sampling flor & meter & 5 & 5 & pm & & & & & \\
\hline Ambient pres & sure & 1049 & 1049 & mbar & & 45 & & & \\
\hline Ambient hum & & $54 \%$ & $30 \%$ & RH & & & & & \\
\hline Ambient Tem & & 61.7 & 80.6 & ${ }^{\circ} \mathrm{F}$ & & 40 & & & \\
\hline B\&K vapor cc & mection & $Y$ & $\mathrm{Y}$ & Y/N & & 35 & & & \\
\hline Back-Gd gas & & & & ppm & & 30 & & & \\
\hline No. $B$ k-Gd se & mples & $\frac{.6,6,6,6,6,6}{5}$ & $\frac{8, .6,6, .5, .5}{5}$ & n & p & 25 & & & \\
\hline Gas analyze & checked: & $8 / 21 / 2013$ & & & $\begin{array}{l}\mathbf{p} \\
\mathbf{m}\end{array}$ & 20 & & & \\
\hline Notes: & Tubing loose on & B\&K, ran Sid & $1 \& 2$ over. & 2 & & 10 & & & \\
\hline & & & 2 & & & & & & \\
\hline & & 7 & ss $8-21-13$ & & & 0 & & & Side \\
\hline 7 & & & & & & Bottom & & & \\
\hline Entries made & & Susan Sande & & & Technical Dat & ata Review performed & & Carmen Arimescu & \\
\hline Signature/da & & $\begin{array}{r}8 / 21 / 2013 \\
\text { Signature on }\end{array}$ & Fle with origina & & Signature/dat & ite & $\begin{array}{l}1 / 27 / 2014 \\
\text { PSP } 122\end{array}$ & & \\
\hline
\end{tabular}




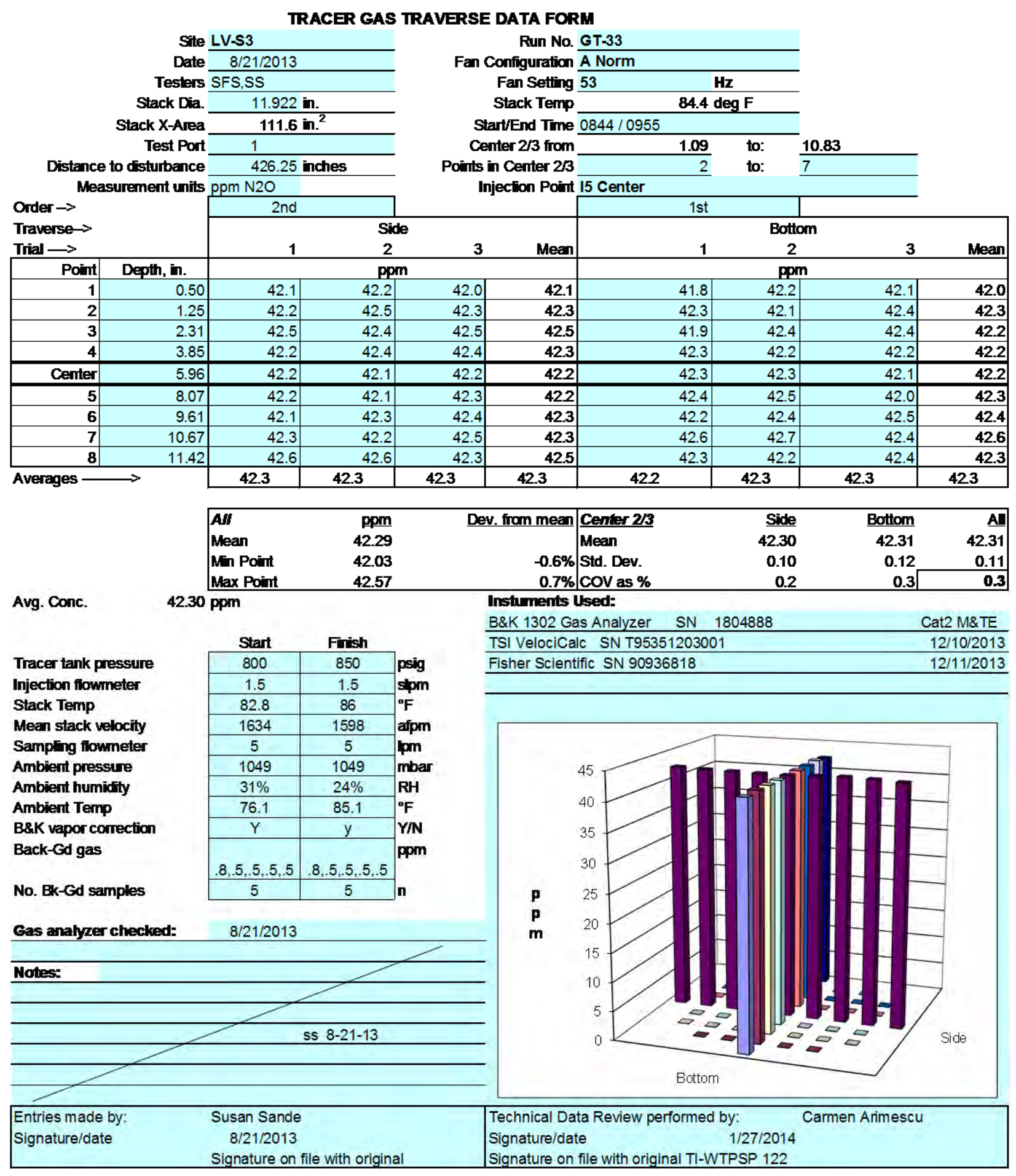




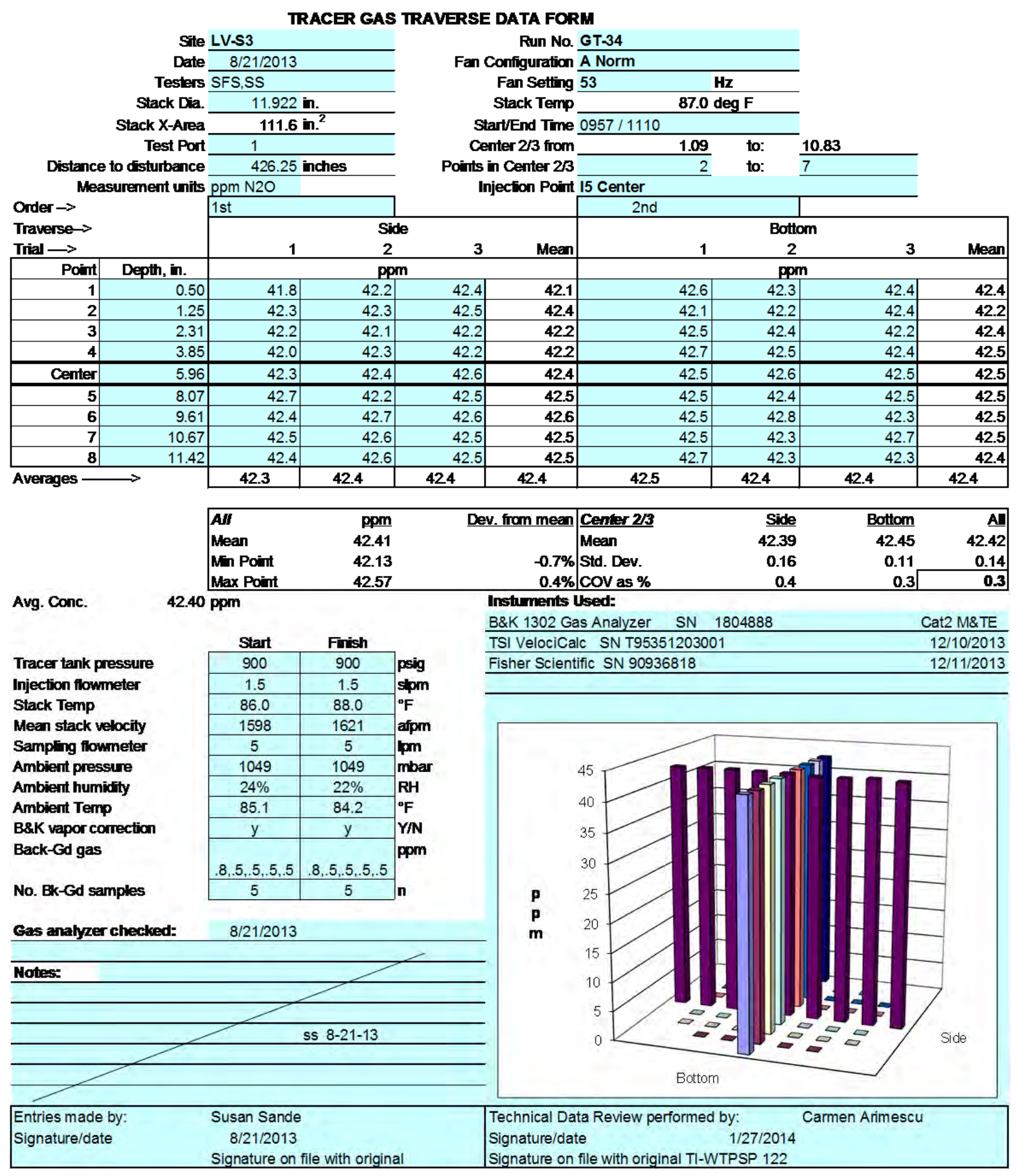




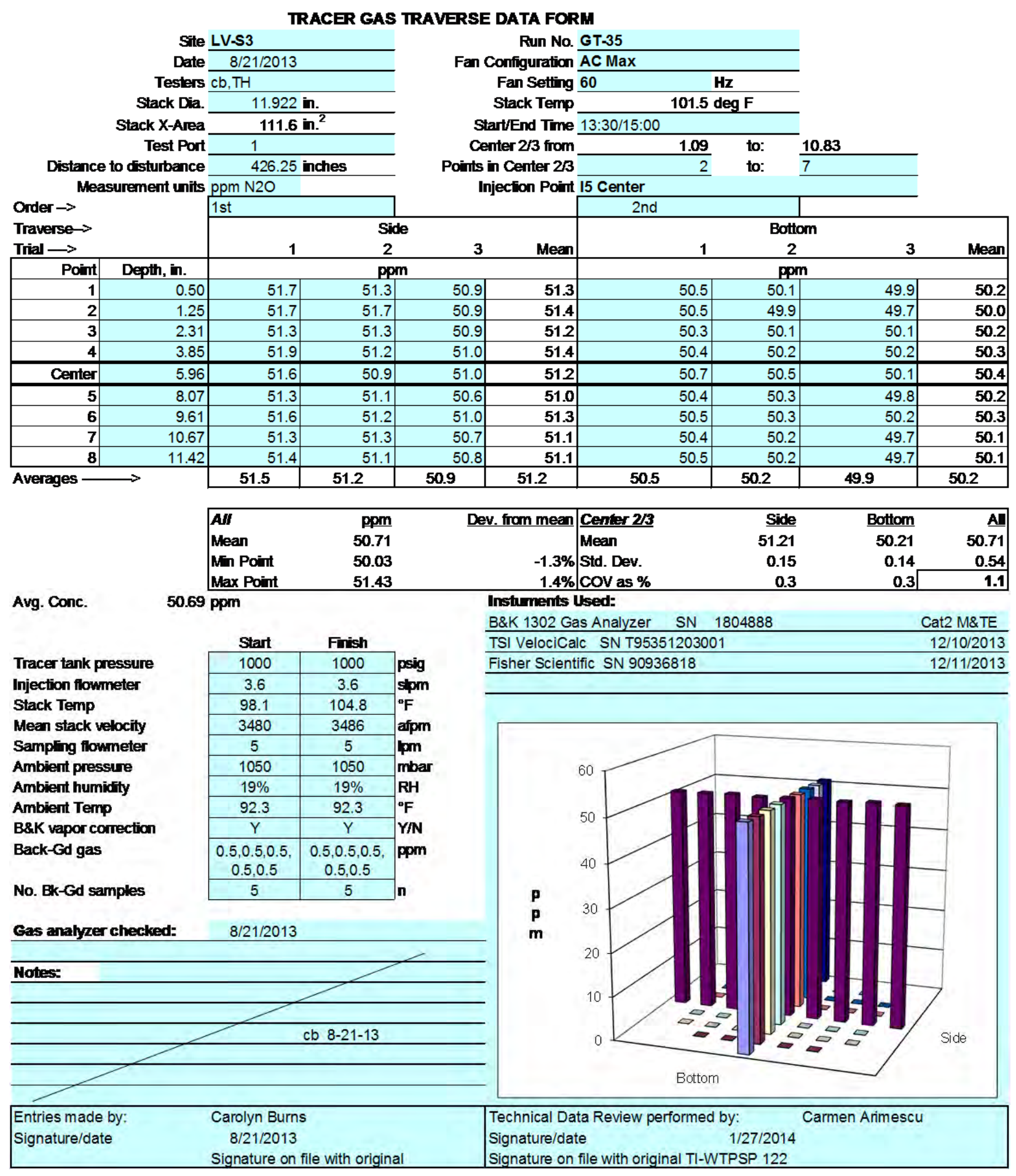




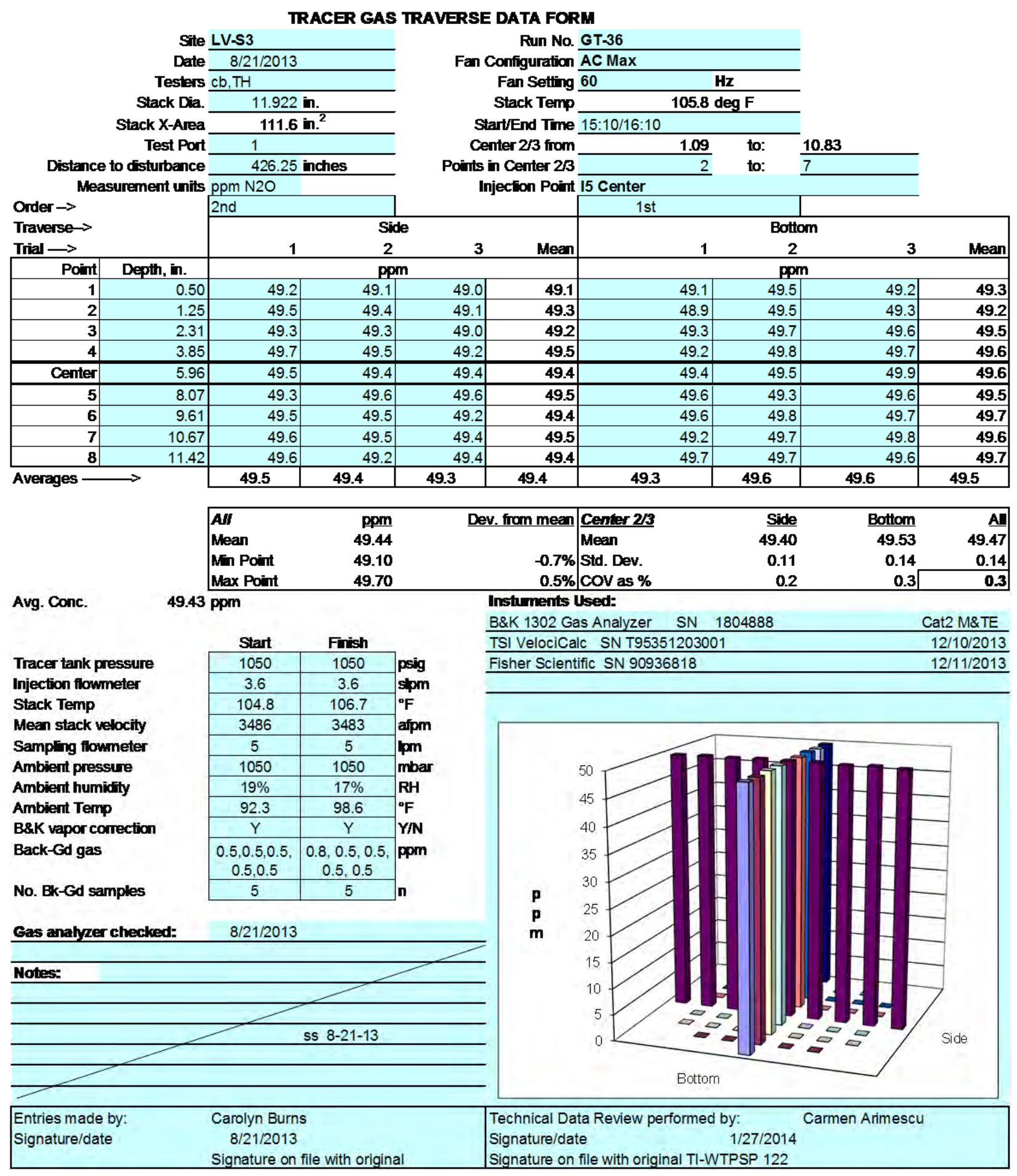




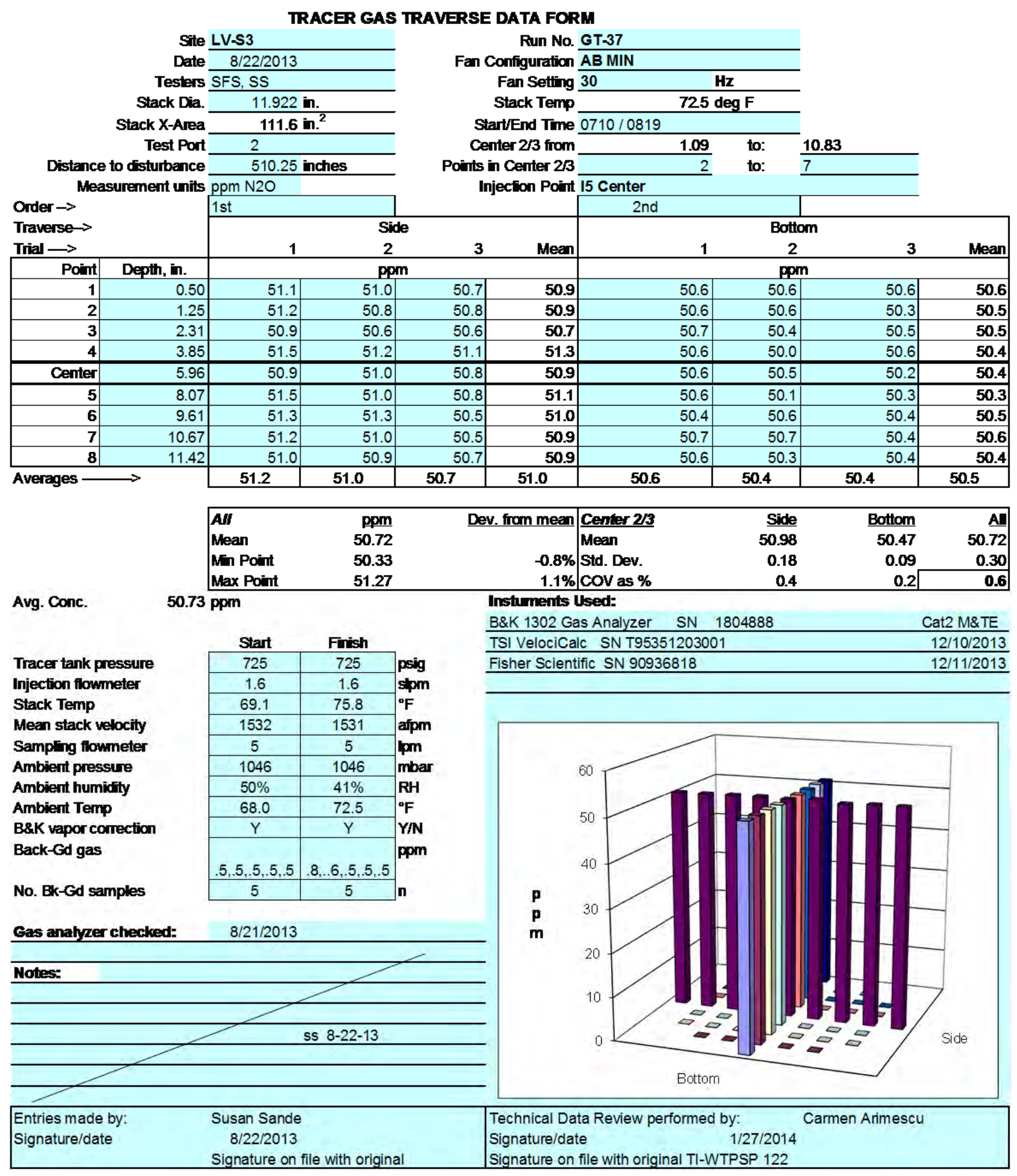




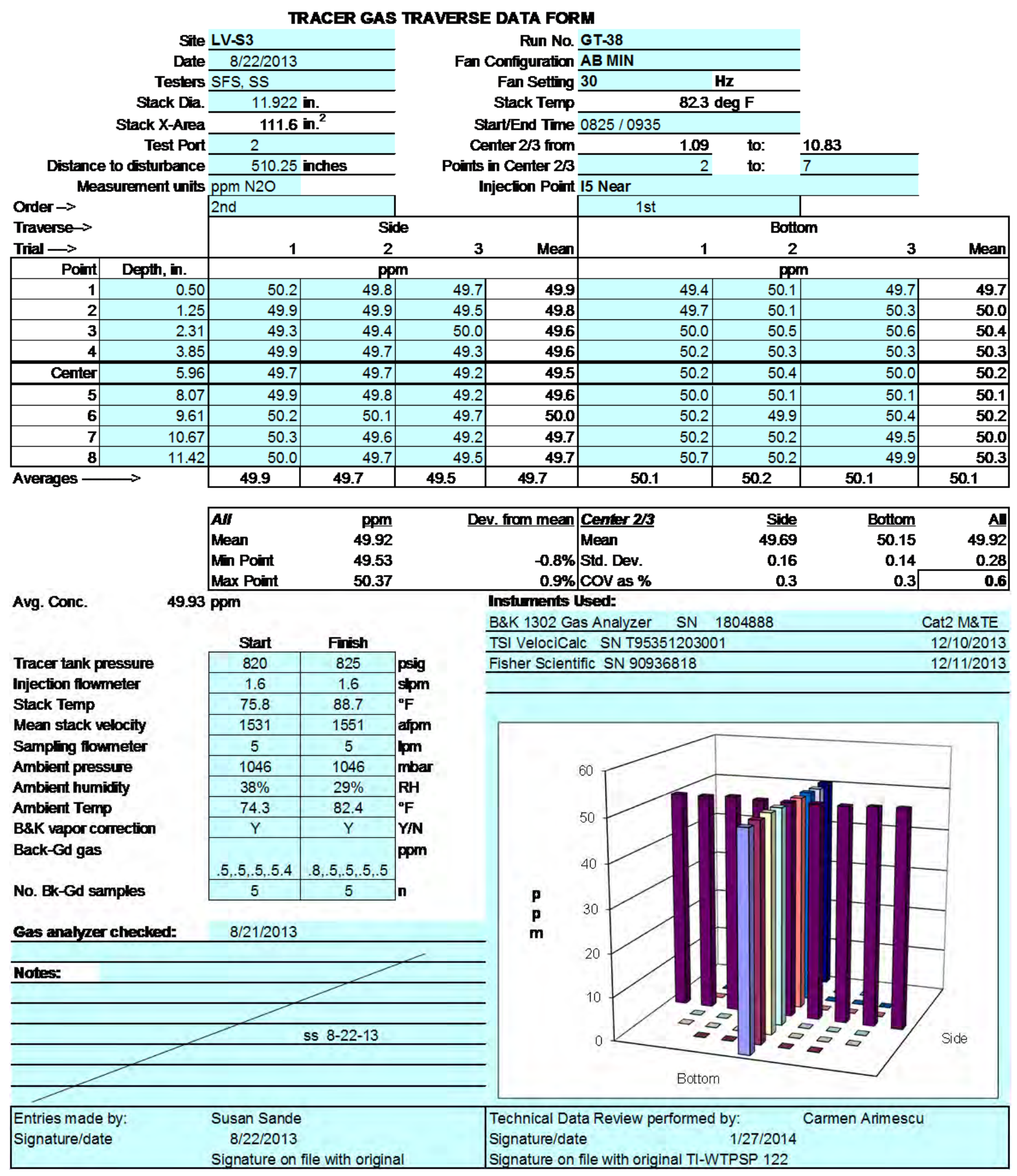




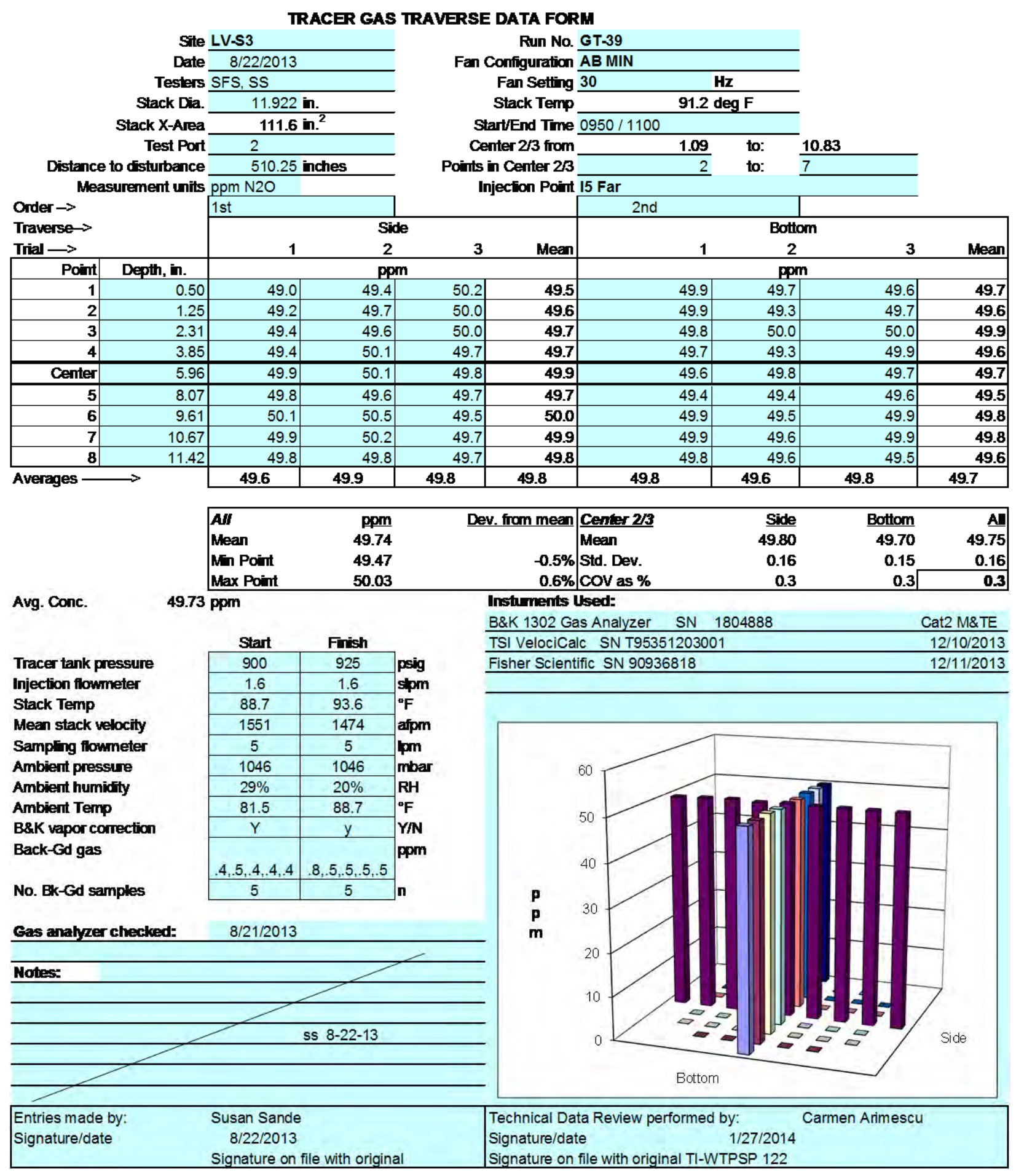




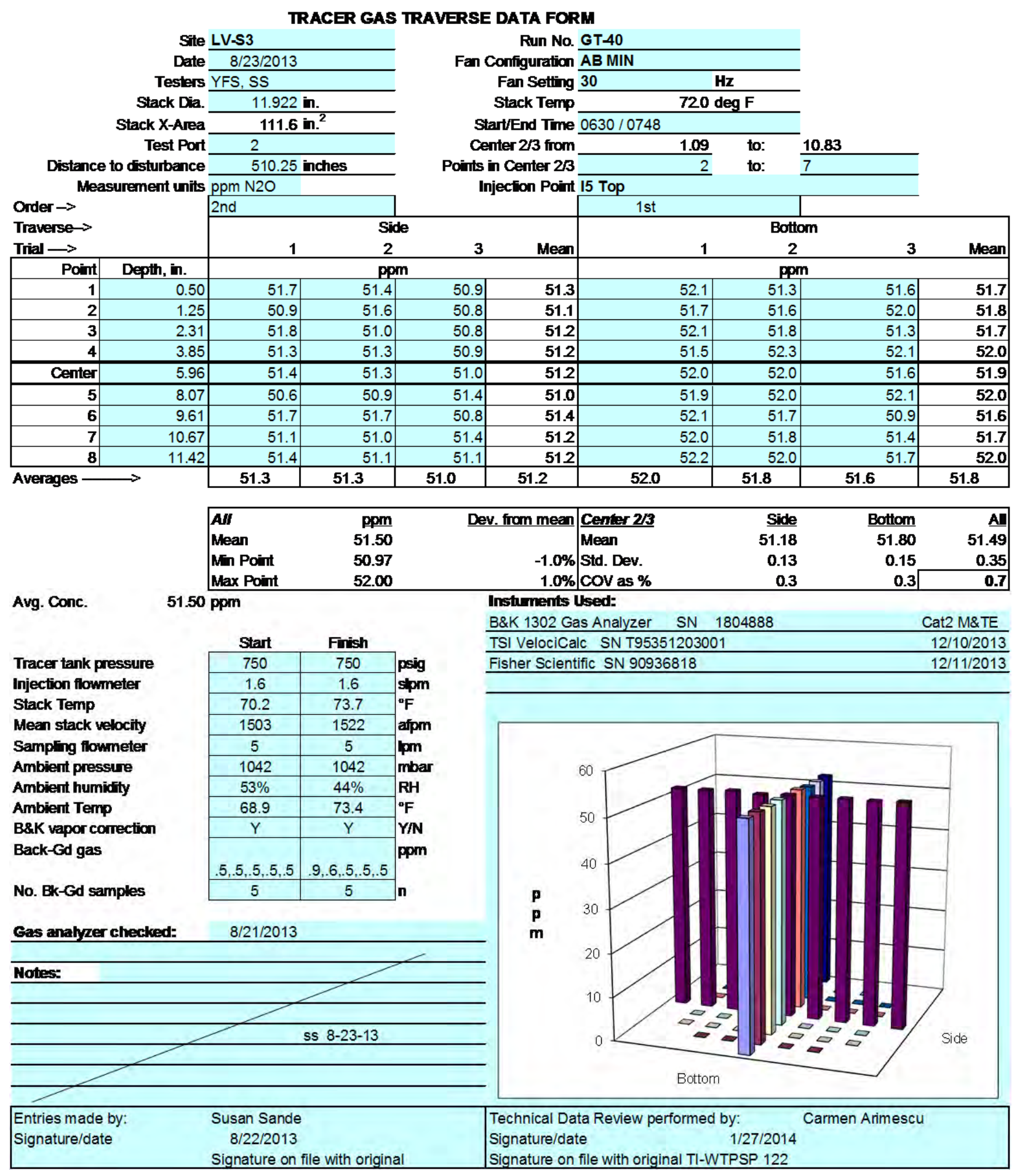




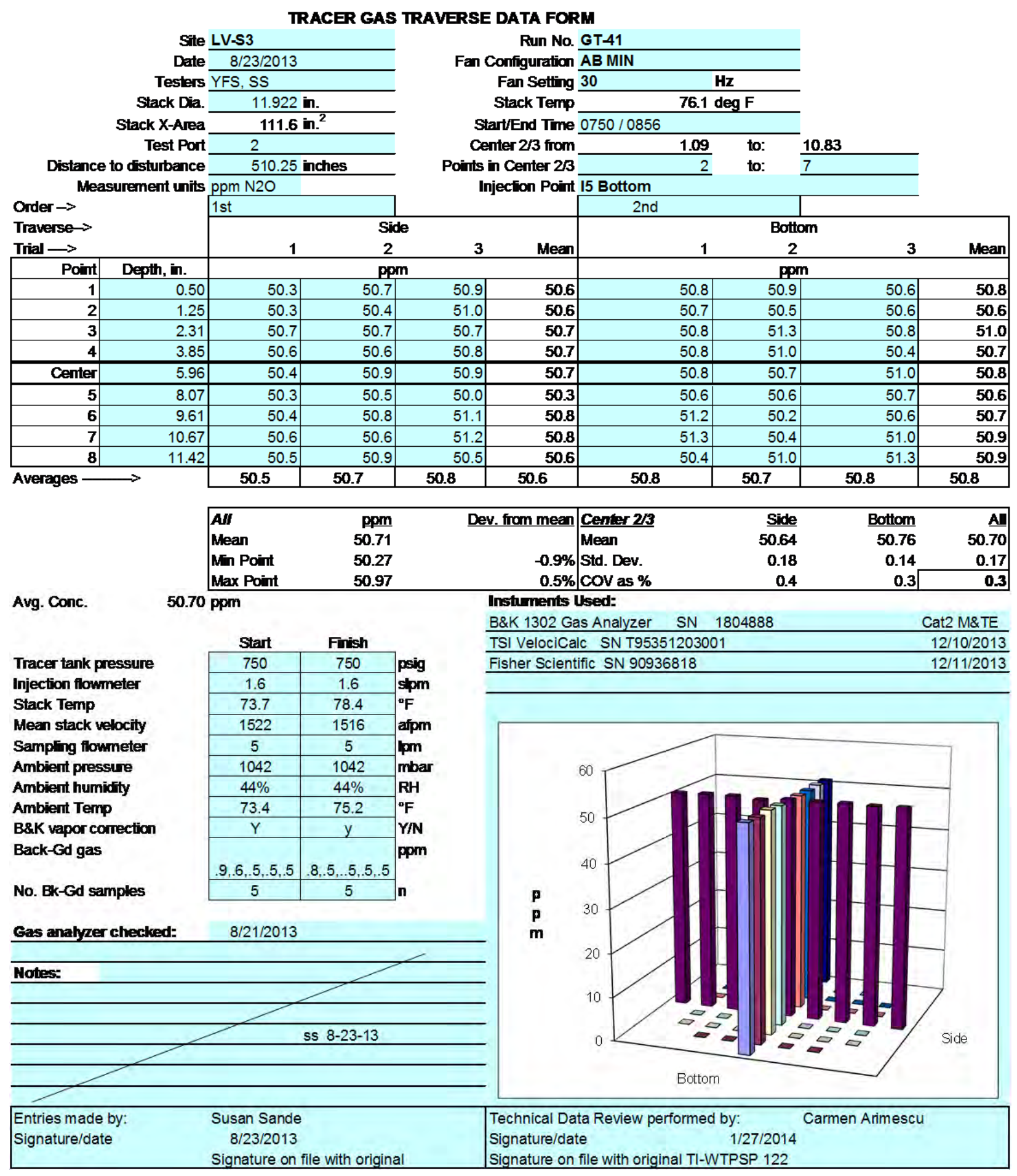




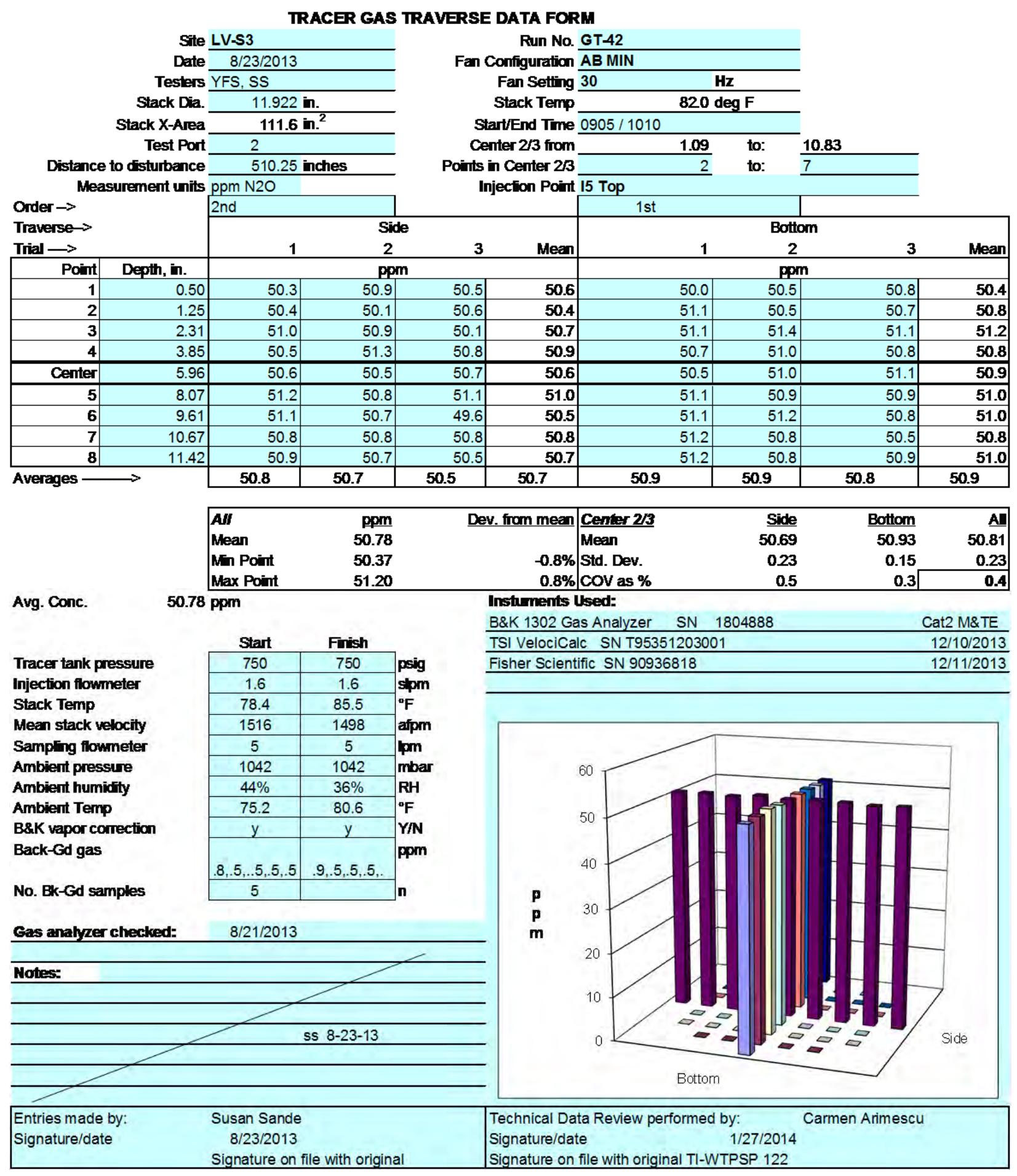




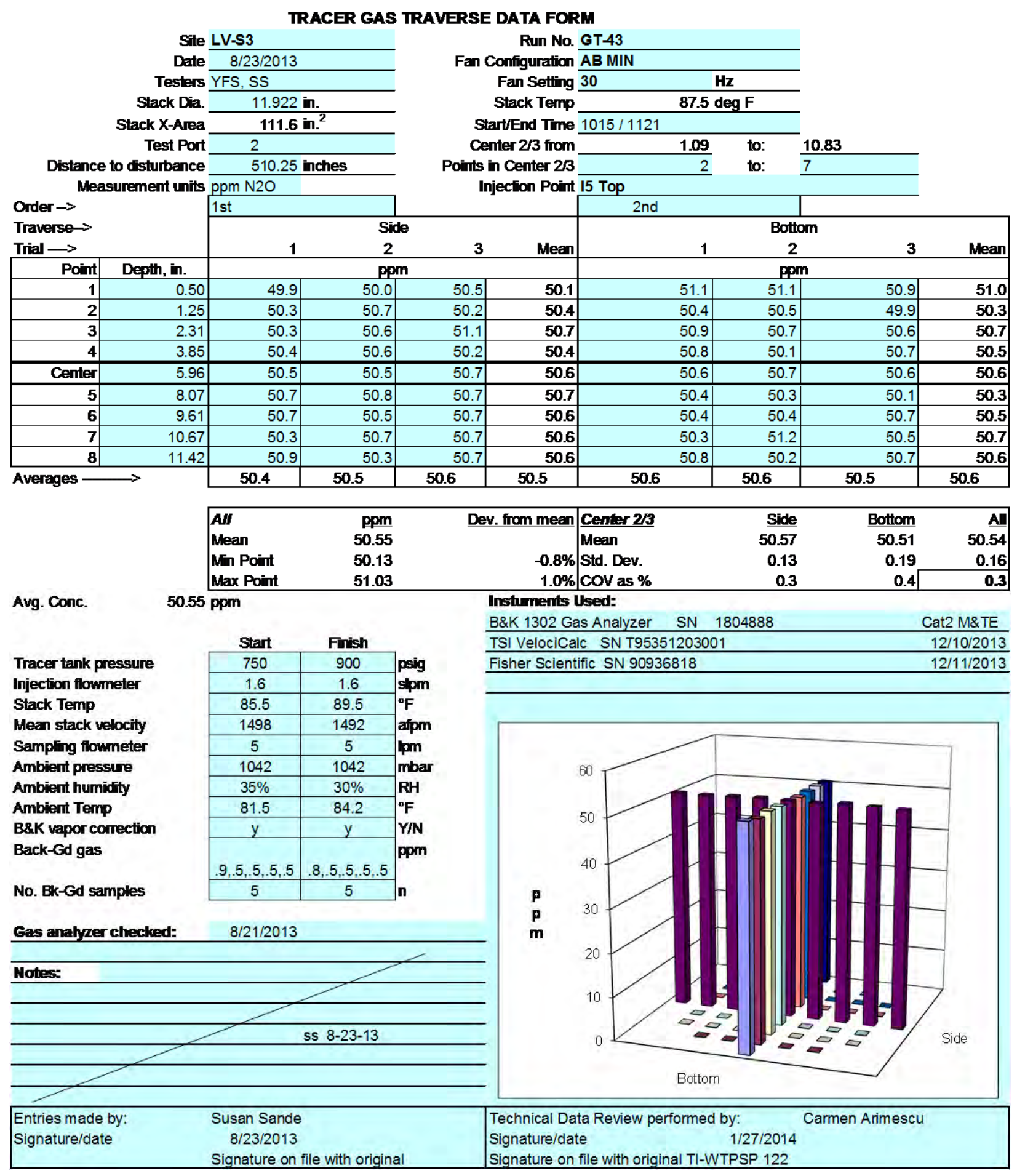




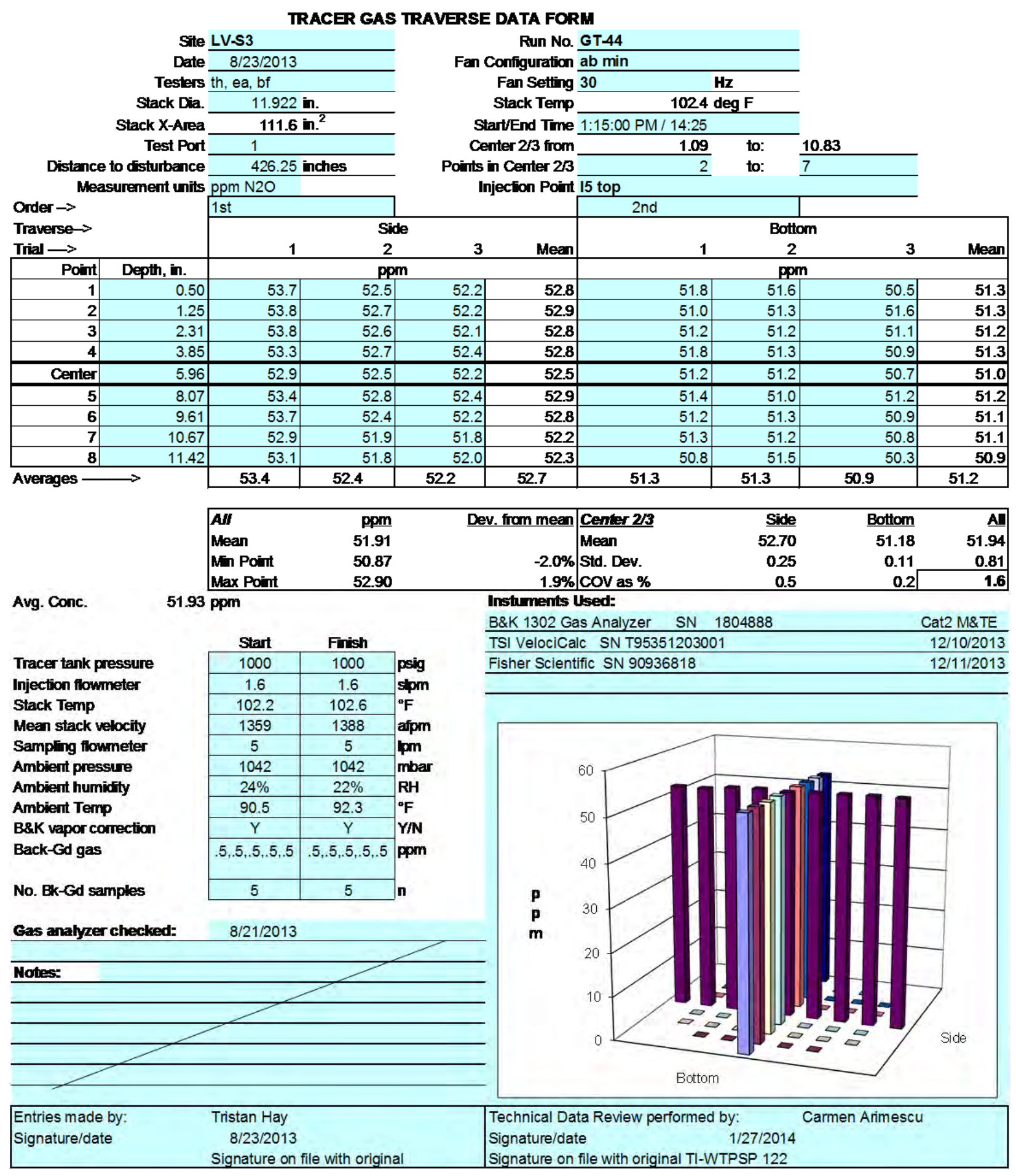




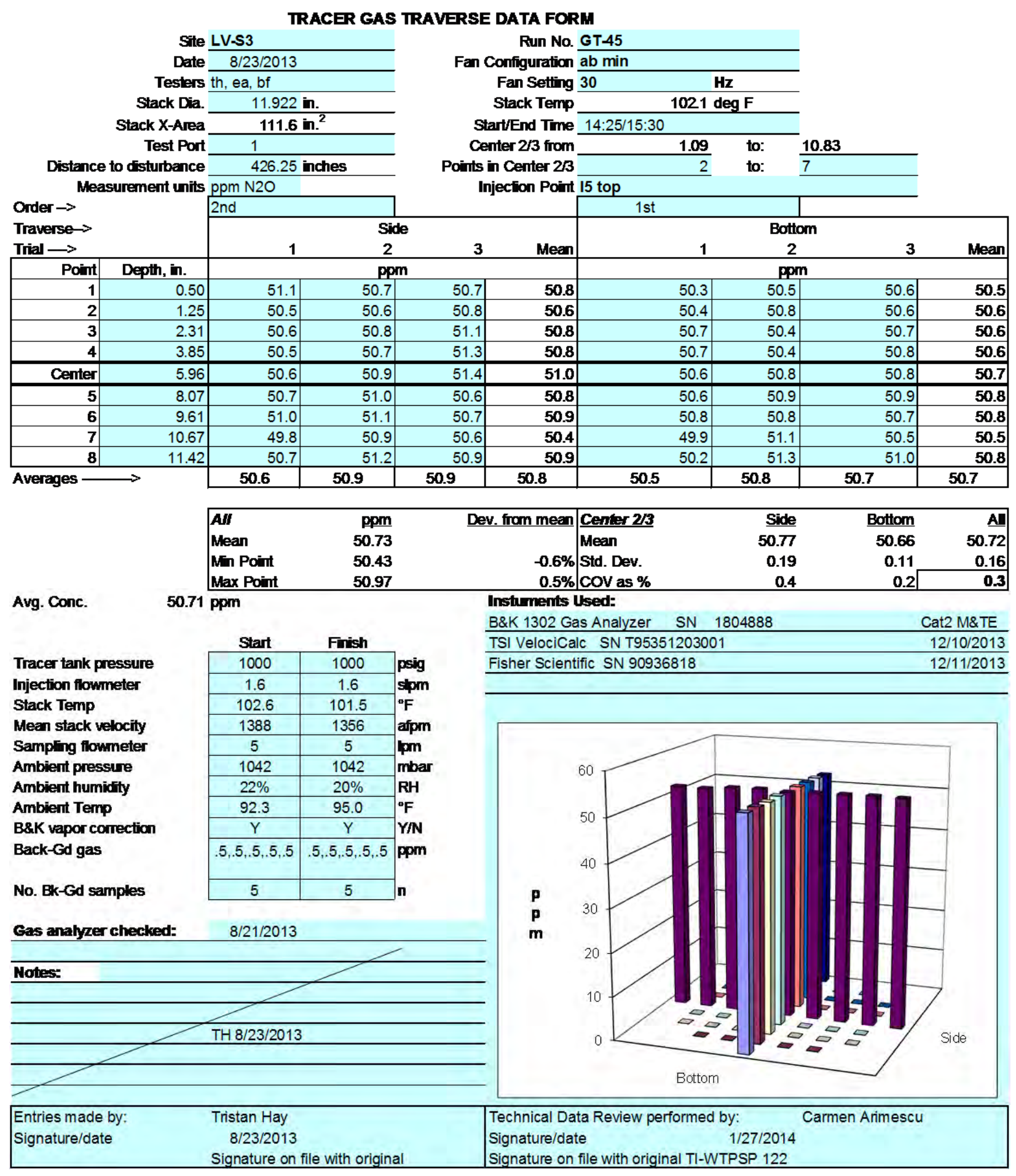




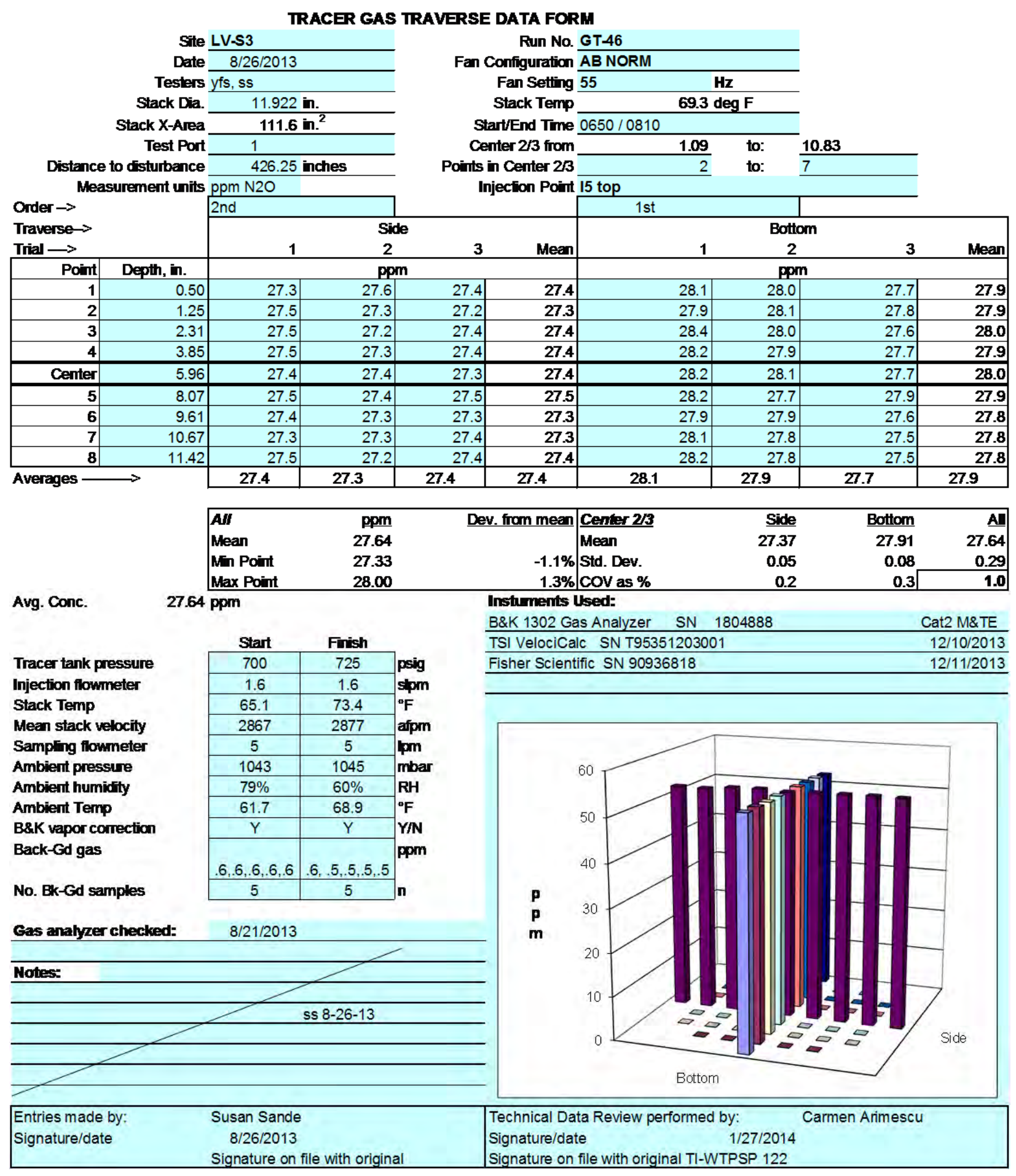




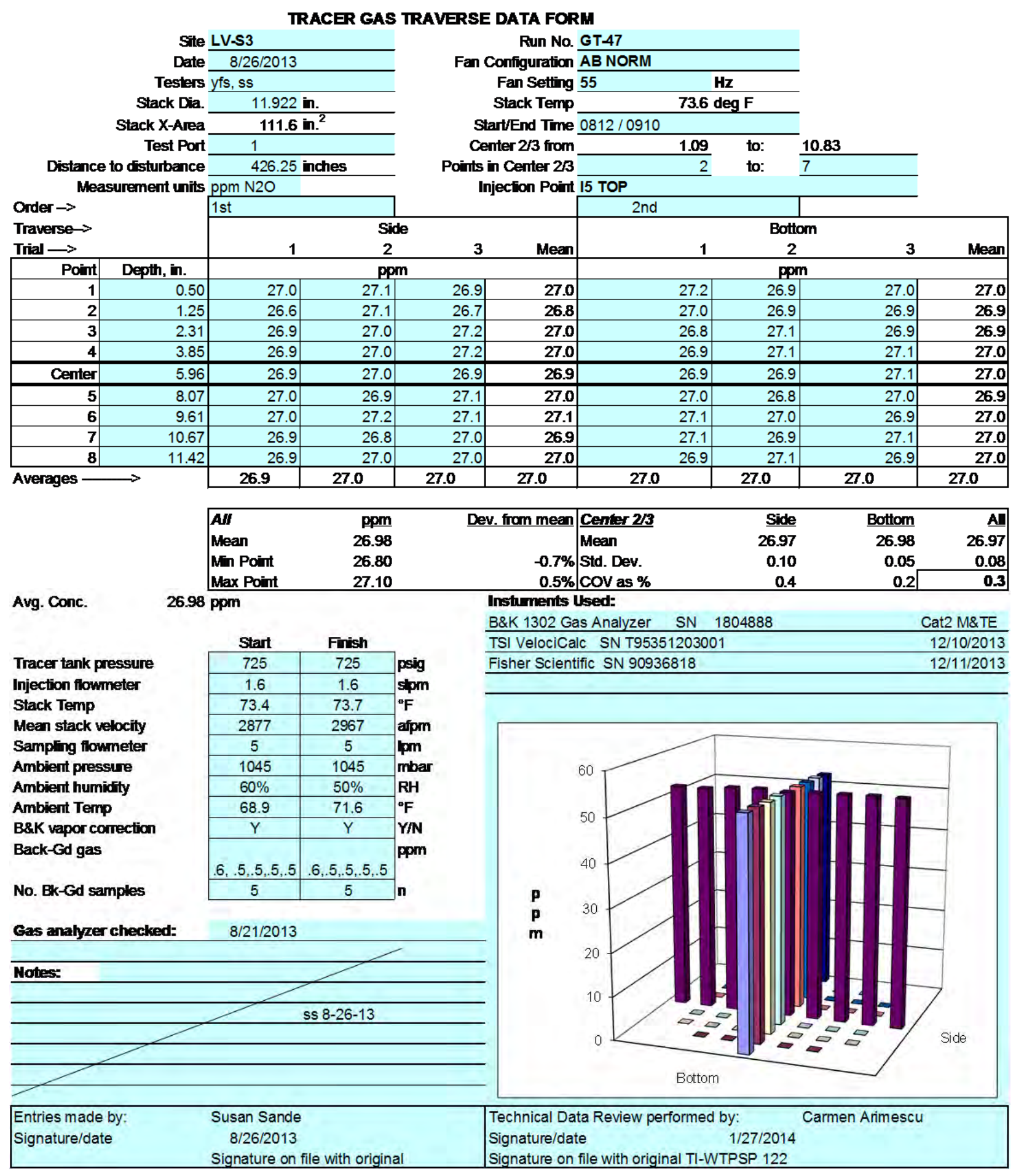




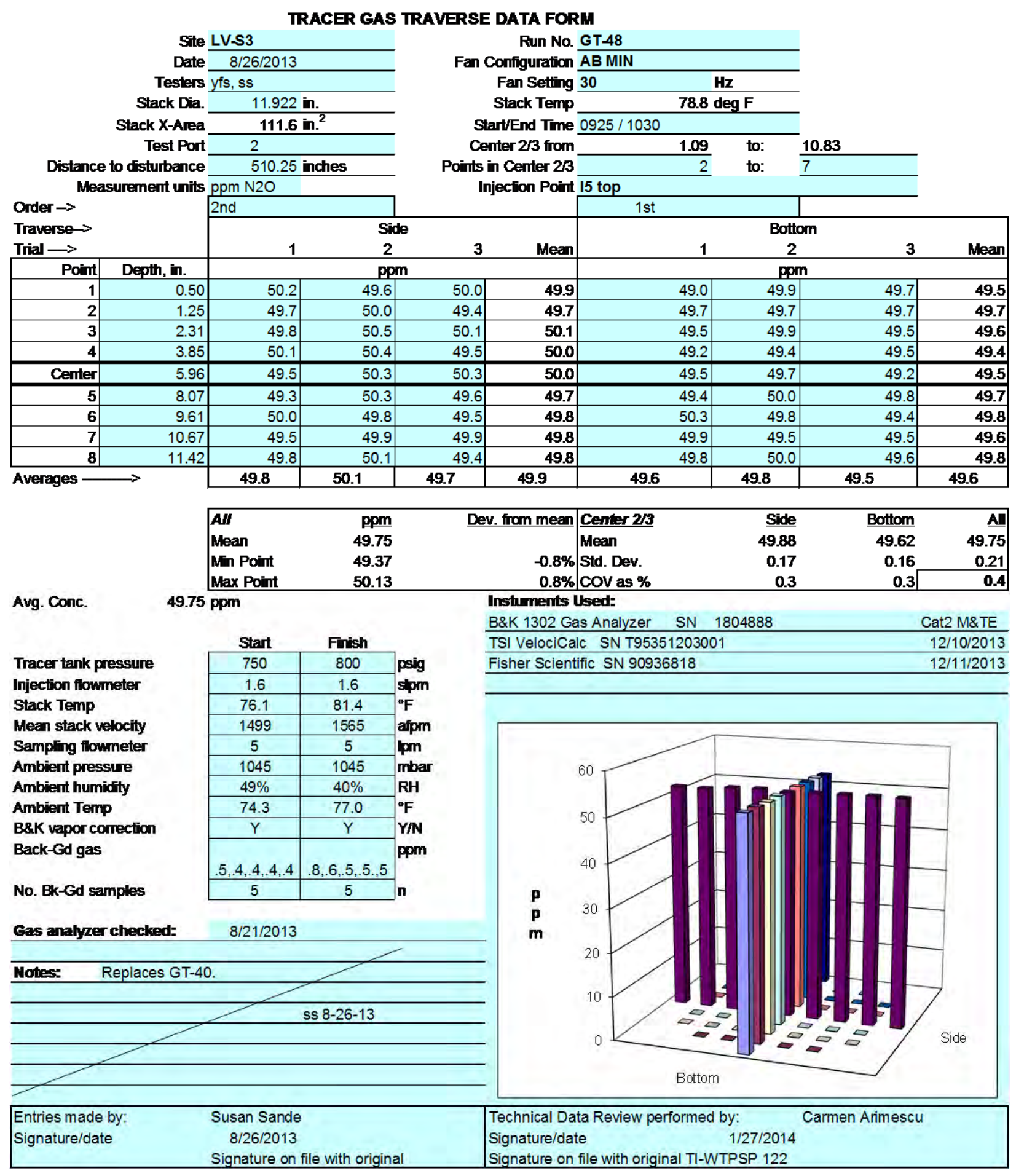




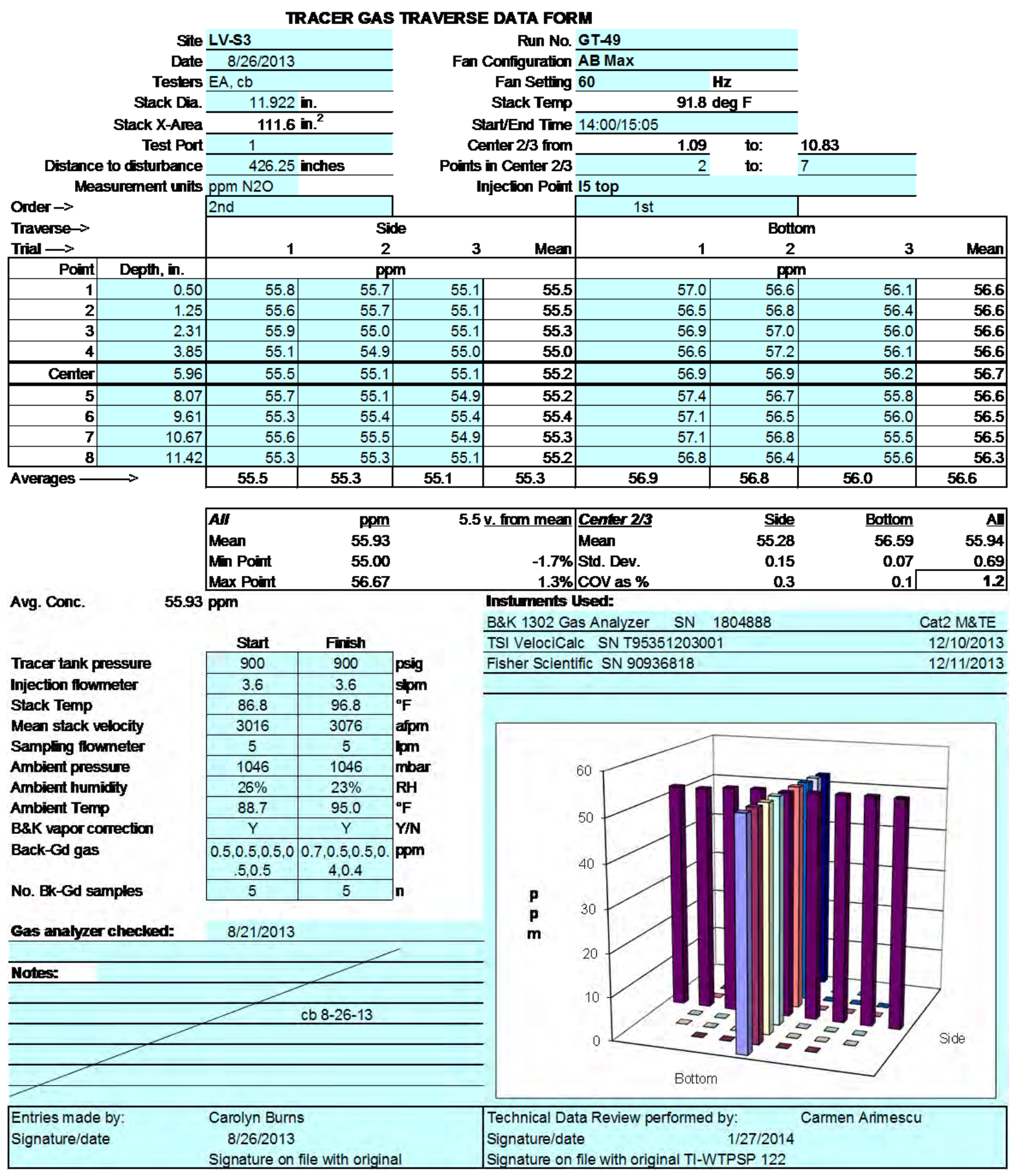




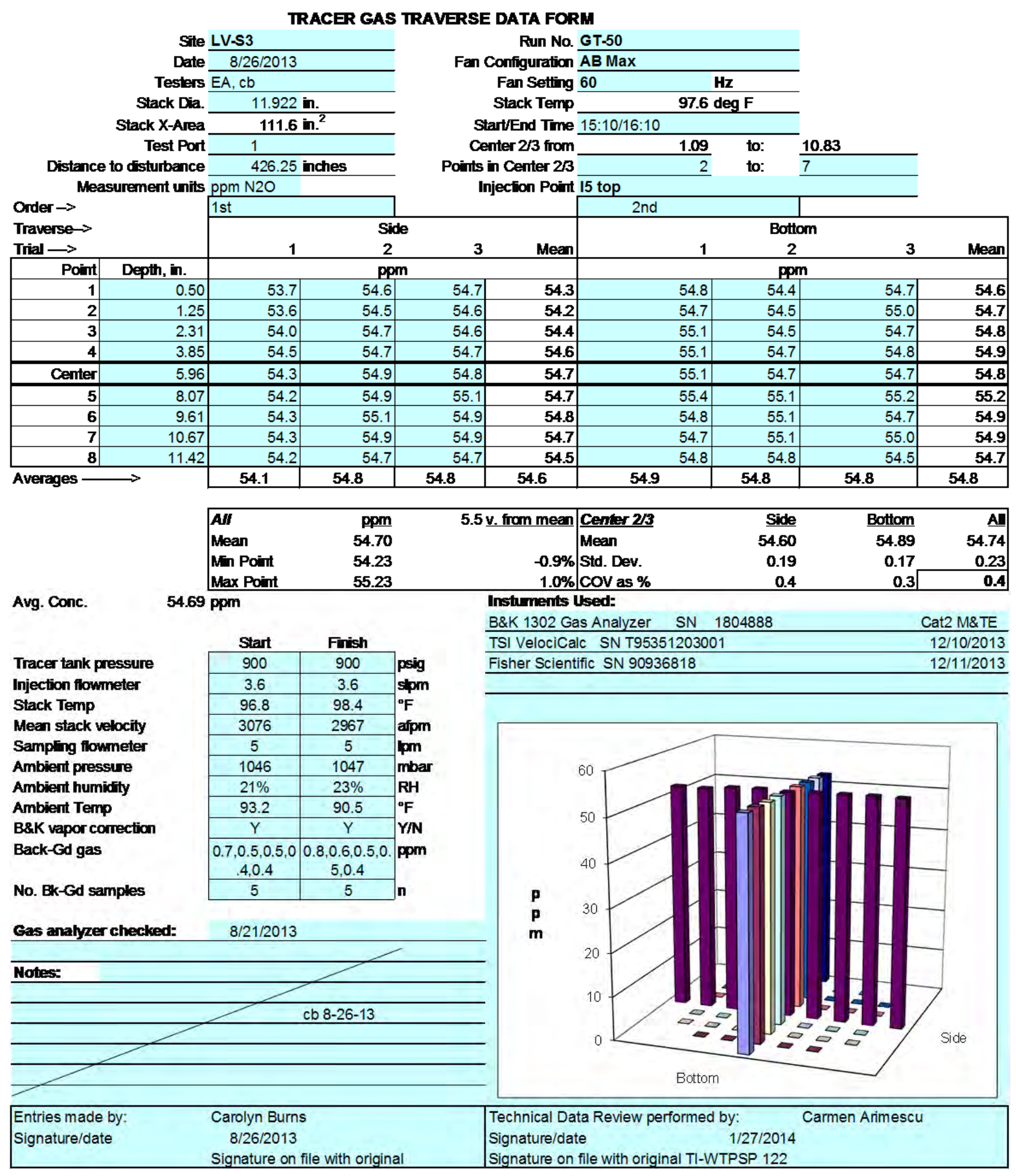




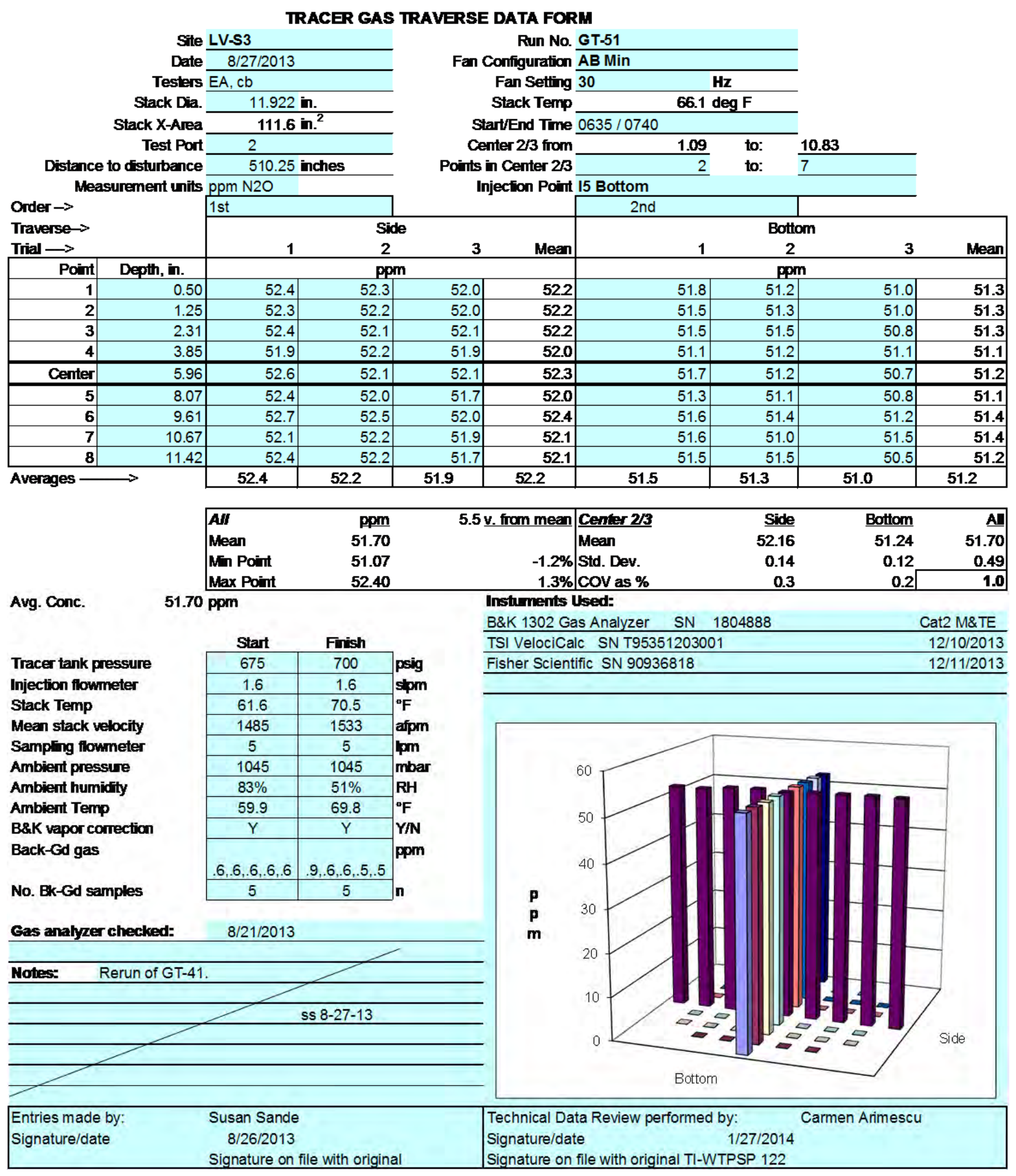




\section{SULFUR HEXAFLUORIDE AND NITROUS OXIDE GAS INSTRUMENT CALIBRATION}

$\begin{aligned} \text { Site } & \text { LV-S3 } \\ \text { Date/Time } & \\ \text { Testers } & \text { EA, SS, SFS }\end{aligned}$

Instrument B\&K Model 1302
Serial No. $\frac{1804888}{\text { WD54623 }}$
Property No.

\begin{tabular}{|c|c|c|}
\hline Setup: & B\&K samp & le inlet tube length \\
\hline & 1012 mbar station pressure & \\
\hline & 70.7 deg F ambient temp & analyzer corrects to $20 \mathrm{deg} C$ \\
\hline & 38 percent $\mathrm{RH}$ ambient humic & \\
\hline & Pre-Test background & compensating for water vapor \\
\hline & $625,456,435,514,486$ & \\
\hline
\end{tabular}

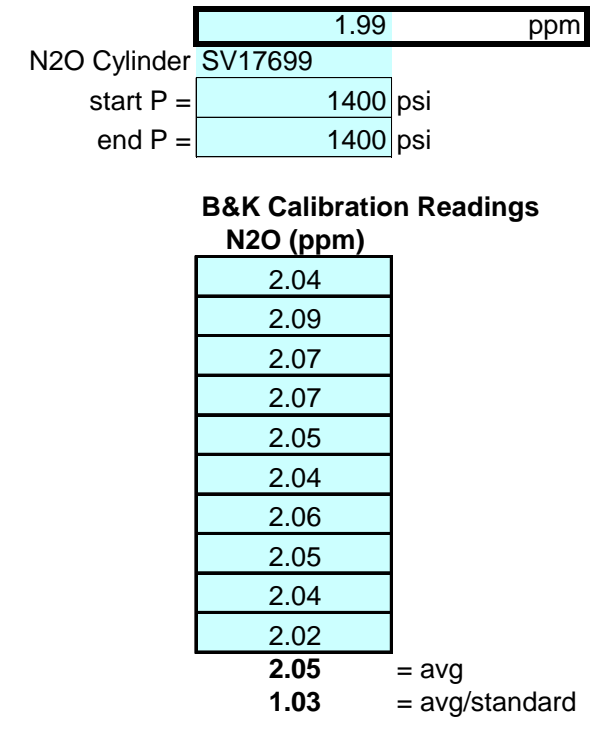

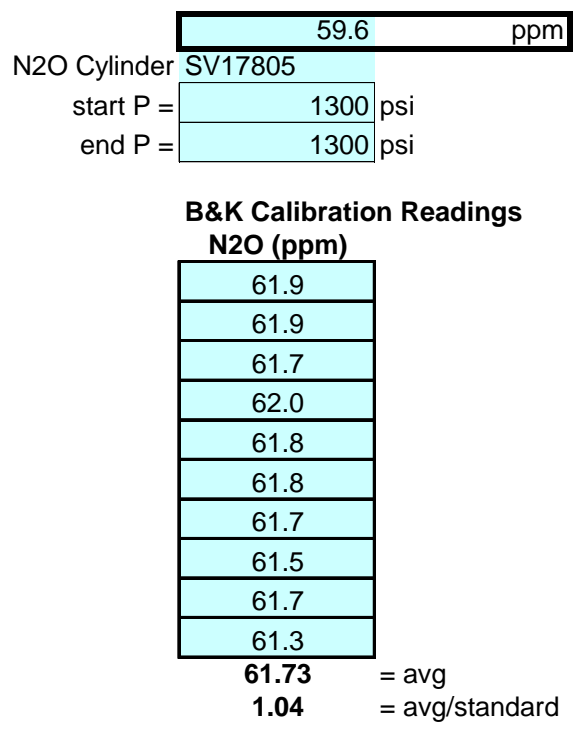

Standards Used:

Expiration date:

\begin{tabular}{llr}
\hline Air Liquide & 1.99 ppm N2O in air, SV17699 & $6 / 1 / 2014$ \\
\hline Air Liquide & 59.6 ppm N2O in air, SV17805 & $6 / 1 / 2014$ \\
\hline Weather Station Used: & $12 / 11 / 2013$
\end{tabular}

Fisher Scientific, S/N $90936818 \quad 12 / 11 / 2013$

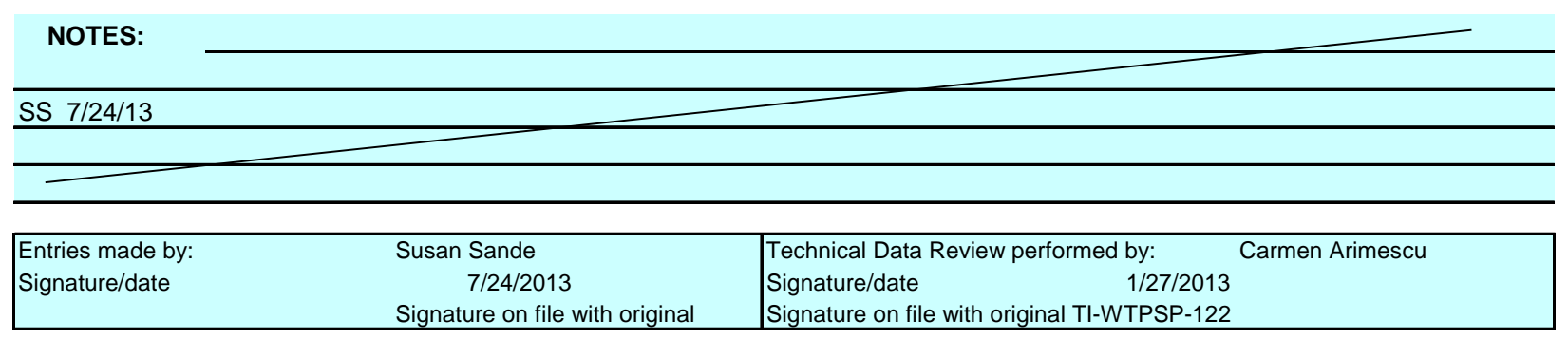




\section{SULFUR HEXAFLUORIDE AND NITROUS OXIDE GAS INSTRUMENT CALIBRATION}

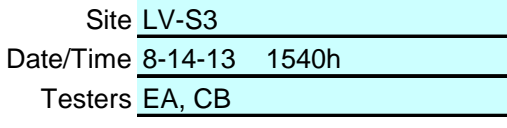

Instrument B\&K Model 1302
Serial No. $\frac{1804888}{\text { WD54623 }}$
Property No.

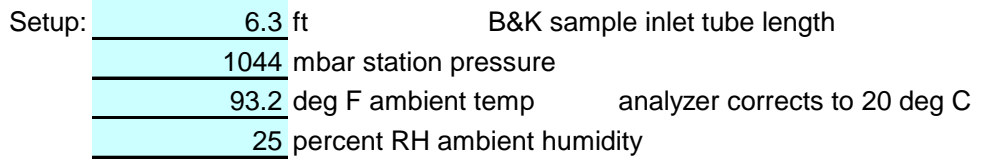

\begin{tabular}{|lll|}
\hline $\begin{array}{l}\text { Pre-Test background } \\
308,333,300,302,309,279\end{array}$ & ppb N2O & compensating for water vapor \\
\hline
\end{tabular}

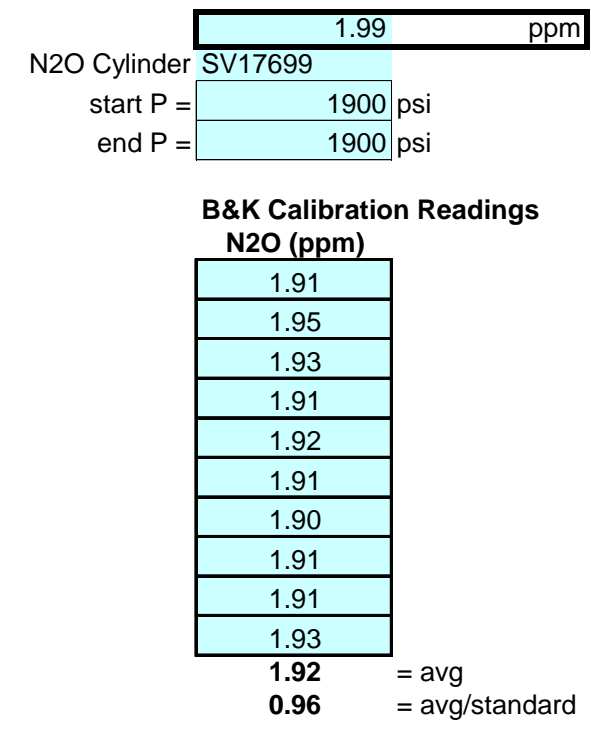

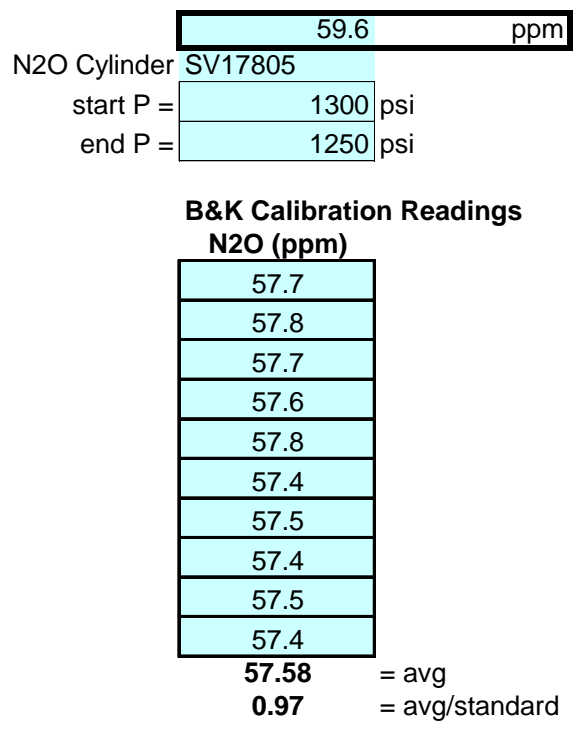

Standards Used:

Expiration date:

\begin{tabular}{llc}
\hline Air Liquide & 1.99 ppm N2O in air, SV17699 & $6 / 1 / 2014$ \\
\hline Air Liquide & 59.6 ppm N2O in air, SV17805 & $6 / 1 / 2014$ \\
\hline Weather Station Used: & $12 / 11 / 2013$
\end{tabular}

Fisher Scientific, S/N 90936818

$12 / 11 / 2013$

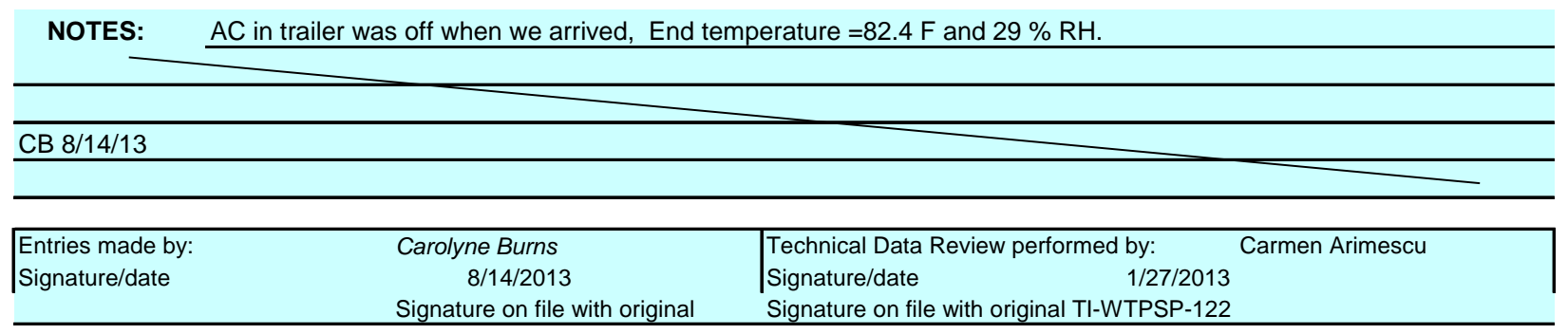




\section{SULFUR HEXAFLUORIDE AND NITROUS OXIDE GAS INSTRUMENT CALIBRATION}

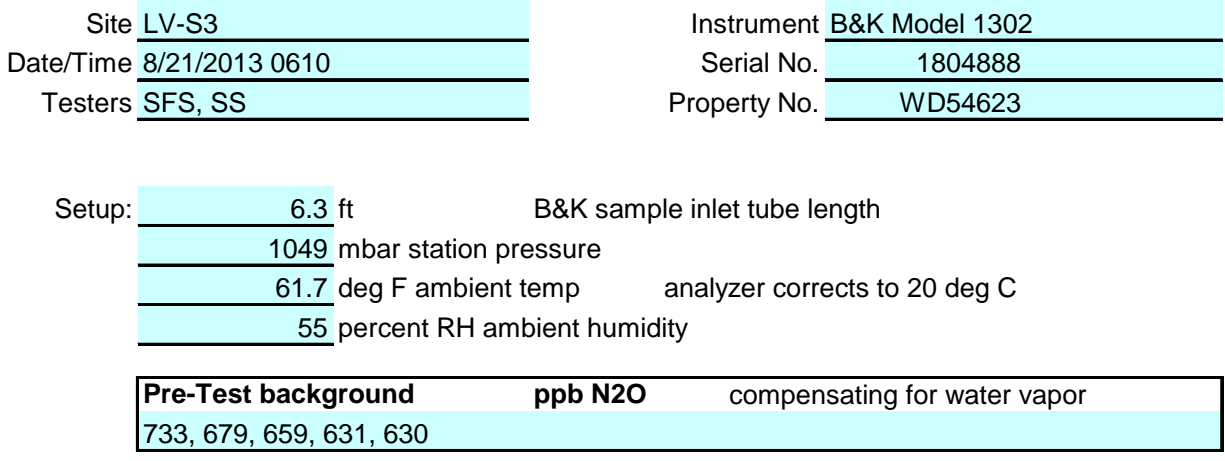

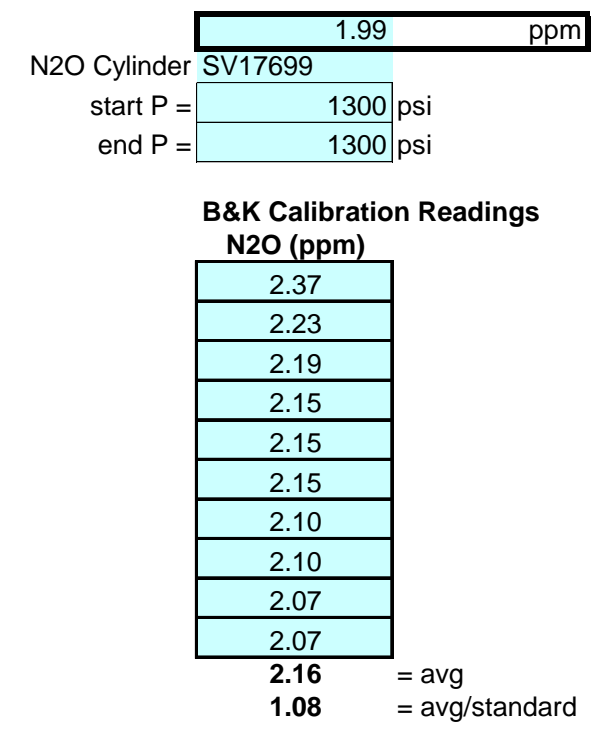

\begin{tabular}{|c|c|c|}
\hline & $\overline{59.6}$ & $\overline{\mathrm{ppm}}$ \\
\hline N2O Cylinder & $\begin{array}{l}\text { SV17805 } \\
\text { SV17 }\end{array}$ & \\
\hline start $P=$ & 1150 & psi \\
\hline end $P=$ & 1150 & psi \\
\hline
\end{tabular}

B\&K Calibration Readings N2O (ppm)

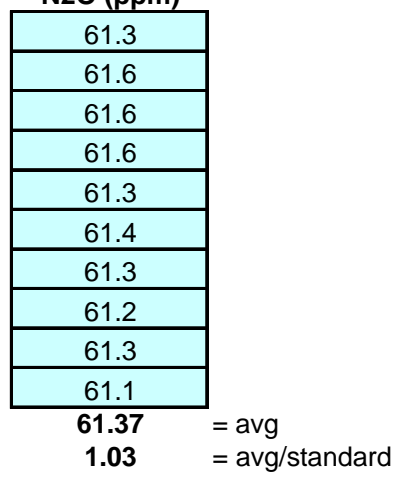

Standards Used:

Air Liquide 1.99 ppm N2O in air, SV17699

Air Liquide 59.6 ppm N2O in air, SV17805

Weather Station Used:

Fisher Scientific, S/N 90936818

$6 / 1 / 2014$

$6 / 1 / 2014$

\begin{tabular}{|l|l|}
\hline NOTES: & \\
\hline SS 8/21/13 & \multicolumn{3}{|c|}{ Carmen Arimescu } \\
\hline \multicolumn{3}{|l|}{ Entries made by: } & $\begin{array}{l}\text { Technical Data Review performed by: } 1 / 27 / 2013 \\
\text { Signature/date }\end{array}$ & $\begin{array}{l}\text { Signature/date } \\
\text { Signature on file with original TI-WTPSP-122 }\end{array}$ \\
\hline
\end{tabular}


Rev. 0

3 Aug. 2006

\section{D.5 LV-S3 Particle Tracer Uniformity Data Sheets}

\section{PARTICLE TRACER TRAVERSE DATA FORM}

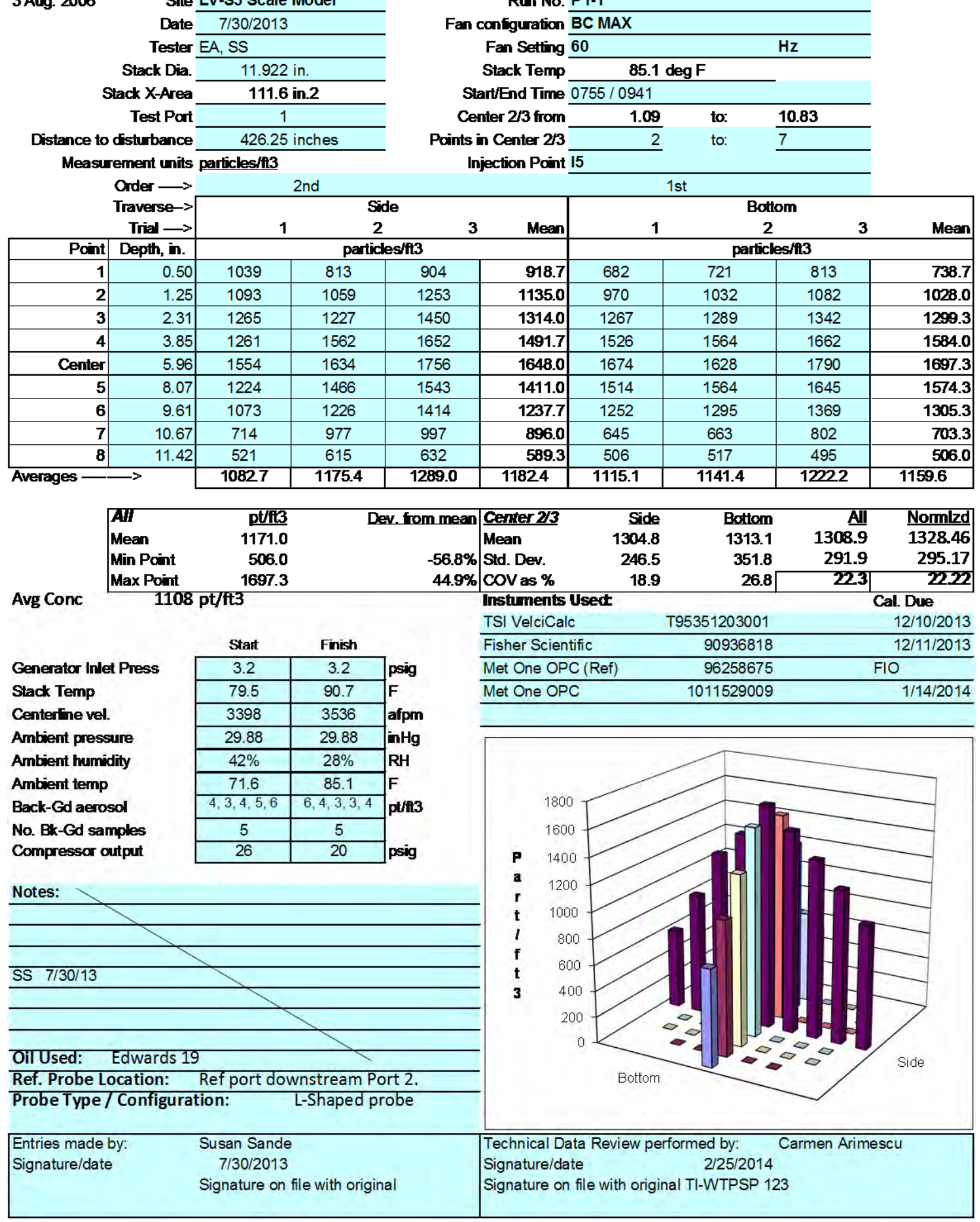


Rev. 0

3 Aug. 2006

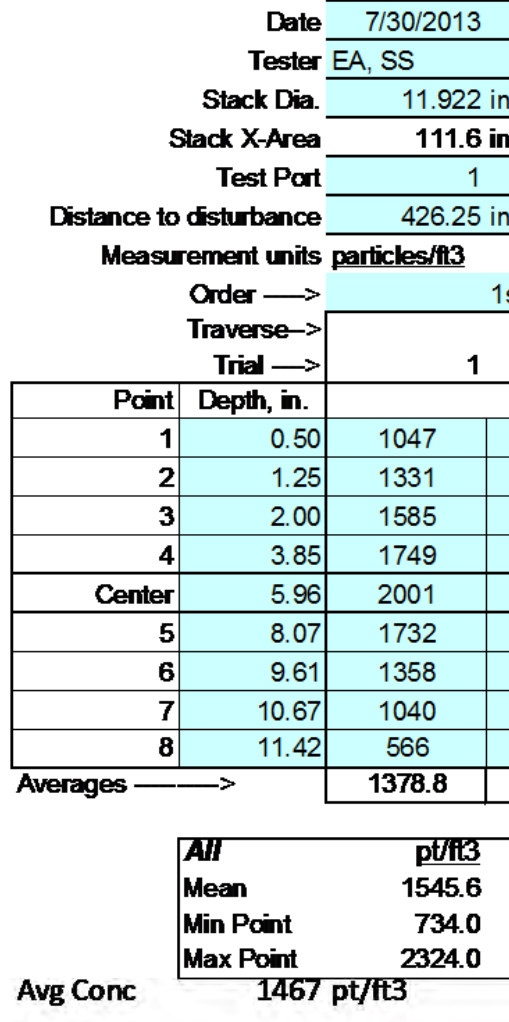

Generator Inlet Press

Stack Temp

Centertine vel.

Ambient pressure

Ambient humidity

Ambient temp

Back-Gd aerosol

No. Bk-Gd samples

Compressor output

\begin{tabular}{|c|c|}
\hline Stat & Finish \\
\hline 3.2 & 3.2 \\
\hline 90.7 & 102.8 \\
\hline 3536 & 3413 \\
\hline 29.88 & 29.94 \\
\hline $28 \%$ & $20 \%$ \\
\hline 85.1 & 92.3 \\
\hline $811,13,7,3$ & $2,9,3,5,2$ \\
\hline 5 & 5 \\
\hline 42 & 20 \\
\hline
\end{tabular}

Notes: Distractions at QAVEPAD, redid side 3 because they seemedlow.

\begin{tabular}{|c|}
\hline SS $7 / 30 / 13$ \\
\hline \multirow{2}{*}{+2} \\
\hline \\
\hline \multirow{2}{*}{ OाTsed: Edwards 19} \\
\hline \\
\hline Ref. Probe Location: $\quad$ Ref port downstream Port 2. \\
\hline Probe Type/Configuration: L-Shaped probe \\
\hline Entries made by: \\
\hline Signature/date \\
\hline Signature on file with original \\
\hline
\end{tabular}

1485.1

1602.0

Fan configuration BC MAX

Fan Setting 60

Stack Temp

$96.75 \operatorname{deg} \mathrm{F}$

Start/End Time $0955 / 1200$

Center $2 / 3$ from

Points in Center $2 / 3$

Injection Point 15

2nd

\begin{tabular}{lll}
\hline 1.09 & to: & 10.83 \\
\hline 15 & & to:
\end{tabular}

$\mathrm{Hz}$

(1)

\begin{tabular}{|r|c|c|c|r|}
\hline \multirow{2}{*}{ Meen } & \multicolumn{4}{|c|}{$\begin{array}{c}\text { Bottom } \\
\mathbf{2}\end{array}$} \\
\hline & \multicolumn{4}{|c|}{ perticles/fl3 } \\
\hline $\mathbf{1 0 9 9 . 0}$ & 1232 & 1159 & 1170 & $\mathbf{1 1 8 7 . 0}$ \\
\hline $\mathbf{1 3 9 6 . 3}$ & 1497 & 1439 & 1425 & $\mathbf{1 4 5 3 . 7}$ \\
\hline $\mathbf{1 6 3 4 . 3}$ & 1837 & 1770 & 1812 & $\mathbf{1 8 0 6 . 3}$ \\
\hline $\mathbf{1 9 2 0 . 3}$ & 2088 & 2105 & 2109 & $\mathbf{2 1 0 0 . 7}$ \\
\hline $\mathbf{2 0 3 1 . 3}$ & 2379 & 2355 & 2238 & $\mathbf{2 3 2 4 . 0}$ \\
\hline $\mathbf{1 8 4 5 . 7}$ & 2159 & 2091 & 2123 & $\mathbf{2 1 2 4 . 3}$ \\
\hline $\mathbf{1 4 8 5 . 0}$ & 1764 & 1731 & 1692 & $\mathbf{1 7 2 9 . 0}$ \\
\hline $\mathbf{1 1 7 4 . 3}$ & 924 & 977 & 989 & $\mathbf{9 6 3 . 3}$ \\
\hline $\mathbf{8 1 1 . 3}$ & 692 & 721 & 789 & $\mathbf{7 3 4 . 0}$ \\
\hline $\mathbf{1 4 8 8 . 6}$ & $\mathbf{1 6 1 9 . 1}$ & $\mathbf{1 5 9 4 . 2}$ & $\mathbf{1 5 9 4 . 1}$ & $\mathbf{1 6 0 2 . 5}$ \\
\hline
\end{tabular}

Center 2/3

Side

\begin{tabular}{rrr|}
\hline Bottom & All & Normlzd \\
\hline 1785.9 & 1713.5 & 1831.69 \\
464.4 & 386.5 & 399.51 \\
26.0 & 22.6 & 21.81 \\
\hline
\end{tabular}

\begin{tabular}{l|lr}
$-525 \%$ & Std. Dev. & 309.4 \\
$50.4 \%$ & COV as \% & 18.9
\end{tabular}

Instuments Used

TSI VelciCalc

Fisher Scientific

Met One OPC (Ref)

Met One OPC

\begin{tabular}{ll}
1011529009 & $1 / 14 / 2014$ \\
\hline
\end{tabular}

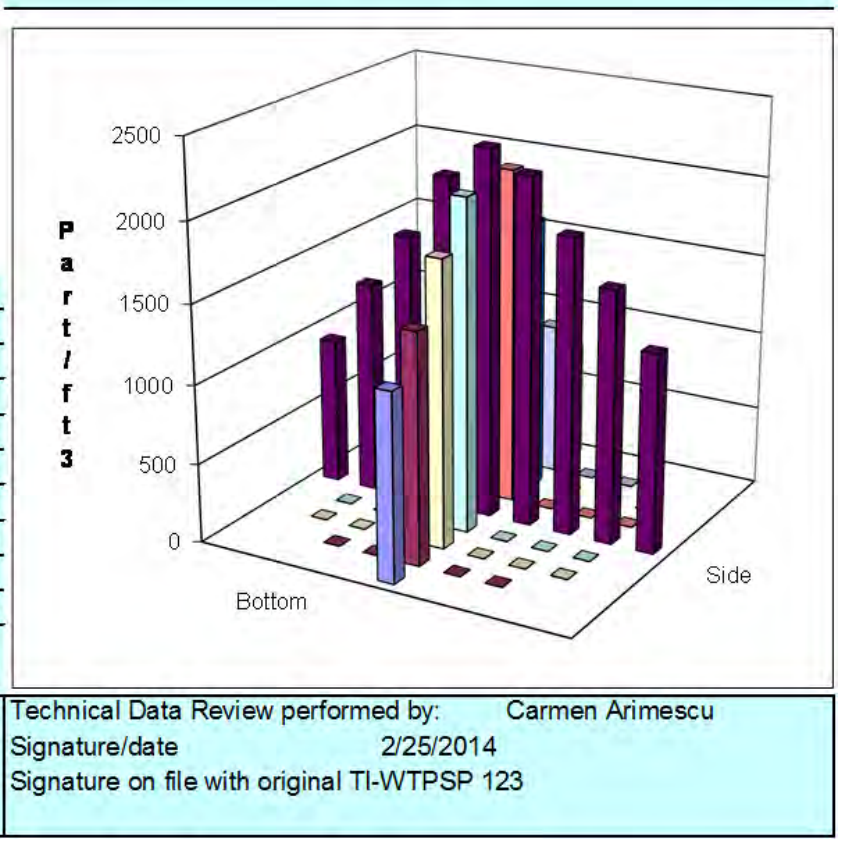


Rev. 0

3 Aug. 2006

\begin{tabular}{|c|c|c|}
\hline & Date & $7 / 30 / 2013$ \\
\hline \multicolumn{3}{|c|}{ Tester $\mathrm{cb}, \mathrm{TH}$} \\
\hline \multirow{2}{*}{\multicolumn{2}{|c|}{$\begin{array}{r}\text { Stack Dia. } \\
\text { Stack X-Area }\end{array}$}} & 11.922 in \\
\hline & & 111.6 in \\
\hline \multicolumn{2}{|r|}{ Test Port } & 1 \\
\hline \multicolumn{2}{|c|}{ Distance to disturbance } & 426.25 in \\
\hline \multicolumn{3}{|c|}{ Measurement units particles/t13 } \\
\hline \multirow{3}{*}{\multicolumn{2}{|c|}{$\begin{array}{c}\text { Order } \longrightarrow \\
\text { Traverse } \longrightarrow \\
\quad \text { Trial } \longrightarrow\end{array}$}} & \multirow[t]{3}{*}{ 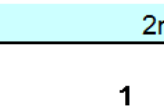 } \\
\hline & & \\
\hline & & \\
\hline \multicolumn{2}{|c|}{\begin{tabular}{l|l} 
Pcint & Depth, in. \\
\end{tabular}} & \\
\hline 1 & 0.50 & 712 \\
\hline 2 & 1.25 & 610 \\
\hline 3 & 2.00 & 945 \\
\hline 4 & 3.85 & 1246 \\
\hline Center & 5.96 & 1316 \\
\hline 5 & 8.07 & 1221 \\
\hline 6 & 9.61 & 902 \\
\hline 7 & 10.67 & 769 \\
\hline 8 & 11.42 & 526 \\
\hline \multicolumn{2}{|c|}{ Averages $\longrightarrow$} & 916.3 \\
\hline \multirow{4}{*}{\multicolumn{2}{|c|}{\begin{tabular}{|l}
$A I$ \\
Mean \\
Min Point \\
Max Point
\end{tabular}}} & $\mathrm{pt} / \mathrm{ti} 3$ \\
\hline & & 10826 \\
\hline & & 577.3 \\
\hline & & 1656.7 \\
\hline \multicolumn{3}{|l|}{ Avg Conc } \\
\hline
\end{tabular}

Generator Inlet Press

Stack Temp

Centerline vel bottom C

Ambient pressure

Ambient humidity

Ambient temp

Back-Gd aerosol

No. Bk-Gd samples

Compressar output

\begin{tabular}{|c|c|}
\hline Stat & Finish \\
\hline 2.5 & 2.5 \\
\hline 102.6 & 105.5 \\
\hline 4035 & 4002 \\
\hline 29.97 & 29.97 \\
\hline $20 \%$ & $18 \%$ \\
\hline 95.9 & 94.1 \\
\hline $7,4,3,4,1$ & $10,1,5,4,1$ \\
\hline 5 & 5 \\
\hline 16 & 24 \\
\hline
\end{tabular}

Notes:

At start 3643 afpm Bottom \# 7 port \#2

Velocity was a little high at $60 \mathrm{~Hz}$

cb $7 / 30 / 13$

start velocity 4035 / end 4002 at center

OाTsed: Edwards 19

Ref. Probe Location: Ref port downstream Port 2.

Probe Type/Configuration: L-Shaped probe

1093.0
Run No. PT-3

Fan configuration BC MAX

Fan Setting 60

Stack Temp

Start/End Time 13:50/15:36

Center $2 / 3$ from

Points in Center $2 / 3$

Injection Point 15
$04.05 \operatorname{deg} F$

1.09

2 to

to:

st

\begin{tabular}{|c|c|c|c|c|}
\hline \multirow{3}{*}{ Mean } & \multicolumn{4}{|c|}{ Bottom } \\
\hline & \multicolumn{2}{|c|}{1} & 2 & Mean \\
\hline & \multicolumn{4}{|c|}{ particles/f3 } \\
\hline 767.0 & 754 & 817 & 811 & 794.0 \\
\hline 781.0 & 1008 & 1004 & 966 & 992.7 \\
\hline 10120 & 1259 & 1239 & 1275 & 1257.7 \\
\hline 1266.0 & 1501 & 1548 & 1541 & 1530.0 \\
\hline 1325.3 & 1637 & 1684 & 1649 & $\begin{array}{l}1656.7 \\
\end{array}$ \\
\hline 12523 & 1594 & 1582 & 1497 & 1557.7 \\
\hline 1061.7 & 1328 & 1266 & 1274 & 1289.3 \\
\hline 885.0 & 806 & 747 & 734 & 762.3 \\
\hline 719.3 & 596 & 553 & 583 & 577.3 \\
\hline 1007.7 & 1164.8 & 1160.0 & 1147.8 & 1157.5 \\
\hline
\end{tabular}

$\mathrm{Hz}$

$\frac{10}{7}$


Rev. 0

3 Aug. 2006

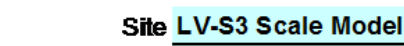

Date 7/31/2013

Tester EA, SS, SFS

Stack Dia. 11.922 in.

Stack X-Area 111.6 in.2

Test Port

Distance to disturbance

Measurement units particles/fi3

Order $\longrightarrow$

Traverse-

Trial $\longrightarrow$

\begin{tabular}{|r|r|}
\hline Point & Depth, in. \\
\hline $\mathbf{1}$ & 0.50 \\
\hline $\mathbf{2}$ & 1.2 \\
\hline $\mathbf{3}$ & 2.00 \\
\hline $\mathbf{4}$ & 3.8 \\
\hline Center & 5.9 \\
\hline $\mathbf{5}$ & 8.07 \\
\hline $\mathbf{6}$ & 9.61 \\
\hline $\mathbf{7}$ & 10.67 \\
\hline $\mathbf{8}$ & 11.4 \\
\hline
\end{tabular}

Averages

PARTCLE TRACER TRAVERSE DATA FORY

Run No. PT-4

Fan configuration BC NORM

Fan Setting 55

Stack Temp

Start/End Time $0600 / 0755$

Center $2 / 3$ from

Points in Center $2 / 3$ Injection Point 15

$72.05 \operatorname{deg} F$

1.09 to: 10.83

426.25 inches

2nd

Side

2

to:

1st
$3 \quad 1$

Bottom

\begin{tabular}{|c|c|c|r|r|c|c|r|}
\hline \multicolumn{4}{c}{$\mathbf{2}$} & $\mathbf{3}$ & Mean & \multicolumn{3}{c}{$\mathbf{1}$} & \multicolumn{2}{c|}{ particles/f13 } & Mean \\
\hline 513 & 673 & 765 & $\mathbf{6 5 0 . 3}$ & 643 & 586 & 598 & $\mathbf{6 0 9 . 0}$ \\
\hline 698 & 986 & 928 & $\mathbf{8 7 0 . 7}$ & 777 & 809 & 817 & $\mathbf{8 0 1 . 0}$ \\
\hline 1052 & 1186 & 1100 & $\mathbf{1 1 1 2 . 7}$ & 971 & 1010 & 1035 & $\mathbf{1 0 0 5 . 3}$ \\
\hline 1249 & 1278 & 1282 & $\mathbf{1 2 6 9 . 7}$ & 1152 & 1213 & 1322 & $\mathbf{1 2 2 9 . 0}$ \\
\hline 1361 & 1330 & 1388 & $\mathbf{1 3 5 9 . 7}$ & 1230 & 1375 & 1452 & $\mathbf{1 3 5 2 . 3}$ \\
\hline 1186 & 1218 & 1295 & $\mathbf{1 2 3 3 . 0}$ & 1302 & 1197 & 1296 & $\mathbf{1 2 6 5 . 0}$ \\
\hline 977 & 1060 & 1069 & $\mathbf{1 0 3 5 . 3}$ & 1056 & 981 & 1084 & $\mathbf{1 0 4 0 . 3}$ \\
\hline 670 & 835 & 855 & $\mathbf{7 8 6 . 7}$ & 662 & 579 & 719 & $\mathbf{6 5 3 . 3}$ \\
\hline 499 & 566 & 469 & $\mathbf{5 1 1 . 3}$ & 482 & 447 & 542 & $\mathbf{4 9 0 . 3}$ \\
\hline $\mathbf{9 1 1 . 7}$ & $\mathbf{1 0 1 4 . 7}$ & $\mathbf{1 0 1 6 . 8}$ & $\mathbf{9 8 1 . 0}$ & $\mathbf{9 1 9 . 4}$ & $\mathbf{9 1 0 . 8}$ & $\mathbf{9 8 5 . 0}$ & $\mathbf{9 3 8 . 4}$ \\
\hline
\end{tabular}

\begin{tabular}{|lrr|lrrrr|}
\hline All & pt/ft3 & Dev. from mean & Center 2/3 & Side & Bottom & Al & Normkd \\
Mean & 959.7 & & Mean & 1095.4 & 1049.5 & 1072.4 & 1075.27 \\
Min Point & 490.3 & $-48.9 \%$ & Std. Dev. & 211.7 & 255.3 & 226.6 & 227.00 \\
Max Point & 1359.7 & $41.7 \%$ & Cov as \% & 19.3 & 24.3 & 21.1 & 21.11 \\
\hline
\end{tabular}

Avg Conc $910 \mathrm{pt} / \mathrm{ft} 3$

Generator Inlet Press Stack Temp

Centerline vel. center

Ambient pressure

Ambient humidity

Ambient temp

Back-Gd aerosol

No. Bk-Gd samples

Compressor output

\begin{tabular}{|c|c|}
\hline Start & Finish \\
\hline 3.0 & 3.0 \\
\hline 66.9 & 77.2 \\
\hline 3150 & 3116 \\
\hline 29.97 & 29.97 \\
\hline $50 \%$ & $42 \%$ \\
\hline 65.3 & 73.4 \\
\hline $1,2,0,2,4$ & $1,0,1,1,0$ \\
\hline 5 & 5 \\
\hline 30 & 36 \\
\hline
\end{tabular}

Notes:

\begin{tabular}{|c|c|}
\hline & 2 \\
\hline \multicolumn{2}{|l|}{ S5 $7 / 31 / 2013$} \\
\hline \multicolumn{2}{|l|}{ 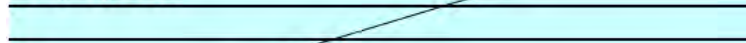 } \\
\hline \\
\hline 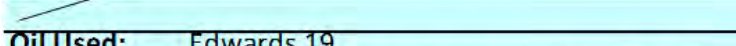 & \\
\hline \multicolumn{2}{|c|}{ Ref. Probe Location: Ref port downstream Port 2.} \\
\hline \multicolumn{2}{|l|}{ Probe Type/Configuration: } \\
\hline Entries made by: & Susan Sande \\
\hline \multirow[t]{2}{*}{ Signature/date } & $7 / 31 / 2013$ \\
\hline & Signature on file with original \\
\hline
\end{tabular}

\begin{tabular}{lrc}
\hline Instuments Used: & & \multicolumn{1}{c}{ Cal. Due } \\
TSI VelciCalc & T95351203001 & $12 / 10 / 2013$ \\
\hline Fisher Scientific & 90936818 & $12 / 11 / 2013$ \\
\hline Met One OPC (Ref) & 96258675 & FIO \\
\hline Met One OPC & 1011529009 & $1 / 14 / 2014$ \\
\hline
\end{tabular}

Technical Data Review performed by: Carmen Arimescu


Rev. 0

3 Aug. 2006

\begin{tabular}{|c|c|}
\hline \multicolumn{2}{|c|}{ Site LV-S3 Scale Model } \\
\hline Date & $7 / 31 / 2013$ \\
\hline Tester & $A, S S, S F S$ \\
\hline Stack Dia. & 11.922 in. \\
\hline Stack X-Area & 111.6 in.2 \\
\hline Test Port & 1 \\
\hline to disturbance & 426.25 inches \\
\hline
\end{tabular}

Measurement units particles/f13

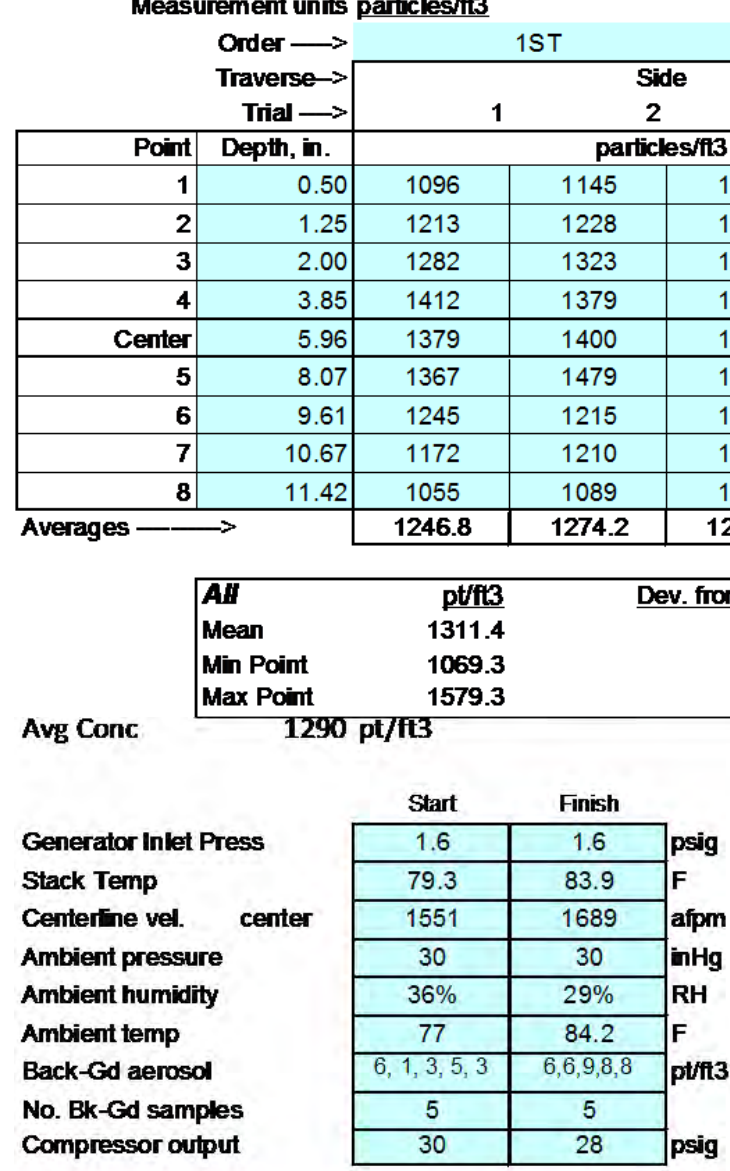

Notes:
Fan configuration BC MIN

$$
\text { Fan Setting } 30
$$

Stack Temp

Start/End Time 0802 / 0952

Center 2/3 from

Points in Center $2 / 3$ Injection Point 15

\begin{tabular}{lll}
\hline & & $\mathrm{Hz}$ \\
\hline $\mathbf{8 1 . 6} \operatorname{deg} \mathrm{F}$ & \\
\hline$\frac{1.09}{2}$ & to: & $\mathbf{1 0 . 8 3}$ \\
\hline & to: & \\
\hline
\end{tabular}

\begin{tabular}{|c|c|c|c|c|c|}
\hline \multicolumn{6}{|c|}{$2 \mathrm{ND}$} \\
\hline \multirow{2}{*}{3} & & \multicolumn{4}{|c|}{ Bottom } \\
\hline & \multirow[t]{2}{*}{ Mean } & \multicolumn{2}{|c|}{1} & 2 & \multirow[t]{2}{*}{ Mean } \\
\hline & & \multicolumn{3}{|c|}{ particles/ft3 } & \\
\hline & 1133.3 & 1150 & 1213 & 1256 & 1206.3 \\
\hline & 1222.3 & 1240 & 1291 & 1347 & 1292.7 \\
\hline & 1265.0 & 1360 & 1390 & 1407 & 1385.7 \\
\hline & 1389.7 & 1446 & 1434 & 1597 & 1492.3 \\
\hline & 1384.7 & 1588 & 1577 & 1573 & 1579.3 \\
\hline & 1403.3 & 1546 & 1461 & 1567 & 1524.7 \\
\hline & 1246.3 & 1494 & 1493 & 1458 & 1481.7 \\
\hline & 1200.0 & 1208 & 1204 & 1273 & 1228.3 \\
\hline & 1069.3 & 978 & 1127 & 1193 & 1099.3 \\
\hline & 1257.1 & 1334.4 & 1354.4 & 1407.9 & 1365.6 \\
\hline
\end{tabular}

\begin{tabular}{l|r}
1250.3 & 1257 \\
\hline
\end{tabular}

\begin{tabular}{r|lrrrr|}
\hline & Center 2/3 & Side & Bottom & Al & Normkd \\
\hline Mean & 1301.6 & 1426.4 & 1364.0 & 1455.50 \\
$20.4 \%$ & Std. Dev. & 87.6 & 128.6 & 123.9 & 114.67 \\
Cov as \% & 6.7 & 9.0 & 9.1 & 7.88 \\
\hline
\end{tabular}

\begin{tabular}{|c|c|c|}
\hline Instuments Used: & & Cal. Due \\
\hline TSI VelciCalc & T95351203001 & $12 / 10 / 2013$ \\
\hline Fisher Scientific & 90936818 & $12 / 11 / 2013$ \\
\hline Met One OPC (Ref) & 96258675 & FIO \\
\hline Met One OPC & 1011529009 & $1 / 14 / 2014$ \\
\hline
\end{tabular}

Technical Data Review performed by: Carmen Arimescu


Rev. 0

3 Aug. 2006

\begin{tabular}{|c|c|}
\hline \multicolumn{2}{|c|}{ Site LV-S3 Scale Model } \\
\hline Date & $7 / 31 / 2013$ \\
\hline Tester & A, SS, SFS \\
\hline Stack Dia. & 11.922 in. \\
\hline Stack X-Area & 111.6 in. 2 \\
\hline Test Port & 1 \\
\hline to disturbance & 426.25 inches \\
\hline
\end{tabular}

Measurement units particles/fi3

\begin{tabular}{|c|c|c|c|c|c|c|c|c|c|}
\hline Distance to & disturbance & 426.2 & ches & Point & Center $2 / 3$ & & to: & & \\
\hline Meast & rement units & articles/fi3 & & & ction Point & & & & \\
\hline & Order $\longrightarrow$ & & & & & & & & \\
\hline & Traverse $\rightarrow$ & & & & & & & & \\
\hline & Trial $\longrightarrow$ & & & & Mean & & & & Mean \\
\hline Point & Depth, in. & & part & & & & & & \\
\hline 1 & 0.50 & 1338 & 1175 & 1068 & 1193.7 & 1053 & 1125 & 1148 & 1108.7 \\
\hline 2 & 1.25 & 1408 & 1327 & 1309 & 1348.0 & 1220 & 1315 & 1360 & 1298.3 \\
\hline 3 & 2.00 & 1501 & 1403 & 1341 & 1415.0 & 1499 & 1475 & 1521 & 1498.3 \\
\hline 4 & 3.85 & 1600 & 1478 & 1554 & 1544.0 & 1536 & 1666 & 1801 & 1667.7 \\
\hline Center & 5.96 & 1726 & 1626 & 1665 & 1672.3 & 1675 & 1754 & 1916 & 1781.7 \\
\hline 5 & 8.07 & 1753 & 1577 & 1510 & 1613.3 & 1650 & 1786 & 1829 & 1755.0 \\
\hline 6 & 9.61 & 1458 & 1422 & 1286 & 1388.7 & 1496 & 1621 & 1557 & 1558.0 \\
\hline 7 & 10.67 & 1209 & 1192 & 1218 & 1206.3 & 1161 & 1250 & 1233 & 1214.7 \\
\hline 8 & 11.42 & 934 & 1141 & 982 & 1019.0 & 963 & 1058 & 1029 & 1016.7 \\
\hline Averages - & - & 1436.3 & 1371.2 & 1325.9 & 1377.8 & 1361.4 & 1450.0 & 1488.2 & 1433.2 \\
\hline
\end{tabular}

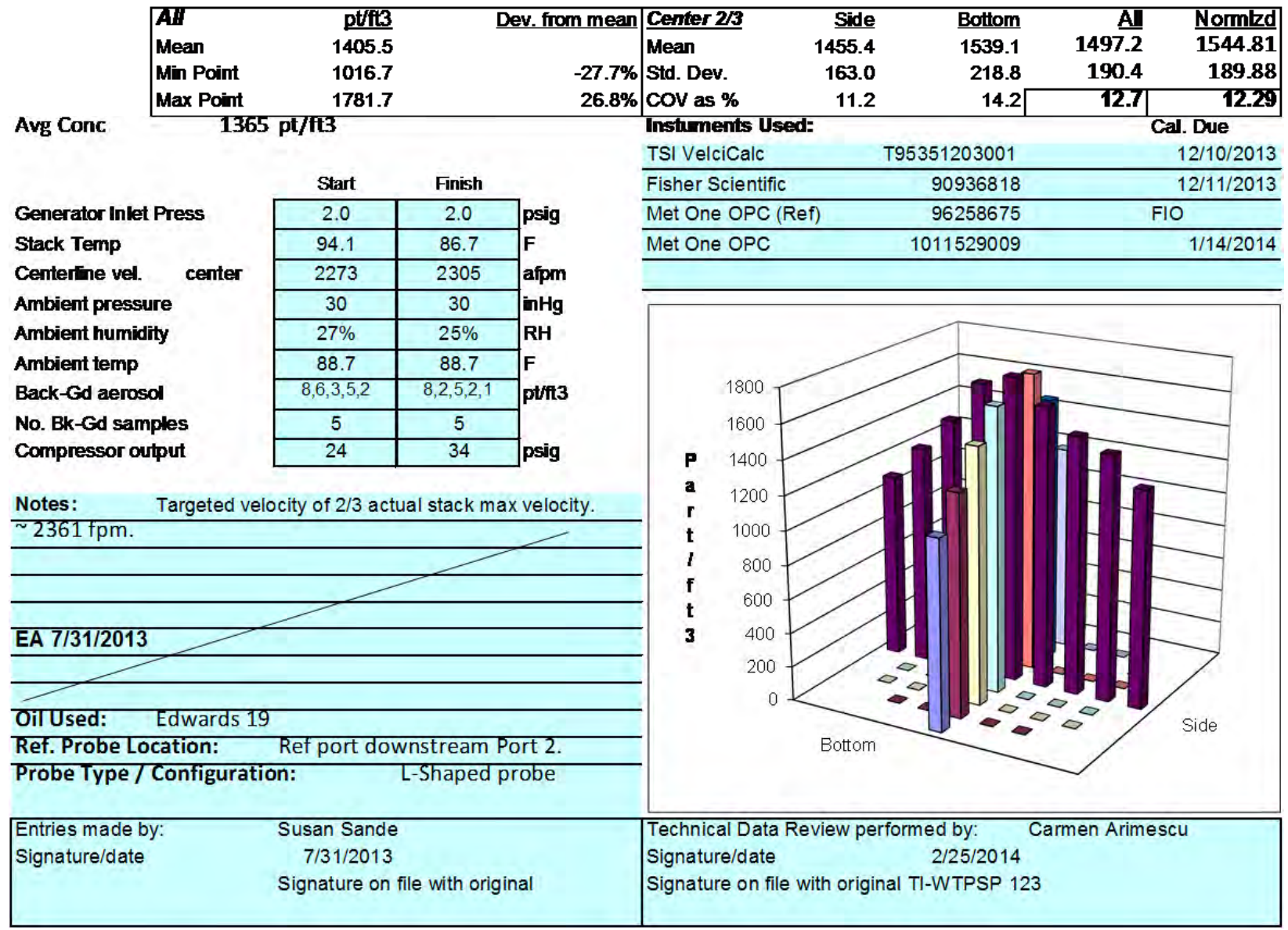

Run No. PT-6

Fan configuration $\mathrm{BC}$

Fan Setting 40

Stack Temp

Start/End Time $1050 / 122$

Center 2/3 from

Points in Center 2/3 1.09 to: 10.83 $0.4 \operatorname{deg} F$ $\mathrm{Hz}$ (n) Injection Point 15

$\begin{array}{ll}2 & \text { to: } \quad \frac{10 .}{7} \\ & \text { to: }\end{array}$

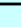
. (2)

\section{PARTICLE TRACER TRAVERSE DATA FORU}


Rev. 0

3 Aug. 2006

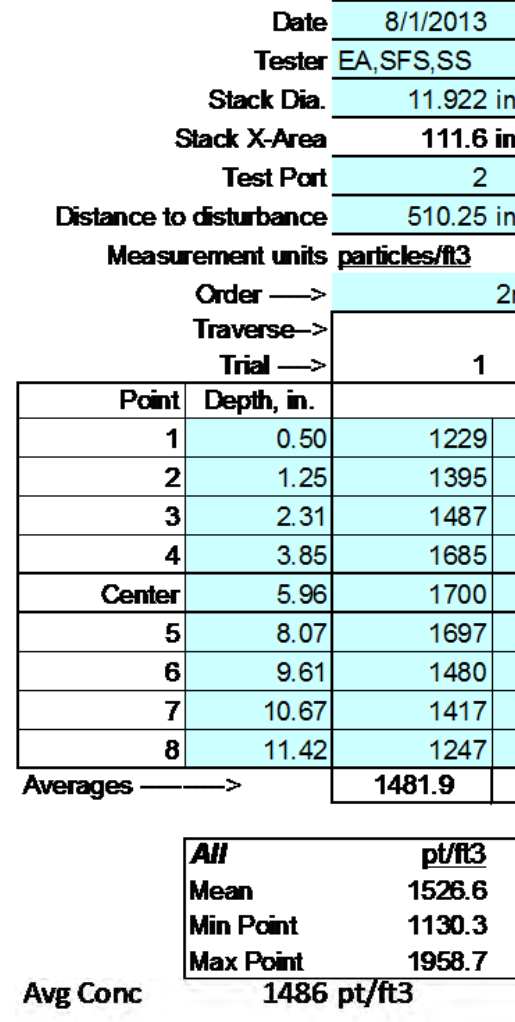

Generator Inlet Press Stack Temp

Centerline vel.

Ambient pressure

Ambient humidity

Ambient temp

Back-Gd aerosol

No. Bk-Gd samples

Compresscr output

\begin{tabular}{|c|c|}
\hline Stat & Finish \\
\hline 3.0 & 3.0 \\
\hline 65.6 & 67.4 \\
\hline 2103 & 2159 \\
\hline 29.88 & 29.91 \\
\hline $54 \%$ & $68 \%$ \\
\hline 67.1 & 65.3 \\
\hline $3,2,15,8,5$ & $6,2,2,4,2$ \\
\hline 5 & 5 \\
\hline 30 & 30 \\
\hline
\end{tabular}

Notes:

\begin{tabular}{l}
\hline Edwards 19 \\
\hline Oil Used: $\quad$ Ref port downstream Port 2. \\
\hline Probe Type /Configuration: $\quad$ L-Shaped probe
\end{tabular}

\begin{tabular}{|lc|l}
\hline $\begin{array}{l}\text { Entries made by: } \\
\text { Signature/date }\end{array}$ & Susan Sande & Tech \\
& $8 / 1 / 2013$ \\
& Signature on file with original & Sign \\
\end{tabular}

117

Dev. from

$-26.0 \%$

DATA

Fan configuration BC

Fan Setting 40

Stack Temp

$66.5 \operatorname{deg} F$

Start/End Time $0620 / 0800$

Center $2 / 3$ from

Points in Center $2 / 3$

Injection Point 15

\begin{tabular}{llll} 
& 1.09 & to: & 10.83 \\
\hline 15 & 2 & to: & \\
\hline
\end{tabular}

to:

Hz

(2)

\begin{tabular}{|r|r|r|r|}
\hline \multicolumn{4}{|c|}{ 1st } \\
\hline \multicolumn{4}{|c|}{$\begin{array}{c}\text { Bottom } \\
\mathbf{2}\end{array}$} \\
\hline 1146 & 1303 & 1166 & $\mathbf{1 2 0 5 . 0}$ \\
\hline 1372 & 1401 & 1333 & $\mathbf{1 3 6 8 . 7}$ \\
\hline 1521 & 1564 & 1527 & $\mathbf{1 5 3 7 . 3}$ \\
\hline 1848 & 1831 & 1973 & $\mathbf{1 8 8 4 . 0}$ \\
\hline 1854 & 1980 & 2042 & $\mathbf{1 9 5 8 . 7}$ \\
\hline 1881 & 1838 & 1852 & $\mathbf{1 8 5 7 . 0}$ \\
\hline 1622 & 1683 & 1653 & $\mathbf{1 6 5 2 . 7}$ \\
\hline 1469 & 1389 & 1437 & $\mathbf{1 4 3 1 . 7}$ \\
\hline 1339 & 1328 & 1303 & $\mathbf{1 3 2 3 . 3}$ \\
\hline $\mathbf{1 5 6 1 . 3}$ & $\mathbf{1 5 9 0 . 8}$ & $\mathbf{1 5 8 7 . 3}$ & $\mathbf{1 5 7 9 . 8}$ \\
\hline
\end{tabular}

$\begin{array}{r}\text { Side } \\ 1556.0 \\ 159.9 \\ 10.3 \\ \hline\end{array}$

\begin{tabular}{rrr|}
\hline Bottom & All & Normlzd \\
\hline 1670.0 & 1613.0 & 1710.41 \\
234.4 & 201.6 & 205.05 \\
\cline { 2 - 3 } 14.0 & 125 & 11.99 \\
\hline
\end{tabular}
Instuments Usect

\begin{tabular}{lrc} 
TSI VelciCalc & T95351203001 & $12 / 10 / 2013$ \\
\hline Fisher Scientific & 90936818 & $12 / 11 / 2013$ \\
\hline Met One OPC (Ref) & 96258675 & FIO \\
\hline Met One OPC & 1011529009 & $1 / 14 / 2014$ \\
\hline
\end{tabular}
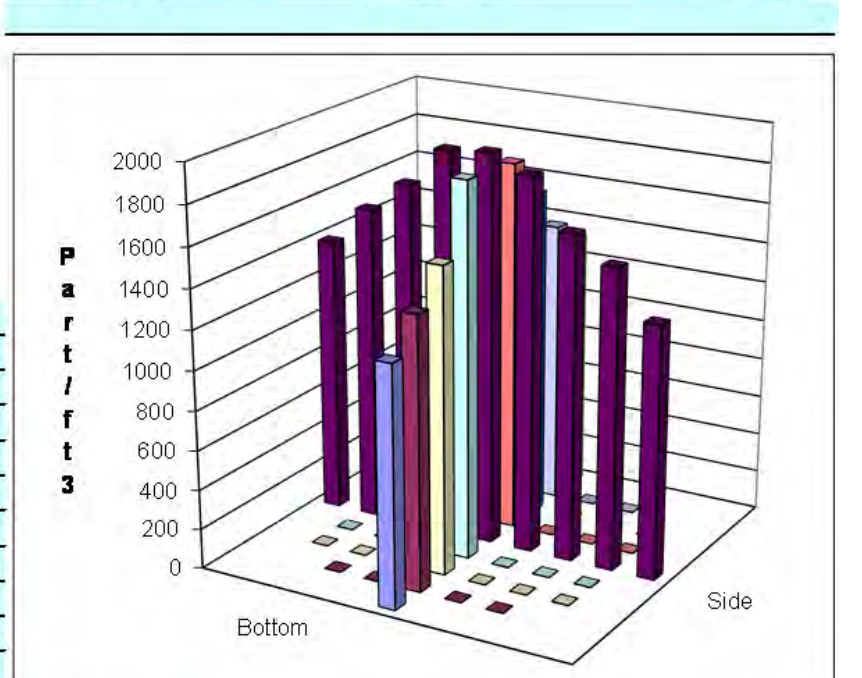

Technical Data Review performed by: Carmen Arimescu

Signature/date

2/25/2014

Signature on file with original TI-WTPSP 123 
Rev. 0

3 Aug. 2006

Site LV-S3 Scale Model

Date 8/1/2013

Tester EA,SFS,SS

Stack Dia. 11.922 in

Stack X-Aree

111.6 in. 2

Test Port

Distance to distumance 510.25 inches

Measurement units particles/ti3

Order $\longrightarrow$

Traverse->

Trial $\longrightarrow$

\begin{tabular}{|r|r|}
\hline Pcint & Depth, in. \\
\hline $\mathbf{1}$ & 0.50 \\
\hline $\mathbf{2}$ & 1.25 \\
$\mathbf{3}$ & 2.31 \\
\hline $\mathbf{4}$ & 3.85 \\
\hline Center & 5.96 \\
\hline $\mathbf{5}$ & 8.07 \\
\hline $\mathbf{6}$ & 9.61 \\
\hline $\mathbf{7}$ & 10.67 \\
\hline $\mathbf{8}$ & \multicolumn{2}{|c}{11.42} \\
\hline
\end{tabular}

1 st Side

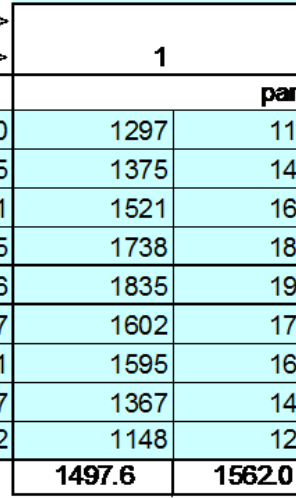

-

-

-

-

-

Side

$\frac{2}{\text { particles/fi3 }}$

\begin{tabular}{|l|l|l|}
\hline 186 & 1274 & \\
\hline 409 & 1373 & \\
\hline
\end{tabular}

\begin{tabular}{|l|l|}
1409 & 1373 \\
\hline 614 & 1502 \\
\hline
\end{tabular}

\begin{tabular}{l|l|}
1806 & 1752 \\
\hline
\end{tabular}

\begin{tabular}{l|l|}
\hline 90 & 1880 \\
\hline 1745 & 1920 \\
\hline
\end{tabular}

\begin{tabular}{|l|l|}
1650 & 1490 \\
\hline 1447
\end{tabular}

\begin{tabular}{|r|r|}
1447 & 1396 \\
\hline 1211 & 1196 \\
\hline
\end{tabular}

\begin{tabular}{|lr}
\hline All & $\mathrm{pt} / \mathrm{tt} 3$ \\
Mean & 15829 \\
Min Point & 1170.3 \\
Max Point & 2054.3 \\
\hline
\end{tabular}

Avg Conc $1534 \mathrm{pt} / \mathrm{tt} 3$

Generator Inlet Press

Stack Temp

Centerline vel.

Ambient pressure

Ambient humidity

Ambient temp

Back-Gd aerosol

No. Bk-Gd samples

Compressar output

\begin{tabular}{|c|c|c|}
\hline Stat & Finish & \\
\hline 3 & 3 & psig \\
\hline 67.4 & 72.1 & $\mathrm{~F}$ \\
\hline 2159 & 2300 & afpn \\
\hline 29.91 & 29.88 & $\mathrm{inHs}$ \\
\hline $66 \%$ & $51 \%$ & $\mathrm{RH}$ \\
\hline 66.2 & 70.7 & $\mathrm{~F}$ \\
\hline $0,1,3,2,1$ & $6,4,3,2,5$ & $\mathrm{t} / \mathrm{At}$ \\
\hline 5 & 5 & \\
\hline 31 & 30 & psig \\
\hline
\end{tabular}

Notes:

\begin{tabular}{l}
\hline \multicolumn{2}{|c|}{ SS 8/1/13 } \\
\hline \\
\hline
\end{tabular}

Dev. from mean
$-26.1 \%$
$29.8 \%$

Run No. PT-8

Fan configuration $\mathrm{BC}$

Fan Setting 40

Stack Temp

$69.75 \operatorname{deg} F$

Start/End Time $0804 / 0945$

Center $2 / 3$ from

1.09

Points in Center $2 / 3$

Injection Point 15

2nd

\begin{tabular}{|r|r|r|r|r|}
\cline { 2 - 5 } & \multicolumn{5}{|c|}{ 2nd } \\
\hline & \multicolumn{5}{|c|}{$\begin{array}{c}\text { Bottom } \\
\mathbf{2}\end{array}$} \\
\hline & $\mathbf{4}$ & $\mathbf{3}$ & Mean \\
\hline $\mathbf{1 2 5 2 3}$ & 1172 & 1157 & 1182 & $\mathbf{1 1 7 0 . 3}$ \\
\hline $\mathbf{1 3 8 5 . 7}$ & 1360 & 1471 & 1451 & $\mathbf{1 4 2 7 . 3}$ \\
\hline $\mathbf{1 5 4 5 . 7}$ & 1638 & 1662 & 1686 & $\mathbf{1 6 6 2 . 0}$ \\
\hline $\mathbf{1 7 6 5 . 3}$ & 1852 & 1885 & 1931 & $\mathbf{1 8 8 9 . 3}$ \\
\hline $\mathbf{1 9 0 1 . 7}$ & 2035 & 2064 & 2064 & $\mathbf{2 0 5 4 . 3}$ \\
\hline $\mathbf{1 7 5 5 . 7}$ & 1901 & 1914 & 1957 & $\mathbf{1 9 2 4 . 0}$ \\
\hline $\mathbf{1 5 7 8 . 3}$ & 1794 & 1710 & 1779 & $\mathbf{1 7 6 1 . 0}$ \\
\hline $\mathbf{1 4 0 3 . 3}$ & 1460 & 1587 & 1508 & $\mathbf{1 5 1 8 . 3}$ \\
\hline $\mathbf{1 1 8 5 . 0}$ & 1293 & 1329 & 1318 & $\mathbf{1 3 1 3 . 3}$ \\
\hline $\mathbf{1 5 3 0 . 3}$ & $\mathbf{1 6 1 1 . 7}$ & $\mathbf{1 6 4 2 1}$ & $\mathbf{1 6 5 2 9}$ & $\mathbf{1 6 3 5 . 6}$ \\
\hline
\end{tabular}

Side

Mean 1619.4

Bottom

1748.0

226.5

All Normlzd

Std. Dev. $\quad 194.9$

13.0

Instuments Usect

12.0

TSI VelciCalc

\begin{tabular}{lrc} 
TSI VelciCalc & T95351203001 & $12 / 10 / 2013$ \\
\hline Fisher Scientific & 90936818 & $12 / 11 / 2013$ \\
\hline Met One OPC (Ref) & 96258675 & FIO \\
\hline Met One OPC & 1011529009 & $1 / 14 / 2014$ \\
\hline
\end{tabular}

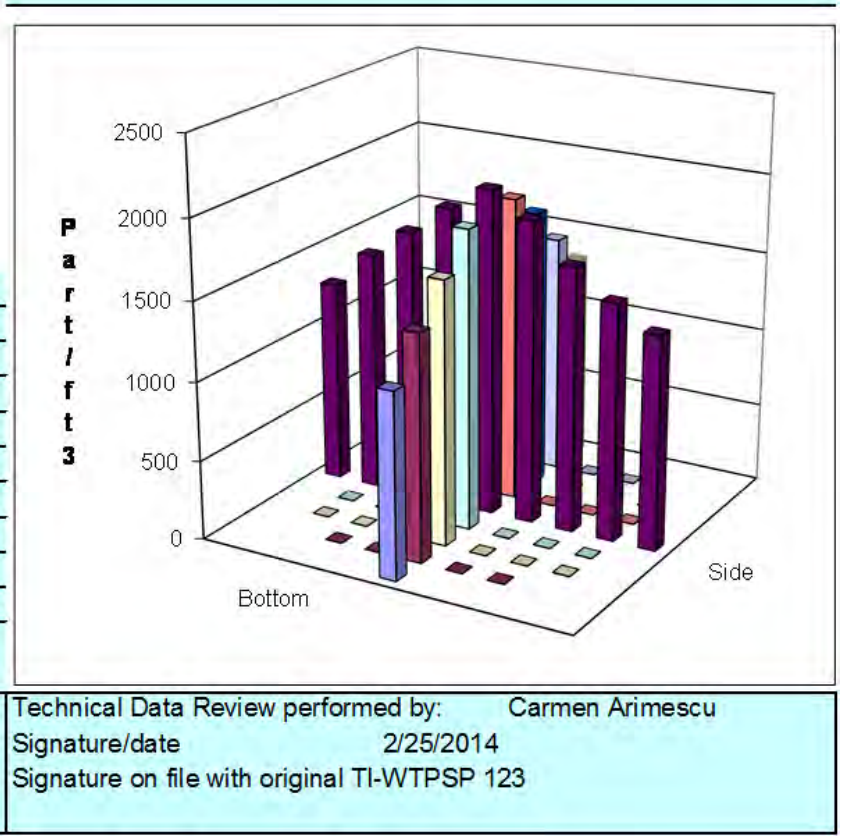

D.131 
Rev. 0

3 Aug. 2006

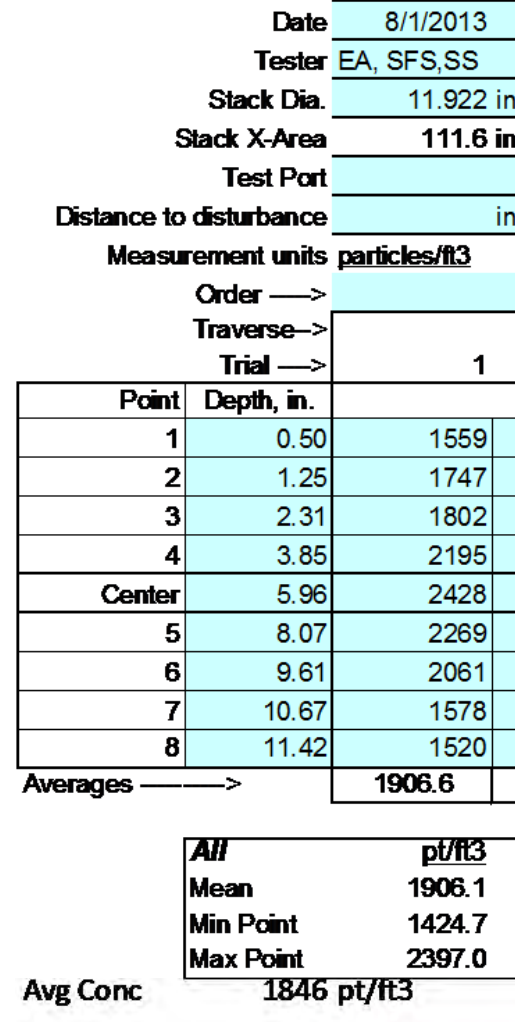

Generator Inlet Press

Stack Temp

Centerfine vel.

Ambient pressure

Ambient humidity

Ambient temp

Back-Gd aerosol

No. Bk-Gd samples

Compressor output

\begin{tabular}{|c|c|}
\hline Stat & Finish \\
\hline 3.0 & 3.0 \\
\hline 72.1 & 79.9 \\
\hline 2300 & 2170 \\
\hline 29.88 & 29.88 \\
\hline $48 \%$ & $35 \%$ \\
\hline 70.7 & 79.7 \\
\hline $4,2,0,0,3$ & $3,1,3,2,5$ \\
\hline 5 & 5 \\
\hline 30 & 36 \\
\hline
\end{tabular}

Notes:

2

1577

1577

PARTICLE TRACER TRAVERSE DATA FORM

Run No. PT-9

Fan configuration B\&C

Fan Setting 40

Stack Temp

$76 \operatorname{deg} F$

Start/End Time $0950 / 1140$

Center $2 / 3$ from

Points in Center 233

Injection Point 15

2nd 1st $\begin{array}{rll}1.09 & \text { to: } & 10.83 \\ 2 & \text { to: } & \end{array}$

\begin{tabular}{|r|r|r|r|r|}
\cline { 2 - 4 } Meen & \multicolumn{5}{|c|}{$\begin{array}{c}\text { Bottom } \\
\mathbf{2}\end{array}$} & $\mathbf{4}$ & $\mathbf{3}$ & Mean \\
\hline $\mathbf{1 5 5 3 . 0}$ & 1420 & 1479 & 1480 & $\mathbf{1 4 5 9 . 7}$ \\
\hline $\mathbf{1 7 3 7 . 0}$ & 1672 & 1661 & 1639 & $\mathbf{1 6 5 7 . 3}$ \\
\hline $\mathbf{1 9 3 7 . 3}$ & 1879 & 1899 & 1929 & $\mathbf{1 9 0 2 . 3}$ \\
\hline $\mathbf{2 2 1 3 . 3}$ & 2287 & 2120 & 2238 & $\mathbf{2 2 1 5 . 0}$ \\
\hline $\mathbf{2 3 7 9 . 0}$ & 2376 & 2289 & 2526 & $\mathbf{2 3 9 7 . 0}$ \\
\hline $\mathbf{2 2 6 5 . 3}$ & 2171 & 2182 & 2318 & $\mathbf{2 2 2 3 . 7}$ \\
\hline $\mathbf{2 0 2 4 . 7}$ & 1865 & 1899 & 2063 & $\mathbf{1 9 4 2 . 3}$ \\
\hline $\mathbf{1 7 5 1 . 3}$ & 1755 & 1702 & 1736 & $\mathbf{1 7 3 1 . 0}$ \\
\hline $\mathbf{1 4 9 6 . 0}$ & 1438 & 1417 & 1419 & $\mathbf{1 4 2 4 . 7}$ \\
\hline $\mathbf{1 9 2 8 . 6}$ & $\mathbf{1 8 7 3 . 7}$ & $\mathbf{1 8 4 9 . 8}$ & $\mathbf{1 9 2 7 . 6}$ & $\mathbf{1 8 8 3 . 7}$ \\
\hline
\end{tabular}

3

55.8

Dev. from mean

\begin{tabular}{lr}
\hline Center 2/3 & Side \\
\hline Meen & 2044.0 \\
Sid. Dev. & 252.2 \\
COV as \% & 12.3 \\
\hline Instiments Used & \\
TSI VelciCalc & T9 \\
\hline
\end{tabular}

\begin{tabular}{rrrr|}
\hline Bottom & All & Normlzd \\
\hline 2009.8 & 2026.9 & 2034.64 \\
275.6 & 254.4 & 255.97 \\
\cline { 2 - 3 } & 13.7 & 12.6 & 12.58 \\
\hline
\end{tabular}

\begin{tabular}{lrr} 
TSI VelciCalc & T95351203001 & \multicolumn{1}{c}{$\begin{array}{c}\text { Cal Due } \\
12 / 10 / 2013\end{array}$} \\
\hline Fisher Scientific & 90936818 & $12 / 11 / 2013$ \\
\hline Met One OPC (Ref) & 96258675 & $\mathrm{FIO}$ \\
\hline Met One OPC & 1011529009 & $1 / 14 / 2014$ \\
\hline
\end{tabular}

Signature/date


Rev. 0

3 Aug. 2006

PARTICLE TRACER TRAVERSE DATA FORM

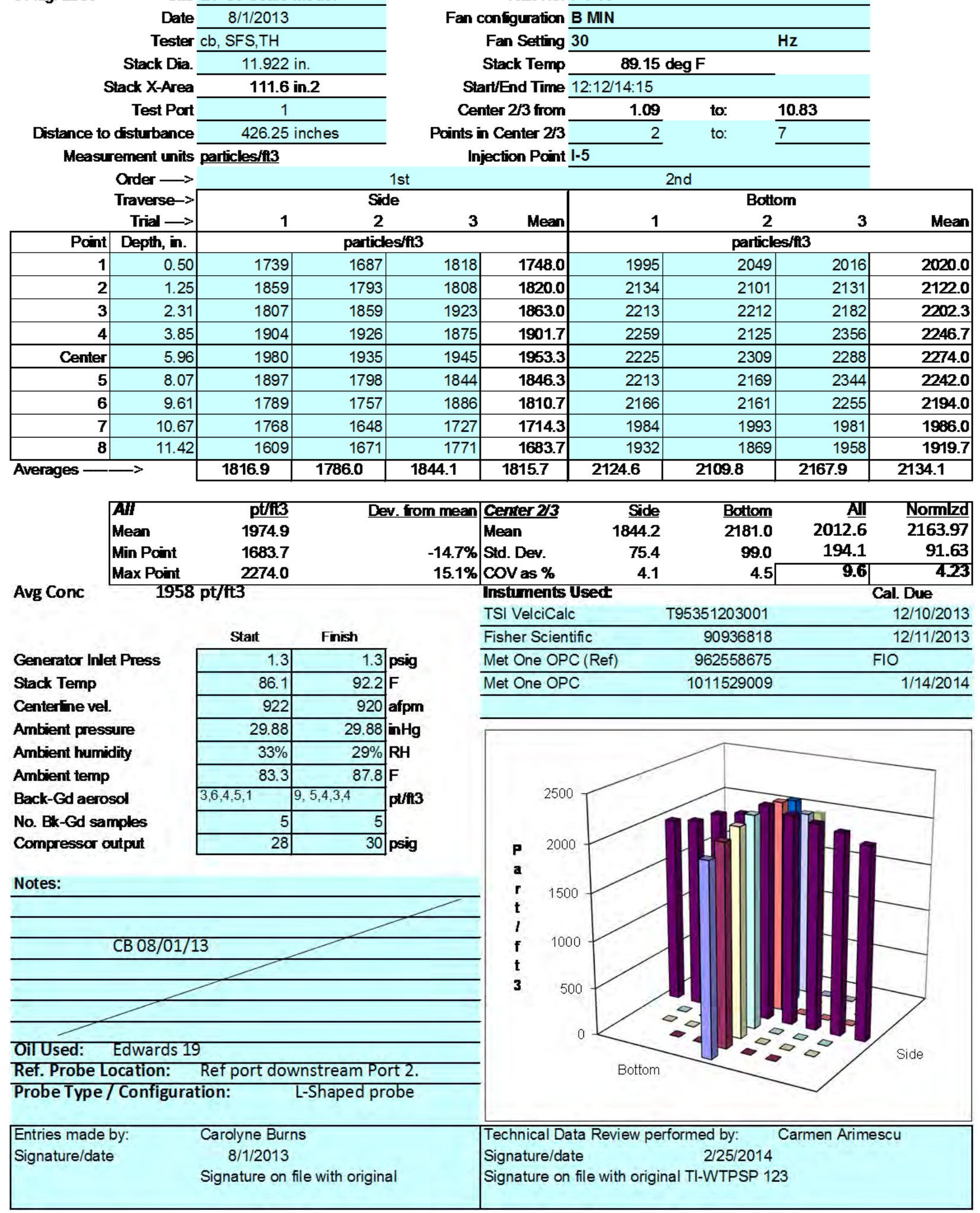


Rev. 0

3 Aug. 2006

Site LV-S3 Scale Model

Date 8/2/2013

Tester SS,SFS

Stack Dia.

11.922 in

Stack X-Area

Test Part

111.6 in.

Distance to disturbance

Measurement units particles/fi3 426.25 inches

\begin{tabular}{|r|r|}
\multicolumn{1}{c}{$\begin{array}{c}\text { Order } \\
\text { Traverse-> } \\
\text { Trial } \longrightarrow\end{array}$} \\
\hline Point & Depth, in. \\
\hline $\mathbf{1}$ & 0.50 \\
\hline $\mathbf{2}$ & 1.25 \\
\hline $\mathbf{3}$ & 2.31 \\
\hline $\mathbf{4}$ & 3.85 \\
\hline Center & 5.96 \\
\hline $\mathbf{5}$ & 8.07 \\
\hline $\mathbf{6}$ & 9.61 \\
\hline $\mathbf{7}$ & 10.67 \\
\hline $\mathbf{8}$ & 11.42 \\
\hline
\end{tabular}

2nd

\section{TRACER TRAVERSE DATA FORM}

\section{Run No. PT-11}

Fan configuration $C$ MIN

Fan Setting 30

Stack Temp

$66.3 \operatorname{deg} F$

Start/End Time $0620 / 0830$

Center $2 / 3$ from

1.09

Points in Center $2 / 3$

Injection Pcint I-5

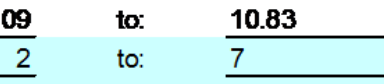

1st Side

\begin{tabular}{lc}
1 & 2 \\
\hline & particles/ $\mathrm{ft}^{3}$
\end{tabular}

3

\begin{tabular}{|r|r|r|r|r|}
\hline & \multicolumn{5}{|c|}{$\begin{array}{c}\text { Bottom } \\
\mathbf{2}\end{array}$} \\
\hline & $\mathbf{5}$ & $\mathbf{3}$ & Mean \\
\hline $\mathbf{1 2 9 4 . 7}$ & 1445 & 1336 & 1387 & $\mathbf{1 3 8 9 . 3}$ \\
\hline $\mathbf{1 3 5 3 . 0}$ & 1446 & 1492 & 1431 & $\mathbf{1 4 5 6 . 3}$ \\
\hline $\mathbf{1 4 2 7 . 3}$ & 1622 & 1485 & 1544 & $\mathbf{1 5 5 0 . 3}$ \\
\hline $\mathbf{1 4 7 7 . 0}$ & 1598 & 1634 & 1606 & $\mathbf{1 6 1 2 . 7}$ \\
\hline $\mathbf{1 5 2 8 . 3}$ & 1650 & 1689 & 1660 & $\mathbf{1 6 6 6 . 3}$ \\
\hline $\mathbf{1 4 7 0 . 3}$ & 1750 & 1620 & 1673 & $\mathbf{1 6 8 1 . 0}$ \\
\hline $\mathbf{1 4 7 6 . 0}$ & 1620 & 1621 & 1563 & $\mathbf{1 6 0 1 . 3}$ \\
\hline $\mathbf{1 3 6 5 . 0}$ & 1380 & 1410 & 1381 & $\mathbf{1 3 9 0 . 3}$ \\
\hline $\mathbf{1 2 2 5 . 3}$ & 1278 & 1368 & 1287 & $\mathbf{1 3 1 1 . 0}$ \\
\hline $\mathbf{1 4 0 1 . 9}$ & $\mathbf{1 5 3 2 . 1}$ & $\mathbf{1 5 1 7 . 2}$ & $\mathbf{1 5 0 3 . 6}$ & $\mathbf{1 5 1 7 . 6}$ \\
\hline
\end{tabular}

\begin{tabular}{|lr|}
\hline All & $\mathrm{pt} / \mathrm{ft} 3$ \\
Mean & 1459.8 \\
Min Point & 1225.3 \\
Max Point & 1681.0 \\
\hline \multicolumn{2}{|c|}{$1443 \mathrm{pt} / \mathrm{ft} 3$}
\end{tabular}

Avg Conc $1443 \mathrm{pt} / \mathrm{ft} 3$

Generator Inlet Press

Stack Temp

Centerine vel.

Ambient pressure

Ambient humidity

Ambient temp

Back-Gd aerosol

No. Bk-Gd samples

Compressor output

\begin{tabular}{|c|c|c|}
\hline Statt & Finish & \\
\hline 1.75 & 1.75 & psig \\
\hline 64.8 & 67.8 & $F$ \\
\hline 1202 & 1202 & a \\
\hline 29.85 & 29.91 & $\mathrm{GH}$ \\
\hline $54 \%$ & $51 \%$ & RH \\
\hline 64.4 & 67.1 & $\mathrm{~F}$ \\
\hline $3,11,4,6,1$ & $2,3,3,2,3$ & $\mathrm{pt} / \mathrm{tr}$ \\
\hline 5 & 5 & \\
\hline 30 & 34 & $\operatorname{sig}$ \\
\hline
\end{tabular}

Notes:

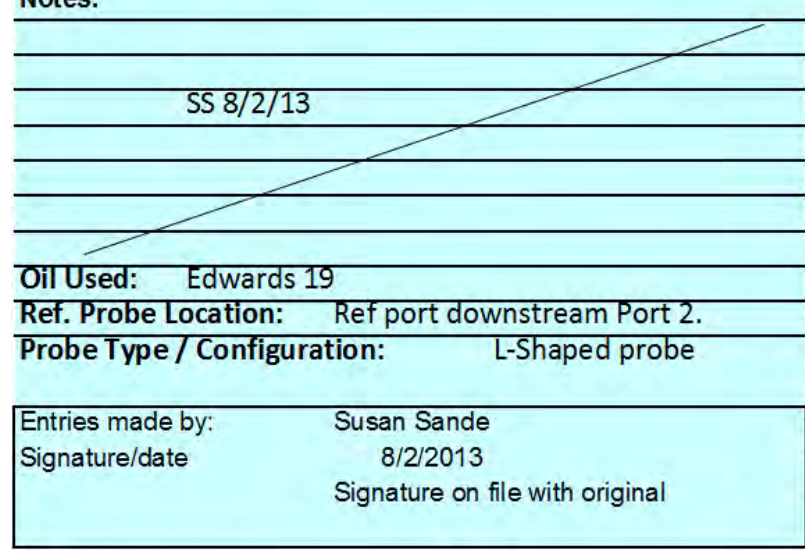

Center 213

Mean

$-16.1 \%$ Std. Dev.

$15.2 \%$ COV as \%

Instuments Used

$\begin{array}{r}\text { Side } \\ 1442.4 \\ 64.2 \\ 4.4 \\ \hline\end{array}$

TSI VelciCalc

\begin{tabular}{l} 
Fisher Scientific \\
\hline Met One OPC (Ref) \\
\hline Met One OPC
\end{tabular}

T95351203001

\begin{tabular}{rrrr|}
\hline Bottom & All & Normlzd \\
\hline 1565.5 & 1504.0 & 1569.07 \\
107.9 & 106.5 & 87.44 \\
\cline { 2 - 3 } & 7.1 & 5.57 \\
\hline
\end{tabular}

6.9

7.1

Met One OPC $\quad 1011529009 \quad 1 / 14 / 2014$

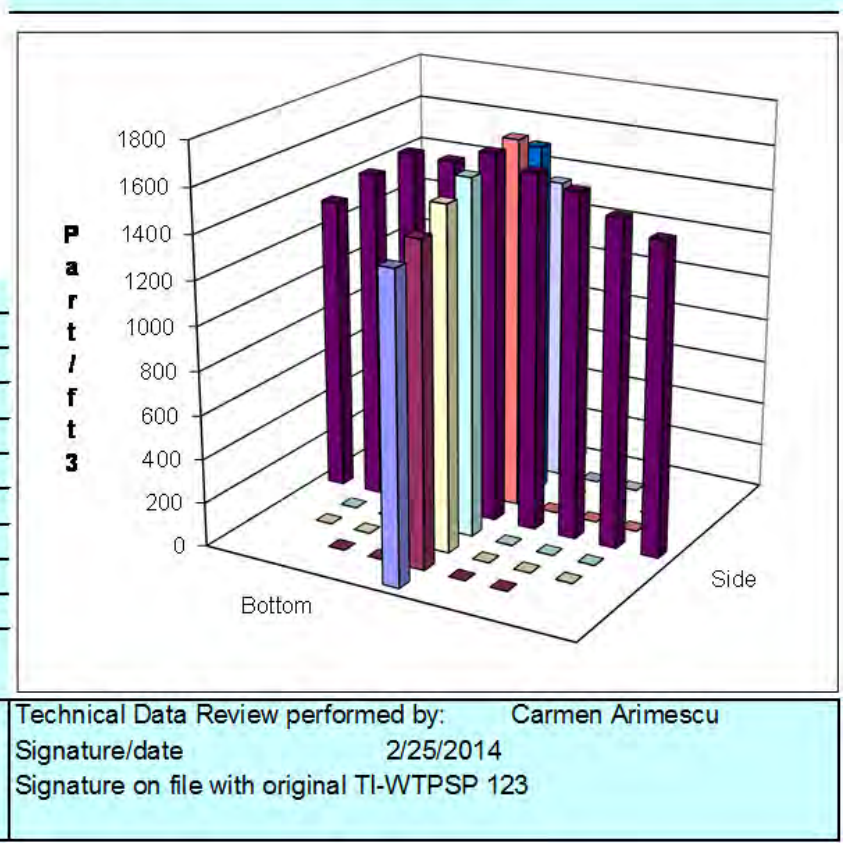


Rev. 0

3 Aug. 2006

PARTICLE TRACER TRAVERSE DATA FORM

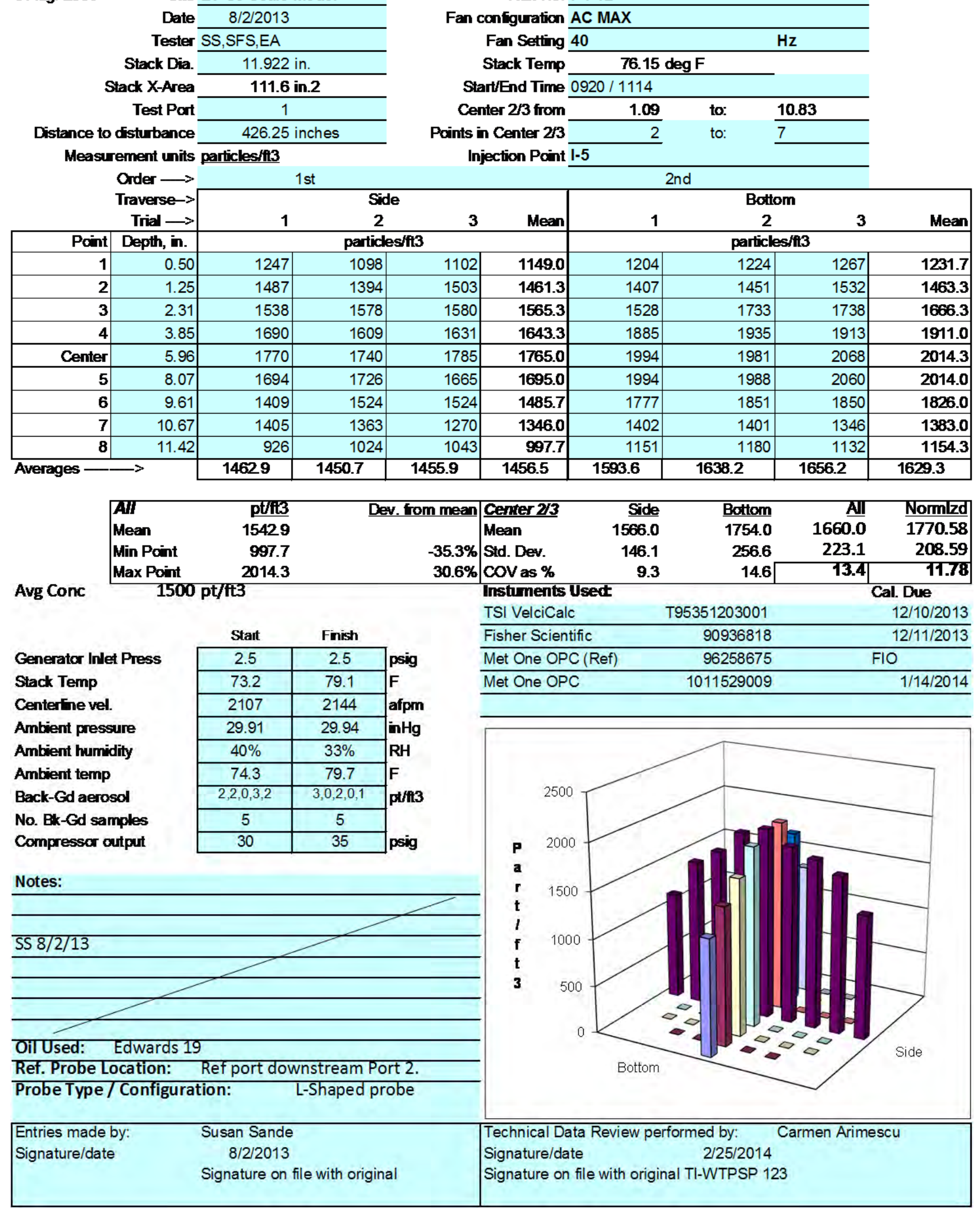


Rev. 0

3 Aug. 2006

\begin{tabular}{|c|c|c|}
\hline & Date & $8 / 2 / 2013$ \\
\hline & Tester & $\mathrm{TH}, \mathrm{cb}$ \\
\hline & Stack Dia. & 11.922 in. \\
\hline & Stack X-Area & 111.6 in. \\
\hline & Test Part & 1 \\
\hline Distance to & distumance & 426.25 inc \\
\hline Measure & rement units & perticles/t13 \\
\hline & Order $\longrightarrow$ & $2 n$ \\
\hline & Traverse-> & \\
\hline & & 1 \\
\hline Point & Depth, in. & \\
\hline 1 & 0.50 & 1266 \\
\hline 2 & 1.25 & 1396 \\
\hline 3 & 2.31 & 1601 \\
\hline 4 & 3.85 & 1705 \\
\hline Center & 5.96 & 1741 \\
\hline 5 & 8.07 & 1630 \\
\hline 6 & 9.61 & 1541 \\
\hline 7 & 10.67 & 1434 \\
\hline 8 & 11.42 & 1229 \\
\hline Averages - & $\longrightarrow$ & 1504.8 \\
\hline & $\boldsymbol{A I I}$ & pt/fi3 \\
\hline & Mean & 1534.1 \\
\hline & Min Pcint & 1237.7 \\
\hline & Max Point & 1837.7 \\
\hline Avg Conc & 150 & t3 \\
\hline
\end{tabular}

Generator Inlet Press

Stack Temp

Centerfine vel side \#7

Ambient pressure

Ambient humidity

Ambient temp

Back-Gd aerosol

No. Bk-Gd samples

Compressor output

\begin{tabular}{|c|c|}
\hline Stat & Finish \\
\hline 2.0 & 2.0 \\
\hline 80.9 & 72.7 \\
\hline 1883 & 1862 \\
\hline 29.94 & 29.97 \\
\hline $34 \%$ & $41 \%$ \\
\hline 77.0 & 77.9 \\
\hline $9,12,6,7,5$ & $5,2,3,3,1$ \\
\hline 5 & 5 \\
\hline 30 & 34 \\
\hline
\end{tabular}

Notes:

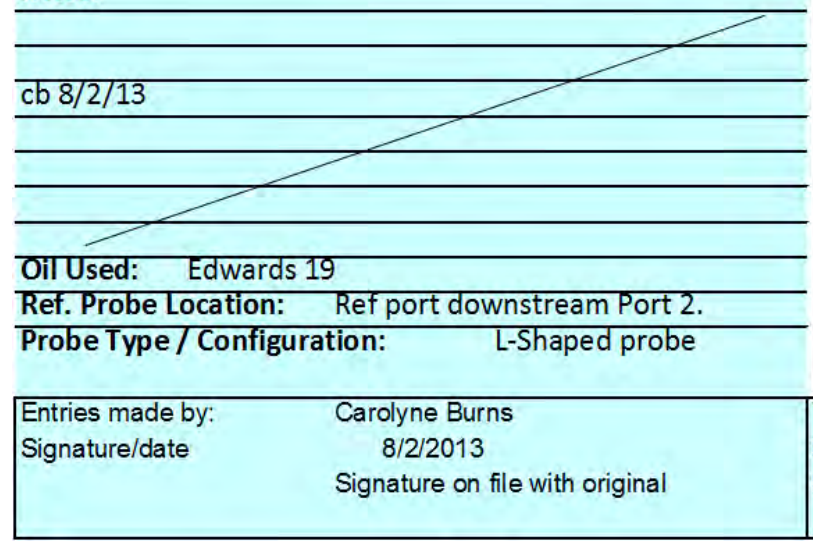

Side

$\frac{2}{1336}$

663.6

Dev. from mean

PARTICLE TRACER TRAVERSE DATA FORM

Run No. PT-13

Fan configuration $A$

Fan Setting 60

Stack Temp

Start/End Time 13:45/15:4

Center $2 / 3$ from

Points in Center 273

Injection Point I-5

$76.8 \mathrm{deg} F$

$\mathrm{Hz}$

1.09

$\begin{array}{rll}1.09 & \text { to: } & 10.83 \\ 2 & \text { to: } & \end{array}$

1st

3

Meen

(298

1st

1 Bottom

\begin{tabular}{|l|r|r|r|r|r|}
\hline 417 & $\mathbf{1 3 3 9 . 7}$ & 1293 & 1319 & 1124 & $\mathbf{1 2 4 5 . 3}$ \\
\hline 507 & $\mathbf{1 4 6 4 . 0}$ & 1488 & 1505 & 1308 & $\mathbf{1 4 3 3 . 7}$ \\
\hline 522 & $\mathbf{1 5 9 0 . 7}$ & 1566 & 1547 & 1524 & $\mathbf{1 5 4 5 . 7}$ \\
\hline 670 & $\mathbf{1 7 0 8 . 0}$ & 1722 & 1715 & 1590 & $\mathbf{1 6 7 5 . 7}$ \\
\hline 689 & $\mathbf{1 7 4 5 . 3}$ & 1897 & 1894 & 1722 & $\mathbf{1 8 3 7 . 7}$ \\
\hline 743 & $\mathbf{1 6 9 6 . 0}$ & 1745 & 1836 & 1675 & $\mathbf{1 7 5 2 . 0}$ \\
\hline 619 & $\mathbf{1 5 5 9 . 3}$ & 1765 & 1736 & 1552 & $\mathbf{1 6 8 4 . 3}$ \\
\hline 515 & $\mathbf{1 4 6 3 . 7}$ & 1382 & 1430 & 1267 & $\mathbf{1 3 5 9 . 7}$ \\
\hline 229 & $\mathbf{1 2 7 5 . 3}$ & 1253 & 1275 & 1185 & $\mathbf{1 2 3 7 . 7}$ \\
\hline $\mathbf{7}$ & $\mathbf{1 5 3 8 . 0}$ & $\mathbf{1 5 6 7 . 9}$ & $\mathbf{1 5 8 4 . 1}$ & $\mathbf{1 4 3 8 . 6}$ & $\mathbf{1 5 3 0 . 2}$ \\
\hline
\end{tabular}

\begin{tabular}{|rrrr|} 
Side & Bottom & All & Normlzd \\
1603.9 & 16127 & 1608.3 & 1650.69 \\
116.0 & 173.1 & 141.6 & 149.21 \\
7.2 & 10.7 & 8.8 & 9.04 \\
\hline
\end{tabular}

Std. Dev. $\quad 116.0$

Instuments Usect

\begin{tabular}{rr|r}
10.7 & 8.8 & 9.0 \\
& & Cal. Due
\end{tabular}

\begin{tabular}{lrc} 
TSI VelciCalc & T95351203001 & $12 / 10 / 2013$ \\
\hline Fisher Scientific & 90936818 & $12 / 11 / 2013$ \\
\hline Met One OPC (Ref) & 96258675 & FIO \\
\hline Met One OPC & 1011529009 & $1 / 14 / 2014$ \\
\hline
\end{tabular}

Technical Data Review performed by: Carmen Arimescu


Rev. 0

3 Aug. 2006

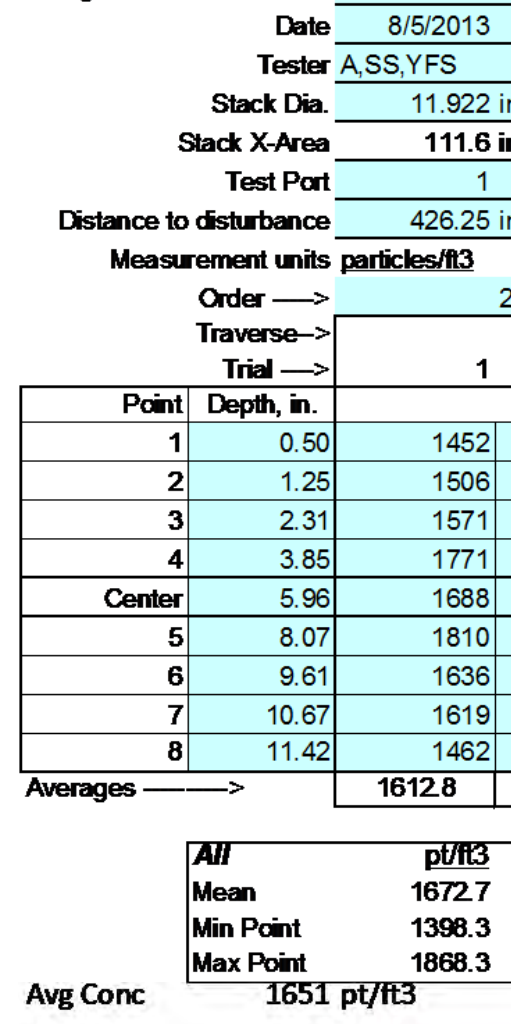

Generator Inlet Press

Stack Temp

Centerine vel side \#7

Ambient pressure

Ambient humidity

Ambient temp

Back-Gd aerosol

No. Bk-Gd samples

Compressor output

\begin{tabular}{|c|c|}
\hline Stat & Finish \\
\hline 1.7 & 1.7 \\
\hline 70.4 & 87.4 \\
\hline 1295 & 1330 \\
\hline 29.88 & 29.88 \\
\hline $52 \%$ & $34 \%$ \\
\hline 69.8 & 81.5 \\
\hline $5,2,6,0,3$ & $5,2,4,0,5$ \\
\hline 5 & 5 \\
\hline 30 & 32 \\
\hline
\end{tabular}

Notes:

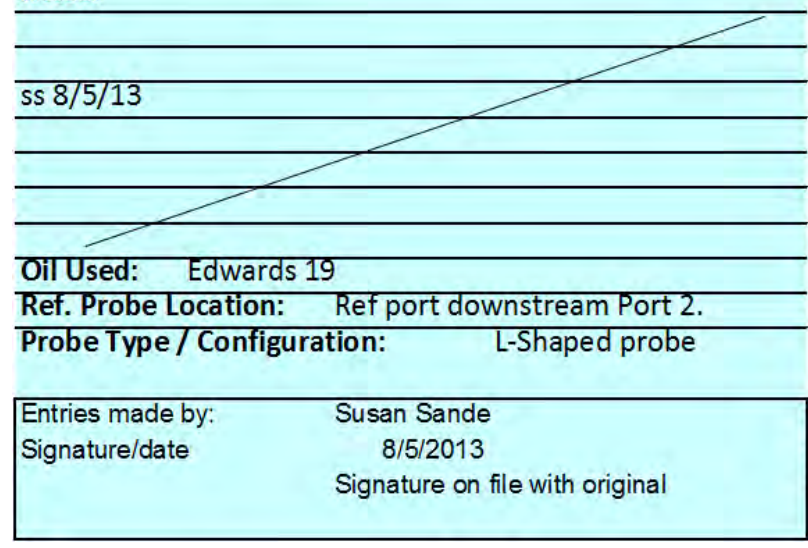

718.8

Side

$\frac{2}{\text { particles/ft3 }}$

3

Dev. from mean
$-16.4 \%$
$11.7 \%$

PARTICLE TRACER TRAVERSE DATA FORM

Run No. PT-14

Fan confguration A\&C MIN

Fan Setting 25

Stack Temp

$78.9 \operatorname{deg} F$

Start/End Time $0620 / 0820$

Center $2 / 3$ from

Points in Center 273

Injection Point I-5

$\begin{array}{rll}1.09 & \text { to: } & 10.83 \\ 2 & \text { to: } & \end{array}$

\begin{tabular}{|r|r|r|r|r|}
\cline { 2 - 4 } Meen & \multicolumn{5}{|c|}{ 1st } \\
\hline & $\mathbf{5}$ & \multicolumn{3}{c|}{$\begin{array}{c}\text { Boftom } \\
\text { perticles/ti3 }\end{array}$} \\
\hline $\mathbf{1 4 9 1 . 7}$ & 1378 & 1484 & 1440 & $\mathbf{1 4 3 4 . 0}$ \\
\hline $\mathbf{1 6 2 7 . 0}$ & 1463 & 1530 & 1604 & $\mathbf{1 5 3 2 . 3}$ \\
\hline $\mathbf{1 7 1 0 . 0}$ & 1636 & 1698 & 1720 & $\mathbf{1 6 8 4 . 7}$ \\
\hline $\mathbf{1 7 8 4 . 3}$ & 1795 & 1904 & 1858 & $\mathbf{1 8 5 2 . 3}$ \\
\hline $\mathbf{1 8 5 2 0}$ & 1737 & 1875 & 1911 & $\mathbf{1 8 4 1 . 0}$ \\
\hline $\mathbf{1 8 6 8 . 3}$ & 1774 & 1833 & 1820 & $\mathbf{1 8 0 9 . 0}$ \\
\hline $\mathbf{1 7 3 0 . 7}$ & 1667 & 1739 & 1803 & $\mathbf{1 7 3 6 . 3}$ \\
\hline $\mathbf{1 7 0 1 . 0}$ & 1434 & 1507 & 1537 & $\mathbf{1 4 9 2 . 7}$ \\
\hline $\mathbf{1 5 6 2 7}$ & 1395 & 1382 & 1418 & $\mathbf{1 3 9 8 . 3}$ \\
\hline $\mathbf{1 7 0 3 . 1}$ & $\mathbf{1 5 8 6 . 6}$ & $\mathbf{1 6 6 1 . 3}$ & $\mathbf{1 6 7 9 . 0}$ & $\mathbf{1 6 4 2 . 3}$ \\
\hline
\end{tabular}

Canter 23

\begin{tabular}{|lrrrr|} 
Center 23 & Side & Bottom & All & Norm $\mid z d$ \\
\hline Mean & 1753.3 & 1706.9 & 1730.1 & 1735.22 \\
Std. Dev. & 86.6 & 145.7 & 117.6 & 117.15 \\
COV as \% & 4.9 & 8.5 & 6.8 & 6.75 \\
\hline
\end{tabular}

\section{Instuments Used}

TSI VelciCalc

\begin{tabular}{lrc}
\hline Fisher Scientific & 90936818 & $12 / 10 / 2013$ \\
\hline Met One OPC (Ref) & 96258675 & \multicolumn{1}{c}{$12 / 11 / 2013$} \\
\hline Met One OPC & 1011529009 & $1 / 14 / 2014$ \\
\hline
\end{tabular}

\begin{tabular}{lll}
\hline Met One OPC & 1011529009 & $1 / 14 / 2014$ \\
\hline
\end{tabular}

Technical Data Review performed by: Carmen Arimescu


Rev. 0

3 Aug. 2006

Site LV-S3 Scale Model

Date 8/5/2013

Tester A,SS,YFS

Stack Dia. 11.922 in

Stack X-Area

Test Port

Distance to disturbance 510.25 inches

Measurement units particles/ti3

Order $\longrightarrow$

Traverse-

Trial $\rightarrow$

\begin{tabular}{|r|r|}
\hline Pcint & Depth, in. \\
\hline $\mathbf{1}$ & 0.50 \\
\hline $\mathbf{2}$ & 1.25 \\
\hline $\mathbf{3}$ & 2.31 \\
\hline $\mathbf{4}$ & 3.85 \\
\hline Center & 5.96 \\
\hline $\mathbf{5}$ & 8.07 \\
\hline $\mathbf{6}$ & 9.61 \\
\hline $\mathbf{7}$ & 10.67 \\
\hline $\mathbf{8}$ & \multicolumn{2}{|c}{11.42} \\
\hline
\end{tabular}

\begin{tabular}{|l|l}
\hline & \\
\hline 0 & \\
\hline 5 & \\
\hline 1 & \\
\hline 5 & \\
\hline 6 & \\
\hline 7 & \\
\hline 1 & \\
\hline 7 & \\
\hline 2 & \\
\hline & 1
\end{tabular}

111.6 in. 2

2

2nd

(2)

-

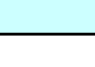

es

1 Side

particles/fi3

\begin{tabular}{|l|r|r|}
1064 & 1031 & 1097 \\
\hline 1098 & 1153 & 1150 \\
\hline 1194 & 1320 & 1277 \\
\hline 1303 & 1325 & 1336 \\
\hline 1202 & 1361 & 1414 \\
\hline 1312 & 1398 & 1296 \\
\hline 1296 & 1357 & 1360 \\
\hline 1258 & 1262 & 1258 \\
\hline 1153 & 1283 & 1270 \\
\hline $\mathbf{0 8 . 9}$ & $\mathbf{1 2 7 6 . 7}$ & $\mathbf{1 2 7 3 . 1}$ \\
\hline
\end{tabular}

\section{AVERE DATA FORM}

Fan configuration $\mathrm{A}$ Norm

Fan Setting 60

Stack Temp

Start/End Time 0824 / 102

Center $2 / 3$ from

Points in Center $2 / 3$

Injection Point I-5
$93.65 \operatorname{deg} \mathrm{F}$

1.09

2

to: $\quad \frac{10.83}{7}$

1st

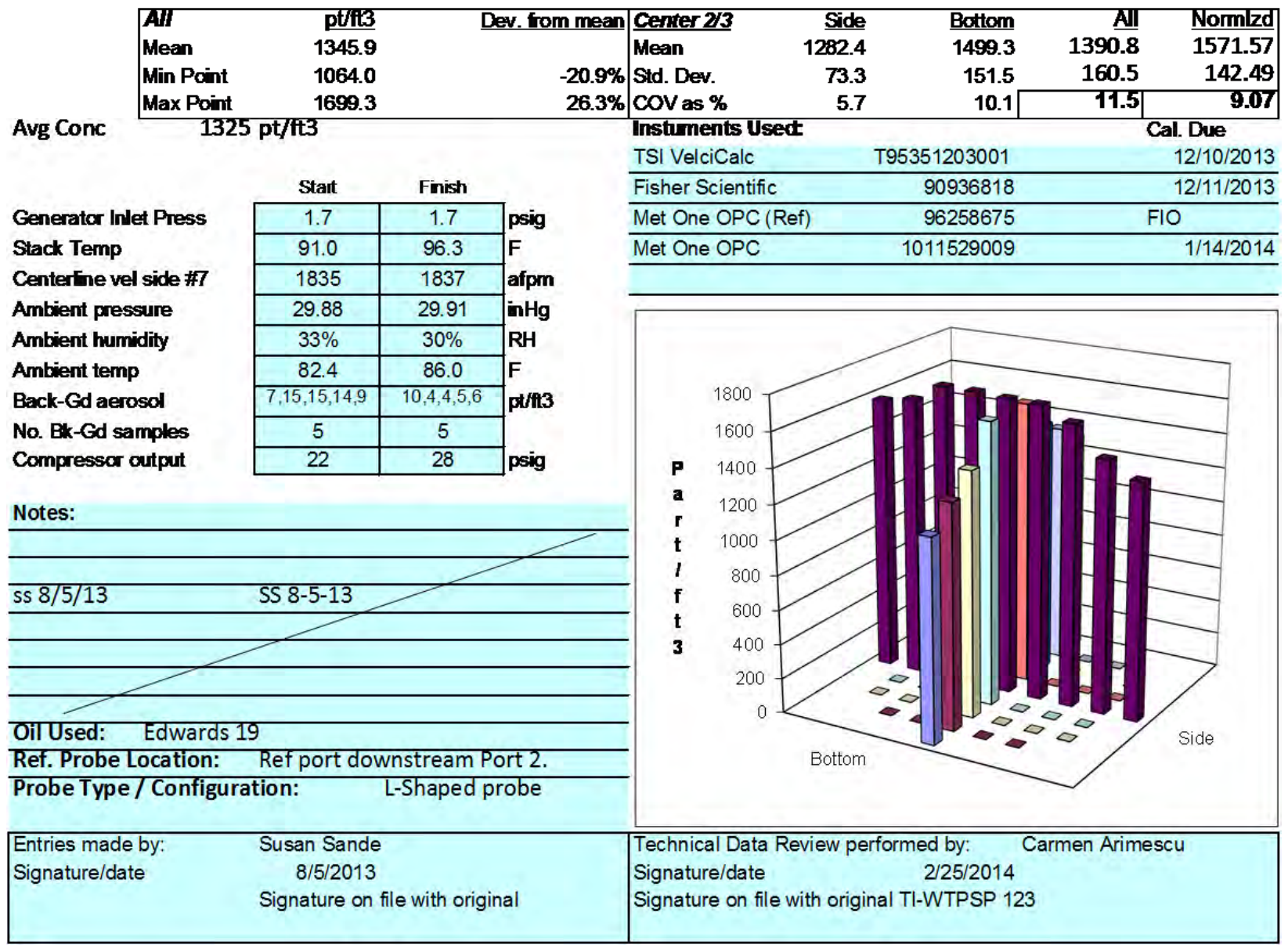


Rev. 0

3 Aug. 2006

Site LV-S3 Scale Model

Date 8/5/2013

Tester A, SS,YFS

Stack Dia 11.922 in

Stack X-Area

Test Part

111.6 in. 2

Distance to disturbance

Measurement units particles/ft3 510.25 inches

\begin{tabular}{|r|r|}
\multicolumn{1}{c}{$\begin{array}{c}\text { Order } \\
\text { Traverse-> } \\
\text { Trial } \longrightarrow\end{array}$} \\
\hline Point & Depth, in. \\
\hline $\mathbf{1}$ & 0.50 \\
\hline $\mathbf{2}$ & 1.25 \\
\hline $\mathbf{3}$ & 2.31 \\
\hline $\mathbf{4}$ & 3.85 \\
\hline Center & 5.96 \\
\hline $\mathbf{5}$ & 8.07 \\
\hline $\mathbf{6}$ & 9.61 \\
\hline $\mathbf{7}$ & 10.67 \\
\hline $\mathbf{8}$ & 11.42 \\
\hline
\end{tabular}

\begin{tabular}{|lr}
\hline All & $\mathrm{pt} / \mathrm{ft} 3$ \\
Mean & 1337.6 \\
Min Point & 10520 \\
Max Point & 1631.7 \\
\hline
\end{tabular}

Avg Conc $1315 \mathrm{pt} / \mathrm{ft3}$

Generator Inlet Press Stack Temp

Centerline vel side \#7

Ambient pressure

Ambient humidity

Ambient temp

Back-Gd aerosol

No. Bk-Gd samples

Compressor output

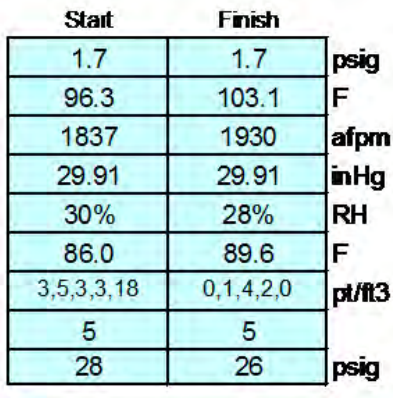

Notes:

\begin{tabular}{l} 
ss $8 / 5 / 13$ \\
$\begin{array}{l}\text { Entries made by: } \\
\text { Signature/date }\end{array}$ \\
$\begin{array}{c}\text { SilUsed: Edwards } 19 \\
\text { Probe Type / Configuration: } \\
\text { Signature on file with original }\end{array}$ \\
\hline
\end{tabular}

1st

Side

\begin{tabular}{l|r|} 
& 123 \\
\hline
\end{tabular}

Dev. from mean
$-21.4 \%$
$220 \%$

Run No. PT-16

Fan configuration A Norm

Fan Setting 60

Stack Temp

$99.7 \operatorname{deg} \mathrm{F}$

Start/End Time $1030 / 1215$

Center $2 / 3$ from

Points in Center $2 / 3$

Injection Pcint 1-5

$\begin{array}{lll}2 & \text { to: } & \mathbf{1 0 . 8 3} \\ & \text { to: } & \end{array}$

2nd

Bottom

3 Mean

1

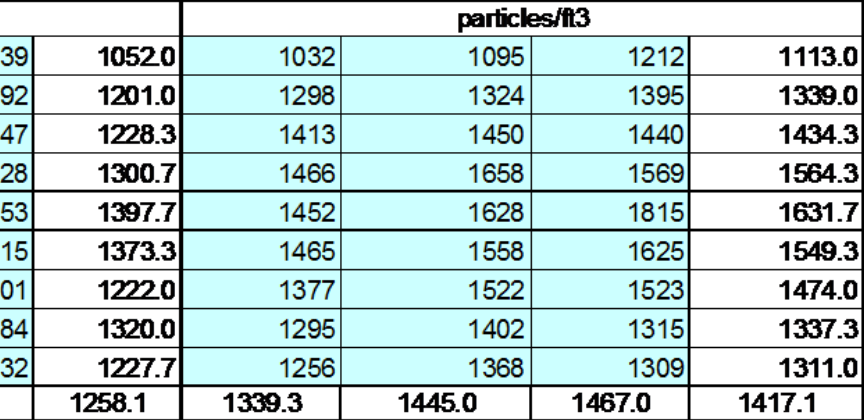

$\begin{array}{r}\text { Side } \\ 1291.9 \\ 77.3 \\ 6.0 \\ \hline\end{array}$

\begin{tabular}{rrrr|}
\hline Bottom & All & Normlzd \\
\hline 1475.7 & 1383.8 & 1491.93 \\
113.4 & 133.4 & 99.89 \\
7.7 & 9.6 & 6.70 \\
\hline
\end{tabular}

\section{Instuments Usedt}

TSI VelciCalc

\begin{tabular}{lrc} 
TSI VelciCalc & T95351203001 & $12 / 10 / 2013$ \\
\hline Fisher Scientific & 90936818 & $12 / 11 / 2013$ \\
\hline Met One OPC (Ref) & 96258675 & \multicolumn{1}{c}{ FIO } \\
\hline Met One OPC & 1011529009 & $1 / 14 / 2014$ \\
\hline
\end{tabular}
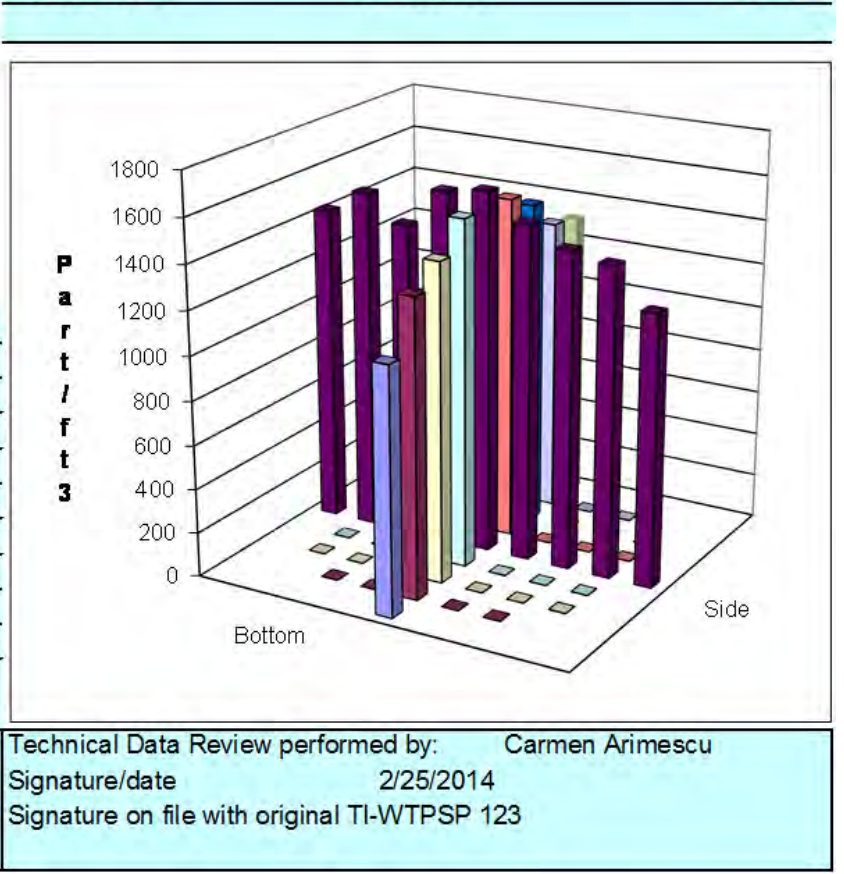
Rev. 0

3 Aug. 2006

\begin{tabular}{|c|c|c|}
\hline & Date & $8 / 5 / 2013$ \\
\hline \multicolumn{3}{|c|}{ Tester $\mathrm{cb}, \mathrm{TH}$} \\
\hline \multirow{2}{*}{\multicolumn{2}{|c|}{$\begin{array}{r}\text { Stack Dia. } \\
\text { Stack X-Area }\end{array}$}} & 11.922 in \\
\hline & & 111.6 in \\
\hline \multicolumn{2}{|r|}{ Test Port } & 2 \\
\hline \multicolumn{2}{|c|}{ Distance to disturbance } & 510.25 in \\
\hline \multicolumn{3}{|c|}{ Measurement units perticles/ti3 } \\
\hline \multicolumn{2}{|r|}{ Order $\longrightarrow$} & $2 r$ \\
\hline \multicolumn{2}{|r|}{$\begin{array}{c}\text { Traverse }> \\
\quad \text { Trial } \longrightarrow\end{array}$} & 1 \\
\hline \multicolumn{2}{|c|}{\begin{tabular}{|l|l|} 
Point & Depth, in.
\end{tabular}} & \\
\hline 1 & 0.50 & 1179 \\
\hline 2 & 1.25 & 1290 \\
\hline 3 & 2.31 & 1363 \\
\hline \multirow{2}{*}{\begin{tabular}{r|}
4 \\
enter
\end{tabular}} & 3.85 & 1521 \\
\hline & 5.96 & 1501 \\
\hline 5 & 8.07 & 1562 \\
\hline \multirow{2}{*}{$\begin{array}{l}6 \\
7\end{array}$} & 9.61 & 1460 \\
\hline & 10.67 & 1397 \\
\hline \begin{tabular}{l|}
7 \\
8
\end{tabular} & 11.42 & 1284 \\
\hline \multicolumn{2}{|c|}{ Averages $\longrightarrow \longrightarrow$} & 1395.2 \\
\hline \multirow{5}{*}{\multicolumn{2}{|c|}{$\begin{array}{ll}\text { Conc } & \frac{\mid A I I}{\text { Mean }} \\
\text { Min Point } \\
\text { Max Point }\end{array}$}} & pt/ft3 \\
\hline & & 1438.3 \\
\hline & & 12027 \\
\hline & & 1784.7 \\
\hline & & \\
\hline
\end{tabular}

Generator Inlet Press Stack Temp

Centerline vel side \#7

Ambient pressure

Ambient humidity

Ambient temp

Back-Gd aerosol

No. Bk-Gd samples

Compresscr output

\begin{tabular}{|c|c|}
\hline Stat & Finish \\
\hline 1.7 & 1.7 \\
\hline 107.9 & 110.6 \\
\hline 2009 & 1810 \\
\hline 29.91 & 29.53 \\
\hline $27 \%$ & $21 \%$ \\
\hline 91.4 & 95 \\
\hline $4,3,0,6,6$ & $0,5,3,0,1$ \\
\hline 5 & 5 \\
\hline 14 & 24 \\
\hline
\end{tabular}

Notes:

$\mathrm{cb8/5/13}$

\section{OाUsed: Edwards 19}

Ref. Probe Location: Ref port downstream Port 2.

Probe Type/Configuration: L-Shaped probe

\begin{tabular}{|lc|}
\hline Entries made by: & Carolyn Burns \\
Signature/date & $8 / 5 / 2013$ \\
& Signature on file with original \\
\hline
\end{tabular}

Dev. from mean

Run No. PT-17

Fan configuration A Norm

Fan Setting 60

Stack Temp

$109.25 \operatorname{deg} F$

Start/End Time 13:25/14:30

Center $2 / 3$ from

Points in Center 233

Injection Point $\mathrm{I}-5$

\begin{tabular}{|c|c|c|}
\hline 1.09 & to: & 10.83 \\
\hline 2 & to: & 7 \\
\hline
\end{tabular}

1 st

\begin{tabular}{|r|r|r|r|r|}
\cline { 2 - 4 } Meen & \multicolumn{5}{|c|}{ 1st } \\
\hline & $\mathbf{5}$ & \multicolumn{4}{c}{ Bottom } \\
\hline $\mathbf{1 2 0 2 7}$ & 1307 & 1161 & 1298 & $\mathbf{1 2 5 5 . 3}$ \\
\hline $\mathbf{1 3 0 2 3}$ & 1392 & 1376 & 1390 & $\mathbf{1 3 8 6 . 0}$ \\
\hline $\mathbf{1 3 6 6 . 3}$ & 1502 & 1516 & 1552 & $\mathbf{1 5 2 3 . 3}$ \\
\hline $\mathbf{1 4 9 2 7}$ & 1690 & 1672 & 1714 & $\mathbf{1 6 9 2 . 0}$ \\
\hline $\mathbf{1 4 9 5 . 0}$ & 1740 & 1785 & 1829 & $\mathbf{1 7 8 4 . 7}$ \\
\hline $\mathbf{1 4 9 5 . 0}$ & 1743 & 1609 & 1753 & $\mathbf{1 7 0 1 . 7}$ \\
\hline $\mathbf{1 3 7 7 . 0}$ & 1487 & 1509 & 1617 & $\mathbf{1 5 3 7 . 7}$ \\
\hline $\mathbf{1 2 9 0 . 3}$ & 1422 & 1257 & 1491 & $\mathbf{1 3 9 0 . 0}$ \\
\hline $\mathbf{1 2 9 4 . 0}$ & 1381 & 1220 & 1307 & $\mathbf{1 3 0 2 . 7}$ \\
\hline $\mathbf{1 3 6 8 . 4}$ & $\mathbf{1 5 1 8 . 2}$ & $\mathbf{1 4 5 6 . 1}$ & $\mathbf{1 5 5 0 . 1}$ & $\mathbf{1 5 0 8 . 1}$ \\
\hline
\end{tabular}

\begin{tabular}{|lrrrr|} 
Center 2/3 & Side & Bottom & $\underline{\text { All }}$ & Normlzd \\
\hline Meen & 1402.7 & 1573.6 & 1488.1 & 1624.03 \\
Std. Dev. & 91.1 & 156.9 & 151.9 & 139.85 \\
\hline COV as \% & 6.5 & 10.0 & 10.2 & 8.61 \\
\hline
\end{tabular}

\section{Instuments Usect}

TSI VelciCalc $\quad$ T95351203001

$12 / 10 / 2013$

\begin{tabular}{lrc}
\hline Fisher Scientific & 90936818 & \multicolumn{1}{c}{$12 / 11 / 2013$} \\
\hline Met One OPC (Ref) & 96258675 & FIO \\
\hline Met One OPC & 1011529009 & $1 / 14 / 2014$ \\
\hline
\end{tabular}
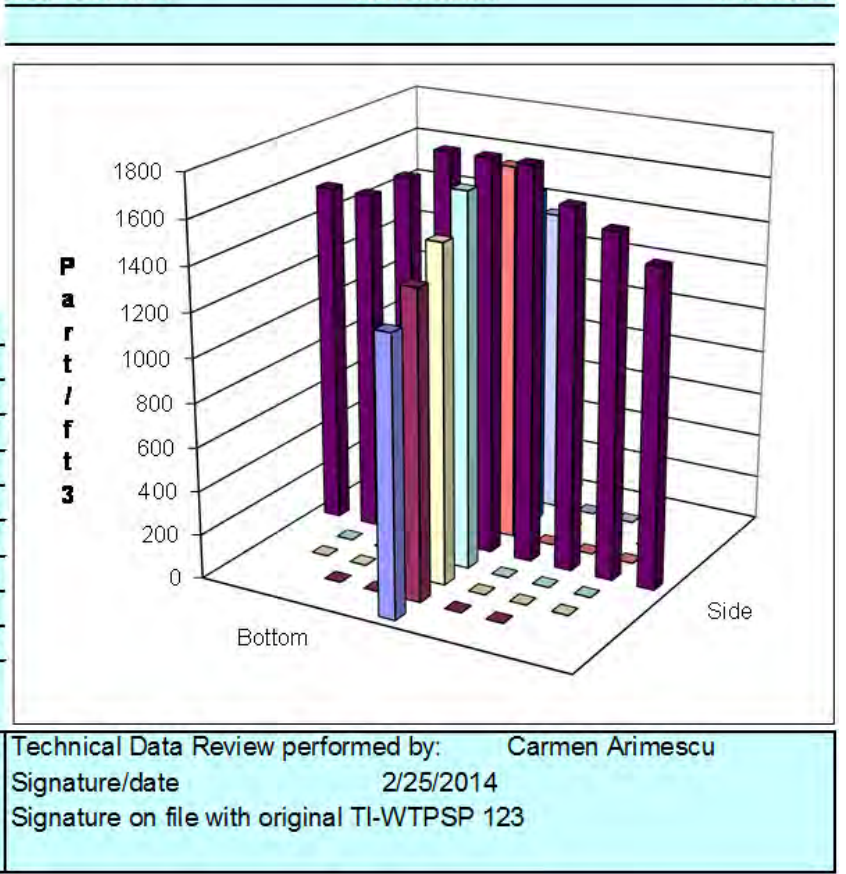

D.140 
Rev. 0

3 Aug. 2006

PARTICLE TRACER TRAVERSE DATA FORM

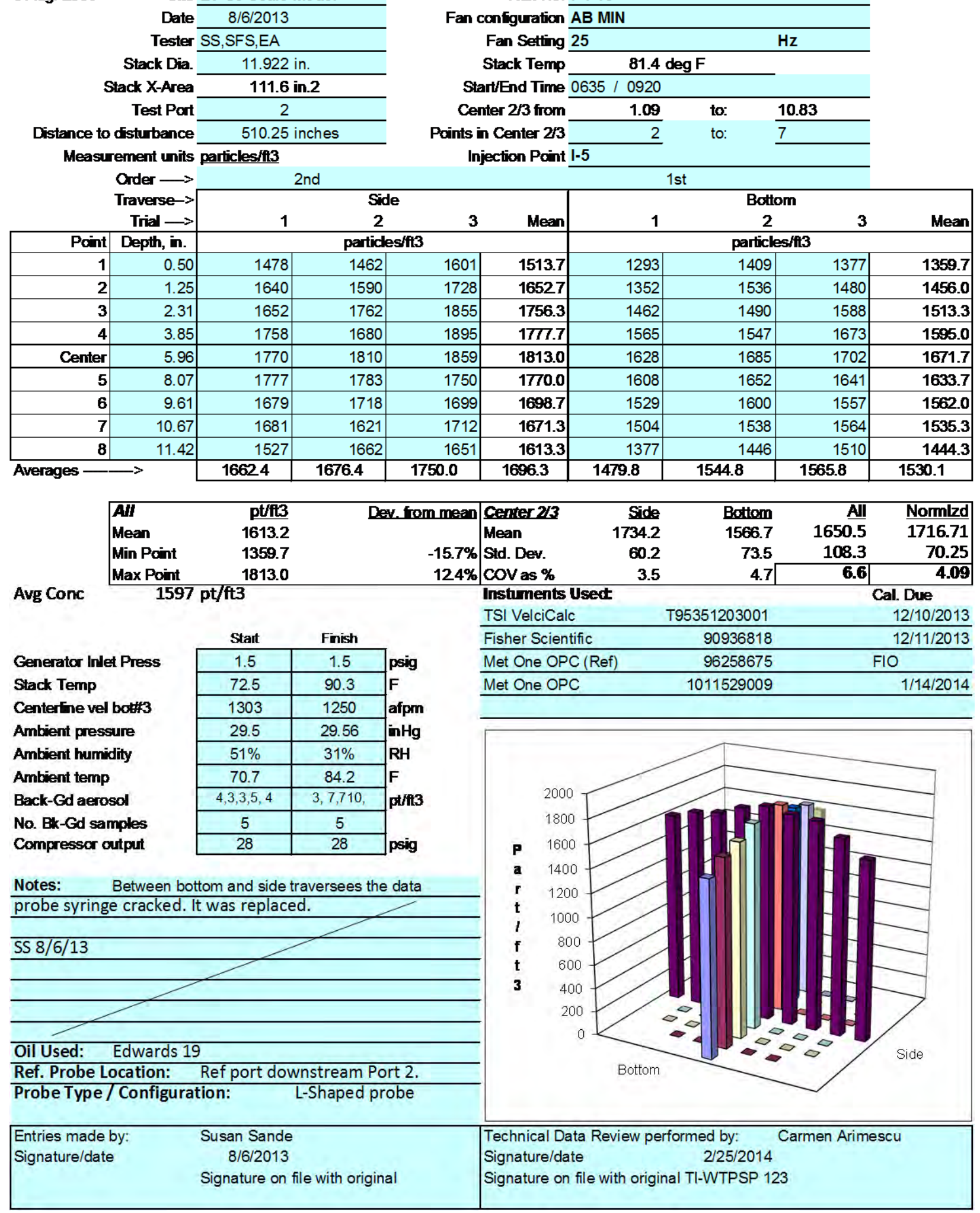


Rev. 0

3 Aug. 2006

Site LV-S3 Scale Model

Date 8/6/2013

Tester SS,SFS

Stack Dia

11.922 in

Stack X-Area

Test Part

111.6 in. 2

Distance to disturbance

Measurement units particles/ft3 510.25 inches

\begin{tabular}{|r|r|}
\multicolumn{2}{c}{$\begin{array}{c}\text { Order } \\
\text { Traverse-> } \\
\text { Trial } \longrightarrow\end{array}$} \\
\hline Point & Depth, in. \\
\hline $\mathbf{1}$ & 0.50 \\
\hline $\mathbf{2}$ & 1.25 \\
\hline $\mathbf{3}$ & 2.31 \\
\hline $\mathbf{4}$ & 3.85 \\
\hline Center & 5.96 \\
\hline $\mathbf{5}$ & 8.07 \\
\hline $\mathbf{6}$ & 9.61 \\
\hline $\mathbf{7}$ & 10.67 \\
\hline $\mathbf{8}$ & 11.42 \\
\hline
\end{tabular}

\begin{tabular}{|lr}
\hline All & $\mathrm{pt} / \mathrm{ft} 3$ \\
Mean & 2065.6 \\
Min Point & 17320 \\
\hline
\end{tabular}

Avg Conc

Min Point

2421.0

$$
2039 \mathrm{pt} / \mathrm{ft} 3
$$

Generator Inlet Press

Stack Temp

Centerline vel bo\#3

Ambient pressure

Ambient humidity

Ambient temp

Back-Gd aerosol

No. Bk-Gd samples

Compressor output

\begin{tabular}{|c|c|}
\hline Stat & Finish \\
\hline 1.5 & 1.5 \\
\hline 90.3 & 103.3 \\
\hline 1250 & 1243 \\
\hline 29.56 & 29.56 \\
\hline $31 \%$ & $23 \%$ \\
\hline 84.2 & 91.4 \\
\hline $9,7,9,8,24,11$ & $2,9,2,4,7$ \\
\hline 6 & 5 \\
\hline 28 & 24 \\
\hline
\end{tabular}

Notes:

SS8/6/13

Oil Used: Edwards 19

Ref. Probe Location: Ref port downstream Port 2.

Probe Type/Configuration:

L-Shaped probe

Entries made by:

Signature/date

Susan Sande
$8 / 6 / 2013$
Signature on file with original

Dev. from mean

Run No. PT-19

Fan configuration AB MIN

Fan Setting 25

Stack Temp

$96.8 \operatorname{deg} F$

Start/End Time $0924 / 1100$

Center $2 / 3$ from

Points in Center $2 / 3$

Injection Point I-5

1.09

to:

to: $\mathbf{1 0 . 8 3}$

to: 7

2nd

\begin{tabular}{|r|r|r|r|r|}
\cline { 2 - 4 } \multicolumn{2}{c|}{ 2nd } \\
\hline & \multicolumn{5}{|c|}{$\begin{array}{c}\text { Bottom } \\
\mathbf{2}\end{array}$} \\
\hline $\mathbf{1 7 3 2 0}$ & 2045 & 1978 & 2047 & $\mathbf{2 0 2 3 . 3}$ \\
\hline $\mathbf{1 9 1 8 . 0}$ & 2075 & 2067 & 2151 & $\mathbf{2 0 9 7 . 7}$ \\
\hline $\mathbf{1 9 4 5 . 3}$ & 2155 & 2183 & 2178 & $\mathbf{2 1 7 2 . 0}$ \\
\hline $\mathbf{2 0 9 0 . 7}$ & 2378 & 2376 & 2509 & $\mathbf{2 4 2 1 . 0}$ \\
\hline $\mathbf{2 1 5 8 . 0}$ & 2349 & 2452 & 2390 & $\mathbf{2 3 9 7 . 0}$ \\
\hline $\mathbf{2 0 8 5 . 3}$ & 2295 & 2375 & 2352 & $\mathbf{2 3 4 0 . 7}$ \\
\hline $\mathbf{1 8 9 8 . 0}$ & 2144 & 2194 & 2192 & $\mathbf{2 1 7 6 . 7}$ \\
\hline $\mathbf{1 8 6 7 . 3}$ & 2071 & 2079 & 2116 & $\mathbf{2 0 8 8 . 7}$ \\
\hline $\mathbf{1 8 3 2 0}$ & 1957 & 1972 & 1884 & $\mathbf{1 9 3 7 . 7}$ \\
\hline $\mathbf{1 9 4 7 . 4}$ & $\mathbf{2 1 6 3 . 2}$ & $\mathbf{2 1 8 6 . 2}$ & $\mathbf{2 2 0 2 1}$ & $\mathbf{2 1 8 3 . 9}$ \\
\hline
\end{tabular}

Center 213

Mean

Side

1994.7

Bottom

22420

Std. Dev.

114.0

Instuments Usect

5.7

141.0

2118 .

All Normlzd

TSI VelciCalc

Fisher Scientific

Met One OPC (Ref)

Met One OPC

6.3

177.9

2228.76

129.48

\begin{tabular}{lrl} 
& 1011529009 & $1 / 14 / 2014$ \\
\hline
\end{tabular}

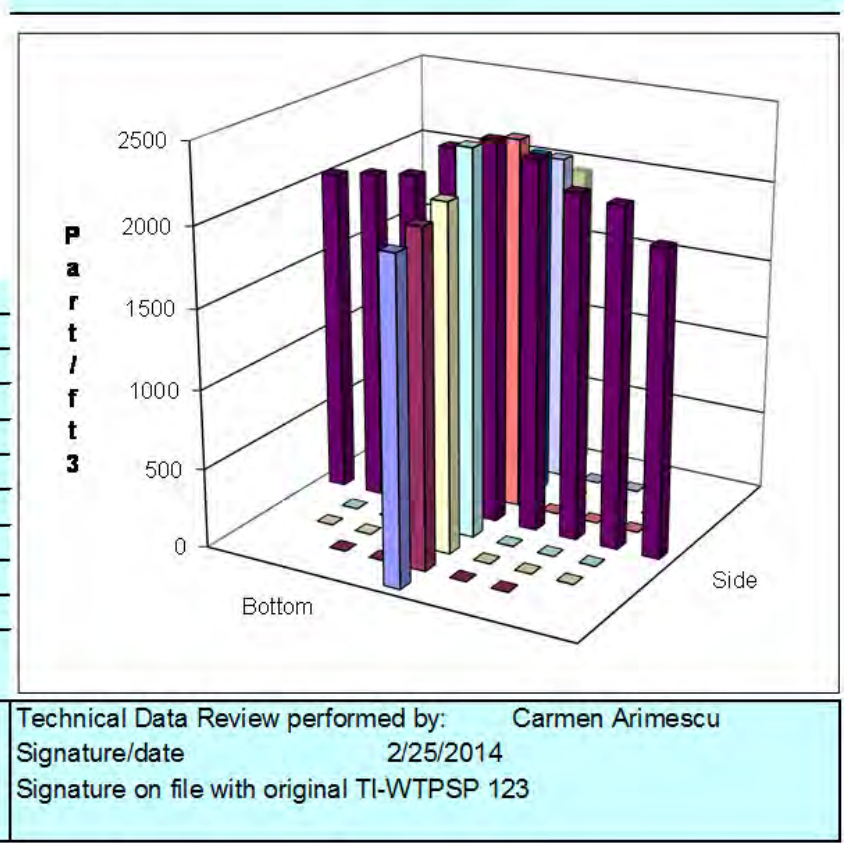


Rev. 0

3 Aug. 2006

PARTICLE TRACER TRAVERSE DATA FORM

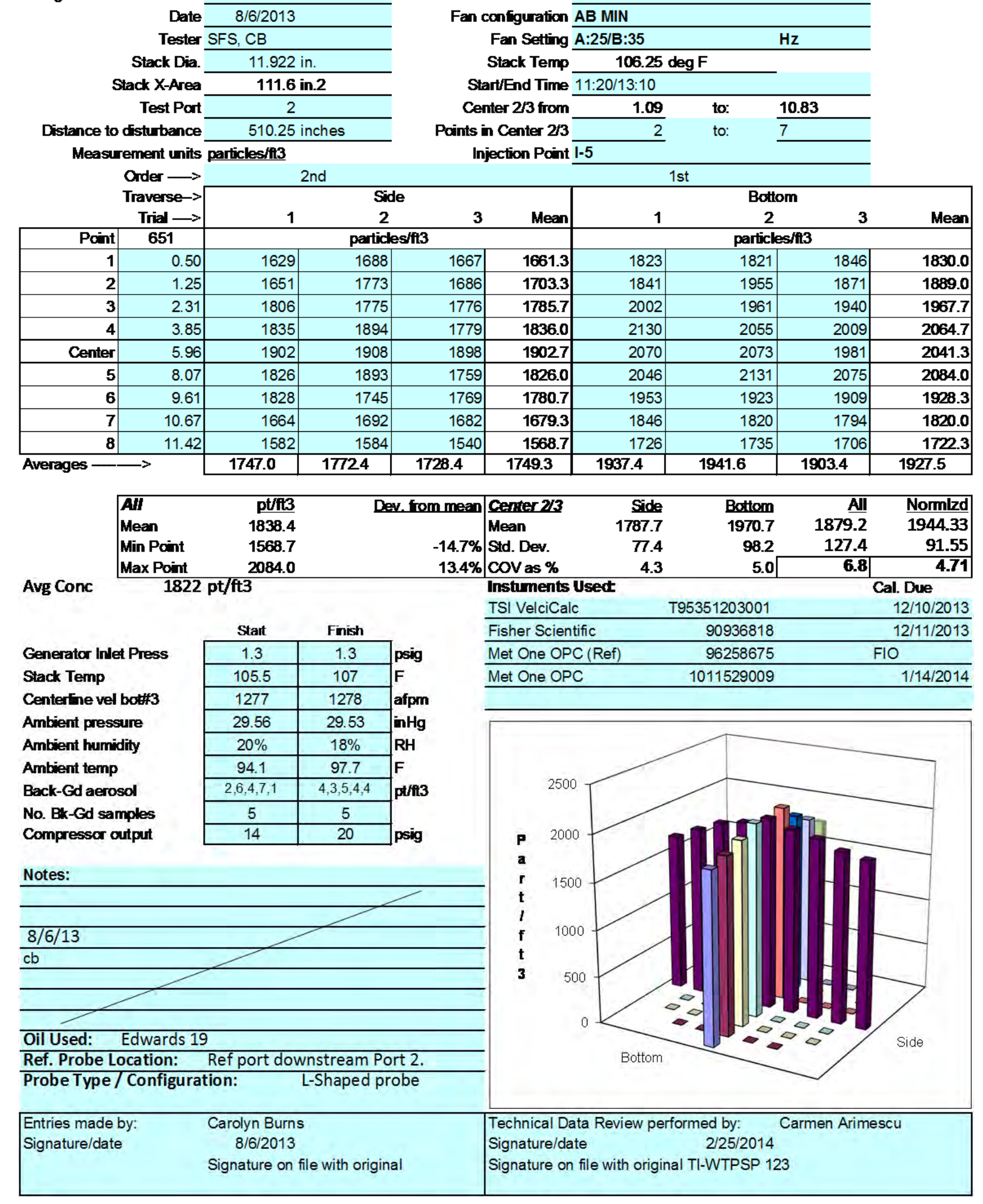


Rev. 0

3 Aug. 2006

PARTICLE TRACER TRAVERSE DATA FORM

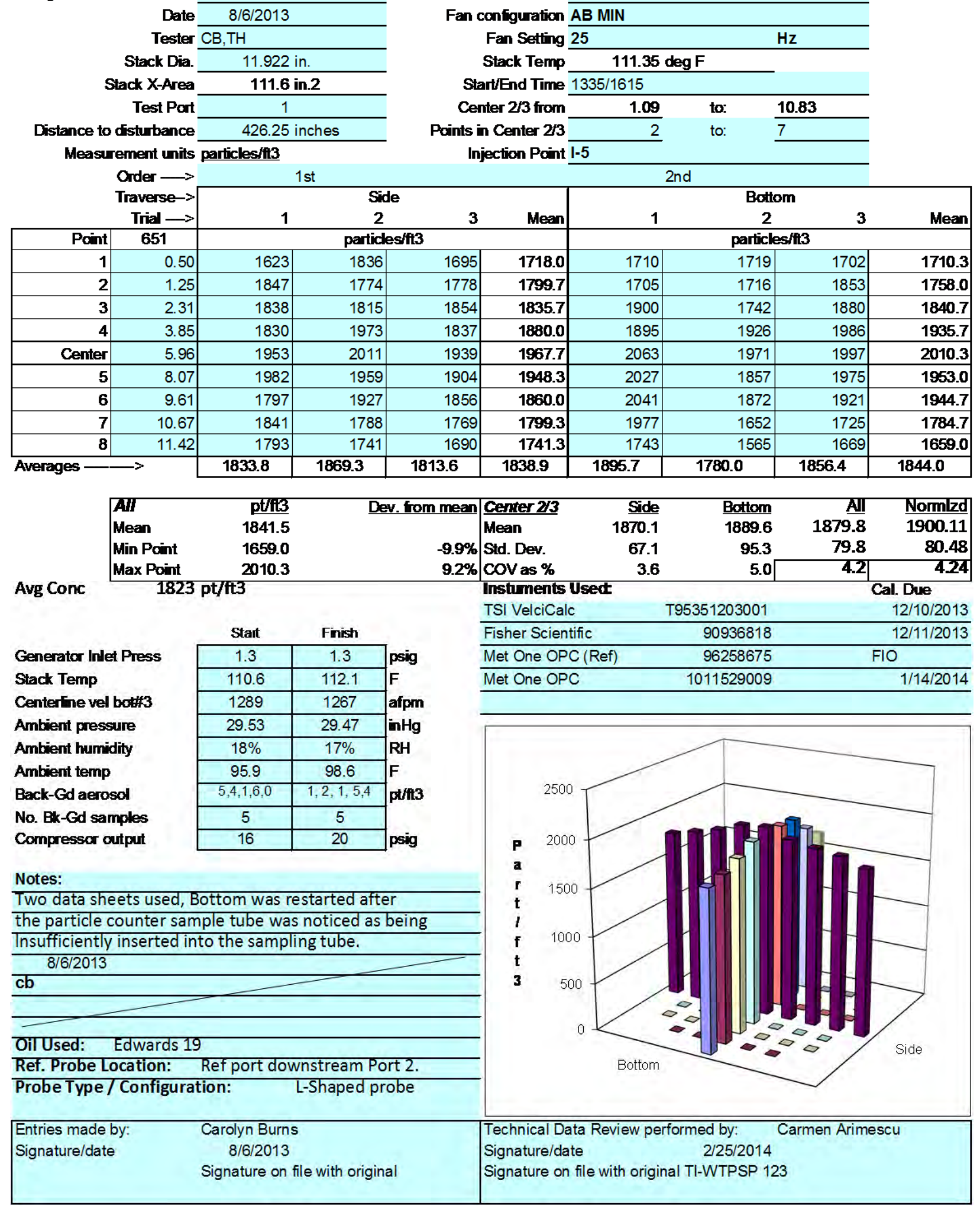


Rev. 0

3 Aug. 2006

PARTICLE TRACER TRAVERSE DATA FORM

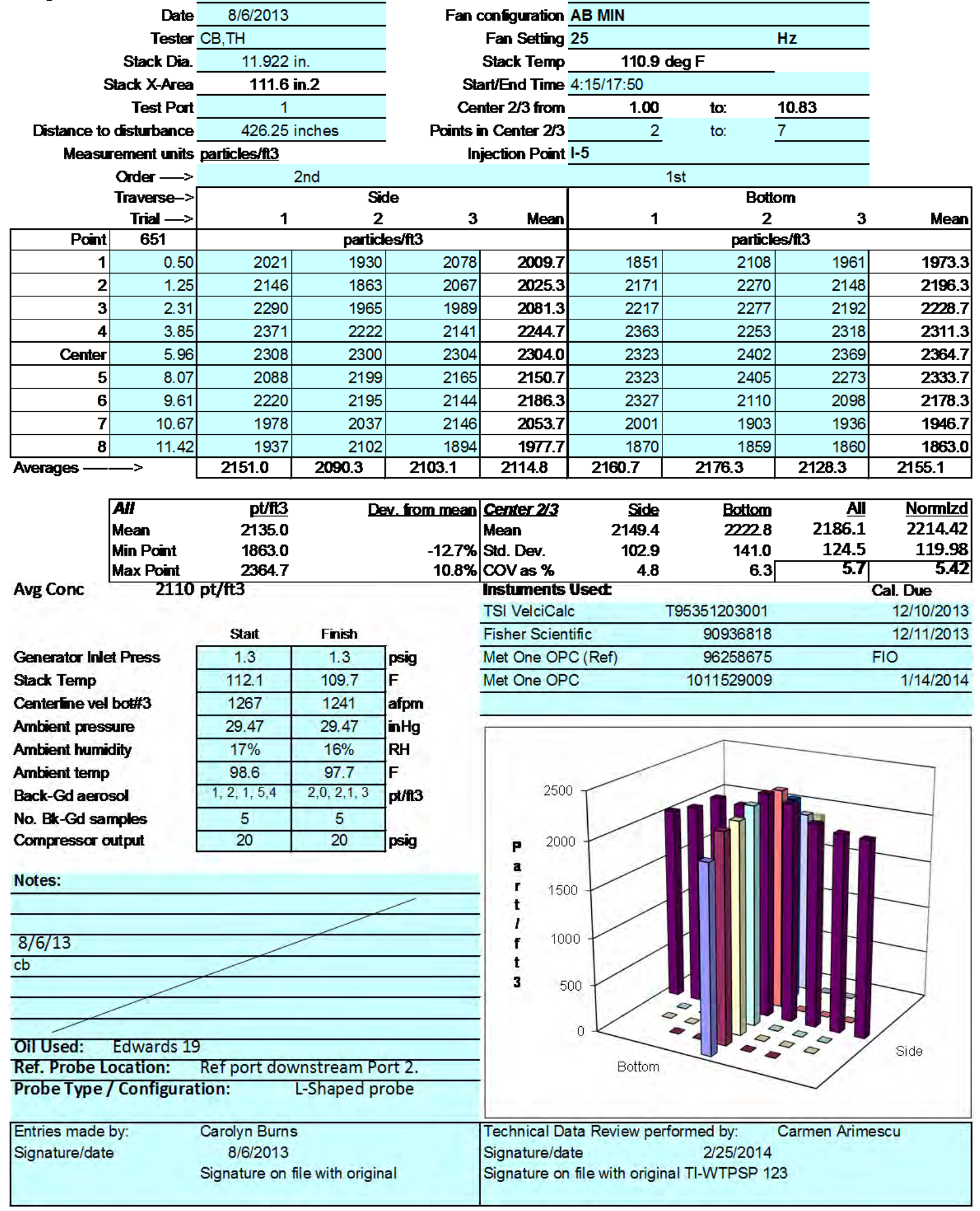


Rev. 0

3 Aug. 2006

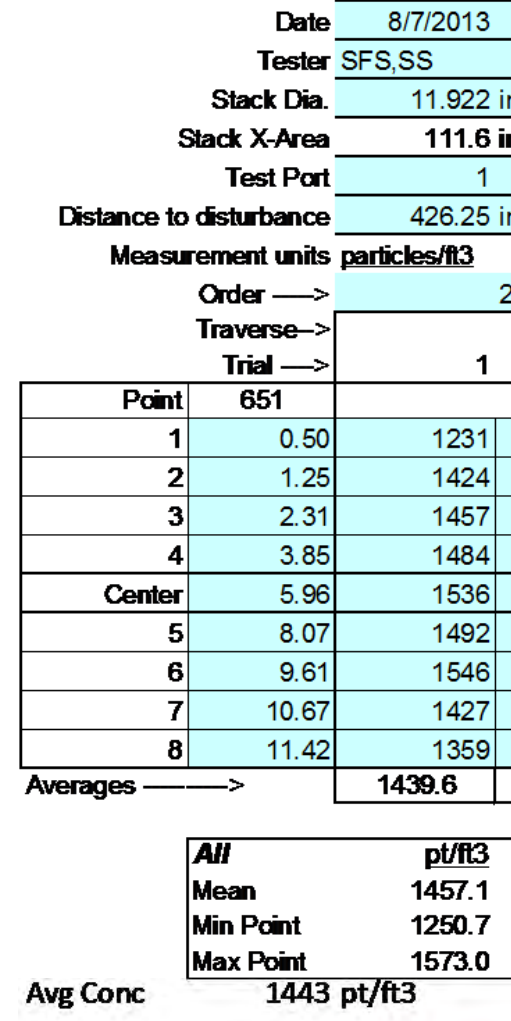

Generator Inlet Press Stack Temp

Centerline vel bo\#3

Ambient pressure

Ambient humidity

Ambient temp

Back-Gd aerosol

No. Bk-Gd samples

Compresscr output

\begin{tabular}{|c|c|}
\hline Stat & Finish \\
\hline 1.6 & 1.6 \\
\hline 70.2 & 84.9 \\
\hline 1325 & 1254 \\
\hline 29.5 & 29.53 \\
\hline $50 \%$ & $32 \%$ \\
\hline 67.1 & 78.8 \\
\hline $1,1,1,4,0$ & $3,2,7,3,3$ \\
\hline 5 & 5 \\
\hline 28 & 30 \\
\hline
\end{tabular}

Notes:

$8 / 7 / 13$

Oil Used: Edwards 19

Ref. Probe Location: Ref port downstream Port 2.

Probe Type/Configuration: L-Shaped probe

\begin{tabular}{|lc|}
\hline $\begin{array}{l}\text { Entries made by: } \\
\text { Signature/date }\end{array}$ & Susan Sande \\
& $8 / 7 / 2013$ \\
& Signature on file with original \\
\hline
\end{tabular}

Rin No.

\begin{tabular}{|c|c|c|c|c|c|}
\hline \multicolumn{5}{|c|}{ Run No. PT-23 } & \\
\hline \multicolumn{5}{|c|}{ Fan configuration $\underline{A B}$ MIN } & \\
\hline \multicolumn{3}{|c|}{ Fan Setting 25} & \multicolumn{2}{|r|}{$\mathrm{Hz}$} & \\
\hline \multicolumn{2}{|c|}{ Stack Temp } & \multicolumn{2}{|c|}{$77.55 \operatorname{deg} F$} & & \\
\hline \multicolumn{5}{|c|}{ Start/End Time $0625 / 0825$} & \\
\hline \multicolumn{2}{|c|}{ Center $2 / 3$ from } & 1.09 & to: & 10.83 & \\
\hline \multicolumn{2}{|c|}{ Points in Center $2 / 3$} & $\underline{2}$ & to: & \multirow[t]{2}{*}{$\underline{7}$} & \\
\hline \multicolumn{4}{|c|}{ Injection Point I-5 } & & \\
\hline \multicolumn{6}{|c|}{ 1st } \\
\hline \multirow{3}{*}{3} & \multirow{3}{*}{ Meen } & \multicolumn{3}{|c|}{ Bottom } & \multirow{3}{*}{ Mean } \\
\hline & & 1 & 2 & 3 & \\
\hline & & \multicolumn{3}{|c|}{ particles/ft3 } & \\
\hline 1378 & 1329.7 & 1265 & 1299 & 1336 & 1300.0 \\
\hline 1501 & 1465.7 & 1261 & 1411 & 1421 & 1364.3 \\
\hline 1638 & 1539.0 & 1356 & 1449 & 1527 & 1444.0 \\
\hline 1566 & 15623 & 1425 & 1512 & 1584 & 1507.0 \\
\hline 1668 & 1573.0 & 1522 & 1543 & 1610 & 1558.3 \\
\hline 1619 & 1564.0 & 1500 & 1551 & 1552 & 1534.3 \\
\hline 1554 & 1523.3 & 1413 & 1508 & 1550 & 1490.3 \\
\hline 1573 & 1498.0 & 1253 & 1306 & 1421 & 1326.7 \\
\hline 1494 & 1396.3 & 1244 & 1243 & 1265 & 1250.7 \\
\hline 4.6 & 1494.6 & 1359.9 & 1424.7 & 1474.0 & 1419.5 \\
\hline
\end{tabular}

\begin{tabular}{r|lrrrr|}
\hline Dev. from mean & Center 23 & Side & Bottom & All & Normlzd \\
\cline { 2 - 6 } & Mean & 1532.2 & 1460.7 & 1496.5 & 1503.33 \\
$8.0 \%$ & Std. Dev. & 39.4 & 87.1 & 74.8 & 71.97 \\
\hline
\end{tabular}
Instuments Used

\begin{tabular}{lrr} 
TSI VelciCalc & T95351203001 & \multicolumn{1}{c}{$12 / 10 / 2013$} \\
\hline Fisher Scientific & 90936818 & $12 / 11 / 2013$ \\
\hline Met One OPC (Ref) & 96258675 & FIO \\
\hline Met One OPC & 1011529009 & $1 / 14 / 2014$ \\
\hline
\end{tabular}

Signature on file with original TI-WTPSP 123


Rev. 0

3 Aug. 2006

Site LV-S3 Scale Model

Date 8/7/2013

Tester SFS,SS

Stack Dia

11.922 in

Stack X-Area

Test Part

111.6 in.

Distance to distumance

Measurement units particles/ti? 426.25 inches

\begin{tabular}{|r|r|}
\multicolumn{1}{|c}{$\begin{array}{r}\text { Order } \longrightarrow \\
\text { Traverse } \rightarrow \\
\text { Trial } \longrightarrow\end{array}$} \\
\hline Point & \multicolumn{1}{c|}{$\mathbf{6 5 1}$} \\
\hline $\mathbf{1}$ & 0.50 \\
\hline $\mathbf{2}$ & 1.25 \\
\hline $\mathbf{3}$ & 2.31 \\
\hline $\mathbf{4}$ & 3.85 \\
\hline Center & 5.96 \\
\hline $\mathbf{5}$ & 8.07 \\
\hline $\mathbf{6}$ & 9.61 \\
\hline $\mathbf{7}$ & 10.67 \\
\hline $\mathbf{8}$ & 11.42 \\
\hline
\end{tabular}

$\rightarrow$

$1 \mathrm{st}$

1st Side

\begin{tabular}{r|r|r|}
\hline & \multicolumn{1}{|c}{ per } \\
\hline 50 & 1049 & 12 \\
\hline $\mathbf{2 5}$ & 1128 & 11 \\
\hline 31 & 1369 & 14 \\
\hline $\mathbf{8 5}$ & 1579 & 15 \\
\hline $\mathbf{0 7}$ & 1583 & 16 \\
\hline 61 & 1589 & 16 \\
\hline 67 & 1424 & 14 \\
\hline 42 & 1273 & 12 \\
\hline & $\mathbf{1 3 3 1 . 4}$ & 11 \\
\hline
\end{tabular}

\section{CER TRAVERSE DATA FORM}

Run No. PT-24

Fan configuration $A B$ Max

Fan Setting 45

Stack Temp

Start/End Time 08:55/10:37

Center $2 / 3$ from

Points in Center $2 / 3$

Injection Point I-5
$0: 37$

1.09 to

2 to:

to: 7

2nd

23

\begin{tabular}{|lr|}
\hline All & pt/fit3 \\
Mean & 1497.0 \\
Min Point & 1078.3 \\
Max Point & 2049.0 \\
\hline
\end{tabular}

Avg Conc $1453 \mathrm{pt} / \mathrm{ft} 3$

Generator Inlet Press

Stack Temp

Centerfine vel bo\#3

Ambient pressure

Ambient humidity

Ambient temp

Back-Gd aerosol

No. Bk-Gd samples

Compressar output

\begin{tabular}{|c|c|c|}
\hline Stat & Finish & \\
\hline 2.3 & 2.3 & psig \\
\hline 88.10 & 100.3 & $\mathrm{~F}$ \\
\hline 2458 & 2571 & afpn \\
\hline 29.53 & 29.56 & $\mathrm{inHg}$ \\
\hline $27 \%$ & $20 \%$ & RH \\
\hline 84.2 & 91.4 & $\mathrm{~F}$ \\
\hline $7,0,1,2,3$ & $3,2,3,1,0$ & $\mathrm{t} / \mathrm{t}$ \\
\hline 5 & 5 & \\
\hline 23 & 23 & psig \\
\hline
\end{tabular}

Notes:

\begin{tabular}{l} 
SS \\
\hline $8 / 7 / 13$ \\
\hline
\end{tabular}

OाUsed: Edwards 19

Ref. Probe Location: Ref port downstream Port 2.

Probe Type/Configuration: L-Shaped probe

\begin{tabular}{|lc|}
\hline Entries made by: & Susan Sande \\
Signature/date & $8 / 7 / 2013$ \\
& Signature on file with original \\
& \\
\hline
\end{tabular}

\begin{tabular}{|r|r|r|r|r|}
\cline { 2 - 4 } Meen & \multicolumn{5}{|c|}{ 2nd } \\
\hline & $\mathbf{5}$ & \multicolumn{3}{c|}{$\begin{array}{c}\text { Boftom } \\
\text { perticles/ti3 }\end{array}$} \\
\hline $\mathbf{1 1 2 2 7}$ & 1250 & 1319 & 1246 & $\mathbf{1 2 7 1 . 7}$ \\
\hline $\mathbf{1 2 0 4 . 0}$ & 1450 & 1683 & 1471 & $\mathbf{1 5 3 4 . 7}$ \\
\hline $\mathbf{1 4 5 1 . 7}$ & 1663 & 1673 & 1699 & $\mathbf{1 6 7 8 . 3}$ \\
\hline $\mathbf{1 5 5 5 . 3}$ & 1861 & 1846 & 1789 & $\mathbf{1 8 3 2 . 0}$ \\
\hline $\mathbf{1 6 4 2 7}$ & 2039 & 2091 & 2017 & $\mathbf{2 0 4 9 . 0}$ \\
\hline $\mathbf{1 6 5 5 . 3}$ & 1931 & 1896 & 1991 & $\mathbf{1 9 3 9 . 3}$ \\
\hline $\mathbf{1 4 6 7 . 3}$ & 1785 & 1709 & 1702 & $\mathbf{1 7 3 2 . 0}$ \\
\hline $\mathbf{1 3 0 8 . 3}$ & 1305 & 1269 & 1341 & $\mathbf{1 3 0 5 . 0}$ \\
\hline $\mathbf{1 0 7 8 . 3}$ & 1186 & 1063 & 1104 & $\mathbf{1 1 1 7 . 7}$ \\
\hline $\mathbf{1 3 8 7 . 3}$ & $\mathbf{1 6 0 7 . 8}$ & $\mathbf{1 6 1 6 . 6}$ & $\mathbf{1 5 9 5 . 6}$ & $\mathbf{1 6 0 6 . 6}$ \\
\hline
\end{tabular}

Center 2/3

Mean

$-28.0 \%$ Std. Dev.

Side

1469.2

Bottom

1724.3

250.7

COV as \%

Instuments Usect

TSI VelciCalc

\begin{tabular}{l} 
Fisher Scientific \\
\hline Met One OPC (Ref) \\
\hline Met One OPC
\end{tabular}

11.4

14.5

\begin{tabular}{rr|}
\hline All & Norm $17 \mathrm{zd}$ \\
\hline 1596.8 & 1778.50 \\
243.9 & 228.83 \\
\hline 15.3 & 12.87 \\
\hline
\end{tabular}

T95351203001

Cal. Due

$12 / 10 / 2013$

$12 / 11 / 2013$

\begin{tabular}{lll}
\hline Met One OPC & 1011529009 & $1 / 14 / 2014$ \\
\hline
\end{tabular}

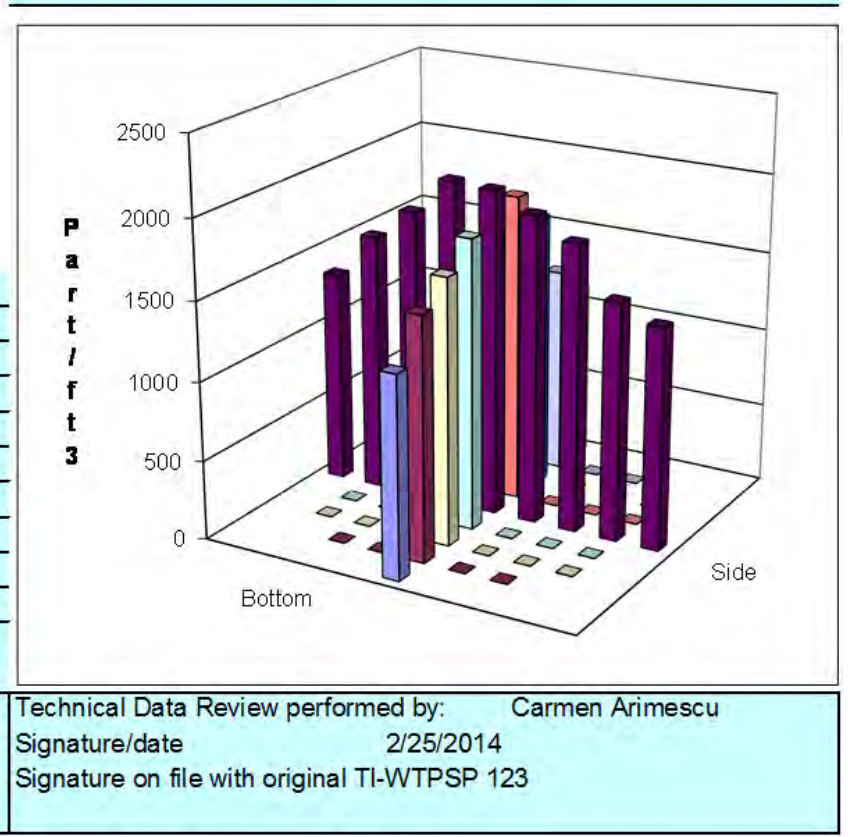


Rev. 0

3 Aug. 2006
Site LV-S3 Scale Model

Date 8/7/2013

Tester SFS,SS

Stack Dia.

Stack X-Area

Test Part

11.922 in

111.6 in. 2

1

Distance to disturbance 426.25 inches

Measurement units particles/ti3

Order $\longrightarrow$

Traverse-

Trial $\rightarrow$

$2 n$

\section{ERACE} -

-

-

$+$

(n)

Side

\section{CER TRAVERSE DATA FORM}

\begin{tabular}{|c|c|c|c|c|c|}
\hline \multicolumn{5}{|c|}{ Run No. PT-25 } & \\
\hline \multicolumn{5}{|c|}{ Fan configuration AB Norm } & \\
\hline \multicolumn{3}{|c|}{ Fan Setting 38} & \multicolumn{2}{|r|}{$\mathrm{Hz}$} & \\
\hline \multicolumn{2}{|c|}{ Stack Temp } & \multicolumn{2}{|c|}{$103.6 \operatorname{deg} F$} & & \\
\hline \multicolumn{5}{|c|}{ Start/End Time 10:44/12:20 } & \\
\hline \multicolumn{2}{|c|}{ Center $2 / 3$ from } & 1.09 & to: & 10.83 & \\
\hline \multicolumn{2}{|c|}{ Points in Center $2 / 3$} & 2 & to: & 7 & \\
\hline \multicolumn{5}{|c|}{ Injection Pcint I-5 } & \\
\hline \multicolumn{6}{|c|}{ 1st } \\
\hline \multirow{3}{*}{3} & \multirow{3}{*}{ Meen } & \multicolumn{3}{|c|}{ Bottom } & \multirow{3}{*}{ Mean } \\
\hline & & 1 & 2 & 3 & \\
\hline & & \multicolumn{3}{|c|}{ particles/ft3 } & \\
\hline 1606 & 16620 & 1493 & 1714 & 1717 & 1641.3 \\
\hline 1837 & 1851.7 & 1762 & 1876 & 1945 & 1861.0 \\
\hline 1894 & 1953.7 & 2057 & 2112 & 2077 & 2082.0 \\
\hline 2141 & 2090.3 & 2348 & 2344 & 2394 & 2362.0 \\
\hline 2207 & 2223.7 & 2312 & 2434 & 2362 & 2369.3 \\
\hline 2174 & 2125.7 & 2237 & 2381 & 2393 & 2337.0 \\
\hline 1992 & 19723 & 2116 & 2224 & 2142 & 2160.7 \\
\hline 1840 & 1828.3 & 1624 & 1682 & 1638 & 1648.0 \\
\hline 1583 & 1550.7 & 1437 & 1526 & 1500 & 1487.7 \\
\hline 919.3 & 1917.6 & 1931.8 & 20326 & 2018.7 & 1994.3 \\
\hline
\end{tabular}


Rev. 0

3 Aug. 2006

\begin{tabular}{|c|c|c|}
\hline & Date & $8 / 7 / 2013$ \\
\hline & Tester & b, TH \\
\hline & Stack Dia. & 11.922 in \\
\hline & Stack X-Area & 111.6 in \\
\hline & Test Part & 1 \\
\hline Distance to & disturbance & 426.25 in \\
\hline Measu & rement units & articles/fi3 \\
\hline & Order $\longrightarrow$ & 1 \\
\hline & Traverse $->$ & \\
\hline & Trial $\longrightarrow$ & 1 \\
\hline Point & 651 & \\
\hline 1 & 0.50 & 1776 \\
\hline 2 & 1.25 & 1980 \\
\hline 3 & 2.31 & 2118 \\
\hline 4 & 3.85 & 2331 \\
\hline Center & 5.96 & 2456 \\
\hline 5 & 8.07 & 2304 \\
\hline 6 & 9.61 & 2078 \\
\hline 7 & 10.67 & 1951 \\
\hline 8 & 11.42 & 1812 \\
\hline Averages - & $\longrightarrow$ & 2089.6 \\
\hline & AWI & $\mathrm{pt} / \mathrm{it} 3$ \\
\hline & Mean & 2190.2 \\
\hline & Min Point & 1727.0 \\
\hline & Max Point & 2661.3 \\
\hline Avg Conc & 2141 & $7 \mathrm{ft3}$ \\
\hline
\end{tabular}

Generator Inlet Press

Stack Temp

Centerine vel bot\#3

Ambient pressure

Ambient humidity

Ambient temp

Back-Gd aerosol

No. Bk-Gd samples

Compressar output

\begin{tabular}{|c|c|}
\hline Stat & Finish \\
\hline 2.1 & 2.1 \\
\hline 109.2 & 109.1 \\
\hline 1901 & 2005 \\
\hline 29.5 & 29.47 \\
\hline $18 \%$ & $18 \%$ \\
\hline 96.8 & 96.8 \\
\hline $1,3,3,1,1$ & $1,1,3,1,4$ \\
\hline 5 & 5 \\
\hline 18 & 14 \\
\hline
\end{tabular}

Notes:

Cb
Ref. Probe Location: Ref port downstream Port 2.
Probe Type /Configuration: L-Shaped probe

\begin{tabular}{|lc|l|}
\hline Entries made by: & Carolyn Burns & Tech \\
Signature/date & $8 / 7 / 2013$ & Si \\
& Signature on file with original & Sign \\
& & \\
\hline
\end{tabular}

1 st

Side

\begin{tabular}{l|r|}
2168.0 & 2229.4 \\
\hline
\end{tabular}

\begin{tabular}{r|r|r|}
$-21.1 \%$ & \\
$21.5 \%$ & &
\end{tabular}

Fan configuration $\mathrm{AB}$ Norm

Fan Setting 38

Stack Temp

Start/End Time 13:40/15:25

Center 2/3 from

Points in Center 233

Injection Point I-5

$\begin{array}{rll}1.09 & \text { to: } & 10.83 \\ 2 & \text { to: } & \end{array}$

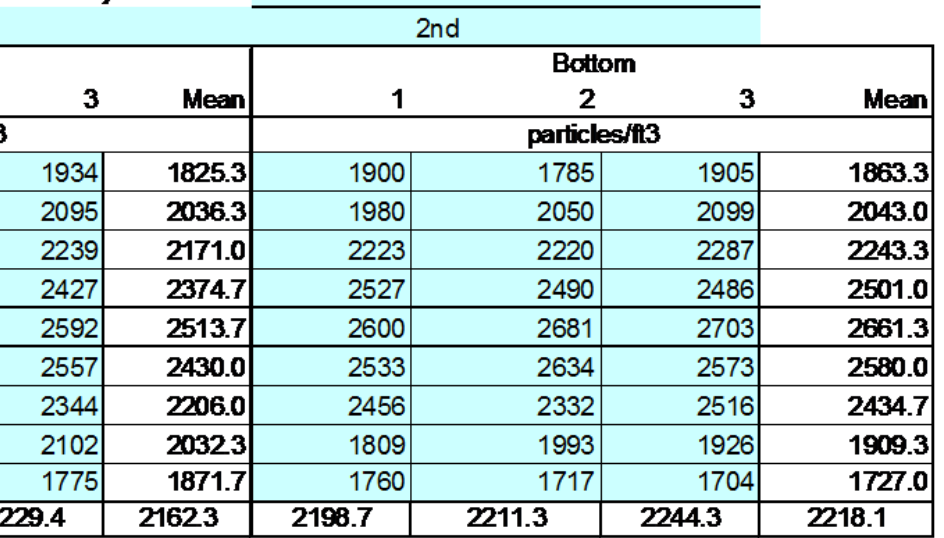

21623

\begin{tabular}{|lrrrr|} 
Center 23 & Side & $\underline{\text { Bottom }}$ & All & Normlzd \\
\hline Meen & 2252.0 & 2339.0 & 2295.5 & 2361.62 \\
Std. Dev. & 190.9 & 2825 & 236.0 & 237.14 \\
\hline COV as \% & 8.5 & 121 & 10.3 & 10.04 \\
\hline
\end{tabular}
Instuments Usect

\begin{tabular}{lrr} 
TSI VelciCalc & T95351203001 & \multicolumn{1}{c}{ Cal. Due } \\
$12 / 10 / 2013$ \\
\hline Fisher Scientific & 90936818 & $12 / 11 / 2013$ \\
\hline Met One OPC (Ref) & 96258675 & $\mathrm{FIO}$ \\
\hline Met One OPC & 1011529009 & $1 / 14 / 2014$ \\
\hline & & \\
\hline
\end{tabular}

Signature/date


Rev. 0

3 Aug. 2006

\begin{tabular}{|c|c|c|}
\hline & Date & $8 / 7 / 2013$ \\
\hline \multicolumn{3}{|c|}{ Tester $\mathrm{cb}, \mathrm{TH}$} \\
\hline \multirow{2}{*}{\multicolumn{2}{|c|}{$\begin{array}{r}\text { Stack Dia. } \\
\text { Stack X-Area }\end{array}$}} & 11.922 in. \\
\hline & & 111.6 in \\
\hline \multicolumn{2}{|r|}{ Test Port } & 1 \\
\hline \multicolumn{2}{|c|}{ Distance to disturbance } & $426.25 \mathrm{in}$ \\
\hline \multicolumn{3}{|c|}{ Measurement units particles/fi3 } \\
\hline \multirow{2}{*}{\multicolumn{2}{|c|}{$\begin{array}{l}\text { Order } \longrightarrow \\
\text { Traverse-> }\end{array}$}} & $2 n$ \\
\hline & & \\
\hline \multicolumn{2}{|r|}{ Trial $\longrightarrow$} & 1 \\
\hline \multicolumn{2}{|l|}{ Point } & \\
\hline 1 & 0.50 & 1934 \\
\hline 2 & 1.25 & 2203 \\
\hline \multirow{2}{*}{3} & 2.31 & 2286 \\
\hline & 3.85 & 2395 \\
\hline $\begin{array}{r}4 \\
\text { ter }\end{array}$ & 5.96 & 2629 \\
\hline 5 & 8.07 & 2579 \\
\hline 6 & 9.61 & 2384 \\
\hline 7 & 10.67 & 1940 \\
\hline \multirow{2}{*}{$\begin{array}{r}8 \\
\text { Averages } \\
\end{array}$} & 11.42 & 1939 \\
\hline & Averages $\longrightarrow$ & 2254.3 \\
\hline \multirow{4}{*}{\multicolumn{2}{|c|}{\begin{tabular}{|l|} 
All \\
Mean \\
Min Point \\
Max Point \\
\end{tabular}}} & $\mathrm{pt} / \mathrm{tit}$ \\
\hline & & 2288.2 \\
\hline & & 1759.3 \\
\hline & & 2705.0 \\
\hline \multicolumn{3}{|c|}{ Avg Conc $2240 \mathrm{pt} / \mathrm{ft} 3$} \\
\hline
\end{tabular}

Generator Inlet Press

Stack Temp

Centerine vel bot\#3

Ambient pressure

Ambient humidity

Ambient temp

Back-Gd aerosol

No. Bk-Gd samples

Compressar output

\begin{tabular}{|c|c|}
\hline Statt & Finish \\
\hline 2.1 & 2.1 \\
\hline 109.1 & 106.9 \\
\hline 2005 & 1883 \\
\hline 29.47 & 29.47 \\
\hline $17 \%$ & $16 \%$ \\
\hline 97.7 & 104 \\
\hline $1,1,3,1,4$ & $1,3,0,2,0$ \\
\hline 5 & 5 \\
\hline 14 & 20 \\
\hline
\end{tabular}

Notes:

Cb
Ref. Probe Location: Ref port downstream Port 2.
Probe Type /Configuration: L-Shaped probe

\begin{tabular}{|lc|l|}
\hline Entries made by: & Carolyn Burns & Tech \\
Signature/date & $8 / 7 / 2013$ & Sign \\
& Signature on file with original & Sign \\
& \\
\hline
\end{tabular}

2nd

Side

64.6

\begin{tabular}{l|l|} 
& 2337.0 \\
\hline
\end{tabular}

Dev. from mean

Run No. PT-27

Fan configuration $A B$ Norm

Fan Setting 38

Stack Temp

Start/End Time 15:30/16:50

Center 233 from

Points in Center 233

Injection Point I-5

$108 \operatorname{deg} F$

$\mathrm{Hz}$

50

$\begin{array}{rll}1.09 & \text { to: } & 10.83 \\ 2 & \text { to: } & \end{array}$

\begin{tabular}{|c|c|c|c|c|}
\hline \multirow{2}{*}{ Mean } & \multicolumn{3}{|c|}{ Bottom } & \multirow[b]{2}{*}{ Mean } \\
\hline & \multirow[t]{2}{*}{1} & 2 & 3 & \\
\hline & & \multicolumn{2}{|c|}{ particles/ft3 } & \\
\hline 2053.7 & 1694 & 1918 & 1910 & 1840.7 \\
\hline 2204.0 & 2027 & 2058 & 2163 & 2082.7 \\
\hline 22927 & 2361 & 2394 & 2490 & 2415.0 \\
\hline 2437.3 & 2566 & 2564 & 2615 & 2581.7 \\
\hline 2645.0 & 2674 & 2807 & 2634 & 2705.0 \\
\hline 2530.7 & 2687 & 2713 & 2667 & 2689.0 \\
\hline 24020 & 2480 & 2473 & 2465 & 2472.7 \\
\hline 2014.3 & 2083 & 2076 & 2061 & 2073.3 \\
\hline 1988.0 & 1779 & 1674 & 1825 & 1759.3 \\
\hline 2285.3 & 2261.2 & 2297.4 & 2314.4 & 2291.0 \\
\hline
\end{tabular}

\begin{tabular}{|lrrrr|} 
Center 23 & Side & Bottom & $\underline{\text { All }}$ & Norm lzd \\
\hline Mean & 2360.9 & 2431.3 & 2396.1 & 2422.87 \\
Std. Dev. & 210.7 & 263.2 & 232.0 & 231.26 \\
COV as \% & 8.9 & 10.8 & 9.7 & 9.54 \\
\hline
\end{tabular}

Instuments Usect

\begin{tabular}{lrr} 
TSI VelciCalc & T95351203001 & \multicolumn{1}{c}{ Cal Due } \\
\hline Fisher Scientific & 90936818 & $12 / 11 / 2013$ \\
\hline Met One OPC (Ref) & 96258675 & $\mathrm{FIO}$ \\
\hline Met One OPC & 1011529009 & $1 / 14 / 2014$ \\
\hline
\end{tabular}

Signature on file with original TI-WTPSP 123


Rev. 0

3 Aug. 2006

Site LV-S3 Scale Model

Date 8/8/2013

Tester SS,SFS

Stack Dia

11.922

Stack X-Area

Test Part

111.6 in 2

Distance to distumance

Measurement units particles/fi3

510.25 inches

\begin{tabular}{|r|r|}
$\begin{array}{r}\text { Measurement units } \\
\text { Order } \longrightarrow \\
\text { Traverse-> } \\
\text { Trial } \longrightarrow\end{array}$ \\
\hline Pcint & Depth, in. \\
\hline $\mathbf{1}$ & 0.50 \\
\hline $\mathbf{2}$ & 1.25 \\
\hline $\mathbf{3}$ & 2.31 \\
\hline $\mathbf{4}$ & 3.85 \\
\hline Center & 5.96 \\
\hline $\mathbf{5}$ & 8.07 \\
\hline $\mathbf{6}$ & 9.61 \\
\hline $\mathbf{7}$ & 10.67 \\
\hline $\mathbf{8}$ & 11.42 \\
\hline
\end{tabular}

2nd

1

Side

12

2

\begin{tabular}{|r|r|}
693 & 625 \\
\hline 812 & 811 \\
\hline 999 & 104 \\
1130 & 1228 \\
\hline 1261 & 1309 \\
\hline 1209 & 1260 \\
\hline 1059 & 1050 \\
\hline 846 & 851 \\
\hline 675 & 729 \\
\hline $\mathbf{4 . 9}$ & $\mathbf{9 8 9 . 7}$ \\
\hline
\end{tabular}

625

67

\begin{tabular}{|r|}
845 \\
\hline
\end{tabular}

\begin{tabular}{l}
1059 \\
\hline
\end{tabular}

\begin{tabular}{|l|l|}
\hline 09 & 1354 \\
\hline & 1330 \\
\hline
\end{tabular}

\begin{tabular}{r|r|}
\hline 50 & 1124 \\
\hline 851 & 892 \\
\hline
\end{tabular}

\begin{tabular}{r|r}
\hline 29 & 64 \\
\hline
\end{tabular}

\begin{tabular}{|lr|}
\hline All & $\mathrm{pt} / \mathrm{ft} 3$ \\
Mean & 969.1 \\
Min Point & 618.0 \\
Max Point & 1309.0 \\
\hline \multicolumn{2}{|c|}{$927 \mathrm{pt} / \mathrm{ft} 3$} \\
\hline
\end{tabular}

Avg Conc

$$
927 \mathrm{pt} / \mathrm{tt} 3
$$

Generator Inlet Press

Stack Temp

Centerline vel.

Ambient pressure

Ambient humidity

Ambient temp

Back-Gd aerosol

No. Bk-Gd samples

Compressor output

\begin{tabular}{|c|c|c|}
\hline Start & Finish & \\
\hline 3.2 & 3,2 & 2 psig \\
\hline 70.3 & 90.2 & $2 \mathrm{~F}$ \\
\hline 2867 & 3375 & 5] \\
\hline 29.50 & 29.53 & $3 \mathrm{nHg}$ \\
\hline $58 \%$ & $31 \%$ & $\mathrm{RH}$ \\
\hline 67.1 & 83.3 & $3 \mathrm{~F}$ \\
\hline $4,3,4,7,4$ & $1,3,1,4,1$ & $\mathrm{pt} / \mathrm{ft}$ \\
\hline 5 & 5 & 5 \\
\hline 25 & 28 & psix \\
\hline
\end{tabular}

Notes:

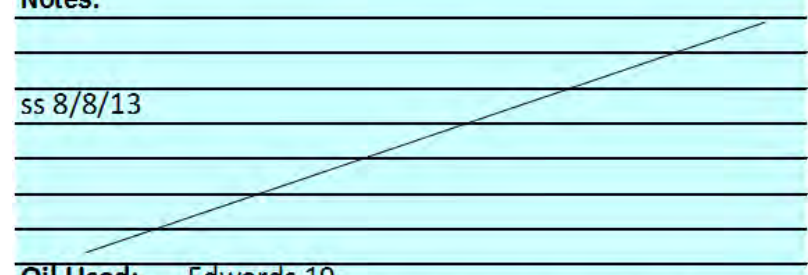

OilUsed: Edwards 19

Ref. Probe Location: Ref port downstream Port 2.

Probe Type/Configuration: L-Shaped probe

\begin{tabular}{|l|c|l}
\hline $\begin{array}{l}\text { Entries made by: } \\
\text { Signature/date }\end{array}$ & Susan Sande & Tech \\
& $8 / 8 / 2013$ & Signature on file with original \\
& Si
\end{tabular}

Run

Fan configuration $A B$ Max

Fan Setting 60

Stack Temp

$80.25 \operatorname{deg} F$

Start/End Time 06:30/9:00

Center $2 / 3$ from

Points in Center 233 1.09 to:

to: $\quad 10.83$

Injection Pcint 1-5

2 to: 7

1st 3

\begin{tabular}{|r|r|r|r|r|}
\hline & \multicolumn{5}{|c|}{$\begin{array}{c}\text { Bottom } \\
\mathbf{2}\end{array}$} \\
\hline $\mathbf{6 6 4 . 3}$ & $\mathbf{5}$ & $\mathbf{3}$ & Mean \\
\hline $\mathbf{8 2 2 7}$ & 765 & 638 & 675 & $\mathbf{6 6 2 . 3}$ \\
\hline $\mathbf{1 0 3 4 . 0}$ & 960 & 785 & 842 & $\mathbf{7 9 7 . 3}$ \\
\hline $\mathbf{1 2 0 5 . 0}$ & 1170 & 988 & 1005 & $\mathbf{9 8 4 . 3}$ \\
\hline $\mathbf{1 3 0 8 . 0}$ & 1303 & 1192 & 1217 & $\mathbf{1 1 9 3 . 0}$ \\
\hline $\mathbf{1 2 6 6 . 3}$ & 1157 & 1315 & 1309 & $\mathbf{1 3 0 9 . 0}$ \\
\hline $\mathbf{1 0 7 7 . 7}$ & 944 & 1170 & 1176 & $\mathbf{1 1 6 7 . 7}$ \\
\hline $\mathbf{8 6 3 . 0}$ & 795 & 1016 & 1045 & $\mathbf{1 0 0 1 . 7}$ \\
\hline $\mathbf{6 8 2 0}$ & 587 & 763 & 802 & $\mathbf{7 8 6 . 7}$ \\
\hline $\mathbf{9 9 1 . 4}$ & & 667 & 600 & $\mathbf{6 1 8 . 0}$ \\
\hline
\end{tabular}

Dev. from mean
$-36.2 \%$
$35.1 \%$

$\begin{array}{r}\text { Side } \\ 1082.4 \\ 190.5 \\ 17.6 \\ \hline\end{array}$

\begin{tabular}{rrr|}
\hline Bottom & All & Normlzd \\
\hline 1034.2 & 1058.3 & 1058.72 \\
199.8 & 189.2 & 189.31 \\
19.3 & 17.9 & 17.88 \\
\hline
\end{tabular}
Instuments Used

\begin{tabular}{lrc} 
TSI VelciCalc & T95351203001 & $12 / 10 / 2013$ \\
\hline Fisher Scientific & 90936818 & $12 / 11 / 2013$ \\
\hline Met One OPC (Ref) & 96258675 & FIO \\
\hline Met One OPC & 1011529009 & $1 / 14 / 2014$ \\
\hline
\end{tabular}

Signature on file with original TI-WTPSP 123


Rev. 0

3 Aug. 2006

PARTICLE TRACER TRAVERSE DATA FORM

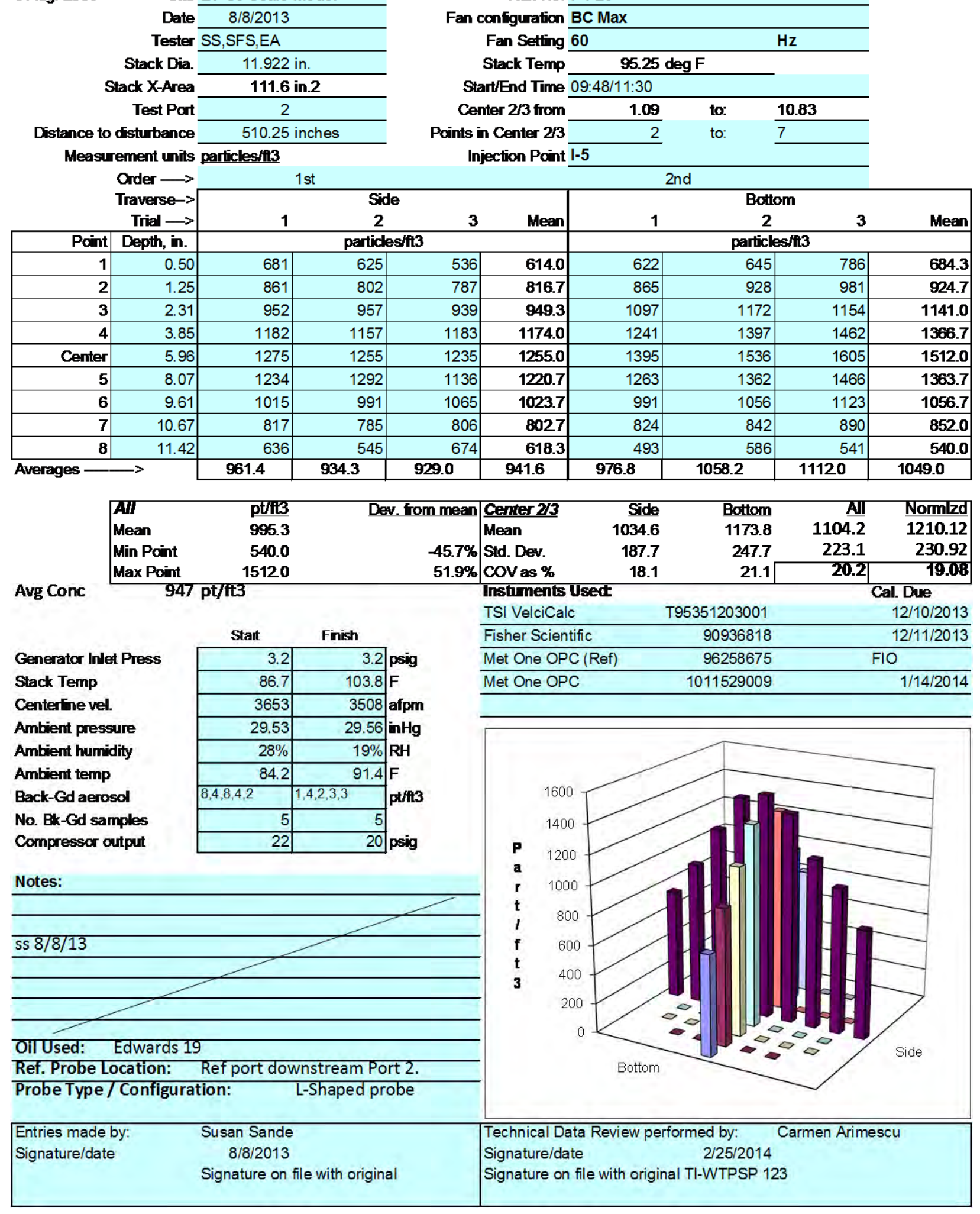


Rev. 0

3 Aug. 2006

Site LV-S3 Scale Model

Date 8/9/2013

Tester YFS, SS

Stack Dia

11.922 in

Stack X-Area

111.6 in.

Test Port

2

Distance to disturbance 510.25 inches

Measurement units particles/ti3

Order $\longrightarrow$

Traverse-

Trial $\rightarrow$

\begin{tabular}{|r|r|}
\hline Point & Depth, in. \\
\hline $\mathbf{1}$ & 0.5 \\
\hline $\mathbf{2}$ & 1.2 \\
$\mathbf{3}$ & 2.31 \\
\hline $\mathbf{4}$ & 3.8 \\
\hline Center & 5.9 \\
\hline $\mathbf{5}$ & 8.07 \\
\hline $\mathbf{6}$ & 9.6 \\
\hline $\mathbf{7}$ & 10.67 \\
\hline $\mathbf{8}$ & 11.4 \\
\hline
\end{tabular}

Averages - 2nd 2nd 79

$12 \quad 2$

23

\begin{tabular}{|r|}
\hline \multicolumn{1}{|c|}{ Mean } \\
\hline \multicolumn{1}{|c|}{} \\
\hline 588.7 \\
\hline 786.3 \\
\hline 877.7 \\
\hline 993.0 \\
\hline 1147.3 \\
\hline 1047.7 \\
\hline 798.3 \\
\hline 708.7 \\
\hline 477.0 \\
\hline 825.0 \\
\hline
\end{tabular}

\begin{tabular}{|r|r|r|}
\hline 516 & 619 & 631 \\
771 & 795 & 793 \\
\hline 912 & 907 & 81 \\
\hline 984 & 974 & 1021 \\
\hline 1099 & 1139 & 120 \\
\hline 920 & 1045 & 117 \\
\hline 862 & 685 & 848 \\
\hline 644 & 753 & 729 \\
\hline 433 & 489 & 509 \\
\hline $\mathbf{3}$ & $\mathbf{8 2 2 . 9}$ & $\mathbf{8 5 8 . 6}$ \\
\hline
\end{tabular}

Run No. PT-31

Fan configuration $B C$

Fan Setting 55

Stack Temp

Start/End Time $0638 / 0848$

Center $2 / 3$ from

Points in Center 233

Injection Point I-5
$77.95 \mathrm{deg} F$

$\mathrm{Hz}$

48

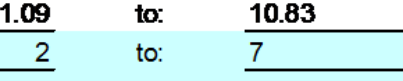

$1 \mathrm{st}$

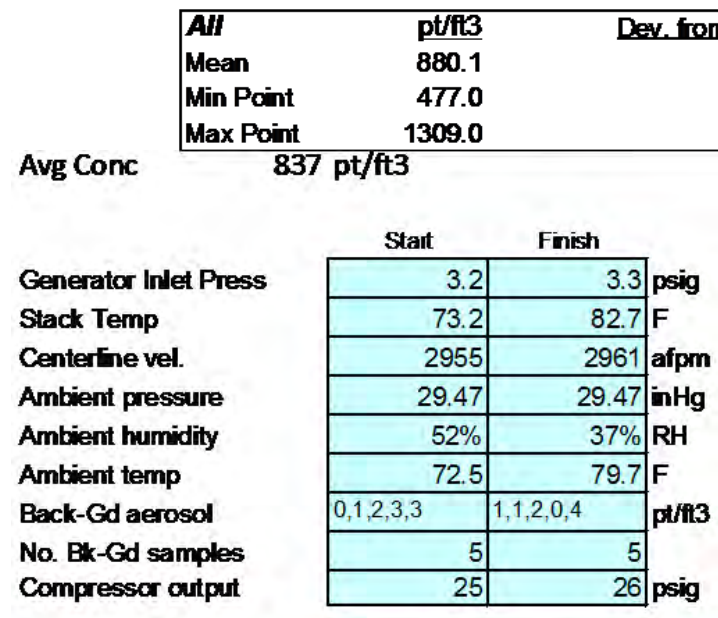

Notes:

SS 8/9/13

Oil Used: Edwards 19

Ref. Probe Location: Ref port downstream Port 2.

Probe Type/Configuration: L-Shaped probe

\begin{tabular}{r|r}
$-45.8 \%$ & \\
$48.7 \%$ &
\end{tabular}

Mean
Std. Dev.

\section{Side}

908.4

COV as \%

158.9
17.5

Instuments Usect

TSI VelciCalc

Fisher Scientific

Met One OPC (Ref)

Met One OPC

Bottom

12

2

3

particles/fl3

\begin{tabular}{|r|r|r|}
\hline 620 & 658 & $\mathbf{6 5 5 . 7}$ \\
\hline 722 & 828 & $\mathbf{7 9 2 . 7}$ \\
\hline 998 & 956 & $\mathbf{1 0 2 2 . 0}$ \\
\hline 1181 & 1183 & $\mathbf{1 2 1 6 . 0}$ \\
\hline 1304 & 1334 & $\mathbf{1 3 0 9 . 0}$ \\
\hline 1144 & 1185 & $\mathbf{1 1 8 8 . 7}$ \\
\hline 943 & 999 & $\mathbf{9 7 0 . 7}$ \\
\hline 760 & 799 & $\mathbf{7 8 3 . 7}$ \\
\hline 468 & 445 & $\mathbf{4 7 9 . 0}$ \\
\hline $\mathbf{4}$ & $\mathbf{9 3 1 . 9}$ & $\mathbf{9 3 5 . 3}$ \\
\hline
\end{tabular}

\begin{tabular}{ll}
1011529009 & $1 / 14 / 2014$ \\
\hline
\end{tabular}

Signature on file with original Tl-WTPSP


Rev. 0

3 Aug. 2006

Site LV-S3 Scale Model

Date 8/9/2013

Tester YFS,SS

Stack Dia

11.922 in

Stack X-Area

Test Part

111.6 in.

Distance to distumbance

Measurement units particles/ft3 426.25 inches

\begin{tabular}{|r|r|}
\multicolumn{1}{|c}{$\begin{array}{r}\text { Order } \\
\text { Traverse-> } \\
\text { Trial } \longrightarrow\end{array}$} \\
\hline Point & Depth, $\mathbf{i n}$ \\
\hline $\mathbf{1}$ & 0.50 \\
\hline $\mathbf{2}$ & 1.25 \\
\hline $\mathbf{3}$ & 2.31 \\
\hline $\mathbf{4}$ & 3.85 \\
\hline Center & 5.96 \\
\hline $\mathbf{5}$ & 8.07 \\
\hline $\mathbf{6}$ & 9.61 \\
\hline $\mathbf{7}$ & 10.67 \\
\hline $\mathbf{8}$ & 11.42 \\
\hline &
\end{tabular}

\begin{tabular}{|lr}
\hline AII & $\mathrm{pt} / \mathrm{ft3}$ \\
Mean & 1177.5 \\
Min Point & 821.7
\end{tabular}

Avg Conc

Max Point

1646.3

$$
1144 \mathrm{pt} / \mathrm{ft3}
$$

Generator Inlet Press

Stack Temp

Centerine vel.

Ambient pressure

Ambient humidity

Ambient temp

Back-Gd aerosol

No. Bk-Gd samples

Compressar output

\begin{tabular}{|c|c|c|}
\hline Statt & Finish & \\
\hline 2.2 & 2.2 & psig \\
\hline 81.5 & 102.7 & $7 \mathrm{~F}$ \\
\hline 2231 & 1983 & 3 \\
\hline 29.83 & 30.86 & 3 \\
\hline $36 \%$ & $26 \%$ & RH \\
\hline 80.6 & 91.4 & $7 / \mathrm{F}$ \\
\hline $2,15,19,11,9$ & $0,1,1,1,0$ & $\theta_{11}$ \\
\hline 5 & 5 & \\
\hline 22 & 22 & sig \\
\hline
\end{tabular}

Notes:

SS 8/9/13

Oil Used: Edwards 19

Ref. Probe Location: Ref port downstream Port 2.

Probe Type/Configuration:

L-Shaped probe

Entries made by:

Signature/date

Susan Sande
$8 / 9 / 2013$
Signature on file with original

Run No. PT-32

Fan configuration $\mathrm{BC}$

Fan Setting 41

Stack Temp

Start/End Time 0850

Center $2 / 3$ from

Points in Center $2 / 3$

Injection Point 1-5

$92.1 \operatorname{deg} F$

$\mathrm{Hz}$

$\begin{array}{rll}1.09 & \text { to: } & 10.83 \\ 2 & \text { to: } & \end{array}$

2nd

\begin{tabular}{l|l} 
Bottom \\
\hline
\end{tabular}

$3 \quad$ Mean $\quad 1$

1

\begin{tabular}{|r|r|r|r|r|r|}
\hline \multicolumn{2}{|c|}{} & \multicolumn{4}{|c|}{ particles/t3 } \\
\hline 69 & $\mathbf{8 3 4 . 3}$ & 1020 & 1070 & 964 & $\mathbf{1 0 1 8 . 0}$ \\
\hline 74 & $\mathbf{9 1 6 . 0}$ & 1125 & 1175 & 1177 & $\mathbf{1 1 5 9 . 0}$ \\
\hline 86 & $\mathbf{1 0 7 3 . 0}$ & 1370 & 1386 & 1358 & $\mathbf{1 3 7 1 . 3}$ \\
\hline 14 & $\mathbf{1 1 8 6 . 7}$ & 1499 & 1512 & 1556 & $\mathbf{1 5 2 2 . 3}$ \\
\hline 57 & $\mathbf{1 2 3 7 . 7}$ & 1644 & 1658 & 1637 & $\mathbf{1 6 4 6 . 3}$ \\
\hline 16 & $\mathbf{1 2 2 3 . 3}$ & 1574 & 1588 & 1632 & $\mathbf{1 5 9 8 . 0}$ \\
\hline 76 & $\mathbf{1 0 9 7 . 3}$ & 1485 & 1472 & 1484 & $\mathbf{1 4 8 0 . 3}$ \\
\hline 28 & $\mathbf{1 0 3 2 3}$ & 1089 & 1027 & 1157 & $\mathbf{1 0 9 1 . 0}$ \\
\hline 28 & $\mathbf{8 2 1 . 7}$ & 911 & 858 & 889 & $\mathbf{8 8 6 . 0}$ \\
\hline & $\mathbf{1 0 4 6 . 9}$ & $\mathbf{1 3 0 1 . 9}$ & $\mathbf{1 3 0 5 . 1}$ & $\mathbf{1 3 1 7 . 1}$ & $\mathbf{1 3 0 8 . 0}$ \\
\hline
\end{tabular}


Rev. 0

3 Aug. 2006

PARTICLE TRACER TRAVERSE DATA FORM

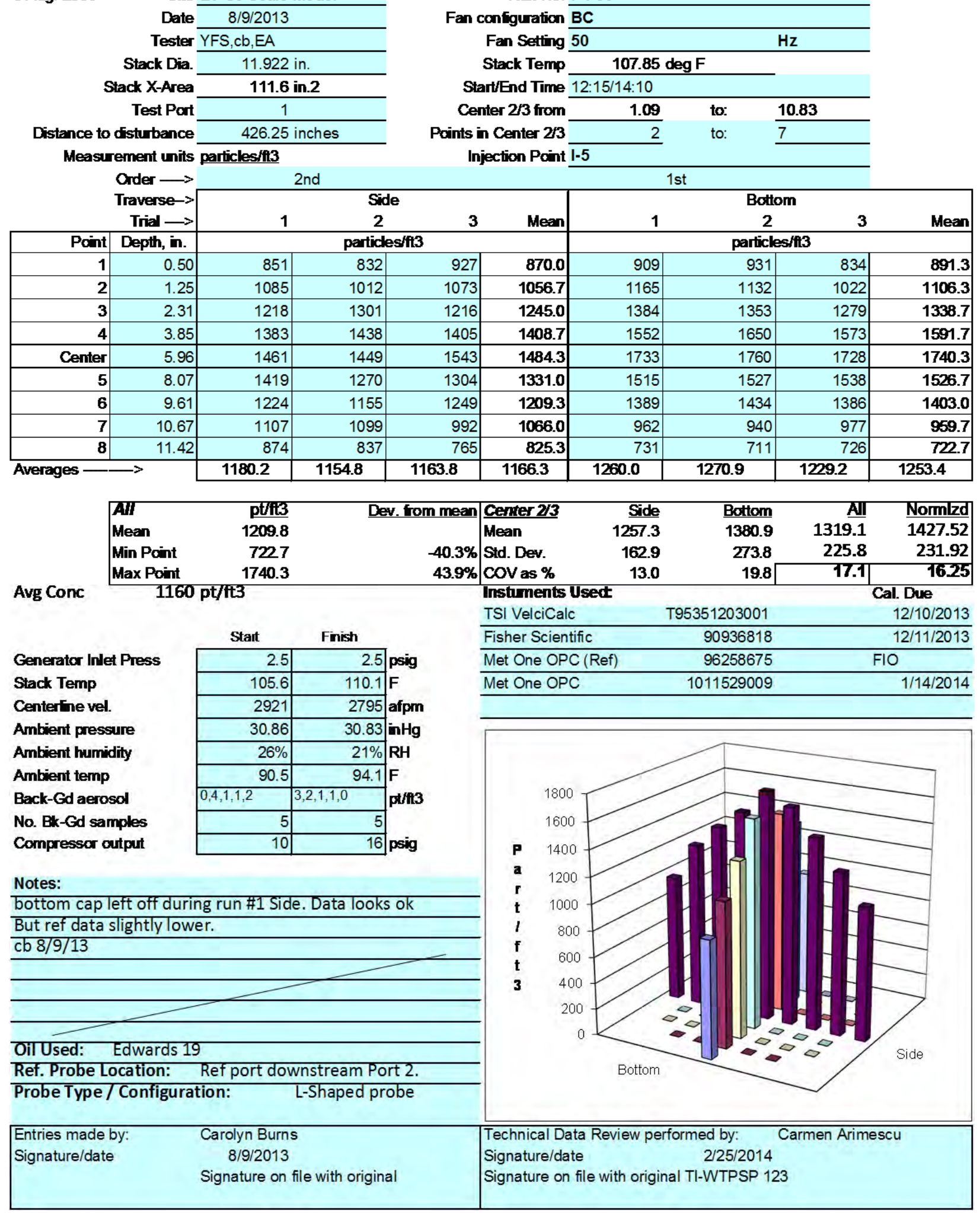


Rev. 0

3 Aug. 2006

PARTICLE TRACER TRAVERSE DATA FORM

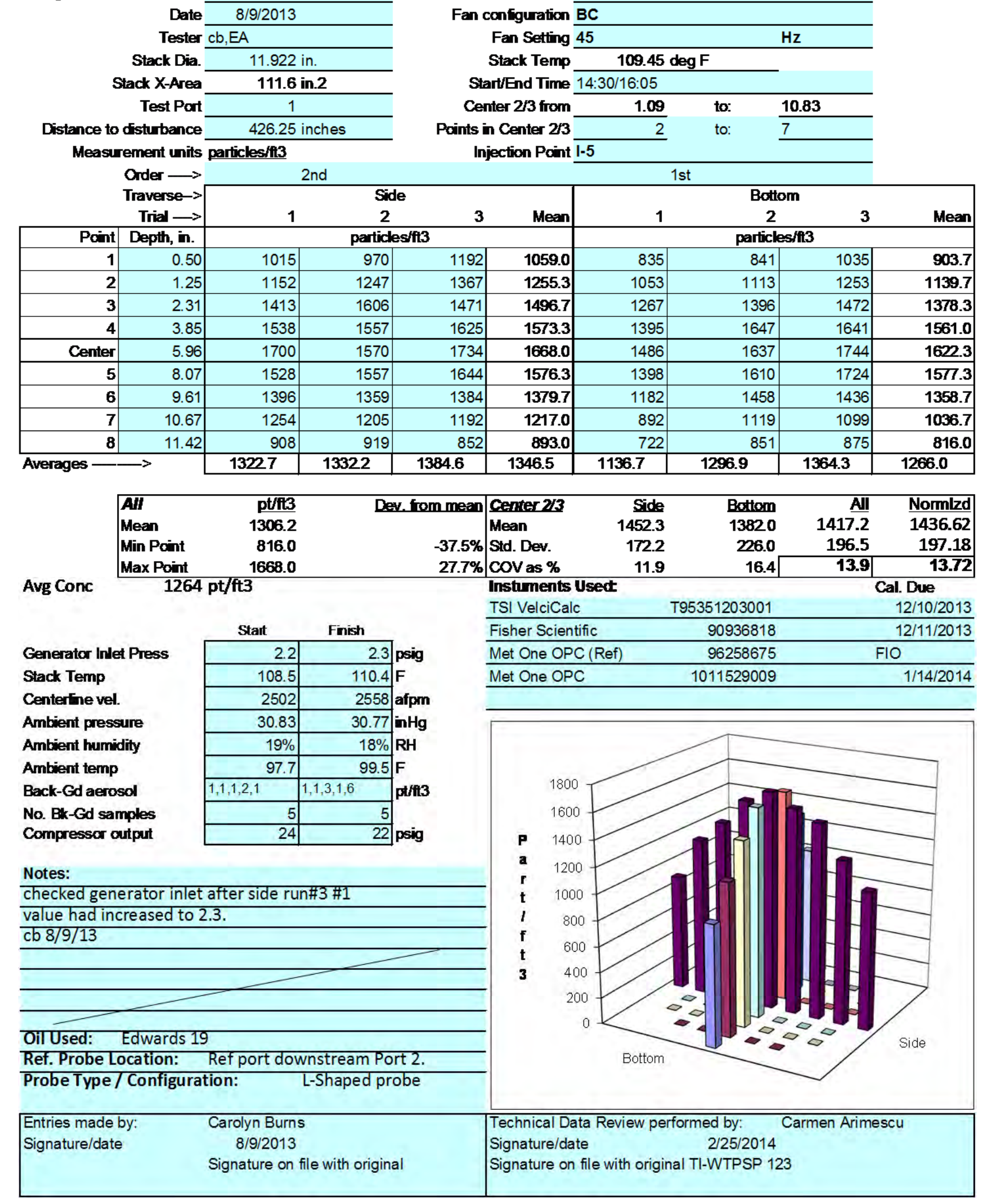


Rev. 0

3 Aug. 2006

Site LV-S3 Scale Model

Date 8/28/2013

Tester SFS,SS

Stack Dia

11.922 in

Stack X-Area

Test Part

111.6 in. 2

Distance to disturbance

Measurement units particles/fi3 510.25 inches

\begin{tabular}{|r|r|}
\multicolumn{2}{c}{$\begin{array}{c}\text { Order } \\
\text { Traverse-> } \\
\text { Trial } \longrightarrow\end{array}$} \\
\hline Point & Depth, in. \\
\hline $\mathbf{1}$ & 0.50 \\
\hline $\mathbf{2}$ & 1.25 \\
\hline $\mathbf{3}$ & 2.31 \\
\hline $\mathbf{4}$ & 3.85 \\
\hline Center & 5.96 \\
\hline $\mathbf{5}$ & 8.07 \\
\hline $\mathbf{6}$ & 9.61 \\
\hline $\mathbf{7}$ & 10.67 \\
\hline $\mathbf{8}$ & 11.42 \\
\hline &
\end{tabular}

2nd

\section{TRACER TRAVERSE DATA FORM}

Run No. PT-35

Fan configuration $A B$ MAX

Fan Setting 60

Stack Temp

74.9 deg $F$

Start/End Time $0630 / 0930$

Center $2 / 3$ from

Points in Center 273

1.09 to:

Injection Point 15

2 to:

to:

1 st

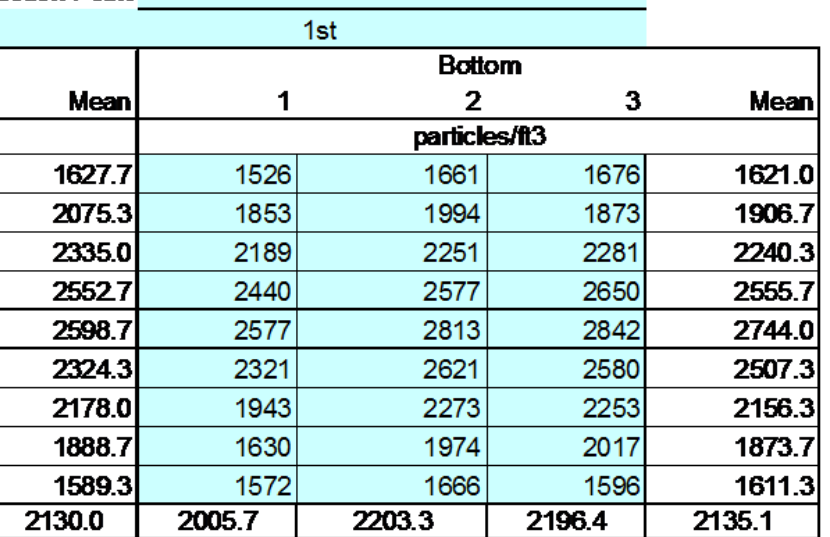

\begin{tabular}{|c|c|c|}
\hline & AII & pt/ft3 \\
\hline & Mean & 21326 \\
\hline & Min Pcint & 1589.3 \\
\hline & Max Point & 2744.0 \\
\hline Avg Conc & 206 & \\
\hline
\end{tabular}

Generator Inlet Press

Stack Temp

Centerine vel.

Ambient pressure

Ambient humidity

Ambient temp

Back-Gd aerosol

No. Bk-Gd samples

Compressar output

\begin{tabular}{|c|c|}
\hline Statt & Finish \\
\hline 3.2 & 3.2 \\
\hline 66.5 & 83.3 \\
\hline 3018 & 3139 \\
\hline 30.77 & 30.74 \\
\hline $69 \%$ & $42 \%$ \\
\hline 64.4 & 76.1 \\
\hline $2,1,1,0,2$ & $1,1,0,2,1$ \\
\hline 5 & 5 \\
\hline 25 & 32 \\
\hline
\end{tabular}

Notes: Redo of PT-28.

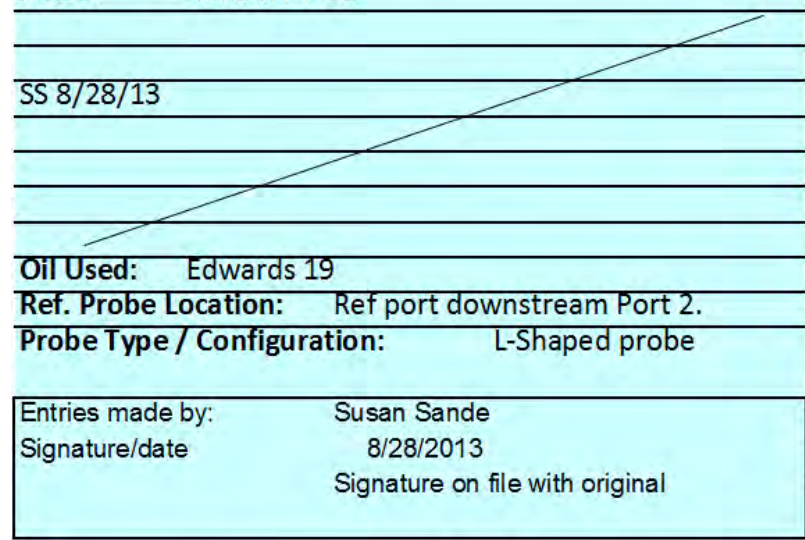

\begin{tabular}{rrrr|} 
Side & Bottom & All & Normlzd \\
2279.0 & 2283.4 & 2281.2 & 2344.92 \\
253.7 & 3327 & 284.3 & 297.15 \\
11.1 & 14.6 & 125 & 12.67 \\
\hline
\end{tabular}

$28.7 \%$ COV as \%

Instuments Usect

TSI VelciCalc $\quad$ T95351203001

$12 / 10 / 2013$

Fisher Scientific

Met One OPC (Ref)

Met One OPC

$12 / 11 / 2013$

$1011529010 \quad 8 / 7 / 2014$

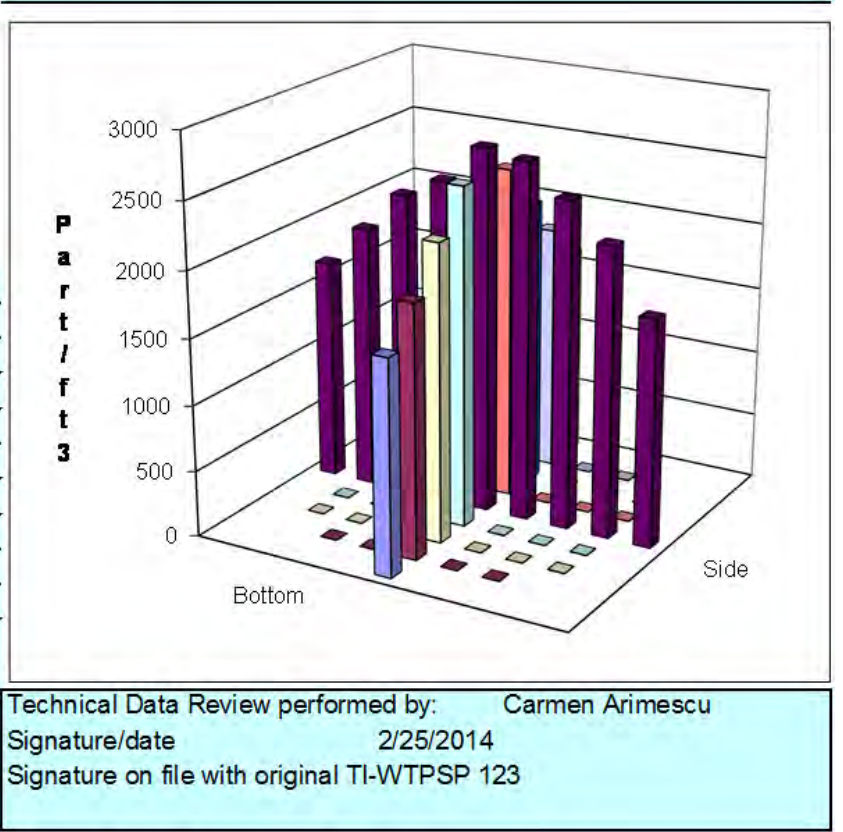


Rev. 0

3 Aug. 2006

Site LV-S3 Scale Model

Date 8/28/2013

Tester SFS,SS

Stack Dia

11.922 in

Stack X-Area

Test Part

111.6 in.

Distance to distumbance

Measurement units particles/ti 426.25 inches

\begin{tabular}{|r|r|}
\multicolumn{1}{|c}{$\begin{array}{r}\text { Order } \longrightarrow \\
\text { Traverse- } \\
\text { Trial } \longrightarrow\end{array}$} \\
\hline Point & Depth, in. \\
\hline $\mathbf{1}$ & 0.50 \\
\hline $\mathbf{2}$ & 1.25 \\
\hline $\mathbf{3}$ & 2.31 \\
\hline $\mathbf{4}$ & 3.85 \\
\hline Center & 5.96 \\
\hline $\mathbf{5}$ & 8.07 \\
\hline $\mathbf{6}$ & 9.61 \\
\hline $\mathbf{7}$ & 10.67 \\
\hline $\mathbf{8}$ & 11.42 \\
\hline &
\end{tabular}

\begin{tabular}{|lr|}
\hline All & $\mathrm{pt} / \mathrm{ft} 3$ \\
Mean & 1088.3 \\
Min Point & 7523 \\
\hline
\end{tabular}

Avg Conc

Max Point

1394.3

$$
1053 \mathrm{pt} / \mathrm{ft} 3
$$

Generator Inlet Press

Stack Temp

Centerine vel.

Ambient pressure

Ambient humidity

Ambient temp

Back-Gd aerosol

No. Bk-Gd samples

Compressor output

\begin{tabular}{|c|c|}
\hline Stat & Finish \\
\hline 2.3 & 2.3 \\
\hline 94.2 & 99.4 \\
\hline 3439 & 3571 \\
\hline 30.74 & 30.74 \\
\hline $30 \%$ & $24 \%$ \\
\hline 85.1 & 89.6 \\
\hline $1,2,1,3,7$ & $1,1,2,0,0$ \\
\hline 5 & 5 \\
\hline 23 & 34 \\
\hline
\end{tabular}

Notes:

SS8/28/13

Oil Used: Edwards 19

Ref. Probe Location: Ref port downstream Port 2.

Probe Type/Configuration:

L-Shaped probe

Entries made by:

Signature/date

Susan Sande
$8 / 28 / 2013$
Signature on file with original

Run No. PT-36

Fan configuration BC MAX

Fan Setting 60

Stack Temp

$96.8 \operatorname{deg} F$

Start/End Time $1040 / 1230$

Center $2 / 3$ from

Points in Center $2 / 3$

Injection Point 16 to:

to: 7

2

$1 \mathrm{st}$

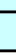

(2)

\begin{tabular}{|c|c|c|c|c|c|}
\hline \multirow[b]{2}{*}{3} & \multirow[b]{2}{*}{ Meen } & \multicolumn{4}{|c|}{ Bottom } \\
\hline & & 1 & 2 & 3 & Mean \\
\hline & & \multicolumn{4}{|c|}{ particles/fi3 } \\
\hline 856 & 874.0 & 868 & 889 & 869 & 875.3 \\
\hline 49 & 1067.0 & 979 & 1055 & 969 & 1001.0 \\
\hline 215 & 1180.7 & 1042 & 1142 & 1172 & 1118.7 \\
\hline 279 & 1284.3 & 1212 & 1314 & 1262 & 1262.7 \\
\hline 419 & 1346.0 & 1371 & 1398 & 1414 & 1394.3 \\
\hline 220 & 12427 & 1349 & 1315 & 1333 & 1332.3 \\
\hline 160 & 1098.0 & 1228 & 1162 & 1192 & 1194.0 \\
\hline 016 & 969.7 & 795 & 900 & 838 & 844.3 \\
\hline 730 & 7523 & 713 & 809 & 736 & 752.7 \\
\hline & 090.5 & 1061.9 & 1109.3 & 1087.2 & 1086.1 \\
\hline
\end{tabular}

\begin{tabular}{r|lrrrr|}
\hline Dev. from mean & Center 2/3 & Side & Bottom & All & Normlzd \\
\cline { 2 - 6 }$-30.9 \%$ & Mean & 1169.8 & 1163.9 & 1166.8 & 1187.84 \\
$28.1 \%$ & Std. Dev. & 132.5 & 1927 & 158.9 & 162.65 \\
\hline COV as \% & 11.3 & 16.6 & 13.6 & 13.69 \\
\hline
\end{tabular}

\section{Instuments Usect}

TSI VelciCalc $\quad$ T95351203001

\begin{tabular}{lrr} 
TSI VelciCalc & T95351203001 & $12 / 10 / 2013$ \\
\hline Fisher Scientific & 90936818 & $12 / 11 / 2013$ \\
\hline Met One OPC (Ref) & 96258675 & FIO \\
\hline Met One OPC & 1011529010 & $8 / 7 / 2014$ \\
\hline
\end{tabular}

Technical Data Review performed by:


Rev. 0

3 Aug. 2006

\begin{tabular}{|c|c|c|c|c|}
\hline & Date & $8 / 28 / 2013$ & & \\
\hline & Tester & $\mathrm{EA}, \mathrm{cb}$ & & \\
\hline & Stack Dia. & 11.922 & & \\
\hline & Stack X-Area & 111.6 & & \\
\hline & Test Part & 1 & & \\
\hline Distance to & distumbance & 426.25 & ches & \\
\hline Measu & rement units & particles/t13 & & \\
\hline & Order $\longrightarrow$ & 1st & & \\
\hline & Traverse-> & & $\mathbf{S}$ & \\
\hline & Trial $\longrightarrow$ & 1 & 2 & \\
\hline Point & Depth, in. & & partic & $e s / f 13$ \\
\hline 1 & 0.50 & 727 & 686 & \\
\hline 2 & 1.25 & 826 & 777 & \\
\hline 3 & 2.31 & 869 & 846 & \\
\hline 4 & 3.85 & 947 & 981 & \\
\hline Center & 5.96 & 917 & 946 & \\
\hline 5 & 8.07 & 865 & 906 & \\
\hline 6 & 9.61 & 792 & 796 & \\
\hline 7 & 10.67 & 756 & 756 & \\
\hline 8 & 11.42 & 610 & 649 & \\
\hline Averages - & $\longrightarrow$ & 8121 & 815.9 & 80 \\
\hline & $A H$ & pt/fi3 & & v. from \\
\hline & Mean & 833.0 & & \\
\hline & Min Point & 623.3 & & \\
\hline & Max Point & 1033.7 & & \\
\hline Avg Conc & 01 & $\mathrm{ft3}$ & & \\
\hline
\end{tabular}

Generator Inlet Press

Stack Temp

Centerline vel.

Ambient pressure

Ambient humidity

Ambient temp

Back-Gd aerosol

No. Bk-Gd samples

Compresscr output

\begin{tabular}{|c|c|c|}
\hline Stat & Finish & \\
\hline 2.3 & 2.3 & psig \\
\hline 104.5 & 105.3 & $F$ \\
\hline 3476 & 3990 & afpm \\
\hline 30.74 & 30.74 & $\mathrm{nH} \mathrm{Hg}$ \\
\hline $20 \%$ & $21 \%$ & $\mathrm{RH}$ \\
\hline 93.2 & 92.3 & $F$ \\
\hline $1,2,0,0,0$, & $1,3,0,4,0$ & pt/f13 \\
\hline 5 & 5 & \\
\hline 23 & 30 & psig \\
\hline
\end{tabular}

Notes:

$\mathrm{cb8/28/2013}$

OाUsed: Edwards 19

Ref. Probe Location: Ref port downstream Port 2.

Probe Type/Configuration: L-Shaped probe

\begin{tabular}{|lc|}
\hline Entries made by: & Carolyn Burns \\
Signature/date & $8 / 28 / 2013$ \\
& Signature on file with original \\
\hline
\end{tabular}

62

\begin{tabular}{r|r|}
$-25.2 \%$ & \\
$24.1 \%$ &
\end{tabular}

Rut No.

Fan configuration $\mathrm{BC}$ Max

Fan Setting 60

Stack Temp

$104.9 \operatorname{deg} \mathrm{F}$

Start/End Time 13:30/15:20

Center $2 / 3$ from

Points in Center 233

Injection Point I-7

$\begin{array}{rll}1.09 & \text { to: } & 10.83 \\ 2 & \text { to: } & \end{array}$

2nd

Bottom

\begin{tabular}{|c|c|c|c|c|}
\hline \multirow[t]{2}{*}{ Meen } & 1 & 2 & 3 & \multirow[t]{2}{*}{ Mean } \\
\hline & \multicolumn{3}{|c|}{ particles/ft3 } & \\
\hline 686.0 & 645 & 672 & 704 & 673.7 \\
\hline 768.7 & 779 & 802 & 735 & 772.0 \\
\hline 859.7| & 897 & 936 & 890 & $\mathbf{9 0 7 . 7}$ \\
\hline 958.0 & 1016 & 1031 & 999 & 1015.3 \\
\hline 949.0 & 1036 & 976 & 1089 & 1033.7 \\
\hline 904.7 & 1030 & 976 & 1019 & 1008.3 \\
\hline 798.7 & 911 & 953 & 972 & 945.3 \\
\hline 750.7 & 702 & 722 & 714 & 712.7 \\
\hline 627.0 & 637 & 636 & 597 & 623.3 \\
\hline 11.4 & 850.3 & 856.0 & 857.7 & 854.7 \\
\hline
\end{tabular}

Center $2 / 3$
Mean
Std. Dev.
COV as \%
Instuments

$\begin{array}{r}\text { Side } \\ 855.6 \\ 85.1 \\ 9.9 \\ \hline\end{array}$

\begin{tabular}{rrr|}
\hline Bottom & \multicolumn{1}{c}{ All } & Normlzd \\
913.6 & 884.6 & 922.76 \\
126.0 & 107.6 & 106.67 \\
\cline { 3 - 3 } 13.8 & 122 & 11.56
\end{tabular}
Instuments Usect

$\begin{array}{lr}\text { TSI VelciCalc } & \text { T95351203001 }\end{array}$

\begin{tabular}{lrr} 
& T95351203001 & $12 / 10 / 2013$ \\
\hline Fisher Scientific & 90936818 & $12 / 11 / 2013$ \\
\hline Met One OPC (Ref) & 96258675 & FIO \\
\hline Met One OPC & 1011529010 & $8 / 7 / 2014$ \\
\hline
\end{tabular}

Technical Data Review performed by: Carmen Arimescu


Run No. PT-38

Fan configuration $\mathrm{BC}$ Max

Fan Setting 60

Stack Temp

Start/End Time 15:30/17:20

Center $2 / 3$ from

Points in Center $2 / 3$

Injection Point I-8
Rev. 0

3 Aug. 2006

Site LV-S3 Scale Model

Date 8/28/2013

Tester EA, cb,JG

Stack Dia

11.922 in

Stack X-Area

111.6 in. 2

Test Port

1

Distance to distumbence 426.25 inches

Measurement units particles/t13

Order $\longrightarrow$ 2nd

\begin{tabular}{|c|c|c|c|}
\hline \multicolumn{2}{|r|}{$\begin{array}{r}\text { Traverse-> } \\
\text { Trial } \longrightarrow\end{array}$} & \multicolumn{2}{|c|}{1} \\
\hline Point & Depth, in. & & par \\
\hline 1 & 0.50 & 733 & \\
\hline 2 & 1.25 & 823 & $\varepsilon$ \\
\hline 3 & 2.31 & 850 & \\
\hline 4 & 3.85 & 916 & 8 \\
\hline Center & 5.96 & 920 & 8 \\
\hline 5 & 8.07 & 846 & 8 \\
\hline 6 & 9.61 & 822 & 0 \\
\hline 7 & 10.67 & 767 & 7 \\
\hline 8 & 11.42 & 698 & 7 \\
\hline Averages - & $\rightarrow$ & 819.4 & 802.2 \\
\hline
\end{tabular}

$102.8 \operatorname{deg} F$

$\mathrm{Hz}$

20

1.09 to

2 to: 7

\begin{tabular}{|r|r|r|r|r|}
\cline { 2 - 5 } Meen & \multicolumn{5}{c}{} \\
\hline & $\mathbf{5}$ & \multicolumn{3}{c|}{$\begin{array}{c}\text { Boftom } \\
\mathbf{2}\end{array}$} \\
\hline $\mathbf{7 3 4 . 7}$ & 784 & 777 & $\mathbf{3}$ & Mean \\
\hline $\mathbf{8 0 3 . 0}$ & 791 & 788 & 808 & $\mathbf{7 8 6 . 7}$ \\
\hline $\mathbf{8 4 6 . 7}$ & 899 & 878 & 915 & $\mathbf{8 9 7 . 3}$ \\
\hline $\mathbf{8 5 2 3}$ & 931 & 954 & 928 & $\mathbf{9 3 7 . 7}$ \\
\hline $\mathbf{8 7 8 . 3}$ & 941 & 989 & 989 & $\mathbf{9 7 3 . 0}$ \\
\hline $\mathbf{8 4 7 . 0}$ & 933 & 960 & 941 & $\mathbf{9 4 4 . 7}$ \\
\hline $\mathbf{8 2 4 . 7}$ & 950 & 943 & 874 & $\mathbf{9 2 2 . 3}$ \\
\hline $\mathbf{7 7 6 . 7}$ & 733 & 799 & 782 & $\mathbf{7 7 1 . 3}$ \\
\hline $\mathbf{7 0 6 . 0}$ & 744 & 734 & 731 & $\mathbf{7 3 6 . 3}$ \\
\hline $\mathbf{8 0 7 . 7}$ & $\mathbf{8 5 6 . 2}$ & $\mathbf{8 6 9 . 1}$ & $\mathbf{8 6 3 . 0}$ & $\mathbf{8 6 2 8}$ \\
\hline
\end{tabular}

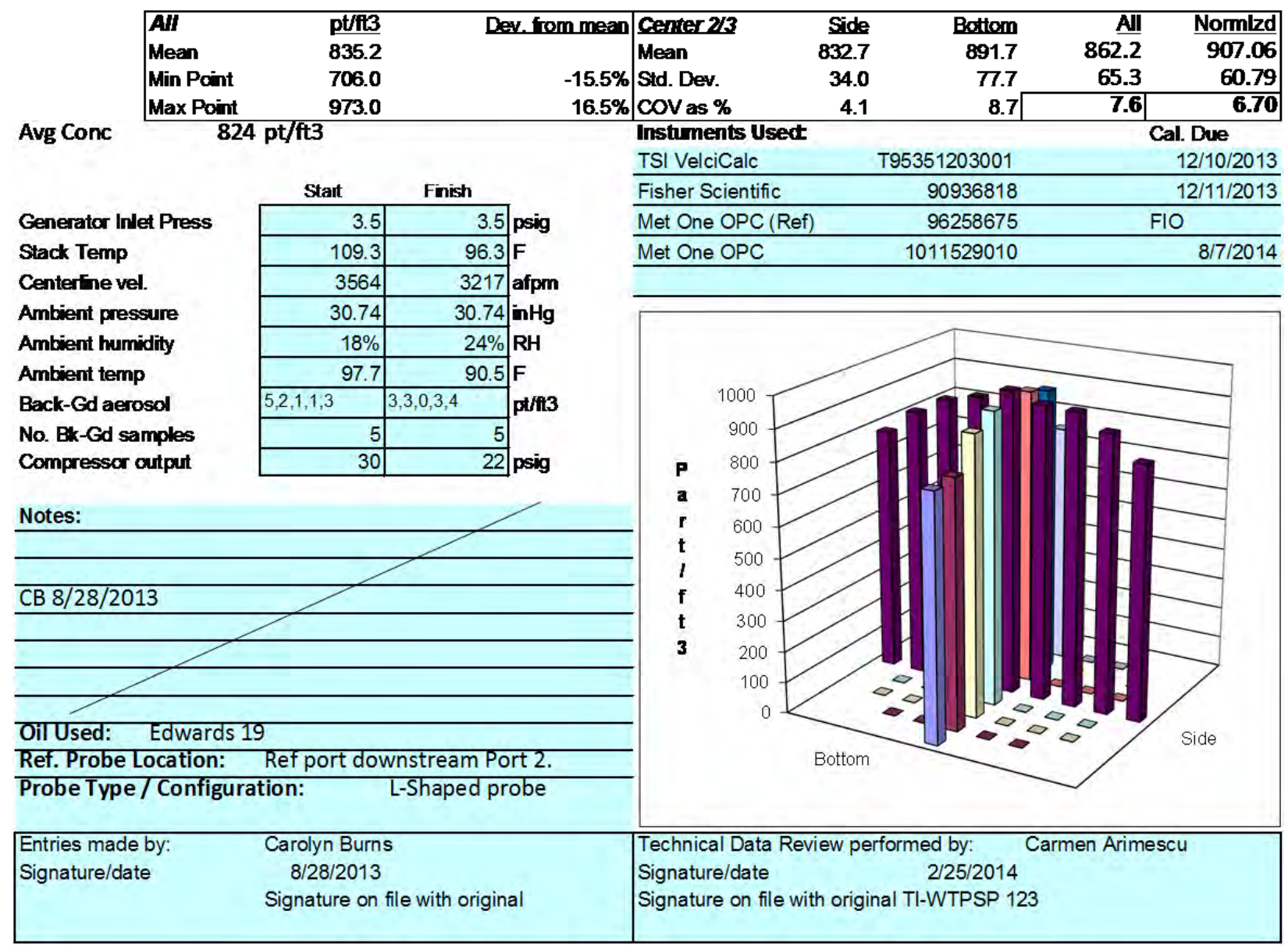


Rev. 0

3 Aug. 2006

Site LV-S3 Scale Model

Date 8/29/2013

Tester SFS, SS

Stack Dia.

11.922 in

Stack X-Area

111.6 in.

Test Port

1

Distance to distumbence 426.25 inches

Measurement units particles/ti3

Order $\longrightarrow$ 2nd

Traverse->

Trial $\longrightarrow$

\begin{tabular}{|r|r|}
\hline Point & Depth, in. \\
\hline $\mathbf{1}$ & 0.5 \\
\hline $\mathbf{2}$ & 1.2 \\
$\mathbf{3}$ & 2.3 \\
\hline $\mathbf{4}$ & 3.8 \\
\hline Center & 5.9 \\
\hline $\mathbf{5}$ & 8.07 \\
\hline $\mathbf{6}$ & 9.61 \\
\hline $\mathbf{7}$ & 10.6 \\
\hline $\mathbf{8}$ & 11.4 \\
\hline
\end{tabular}

\begin{tabular}{l|l}
\hline Averages $\longrightarrow$ & \\
\hline &
\end{tabular}

PARTICLE TRACER TRAVERSE DATA FORM

Run No. PT-39

Fan configuration $\mathrm{BC}$ Max

Fan Setting 60

Stack Temp

Start/End Time $0850 / 104$

Center $2 / 3$ from

Points in Center $2 / 3$

Injection Point I-9
$88.15 \operatorname{deg} F$

$\mathrm{Hz}$

40

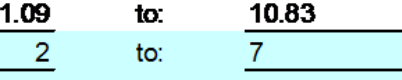

$1 \mathrm{st}$

\begin{tabular}{|lrr|lrrrr|}
\hline All & pt/ft3 & Dev. from mean & Center 2/3 & Side & Bottom & All & Normlzd \\
Mean & 758.7 & Mean & 788.2 & 777.5 & 782.8 & 788.70 \\
Min Point & 639.3 & $-15.7 \%$ & Std. Dev. & 39.1 & 53.8 & 45.5 & 45.62 \\
Max Point & 8527 & $124 \%$ & COV as \% & 5.0 & 6.9 & 5.8 & 5.78 \\
\hline
\end{tabular}

Avg Conc $748 \mathrm{pt} / \mathrm{ft} 3$

Generator Inlet Press

Stack Temp

Centerfine vel.

Ambient pressure

Ambient humidity

Ambient temp

Back-Gd aerosol

No. Bk-Gd samples

Compressor output

\begin{tabular}{|c|c|c|}
\hline Stat & Finish & \\
\hline 3.9 & 3.9 & 9 psigi \\
\hline 81.3 & 95 & $5 \mid \mathrm{F}$ \\
\hline 3557 & 3489 & 9 afp \\
\hline 30.77 & 30.8 & 8 \\
\hline $41 \%$ & $28 \%$ & RH \\
\hline 78.8 & 89.6 & 6 \\
\hline $5,0,2,1,1$ & $2,1,0,2,3$ & $\mathrm{pt} / \mathrm{t}$ \\
\hline 5 & 5 & \\
\hline 24 & 30 & psig \\
\hline
\end{tabular}

Notes: Ref probe was found to be off perpendicular Corrected at run Bottom 3 .

\begin{tabular}{l} 
Corrected at run Bottom 3. \\
ss 8/29/13 \\
OilUsed: Edwards 19 \\
$\begin{array}{l}\text { Ref. Probe Location: Ref port downstream Port 2. } \\
\begin{array}{l}\text { Entries made by: } \\
\text { Signature/date }\end{array} \\
\text { Susan Sande } \\
8 / 29 / 2013 \\
\text { Signature on file with original }\end{array}$ \\
\hline
\end{tabular}

\section{Instuments Usedt}

TSI VelciCalc

Fisher Scientific

Met One OPC (Ref)

Met One OPC

T95351203001

Cal. Due

$12 / 10 / 2013$

$12 / 11 / 2013$

\begin{tabular}{ll}
1011529010 & $8 / 7 / 2014$ \\
\hline
\end{tabular}

Technical Data Review performed by:
Signature/date
Signature on file with original TI-WTPSP 123


Rev. 0

3 Aug. 2006

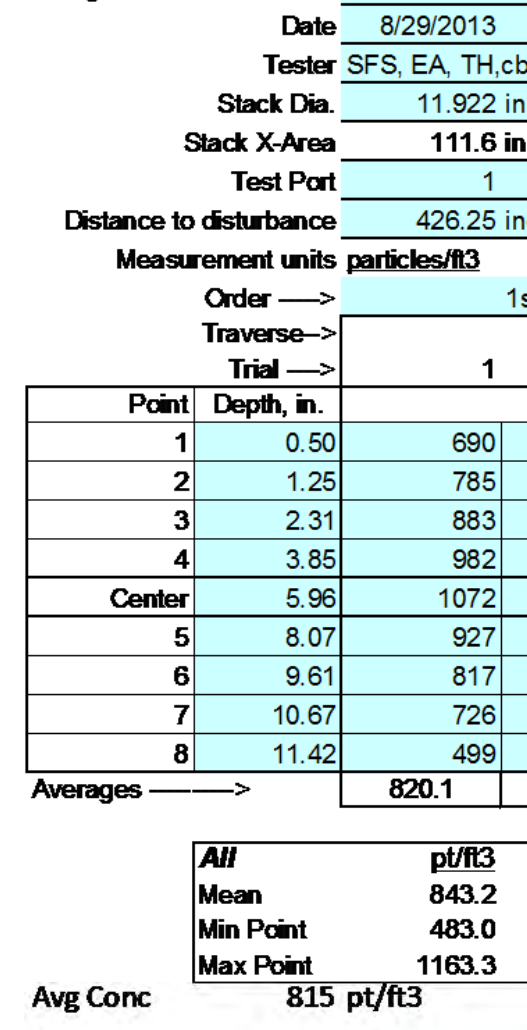

Site LV-S3 Scale

Tester SFS, EA, TH,cb

Measurement units particles/ti3

Order $\longrightarrow$

rial $\rightarrow$
PARTICLE
Site LV-S3 Scale Model

$\begin{array}{rc}\text { Stack Dia. } & 11.922 \text { in. } \\ \text { Stack X-Area } & \mathbf{1 1 1 . 6} \text { in.2 } \\ \text { Test Port } & 1\end{array}$

Generator Inlet Press

Stack Temp

Centerline vel.

Ambient pressure

Ambient humidity

Ambient temp

Back-Gd aerosol

No. Bk-Gd samples

Compressor output

\begin{tabular}{|c|c|c|}
\hline Stat & Finish & \\
\hline 1.5 & 1.5 & psix \\
\hline 94 & 94.5 & \\
\hline 3442 & 3259 & 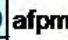 \\
\hline 30.8 & 30.83 & 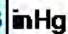 \\
\hline $25 \%$ & $23 \%$ & \\
\hline 92.3 & 95 & 1 \\
\hline $2,3,0,0,3$ & $0,2,1,1,1$ & 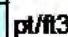 \\
\hline 5 & 5 & \\
\hline 30 & 40 & psig \\
\hline
\end{tabular}

Notes:

CB 08/29/2013

Oil Used: Edwards 19

Ref. Probe Location: Ref port downstream Port 2.

Probe Type/Configuration: L-Shaped probe

\begin{tabular}{|lc|}
\hline $\begin{array}{l}\text { Entries made by: } \\
\text { Signature/date }\end{array}$ & Carolyn Burns \\
& $8 / 29 / 2013$ \\
& Signature on file with original \\
\hline
\end{tabular}

Run No. PT-40

Fan configuration $\mathrm{BC}$ Max

Fan Setting 60

Stack Temp

$94.25 \operatorname{deg} \mathrm{F}$

Start/End Time $\frac{1100 / 1242}{1.09}$

Center $2 / 3$ from

Points in Center $2 / 3$

Injection Point 15

\begin{tabular}{|c|c|c|}
\hline 1.09 & to: & 10.83 \\
\hline 2 & to: & 7 \\
\hline
\end{tabular}

\begin{tabular}{|c|c|c|c|c|}
\hline \multicolumn{4}{|c|}{ 2nd } & \multirow[b]{3}{*}{ Mean } \\
\hline \multirow{3}{*}{ Meen } & \multicolumn{3}{|c|}{ Bottom } & \\
\hline & \multirow[t]{2}{*}{1} & 2 & \multirow[t]{2}{*}{3} & \\
\hline & & particles/ft3 & & \\
\hline 651.7 & 625 & 617 & 745 & 662.3 \\
\hline 784.3 & 738 & 801 & 794 & $7 \pi 7.7$ \\
\hline 869.3 & 948 & 892 & 941 & 927.0 \\
\hline 9923 & 1094 & 1113 & 1108 & 1105.0 \\
\hline 1017.3 & 1109 & 1124 & 1131 & 1121.3 \\
\hline 940.7 & 1106 & 1173 & 1211 & 1163.3 \\
\hline 798.0 & 953 & 963 & 1018 & 978.0 \\
\hline 705.3 & 669 & 692 & 648 & 669.7 \\
\hline 483.0 & 536 & 498 & 560 & 531.3 \\
\hline 804.7 & 864.2 & 874.8 & 906.2 & 881.7 \\
\hline
\end{tabular}

\begin{tabular}{|r|lrrrr|}
\hline Dev. from mean & Center 2/3 & Side & Bottom & All & Normlzd \\
\cline { 2 - 5 } & Mean & 872.5 & 963.1 & 917.8 & 962.41 \\
$38.0 \%$ & Std. Dev. & 116.4 & 185.9 & 156.2 & 153.43 \\
\hline Cov as \% & 13.3 & 19.3 & 17.0 & 15.94 \\
\hline
\end{tabular}

Instuments Usect

Instuments Usect Cal. Due

\begin{tabular}{lrr} 
TSI VelciCalc & T95351203001 & $12 / 10 / 2013$ \\
\hline
\end{tabular}

\begin{tabular}{lrc}
\hline Fisher Scientific & 90936818 & \multicolumn{1}{c}{$12 / 11 / 2013$} \\
\hline Met One OPC (Ref) & 96258675 & \multicolumn{2}{c}{ FIO } \\
\hline Met One OPC & 1011529010 & $8 / 7 / 2014$ \\
\hline
\end{tabular}

Technical Data Review performed by: Carmen Arimescu


Rev. 0

3 Aug. 2006

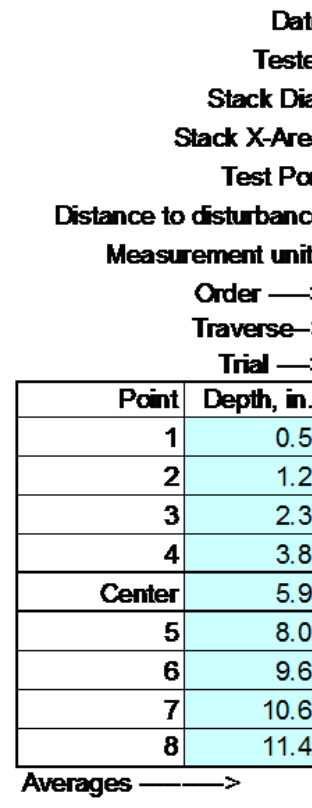

PARTICLE TRACER TRAVERSE DATA FORM

Site LV-S3 Scale Model

Date 8/29/2013

Tester $\mathrm{TH}, \mathrm{cb}$

11.922 in

$X$-Areo

111.6 in

$\frac{111.6 \text { in. } 2}{1}$

perticles/fi3

$2 \mathrm{nd}$
Run No. PT-41

Fan configuration $\mathrm{BC}$ Max

Fan Setting 55

Stack Temp

Start/End Time 1300/1442

Center $2 / 3$ from

Points in Center $2 / 3$

Injection Point 15
$100.55 \operatorname{deg} F$

$\mathrm{Hz}$

442

$\begin{array}{rll}1.09 & \text { to: } & 10.83 \\ 2 & \text { to: } & \underline{7}\end{array}$

1 st

\begin{tabular}{|r|r|r|r|}
\hline \multicolumn{5}{|c}{1 st } \\
\hline \multicolumn{5}{|c|}{$\begin{array}{c}\text { Bottom } \\
\mathbf{2}\end{array}$} \\
\hline 579 & 519 & $\mathbf{3}$ & Mean \\
\hline 685 & 622 & 634 & $\mathbf{5 3 9 . 7}$ \\
\hline 762 & 715 & 743 & $\mathbf{6 4 7 . 0}$ \\
\hline 854 & 815 & 829 & $\mathbf{8 4 0 . 0}$ \\
\hline 853 & 928 & 879 & $\mathbf{8 8 6 . 7}$ \\
\hline 838 & 870 & 873 & $\mathbf{8 6 0 . 3}$ \\
\hline 762 & 761 & 727 & $\mathbf{7 5 0 . 0}$ \\
\hline 552 & 503 & 522 & $\mathbf{5 2 5 . 7}$ \\
\hline 489 & 437 & 498 & $\mathbf{4 7 4 . 7}$ \\
\hline $\mathbf{7 0 8 . 2}$ & $\mathbf{6 8 5 . 6}$ & $\mathbf{6 9 1 . 8}$ & $\mathbf{6 9 5 . 2}$ \\
\hline
\end{tabular}

Avg Conc \begin{tabular}{lll}
\hline All & $\mathrm{pt} / \mathrm{ti} 3$ \\
Mean & 6926 \\
Min Point & 474.7 \\
Max Point & 886.7 \\
\hline \multicolumn{2}{|c|}{$673 \mathrm{pt} / \mathrm{ft3}$}
\end{tabular}

Generator Inlet Press

Stack Temp

Centerthe vel.

Ambient pressure

Ambient humidity

Ambient temp

Back-Gd aerosol

No. Bk-Gd samples

Compressor output

\begin{tabular}{|c|c|c|}
\hline Stat & Finish & \\
\hline 1.2 & 1.1 & psig \\
\hline 93.3 & 107.8 & $F$ \\
\hline 3006 & 2932 & afpm \\
\hline 30.83 & 30.83 & $i n \mathrm{Hg}$ \\
\hline $24 \%$ & $20 \%$ & $\mathrm{RH}$ \\
\hline 93.2 & 96.8 & $F$ \\
\hline $0,1,3,0,0$ & $0,1,2,0,0$ & pt/ft3 \\
\hline 5 & 5 & \\
\hline 26 & 20 & psig \\
\hline
\end{tabular}

Notes:

Sun came out then behind the clouds throughout test cover was missing on cap used for velocity check

for duration of test. Ref may be lower than actual due

to air flow out of duct.

CB 08/29/2013

OाUsed: Edwards 19

Ref. Probe Location: Ref port downstream Port 2.

Probe Type/Configuration: L-Shaped probe

\begin{tabular}{|lc|}
\hline Entries made by: & Carolyn Burns \\
Signature/date & $8 / 29 / 2013$ \\
& Signature on file with original \\
\hline
\end{tabular}

\begin{tabular}{r|}
$-31.5 \%$ \\
$28.0 \%$
\end{tabular}

\begin{tabular}{rrrr|} 
Side & Bottom & All & Normlzd \\
731.9 & 748.9 & 740.4 & 775.54 \\
68.6 & 128.3 & 99.2 & 104.71 \\
& 17.1 & 13.4 & 13.50 \\
\hline
\end{tabular}

OV as $\%$

Instuments Usedt

TSI VelciCalc

\begin{tabular}{lrr}
\hline Fisher Scientific & 90936818 & \multicolumn{1}{c}{$12 / 11 / 2013$} \\
\hline Met One OPC (Ref) & 96258675 & \multicolumn{2}{c}{ FIO } \\
\hline Met One OPC & 1011529010 & $8 / 7 / 2014$ \\
\hline
\end{tabular}

Technical Data Review performed by:


Rev. 0

3 Aug. 2006
Site LV-S3 Scale Model

Date 8/30/2013

Tester YFS, EA

Stack Dia.

Stack X-Area

Test Part

11.922 in

111.6 in. 2

Distance to disturbance

Measurement units particles/ft3 426.25 inches

Order $\longrightarrow$

Traverse-

Trial $\rightarrow$

\begin{tabular}{|r|r|}
\hline Point & Depth, in \\
\hline $\mathbf{1}$ & 0.50 \\
\hline $\mathbf{2}$ & 1.2 \\
\hline $\mathbf{3}$ & 2.31 \\
\hline $\mathbf{4}$ & 3.8 \\
\hline Center & 5.9 \\
\hline $\mathbf{5}$ & 8.07 \\
\hline $\mathbf{6}$ & 9.61 \\
\hline $\mathbf{7}$ & 10.67 \\
\hline $\mathbf{8}$ & 11.42 \\
\hline
\end{tabular}

Averages - particles/fl3

\begin{abstract}
2nd
\end{abstract}

nd

\section{RACER TRAVERSE DATA FORM}

Fan configuration B\&C Max

Fan Setting 60

Stack Temp

$96.85 \mathrm{deg} F$

Start/End Time $1100 / 1300$

Center $2 / 3$ from

Points in Center $2 / 3$

Injection Pcint

\begin{tabular}{lll}
\hline$\frac{1.09}{2}$ & to: & $\mathbf{1 0 . 8 3}$ \\
\hline 15 & to: & \\
\hline 1 st &
\end{tabular}

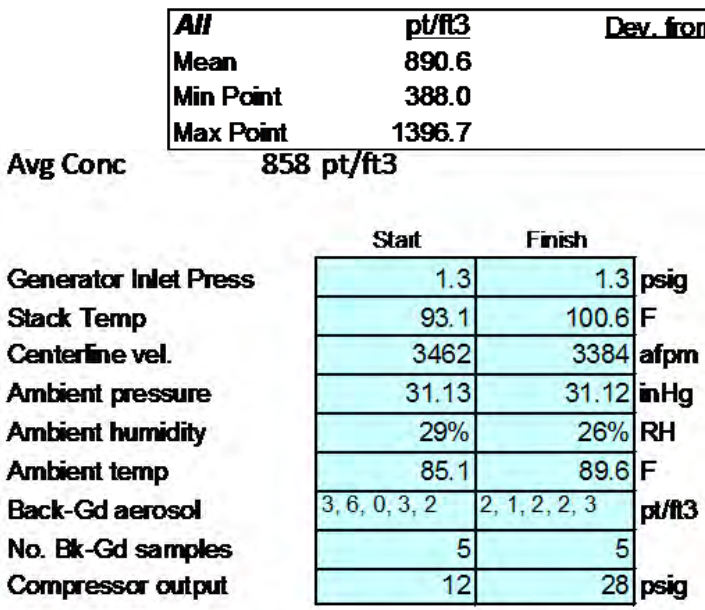

Notes: Velocity measured at Bottom 7.

During middle of 2 nd side traverse

seems that particle generation lowered.

Plastic particle generator (old style).

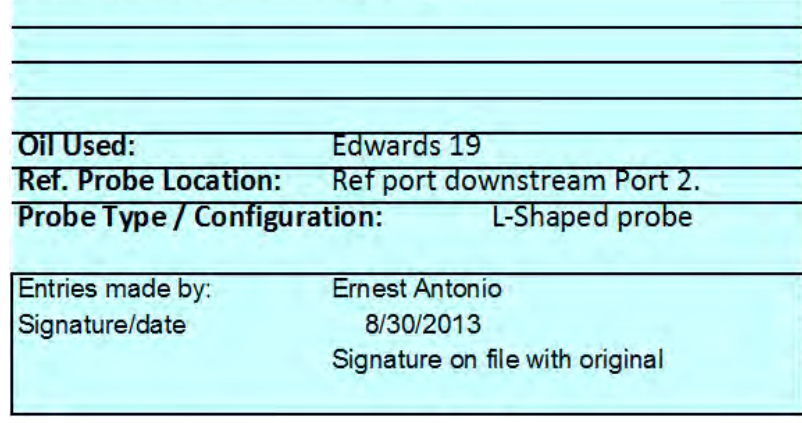

$-56.4 \%$
$56.8 \%$

\begin{tabular}{|r|r|r|r|r|}
\hline \multicolumn{5}{|c|}{$\begin{array}{c}\text { Bottom } \\
\mathbf{2}\end{array}$} \\
\hline $\mathbf{7}$ & $\mathbf{5}$ & $\mathbf{3}$ & Mean \\
\hline $\mathbf{7}$ & 705 & 777 & 819 & $\mathbf{7 6 7 . 0}$ \\
\hline $\mathbf{3}$ & 898 & 972 & 987 & $\mathbf{9 5 2 . 3}$ \\
\hline $\mathbf{3}$ & 1098 & 1129 & 1090 & $\mathbf{1 1 0 5 . 7}$ \\
\hline $\mathbf{3}$ & 1249 & 1321 & 1354 & $\mathbf{1 3 0 8 . 0}$ \\
\hline $\mathbf{3}$ & 1352 & 1357 & 1481 & $\mathbf{1 3 9 6 . 7}$ \\
\hline $\mathbf{0}$ & 1289 & 1344 & 1342 & $\mathbf{1 3 2 5 . 0}$ \\
\hline & 1120 & 1120 & 1218 & $\mathbf{1 1 5 2 . 7}$ \\
\hline $\mathbf{1 0 0 4 . 8}$ & $\mathbf{5 7 1}$ & $\mathbf{1 0 4 2 6}$ & 847 & $\mathbf{7 8 3 . 3}$ \\
\hline
\end{tabular}

.1

-


Rev. 0

3 Aug. 2006

\begin{tabular}{|c|c|}
\hline \multicolumn{2}{|c|}{ Site LV-S3 Scale Model } \\
\hline Date & $9 / 5 / 2013$ \\
\hline Tester & G, YFS \\
\hline Stack Dia. & 11.922 in. \\
\hline Stack X-Area & 111.6 in.2 \\
\hline Test Port & 1 \\
\hline to disturbance & 426.25 inches \\
\hline
\end{tabular}

Measurement units particles/fi3

Order $\longrightarrow$

Traverse-

Trial $\longrightarrow$

\begin{tabular}{|r|r|}
\hline Point & Depth, in. \\
\hline $\mathbf{1}$ & 0.5 \\
\hline $\mathbf{2}$ & 1.2 \\
$\mathbf{3}$ & 2.3 \\
\hline $\mathbf{4}$ & 3.8 \\
\hline Center & 5.9 \\
\hline $\mathbf{5}$ & 8.0 \\
\hline $\mathbf{6}$ & 9.6 \\
\hline $\mathbf{7}$ & 10.6 \\
\hline $\mathbf{8}$ & 11.4 \\
\hline
\end{tabular}

Averages -

PARTICLE TRACER TRAVERSE DATA FORU

Run No. PT-43

Fan configuration $\mathrm{BC}$ Max

Fan Setting 60

Stack Temp

Start/End Tme 9:37

Center 2/3 from

Points in Center 2/3

Injection Point 15

$\mathrm{Hz}$

$\operatorname{deg} F$

$\frac{1.09}{2}$ to:

\begin{tabular}{l|ll}
\cline { 2 - 3 } & \multicolumn{2}{c}{1 st } \\
\hline
\end{tabular}

3 Mean 1

2

\begin{tabular}{|c|c|c|c|c|c|c|c|}
\hline 1 & 2 & 3 & Mean & 1 & 2 & 3 & Mean \\
\hline \multicolumn{4}{|c|}{ particles/f13 } & \multicolumn{4}{|c|}{ particles/fi3 } \\
\hline 119 & 57 & 48 & 74.7 & 380 & 232 & 269 & 293.7 \\
\hline 167 & 81 & 80 & 109.3 & 401 & 277 & 254 & 310.7 \\
\hline 198 & 87 & 108 & 131.0 & 436 & 327 & 318 & 360.3 \\
\hline 165 & 107 & 96 & 122.7 & 495 & 344 & 360 & 399.7 \\
\hline 178 & 115 & 100 & 131.0 & 502 & 364 & 386 & 417.3 \\
\hline 156 & 94 & 117 & 122.3 & 458 & 328 & 323 & 369.7 \\
\hline 126 & 85 & 88 & 99.7 & 393 & 291 & 280 & 321.3 \\
\hline 117 & 91 & 68 & 92.0 & 246 & 207 & 181 & 211.3 \\
\hline 90 & 59 & 50 & 66.3 & 182 & 175 & 148 & 168.3 \\
\hline 2 & 86.2 & 83.9 & 105.4 & 388.1 & 282.8 & 279.9 & 316.9 \\
\hline
\end{tabular}

\begin{tabular}{|c|c|c|c|c|c|c|c|}
\hline 1 & 2 & 3 & Mean & 1 & 2 & 3 & Mean \\
\hline \multicolumn{4}{|c|}{ particles/f13 } & \multicolumn{4}{|c|}{ particles/fi3 } \\
\hline 119 & 57 & 48 & 74.7 & 380 & 232 & 269 & 293.7 \\
\hline 167 & 81 & 80 & 109.3 & 401 & 277 & 254 & 310.7 \\
\hline 198 & 87 & 108 & 131.0 & 436 & 327 & 318 & 360.3 \\
\hline 165 & 107 & 96 & 122.7 & 495 & 344 & 360 & 399.7 \\
\hline 178 & 115 & 100 & 131.0 & 502 & 364 & 386 & 417.3 \\
\hline 156 & 94 & 117 & 122.3 & 458 & 328 & 323 & 369.7 \\
\hline 126 & 85 & 88 & 99.7 & 393 & 291 & 280 & 321.3 \\
\hline 117 & 91 & 68 & 92.0 & 246 & 207 & 181 & 211.3 \\
\hline 90 & 59 & 50 & 66.3 & 182 & 175 & 148 & 168.3 \\
\hline 2 & 86.2 & 83.9 & 105.4 & 388.1 & 282.8 & 279.9 & 316.9 \\
\hline
\end{tabular}

\begin{tabular}{|lr|}
\hline All & $\mathrm{pt} / \mathrm{tt} 3$ \\
Mean & 211.2 \\
Min Point & 66.3 \\
Max Point & 417.3 \\
\hline
\end{tabular}

$$
\text { Avg Conc } 203 \mathrm{pt} / \mathrm{ft} 3
$$

Generator Inlet Press

Stack Temp

Centerine vel.

Ambient pressure

Ambient humidity

Ambient temp

Back-Gd aerosol

No. Bk-Gd samples

\begin{tabular}{|c|c|c|}
\hline Start & Finish & \\
\hline 4.2 & 4.9 & 9 psig \\
\hline 88.6 & 95.3 & $\mathrm{~F}$ \\
\hline 3796 & 3213 & \\
\hline 30.92 & 30.92 & \\
\hline $32 \%$ & $31 \%$ & $\mathbf{R H}$ \\
\hline 83.3 & 87.8 & \\
\hline $0,1,1,0,0$ & $3,1,0,1,0$ & \\
\hline 5 & & 5 \\
\hline 33 & 29 & \\
\hline
\end{tabular}

Compressor output

Notes:

1. Ref and Measure OPC in concentration mode. 2. After first traverse replaced A G Nozzle. 3. Plastic Aerosol Generator used.

\begin{tabular}{l}
\hline JAG 9/5/13 \\
\hline Oil Used: Edwards 19 \\
\hline Ref. Probe Location: Ref port downstream Port 2. \\
\hline Probe Type / Configuration: L-Shaped probe
\end{tabular}

Entries made by: Signature/date

J Glissmeyer
$\quad 9 / 5 / 2013$
Signature on file with original

\begin{tabular}{r|lrrrr|}
\hline & Center 2/3 & Side & Bottom & \multicolumn{1}{|c|}{. } & Normzd \\
Mean & 115.4 & 341.5 & 228.5 & 354.60 \\
$98.6 \%$ & Std. Dev. & 15.4 & 69.0 & 126.7 & 59.10 \\
$97.6 \%$ & Cov as \% & 13.3 & 20.2 & 55.5 & 16.67 \\
\hline
\end{tabular}

\begin{tabular}{lrc} 
Instuments Used: & T95351203001 & Cal. Due \\
TSI VelciCalc & 90936818 & $12 / 10 / 2013$ \\
\hline Fisher Scientific & 96258675 & $12 / 11 / 2013$ \\
\hline Met One OPC (Ref) & 1011529010 & \multicolumn{2}{c}{ FIO } \\
\hline Met One OPC & & $8 / 7 / 2014$ \\
\hline
\end{tabular}

Signature/date


Rev. 0

3Aug. 2006

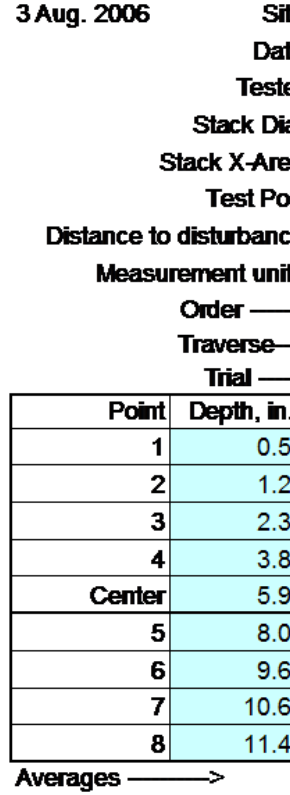

PARTICLE TRACER TRAVERSE DATA FORY

Site LV-S3 Scale Model

Date 9/9/2013

Tester JG,cb

Stack Dia. 11.922 in

XArea 111.6 in. 2

Art 426.25 inches

particles/fi3

Run No. PT-44

Fan configuration $\mathrm{BC}$ Max

Fan Setting 60

Stack Temp

$\mathrm{Hz}$

Start/End Tme 9:15/11:10

Center $2 / 3$ from

Points in Center $2 / 3$

Injection Point 15

1 st

(1) Bottom

2

2

3 Mean

\begin{tabular}{|r|r|r|r|}
783 & $\mathbf{7 7 2}$ & 806 & $\mathbf{7 8 7 . 0}$ \\
\hline 927 & 1001 & 1121 & $\mathbf{1 0 1 6 . 3}$ \\
\hline 1138 & 1279 & 1149 & $\mathbf{1 1 8 8 . 7}$ \\
\hline
\end{tabular}

\begin{tabular}{|l|r|r|r|}
1138 & 1279 & 1149 & $\mathbf{1 1 8 8 . 7}$ \\
\hline
\end{tabular}

\begin{tabular}{l|r|r|r|}
1379 & 1387 & 1383 & $\mathbf{1 3 8 3 . 0}$ \\
\hline
\end{tabular}

\begin{tabular}{l|r|}
1505 & 1518 \\
\hline
\end{tabular}

\begin{tabular}{l|l|}
1505 & 1518 \\
\hline 1345 & 1458 \\
\hline 1176 & 1178 \\
\hline
\end{tabular}

\begin{tabular}{r|r|}
\hline 1176 & 1178 \\
\hline 889 & 11 \\
\hline 847 & 844 \\
\hline 109.9 & $\mathbf{1 1 7 6 . 8}$ \\
\hline
\end{tabular}

\begin{tabular}{r|r}
\hline 8 & 156 \\
\hline 8 & 1436 \\
\hline 8 & 116 \\
\hline 4 & 951 \\
\hline 4 & 637 \\
\hline
\end{tabular}

\begin{tabular}{l|l|}
1564 & 1529.0 \\
\hline
\end{tabular}

\begin{tabular}{r|r|}
\hline 1413.0 \\
\hline 11736
\end{tabular}

\begin{tabular}{r|r|}
1164 & 1172.7 \\
\hline 951 & $\mathbf{9 9 8 . 0}$
\end{tabular}

\begin{tabular}{l|r}
637 & 776.0 \\
\hline 6 & 1140.4 \\
\hline
\end{tabular}

\begin{tabular}{|r|}
\hline \\
\hline 11 \\
\hline 130 \\
\hline 14 \\
\hline 1051.4 \\
\hline
\end{tabular}

122

\begin{tabular}{|c|c|c|c|}
\hline \multicolumn{4}{|c|}{ particles/fi3 } \\
\hline 725 & 683 & 632 & 680.0 \\
\hline 910 & 808 & 860 & 859.3 \\
\hline 1148 & 949 & 968 & 1021.7 \\
\hline 1300 & 1149 & 1108 & 1185.7 \\
\hline 1425 & 1217 & 1267 & 1303.0 \\
\hline 1370 & 1212 & 1203 & 1261.7 \\
\hline 1191 & 1001 & 1003 & 1065.0 \\
\hline 814 & 719 & 746 & 759.7 \\
\hline 580 & 548 & 533 & 553.7 \\
\hline & & & 35.5 \\
\hline
\end{tabular}

\begin{tabular}{|lrr|lrrr|r|}
\hline Alf & ptfit3 & Dev. from mean & Center 2/3 & Side & Bottom & Al & Normbd \\
Mean & 1053.0 & & 1243.0 & 1065.1 & 1154.0 & 1246.42 \\
Min Point & 553.7 & $-47.4 \%$ & Std. Dev. & 204.0 & 203.0 & 216.2 & 213.09 \\
Max Point & 1529.0 & $45.2 \%$ & Cov as $\%$ & 16.4 & 19.1 & 18.7 & 17.10 \\
\hline
\end{tabular}

Avg Conc $1008 \mathrm{pt} / \mathrm{ft3}$

\begin{tabular}{|c|c|c|c|}
\hline & Start & Finish & \\
\hline Generator Inlet Press & 1.5 & 1.5 & psig \\
\hline Stack Temp & 80.9 & 95.4 & $F$ \\
\hline Centerline vel. & 3450 & 3362 & afpm \\
\hline Ambient pressure & 30.98 & 31.01 & $\mathrm{nhH}$ \\
\hline Ambient humidity & $31 \%$ & $28 \%$ & RH \\
\hline Ambient temp & 79.7 & 84.2 & $F$ \\
\hline Back-Gd aerosol & $1,1,5,2,1$ & $0,3,0,0,0$ & $\mathrm{pt} / \mathrm{ft} 3$ \\
\hline No. Bk-Gd samples & 5 & 5 & \\
\hline Compressor output & 32 & 30 & psig \\
\hline
\end{tabular}

Notes:

Added cap of the Port2 cover after the 3rd reading on run 1 side.

\section{CB 09/09/2013}

Oilused: Edwards 19

Ref. Probe Location: Ref port downstream Port 2.

Probe Type/Configuration: L-Shaped probe

\begin{tabular}{|l|l|l|}
\hline $\begin{array}{l}\text { Entries made by: } \\
\text { Signature/date }\end{array}$ & $\begin{array}{l}\text { Carolyn Burns } \\
9 / 9 / 2013 \\
\text { Signature on file with original }\end{array}$ & $\begin{array}{l}\text { Technical Data Review performed by: } \\
\text { Signature/date } \\
\text { Signature on file with original TI-WTPSP 123 }\end{array}$ \\
\end{tabular}


Rev. 0

3 Aug. 2006

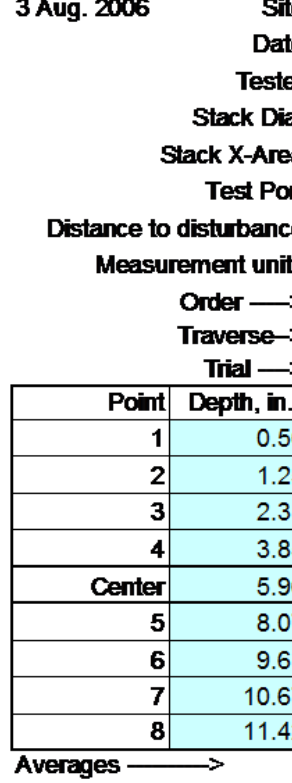

PARTCLE TRACER TRAVERSE DATA FORU
Site LV-S3 Scale Model

Date 9/9/2013

Tester JG,cb

111.6 in.2

ort $\frac{1}{426.25 \text { inches }}$

426.25 inches ts particles/ft3

\begin{tabular}{|c|c|}
\hline & Side \\
\hline 1 & 2 \\
\hline
\end{tabular}

\begin{tabular}{|c|c|}
\hline & particles/fi3 \\
\hline 1143 & 1109 \\
\hline
\end{tabular}

Fan configuration BC Norm

Fan Setting 55

Stack Temp

$\mathrm{Hz}$

Start/End Tme 11:30/13:15

Center $2 / 3$ from

Points in Center $2 / 3$ Injection Point 15

2nd

3. Bottom

\begin{tabular}{|c|c|c|c|c|}
\hline Mean & 1 & 2 & 3 & Mean \\
\hline & \multicolumn{4}{|c|}{ particles/fi3 } \\
\hline 1122.3 & 1079 & 1044 & 977 & 1033.3 \\
\hline 1291.7 & 1241 & 1199 & 1177 & 1205.7 \\
\hline 1521.0 & 1453 & 1460 & 1373 & 1428.7 \\
\hline 1695.0 & 1612 & 1686 & 1649 & 1649.0 \\
\hline 1784.7 & 1883 & 1908 & 1760 & 1850.3 \\
\hline 1736.0 & 1796 & 1778 & 1717 & 1763.7 \\
\hline 1552.7 & 1631 & 1567 & 1425 & 1541.0 \\
\hline 1352.7 & 1199 & 1231 & 1176 & 1202.0 \\
\hline 1097.3 & 1208 & 938 & 952 & 1032.7 \\
\hline 61.5 & 1455.8 & 1423.4 & 1356.2 & 1411.8 \\
\hline
\end{tabular}

\begin{tabular}{|lr}
\hline All & $p t / f t 3$ \\
Mean & 1436.6 \\
Min Point & 1032.7 \\
Max Point & 1850.3 \\
\hline \multicolumn{2}{|l|}{1389 pt/ft3 }
\end{tabular}

Avg Conc $1389 \mathrm{pt} / \mathrm{ft} 3$

\begin{tabular}{|c|c|c|}
\hline 1143 & 1109 & 1115 \\
\hline 1177 & 1390 & 1308 \\
\hline 1472 & 1635 & 1456 \\
\hline 1679 & 1642 & 1764 \\
\hline 1710 & 1778 & 1866 \\
\hline 1792 & 1677 & 1739 \\
\hline 1614 & 1624 & 1420 \\
\hline 1422 & 1379 & 1257 \\
\hline 1138 & 1002 & 1152 \\
\hline 0.8 & 1470.7 & 1453.0 \\
\hline
\end{tabular}

\begin{tabular}{|c|c|c|}
\hline $\begin{array}{r}\text { ptit3 } \\
1436.6 \\
1032.7 \\
1850.3 \\
\end{array}$ & & av. from \\
\hline pt/ft3 & & \\
\hline Start & Frish & \\
\hline 1.5 & 1.5 & psig \\
\hline 96.1 & 101.4 & $F$ \\
\hline 3088 & 3204 & afpm \\
\hline 31.01 & 30.98 & $\mathbf{i n H g}$ \\
\hline $28 \%$ & $21 \%$ & $\mathbf{R H}$ \\
\hline 84.2 & 94.1 & $\mathbf{F}$ \\
\hline $0,3,0,0,0$ & $0,0,3,0,0$ & pt/fi3 \\
\hline 5 & 5 & \\
\hline 26 & 40 & psig \\
\hline
\end{tabular}

Generator Inlet Press Stack Temp

Centerine vel.

Ambient pressure

Ambient humidity

Ambient temp

Back-Gd aerosol

No. Bk-Gd samples

Compressor output

Notes:

\begin{tabular}{l|}
\hline \\
\hline
\end{tabular}

\begin{tabular}{rrrr|} 
Side & Bottom & A & Nomkd \\
1562.0 & 1520.0 & 1541.0 & 1569.74 \\
189.8 & 256.2 & 217.7 & 225.46 \\
12.2 & 16.9 & 14.1 & 14.36 \\
\hline
\end{tabular}

Cal. Due

\begin{tabular}{lcc} 
hostuments Used: & & Cal. Due \\
TSI VelciCalc & T95351203001 & $12 / 10 / 2013$ \\
\hline
\end{tabular}

\begin{tabular}{lrc} 
TSI VelciCalc & T95351203001 & $12 / 10 / 2013$ \\
\hline Fisher Scientific & 90936818 & $12 / 11 / 2013$ \\
\hline Met One OPC (Ref) & 96258675 & FIO \\
\hline Met One OPC & 1011529010 & $8 / 7 / 2014$ \\
\hline
\end{tabular}

Technical Data Review performed by:
Signature/date
Signature on file with original T-WTPSP 123
$\mathbf{r}$


Rev. 0

3 Aug. 2006

\begin{tabular}{|c|c|}
\hline \multicolumn{2}{|c|}{ Site LV-S3 Scale Model } \\
\hline Date & $9 / 10 / 2013$ \\
\hline Tester & $S S, c b$ \\
\hline Stack Dia. & 11.922 in. \\
\hline Stack X-Area & 111.6 in. 2 \\
\hline Test Port & 1 \\
\hline to disturbance & 426.25 inches \\
\hline
\end{tabular}

Distance to disturbance 426.25 inches

Measurement units particles/fi3

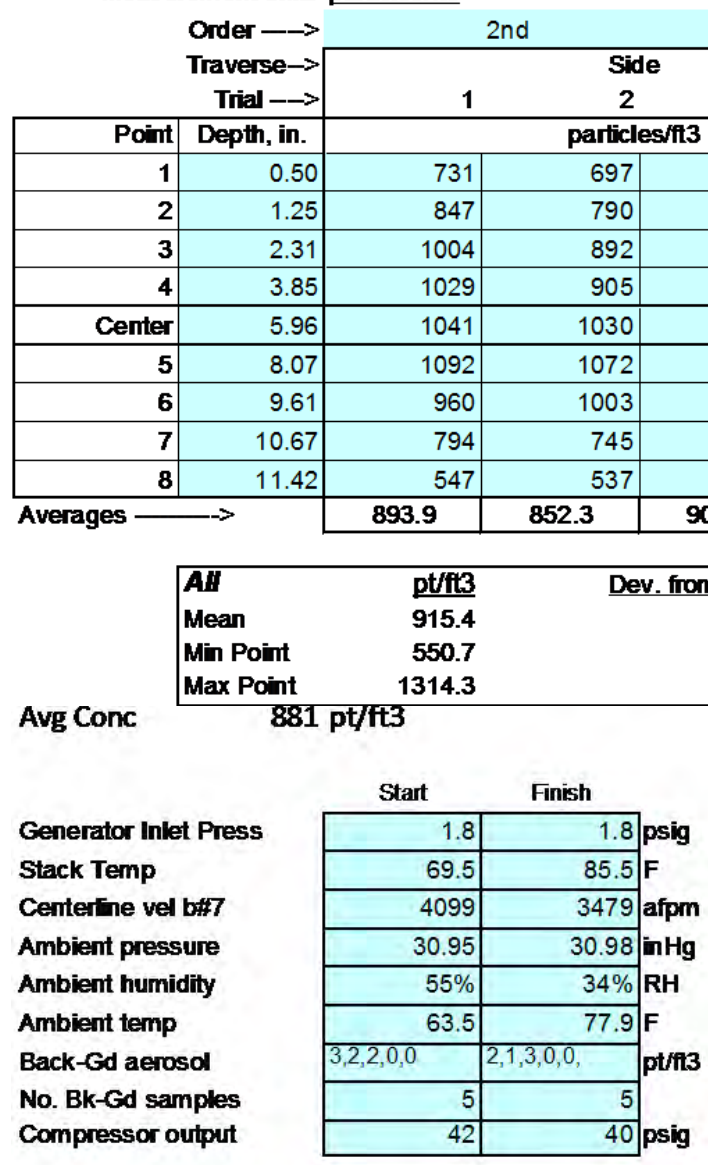

Notes:

CB $9 / 10 / 13$

CB 9/10/13

OाUsed: Edwards 19

Ref. Probe Location: Ref port downstream Port 2.

Probe Type/Configuration: L-Shaped probe

\begin{tabular}{|lc|}
\hline Entries made by: & Carolyn Burns \\
Signature/date & $9 / 10 / 2013$ \\
& Signature on file with original
\end{tabular}

Run No.

Fan configun No. PT-46

configuration $\mathrm{BC}$ Max

Fan Setting 60

Stack Temp

Start/End Time 7:20/9:30

Center $2 / 3$ from

Points in Center 2/3 Injection Point 15

\begin{tabular}{lll}
\hline \multicolumn{2}{|l}{ 77.5 deg $F$} & $\mathrm{~Hz}$ \\
\hline$\frac{1.09}{2}$ & to: & 10.83 \\
\hline & to: & 7
\end{tabular}

1 st

3. Bottom

\begin{tabular}{|r|r|r|r|r|r|}
\hline 3 & Mean & $\mathbf{4}$ & $\mathbf{2}$ & $\mathbf{3}$ & Mean \\
\hline \multicolumn{2}{|c|}{} & & \multicolumn{4}{|c|}{ particles/f3 } \\
\hline 600 & $\mathbf{6 7 6 . 0}$ & 646 & 685 & 558 & $\mathbf{6 2 9 . 7}$ \\
\hline 778 & $\mathbf{8 0 5 . 0}$ & 849 & 827 & 758 & $\mathbf{8 1 1 . 3}$ \\
\hline 911 & $\mathbf{9 3 5 . 7}$ & 1090 & 1047 & 889 & $\mathbf{1 0 0 8 . 7}$ \\
\hline 111 & $\mathbf{1 0 1 5 . 0}$ & 1320 & 1180 & 1107 & $\mathbf{1 2 0 2 . 3}$ \\
\hline 108 & $\mathbf{1 0 5 9 . 7}$ & 1511 & 1253 & 1179 & $\mathbf{1 3 1 4 . 3}$ \\
\hline 186 & $\mathbf{1 1 1 6 . 7}$ & 1422 & 1251 & 1035 & $\mathbf{1 2 3 6 . 0}$ \\
\hline 951 & $\mathbf{9 7 1 . 3}$ & 1160 & 1028 & 956 & $\mathbf{1 0 4 8 . 0}$ \\
\hline 874 & $\mathbf{8 0 4 . 3}$ & 792 & 730 & 661 & $\mathbf{7 2 7 . 7}$ \\
\hline 612 & $\mathbf{5 6 5 . 3}$ & 561 & 584 & 507 & $\mathbf{5 5 0 . 7}$ \\
\hline & $\mathbf{8 8 3 . 2}$ & $\mathbf{1 0 3 9 . 0}$ & $\mathbf{9 5 3 . 9}$ & $\mathbf{8 5 0 . 0}$ & $\mathbf{9 4 7 . 6}$ \\
\hline
\end{tabular}

\begin{tabular}{l|l}
\hline & Center 2/3 \\
$-39.8 \%$ & Mean \\
$43.6 \%$ & Std. Dev. \\
CoV as \%
\end{tabular}

\begin{tabular}{rr} 
Side & Bottom \\
958.2 & 1049 \\
120.0 & 219 \\
12.5 & 21 \\
\hline
\end{tabular}

\begin{tabular}{rr|r|}
\hline Bottom & All & Normbd \\
\hline 1049.8 & 1004.0 & 1119.15 \\
219.9 & 176.7 & 194.27 \\
& 17.6 & 17.36 \\
\hline
\end{tabular}

\begin{tabular}{lrr} 
Instuments Used: & T95351203001 & \multicolumn{1}{c}{ Cal. Due } \\
TSI VelciCalc & 90936818 & $12 / 10 / 2013$ \\
\hline Fisher Scientific & 96258675 & $12 / 11 / 2013$ \\
\hline Met One OPC (Ref) & 1011529010 & FIO \\
\hline Met One OPC & & $8 / 7 / 2014$ \\
\hline
\end{tabular}

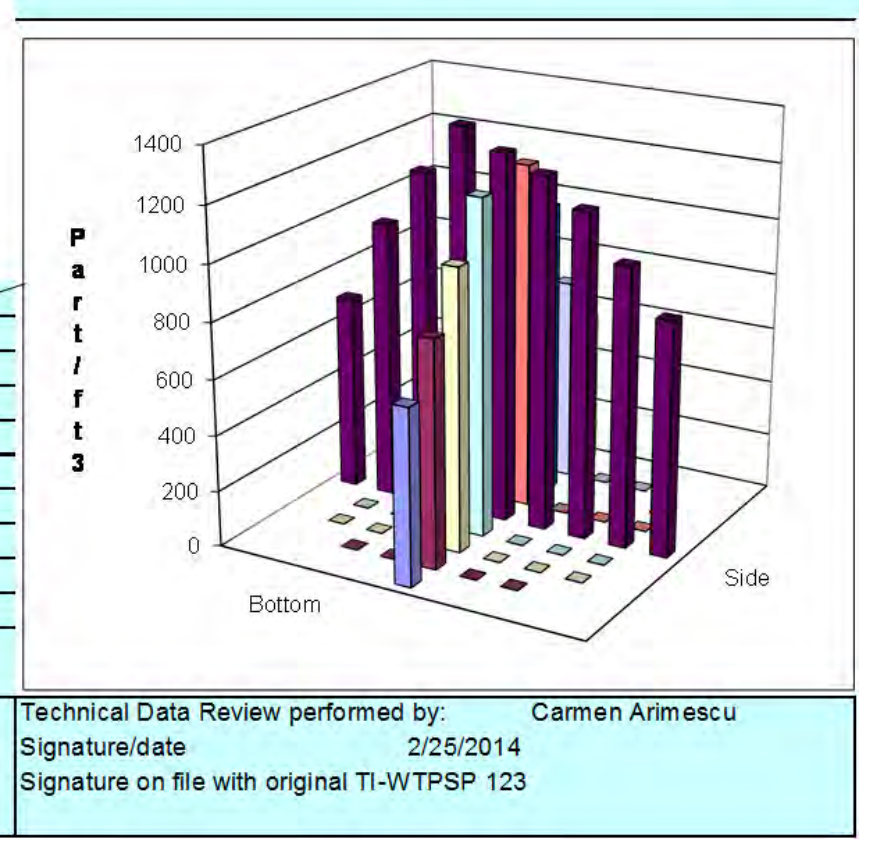


Rev. 0

3 Aug. 2006

\begin{tabular}{|c|c|}
\hline \multicolumn{2}{|c|}{ Site LV-S3 Scale Model } \\
\hline Date & $9 / 10 / 2013$ \\
\hline Tester & SS,TH \\
\hline Stack Dia. & 11.922 in. \\
\hline Stack X-Area & 111.6 in. 2 \\
\hline Test Port & 1 \\
\hline to disturbance & 426.25 inches \\
\hline
\end{tabular}

Distance to disturbance

Measurement units particles/ft3

\begin{tabular}{|c|c|c|c|c|c|c|c|c|c|}
\hline & & \multirow{2}{*}{\multicolumn{3}{|c|}{ 1st }} & \multirow{2}{*}{\multicolumn{5}{|c|}{ 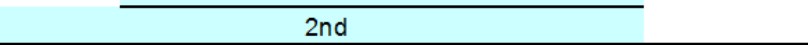 }} \\
\hline & \multirow{3}{*}{$\begin{array}{l}\text { Order } \longrightarrow \\
\text { Traverse } \rightarrow \\
\quad \text { Trial } \longrightarrow\end{array}$} & & & & & & & & \\
\hline & & \multirow[b]{2}{*}{1} & \multicolumn{2}{|c|}{ Side } & \multirow{3}{*}{ Mean } & \multicolumn{3}{|c|}{ Bottom } & \multirow[b]{2}{*}{ Mean } \\
\hline & & & 2 & 3 & & \multirow[t]{2}{*}{1} & \multicolumn{2}{|c|}{2} & \\
\hline Point & Depth, in. & \multicolumn{3}{|c|}{ particles/fi3 } & & & \multicolumn{2}{|c|}{ particles/f13 } & \\
\hline 1 & 0.50 & 860 & 828 & 876 & 854.7 & 836 & 848 & 857 & 847.0 \\
\hline 2 & 1.25 & 905 & 902 & 933 & 913.3 & 860 & 927 & 912 & 899.7 \\
\hline 3 & 2.31 & 958 & 972 & 951 & 960.3 & 968 & 1003 & 962 & 977.7 \\
\hline 4 & 3.85 & 960 & 1036 & 1009 & 1001.7 & 1019 & 986 & 1069 & 1024.7 \\
\hline Center & 5.96 & 984 & 1004 & 1045 & 1011.0 & 1018 & 1040 & 1105 & 1054.3 \\
\hline 5 & 8.07 & 961 & 957 & 999 & 972.3 & 1036 & 1054 & 1061 & 1050.3 \\
\hline 6 & 9.61 & 929 & 955 & 941 & 941.7 & 1016 & 1010 & 1061 & 1029.0 \\
\hline 7 & 10.67 & 907 & 886 & 898 & 897.0 & 910 & 870 & 961 & 913.7 \\
\hline 8 & 11.42 & 846 & 794 & 853 & 831.0 & 814 & 805 & 863 & 827.3 \\
\hline \multicolumn{2}{|c|}{ Averages $\longrightarrow$} & 923.3 & 926.0 & 945.0 & 931.4 & 941.9 & 949.2 & 983.4 & 958.2 \\
\hline
\end{tabular}

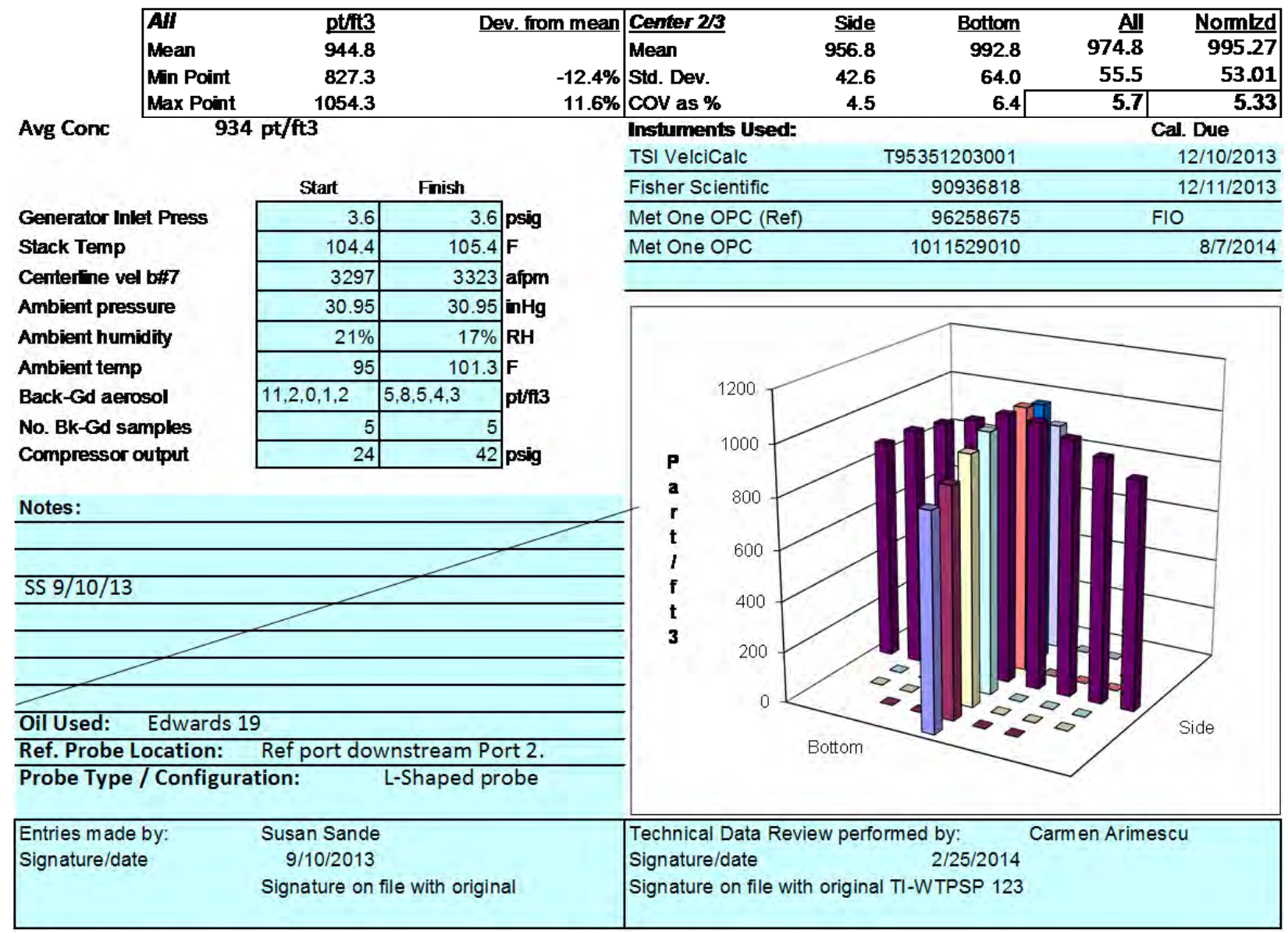


Rev. 0

3 Aug. 2006

PARTCLE TRACER TRAVERSE DATA FORY

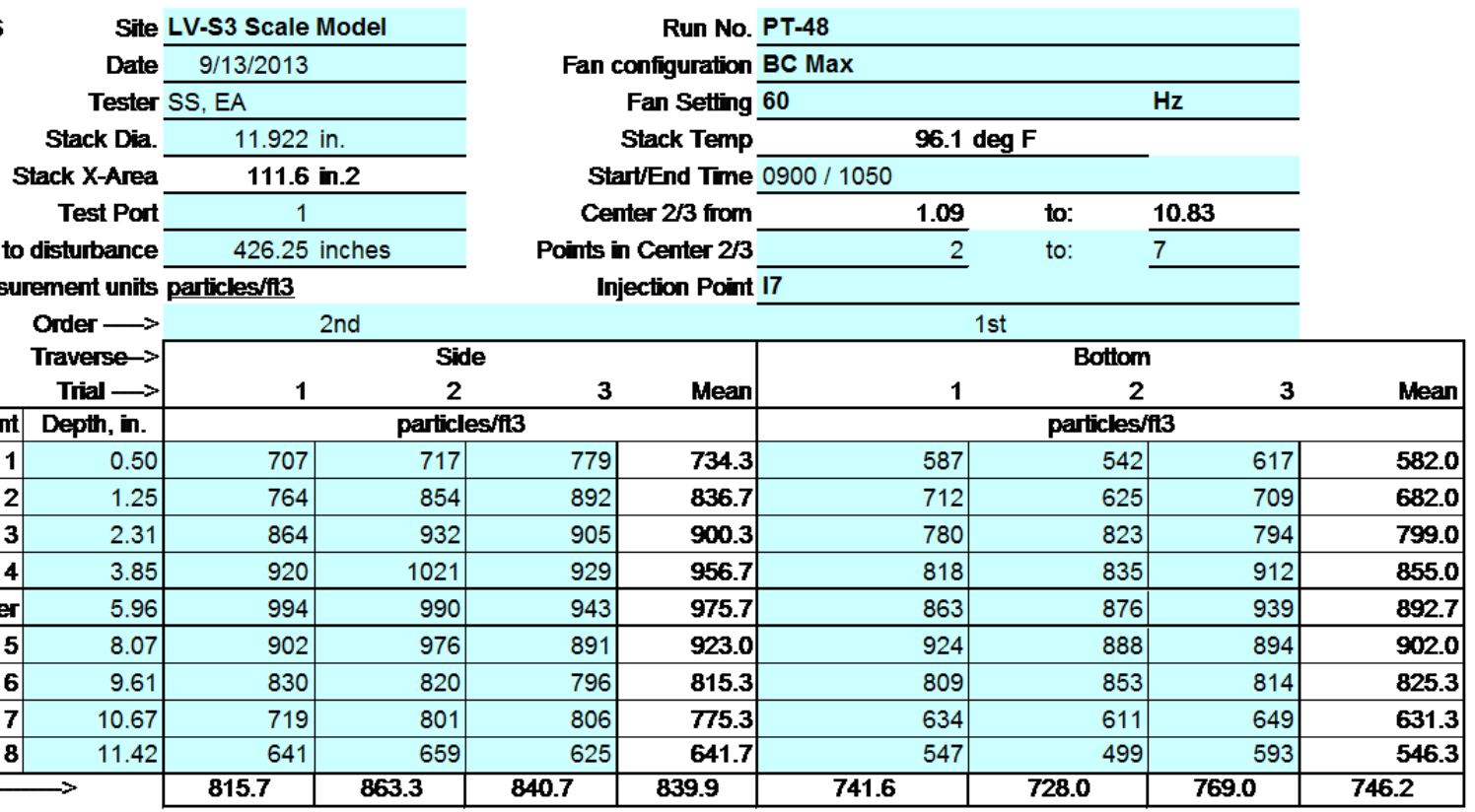

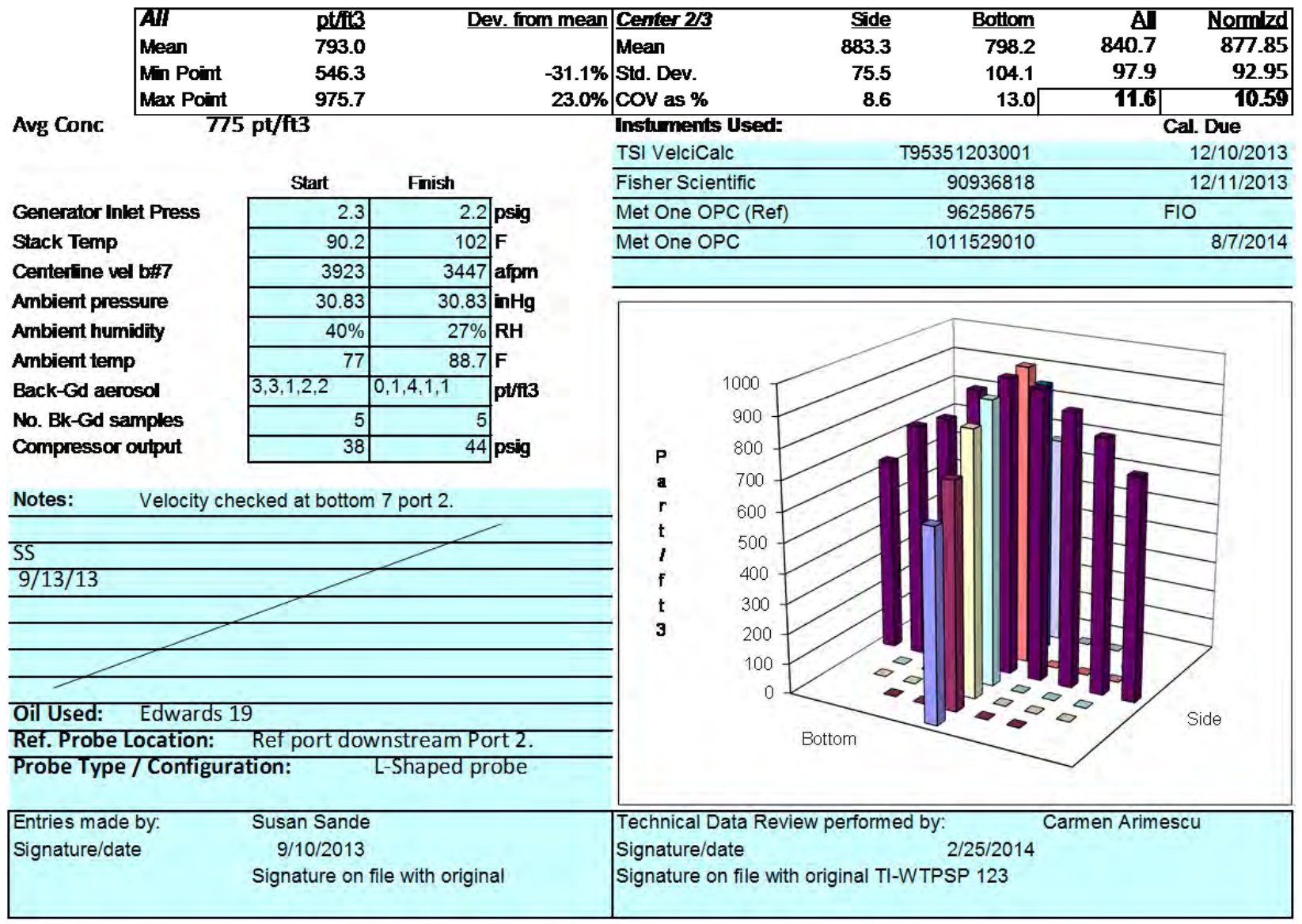


Rev. 0

3 Aug. 2006

\begin{tabular}{|c|c|}
\hline \multicolumn{2}{|c|}{ Site LV-S3 Scale Model } \\
\hline Date & $9 / 13 / 2013$ \\
\hline Tester & S, EA \\
\hline Stack Dia. & 11.922 in. \\
\hline Stack X-Area & 111.6 in. 2 \\
\hline Test Port & 1 \\
\hline to disturbance & 426.25 inches \\
\hline
\end{tabular}
Measurement units particles/fi3
PARTICLE TRACER TRAVERSE DATA FORU

Run No. PT-49

Fan configuration BC min

Fan Setting 25

Stack Temp

Start/End Tme $1320 / 1510$

Center 2/3 from

Points in Center 2/3 Injection Point 17

\begin{tabular}{|c|c|c|c|c|c|c|c|c|c|}
\hline \multirow{3}{*}{\multicolumn{2}{|c|}{ Order $-\longrightarrow$}} & \multicolumn{3}{|c|}{ 1st } & \multicolumn{5}{|c|}{ 2nd } \\
\hline & & \multicolumn{3}{|c|}{ Side } & \multirow[b]{2}{*}{ Mean } & \multicolumn{3}{|c|}{ Bottom } & \multirow[b]{2}{*}{ Mean } \\
\hline & & 1 & 2 & 3 & & 1 & 2 & 3 & \\
\hline Point & Depth, in. & \multicolumn{4}{|c|}{ particles/ft3 } & \multicolumn{4}{|c|}{ particles/ft3 } \\
\hline 1 & 0.50 & 977 & 929 & 1003 & 969.7 & 1139 & 994 & 1059 & 1064.0 \\
\hline 2 & 1.25 & 1090 & 1055 & 1090 & 1078.3 & 1072 & 1024 & 1150 & 1082.0 \\
\hline 3 & 2.31 & 1124 & 1107 & 1051 & 1094.0 & 1152 & 1112 & 1146 & 1136.7 \\
\hline 4 & 3.85 & 1135 & 1104 & 1093 & 1110.7 & 1183 & 1215 & 1163 & 1187.0 \\
\hline Center & 5.96 & 1152 & 1167 & 1173 & 1164.0 & 1193 & 1299 & 1243 & 1245.0 \\
\hline 5 & 8.07 & 1122 & 1050 & 1067 & 1079.7 & 1230 & 1228 & 1200 & 1219.3 \\
\hline 6 & 9.61 & 1025 & 1098 & 1034 & 1052.3 & 1133 & 1201 & 1211 & 1181.7 \\
\hline 7 & 10.67 & 1038 & 950 & 1051 & 1013.0 & 1053 & 1156 & 1140 & 1116.3 \\
\hline 8 & 11.42 & 942 & 968 & 984 & 964.7 & 1042 & 1084 & 1007 & 1044.3 \\
\hline Averages - & & 1067.2 & 1047.6 & 1060.7 & $\begin{array}{l}1058.5 \\
\end{array}$ & 1133.0 & 1145.9 & 1146.6 & $\begin{array}{l}1141.8 \\
\end{array}$ \\
\hline
\end{tabular}

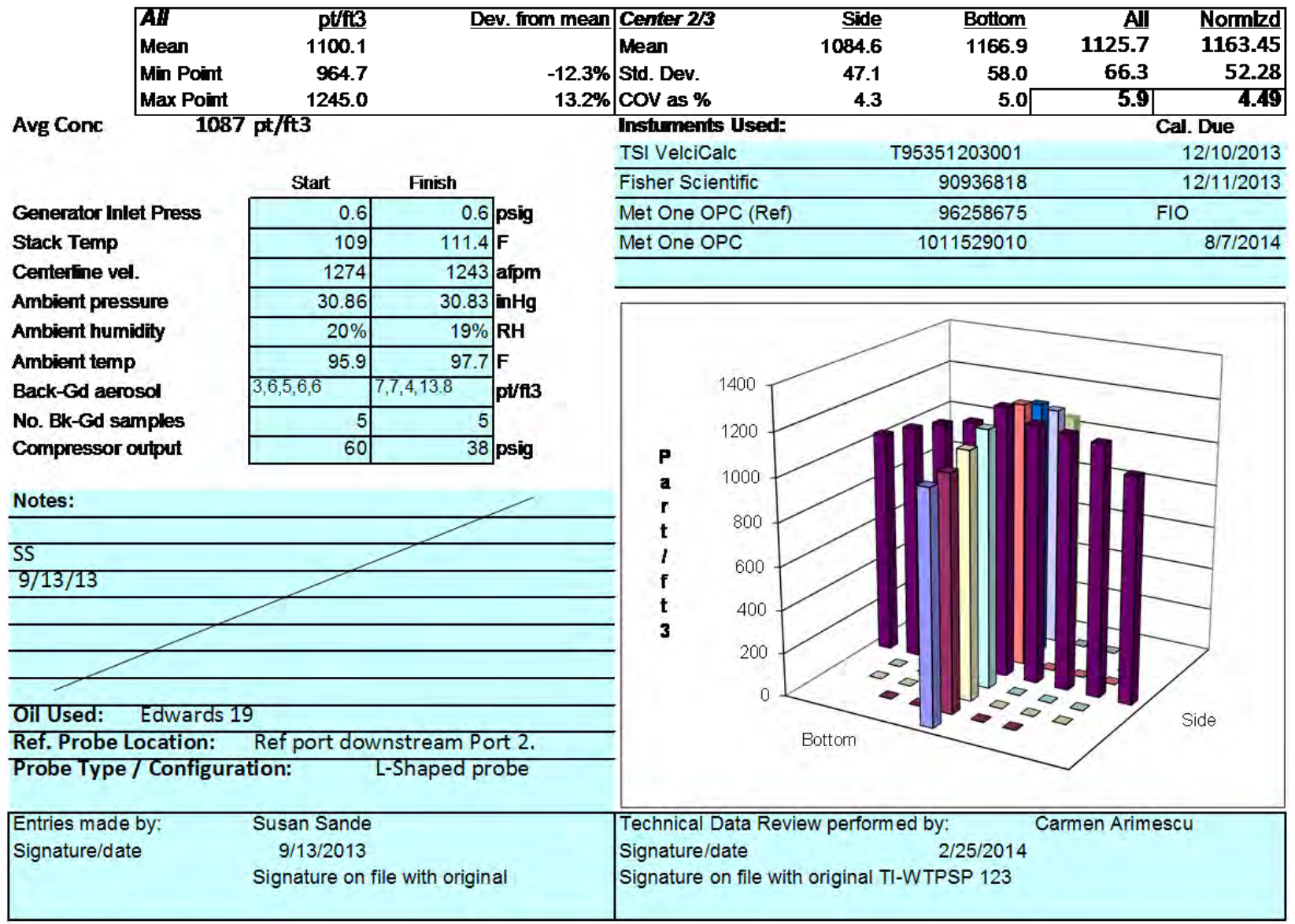


Rev. 0

3 Aug. 2006

\begin{tabular}{|c|c|c|}
\hline & Date & $9 / 16 / 2013$ \\
\hline \multicolumn{3}{|c|}{ Tester $\mathrm{cb}, \mathrm{TH}$} \\
\hline \multirow{2}{*}{\multicolumn{2}{|c|}{$\begin{array}{r}\text { Stack Dia. } \\
\text { Stack X-Area }\end{array}$}} & $11.922 \mathrm{in}$ \\
\hline & & 111.6 in. 2 \\
\hline \multicolumn{2}{|r|}{ Test Port } & 2 \\
\hline \multicolumn{2}{|c|}{ Distance to distumbance } & $510.25 \mathrm{inc}$ \\
\hline \multicolumn{3}{|c|}{ Measurement units particles/ti3 } \\
\hline & $2 n$ \\
\hline \multicolumn{2}{|r|}{$\begin{array}{r}\text { Traverse- }> \\
\text { Trial } \longrightarrow\end{array}$} & 1 \\
\hline \multicolumn{2}{|c|}{\begin{tabular}{l|l|} 
Pcint & Depth, in. \\
\end{tabular}} & \\
\hline 1 & 0.50 & 770 \\
\hline \multirow{2}{*}{2} & 1.25 & 839 \\
\hline & 2.31 & 955 \\
\hline $\begin{array}{l}3 \\
4\end{array}$ & 3.85 & 1023 \\
\hline Center & 5.96 & 1085 \\
\hline 5 & 8.07 & 1058 \\
\hline 6 & 9.61 & 963 \\
\hline 7 & 10.67 & 954 \\
\hline \multirow{2}{*}{$\begin{array}{r}8 \\
\text { Averages } \\
\end{array}$} & 11.42 & 827 \\
\hline & Averages $\longrightarrow \longrightarrow$ & 941.6 \\
\hline \multirow{4}{*}{\multicolumn{2}{|c|}{\begin{tabular}{|l|}
$\boldsymbol{A} H$ \\
Mean \\
Min Point \\
Max Point \\
\end{tabular}}} & pt/fi3 \\
\hline & & 933.7 \\
\hline & & 739.3 \\
\hline & & 1133.3 \\
\hline \multicolumn{2}{|l|}{ Avg Conc } & $7 \mathrm{ft} 3$ \\
\hline
\end{tabular}

PARTICLE TRACER TRAVERSE DATA FORM

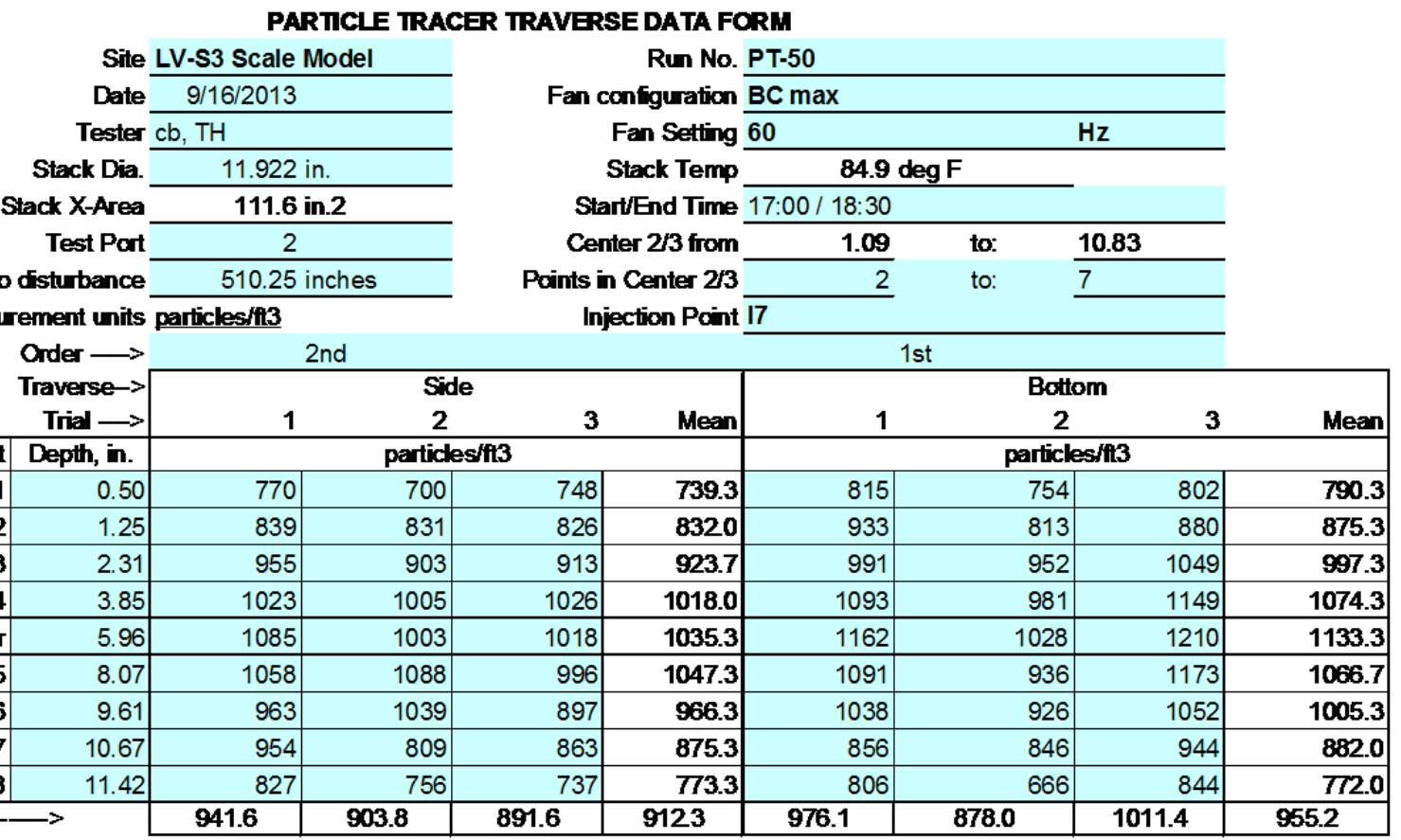

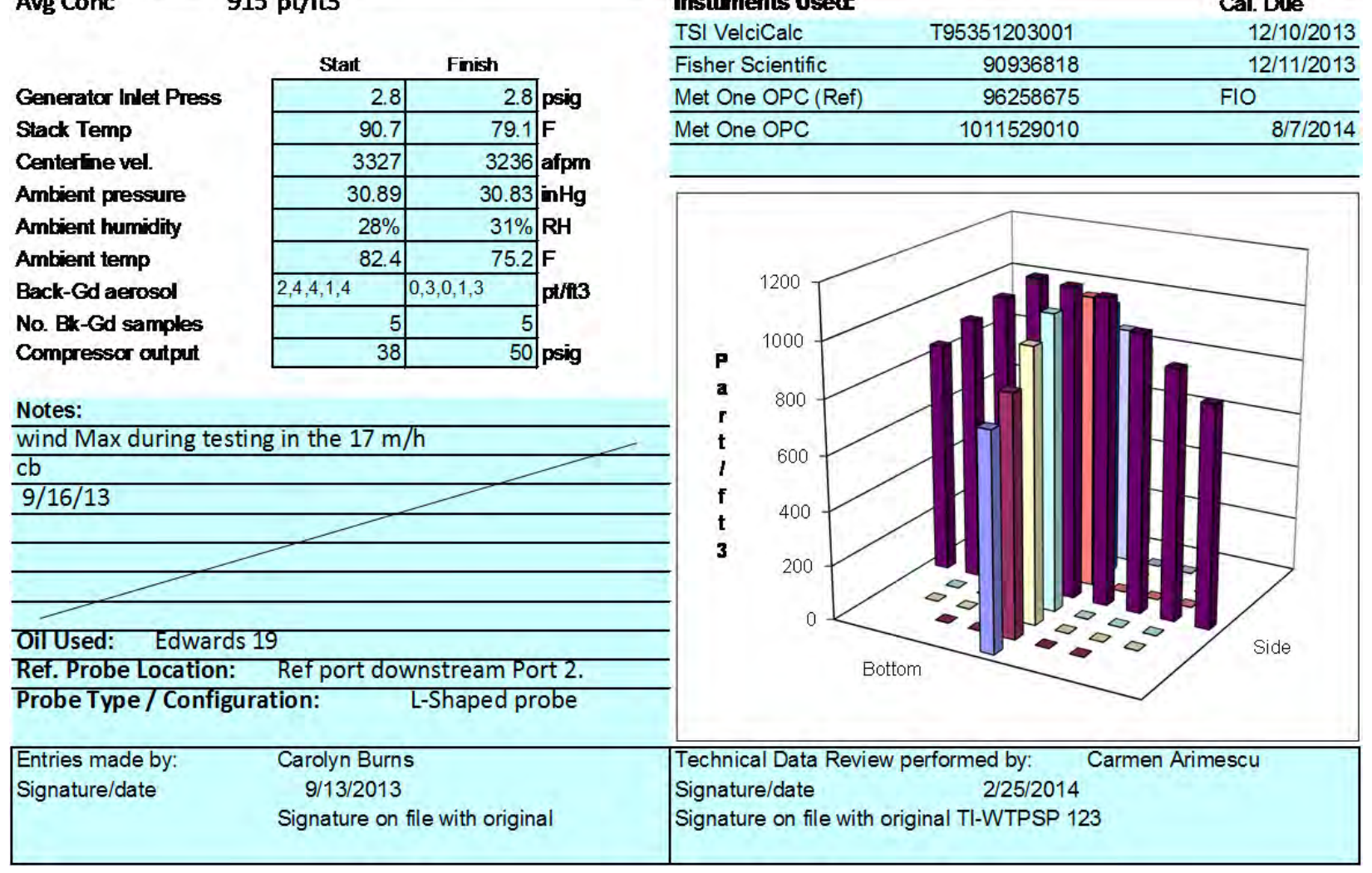


Rev. 0

3 Aug. 2006

Site LV-S3 Scale Model

Date 9/17/2013

Tester SS, SFS

Stack Dia.

11.922 in

Stack X-Area

111.6 in.

Test Port

2

Distance to disturbance 510.25 inches

Measurement units particles/ti 3

Order $\longrightarrow$

Traverse->

Trial $\rightarrow$

\begin{tabular}{|r|r|}
\hline Point & Depth, in. \\
\hline $\mathbf{1}$ & 0.5 \\
\hline $\mathbf{2}$ & 1.2 \\
$\mathbf{3}$ & 2.3 \\
\hline $\mathbf{4}$ & 3.8 \\
\hline Center & 5.9 \\
\hline $\mathbf{5}$ & 8.07 \\
\hline $\mathbf{6}$ & 9.6 \\
\hline $\mathbf{7}$ & 10.6 \\
\hline $\mathbf{8}$ & 11.4 \\
\hline
\end{tabular}

Averages -

\begin{tabular}{|lr}
\hline AII & $\mathrm{pt} / \mathrm{ft} 3$ \\
Mean & 1898.2 \\
Min Point & 1611.0
\end{tabular}

Max Point

2188.7

2nd

PARTICLE TRACER TRAVERSE DATA FORM

Run No. PT-51

Fan configuration $\mathrm{BC}$ min

Fan Setting 25

Stack Temp

$80.5 \operatorname{deg} F$

Start/End Time 0915/1130

Center $2 / 3$ from

Points in Center 23

Injection Point 17

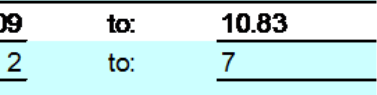
Side

3

\begin{tabular}{|r|r|r|r|r|}
\hline & \multicolumn{5}{|c|}{$\begin{array}{c}\text { Bottom } \\
\mathbf{2}\end{array}$} \\
\hline & $\mathbf{4}$ & $\mathbf{3}$ & Mean \\
\hline $\mathbf{1 6 3 7 . 3}$ & 1598 & 1616 & 1619 & $\mathbf{1 6 1 1 . 0}$ \\
\hline $\mathbf{1 8 9 1 . 3}$ & 1647 & 1687 & 1802 & $\mathbf{1 7 1 2 . 0}$ \\
\hline $\mathbf{1 9 9 6 . 3}$ & 1787 & 1712 & 1823 & $\mathbf{1 7 7 4 . 0}$ \\
\hline $\mathbf{2 0 0 4 . 3}$ & 1711 & 1763 & 1819 & $\mathbf{1 7 6 4 . 3}$ \\
\hline $\mathbf{2 1 3 2 7}$ & 1801 & 1771 & 1938 & $\mathbf{1 8 3 6 . 7}$ \\
\hline $\mathbf{2 1 6 7 . 3}$ & 1772 & 1912 & 1901 & $\mathbf{1 8 6 1 . 7}$ \\
\hline $\mathbf{2 1 8 8 . 7}$ & 1765 & 1778 & 1795 & $\mathbf{1 7 7 9 . 3}$ \\
\hline $\mathbf{2 1 8 1 . 0}$ & 1724 & 1748 & 1923 & $\mathbf{1 7 9 8 . 3}$ \\
\hline $\mathbf{2 1 3 6 . 3}$ & 1679 & 1640 & 1768 & $\mathbf{1 6 9 5 . 7}$ \\
\hline $\mathbf{2 0 3 7 . 3}$ & $\mathbf{1 7 2 0 . 4}$ & $\mathbf{1 7 3 6 . 3}$ & $\mathbf{1 8 2 0 . 9}$ & $\mathbf{1 7 5 9 . 2}$ \\
\hline & & & & \\
\hline
\end{tabular}

\begin{tabular}{r|r}
2094 & 2378 \\
$\mathbf{2 0 3 2 7}$ & $\mathbf{2 3 0 0}$.2 \\
\hline
\end{tabular}

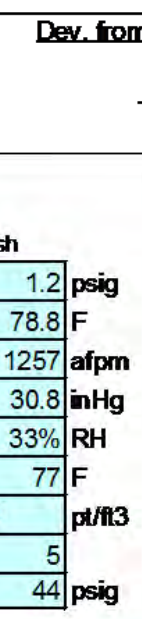

Generator Inlet Press

Stack Temp

Centerline vel.

Ambient pressure

Ambient humidity

Ambient temp

Back-Gd aerosol

No. Bk-Gd samples

Compressor output

Notes:

SS

Oil Used: Edwards 19

Ref. Probe Location: Ref port downstream Port 2.

Probe Type/Configuration:

L-Shaped probe

Entries made by:

Signature/date

Susan Sande
9/17/2013
Signature on file with original

Center 2/3

Mean

$-15.1 \%$ Std. Dev.

$15.3 \%$ COV as \%

Instuments Usect

Side
2080.2
116.0
5.6

TSI VelciCalc

Fisher Scientific

Met One OPC (Ref)

Met One OPC

\begin{tabular}{rrr|} 
Bottom & All & Normlzd \\
\hline 1789.5 & 1934.9 & 2079.05 \\
49.1 & 173.5 & 87.83 \\
27 & $\mathbf{9 . 0}$ & 4.22 \\
\hline
\end{tabular}

27

T95351203001

Cal. Due

$12 / 10 / 2013$

\begin{tabular}{lll} 
Met One OPC & 1011529010 & $8 / 7 / 2014$ \\
\hline
\end{tabular}

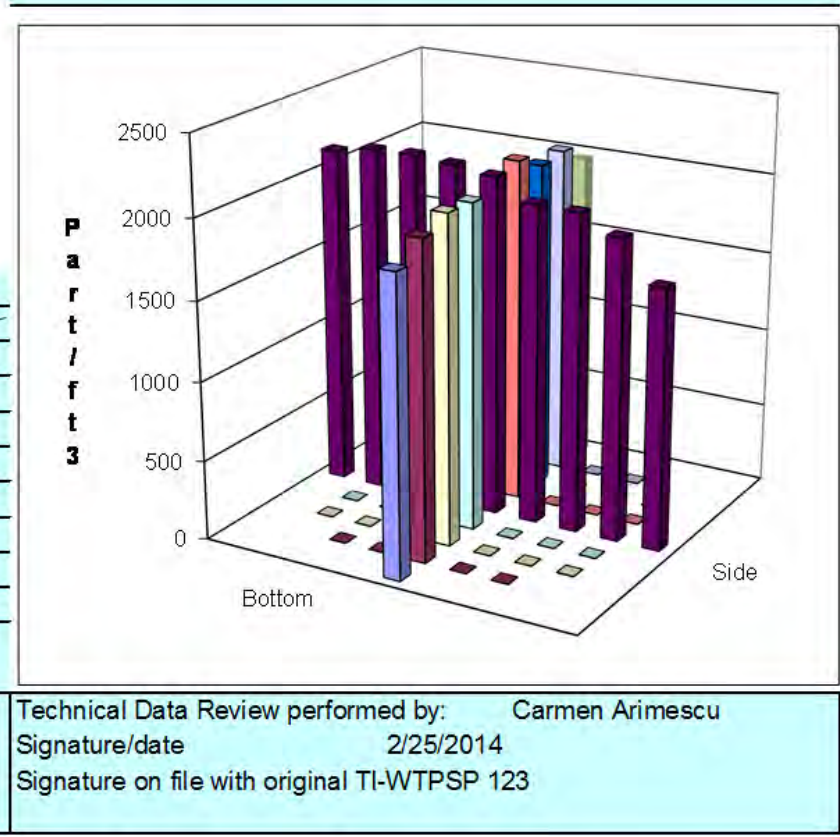


Rev. 0

3 Aug. 2006

Site LV-S3 Scale Model

Date 9/17/2013

Tester SFS, TH

Stack Dia

Stack X-Area

Test Part

11.922 in

111.6 in. 2

Distance to disturbance

Measurement units particles/fi3

510.25 inches

\begin{tabular}{|r|r|}
\multicolumn{1}{|c}{$\begin{array}{r}\text { Order } \\
\text { Traverse- } \\
\text { Trial } \longrightarrow\end{array}$} \\
\hline Point & Depth, $\mathbf{i n}$ \\
\hline $\mathbf{1}$ & 0.50 \\
\hline $\mathbf{2}$ & 1.25 \\
\hline $\mathbf{3}$ & 2.31 \\
\hline $\mathbf{4}$ & 3.85 \\
\hline Center & 5.96 \\
\hline $\mathbf{5}$ & 8.07 \\
\hline $\mathbf{6}$ & 9.61 \\
\hline $\mathbf{7}$ & 10.67 \\
\hline $\mathbf{8}$ & 11.42 \\
\hline &
\end{tabular}

\begin{tabular}{l|l} 
Averages $\longrightarrow$ & $\mathbf{8 6 1 . 3}$ \\
\hline
\end{tabular}

1 st

PARTICLE TRACER TRAVERSE DATA FORM

Run No. PT-52

Fan configuration AC MAX

Fan Setting 60

Stack Temp $78.35 \mathrm{deg} F$

$\mathrm{Hz}$

Start/End Time 12:35 14:12

Center 2/3 from

Points in Center 233

Injection Point 17

\begin{aligned} $\mathbf{1 . 0 9} &$ to: $14: 12 \\$\hline 2 & to: $\quad \underline{\mathbf{1 0 . 8 3}}\end{aligned}$

2nd

1st Side

\begin{tabular}{|ll|}
\hline AII & pt/ft3 \\
Mean & 814.1 \\
Min Point & 6120 \\
Max Point & 977.0 \\
\hline
\end{tabular}

Avg Conc $796 \mathrm{pt} / \mathrm{ft} 3$

Generator Inlet Press

Stack Temp

Centerfine vel.

Ambient pressure

Ambient humidity

Ambient temp

Back-Gd aerosol

No. Bk-Gd samples

Compressor output

\begin{tabular}{|c|c|c|}
\hline Stat & Finish & \\
\hline 2.9 & 2.9 & psig \\
\hline 79.1 & 77.6 & $F$ \\
\hline 3365 & 3384 & afpm \\
\hline 30.8 & 30.8 & $\mathrm{inHg}$ \\
\hline $31 \%$ & $38 \%$ & RH \\
\hline 77.9 & 73.4 & $7 \mathrm{~F}$ \\
\hline $0,1,1,0,1$ & $1,1,0,0,0$ & pt/f3 \\
\hline 5 & 5 & \\
\hline 40 & 48 & psig \\
\hline
\end{tabular}

Notes:

SS $9 / 17 / 13$

Oil Used: Edwards 19

Ref. Probe Location: Ref port downstream Port 2.

Probe Type/Configuration: L-Shaped probe

\begin{tabular}{|lc|}
\hline Entries made by: & Tristan Hay \\
Signature/date & $9 / 17 / 2013$ \\
& Signature on file with original \\
& \\
\hline
\end{tabular}

\begin{tabular}{|r|lrrrr|}
\hline Dev. from mean & Center 2/3 & Side & Bottom & \multicolumn{1}{c|}{ All } & Normlzd \\
\cline { 2 - 5 } & Mean & 897.2 & 816.3 & 856.7 & $\mathbf{8 6 6 . 7 5}$ \\
$24.8 \%$ & Std. Dev. & 86.9 & 97.2 & 98.0 & 95.18 \\
\hline CoV as \% & 9.7 & 11.9 & 11.4 & 10.98 \\
\hline
\end{tabular}

Instuments Usedt

\begin{tabular}{lrr} 
TSI VelciCalc & T95351203001 & \multicolumn{1}{c}{$12 / 10 / 2013$} \\
\hline Fisher Scientific & 90936818 & $12 / 11 / 2013$ \\
\hline Met One OPC (Ref) & 96258675 & \multicolumn{2}{c}{ FIO } \\
\hline Met One OPC & 1011529010 & \multicolumn{2}{c}{$8 / 7 / 2014$} \\
\hline
\end{tabular}

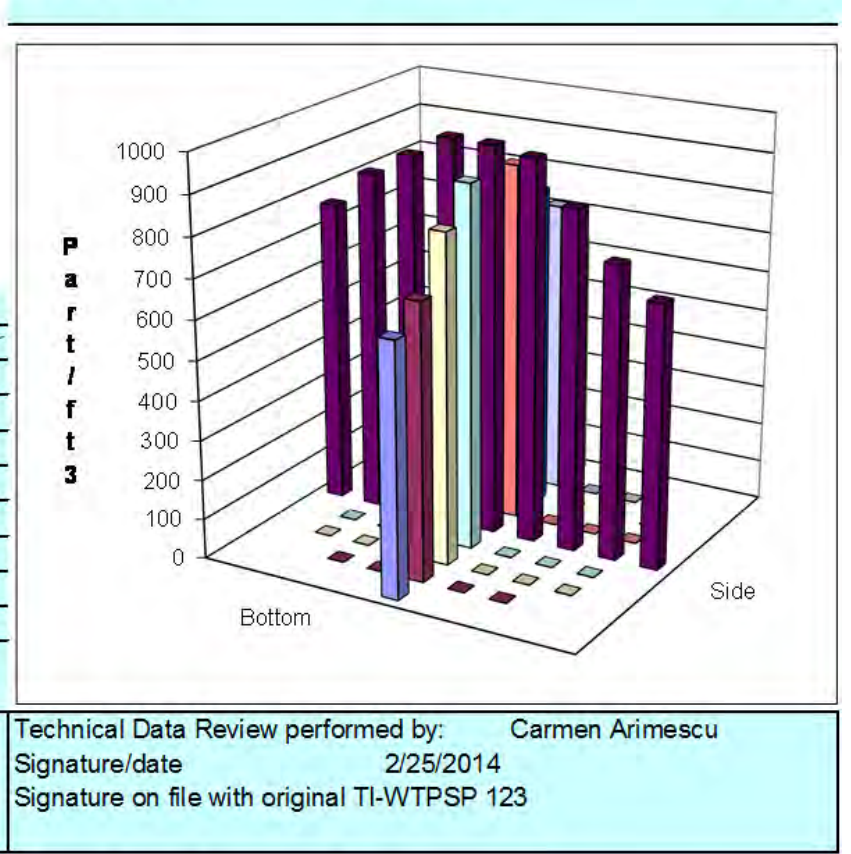


Rev. 0

3 Aug. 2006

Site LV-S3 Scale Model

Date 9/18/2013

Tester SFS,SS

Stack Dia

11.922 in

Stack X-Area

Test Part

111.6 in. 2

Distance to disturbance

Measurement units particles/ti3 510.25 inches

\begin{tabular}{|r|r|}
\multicolumn{1}{|c}{$\begin{array}{r}\text { Order } \longrightarrow \\
\text { Traverse } \rightarrow \\
\text { Trial } \longrightarrow\end{array}$} \\
\hline Point & Depth, in. \\
\hline $\mathbf{1}$ & 0.50 \\
\hline $\mathbf{2}$ & 1.25 \\
\hline $\mathbf{3}$ & 2.31 \\
\hline $\mathbf{4}$ & 3.85 \\
\hline Center & 5.96 \\
\hline $\mathbf{5}$ & 8.07 \\
\hline $\mathbf{6}$ & 9.61 \\
\hline $\mathbf{7}$ & 10.67 \\
\hline $\mathbf{8}$ & 11.42 \\
\hline
\end{tabular}

2nd

\section{TRACER TRAVERSE DATA FORM}

Run No. PT-53

Fan configuration AC MIN

Fan Setting 25

Stack Temp

$64.75 \operatorname{deg} F$

Start/End Time $0730 / 0920$

Center $2 / 3$ from

Points in Center 23

1.09 to:

to: $\quad \mathbf{1 0 . 8 3}$

Injection Point 17

2 to:

o: $\quad \underline{7}$

1st

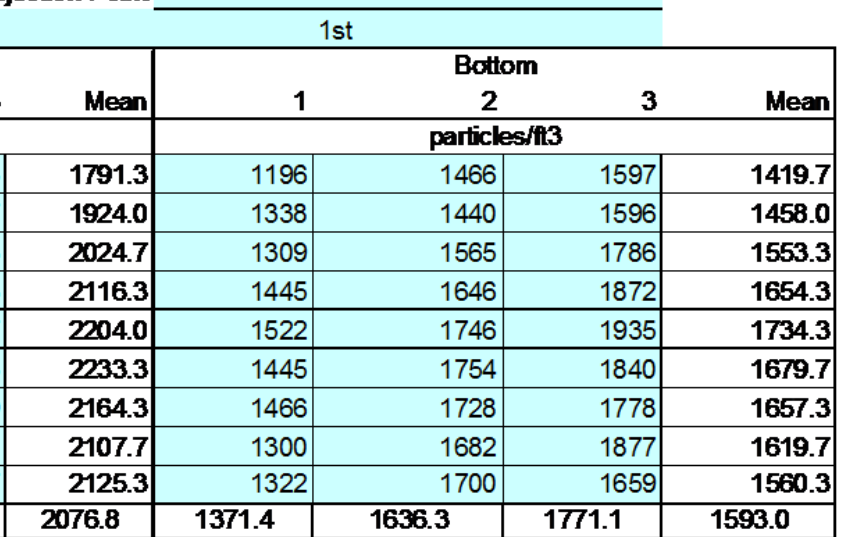

\begin{tabular}{|c|c|c|}
\hline & AH & $\mathrm{pt} / \mathrm{it3}$ \\
\hline & Mean & 1834.9 \\
\hline & Min Point & 1419.7 \\
\hline & Max Point & 2233.3 \\
\hline Avg Conc & 1818 & \\
\hline
\end{tabular}

Generator Inlet Press
Stack Temp
Centerfine vel.
Ambient pressure
Ambient humidity
Ambient temp
Back-Gd aerosol
No. Bk-Gd samples
Compressor output

\begin{tabular}{|c|c|c|}
\hline Stat & Finish & \\
\hline 1.5 & 1.5 & 5 psig \\
\hline 56.6 & 72.9 & $9 \mathrm{~F}$ \\
\hline 1261 & 1241 & 1 afpm \\
\hline 30.92 & 30.95 & $5[\mathrm{nHg}$ \\
\hline $50 \%$ & $35 \%$ & RH \\
\hline 55.4 & 66.2 & $2 \mathrm{~F}$ \\
\hline $3,1,1,3,3$ & $3,4,5,8,6$ & pt/fi3 \\
\hline 5 & 5 & 5 \\
\hline 41 & 47 & 7 |psig \\
\hline
\end{tabular}

Notes:

SS $9 / 18 / 13$

OाUsed: Edwards 19

Ref. Probe Location: Ref port downstream Port 2.

Probe Type /Configuration: L-Shaped probe

\begin{tabular}{|lc|}
\hline $\begin{array}{l}\text { Entries made by: } \\
\text { Signature/date }\end{array}$ & Susan Sande \\
& $9 / 18 / 2013$ \\
& Signature on file with original \\
\hline
\end{tabular}

Side

$-226 \%$

\begin{tabular}{rrrr|}
\hline Bottom & All & Normlzd \\
\hline 16224 & 1866.5 & 2086.17 \\
91.2 & 270.8 & 110.17 \\
\hline 5.6 & 14.5 & 5.28 \\
\hline
\end{tabular}

Std. Dev. $\quad 107.2$

COV as \%

Instuments Used

TSI VelciCalc

5.1

\begin{tabular}{lrr} 
TSI VelciCalc & T95351203001 & $12 / 10 / 2013$ \\
\hline Fisher Scientific & 90936818 & $12 / 11 / 2013$ \\
\hline Met One OPC (Ref) & 96258675 & \multicolumn{1}{l}{ FIO } \\
\hline Met One OPC & 1011529010 & $8 / 7 / 2014$ \\
\hline
\end{tabular}

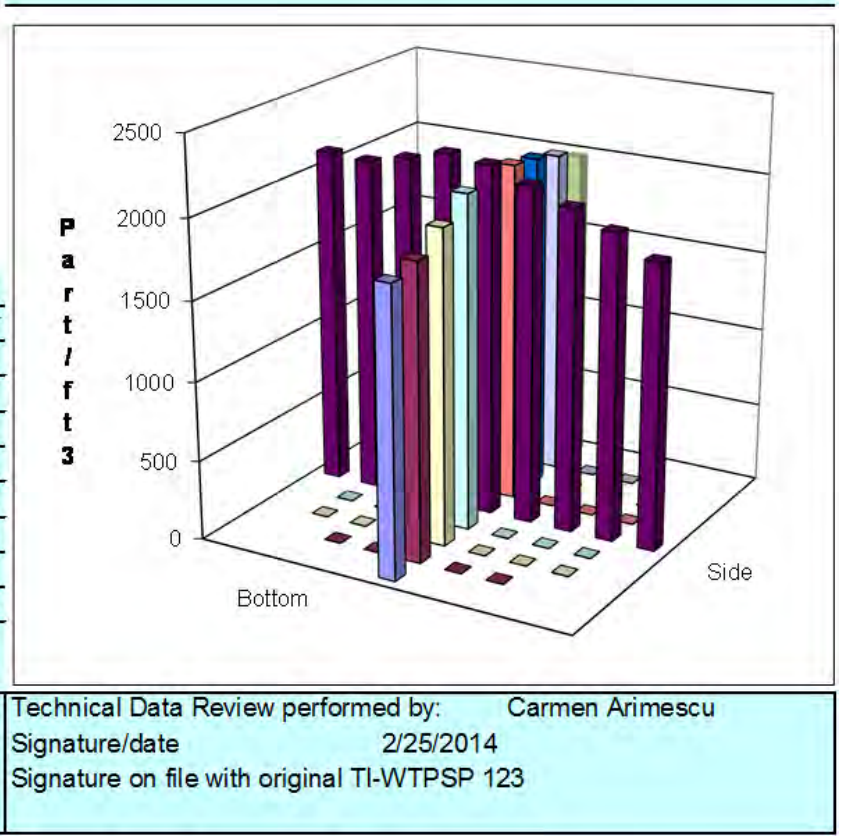


Rev. 0

3 Aug. 2006

Site LV-S3 Scale Model

Date 9/18/2013

Tester SFS,SS

Stack Dia

11.922 in

Stack X-Area

111.6 in. 2

Test Port

1

Distance to distumbence 426.25 inches

Measurement units particles/ti3

Order $\longrightarrow$

Traverse->

Trial $\rightarrow$

Averages

2nd

\begin{tabular}{|c|c|c|}
\hline & $\begin{array}{r}\text { Order } \longrightarrow \\
\text { Traverse-> } \\
\quad \text { Trial } \longrightarrow\end{array}$ & 1 \\
\hline Point & Depth, in. & \\
\hline 1 & 0.50 & 2413 \\
\hline 2 & 1.25 & 2544 \\
\hline 3 & 2.31 & 2598 \\
\hline 4 & 3.85 & 2794 \\
\hline Center & 5.96 & 2949 \\
\hline 5 & 8.07 & 2950 \\
\hline 6 & 9.61 & 2787 \\
\hline 7 & 10.67 & 2721 \\
\hline 8 & 11.42 & 2615 \\
\hline \multicolumn{2}{|c|}{ Averages $\longrightarrow \longrightarrow$} & 2707.9 \\
\hline & $\boldsymbol{A H}$ & pt/fit3 \\
\hline & Mean & 2738.4 \\
\hline & Min Pcint & 2493.7 \\
\hline & Max Point & 2967.3 \\
\hline Avg Conc & 2724 & $\mathrm{t} / \mathrm{ft3}$ \\
\hline
\end{tabular}

PARTICLE TRACER TRAVERSE DATA FORM

Run No. PT-54

Fan configuration AC MIN

Fan Setting 25

Stack Temp

$77.7 \operatorname{deg} \mathrm{F}$

Start/End Time $0930 / 1110$

Center $2 / 3$ from

Points in Center $2 / 3$

Injection Point 17

\begin{tabular}{|c|c|c|}
\hline 1.09 & to: & 10.83 \\
\hline 2 & to: & 7 \\
\hline
\end{tabular}

\begin{tabular}{|r|r|r|r|r|}
\cline { 2 - 4 } Mean & \multicolumn{5}{|c|}{ 1st } \\
\hline & $\mathbf{5}$ & \multicolumn{3}{c|}{ Porticlom } \\
\hline $\mathbf{2 4 9 3 . 7}$ & 2362 & 2527 & 2672 & $\mathbf{2 5 2 0 . 3}$ \\
\hline $\mathbf{2 6 2 7 . 0}$ & 2571 & 2754 & 2752 & $\mathbf{2 6 9 2 . 3}$ \\
\hline $\mathbf{2 6 6 5 . 7}$ & 2845 & 2868 & 2851 & $\mathbf{2 8 5 4 . 7}$ \\
\hline $\mathbf{2 8 4 6 . 3}$ & 2919 & 2914 & 2925 & $\mathbf{2 9 1 9 . 3}$ \\
\hline $\mathbf{2 7 5 3 . 0}$ & 2993 & 2946 & 2934 & $\mathbf{2 9 5 7 . 7}$ \\
\hline $\mathbf{2 7 6 6 . 3}$ & 2949 & 2947 & 3006 & $\mathbf{2 9 6 7 . 3}$ \\
\hline $\mathbf{2 8 2 8 . 3}$ & 2870 & 2926 & 2882 & $\mathbf{2 8 9 2 . 7}$ \\
\hline $\mathbf{2 7 2 2 3}$ & 2636 & 2765 & 2600 & $\mathbf{2 6 6 7 . 0}$ \\
\hline $\mathbf{2 6 0 2 7}$ & 2457 & 2517 & 2572 & $\mathbf{2 5 1 5 . 3}$ \\
\hline $\mathbf{2 7 0 0 . 6}$ & $\mathbf{2 7 3 3 . 6}$ & $\mathbf{2 7 9 6 . 0}$ & $\mathbf{2 7 9 9 . 3}$ & $\mathbf{2 7 7 6 . 3}$ \\
\hline
\end{tabular}

\section{Generator Inlet Press \\ Stack Temp \\ Centerline vel. \\ Ambient pressure \\ Ambient humidity \\ Ambient temp \\ Back-Gd aerosol \\ No. Bk-Gd samples \\ Compressor output}

\begin{tabular}{|c|c|c|}
\hline Stat & Finish & \\
\hline 1.5 & 1.5 & 5 psig \\
\hline 72.9 & 82.5 & $5 \mid \mathrm{F}$ \\
\hline 1261 & 1328 & $8 \mid \mathbf{a f p m}$ \\
\hline 30.95 & 31.01 & $1 \mathrm{hHg}$ \\
\hline $35 \%$ & $24 \%$ & RH \\
\hline 66.2 & 75.2 & $2 \mathrm{~F}$ \\
\hline $4,4,3,0,2$ & $0,3,3,1,3$ & $\mathrm{pt} / \mathrm{f} \mathbf{3}$ \\
\hline 5 & 5 & 5 \\
\hline 47 & 44 & 4 psig \\
\hline
\end{tabular}

\section{Dev. from mean Center 2/3 \\ Mean \\ $-8.9 \%$ Std. Dev. \\ COV as \%}

Side
2744.1
80.1

Instuments Used

TSI VelciCalc

2.9

\begin{tabular}{l} 
Fisher Scientific \\
\hline Met One OPC (Ref) \\
\hline Met One OPC
\end{tabular}

\begin{tabular}{rrr|} 
Bottom & All & Normlzd \\
\hline 2850.1 & 2797.1 & 2899.15 \\
1227 & 113.7 & 113.80 \\
4.3 & 4.1 & 3.93 \\
\hline
\end{tabular}

Notes:

SS $9 / 18 / 13$

OाUsed: Edwards 19

Ref. Probe Location: Ref port downstream Port 2.

Probe Type/Configuration: L-Shaped probe

\begin{tabular}{|lc|}
\hline $\begin{array}{l}\text { Entries made by: } \\
\text { Signature/date }\end{array}$ & Susan Sande \\
& $9 / 18 / 2013$ \\
& Signature on file with original \\
\end{tabular}

Technical Data Review performed by:


Rev. 0

3 Aug. 2006

Site LV-S3 Scale Model

Date 9/18/2013

Tester SFS,SS

Stack Dia

11.922 in

Stack X-Area

Test Part

111.6 in.

Distance to disturbance

Measurement units particles/ti?

426.25 inches

\begin{tabular}{|r|r|}
\multicolumn{1}{|c}{$\begin{array}{r}\text { Order } \\
\text { Traverse-> } \\
\text { Trial } \longrightarrow\end{array}$} \\
\hline Point & Depth, $\mathbf{i n}$ \\
\hline $\mathbf{1}$ & 0.50 \\
\hline $\mathbf{2}$ & 1.25 \\
\hline $\mathbf{3}$ & 2.31 \\
\hline $\mathbf{4}$ & 3.85 \\
\hline Center & 5.96 \\
\hline $\mathbf{5}$ & 8.07 \\
\hline $\mathbf{6}$ & 9.61 \\
\hline $\mathbf{7}$ & 10.67 \\
\hline $\mathbf{8}$ & 11.42 \\
\hline &
\end{tabular}

1 st

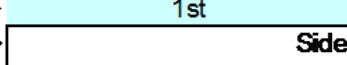

$\rightarrow-1$ partic

Side

\begin{tabular}{r|r|}
\hline 978 & 967 \\
\hline 974 & 1116 \\
\hline 1138
\end{tabular}

138

$1241 \quad 1220$

1220

\begin{tabular}{r|r|}
1119 & 962 \\
\hline 1068 & 1017 \\
\hline 948 & 948
\end{tabular}

948

\begin{tabular}{l|r}
\hline Averages $\longrightarrow \longrightarrow$ & 867 \\
&
\end{tabular}

867

\section{CER TRAVERSE DATA FORM}

Run No. PT-55

Fan configuration AC MAX

Fan Setting 60

Stack Temp

$8285 \operatorname{deg} \mathrm{F}$

Start/End Time $1120 / 1312$

Center $2 / 3$ from

Points in Center 273

Injection Point 17

1.09

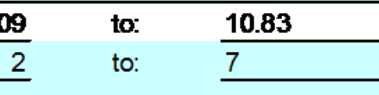

2

3

\begin{tabular}{|r|r|r|}
\hline 908 & 852 & $\mathbf{8 6 6 . 7}$ \\
\hline 967 & 895 & $\mathbf{9 4 6 . 7}$ \\
\hline 1116 & 1091 & $\mathbf{1 0 6 0 . 3}$ \\
\hline 1157 & 1176 & $\mathbf{1 1 5 7 . 0}$
\end{tabular}

1176

1183

\begin{tabular}{|r|r|}
\hline 1157.0 \\
\hline 83 & 12147 \\
\hline
\end{tabular}

\begin{tabular}{r|r|r|}
948 & 948 & 1061 \\
\hline 67 & 833 & 862 \\
\hline
\end{tabular}

\begin{tabular}{|lr}
\hline All & $\mathrm{pt} / \mathrm{tt} 3$ \\
Mean & 1003.0 \\
Min Point & 729.7 \\
Max Point & 1214.7 \\
\hline
\end{tabular}

Avg Conc $978 \mathrm{pt} / \mathrm{ft} 3$

Generator Inlet Press

Stack Temp

Centerline vel.

Ambient pressure

Ambient humidity

Ambient temp

Back-Gd aerosol

No. Bk-Gd samples

Compressar output

\begin{tabular}{|c|c|c|}
\hline Stat & Finish & \\
\hline 2.9 & 2.9 & psig \\
\hline 80.6 & 85.1 & $F$ \\
\hline 3684 & 3353 & afpm \\
\hline 31.01 & 30.98 & $\mathrm{inHg}$ \\
\hline $24 \%$ & $19 \%$ & RH \\
\hline 78.8 & 88.7 & $F$ \\
\hline $0,1,0,1,2$ & $0,0,0,0,0$ & $\mathrm{pt} / \mathrm{n} 3$ \\
\hline 5 & 5 & \\
\hline 38 & 42 & psig \\
\hline
\end{tabular}

Notes:

Notes:

\section{SS $9 / 18 / 13$}

Oil Used: Edwards 19

Ref. Probe Location: Ref port downstream Port 2.

Probe Type/Configuration

L-Shaped probe

Entries made by:

Signature/date

Susan Sande
$9 / 18 / 2013$
Signature on file with original

Dev. from mean|

Center 233

Std. Dev.

COV as \%

Instuments Usect

TSI VelciCalc

\begin{tabular}{lrr}
\hline Fisher Scientific & & $12 / 10 / 2013$ \\
\hline Met One OPC (Ref) & 96258675 & \multicolumn{2}{c}{$12 / 11 / 2013$} \\
\hline Met One OPC & 1011529010 & FIO \\
\hline
\end{tabular}
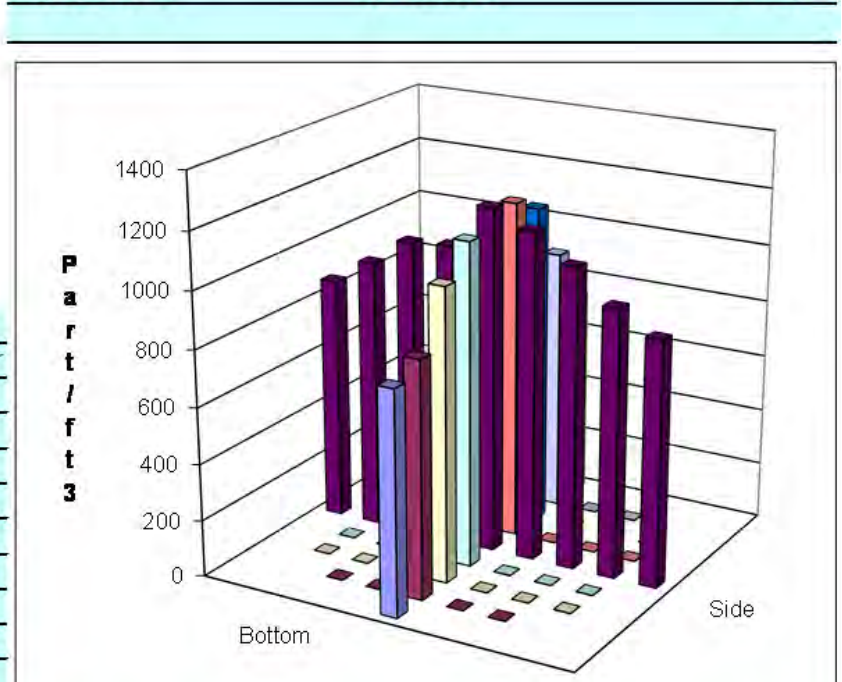

Technical Data Review performed by: Carmen Arimescu

Signature/date

2/25/2014

Signature on file with original TI-WTPSP 123 
Rev. 0

3 Aug. 2006

PARTICLE TRACER TRAVERSE DATA FORM

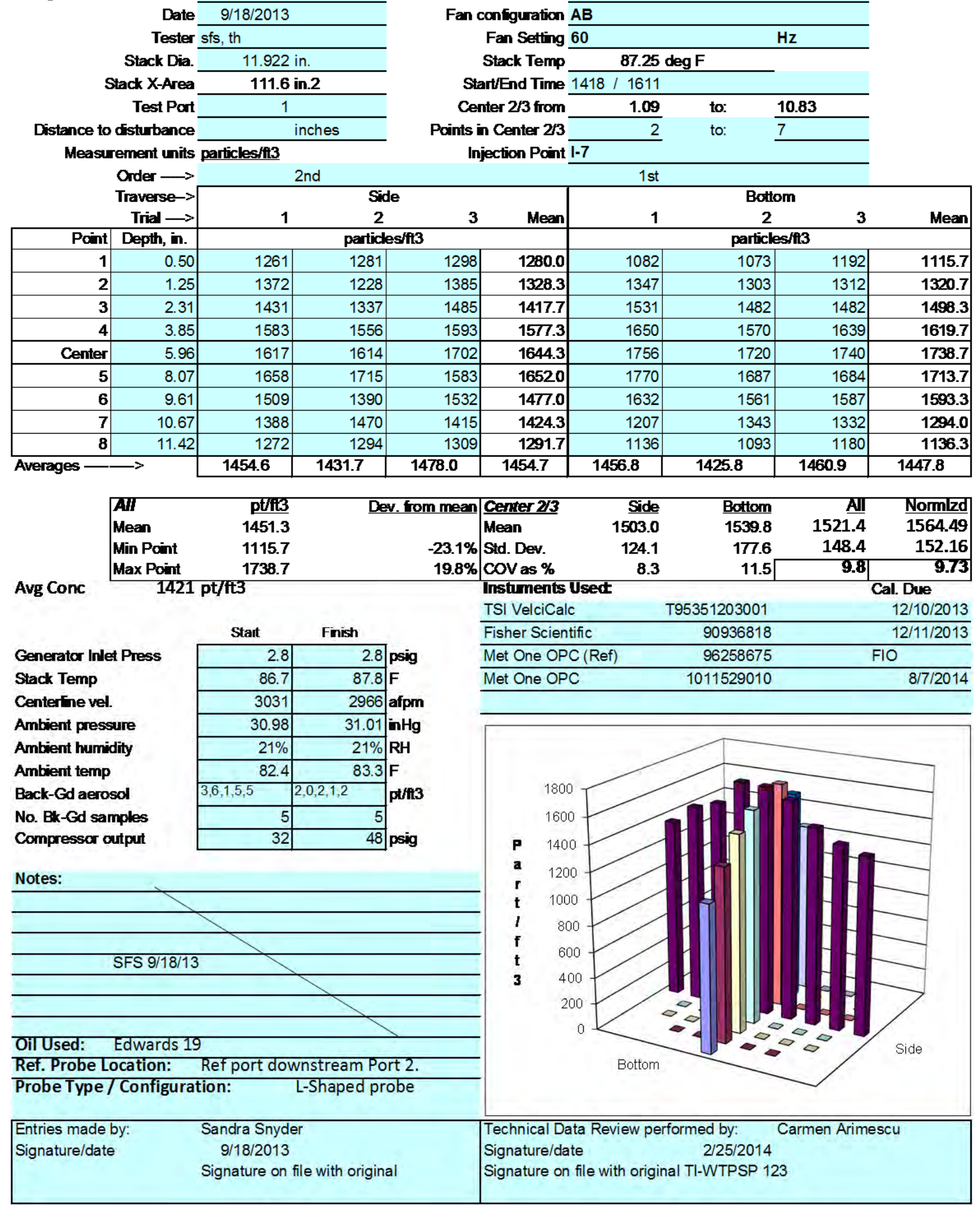


Rev. 0

3 Aug. 2006

Site LV-S3 Scale Model

Date 9/19/2013

Tester sfs, ss

Stack Dia

11.922 in

Stack X-Area

111.6 in. 2

Test Port

1

Distance to distumbence 426.25 inches

Measurement units particles/ti 3

Order $\longrightarrow$

\begin{tabular}{|r|r|}
\multicolumn{1}{c|}{ Order $\longrightarrow$} \\
\hline Point & Depth, in. \\
\hline $\mathbf{1}$ & 0.50 \\
\hline $\mathbf{2}$ & 1.25 \\
\hline $\mathbf{3}$ & 2.31 \\
\hline $\mathbf{4}$ & 3.85 \\
\hline Center & 5.96 \\
\hline $\mathbf{5}$ & 8.07 \\
\hline $\mathbf{6}$ & 9.61 \\
\hline $\mathbf{7}$ & 10.67 \\
\hline $\mathbf{8}$ & 11.42 \\
\hline &
\end{tabular}

2nd

PARTICLE TRACER TRAVERSE DATA FORM

Run No. PT-57

Fan configuration $\mathrm{AB}$

Fan Setting 25

Stack Temp

Start/End Time $0730 / 0940$

Center $2 / 3$ from

Points in Center $2 / 3$

Injection Point $1-7$
$61.65 \operatorname{deg} F$

\author{
40
}

1.09 to

to: $\quad \frac{10.83}{7}$

1 st

\section{Averages 19

\begin{tabular}{|lr}
\hline All & $\mathrm{pt} / \mathrm{tt} 3$ \\
Mean & 1945.9 \\
Min Point & 1720.0 \\
Max Point & 2131.0 \\
\hline
\end{tabular}

Avg Conc

$1931 \mathrm{pt} / \mathrm{ft} 3$

Generator Inlet Press

Stack Temp

Centerfine vel.

Ambient pressure

Ambient humidity

Ambient temp

Back-Gd aerosol

No. Bk-Gd samples

Compressor output

\begin{tabular}{|c|c|c|}
\hline Stat & Finish & \\
\hline 1.5 & 1.5 & 5 psig \\
\hline 50.5 & 72.8 & $8 \mathrm{~F}$ \\
\hline 1169 & 1163 & 3 afpm \\
\hline 31.07 & 31.07 & $7 \mathrm{hH}$ \\
\hline $72 \%$ & $47 \%$ & RH \\
\hline 47.3 & 60.8 & $8 \mid F$ \\
\hline $1,2,1,2,3$ & $1,9,2,5,0$ & $\mathrm{p} / \mathrm{t} \mathbf{t 3}$ \\
\hline 5 & 5 & 5 \\
\hline 40 & 44 & 4 psig \\
\hline
\end{tabular}

Notes: Reran BTM 1\& 2 due to low particle count.

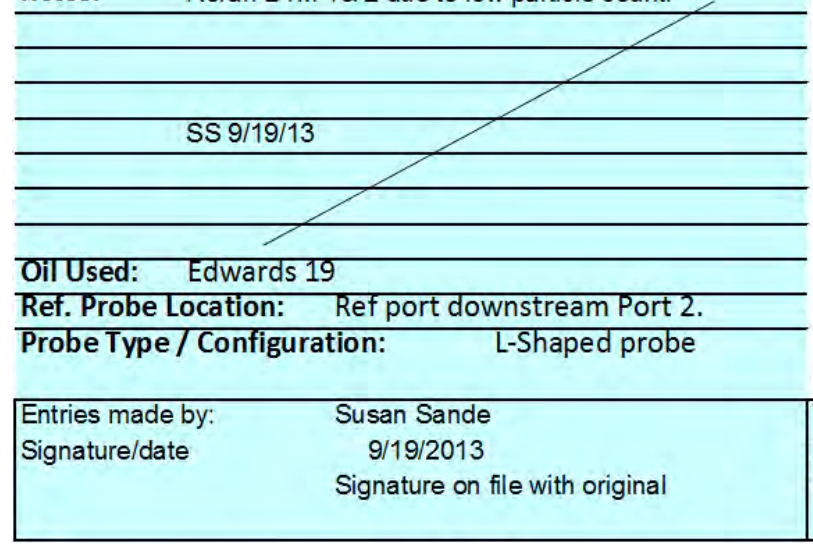

\begin{tabular}{r|lrrrr|} 
Dev. from mean & Center 2/3 & Side & Bottom & $\underline{\text { All }}$ & Normlzd \\
\cline { 2 - 6 } & Mean & 2038.4 & 1917.5 & 1977.9 & 2042.94 \\
$9.5 \%$ & Std. Dev. & 60.6 & 90.2 & 96.9 & 77.43 \\
COV as \% & 3.0 & 4.7 & 4.9 & 3.79 \\
\hline
\end{tabular}

Instuments Used

TSI VelciCalc

\begin{tabular}{lrr} 
TSI VelciCalc & T95351203001 & $12 / 10 / 2013$ \\
\hline Fisher Scientific & 90936818 & \multicolumn{2}{c}{$12 / 11 / 2013$} \\
\hline Met One OPC (Ref) & 96258675 & \multicolumn{2}{c}{ FIO } \\
\hline Met One OPC & 1011529010 & $8 / 7 / 2014$ \\
\hline
\end{tabular}

Signature on file with original TI-WTPSP 123


Rev. 0

3 Aug. 2006

Site LV-S3 Scale Model

Date 9/19/2013

Tester sfs, ss

Stack Dia

11.922 in

Stack X-Area

111.6 in.

Test Port

2

Distance to disturbance 510.25 inches

Order $\longrightarrow$

\begin{tabular}{|r|r|}
\multicolumn{2}{c}{ Order $\longrightarrow$} \\
\hline Point & Depth, $\mathbf{i n}$ \\
\hline $\mathbf{1}$ & 0.50 \\
\hline $\mathbf{2}$ & 1.25 \\
\hline $\mathbf{3}$ & 2.31 \\
\hline $\mathbf{4}$ & 3.85 \\
\hline Center & 5.96 \\
\hline $\mathbf{5}$ & 8.07 \\
\hline $\mathbf{6}$ & 9.61 \\
\hline $\mathbf{7}$ & 10.67 \\
\hline $\mathbf{8}$ & 11.42 \\
\hline &
\end{tabular}

2nd
Run No. PT-58

Fan configuration $\mathrm{AB}$

Fan Setting 25

Stack Temp

Start/End Time 0944 / 1130

Center $2 / 3$ from

Points in Center $2 / 3$

Injection Point I-7
Measurement units particles/ti3

to

2 to: 7

\begin{tabular}{|c|c|c|c|c|}
\hline \multicolumn{4}{|c|}{ 1st } & \multirow[b]{3}{*}{ Mean } \\
\hline \multirow[b]{2}{*}{ Meen } & \multicolumn{3}{|c|}{ Bottom } & \\
\hline & \multirow[t]{2}{*}{1} & 2 & \multirow{2}{*}{3} & \\
\hline & & particles/ft3 & & \\
\hline 1531.7 & 1480 & 1714 & 1688 & 1627.3 \\
\hline 1493.3 & 1677 & 1740 & 1745 & 1720.7 \\
\hline 1574.7 & 1743 & 1822 & 1747 & 1770.7 \\
\hline 1611.0 & 1854 & 1843 & 1818 & 1838.3 \\
\hline 1706.0 & 1911 & 1916 & 1881 & 1902.7 \\
\hline 1649.7 & 1865 & 1879 & 1840 & 1861.3 \\
\hline 1635.0 & 1820 & 1759 & 1784 & 1787.7 \\
\hline 1721.3 & 1780 & 1758 & 1758 & 1765.3 \\
\hline 1563.7 & 1665 & 1622 & 1772 & 1686.3 \\
\hline 1609.6 & 1755.0 & 1783.7 & 1781.4 & 1773.4 \\
\hline
\end{tabular}

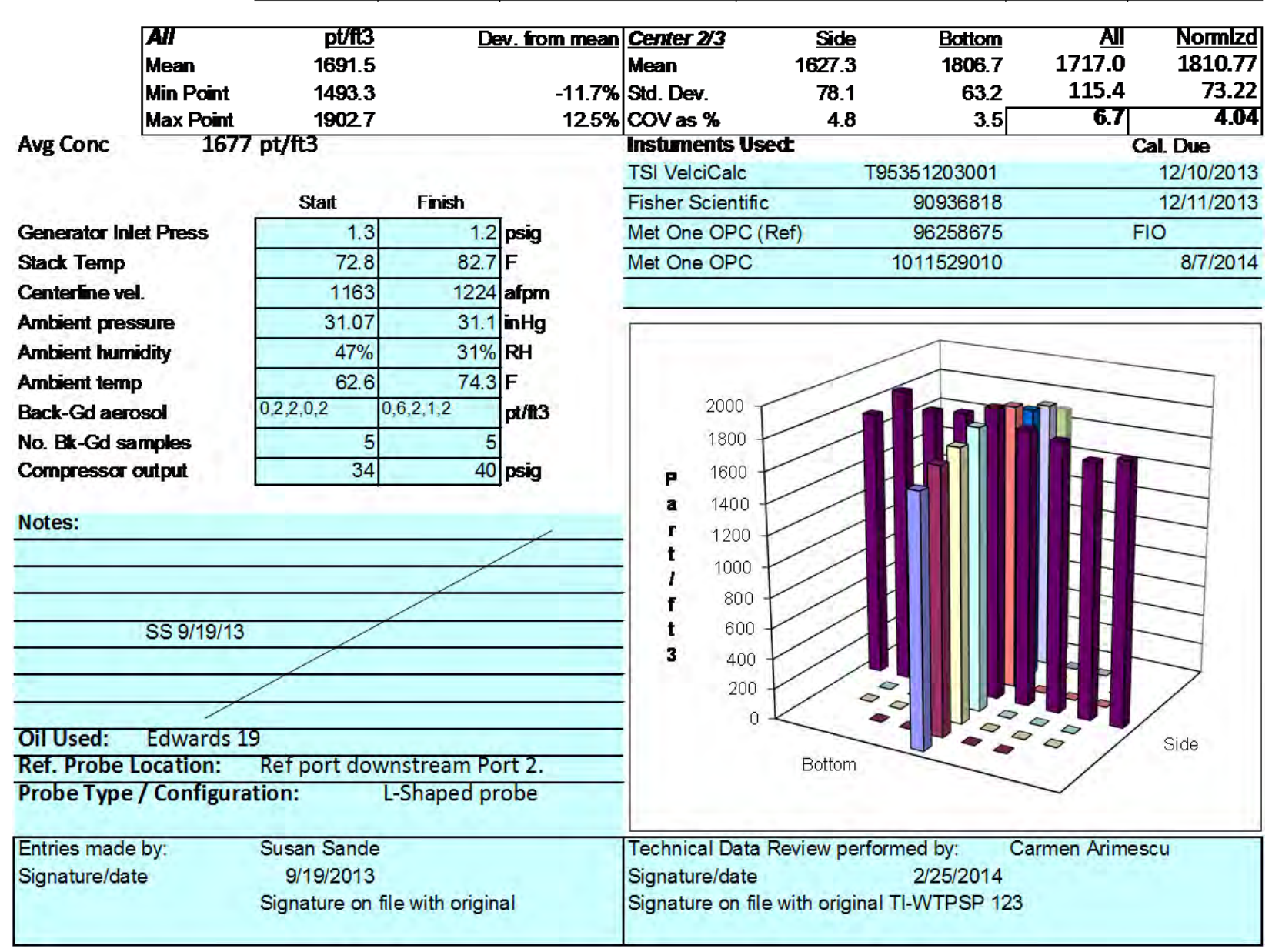


Rev. 0

3 Aug. 2006

\begin{tabular}{|c|c|c|}
\hline & Date & 9/19/2013 \\
\hline \multicolumn{3}{|c|}{ Tester $\mathrm{cb}, \mathrm{TH}$} \\
\hline \multirow{2}{*}{\multicolumn{2}{|c|}{$\begin{array}{r}\text { Stack Dia. } \\
\text { Stack X-Area }\end{array}$}} & 11.922 ir \\
\hline & & $111.6 \mathrm{ir}$ \\
\hline \multicolumn{2}{|r|}{ Test Part } & 2 \\
\hline \multicolumn{2}{|c|}{ Distance to distumbance } & $510.25 \mathrm{ir}$ \\
\hline \multicolumn{3}{|c|}{ Measurement units particles/fi3 } \\
\hline \multicolumn{2}{|r|}{ Order $\longrightarrow$} & 1st \\
\hline \multicolumn{2}{|r|}{ Trial $\longrightarrow$} & 1 \\
\hline Point & Depth, in. & \\
\hline 1 & 0.50 & 533 \\
\hline 2 & 1.25 & 761 \\
\hline 3 & 2.31 & 913 \\
\hline 4 & 3.85 & 1094 \\
\hline Center & 5.96 & 1077 \\
\hline 5 & 8.07 & 1065 \\
\hline 6 & 9.61 & 1010 \\
\hline 7 & 10.67 & 963 \\
\hline 8 & 11.42 & 948 \\
\hline \multicolumn{2}{|c|}{ Averages $\longrightarrow \longrightarrow$} & 929.3 \\
\hline \multicolumn{2}{|r|}{$\boldsymbol{A H}$} & pt/ft3 \\
\hline \multicolumn{2}{|r|}{ Mean } & 1074.2 \\
\hline \multicolumn{2}{|r|}{ Min Point } & 735.0 \\
\hline \multicolumn{2}{|r|}{ Max Point } & 1407.3 \\
\hline \multicolumn{3}{|c|}{ Avg Conc $\quad 1051 \mathrm{pt} / \mathrm{ft3}$} \\
\hline
\end{tabular}

Generator Inlet Press

Stack Temp

Centerfine vel.

Ambient pressure

Ambient humidity

Ambient temp

Back-Gd aerosol

No. Bk-Gd samples

Compresscr output

\begin{tabular}{|c|c|c|}
\hline Stat & Finish & \\
\hline 2.8 & 2.8 & psig \\
\hline 82.1 & 89.4 & $F$ \\
\hline 3028 & 3009 & afpm \\
\hline 31.1 & 31.1 & $=\mathrm{nHg}$ \\
\hline $27 \%$ & $24 \%$ & RH \\
\hline 75.2 & 79.7 & $\mathrm{~F}$ \\
\hline 3.0 .1 .1 .1 & 2.1.0,1,2 & pt/f3 \\
\hline 5 & 5 & \\
\hline 24 & 36 & psig \\
\hline
\end{tabular}

Notes:

CB 09/19/2013

Oil Used: Edwards 19

Ref. Probe Location: Ref port downstream Port 2.

Probe Type/Configuration: L-Shaped probe

\begin{tabular}{|lc|}
\hline $\begin{array}{l}\text { Entries made by: } \\
\text { Signature/date }\end{array}$ & Carolyn Burns \\
& $9 / 19 / 2013$ \\
& Signature on file with original \\
\end{tabular}

Run No. PT-59

Fan configuration $\mathrm{AB}$

Fan Setting 60

Stack Temp

$85.75 \operatorname{deg} F$

Start/End Time 11:55/ 13:45

Center $2 / 3$ from

Points in Center $2 / 3$

Injection Point I-7

1.09

to:

to: $\quad \mathbf{1 0 . 8 3}$

2nd

3

3 Meen 1

1 Bottom

2

particles/fi3

\begin{tabular}{|r|r|r|}
941 & 873 & $\mathbf{9 0 4 . 0}$ \\
\hline 1069 & & 1077
\end{tabular}

\begin{tabular}{|l|r|r|}
1069 & 1077 & $\mathbf{1 0 5 8 . 0}$ \\
\hline
\end{tabular}

\begin{tabular}{|l|l|l|}
1193 & 1183 & $\mathbf{1 1 6 0 . 0}$ \\
\hline
\end{tabular}

\begin{tabular}{l|l|l|}
1325 & 1302 & 1296.3 \\
\hline
\end{tabular}

\begin{tabular}{|l|l|l|}
1413 & 1477 & $\mathbf{1 4 0 3 . 7}$ \\
\hline 1455 & 1402 & 1407.3 \\
\hline
\end{tabular}

\begin{tabular}{|l|l|l|}
1455 & 1402 & $\mathbf{1 4 0 7 . 3}$ \\
\hline
\end{tabular}

\begin{tabular}{|l|l|l|}
1227 & 1240 & $\mathbf{1 2 4 1 . 0}$ \\
\hline
\end{tabular}

\begin{tabular}{|l|r|r|}
1185 & 1126 & $\mathbf{1 1 3 2 . 7}$ \\
\hline
\end{tabular}

8

69.4

Dev from

$-31.6 \%$
$31.0 \%$

147.8

1009

center 23

Side
1013.6
97.3
9.6
$\quad 9$

\begin{tabular}{|r|r|r|}
\hline 1023 & 994 & 1008.7 \\
\hline
\end{tabular}

\begin{tabular}{rrr|}
\hline Bottom & All & Normlzd \\
\hline 12427 & 1128.2 & 1261.10 \\
134.8 & 163.9 & 125.31 \\
\hline 10.8 & 14.5 & 9.94 \\
\hline
\end{tabular}

\section{Instuments Usect}

\begin{tabular}{lrr} 
TSI VelciCalc & T95351203001 & \multicolumn{1}{c}{$12 / 10 / 2013$} \\
\hline Fisher Scientific & 90936818 & $12 / 11 / 2013$ \\
\hline Met One OPC (Ref) & 96258675 & \multicolumn{2}{c}{ FIO } \\
\hline Met One OPC & 1011529010 & \multicolumn{1}{c}{$8 / 7 / 2014$} \\
\hline
\end{tabular}

Sechnical Data Review performed by:


PNNL-23386

WTP-RPT-231, Rev. 0

\section{Distribution*}

No. of

Copies

4 Bechtel National, Inc.

FW Damerow

PA Douglass

MM Mwembeshi

WTP Docs
No. of

Copies
$7 \quad$ Pacific Northwest National Laboratory

H4-02

$\mathrm{H} 4-02$

$\mathrm{H} 4-02$

$\mathrm{H} 4-02$
EJ Antonio

JE Flaherty

JA Glissmeyer

RA Peterson

LM Peurrung

Information Release

Project File (1)
K3-54

K9-30

K3-54

P7 -22

K3-52

K3-52

K3-52

*All distribution will be made electronically.

Distr. 1 


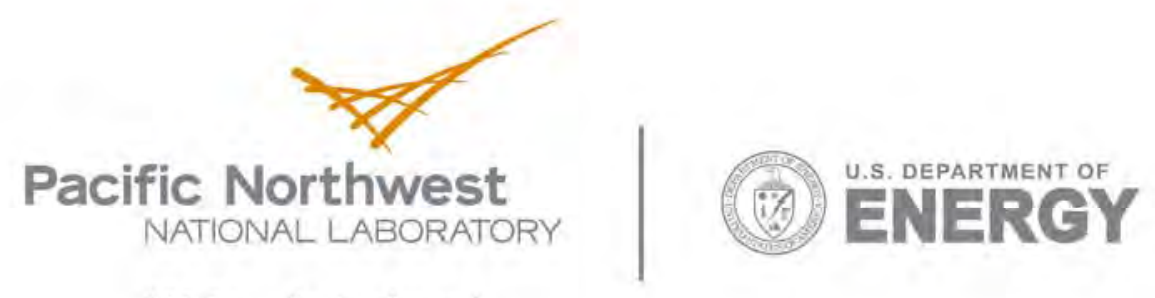

902 Battelle Boulevard

P.O. Box 999

Richland, WA 99352

1-888-375-PNNL (7665)

www.pnl.gov 